\title{
Structural and functional studies on GTPases involved in eukaryal translation initiation
}

\author{
Dissertation \\ zur Erlangung des mathemtisch-natruwissenschaftlichen Doktorgrades \\ "Doctor rerum naturalis" \\ der Georg-August-Universität Göttingen \\ im Promotionsprogramm "Grundprogramm Biologie" \\ der Georg-August University School of Science (GAUSS) \\ vorgelegt von \\ Bernhard Kuhle \\ aus Göttingen \\ Göttingen, 2014
}




\section{$\underline{\text { Betreuungsausschuss }}$}

Prof. Dr. Ralf Ficner, Abteilung für Molekulare Strukturbiologie, Institut für Mikrobiologie und Genetik, Georg August Universität Göttingen

Prof. Dr. Marina Rodnina, Abteilung für Physikalische Biochemie, Max Planck Institut für Biophysikalische Chemie, Göttingen

\section{Mitglieder der Prüfungskommission}

Referent:

Prof. Dr. Ralf Ficner, Abteilung für Molekulare Strukturbiologie, Institut für Mikrobiologie und Genetik, Georg August Universität Göttingen

Korreferent:

Prof. Dr. Marina Rodnina, Abteilung für Physikalische Biochemie, Max Planck Institut für Biophysikalische Chemie, Göttingen

Weitere Mitglieder der Prüfungskommission

Prof. Dr. Reinhard Lührmann, Abteilung für Zelluläre Biochemie, Max Planck Institut für Biophysikalische Chemie, Göttingen

Prof. Dr. Heinz Neumann, Angewandte Synthetische Biologie, Institut für Mikrobiologie und Genetik, Georg August Universität Göttingen

Prof. Dr. Holger Stark, Abteilung für Dreidimensionale Kryo-Elektronenmikroskopie, Max Planck Institut für Biophysikalische Chemie, Göttingen

Prof. Dr. Jörg Stülke, Abteilung für Allgemeine Mikrobiologie, Institut für Mikrobiologie und Genetik, Georg August Universität Göttingen

Tag der mündlichen Prüfung: 16. Oktober 2014 


\section{Preface}

The following thesis summarizes my work on translational GTPases and the mechanism of eukaryal translation initiation, which was performed at the University of Göttingen under the supervision of Prof. Dr. Ralf Ficner. The work led to the following publications:

"elF5B employs a novel domain release mechanism to catalyze ribosomal subunit joining", Kuhle, B. and Ficner, R., 2014, The EMBO Journal, 33: 1177-1191.

"A monovalent cation acts as structural and catalytic cofactor in translational GTPases", Kuhle, B. and Ficner, R., 2014, The EMBO Journal, in press.

"Structural insight into the recognition of amino-acylated initiator tRNA by elF5B in the 80S initiation complex", Kuhle, B. and Ficner, R., 2014, BMC Structural Biology, accepted.

"Translation initiation factor elF3b contains a nine-bladed beta-propeller and interacts with the 40 S ribosomal subunit", Liu, Y., Neumann, P., Kuhle, B., Monecke, T., Schell, S., Chari, A., \& Ficner, R., 2014, Structure, 22: 1-8.

"Analysis of the interaction network between elF2 and elF5 and its implications for the process of eukaryal translation initiation", Kuhle, B., Valerius, O. and Ficner, R., 2014, manuscript in preparation.

"Analysis of the interaction network between elF2 and elF2B $\varepsilon$ and its implications for the mechanism of nucleotide exchange", Kuhle, B., Valerius, O. and Ficner, R., 2014, manuscript in preparation.

"The crystal structures of elF2B $\beta$ and elF2B $\delta$ provide new insights into the regulatory subcomplex of elF2B, the guanine nucleotide exchange factor for elF2", Kuhle, B., and Ficner, R., 2014, manuscript in preparation.

With the exception of Liu et al. (2014), these publications are included in this present thesis, although not in chronological but in topical order. The contributions of the individual authors are given at the beginning of the respective chapters. 


\section{Contents}

Summary

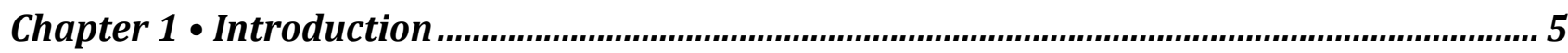

1.1 The ribosome and the universal mechanism of translation .............................................. 6

$1.2 \quad$ Translation initiation ....................................................................................................... 10

1.2.1 Cap-dependent translation initiation in eukarya ……………………………………………..... 10

$1.3 \quad$ GTPases involved in translation ................................................................................... 12

1.3.1 The eukaryal translation initiation factor 2 (eIF2) ……………………………………... 16

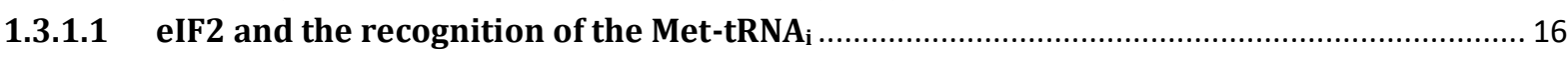

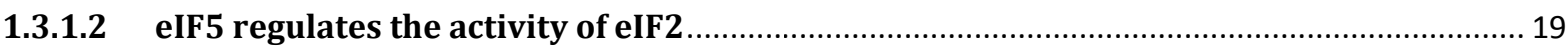

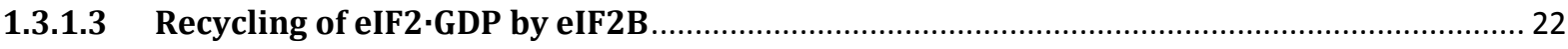

1.3.2 The eukaryal translation initiation factor 5B (eIF5B) …………………………………….... 23

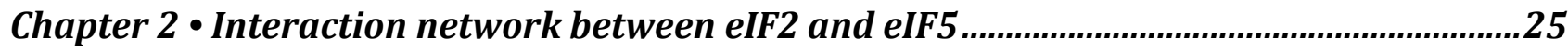

2.1 Introduction ................................................................................................................... 26

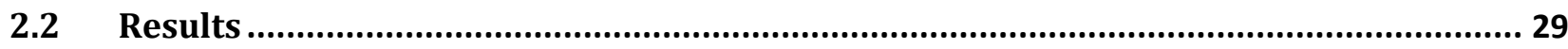

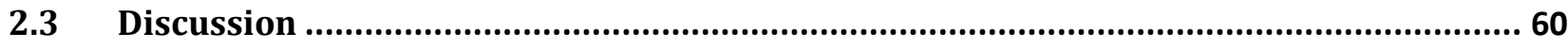

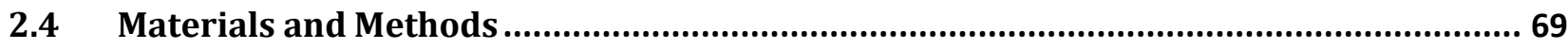

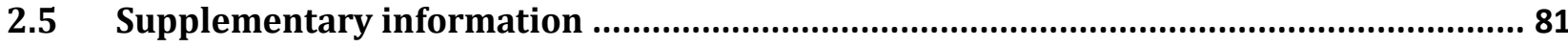

Chapter 3 • Interaction network between eIF2 and eIF2B

3.1 Introduction ..................................................................................................................... 88

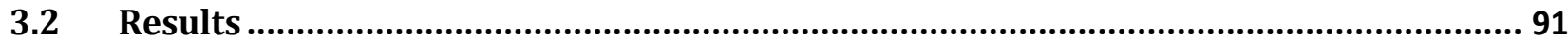

3.3 Discussion ...........................................................................................................103

3.4 Materials and Methods ...................................................................................................112

3.5 Supplementary information ...................................................................................116

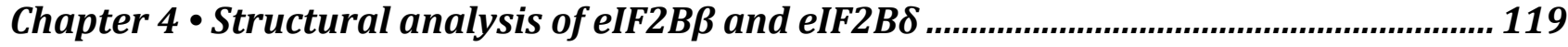

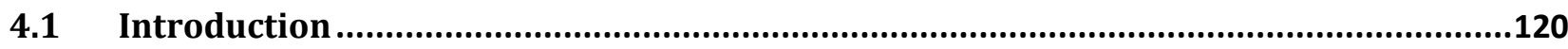

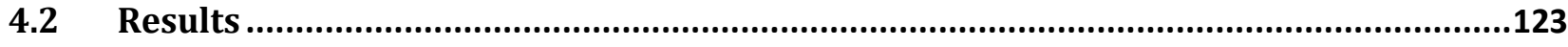

4.3 Discussion ...........................................................................................................136

4.4 Materials and Methods .........................................................................................................141

Chapter 5 • The domain release mechanism of eIF5B ...................................................... 145 


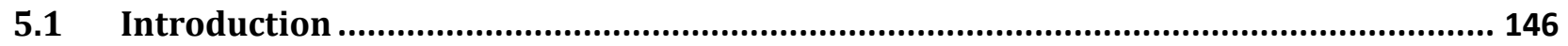

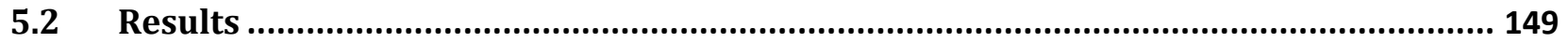

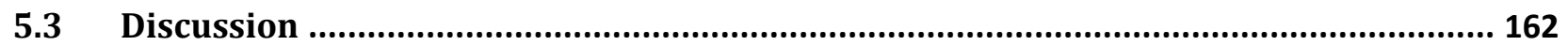

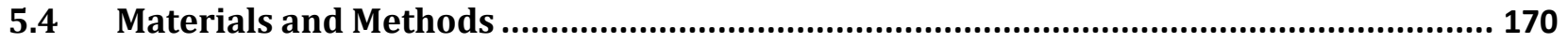

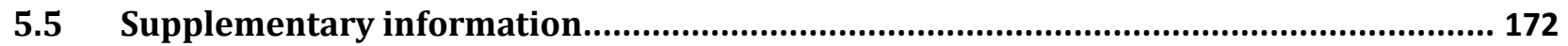

Chapter 6 • Recognition of amino-acylated initiator tRNA by eIF5B in the 80S IC..............185

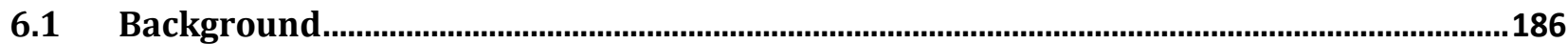

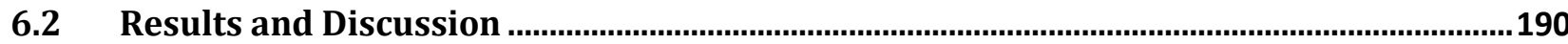

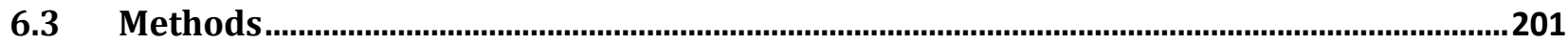

Chapter 7 • A monovalent cation as cofactor in translational GTPases .............................203

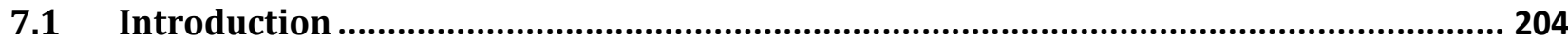

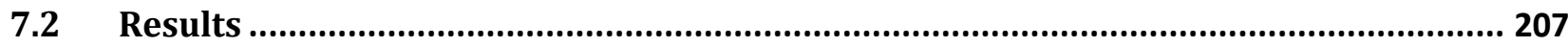

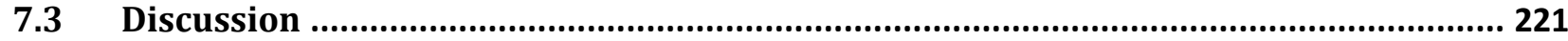

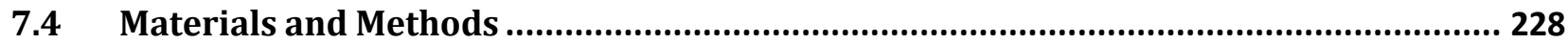

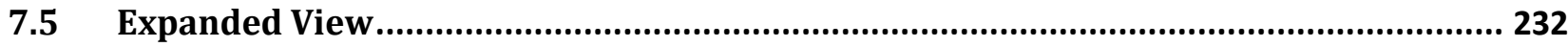

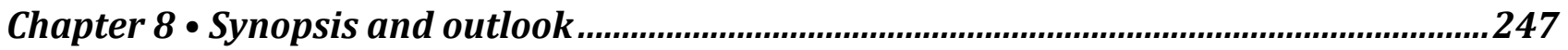

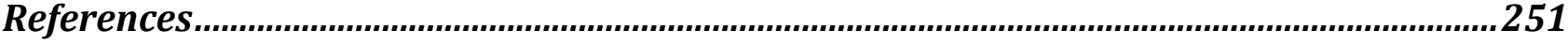

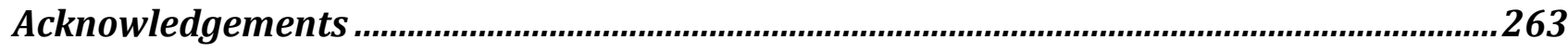

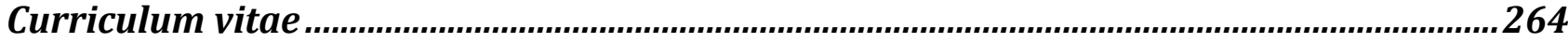




\section{Summary}

Translation, the process of mRNA-encoded protein biosynthesis, is one of the fundamental processes that are universally conserved in extant cellular life. In all its major stages, namely initiation, elongation, termination and recycling, translation depends on a dynamic interplay between the ribosome as the principle place of protein synthesis, mRNA, tRNAs and a number of accessory proteins called translation factors. A subgroup of these translation factors belongs to the universally conserved family of translational GTPases (trGTPases) that use the free energy of GTP hydrolysis to ensure the necessary speed and accuracy of protein synthesis on the ribosome. One of the most complex processes supported by trGTPases, namely by the eukaryal translation initiation factors 2 (elF2) and $5 B$ (elF5B), is the intricate mechanism of cap-dependent translation initiation in eukaryal cells.

The current thesis focuses on the structural and functional characterization of the trGTPases elF2 and elF5B. The first part of this thesis (chapters 2-4) is dedicated to elF2, a structurally unusually complex trGTPase that is responsible for the GTP-dependent delivery of the unique initiator tRNA to the ribosome, and the auxiliary initiation factors that regulate the guaninenucleotide cycle of elF2, namely the specialized GTPase activating protein (GAP) elF5 and the guanine-nucleotide exchange factor (GEF) elF2B. The second part of the thesis (chapters 5-7) encompasses a structural and functional analysis of elF5B, a universally conserved trGTPase that catalyzes ribosomal subunit joining in a GTP-dependent manner to form elongation-competent ribosomes.

Throughout the thesis, the methodological focus lies on the specific mechanistic aspects of elF2 and elF5B function during translation initiation. Both initiation factors will thereby be treated individually in their respective functional contexts of the initiation process, with each of the six chapters dedicated to particular questions at hand. The natural framework for the analysis of the presented findings is however provided by the origin of the functional properties of elF2 and elF5B 
in the historical settings of molecular evolution as a compromise between the innovative processes of structural adaption to biological constellations and the conservative preservation of underlying functional principles. Based on this framework, the specific findings for either trGTPase are used for generalizations in the larger context of translation as an evolved process.

The first chapter forms a general introduction into the mechanism of translation, with a particular focus on the processes that underlie translation initiation in eukarya, to provide an overview of the general mechanistic settings into which elF2 and elF5B are placed. More specific introductions into the particular questions addressed in the present work are given individually in each chapter.

Chapter 2 focuses on the analysis of the interaction network between elF2 and its multifunctional effector protein elF5. A combination of complimentary high- and low-resolution structural approaches with mutational and biochemical methods is used to show how elF5 forms three main contacts with the elF2 complex, and how these interactions affect the contacts of both proteins with initiation factors elF1 and elF3c. A high-resolution crystal structure of theelF $2 \beta \mathrm{N}$ terminal tail in complex with the elF5-CTD and Trp quenching experiments showhowthe recruitment of elF5 to its substrate elF2 is mediated by a flexible peptide-domain interaction that provides the means for the dynamic interplay between eIF2, elF5 and other elFs in the course of the initiation process. A second interaction was found to be formed between the elF5-NTDand the GTP-binding elF2 $\gamma$ subunit. Finally, the third interaction occurs between the elF5-CTD and elF2 $\gamma$ next to the nucleotide binding pocket, indicating that this contact might be responsible for the previously reported function of elF5 in stabilizing the bound guanine-nucleotide on elF2. Together, the presented findings provide a more complete and refined picture of the intricate interactions between elF2 and elF5 that play central roles at various stages of the initiation process.

Chapter 3 focuses on the interactions between elF2 and the catalytic $\varepsilon$-subunit of its GEF elF2B. A combination of structural and biochemical methods was used to gain insight into the interactions between elF2B $\varepsilon$ and its substrate elF 2 to understand the molecular basis for nucleotide exchange. This analysisindicatesthat the catalytic elF2B $\varepsilon$-CTD contacts its substrate elF2 via two main interfaces: a nucleotide-independent high-affinity interaction with the elF2 $\beta$ subunit, and a nucleotide-dependent direct contact to the $\gamma$-subunit, which is responsible for the 
destabilization of the bound nucleotide, most likely in a manner analogous to the mechanisms used by other GEFs. One of the central aspects of the presented findings is their qualitative comparison to those reported for the elF2-elF5 interaction in chapter 2 . The observation of a high degree of structural and functional similarities between the regions in elF2B $\varepsilon$ and elF5 that contact elF2 to modulate its affinity to guanine nucleotides results in the hypothesis that these similaritiesare not merely the result of mechanistic analogies between both proteins but might in fact be the result of an evolutionary homology.

Chapter 4 focuses on structural aspects of the regulatory subcomplex of the elF2-specific exchange factor elF2B which is composed of subunits $\alpha, \beta$ and $\delta$ and which is involved in the regulation of translation by modulating the exchange activity of the elF2B complex. In this chapter the first high-resolution crystal structures of the isolated elF2B $\beta$ and elF2B $\delta$ subunits are presented together with a protocol for the recombinant purification and in vitro reconstitution of the complete elF2B $\alpha \beta \delta$ complex from the fungus Chaetomium thermophilum.

In chapter 5 , the question is addressed how GTP binding and hydrolysis by elF5B mediate its catalytic role during ribosomal subunit joining. Six high resolution crystal structures of elF5B in its apo, GDP- and GTP-bound form are presented. Together with an analysis of the thermodynamics of nucleotide binding, they provide a detailed picture of the nucleotide cycle performed by elF5B. The data suggest that GTP binding induces significant conformational changes in elF5B that activate the factor for ribosome binding and subunit joining. Based on these observations, a domain release mechanism for elF5B activation is proposed, which represents a novel variation from the classical paradigm of GTPase function and suggests a unified picture of subunit joining by a/elF5B and its bacterial ortholog IF2.

Chapter 6 addresses the specific aspect of how domain IV in eIF5B and IF2 interacts with the acceptor stem of the initiator tRNA in the ribosomal context, an interaction that is central to subunit joining as the last checkpoint for the formation of functional ribosomes. A reinterpretation of a $6.6 \AA$ resolution cryo-EM structure of the yeast 80 S initiation complex using the newly determined crystal structures of elF5B from $C$. thermophilum shows that elF5B domain IV interacts extensively with the initiator tRNA, which includes direct recognition of the methionylated $3^{\prime}$ CCAend. The presented findings provide the basis for a possible scenario for the evolution of the 
structurally homologous translational $\beta$ barrel folds in elF5B/IF2 and EF-Tu/alF2 $\gamma$ and their interactions with tRNAs.

Chapter 7 is dedicated to the presentation of the hypothesis of monovalent cation $\left(\mathrm{M}^{+}\right)$ dependency among trGTPases, according to which an $\mathrm{M}^{+}$ion acts as structural and catalytic cofactor in trGTPases. The fundament for this hypothesis is formed by biochemical and highresolution structural data that demonstrate that elF5B, the archaeal elongation factor aEF1A and its bacterial ortholog EF-Tu in their GTP-bound form coordinate an $\mathrm{M}^{+}$in their active sites. In sequence and structure, the coordination shell for the $\mathrm{M}^{+}$ion is highly conserved among trGTPases from bacteria to human, suggesting a universal mechanism of $\mathrm{M}^{+}$-dependent conformational switching and GTP-hydrolysis among trGTPases, which provides considerable explanatory power for previously unresolved questions concerning the function and evolution of trGTPases.

Chapter 8 finally forms a brief synopsis for the presented findings and gives an outlook into the prospects for future work on the various questions that were addressed in the course of this thesis. 


\section{Chapter $1 \bullet$ Introduction}

The metabolism of living organisms depends on an intricate network of mutually interdependent reactions that serve the accumulation of energy and the synthesis of cell material. As functionally most versatile macro molecules, proteins play the central role as catalysts of chemical reactions, for cellular transport or as storage molecules. The synthesis of functional proteins according to genetically encoded information thus constitutes one of the key processes in the metabolism of extant cellular life.

During the biosynthesis of proteins, the flow of the genetic information follows a common principle in all living cells, consisting of two major steps: In the first step, called transcription, the DNA-encoded genetic information is copied to a messenger RNA (mRNA), representing a shortlived mobile version of the genetic information. In the second step, called translation, this mRNA serves as template for protein synthesis, during which the triplet codons of the mRNA are translated into amino-acids that become covalently condensed into the growing polypeptide chain. The principle site of protein synthesis is the ribosome, a large multi-protein-RNA complex, whose activity is complemented by an apparatus of additional proteins, the so-called translation factors that ensure the necessary accuracy and speed during the four major stages of translation: initiation, elongation, termination and recycling. As the critical step of the information flow, in which the level of the genetic information intersects with the functional level of proteins, translation thus depends on the concerted interplay between ribonucleic acids and proteins. The key role of this process for cellular life becomes apparent from the universal evolutionary conservation of the translation machinery in the three domains of life, indicating that the underlying principles of extant protein synthesis evolved long before the onset of speciation. 


\subsection{The ribosome and the universal mechanism of translation}

Protein synthesis is carried out by the ribosome, a large ribonucleo-protein assembly of 2.5 and 4 MDa in size in bacteria/archaea and eukarya, respectively, composed of about two-thirds ribosomal RNA (rRNA) and one-third protein (Fig. 1.1) [2-4]. The overall architecture as well as the structural and functional core of the ribosome is universally conserved throughout evolution from bacteria to human $[5,6]$. The Ribosome comprises two subunits: The small subunit (30S in bacteria/archaea, 40S in eukarya) contains the mRNA binding channel and decoding center (DC), and is responsible for fidelity and processivity of the translation process. The large subunit (50S in bacteria/archaea, 60S in eukarya) contains the peptidyl transferase center (PTC), which constitutes the active site for peptide bond formation on the ribosome from which the nascent peptide chain is ultimately released through an exit tunnel at the back of the large subunit. Together, small and large ribosomal subunits form the complete $70 \mathrm{~S}$ or $80 \mathrm{~S}$ ribosome in bacteria/archaea and eukarya, respectively, that becomes reversibly assembled on mRNAs for protein synthesis [7].

The delivery of amino-acids to the ribosome occurs through transfer RNAs (tRNAs) which successively bind to three tRNA binding sites that are shared between both subunits: the amino$\operatorname{acyl}(A)$, peptidyl $(P)$, and exit $(E)$ site. The A site accommodates the incoming amino-acylated tRNA (aa-tRNA) with its anticodon stem-loop (ASL) oriented toward the mRNA in the DC of the small ribosomal subunit, while the acceptor end with the attached amino-acyl group is positioned next to the PTC of the large subunit (Fig. 1.1). The P site accommodates the peptidyl-tRNA from which the nascent peptide chain is transferred to the A site tRNA. Finally, the E site binds the deacylated tRNA before it dissociates from the ribosome $[7,8]$.

Like the ribosome itself, the principles that underlie the process of ribosomal protein synthesis with its subdivision into initiation, elongation, termination, and recycling are one of the major commonalities of cellular life (Fig. 1.2) [7].

The initiation phase covers all the steps between the dissociation of the ribosomal subunits after a previous translation cycle and its reassembly at the start site of an mRNA with a unique charged initiator tRNA in its $P$ site, a process that is supported by a set of auxiliary initiation factors (IFs). The major steps that have to be accomplished by the initiation machinery are the recruitment of the initiator tRNA and the mRNA to the small ribosomal subunit, identification of the correct 


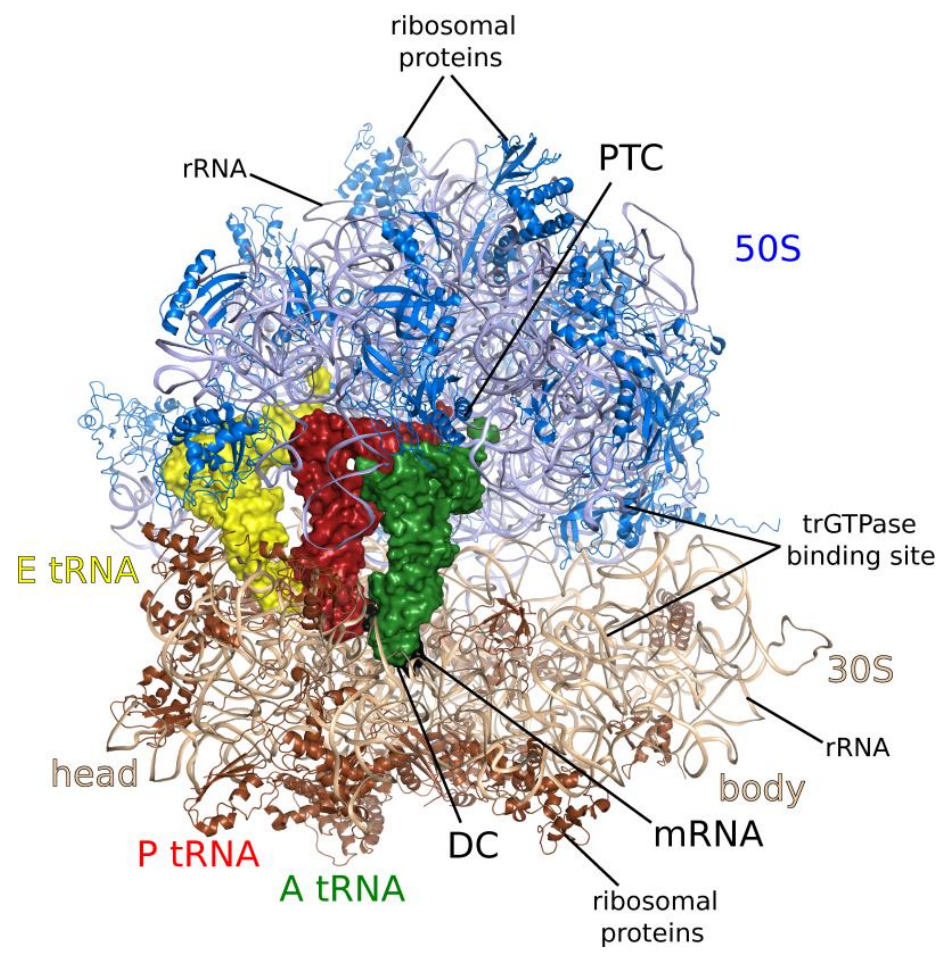

Figure 1.1: Structure of the Ribosome. Overall structure of the bacterial 70S ribosome with mRNA (black) in the decoding center (DC), and tRNAs in A-, P- and E-site (green, red and yellow, respectively) (PDB: 2WDL, 2WDK). The ribosomal RNA (rRNA) and ribosomal proteins are shown in light blue and dark blue for the large 50S ribosomal subunit, and in beige and brown for the small $30 S$ subunit, respectively. The acceptor ends of A- and P-site tRNAs are positioned in the peptidyl-transferase center (PTC) of the 50S subunit, while the anticodon loop of the P site tRNA occupies the DC of the 305 subunit. start site for translation, and finally the joining of the two ribosomal subunits at the start site. The ultimate result is the formation of an elongationcompetent ribosome, ready to accept the first elongator aa-tRNA for peptide bond formation [7, 9]. The critical importance of this process lies in the fact that it sets the reading frame for the subsequent elongation phase and thus decides over the functionality of the synthesized protein.

The elongation phase of translation is a dynamic cyclic process in which tRNAs deliver amino-acids to the ribosome as specified by the mRNA template and sequentially move through the ribosome from the $A$ site to the $P$ site and finally to the $E$ site, thereby adding their amino-acid to the nascent polypeptide chain (Fig. 1.2).

The first step of this cycle is the binding of an aa-tRNA to the ribosomal A site to form a cognate codon-anticodon interaction with the mRNA in the DC of the small subunit. This is followed by peptide bond formation in the PTC between the aa-tRNA in the A site and the peptide attached to the $P$ site-bound tRNA, accompanied by transfer of the peptidyl group to the $A$ site aa-tRNA. Subsequently, in a series of coordinated movements, the mRNA-tRNA complex is translocated by one codon on the ribosome, thereby moving the deacylated tRNA to the E site, while the newly formed peptidyl-tRNA is moved from the $A$ to the $P$ site, leaving the A site free for a new aa-tRNA. In all domains of life, this elongation cycle is supported by two ubiquitous translational GTPases, 
namely elongation factors (EFs) $1 \mathrm{~A} / \mathrm{EF}-\mathrm{Tu}$ and 2/EF-G, which are responsible for the delivery of cognate aa-tRNAs to the ribosome and to promote the translocation of the mRNA-tRNA complex on the ribosome, respectively $[8,10]$.

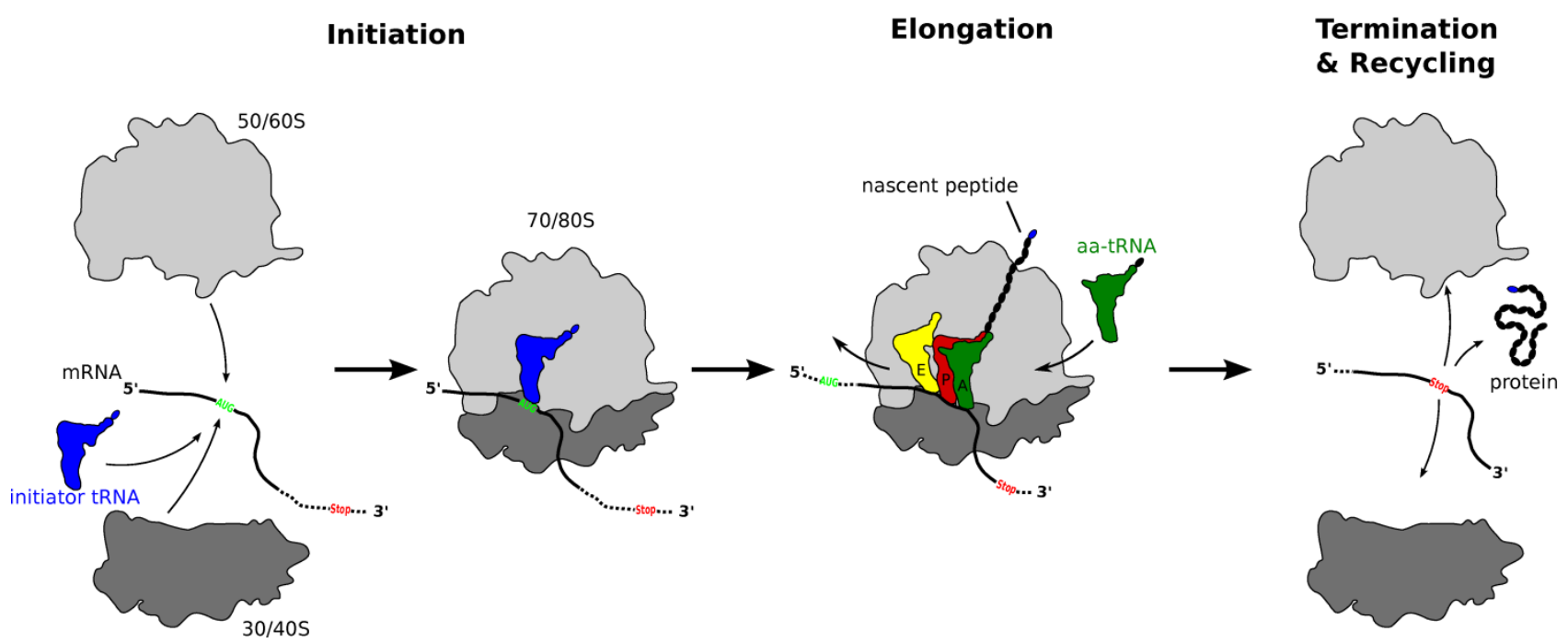

Figure 1.2: Schematic presentation of the basic processes of translation in the three domains of life. The process of translation can be subdivided into initiation, elongation, termination, and recycling. During initiation, the two ribosomal subunits and the specialized initiator tRNA (blue) are recruited to the mRNA to assemble the 70S/80S ribosome with the initiator tRNA positioned in the P site, base paired to the AUG start codon (light green). The 70S/80S ribosome then enters the elongation phase, a cyclic process during which tRNAs (aa-tRNA) deliver amino-acids to the ribosome and move through the ribosome from the $A$ (green) to the $P$ (red) and finally to the E site (yellow), thereby adding their amino-acid to the nascent polypeptide chain as specified by the mRNA template. Translation is terminated upon the encounter of a stop codon (red 'stop') on the mRNA, which results in the release of the synthesized protein and finally in the recycling of the ribosome by dissociation into the individual subunits. During all its stages, the translation process is supported by translation factors, including a number of translational GTPases to ensure the necessary accuracy and speed (not shown for clarity).

Termination of the translation process occurs upon the encounter of an in-frame stop codon in the mRNA, which is recognized by specialized release factors (RFs) to catalyze the hydrolysis of the newly synthesized polypeptide chain from the P site-tRNA. The translation cycle ends in the recycling step with the dissociation of the two ribosomal subunits, which become thus available for a new round of translation initiation (Fig. 1.2) [7, 11]. 


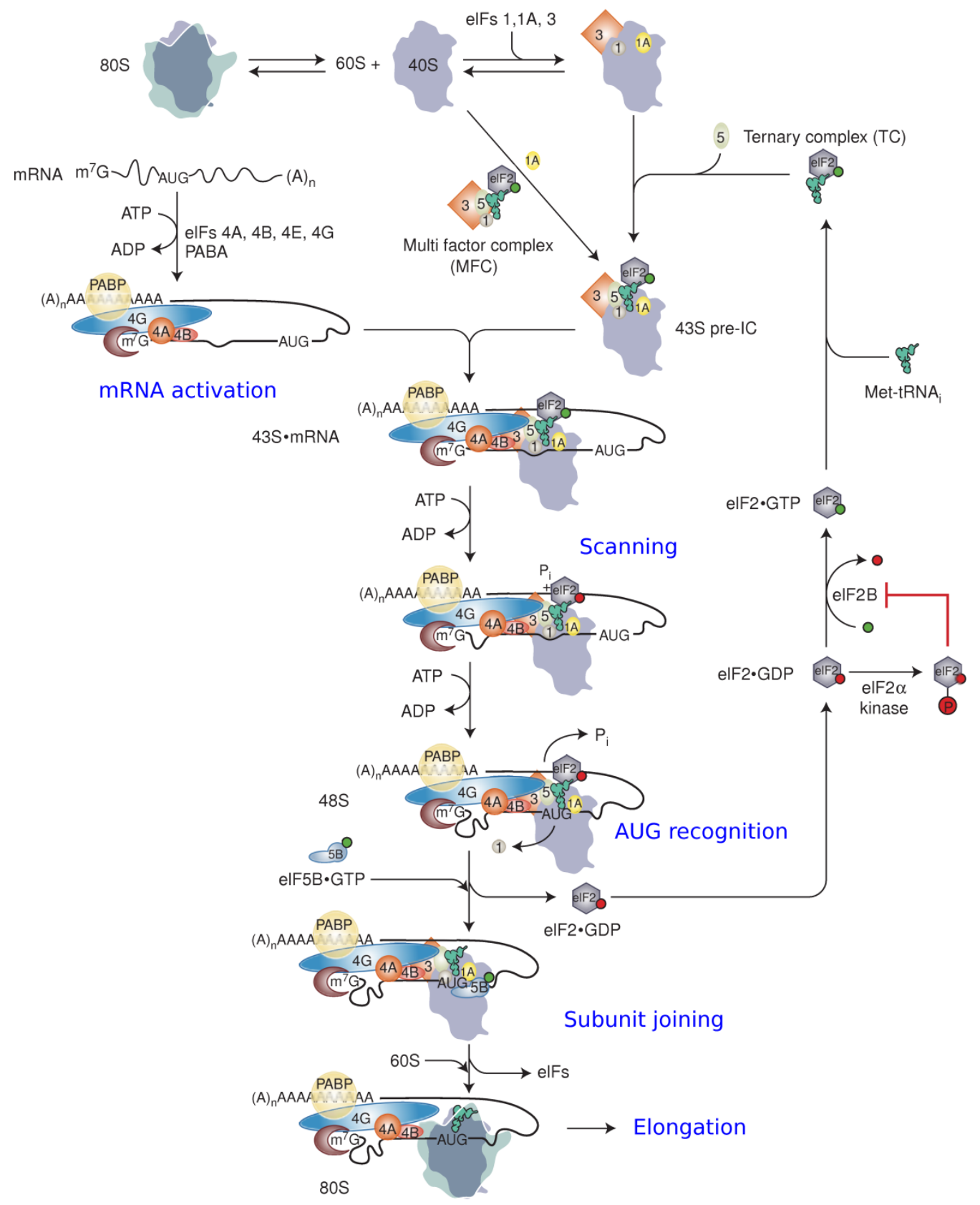

Figure 1.3: Schematic presentation of cap-dependent translation initiation. The mechanism of eukaryal translation initiation covers all steps between dissociation of the $80 \mathrm{~S}$ ribosome from a preceding translation cycle and its reassembly at the start codon of an mRNA. The initiation process starts with the formation of the elF2.GTP.Met-tRNA TC, which is subsequently recruited to the $40 \mathrm{~S}$ subunit with the help of elFs $1,1 \mathrm{~A}, 3$ and 5 to form the $43 \mathrm{~S}$ pre-IC. The $43 \mathrm{~S}$ pre-IC then attaches to the $5^{\prime}$ proximal region of the mRNA through interactions with the cap binding complex elF4F and facilitated by the helicase elF4A. Once bound, the $43 \mathrm{~S}$ pre-IC starts to scan the 5'-UTR of the mRNA in a 5'to- $3^{\prime}$ direction until it reaches the correct AUG start codon. Recognition of the start codon arrests the scanning process and results in the displacement of elF1 and the elF5-stimulated hydrolysis of elF2-bound GTP. elF2.GDP then dissociates from the pre-IC together with elFs $1,5,3$ and $4 \mathrm{~F}$. Joining of the $60 \mathrm{~S}$ subunit is finally promoted by elF5B.GTP, followed by ribosome-induced GTP hydrolysis on elF5B and the dissociation of elF5B.GDP and elF1A to yield the elongation-competent $80 \mathrm{~S}$ ribosome. The figure was modified from Hinnebusch \& Lorsch [12]. 


\subsection{Translation initiation}

Among the different steps of the translation process, translation initiation incurred by far the most extensive divergence in the three domains of life. Hereby, the main differences are found in the mechanisms by which the initiator tRNA and mRNA are recruited to the small ribosomal subunit and the recognition of the correct start site $[9,13]$. In prokayotes, mRNA recruitment entails the direct recognition of the translation initiation region by the 305 subunit through annealing of the Shine-Dalgarno (SD) motif on the mRNA with the complementary anti-SD sequence at the $3^{\prime}$ end of the $16 \mathrm{~S}$ rRNA, placing the start codon directly in the ribosomal P site $[13,14]$. In bacteria, this process, together with the recruitment of the initiator tRNA and joining of the 50S subunit is modulated by three monomeric accessory initiation factors, namely IF1, IF2 and IF3 [15]. In eukarya binding of most mRNAs to the $40 \mathrm{~S}$ subunit does not rely on direct mRNA-rRNA recognition, but instead involves the recruitment of the $40 \mathrm{~S}$ subunit to the capped mRNA $5^{\prime}$ end and a subsequent scanning of the mRNA in the $3^{\prime}$ direction until the correct start site is encountered. Although eukaryal cells contain orthologs of bacterial IF1 and IF2 as well as a functional counterpart to IF3, the eukaryal initiation machinery relies on at least 9 additional initiation factors (elFs) (Table 1.1), many of which are multimeric protein complexes, that together mediate the recruitment of initiator tRNA and mRNA to the 405 subunit, scanning, start codon recognition and finally the joining of the 60 S subunit $[9,16]$.

\subsubsection{Cap-dependent translation initiation in eukarya}

Cap-dependent translation initiation in eukarya can be subdivided into several, partly parallel occurring steps that are orchestrated by the concerted action of the initiation factors (Fig. 1.3) [9, $12,16]$. In a first step, the heterotrimeric GTPase elF2 in its GTP-bound form specifically recognizes and binds the methionylated initiator tRNA (Met-tRNA $\mathrm{A}_{\mathrm{i}}$ ) to form a ternary complex (TC). Supported by elFs $1,1 \mathrm{~A}, 5$ and the multisubunit elF3 complex, the TC binds to the $40 \mathrm{~S}$ subunit to form the $43 \mathrm{~S}$ pre-initiation complex (pre-IC), placing the Met-tRNA $A_{i}$ in the ribosomal P site. The $43 \mathrm{~S}$ pre-IC is then recruited to the 5'-7-mthylguanosine cap of the mRNA in a way facilitated by elF3, the poly $(A)$ binding protein (PABP) and the multimeric cap-binding complex elF4F. Once attached to the $5^{\prime}$ proximal region of the mRNA, the pre-IC starts to scan the $5^{\prime}$ untranslated region (5' UTR) in a $5^{\prime}-3^{\prime}$ 
Table 1.1: Translation factors involved in translation in eukarya, archaea and bacteria

\begin{tabular}{|c|c|c|c|}
\hline $\begin{array}{l}\text { Eukaryal } \\
\text { initiation factor }\end{array}$ & $\begin{array}{l}\text { Archaeal } \\
\text { homolog }\end{array}$ & $\begin{array}{l}\text { Bacterial } \\
\text { homolog }\end{array}$ & Function of the eukaryal homolog \\
\hline elF1 & alF1 & IF3* & $\begin{array}{l}\text { Promotes binding of the elF2.GTP.Met-tRNA } A_{i} \text { complex to the } 40 \mathrm{~S} \\
\text { subunit together with elF1A; promotes ribosomal scanning and } \\
\text { ensures the fidelity of start codon recognition; prevents premature } \\
\text { accommodation of Met-tRNA } A_{i} \text { in the P site; its dissociation is } \\
\text { coupled to elF5-induced GTP hydrolysis by elF } 2 \text { and } P_{i} \text { release. }\end{array}$ \\
\hline elF1A & alF1A & IF1 & $\begin{array}{l}\text { Promotes binding of the elF2.GTP.Met-tRNA }{ }_{i} \text { complex to the } 40 \mathrm{~S} \\
\text { subunit together with elF1; stimulates ribosomal scanning and } \\
\text { promotes start codon recognition cooperatively with elF1. }\end{array}$ \\
\hline elF2 & alF2 & & $\begin{array}{l}\text { Consists of three subunits (elF } 2 \alpha \beta \gamma \text { ); specifically recognizes Met- } \\
\text { tRNA } A_{i} \text { to form an elF2.GTP.Met-tRNA } A_{i} \text { TC; recruits Met-tRNA } A_{i} \text { to the } \\
\text { P site of the } 40 \text { S subunit; hydrolyzes GTP upon start codon } \\
\text { recognition. }\end{array}$ \\
\hline elF2B & & & $\begin{array}{l}\text { Consists of five subunits (elF } 2 B \alpha \beta \gamma \delta \varepsilon \text { ); guanosine-nucleotide } \\
\text { exchange factor (GEF) that promotes exchange of GDP against GTP } \\
\text { on elF2. }\end{array}$ \\
\hline elF3 & & & $\begin{array}{l}\text { Consists of } 13 \text { subunits in mammals; interacts with elF1, elF5 and } \\
\text { elF } 2 \text { to form a multi-factor complex; stimulates recruitment of the } \\
\text { elF2.GTP.Met-tRNA } A_{i} \text { TC to the } 40 \mathrm{~S} \text { subunit; promotes attachment of } \\
43 \mathrm{~S} \text { pre-IC to mRNA; is involved in ribosome dissociation and anti- } \\
\text { association, preventing premature joining of } 40 \mathrm{~S} \text { and } 60 \mathrm{~S} \text { subunits. }\end{array}$ \\
\hline elF4A & alF4A & & $\begin{array}{l}\text { ATP-dependent DEAD-box RNA helicase that unwinds secondary } \\
\text { structures in the } 5 \text { ' leader of the mRNA. }\end{array}$ \\
\hline elF4B & & & Enhances the ATPase and helicase activity of elF4A \\
\hline elF4E & & & Binds to the 5 -cap structure of the mRNA \\
\hline elF4H & & & $\begin{array}{l}\text { Homologous to the } \mathrm{N} \text {-terminal domain of elF4B; enhances the } \\
\text { ATPase and helicase activity of elF4A }\end{array}$ \\
\hline elF5 & & & $\begin{array}{l}\text { Binds elF1, elF2 and elF3; forms nucleation point for the MFC; } \\
\text { promotes recruitment of elF2-GTP.Met-tRNA } A_{i} \text { TC to the } 40 \text { subunit } \\
\text { and stimulates start codon recognition; acts as GTPase activating } \\
\text { protein (GAP) that induces hydrolysis of elF2-bound GTP upon start } \\
\text { codon recognition. }\end{array}$ \\
\hline elF5B & alF5B & IF2 & $\begin{array}{l}\text { Ribosome-dependent GTPase that catalyzes ribosomal subunit } \\
\text { joining }\end{array}$ \\
\hline elF6 & & & $\begin{array}{l}\text { Anti-association factor that binds the } 60 \mathrm{~S} \text { subunit and prevents its } \\
\text { association to the } 40 \mathrm{~S} \text { subunit. }\end{array}$ \\
\hline
\end{tabular}

* The grouping of IF3 with a/elF1 is based on their functional homology and the structural similarity of a/elF1 to the C-terminal domain of IF3 $[17,18]$ and not on a common evolutionary descent. 
direction for the AUG start codon. This process is supported by the small monomeric elFs 1 and $1 \mathrm{~A}$, which bind close to the mRNA channel of the 40S subunit and stabilize the 43S pre-IC in an 'open' conformation that is competent for processive movement along the $\mathrm{mRNA}$, thereby allowing the $\mathrm{P}$ site-bound Met-tRNA $A_{i}$ to sample each of the successive mRNA triplets by using complementarity with its anticodon[19-21]. Base-pairing between anticodon of the Met-tRNA $A_{i}$ and the AUG start codon in the ribosomal $P$ site arrests the scanning process and triggers rearrangements within the pre-IC involving elFs 1, 1A, 2 and 5 that result in the irreversible hydrolysis of the elF2-bound GTP by the gated release of the orthophosphate $\left(\mathrm{P}_{\mathrm{i}}\right)$ from elF2, triggered by the GTPase activating protein (GAP) elF5. Conversion of elF2 to its GDP-bound form commits the pre-IC to the downstream events of the initiation process: The inactive elF2.GDP has a reduced affinity to the Met-tRNA $A_{i}$, resulting in its release together with elF5 and elF1 from the 40S subunit. Joining of the $60 \mathrm{~S}$ ribosomal subunit is finally catalyzed by the ribosome-dependent GTPase elF5B. Supported by elF1A, elF5B binds to the scanning-arrested 435 pre-IC in a GTP-dependent manner and promotes the association of the large 60S ribosomal subunit. 60S binding triggers the GTPase activity in elF5B, resulting in the concerted dissociation of elF5B.GDP and elF1A from the 80S initiation complex (IC) which is thus ready to enter the elongation phase of translation.

\subsection{GTPases involved in translation}

Translation is one of the most fundamental cellular processes that are regulated by guanine nucleotide-binding (G) proteins (GTPases). Apart from translation, G proteins such as the small Raslike GTPases or $G_{\alpha}$ proteins are involved in a wide variety of mechanisms from signal transduction to nucleocytoplasmic transport. The unifying characteristic of $\mathrm{G}$ proteins, which share a common evolutionary origin[22], is their function as molecular switches that alternate between an 'inactive' GDP-bound state and a structurally distinct 'active' GTP-bound state, characterized by the interaction of two conserved dynamic structural elements, switch 1 and switch 2 , with the $\gamma$ phosphate of the bound GTP molecule (Fig. 1.4A) [23-25]. Only the active GTP-bound conformation of the $\mathrm{G}$ protein is able to interact productively with effector molecules or complexes and is therefore a prerequisite for its biological function (Fig. 1.4B). Conversion of the active to the inactive state to 'switch off' the G protein requires the hydrolysis of the bound GTP molecule, 
which is achieved by stimulation of the usually low intrinsic GTPase activity. Common features of these activation mechanisms are the stabilization of the catalytic machinery of the GTPase in a conformation that is productive for GTP hydrolysis and the introduction of additional catalytic elements into the GTPase center that stabilize the transition state of the hydrolysis reaction [23, 25].

A

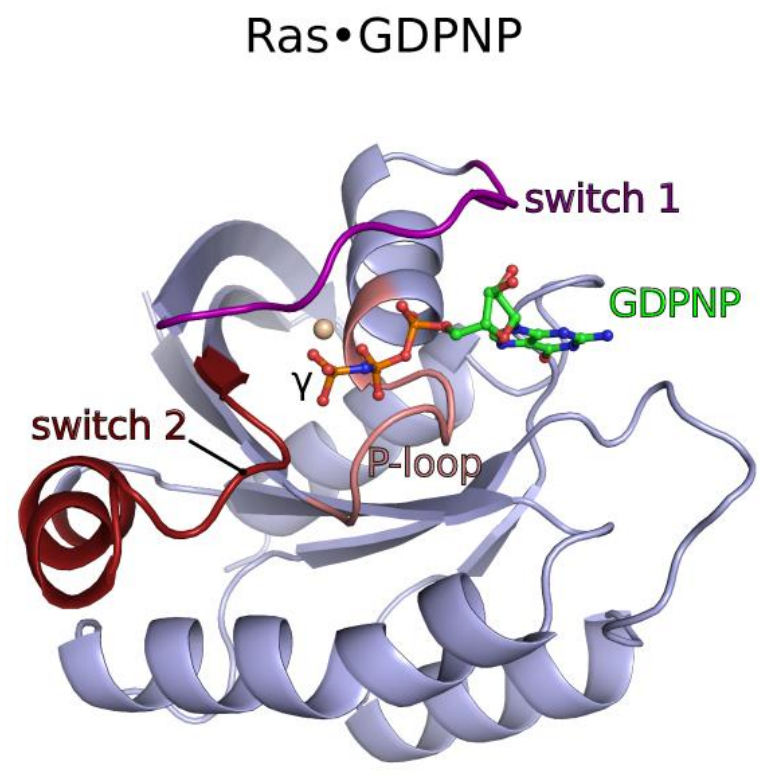

B

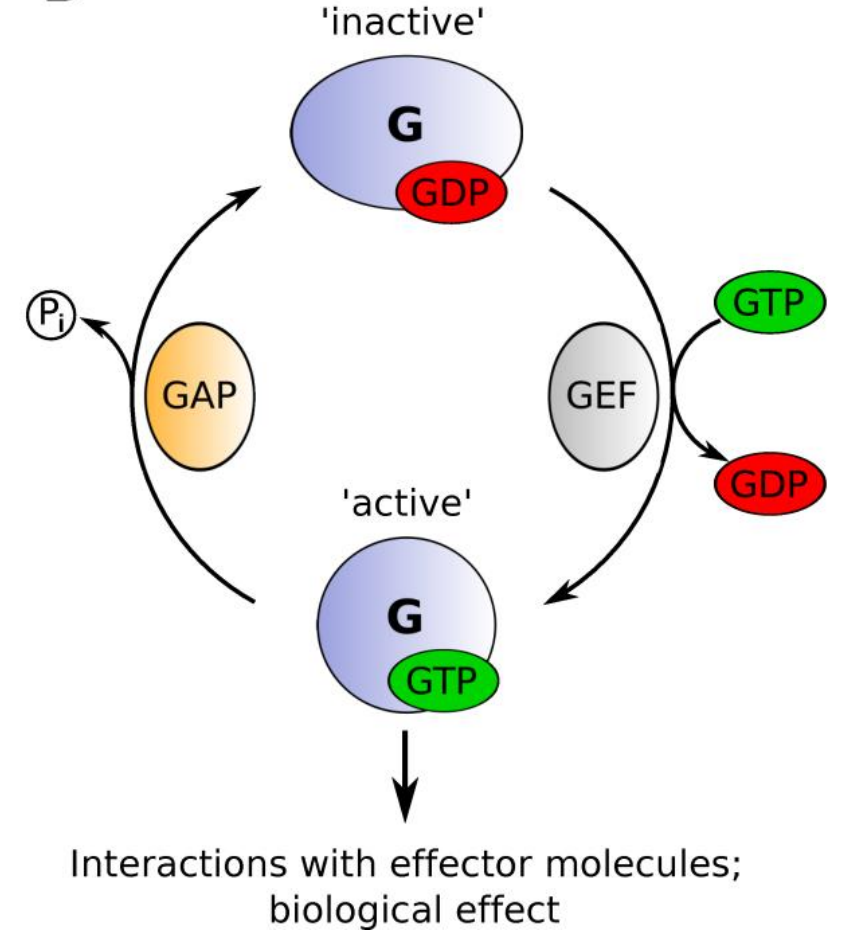

Figure 1.4: G proteins as molecular switches. A) Crystal structure of human Ras (light blue; PDB: 5P21) bound to GDPNP. Ras belongs to the family of small GTPases that consist only of the G domain itself. The phosphate binding loop (P loop), switch 1 and switch 2 are colored in light red, magenta and dark red, respectively. Switch 1 and switch 2 are highly conserved structural elements that specifically recognize the nucleotide state of the $\mathrm{G}$ domain by forming direct contacts to the GTP $\gamma$-phosphate; both switch elements become thus stabilized in a defined conformation that renders the $\mathrm{G}$ domain active for interactions with effector molecules. B) Schematic presentation of the conventional guaninenucleotide cycle of $\mathrm{G}$ proteins. To convert the 'inactive' form of the G protein to its 'active' state, GDP has to be replaced by GTP; in some cases (e.g. Ras, EF-Tu and elF2 $\gamma$ ) this step is accelerated by guanine-nucleotide exchange factors (GEFs). GTP-binding induces the 'active' conformation of the G domain, in which it binds to effector molecules and is able to carry out its biological function in the cell. The GTPase reaction to convert the G protein back to its GDPbound form is usually slow and has to be accelerated by external factors that complement and/or stabilize the active site (here indicated by the collective term GAP for GTPase activating protein). 

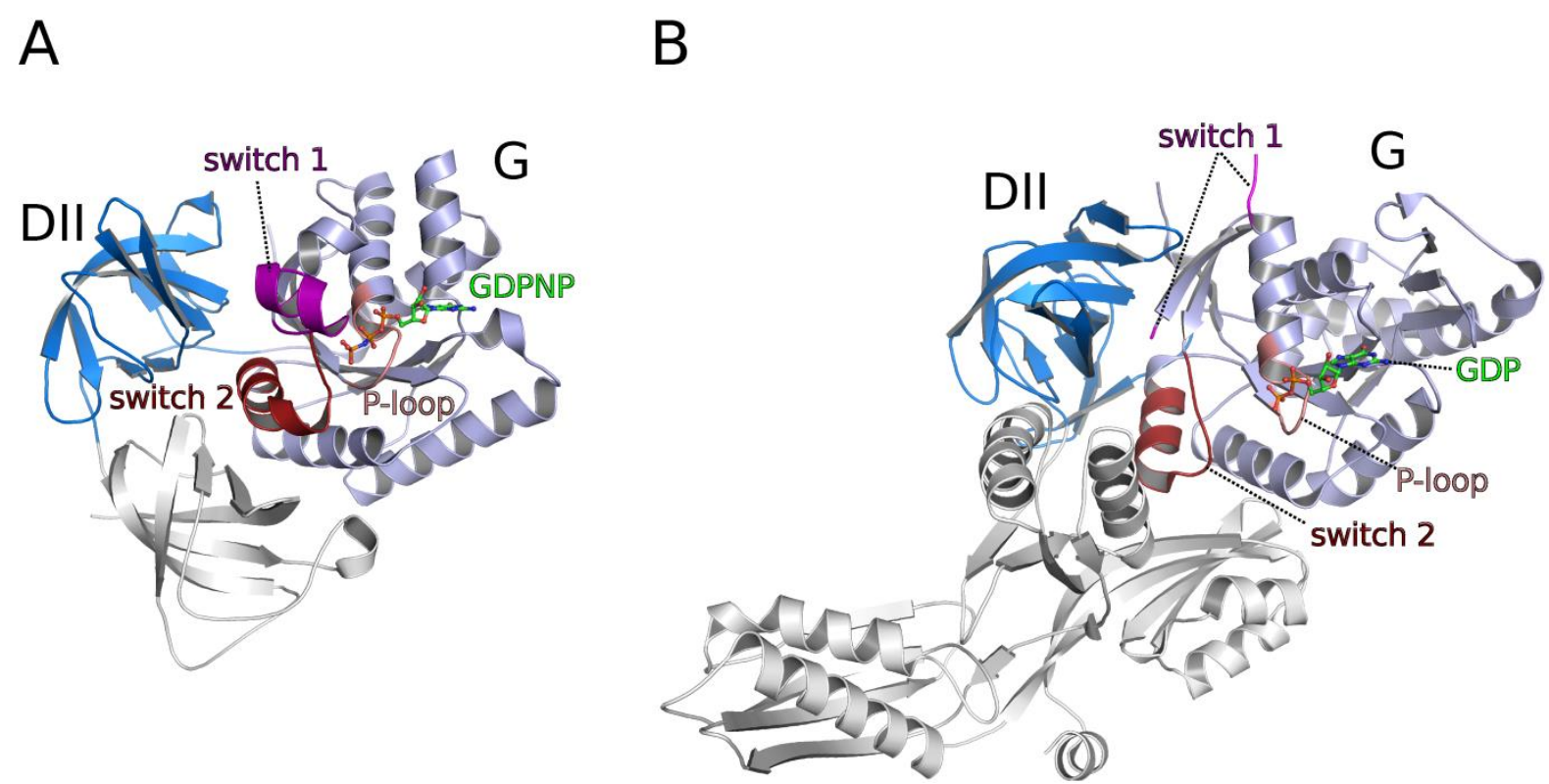

Figure 1.5: The universally conserved structural core in translational GTPases. A, B) Crystal structures of GDPNPbound EF-Tu (A) and GDP-bound EF-G (B) from Thermus thermophilus (PDBs: 1EXM; 1FNM). Both bacterial elongation factors contain a $\mathrm{G}$ domain (G; coloring as in Fig. 1.4A) homologous to Ras-like $G$ proteins and a $\beta$-barrel domain with greek-key topology (DII; dark blue), which are shared with all other trGTPases. G domain and domain II are supplemented with additional domains (shown in grey), which are required for factor specific functions and not evolutionarily universally conserved across the trGTPase lineages.

In all its basic sub-processes from initiation to ribosome recycling, the translation apparatus depends on auxiliary trGTPases (Table 1.2). Three of these factors, namely initiation factor a/elF5B/IF2 and elongation factors a/eEF1 $\alpha / E F-T u$ and a/eEF2/EF-G, are ubiquitous in the three kingdoms of life and are therefore likely to represent the minimal set of trGTPases necessary for mRNA translation that evolved before the emergence of the three primary lineages bacteria, archaea and eukarya [22]. Elongation factor SelB constitutes a special case, as it is represented with homologs in all three domains of life, but not ubiquitously distributed among all species. Additional trGTPases that are not found in all three domains apparently evolved later from the lineages of either a/eEF1 $\alpha /$ EF-Tu or a/eEF2/EF-G $[22,26]$. These include the initiation factor a/elF2, which is only found in archaea and eukarya and the release factors RF3 and eRF3 in bacteria and eukarya, respectively. Despite their different functions during translation, all trGTPases share a common evolutionarily conserved structural core, composed of the G domain and domain II, which is supplemented with additional factor specific domains (Fig. 1.5). The G domain, which is related 
to the $G$ domains of other Ras-related GTPases, forms the functional center in trGTPases that couples GTP binding and its hydrolysis to the specific biological function of the factor during translation. The ribosomal complexes on which the different trGTPases fulfill their role hereby act as the GTPase activating element, which stimulates the usually low intrinsic GTPase activity of trGTPases [27].

Table 1.2: Translational GTPases in eukarya, archaea and bacteria

\begin{tabular}{|c|c|c|c|}
\hline $\begin{array}{l}\text { Eukaryal } \\
\text { initiation factor }\end{array}$ & $\begin{array}{l}\text { Archaeal } \\
\text { homolog }\end{array}$ & $\begin{array}{l}\text { Bacterial } \\
\text { homolog }\end{array}$ & Function \\
\hline elF $2 \gamma$ & alf $2 \gamma$ & & $\begin{array}{l}\text { Forms the central subunit of the heterotrimeric elF2 } \\
\text { complex; recognizes Met-tRNA } A_{i} \text { to form the } \\
\text { elF2.GTP.Met-tRNA } A_{i} \text { TC to recruit the initiator tRNA } A_{i} \text { to } \\
\text { the } 40 \text { subunit; hydrolyzes GTP upon start codon } \\
\text { recognition. }\end{array}$ \\
\hline elF5B & alF5B & IF2 & $\begin{array}{l}\text { Catalyzes ribosomal subunit joining; the bacterial } \\
\text { ortholog also promotes binding of the fMet-tRNA }{ }^{\text {fMet }} \text { to } \\
\text { the } 30 \mathrm{~S} \text { subunit. }\end{array}$ \\
\hline eEF1A & aEF1A & EF-Tu & Delivers aa-tRNAs to the ribosome during elongation. \\
\hline eEF2 & $\mathrm{aEF} 2$ & EF-G & $\begin{array}{l}\text { Translocation of the mRNA/tRNA complex on the } \\
\text { ribosomeduring elongation. }\end{array}$ \\
\hline eSelB & aSelB & bSelB & Delivers Sec-tRNA ${ }^{\text {sec }}$ to the ribosome during elongation \\
\hline \multirow[t]{3}{*}{ eRF3 } & & & $\begin{array}{l}\text { Class- } 2 \text { release factor; binds class } 1 \text { release factors and } \\
\text { promotes termination of the translation process. }\end{array}$ \\
\hline & & $\mathrm{RF} 3$ & $\begin{array}{l}\text { Class- } 2 \text { release factor; binds class } 1 \text { release factors and } \\
\text { promotes termination of the translation process. }\end{array}$ \\
\hline & & LepA & $\begin{array}{l}\text { Thought to promote back-translocation on the } \\
\text { ribosome. }\end{array}$ \\
\hline
\end{tabular}

Two trGTPases that exemplify this split between structural adaptation to new biological constellations and the simultaneous preservation of the underlying principles of function are the eukaryal initiation factors elF2 and elF5B. On the one hand, elF2 constitutes an example for a 
tightly regulated trGTPase, in which the $G$ protein forms a constitutive complex with two accessory subunits, and which requires various additional effector proteins to perform its role during translation initiation. elF5B, on the other hand, is an example for a monomeric, universally conserved trGTPase that fulfills its role during subunit joining without the absolute requirement for additional effector molecules apart from the ribosome itself. Notwithstanding these differences, both proteins follow the basic paradigms of $\mathrm{G}$ protein function as molecular switches.

\subsubsection{The eukaryal translation initiation factor 2 (eIF2)}

elF2 is responsible for the delivery of the Met-tRNA $A_{i}$ to the $40 \mathrm{~S}$ ribosomal subunit and plays a key role during the identification of the correct start site for translation (Fig. 1.3). Like other $\mathrm{G}$ proteins, elF2 thereby oscillates between an 'active' GTP-bound form, in which it is able to specifically recognize and bind the Met-tRNA $A_{i}$ and an 'inactive' GDP-bound form, in which elF2 dissociates from the scanning arrested 435 pre-IC. During this nucleotide cycle, elF2 function is dependent on two accessory protein factors, namely elF5 and eIF2B. While elF5 is essential for elF2 TC binding to the $40 \mathrm{~S}$ subunit and required to trigger the hydrolysis of elF2-bound GTP, elF2B functions as guanine nucleotide exchange factor (GEF) that recycles elF2-GDP back into its GTP-bound form.

\subsubsection{1 eIF2 and the recognition of the Met-tRNA $A_{i}$}

elF2 is a heterotrimeric $G$ protein, comprising the subunits elF $2 \alpha,-\beta$, and $-\gamma$. All three elF2 subunits have orthologs in archaea, namely alF $2 \alpha,-\beta$, and $-\gamma$, whereas no counterparts are known from bacteria [28]. With the exception of elF2 $\alpha$, structural information about elF2 is limited to its archaeal orthologs (Fig. 1.6). The a/elF2 $\alpha$-subunit is composed of two domains: an $\mathrm{N}$-terminal domain that is subdivided into an S1-like OB-fold that forms a rigid body with the following small $\alpha$ helical subdomain, and a $C$-terminal $\alpha / \beta$ domain which contains an additional acidic extension only in the eukaryal orthologs (Fig. 1.6A) [29, 30]. The C-terminal domain of elF2 $\alpha$ is responsible for direct interactions with the $\gamma$-subunit of elF2 [31-33]. The body of a/elF2 $\beta$ is composed of two conserved sub-domains, an $N$-terminal $\alpha / \beta$ sub-domain and a zinc binding sub-domain (ZBD), that are preceded by a single $\mathrm{N}$-terminal $\alpha$-helix $(\alpha 1)$ which is responsible for stable binding to the elF $2 \gamma$ (Fig. 1.6B) $[31,32,34]$. Only the eukaryal elF2 $\beta$ ortholog contains an additional long $\mathrm{N}$-terminal 
extension, which harbors three conserved lysine-rich patches (K-boxes) that are involved in interactions with aromatic/acidic boxes (AA-boxes) in the C-terminal domains of the elF2 effectors elF5 and elF2BE (see next sections) which are likewise not found in archaeal cells [35, 36]. Within the a/elF2 complex, the $\alpha$ - and $\beta$-subunits do not interact directly with each other, but both bind independently to the central $\gamma$-subunit $[31,32]$. a/elF2 $\gamma$ is the $G$ protein within the complex, homologous to the elongation factors EF-Tu, eEF1A and SelB, and accordingly adopts a threedomain architecture composed of a $\mathrm{G}$ domain (domain I) and two $\beta$-barrel domains (domains II and III) with greek key topology (Fig. 1.6C). Crystal structures of alF2 $\gamma$, obtained either in its apo state, in complex with GDP or with the nonhydrolyzable GTP analog GDPNP revealed that the three domains invariably adopt a 'closed' conformation similar to that of the active GDPNP-bound EF-Tu and in stark contrast to the 'open' conformation in GDP-bound EF-Tu [33, 37-39]. A number of eukaryal orthologs contain an additional non-conserved N-terminal extension, which was, however, shown to be dispensable for elF2 function in vivo[40]. Like in other G proteins, the $G$ domain of a/elF2 $\gamma$ harbors the guanine-nucleotide binding site with the highly conserved nucleotide binding motifs [24]. This includes the two mobile switch 1 and switch 2 elements that are thought to change their conformation upon the transition from the GDP-bound form to the GTP-bound state of the $G$ domain, thereby promoting the binding of Met-tRNA $A_{i}$ by elF2. GTPbound a/elF2 $\gamma$ alone is able to bind the Met-tRNA $A_{i}$ directly [32]. However, the affinity of the elF2-GTP complex for the Met-tRNA $A_{i}$ is significantly increased ( $\sim 100$-fold) by each of the accessory subunits elF $2 \alpha$ and elF2 $\beta[32,41]$. Structural analyses suggest that binding of the C-terminal domain of alF $2 \alpha$ to domain II of GTP-bound alF2 $\gamma$ allosterically opens a channel between switch 1 of the $\mathrm{G}$ domain and domain II, thereby allowing the accommodation of the ACC acceptor stem with the attached methionyl group [42, 43]. Further alF2 $\alpha$ - or GTP-induced domain rearrangements are limited and significantly smaller than those observed for the transition between GDP- and GTP-bound forms of EF-Tu. Consistently, it was found that the affinity of MettRNA $_{i}$ is only $\sim 10$-fold greater for elF2.GTP than for the GDP-bound form, and that the affinities of elF2.GTP and elF2.GDP to deacylated tRNA $A_{i}$ is about equal to that of elF2.GDP for Met-tRNA $A_{i}[44$, 45]. This indicates that the thermodynamic coupling between the binding of GTP and Met-tRNA mainly depends on the methionyl moiety, whereas the rest of the binding interface is virtually 
unperturbed [44]. However, the first base pair of the tRNA $A_{i}$ acceptor stem (A1:U72), together with the G31:C39 pair in the anticondon stem loop (ASL) and bases within the T loop form additional signature elements in the initiator tRNA that enhance Met-tRNA $A_{i}$ binding to eIF2.GTP and allow its discrimination against elongator tRNAs[46, 47].
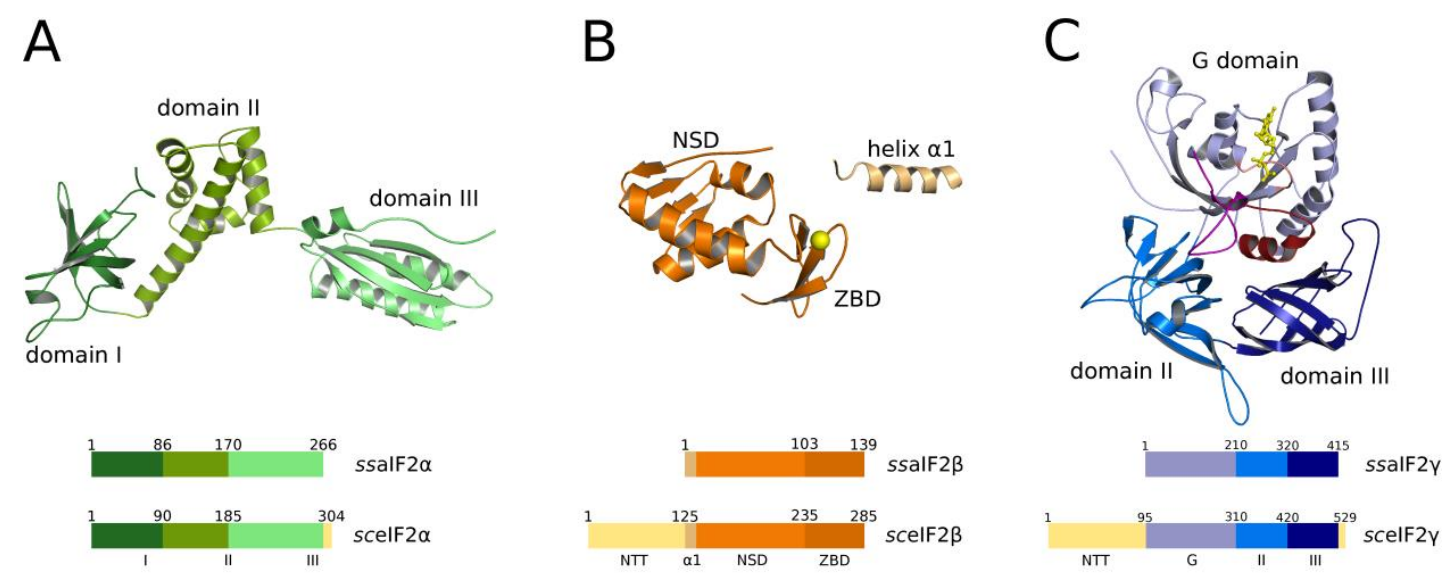

D

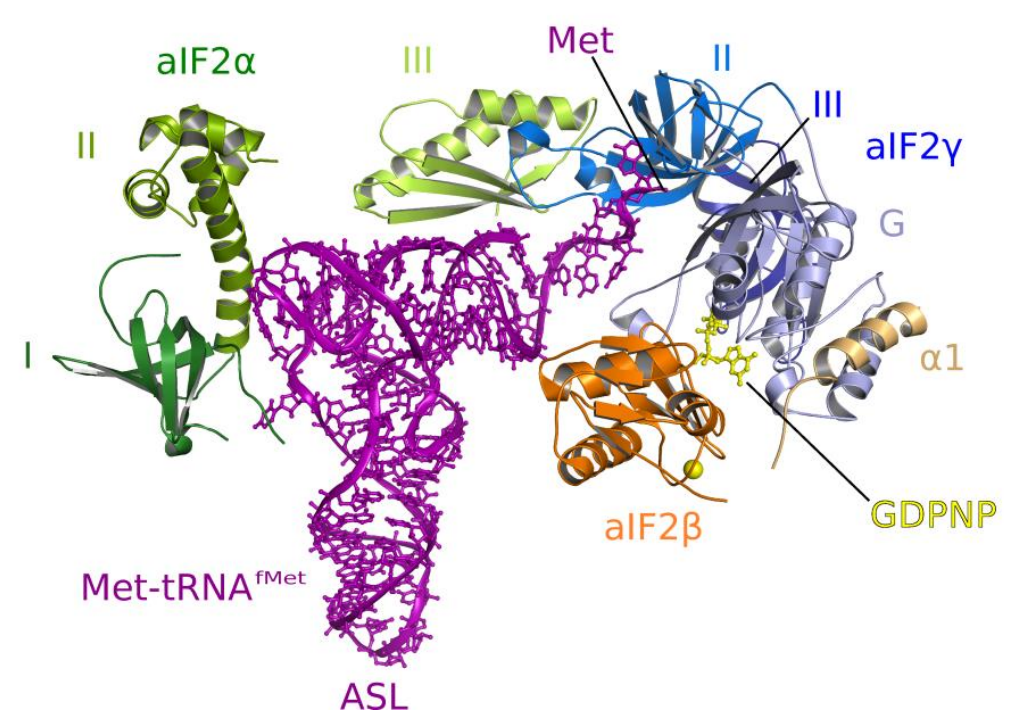

Figure 1.6: Structure of a/elF2. A-C) NMR solution structure of human elF2 $\alpha$ (A) and crystal structures of alF2 $\beta$ (B) and GDP-bound alF2 $\gamma$ (C) as found in the heterotrimeric alF2 complex from S. solfataricus (PDBs: 1Q8K; 2QMU). A comparison of the domain architectures in the eukaryal (sc: S. cerevisiae) and archaeal (ss: S. solfataricus) orthologs is schematically shown below the structures with corresponding color codes; regions idiosyncratic to the eukaryal lineages are indicated in yellow. The zinc ion in alF2 $\beta$ is shown as yellow sphere; the GDP molecule bound to alF2 $\gamma$ is shown as yellow balls and sticks; $G$ domain and domain II of alF2 $\gamma$ are colored as their homologous domains in EF-Tu and EF-G in Fig. 1.5. D) Cartoon representation of the ternary complex of S. solfataricus alF2 with GDPNP and MettRNA $^{\text {fMet }}$. The C-terminal domain of alF2 $\beta$ is not visible in the crystal structure (PDB: 3V11) and was therefore modeled into alF2 according to its position in $2 \mathrm{QMU}$. The color code for the individual subunits corresponds to that in A-C; the Met-tRNA $A_{i}$ is colored in magenta. 
The crystal structure of the TC formed between alF2-GDPNP and E. coli Met-tRNA ${ }^{\text {fMet }}$ revealed that alF2 most likely binds the tRNA $A_{i}$ in a manner significantly different from that observed for elongator tRNA binding by EF-Tu (Fig.1.6D) [42, 48]. The ACC end with the methionyl group is bound in a pocket between switch 1 of the $G$ domain and domain II of alF2 $\gamma$ in the same way as in TCs of EF-Tu. However, a kink in the acceptor stem reorients the rest of the tRNA dramatically relative to alF $2 \gamma$, thereby preventing the direct interaction between domain III of alF2 $\gamma$ and the T stem minor groove of the tRNA as observed in EF-Tu TCs. Instead, the acceptor arm and the T-loop of the tRNA form direct contacts with the C-terminal and $\mathrm{N}$-terminal domains of alF2 $\alpha$, respectively. Although similar interactions to elF2 $\alpha$ were not found for the elF2.GTP.Met$\mathrm{tRNA}_{i}$ complex in directed hydroxyl radical footprinting experiments [49], mutational analyses for elF2 $\gamma$, as well as the cryo-electron microscopy (cryo-EM) model of the mammalian $43 S$ pre-IC suggest a similar mode of Met-tRNA $\mathrm{A}_{i}$ binding in the eIF2 TC, with the difference that the $\mathrm{N}$-terminal S1-like OB-fold domain of elF $2 \alpha$ seems to form no direct contact to tRNA $A_{i}$ but instead contacts the $40 S$ subunit $[45,50]$. How the $\beta$-subunit is positioned in the TC and how it might contribute to MettRNA $A_{i}$ binding by a/elF2 is still not known.

\subsubsection{2 eIF5 regulates the activity of eIF2}

elF5 consists of two distinct structural domains (Fig. 1.7). The N-terminal domain (elF5-NTD) shares sequence and structural homology with the C-terminal portion of a/elF2 $\beta$ and can be further subdivided into an $N$-terminal $\alpha / \beta$ sub-domain (NSD) and a zinc binding subdomain (ZBD) [51]. Unique features of the elF5-NTD that are not found in a/elF2 $\beta$ are a short sequence insertion in the NSD which bears some resemblance with a Walker A box motif (P-loop) of nucleotide-binding proteins, and a flexible $\mathrm{N}$-terminal tail of $\sim 20$ residues that contains a highly conserved arginine residue (Arg15), which was proposed to act as catalytic arginine-finger to trigger the GTPase activity in elF2 on the $43 \mathrm{~S}$ pre-IC (see below) $[52,53]$. The C-terminal domain of elF5 (elF5-CTD) adopts a globular atypical HEAT repeat fold [1,54], which is connected to the elF5-NTD through a long mainly negatively charged linker region (elF5-LR). The overall sequence conservation within the eIF5-CTD is low with the exception of two regions (AA-boxes) that are rich in aromatic and 
acidic side chains and together form a large negatively charged surface area (area I) that is required for interactions with the three lysine-rich K-boxes in the $\mathrm{N}$-terminal domain of elF2 $3[35,55]$.

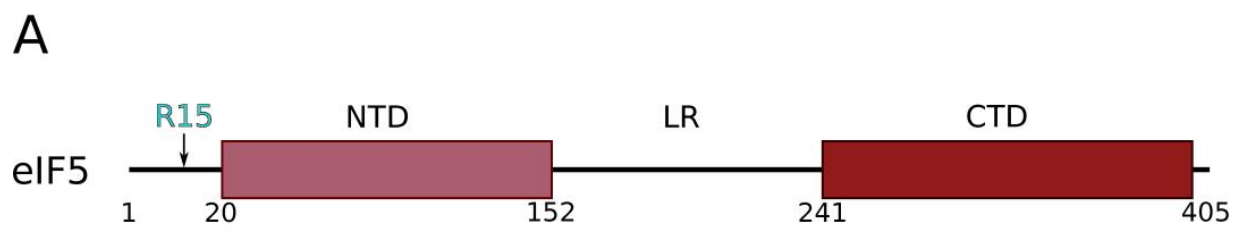

B

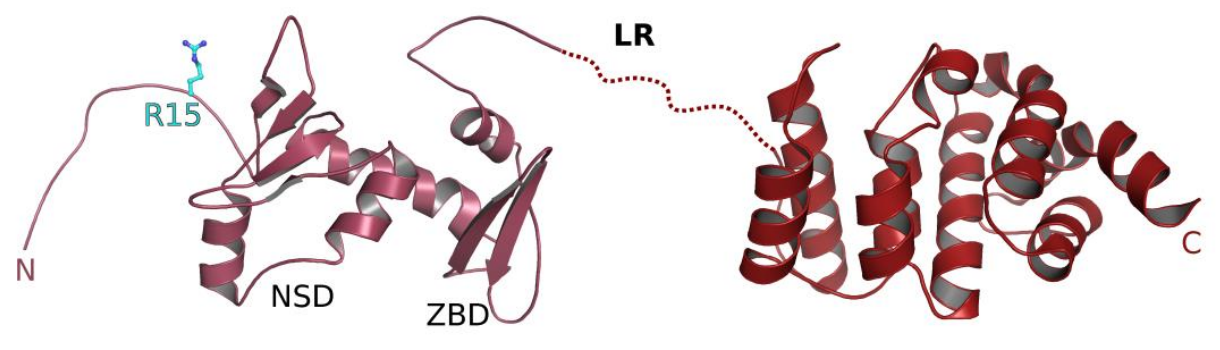

NTD

CTD

Figure 1.7: Domain architecture of elF5. A) Schematic presentation of the domain architecture in elF5. elF5 is thought to fold into an N-terminal domain (NTD; colored wine-red) that contains the putative arginine-finger (R15) in the Nterminal extension, and a HEAT-repeat like C-terminal domain (CTD; colored in dark red). Both domains are connected via a flexible linker region (LR). B) NMR solution structure of the human elF5-NTD (left; PDB: 2G2K) and the yeast elF5CTD (right; PDB: 2FUL), connected by the flexible linker region (dashed line). The putative arginine-finger (R15) is shown as sticks. N-terminal subdomain (NSD) and zinc binding domain (ZBD) of the elF5-NTD are homologous to those in the C-terminal domain of e/alF2 $\beta$ (see Fig. 1.6B). The color code is the same as in (A).

As mentioned above, elF5 supports the recruitment of elF2 TCs to the 40S ribosomal subunit during $43 \mathrm{~S}$ pre-IC formation [56-58]. Binding of the isolated TC to the 40S subunit is slow and its efficient recruitment thus depends on the assistance by other factors, namely elFs 1, 1A, 5 and the elF3 complex [56, 57, 59]. With the exception of elF1A, these factors together with the TC preassemble into a multifactor complex (MFC) in yeast, plants and mammals [59-61] through a network of physical interactions with each other, thereby mutually promoting their binding and the recruitment of the TC to the $40 \mathrm{~S}$ subunit for $43 \mathrm{~S}$ pre-IC formation. Hereby, the eIF5-CTD plays a key role as nucleation point for the other factors by forming a platform for direct and partially cooperative interactions with the $\mathrm{N}$-terminal domains of elF2 $\beta$ (through the K-boxes) and subunit c of the elF3 complex, as well as with elF1 $[56,57,59]$. Consistently, mutations that disrupt any of 
these interactions result in impaired 435 pre-IC formation and reduced growth rates in a manner that can be suppressed through the overexpression of the TC, highlighting their importance for TC recruitment $[58,62]$.

After $43 \mathrm{~S}$ pre-IC formation and its recruitment to the $5^{\prime}$ end of the $\mathrm{mRNA}$, the interaction between elF2 and elF5-CTD continues to play a role in scanning and start codon recognition by antagonizing elF1 binding to the 43S pre-IC, thereby promoting the 'closed' scanning arrested conformation of the pre-IC $[21,63]$. However, the main role of elF5 during scanning and start site recognition is to trigger the intrinsic GTPase activity of elF2.GTP in its capacity as GTPase activating protein (GAP). Several studies suggested that the elF5-NTD acts as classical GAP that provides an arginine-finger (Arg15) in trans which is introduced into the active site of elF2 in the context of the scanning 43S pre-IC and stabilizes the transition state of the GTP hydrolysis reaction [52, 53]. This was supported by the finding that substitutions of the invariant Arg15 in the unstructured $\mathrm{N}$ terminal tail of elF5 are lethal and abolish the elF5-dependent GTPase activation without reducing the ability of elF5 to bind elF2 [51-53, 64]. However, this activating effect of elF5-NTD strongly depends on the structural context of the pre-IC, suggesting that elF5 may also contribute allosterically to GTPase activation in elF2 $\gamma$ in the scanning pre-IC [64]. Finally, it was reported that the elF5-NTD is able to bind isolated elF2 $\gamma$ only in the absence of elF2 $\beta$ [65], which in turn is known to suppress the intrinsic GTPase activity of elF2 $\gamma$ through its ZBD $[21,66,67]$. Based on these observations it was proposed that the elF5-ZBD may displace the structurally homologous elF2 $\beta$ ZBD from elF2 $\gamma$, thereby simultaneously removing the inhibitory effect of elF2 $\beta$ and providing the catalytic Arg15 with access to the nucleotide binding site [21]. AUG recognition would then have to trigger the removal of the elF5-NTD to allow the gated release of the orthophosphate from elF2.GDP/P, which would thus be connected to the release of elF1 from the P-site and accommodation of the Met-tRNA ${ }_{i}$, promoted by the interaction between elF5-CTD and elF2 $\beta$-NTT.

After $\mathrm{P}_{\mathrm{i}}$ release, eIF2.GDP is thought to dissociate from the $43 \mathrm{~S}$ pre-IC in a tight complex with elF5 [68]. Recent studies suggested that within this elF5·elF2·GDP complex the flexible elF5-LR between $\mathrm{N}$ - and C-terminal domains forms a direct contact to elF2 in addition to that between elF5-CTD and eIF2 $\beta$-NTT [69]. This interaction was shown to mediate the stabilization of GDP on elF2. Based on this finding and the observation that a large fraction of cytosolic elF2 is present in a 
complex with elF5, it was proposed that elF5 might serve an additional role as guanine-nucleotide dissociation inhibitor (GDI) that antagonizes the GEF function of elF2B by physically competing with the catalytic elF2Br-CTD (see below) and by suppressing spontaneous GDP release, thereby preventing elF2·GDP recycling and reducing the levels of elF2 TCs in the cell $[68,69]$.

\subsubsection{Recycling of eIF2-GDP by eIF2B}

After its release from the $43 \mathrm{~S}$ pre-IC, elF2·GDP has to be recycled back to elF2·GTP to allow a new round of Met-tRNA $A_{i}$ binding and translation initiation. Like various other Ras-related G proteins, elF2 binds GDP tightly with an affinity in the low nanomolar range, and the rates of spontaneous GDP release are too slow to allow efficient TC formation and translation initiation [44]. Guaninenucleotide exchange on elF2 is therefore catalyzed by the GEF eIF2B, which promotes GDP release from elF2.GDP and thereby facilitates its replacement by GTP $[70,71]$. elF2B is composed of five non-identical subunits $(\alpha, \beta, \gamma, \delta$, and $\varepsilon$ ) and was recently proposed to form a functional decamer of $\sim 600 \mathrm{kDa}$ in solution containing two copies of each subunit $[72,73]$. The minimal catalytically active region of the eIF2B complex was mapped to the $\sim 20 \mathrm{kDa} C$-terminal domain of elF2B $\varepsilon$, which adopts an atypical HEAT repeat fold homologous to that of the elF5-CTD (Fig. 1.8) [1, 74]. Like elF5-CTD, the elF2BE-CTD contains two conserved AA-boxes at its $C$-terminus that are required for strong interactions with the K-boxes of the elF2 $\beta-N T T$ and are thus implicated in the recruitment of the substrate elF2 to its GEF [35]. Moreover, elF2B $\varepsilon$-CTD was found to interact directly with elF2 $\gamma$ through its $\mathrm{N}$-terminal portion, which was shown to contribute directly to nucleotide exchange on elF2 and is thus considered to form the catalytic center [70, 71, 75, 76]. Together with eIF2By, elF2B $\varepsilon$ forms the 'catalytic' subcomplex of elF2B, in which the $\gamma$ subunit

serves an accessory role by promoting the nucleotide exchange activity of the $\varepsilon$ subunit. $A$ regulatory subcomplex is formed by the homologous $\alpha, \beta$, and $\delta$ subunits, which contribute to elF2·GDP binding through interactions with elF2 $\alpha$ [77].

Due to its central position in the guanine-nucleotide cycle of elF2, elF2B is one of the major targets for the regulation of translation initiation. One of the best studied pathways of elF2B inhibition is the phosphorylation of elF $2 \alpha$ at Ser51 (elF2 $\alpha-P$ ) by elF $2 \alpha$ kinases, which in turn 
become activated in response to stress conditions to down-regulate general translation (Fig. 1.1) [78-80].
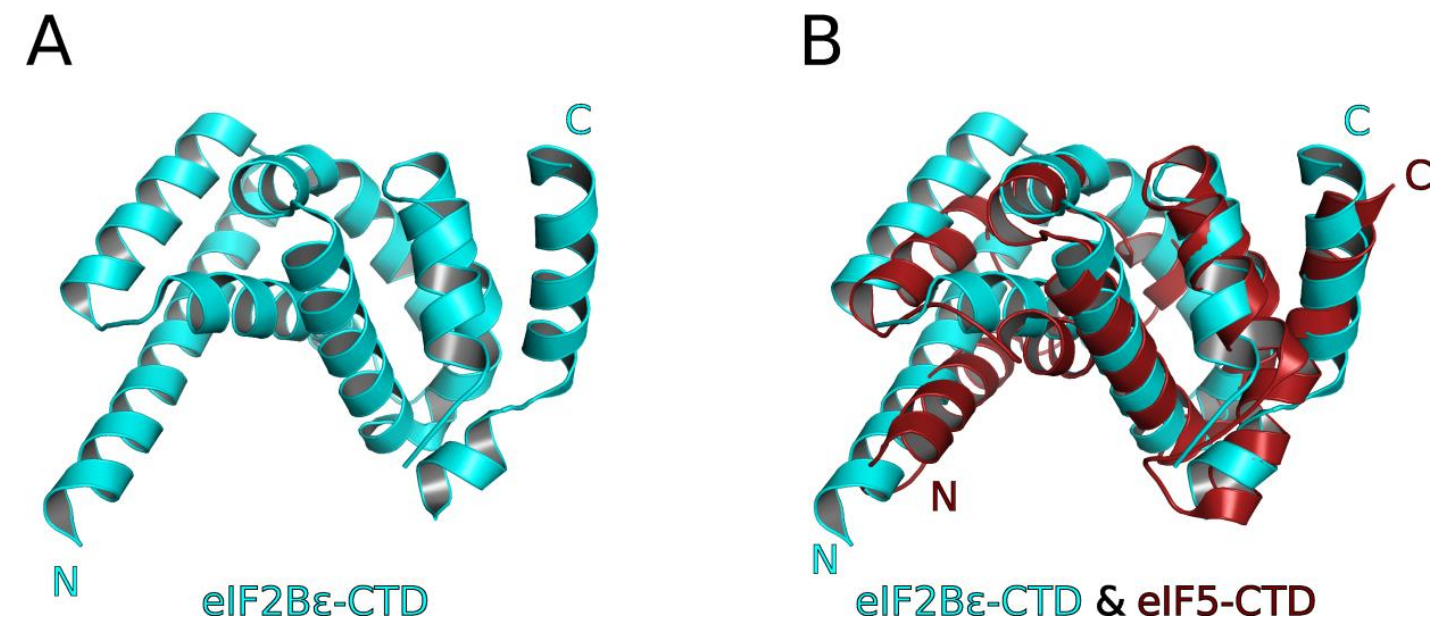

Figure 1.8: Structure of the catalytic domain in elF2BE. A) Crystal structure of the human elF2BE-CTD (PDB: 3JUI), comprising the minimal catalytically active domain within the heteropentameric elF2B complex. B) Structural comparison of the eIF2BE-CTD (cyan) with the elF5-CTD (red). Both proteins adopt a structurally homologous atypical HEAT-repeat fold that provides surfaces for interactions with elF2.

\subsubsection{The eukaryal translation initiation factor 5B (eIF5B)}

elF5B is the second trGTPase involved in translation and, unlike elF2, universally conserved among the three domains of life with the orthologous alF5B and IF2 in archaea and bacteria, respectively [81]. Crystal structures of aIF5B and IF2 revealed a four domain architecture for both proteins with an N-terminal G domain followed by domains II to IV (Fig. 1.9) [82-84]. The G domain and domain II form the structural core, which in sequence and structure is homologous to the corresponding domains in other trGTPases such as EF-Tu, EF-G and elF2 $\gamma$, whereas the $\alpha / \beta$ domain III is idiosyncratic to a/elF5B/IF2. The C-terminal domain IV is connected to the preceding domains through a $\sim 40 \AA ̊$ long $\alpha$-helix and is structurally homologous to domain II; only the aerchaeal and eukaryal ortholgs contain two additional $\alpha$-helices at their very C-terminal end, which are packed against the $\beta$-barrel fold of domain IV. IF2 and elF5B contain an additional N-domain of up to 600 residues, which displays little conservation in sequence and length and, although implicated in 
mediating interactions with the small ribosomal subunit, was shown to be dispensable for elF5B function in yeast [85].

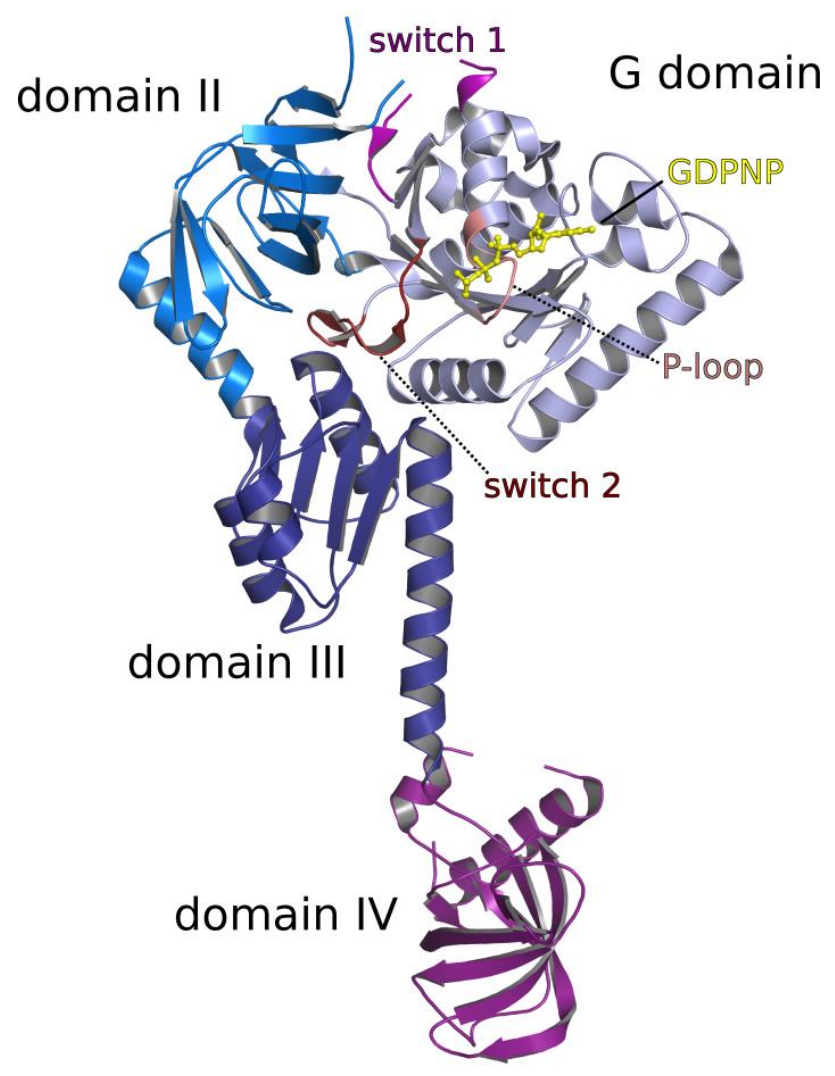

Figure 1.9: Structure of the trGTPase aIF5B. Crystal structure of GDPNP-bound alF5B from M. thermoautotrophicum (PDB: 1G7T). The color code for $G$ domain and domain II are the same as for the homologous domains in EF-Tu, EF-G and alF2 $\gamma$ in Fig. 1.6 and 1.7, with P-loop, switch 1 and switch 2 colored light red, magenta and dark red, respectively. GDPNP is shown as yellow balls and sticks. Domains III and IV are colored in dark blue and purple, respectively.
The principle role of elF5B is to promote subunit joining to form the elongation competent $80 \mathrm{~S}$ initiation complex. Following the dissociation of elF2.GDP and most other initiation factors, elF5B in its GTP-bound form binds to the pre-IC in a manner accelerated by interactions between the $\mathrm{C}$-terminal tail of $\mathrm{A}$ site-bound elF1A and the two a/elF5Bspecific $\alpha$-helices in domain IV [86-89]. Biochemical studies as well as the recent cryo-EM model of the yeast $80 \mathrm{~S}$ pre-IC containing a GDPNP-bound elF5B mutant (T439A) suggest that elF5B.GTP binds to the 435 pre-IC similar to IF2 on bacterial 305 pre-ICs with domain II interacting with the 40S subunit, while domain IV forms a direct contact to the acceptor stem of the MettRNA $A_{i}$ in the P-site $[85,90,91]$. Following its binding to the $43 \mathrm{~S}$ pre-IC, elF5B.GTP

promotes the association of the $60 \mathrm{~S}$ ribosomal subunit, which in turn triggers the intrinsic GTPase activity of elF5B. GTP hydrolysis and the subsequent transition to the GDP-bound state reduce elF5B affinity for the $80 \mathrm{~S}$ ribosome and trigger the concerted release of elF5B.GDP and elF1A from the 80 initiation complex [88]. 


\title{
Chapter $2 \cdot$ Interaction network between eIF2 and eIF5
}

This manuscript is in preparation for submission

\section{Analysis of the interaction network between eIF2 and eIF5 and its implications for the process of eukaryal translation initiation}

\author{
Bernhard Kuhle ${ }^{1, \S}$, Oliver Valerius ${ }^{2}$ and Ralf Ficner ${ }^{1}$ \\ 1 Abteilung für Molekulare Strukturbiologie, Institut für Mikrobiologie und Genetik, Göttinger \\ Zentrum für Molekulare Biowissenschaften, Georg-August-Universität Göttingen, D-37077 \\ Göttingen, Germany \\ ${ }^{2}$ Abteilung für Molekulare Mikrobiologie und Genetik, Institut für Mikrobiologie und Genetik, \\ Georg-August-Universität Göttingen, D-37077 Göttingen, Germany \\ \$To whom correspondence should be addressed: bkuhle@gwdg.de
}

Author contributions:

BK: designed the study and conceived the experiments; performed cloning, protein purification, crystallization, data collection and structure determination, crosslinking experiments, and biochemical studies on nucleotide binding by elF2; analyzed and interpreted the data; wrote the manuscript. OV: performed mass-spectrometry analysis of crosslinks and helped to analyze MSdata. RF: design of the study; helped to draft the manuscript. 


\section{Abstract}

Translation initiation results in the accurate assembly of the elongation-competent ribosome with the unique initiator tRNA positioned in its $P$ site, base paired to the AUG start codon of an mRNA. In eukarya, recruitment of the initiator tRNA (Met-tRNA ${ }_{i}$ ) to the ribosome is mediated by the heterotrimeric initiation factor 2 (elF2), a translational GTPase that requires the multifunctional effector protein elF5 to carry out its function. Here, we present a structural and biochemical analysis of the interaction network between elF2 and elF5. A high-resolution crystal structure of the complex between the elF5-CTD and the elF2 $\beta \mathrm{N}$-terminal tail in combination with Trp quenching experiments shows that recruitment of elF5 to elF2 occurs via a flexible peptide-domain interaction that provides a possible explanation for the dynamic interplay between elF2 and elf5 and other elFs that occurs during start site selection on the ribosome. A second direct interaction was mapped between the elF5-NTD, which is responsible for the stimulation of GTP hydrolysis by elF2 on the ribosome, and the GTP-binding eIF2 $\gamma$ subunit. Finally, the third interaction occurs between the elF5-CTD and elF2 $\gamma$ in the direct vicinity to the nucleotide binding pocket, which is likely to be responsible for the previously reported role of elF5 in stabilizing GDP on elF2. Together, the presented findings provide a more complete and refined picture of the intricate interactions between elF2 and elF5 that play central roles at various stages of the initiation process.

\subsection{Introduction}

In eukarya, translation is initiated by the formation of an elongation-competent 80S ribosome, assembled with the methionlyated initiator tRNA (Met-tRNA $A_{i}$ ) in its P-site base-paired to the AUG start codon of an mRNA. This process depends on the concerted action of at least 12 different auxiliary protein factors (elFs) that mediate the specific binding of mRNA and initiator tRNA, as well as the joining of the two ribosomal subunits by an intricate series of formation, rearrangement, and breaking of interactions and GTP hydrolysis [9, 12].

In the first step of translation initiation, GTP-bound elF2 binds the Met-tRNA $A_{i}$ to form a ternary complex (TC). Together with elFs 1, 1A, 5 and the elF3 complex, the TC is then recruited to the $40 \mathrm{~S}$ ribosomal subunit and placed over the ribosomal P-site, thereby forming the $43 \mathrm{~S}$ pre- 
initiation complex (pre-IC). Assisted by elF3 and the elF4F complex, the 435 pre-IC subsequently binds to the $5^{\prime}$-proximal region of an $\mathrm{mRNA}$, whereby it enters the scanning phase of the initiation process, in which the 5'-untranslated region of the mRNA is searched for the AUG start codon. Upon encounter of the correct initiation site, the scanning process is arrested by the formation of codon-anticodon interactions between the mRNA and initiator tRNA, which triggers a rearrangement of elF1A, dissociation of elF1 and finally the irreversible hydrolysis of the elF2bound GTP, stimulated by elF5. GDP-bound elF2 then dissociates from the scanning-arrested $40 \mathrm{~S}$ subunit together with elF5, and is subsequently recycled back to elF2.GTP by the guaninenucleotide exchange factor (GEF) elF2B. Instead of elF2, GTP-bound elF5B now binds to the 40S.Met-tRNA ${ }_{i}$ complex to promote the joining of the large 605 ribosomal subunit, thus forming the $80 S$ pre-IC. Subunit joining finally triggers GTP hydrolysis in elF5B, resulting in the dissociation of elF1A together with elF5B.GDP and the transition of the $80 \mathrm{~S} \mathrm{IC} \mathrm{into} \mathrm{the} \mathrm{elongation} \mathrm{phase} \mathrm{of}$ translation $[9,12,16]$.

The focus of this work lies on the elucidation of the molecular details of the intricate interaction network between the two central initiation factors elF2 and elF5. elF2 is composed of three subunits (elF2 $\alpha,-\beta$, and $-\gamma$ ), each of which has orthologs in archaea (alF2) but not in bacteria [28]. The elF2 $\gamma$ subunit, a paralog of EF-Tu, belongs to the family of Ras-related $G$ proteins and is responsible for the GTP-dependent binding of Met-tRNA $A_{i}$ to form the TC, assisted by the $\alpha$ - and $\beta$ subunits $[32,41,42]$. The TC does not bind to the $40 \mathrm{~S}$ subunit on its own, but instead depends on the concerted action of additional factors, namely elFs $1,1 \mathrm{~A}, 3$ and $5[12,58,59]$. Hereby, the direct interaction between three lysine-rich segments (K-boxes 1-3) in the eukarya-specific Nterminal tail (NTT) of elF2 $\beta$ and the negatively charged AA-boxes $1 \& 2$ in the C-terminal domain (CTD) of elF5 plays a central role to allow the efficient recruitment of the TC [35]. The elF5-CTD thereby acts as nucleation core that mediates cooperative interactions between elF2 $\beta$-NTT, elF1 and the eIF3c-NTD for the formation of an intermediate multi-factor complex (MFC; composed of elFs 1, 3, 5 and the TC) and finally the proper incorporation of the TC into the 43S pre-IC [56, 57, 59]. Consequently, the loss or weakening of the elF5-CTD-elF2 $\beta-N T T$ interaction results in a significant reduction of TC recruitment to the 40 S subunit $[35,57,92]$. 
Recent studies suggest an additional role for the elF5-CTD·elF2 $\beta$-NTT interaction during scanning and start site recognition by destabilzing eIF1 binding to the pre-IC that seems to involve the loss of interactions between elF1 and elF2 $\beta$-NTT and instead the formation of a stronger interaction of the latter to elF5 in response to start-site recognition $[21,63]$. Together with the displacement of the eIF1A C-terminal tail (CTT) from the P-site by the Met-tRNA $A_{i}$, the resulting release of elF1 triggers conformational rearrangements of the 40S subunit from an 'open' scanning competent conformation to its 'closed' scanning arrested state, ultimately causing the irreversible hydrolysis of the elF2-bound GTP by the gated release of $\mathrm{P}_{\mathrm{i}}[64]$. Here, elF5 again plays a critical role with its N-terminal domain acting as GTPase activating protein (GAP) to trigger the GTPase activity in elF2, most likely by introducing an arginine-residue (Arg15 in yeast elF5) as catalytic residue into the GTPase center $[52,53,64]$.

After their release from the pre-IC, elF2-GDP and elF5 were found to remain tightly associated, giving rise to a large cytosolic pool of elF2-elF5 complexes that significantly exceeds the levels of TC in the cell [68]. This observation suggested that elF5 might serve an additional role in the recycling pathway of elF2-GDP by antagonizing the guanine-nucleotide exchange (GEF) activity of elF2B $[68,69]$. In line with this assumption an evolutionarily conserved region within elF5, called DWEAR-motif, was found to be responsible for a reduced rate of GDP dissociation from elF2. This suggested that elF5 indeed contains a guanine-nucleotide dissociation inhibitor (GDI) activity, in addition to its ability to compete physically with elF2B for their common substrate elF2. Based on these and genetic observations, it was proposed that elF5 provides a regulatory function under stress conditions by antagonizing spontaneous or GEF-catalyzed recycling of elF2.GTP and TC formation in addition to the mechanism of elF2B inhibition by elF2 $\alpha$ phosphorylation [69].

Over the last years, considerable progress has been achieved in the elucidation of the intricate series of molecular events that govern the involvement of elF2 and eIF5 in pre-IC formation, start codon recognition and recycling of elF2 [12]. However, a detailed understanding of these processes is still limited, in part due to the highly dynamic and transient nature of most of the involved interactions that, as a result, escape most biochemical techniques and resist high-resolution structural investigations. By combining different high- and low-resolution structural approaches with mutational and biochemical methods we were able to analyze the complex interaction 
network between elF2 and its multifunctional effector protein elF5, as well as its influence on the association of elF1 and elF3c with the elF5-CTD. We find that elF5 forms three major contact sites with the elF2 complex. One is formed by the N-terminal portion of the elF5-NTD, which harbors the putative catalytic arginine-finger and specifically contacts the elF2 $\gamma$ subunit in the presence and

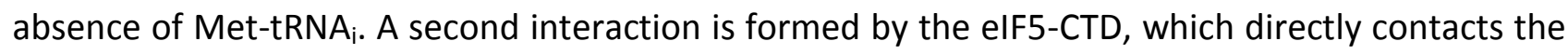
$\gamma$-subunit close to the nucleotide binding site of the $G$ domain. Interestingly, we could show that this interface involves the DWEAR-motif, which seems to form an element of the CTD itself and not, as previously assumed, of the flexible linker region between the $\mathrm{N}$ - and C-terminal domains. Using fluorescence-labeled nucleotide analogs, we show that this interaction, which is formed irrespective of the nucleotide-state of elF2 or the presence of Met-tRNA $A_{i}$, is responsible for the stabilization of elF2-bound guanine-nucleotides. Finally, the third interaction, which is responsible for the high affinity binding of the two factors, is formed between the AA-boxes in elF5-CTD and the K-boxes in the elF2 $\beta$-NTT. The first high-resolution crystal structure of an elF5-CTD-elF2 $\beta$-NTT complex, in combination with fluorescence-based tryptophan quenching and mass-spectrometrycoupled crosslinking experiments, indicates that the two factors interact via a peptide-domain interaction, which allows a dynamic interplay with the simultaneously bound elF1 and elF3c. Together, the presented data provide a more complete and refined picture of interactions occurring between elF2 and its effector protein elF5, with implications for the chain of molecular events that accompany pre-IC formation, start codon recognition and eIF2 recycling.

\subsection{Results}

Contributions of the lysine-rich patches K1, K2 and K3 of the elF2 $\beta-N T T$ to the interactions with elF5-CTD

In order to gain insight into the roles of the individual K-boxes for the interactions between elF2 $\beta$ NTT and eIF5-CTD, complex formation between both proteins was monitored by means of tryptophan quenching. For this purpose an elF5-CTD construct was used comprising residues 241405 which contains two tryptophan residues, Trp245 and Trp391. According to the previously determined crystal structure of elF5(241-405)(PDB: 2FUL) [1]., the universally conserved Trp391 in AA-box 2 lies close to the C-terminus of the CTD and thereby in direct vicinity to the negatively 
A
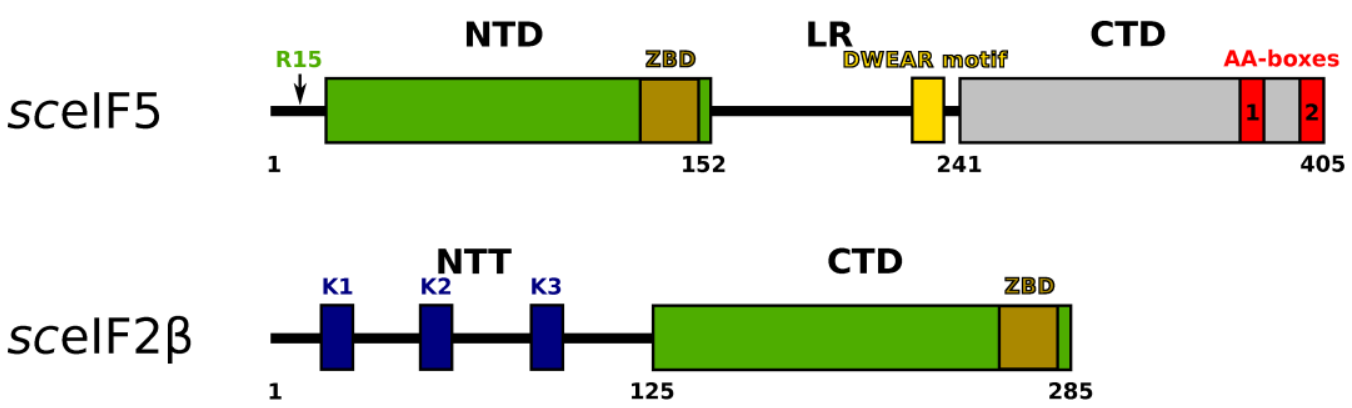

B
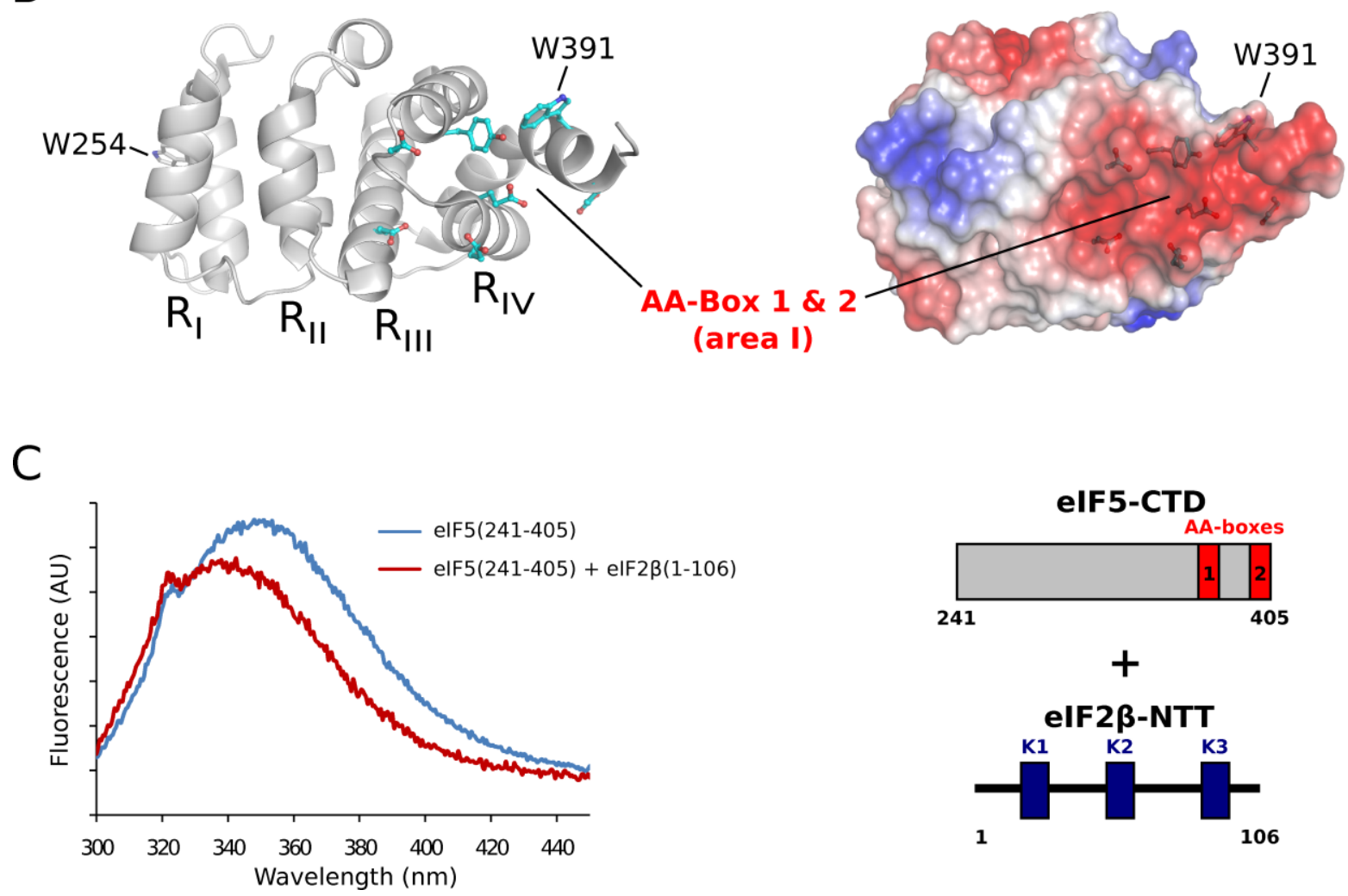

Figure 1. A)Schematic presentation of the proposed domain architecture in elF5 and elF2 $\beta$.B) Crystal structure of the C-terminal HEAT-like domain of elF5 (residues 241-396) from S. cerevisiae(PDB: 2FUL) [1]. The left panel shows the eIF5-CTD in cartoon presentation; the four HEAT-like repeats $\left(R_{1}-R_{I V}\right)$ are indicated, with the conserved Trp245 in $R_{l}$ and Trp391 in $R_{\mathrm{IV}}$. Next to Trp391, conserved residues of the AA-boxes are shown as balls and sticks. The right panel shows the surface charge distribution of eIF5-CTD (negative, red; positive, blue), revealing the highly negatively charged area I, formed by residues of the two AA-boxes, next to Trp391. C) Trp-quenching by elF2 $\beta$ on the elF5-CTD. The chromatogram shows the emission spectrum of elF5(241-405) upon the excitation of the Trp fluorescence at $280 \mathrm{~nm}$ in the absence (blue) and presence (red) of the elF2 $\beta-N T T$.

charged area I, implicated in binding of the K-boxes of elF2 $\beta$ (Fig. 1 and S1). We therefore reasoned that binding of the lysine-rich patches of the elF $2 \beta-\mathrm{NTT}$ in this area should be reflected in the 
fluorescence signal of Trp391, either indirectly by its conformational rearrangement or directly through proton transfer from a nearby positioned $\varepsilon$-amino group.

A

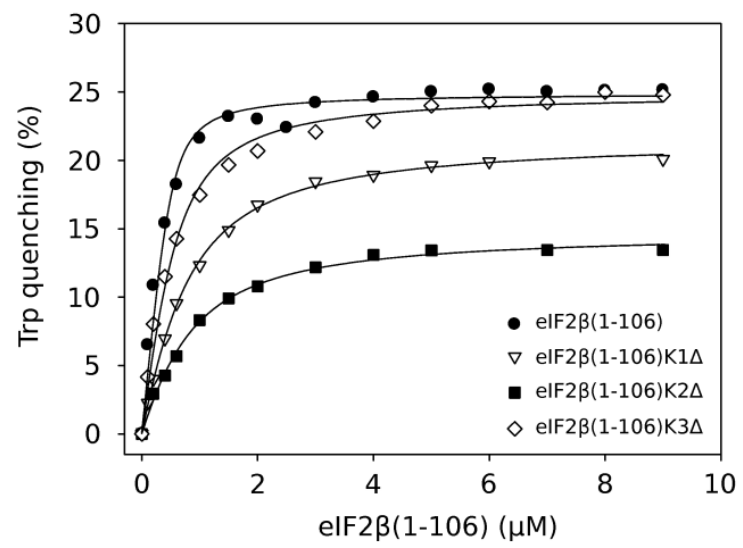

C

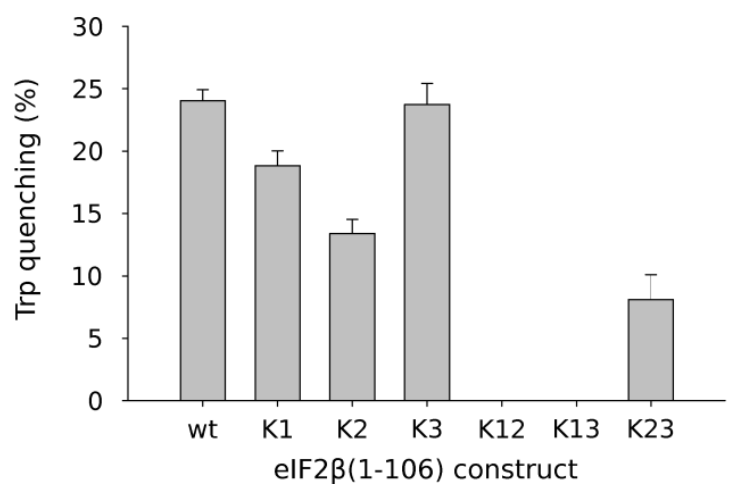

B

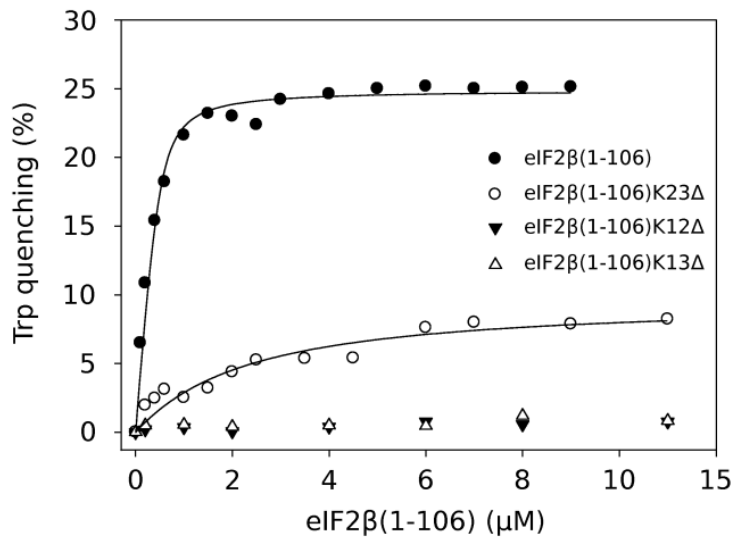

Figure 2. Trp-quenching experiments between elF5-CTD and elF2 $\beta$-NTT. A) Equilibrium titrations of $0.5 \mu \mathrm{M}$ elF5(241405 ) with increasing amounts of wild-type elF $2 \beta(1-106)$ or the three single K-box mutants K1 $\Delta, \mathrm{K} 2 \Delta$, and K3$\Delta$. The Trp quenching signal (in \% of the original fluorescence signal of elF5(241-405)) was plotted against the concentration of the respective elF $2 \beta$-NTT constructs. The black lines show the fit to the data, using a quadratic one-side binding model. B) Equilibrium titration of $0.5 \mu \mathrm{M}$ elF5(241-405) with wild-type elF2 $\beta(1-106)$ or the three double K-box mutants K12 $\Delta$, $\mathrm{K} 13 \Delta$, and $\mathrm{K} 23 \Delta$. C) Comparison of the Trp quenching signals for the seven elF2 $\beta(1-106)$ constructs. Standard deviations are given as error bars.

In line with this assumption, the addition of elF2 $\beta$ (which contains no Trp) to elF5(241-405) results in a significant decrease in the fluorescence signal at $360 \mathrm{~nm}$ (excitation at $290 \mathrm{~nm}$ ), accompanied by a blueshift of the emission maximum from 350 to $340 \mathrm{~nm}$ (Fig. 1C). Both effects are lost in the elF5(241-405)W391F mutant (Fig. S2). Titration of elF5(241-405) with increasing amounts of elF $2 \beta$ gives an estimate for the $K_{\mathrm{d}}$ of $\sim 100 \mathrm{nM}$ and a maximum of $\sim 25 \%$ quenching of 
the initial fluorescence signal at saturating eIF $2 \beta$ concentrations. Similar values are obtained with $N$-terminal fragments of elF2 $\beta$, containing either residues 1-148 ( $K_{d}=95 \mathrm{nM}$ and $\sim 24 \%$ quenching) or 1-106 ( $K_{d}=70 \mathrm{nM}$ and $\sim 24 \%$ quenching) (Fig. $2 \mathrm{~A}$ and Table 1$)$. These values are consistent with the assumption that the NTT provides the entire binding region for interactions of elF2 $\beta$ to elF5CTD and are in good agreement with the reported affinity $\left(K_{d}=40 \mathrm{nM}\right)$ between elF5-CTD and the heterotrimeric elF2 complex [21, 35, 36]. We therefore used the shortest fragment containing all three K-boxes (elF2 $\beta(1-106))$ for the subsequent mutational analysis.

Table 1. Trp quenching experiments between elF5(241-405) and elF2 $\beta$.

\begin{tabular}{|c|c|c|c|}
\hline elF $2 \beta$ construct & $K_{\mathrm{d}}(\mathrm{nM})$ & $\begin{array}{l}\text { Reduction of affinity } \\
\text { relative to wt }\end{array}$ & $\begin{array}{l}\text { Quenching signal } \\
(\%)\end{array}$ \\
\hline elF2 $\beta(F L) w t$ & $100 \pm 44$ & -- & 25 \\
\hline elF2 $\beta(1-148) w t$ & $95 \pm 24$ & -- & 24 \\
\hline elF2$\beta(1-106) w t$ & $70 \pm 20$ & -- & 24 \\
\hline eIF2 $\beta(1-106) K 1 \Delta$ & $825 \pm 55$ & 12-fold & 19 \\
\hline elF2 $\beta(1-106) K 2 \Delta$ & $550 \pm 45$ & 8-fold & 13 \\
\hline $\operatorname{eIF} 2 \beta(1-106) K 3 \Delta$ & $200 \pm 19$ & 3-fold & 24 \\
\hline elF2 $\beta(1-106) K 12 \Delta$ & N.D. & & No signal \\
\hline elF2 $\beta(1-106) K 13 \Delta$ & N.D. & & No signal \\
\hline elF2 $\beta(1-106) K 23 \Delta$ & $2200 \pm 400$ & 30-fold & 8 \\
\hline
\end{tabular}

Either one or two of the K-boxes in the elF2 $\beta$-NTT (Fig. 1A) were deleted by mutation to alternating Ala and Ser residues. Among the single K-box mutants, the most severe effect was observed for $\mathrm{K} 1 \Delta$ exhibiting a $\sim 12$-fold increase in the dissociation constant $\left(K_{d}=825 \mathrm{nM}\right)$ and a simultaneous loss of nearly $20 \%$ of the quenching signal (Fig. $2 \mathrm{~A} / \mathrm{C}$ and Table 1). K2 $\Delta$ resulted in an $\sim 8$-fold loss of binding affinity $\left(K_{d}=550 \mathrm{nM}\right)$, accompanied by a loss of nearly $50 \%$ of the quenching signal. Finally, $\mathrm{K} 3 \Delta$ exhibited the smallest effect with an only 3 -fold reduction in binding affinity $\left(K_{\mathrm{d}}\right.$ $=200 \mathrm{nM}$ ) and no effect at all on the quenching signal. 
Among the double K-box mutants only $\mathrm{K} 23 \Delta$ exhibited a quenching signal ( $30 \%$ of the signal observed for wild-type elF2 $\beta$ ), allowing an estimate of $2.2 \mu \mathrm{M}$ for the $K_{\mathrm{d}}$, corresponding to a $\sim 30$ fold reduction in binding affinity (Fig. $2 \mathrm{~B} / \mathrm{C}$ and Table 2). By contrast, neither K12 $\Delta$ nor K13 $\Delta$ exhibited any quenching signal upon addition to elF5(241-405). For $K 23 \Delta$ and $K 12 \Delta$ these observations are compatible with the effects of the single K-box deletions. However, it is interesting to note that both, the loss of binding affinity and the quenching signal for the $\mathrm{K} 23 \Delta$ double mutant are slightly larger than an entirely additive contribution by K2 and K3 would suggest. Moreover, the sole presence of K2 (in K13 4 ), which according to the single mutations contributes significantly to the binding affinity and $\sim 50 \%$ of the total quenching signal, results in no signal at all. Thus, $\mathrm{K} 3$ actually does seem to provide contributions in $\mathrm{K} 1 \Delta$ and $\mathrm{K} 2 \Delta$, which, however, do not become apparent from its single-deletion.

These results demonstrate that K1 and K2 provide the principal contact surface for elF5-CTD, both with immediate effects on the surrounding of Trp391. This central role of Trp391 in the interactions with the elF2 $\beta-\mathrm{NTT}$ is further supported by the complete loss of the quenching signal and a severe negative impact on the interactions in GST-pull-down assays with elF5(241405)W391A, -W391F (Fig. S2). At the same time, K3 seems to provide only a small contribution - at least in the presence of $\mathrm{K} 1$ and $\mathrm{K} 2$-, which, moreover, does not involve a direct effect on Trp391. Although in this kind of experiments no quenching signal is not necessarily indicative of no binding, our results suggest that $\mathrm{K} 2$ binding is partially assisted by either $\mathrm{K} 1$ or $\mathrm{K} 3$ for its full binding affinity, and that $\mathrm{K} 3$ is able to partially substitute for the loss of K2 in the K $2 \Delta$ mutant, possibly by binding to similar surface residues on elF5-CTD in the absence of $\mathrm{K} 2$.

\section{Crystal structure of elF2 $\beta(39-106)$ bound to elF5(201-405)}

Despite the critical role which the elF5-CTD plays as nucleation point for other elFs during MFC and pre-IC assembly, scanning and start codon recognition, no structural information that would allow direct insight into the molecular details of any of these interactions was so far available. We therefore intended to determine the structure of the eIF5-CTD in complex with the elF2 $\beta$-NTT by means of X-ray crystallography. Based on the results from the Trp-quenching experiments we were particularly interested in the mode of binding of K3, as the contributions of this lysine-patch 
Table 2. Data collection and refinement statistics for the structure of the elF5(201405)·elF2 $\beta(39-106)$ complex from S. cerevisiae

\begin{tabular}{ll}
\hline Crystallization & $0.8 \mathrm{M}\left(\mathrm{NH}_{4}\right) \mathrm{SO}_{4}$, \\
\hline Condition & $0.3 \mathrm{M} \mathrm{LiSO}_{4}$ \\
Temperature $\left({ }^{\circ} \mathrm{C}\right)$ & 20 \\
\hline Data Collection & $\mathrm{P} 3_{2} 12$ \\
\hline Space Group & $\mathrm{a}=74.3 \AA$ \\
Unit Cell & $\mathrm{b}=74.3 \AA$ \\
& $\mathrm{c}=124.8 \AA$ \\
& \\
& $\alpha=90^{\circ}$ \\
& $\beta=90^{\circ}$ \\
& $\gamma=120^{\circ}$ \\
Resolution $(\AA)$ & $2.0(2.1-2.0)$ \\
Observed reflections & $359597(87743)$ \\
Unique reflections & $48223(11831)$ \\
Completeness (\%) & $99.9(99.8)$ \\
$<1>/ \sigma$ & $22.3(3.9)$ \\
$\mathrm{R}_{\text {sym }}(\%)$ & $8.9(42.8)$ \\
\hline Refinement &
\end{tabular}

\section{Refinement}

$\mathrm{R}_{\text {work }} / \mathrm{R}_{\text {free }}(\%) \quad 17.4 / 20.1$

\section{Rmsd from Strd. Stereochemistry}

$\begin{array}{ll}\text { Bond length }(\AA) & 0.007 \\ \text { Bond angles }\left({ }^{\circ}\right) & 0.98\end{array}$

\section{Ramachandran Plot Statistics}

Most favored (\%)

98.5

Allowed regions (\%)

1.5

Disallowed regions (\%)

Values in parentheses refer to the highest resolution shell.

$R_{\text {work }}$ and $R_{\text {free }}$ factors are calculated using the formula $R=\Sigma_{\text {hk }}|| F(o b s)_{h k l} \mid$

- $\left|\mathrm{F}(\mathrm{calc})_{\mathrm{hk}}\right|\left|/ \Sigma_{\mathrm{hkl}}\right| \mathrm{F}(o b s)_{\mathrm{hkl}} \mid$, where $\mathrm{F}(o b s)_{\mathrm{hkl}}$ and $\mathrm{F}(\mathrm{calc})_{\mathrm{hkl}}$ are observed and measured structure factors, respectively. $R_{\text {work }}$ and $R_{\text {free }}$ differ in the set of reflections they are calculated from: $R_{\text {free }}$ is calculated for the test set, whereas $R_{\text {work }}$ is calculated for the working set. seemed to be contingent on the overall binding situation, apparently at odds with its universal conservation among elF $2 \beta$ orthologs across the eukaryal lineages. We therefore purified $\mathrm{N}$-terminal fragments of S. cerevisiae elF2 $\beta$ containing either all three K-boxes (residues 1-106) or only K2 and K3 (residues 39-106) in complex with the C-terminal domain of S. cerevisiae elF5 (containing either residues $241-405$ or 201405) from $E$. coli cells. The longer construct of elF5 including residues 201-240 was chosen in order to gain additional insight into the structural arrangement of the highly conserved 'DWEAR-motif' (residues 220-238), previously assigned to the flexible linker region between elF5-NTD and -CTD and proposed to stabilize GDP binding by elF2 in elF2-elF5 complexes[69] (Fig. S1).

Among the four purified combinations, only the elf5(201405)·elF2 $\beta(39-106)$ complex yielded crystals of sufficient quality to allow structure determination. The final model, refined at a resolution of $2.0 \AA$, contains two elF5-CTD copies per asymmetric unit including residues 201 to 399 (with the additional vector-encoded residues PGLGS at the $\mathrm{N}$ terminus) (Details of data collection and 


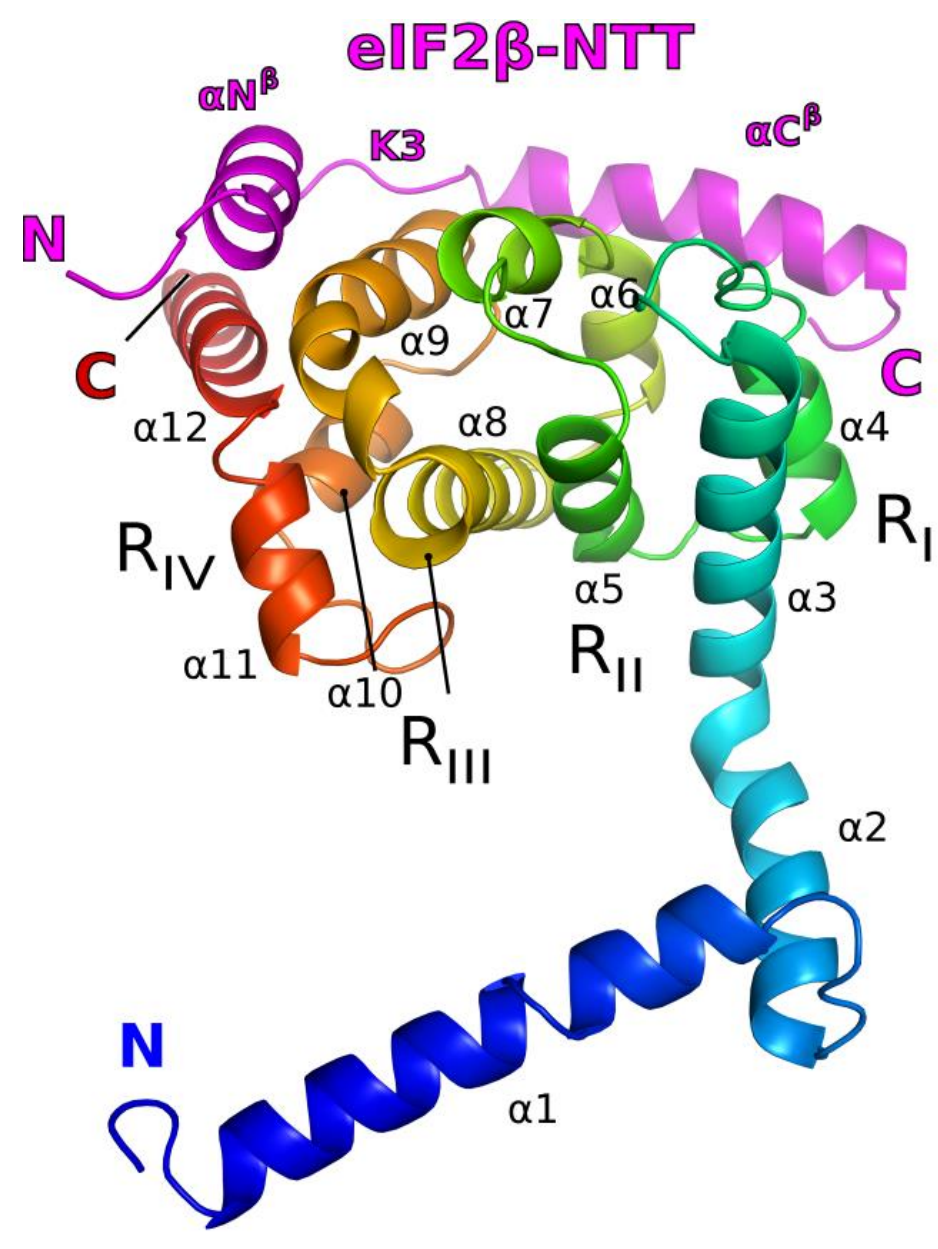

Figure 3. Crystal structure of an elF2 $\beta$-NTT fragment bound to the elF5-CTD. The elF5(201-399).elF2 $\beta(66-106)$ complex is shown in cartoon presentation with elF2 $\beta$ colored pink and the elF5-CTD shown in rainbow coloring ( $\mathrm{N}$-terminus, blue; $\mathrm{C}$-terminus, red). The body of the elF5-CTD is formed by ten $\alpha$-helices ( $\alpha 3-\alpha 12$ ), folding into four atypical HEAT repeats $R_{1}-R_{\mathrm{IV}}$ (compare Fig. 1B). The $N$-terminal helices $\alpha 1$ and $\alpha 2$ protrude from the HEAT-like domain and form crystal contacts to symmetry related molecules. The bound elF2 $\beta$-NTT fragment folds into two $\alpha$-helices $\left(\alpha N^{\beta}\right.$ and $\alpha \mathrm{c}^{\beta}$ ) connected by a loop region containing K-box 3 (K3). The elF2 $\beta$-NTT thereby adopts an extended conformation in which it wraps around the elF5 HEAT-like domain, placing its $\mathrm{N}$-terminus (residue 66) at repeat $\mathrm{R}_{\mathrm{IV}}$, while the C-terminus (residue 106) is placed atop of $R_{1}$. refinements statistics are summarized in

Table 2). Residues 241-399 of both elF5 molecules adopt a fold composed of four antiparallel helical repeats $\left(R_{1}-R_{V_{I}}\right)$ (Fig. 3) that is virtually identical to that of the previously reported yeast elF5CTD structure (rmsd of $0.8 \AA$ over $133 \mathrm{C}_{\alpha}$ atoms) [1]. The remaining $\mathrm{N}$-terminal residues (201-240) fold into two additional $\alpha$-helices ( $\alpha 1$ and $\alpha 2$ ) that protrude from the globular part of the elF5B-CTD and form extensive contacts to symmetry related molecules in the crystal packing. Only one of the two elF5-CTD molecules binds a fragment of elF2 $\beta$ which includes only residues 66106 and folds into two amphipathic $\alpha$ helices (hereafter termed $\alpha \mathrm{N}^{\beta}$ and $\alpha \mathrm{C}^{\beta}$ ), connected by a loop formed by K-box 3 (Fig. 4). Residues 39 to 65, including K2, are not defined in the electron density. 

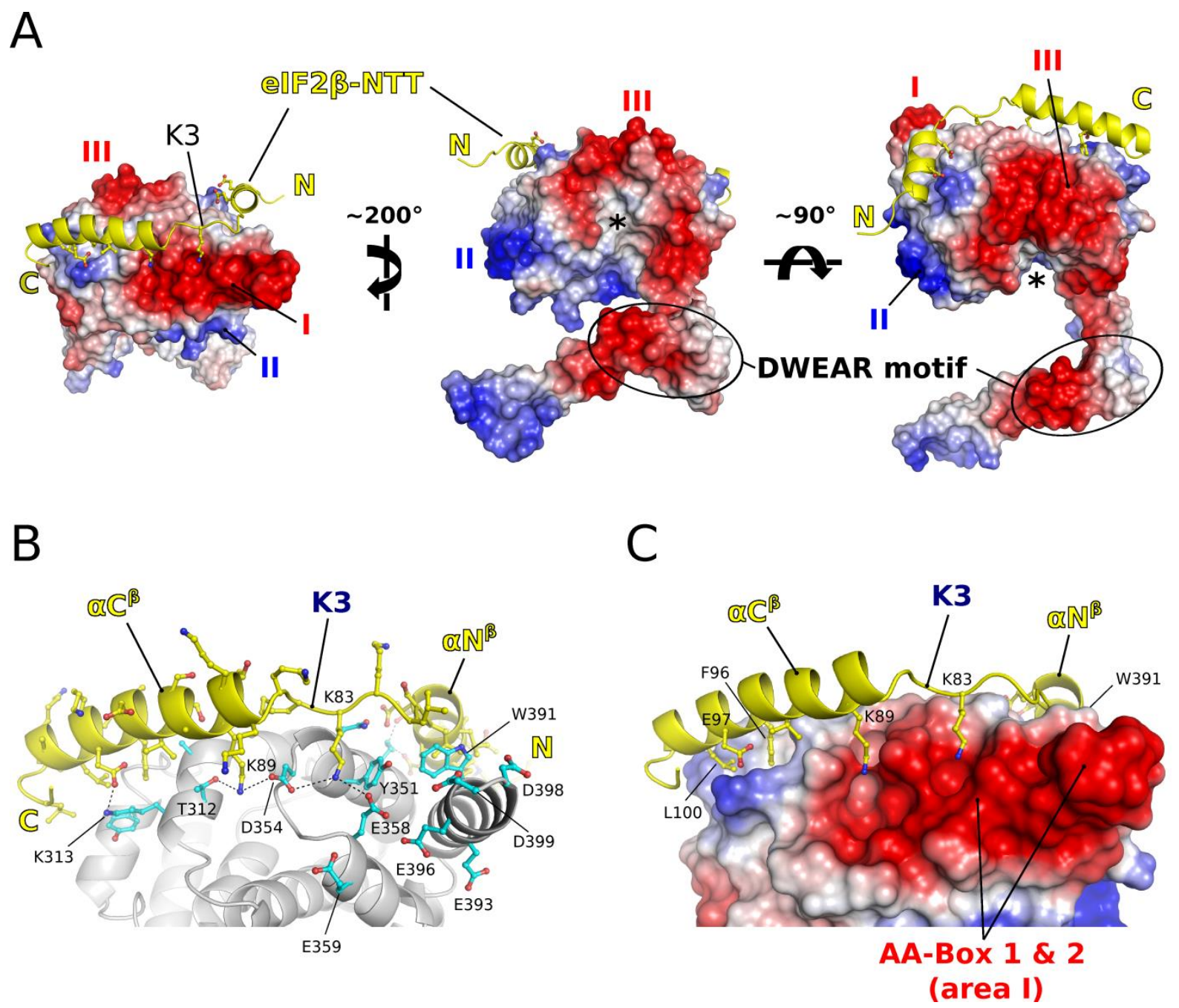

Figure 4. Peptide-domain interaction between the elF2 $\beta-N T T$ and elF5-CTD. A) The elF5(201-399)-elF2 $\beta(66-106)$ complex from different orientations. The elF5-CTD is shown with surface charge presentation (negative, red; positive, blue), demonstrating how the elF2 $\beta$-NTT (yellow cartoon) adopts an extended conformation to associate with the regions between the charged areas I-III through the amphipathic helices $\alpha \mathrm{N}^{\beta}$ and $\alpha \mathrm{C}^{\beta}$, thereby placing $\mathrm{K}$-box 3 (K3) in direct vicinity to the negatively charged area I. The middle and right panel reveal the existence of a hydrophobic cleft adjacent to area III (asterisk). B) Details of the interactions between K-box 3 of elF2 $\beta$ (yellow) and the AA-boxes of elF5 (grey, with conserved AA-box residues and residues involved in direct contacts with elF $2 \beta$ shown as balls and sticks in cyan). Among the seven lysine residues of K-box 3, only Lys83 and Lys89 form stable polar interactions with AA-box 1 (indicated by black dashed lines). C) The surface charge distribution of elF5-CTD reveals that Lys83 and Lys89 are inserted into negatively charged pockets from the periphery of area I, while the major part of the negative surface area remains available for interactions with K-boxes 1 and 2 (not present in the crystal structure).

Helix $\alpha N^{\beta}$ (residues 69-79) lies between helices $\alpha 9$ and $\alpha 12$ of elF5, on one side contacting Lys345 of elF5 through Asp88 and Glu91 and on the other side forming a hydrophobic interface with Pro344, Ile348, Pro387, Phe388 and Trp391. Following helix $\alpha \mathrm{N}^{\beta}$, K3 comes to lie at the very Cterminus of helix $\alpha 9$ of elF5-CTD and thereby in immediate vicinity to the negatively charged 
surface formed by the two conserved AA-boxes in elF5 (area I) (Fig. 4). However, only two of the seven lysine residues, Lys83 and Lys89, form a direct contact to AA-box 1, with Lys83 lying in a pocket formed by Tyr351, Asp354 and Glu358, and Lys89 lying between Thr312 and Asp354 (Fig. $4 \mathrm{~B} / \mathrm{C})$. The remaining lysine residues of K-box 3 are poorly resolved in the electron density, indicating that they either do not participate in the interactions with elF5 or that the formation of stable interactions is prevented by the relatively high salt concentrations at which the crystals were obtained (400 mM $\left(\mathrm{NH}_{4}\right)_{2} \mathrm{SO}_{4}$ ); this may as well be responsible for the inability of $\mathrm{K}$-box 2 to form a stable interaction under these conditions. In agreement with the absence of a strong contribution to the quenching signal in the fluorescence experiments, no lysine residue in $\mathrm{K}$-box 3 is stably positioned in the direct vicinity to Trp391. Finally, helix $\alpha C^{\beta}$ of elF2 $\beta-N T T$ (Lys89-Ala103) is packed on top of and orthogonal to helices $\alpha 7$ and $\alpha 4$ of elF5, with Phe96 and Leu100 positioned in a hydrophobic pocket on the elF5-CTD surface formed by Val270, Tyr273, Ala309 and the aliphatic part of the Lys313 side chain, whose $\varepsilon$-amino group additionally forms a salt bridge to Glu97 of elF2 $\beta$ (Fig. 4B/C).

Thus, the co-crystallized portion of the elF2 $\beta-N T T$ adopts an elongated conformation containing secondary structure elements but no tertiary structure, thereby allowing an extended interaction interface that reaches half around the elF5-CTD (Fig 4A). Interestingly, the elF2 $\beta$-NTT thereby only peripherally contacts the negatively charged area I of elF5 through K-box 3 and mainly occupies those regions lying between the negatively charged areas I and III and the positively charged area II, which is thought to provide the main interface for interactions with elF3c and elF1 [57]. K-box 3 thereby leaves most of area I available for interactions with $\mathrm{K} 1$ and K2, including the highly negatively charged C-terminal end (Glu393-Glu405), as well as Glu358 and Glu359 in AA-box 1 , both of which are critical for high affinity binding of the elF2 $\beta$-NTT $[57,93]$. This proposed binding interface for the elF $2 \beta-N T T$ is in good agreement with the previously reported contact sites for a human elF2 $\beta$-NTT fragment containing K-boxes 2 and 3 on hselF5-CTD, determined by NMR chemical shift assays[63] (Fig. S3B/C). 


\section{The DWEAR-motif as transient element of the elF5-CTD.}

An interesting and yet unrecognized characteristic of charge distribution and morphology on the surface of elF5-CTD is a broad, mainly hydrophobic cleft lying parallel to helix $\alpha 3$ and ending on one side in the acidic area III (Fig. 4A and S3A). The comparison of the available elF5-CTD structures shows that this cleft is conserved between fungi and mammals and may thus play a functional role. In the human ortholog the lower half of the cleft (next to the N-terminus of helix $\alpha 3$ ) is occupied by an $\alpha$-helix formed by 11 residues of the extended C-terminal end (Fig. S4A). However, this extension is idiosyncratic to the human ortholog and not found in fungal elF5, where the Cterminal end is physically incapable of reaching this far, suggesting an alternative role for the hydrophobic cleft. Our structure of yeast elF5(201-405) suggests that this role may in fact be the at least transient - accommodation of helix $\alpha 2$ (residues 229-241) in a manner antiparallel to helix a3 (Fig. 5).

To test this possibility, we took advantage of the technique of genetic code expansion which allows the incorporation of unnatural amino-acids such as the UV-inducible crosslinker p-benzoylL-phynylalanine (Bpa) into specific positions of a protein[94]. The idea was that the accommodation of helix $\alpha 2$ into the hydrophobic cleft of the elF5-CTD should be reflected in a specific crosslinking pattern, where the internal crosslink would be manifested in a faster migration speed during SDS-PAGE due to the formation of the more compact internal lariat topology of the primary structure. For this purpose Bpa was individually introduced in a number of positions of elF5 along the DWEAR-motif (Asp220, Asp221, Trp223, Ala234, Glu238), and in area III (Glu261) (Fig. 5B). The purified elF5(201-405)Bpa constructs were exposed to UV-radiation and the crosslinking products were analyzed by SDS-PAGE. In line with our assumption, UV-exposure resulted in the emergence of additional bands migrating faster than the original elF5(201-405)Bpa constructs (Fig. 5C). No additional bands appeared at higher molecular weight, indicating the absence of dimer or oligomer formation. While no crosslinking was observed for the position Asp220, the strongest crosslink bands were obtained in the cases of E238-, A234-, E261Bpa and to a lesser extend, although still significant, for D221- and W223Bpa. Importantly, MS analysis confirmed that these bands are the product of internal crosslinks in elF5(201-405), and the search for the target peptide of the crosslink suggests proximity of A234- and E238Bpa to helix a8 
$\left({ }^{330} \mathrm{RF}^{331}\right)$ and the loop preceding helix $\alpha 11\left({ }^{372} \mathrm{FVPK}^{375}\right)$, while E221Bpa in area III was found to crosslink to the C-terminal half of helix $\alpha 8\left({ }^{335} \mathrm{HK}^{336}\right)$ (Fig. 5B and Table 3).

A

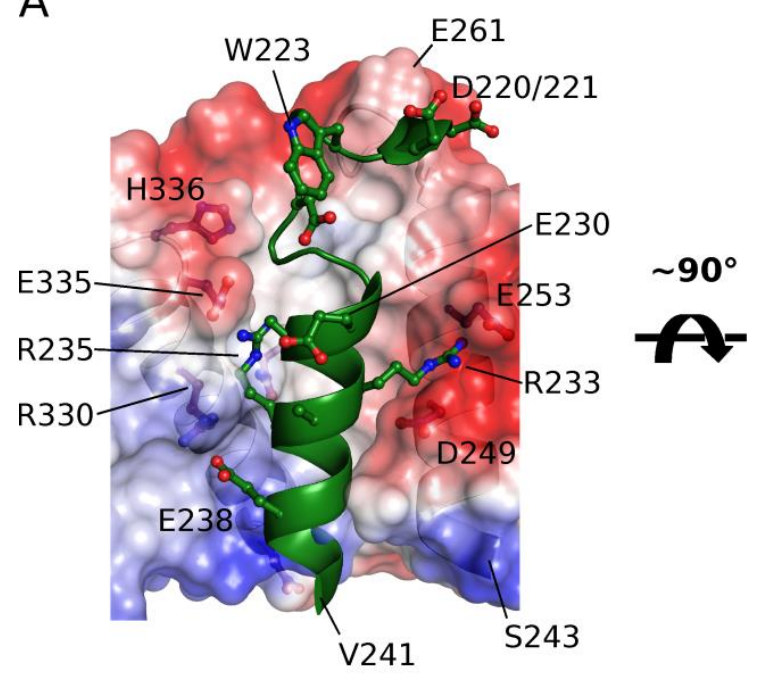

B

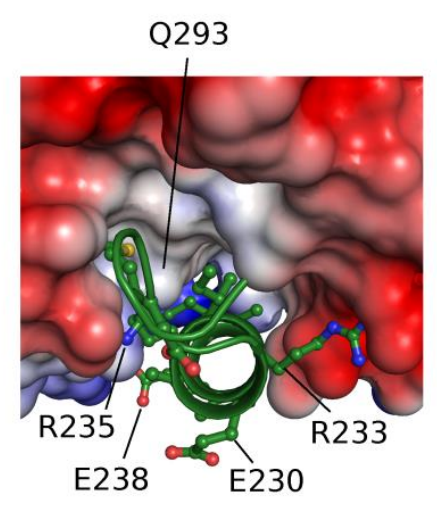

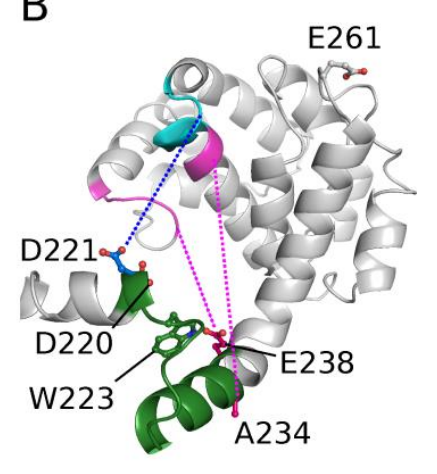

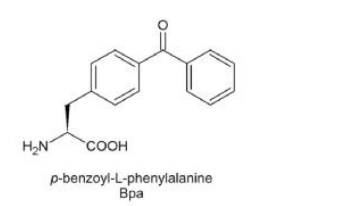

C

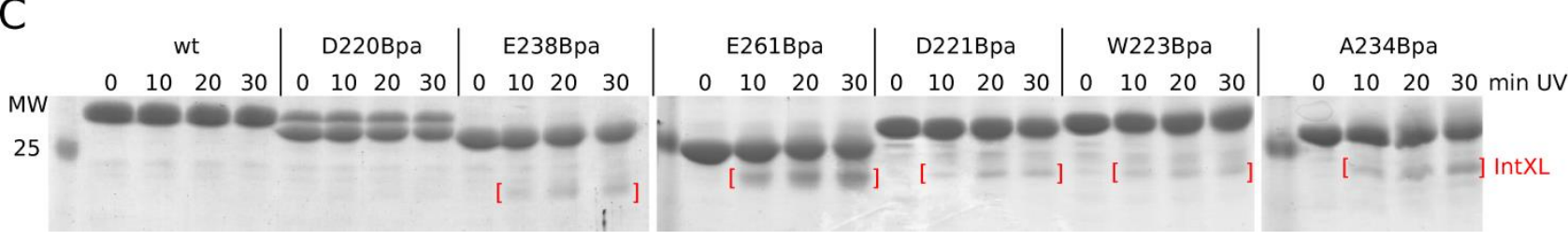

Figure 5. The DWEAR-motif as transient element of the elF5-CTD. A)Proposed model of the DWEAR-motif (green) as part of the C-terminal HEAT-like domain of elF5. According to this model, the amphipathic helix $\alpha 2$ is accommodated into the hydrophobic cleft between helices $\alpha 3, \alpha 5$ and $\alpha 8$, thereby allowing the conserved Arg233, Arg235 and Glu238 of the DWEAR-motif to form ionic interactions with Glu253, Glu335 and Arg330, respectively. At the same time, Met227, Ile232, Ala236, and Leu239 of helix $\alpha 1$ point toward helix $\alpha 3$ thus forming a continuous hydrophobic interface within the cleft. This places the N-terminus of the DWEAR-motif with Asp220, Asp221, Trp223, Asp226, and Glu230 next to Glu261, resulting in an extended negatively charged area III. Arg330, Glu335 and His336 indicate positions within helix $\alpha 8$ that were identified as target peptides for Bpa-crosslinks from Asp221 and Glu238 (see B). Compare also Fig. S4C. B)Positions in elF5-CTD where the UV-inducible crosslinker p-benzoyl-L-phenylalanine (Bpa) was introduced (substituted residues are shown as sticks). The DWEAR-motif is colored green. Peptides that were identified as targets for internal crosslinks from Ala234/Glu238 and Asp221 are colored pink and cyan, respectively (see also Table 3). C) SDS-gels showing the UV- and time-dependent formation of internal crosslink bands (IntXL) for the elF5(201-405)Bpa constructs (5 $\mathrm{MM}$ of the protein were exposed to UV light for the indicated time on ice); crosslinks are indicated by red brackets. 
Table 3. Internal crosslinks in elF5(201-405)Bpa constructs.

\begin{tabular}{|c|c|c|c|c|c|c|}
\hline \multirow[b]{2}{*}{ Construct } & \multicolumn{6}{|c|}{ Crosslinked peptides } \\
\hline & Bpa peptide & Target peptide & Site & Mass (Da) & Error (ppm) & $\mathrm{m} / \mathrm{z}$ \\
\hline \multirow[t]{2}{*}{ D221Bpa } & VKDㅁEWAVDMSE & $\underline{\text { QIK }}$ & $205-207$ & 1962.9116 & -1.9 & 555.7913 \\
\hline & $\underline{b} E W A V D$ & FLGLEHK & $336-337$ & 1712.8161 & -1.0 & 571.6102 \\
\hline \multirow[t]{7}{*}{ A234Bpa } & $\underline{b}$ RAKELE & FVPKEVSK & $372-373$ & 1929.0430 & -2.0 & 483.0162 \\
\hline & $\underline{b} R A K E L E$ & $\underline{\text { RFLGLEHK }}$ & $330-331$ & 1747.0014 & 2.0 & 583.0053 \\
\hline & $\underline{b} R$ & FVPKEEVSK & $375-379$ & 1358.7377 & 0.1 & 453.5840 \\
\hline & EAIR $\underline{B} R$ & FGTK & 367 & 1346.7144 & 0.0 & 449.5763 \\
\hline & $E A I R \underline{b} R$ & FGTK & 367 & 1346.7144 & 0.3 & 673.8601 \\
\hline & $\underline{b} R$ & KFVPK & $374-375$ & 1043.5966 & 0.1 & 348.5370 \\
\hline & $\underline{b} R$ & FVPK & $374-375$ & 915.5028 & -0.3 & 458.2550 \\
\hline \multirow[t]{4}{*}{ E238Bpa } & bLLEVNSE & KFVP互 & $374-375$ & 1558.8025 & -0.4 & 779.9049 \\
\hline & $\underline{b L E}$ & FVPKEVSK & $372-374$ & 1444.7627 & -0.6 & 482.2590 \\
\hline & bLLEVNSE & FVPK & $374-375$ & 1430.7201 & -0.2 & 715.8637 \\
\hline & AKbLE & FVPKK & 374 & 1200.6663 & 0.0 & 600.8367 \\
\hline
\end{tabular}

The likely target positions in the target peptides are underlined.

In theresulting structural model, Ile232, Ala236, and Leu239 point toward helix $\alpha 5$, thus forming a continuous hydrophobic interface, while the outward pointing charged residues of the amphipathic helix $\alpha 2$ would allow the formation of polar interactions to the adjacent regions in helices $\alpha 3$ and $\alpha 8$ and the loop preceding $\alpha 11$. Interestingly, as helix $\alpha 2$ is formed by the C-terminal half of the DWEAR-motif $\left({ }^{231}\right.$ AIRARAKEL $\left.{ }^{239}\right)$ [69], this structural role would provide an explanation for the high degree of conservation of residues such as Arg233, Arg235 and Glu238. At the same time, the $\mathrm{N}$-terminal mainly negatively charged half of the motif $\left({ }^{220}\right.$ DDEWAVDMSEE $\left.^{230}\right)$ would come to lie between the C-terminal ends of helices $\alpha 3$ and $\alpha 8$, respectively, thereby complementing the acidic area III. Importantly, independent support for the manually generated model presented in Figure 5 is provided by the modeling server CluPro[95, 96], which predicts two very similar solutions, when the isolated helix $\alpha 2$ was provided as ligand for the isolated elF5-CTD (Fig. S4C). 
Taken together, these observations indicate that, in contrast to previous assumptions [68, 69], helix $\alpha 2$ and the DWEAR-motif form an at least transient element of the elF5-CTD rather than of the preceding flexible linker region (Fig. $1 \mathrm{~A}$ and $5 \mathrm{~A}$ ). This would in part provide an explanation for its high degree of conservation among elF5 orthologs via a structural role for some of its residues (Fig. S1). At the same time this suggests that the C-terminal domain itself, not the linker region, is involved in the stabilization of the guanine nucleotide bound to the $\mathrm{p}$-subunit of elF2. In order to assess this possibility the effect of different elF5 constructs on the nucleotide binding properties of elF2 were studied using fluorescent derivatives of GDP and GTP (see below).

\section{Mant-GDP and -GTP as fluorescence-labeled GDP/GTP analogs to study nucleotide binding by elF2}

Derivatives of guanine nucleotides, in which the fluorescent mant group is attached to the 2' or $3^{\prime}$ positions of the ribose ring, have been widely used to study the nucleotide binding properties of a variety of GTP-binding proteins. The binding reaction of a mant-nucleotide to a protein can be monitored either by direct excitation of the mant group itself (at $355 \mathrm{~nm}$ ), in which case the binding event is manifested in a change in the quantum yield of the fluorophore at its emission wavelength $(440 \mathrm{~nm})$ due to an environmental change, or indirectly by Foerster Resonance Energy Transfer (FRET) from a nearby tryptophan residue (excited at $280 \mathrm{~nm}$ ) to the mant-nucleotide.

Despite their widespread use, no study exists so far in which mant-nucleotides were used to monitor guanine nucleotide binding to elF2. To test the feasibility of such experiments, both options for the excitation of the mant group were tested for mant-GDP and mant-GTP in the presence of elF2 (Fig. 6). For both nucleotide derivatives, their binding to elF2 resulted in a significant fluorescence change upon direct excitation. Likewise, a substantial energy transfer between tryptophan residues and the mant group was observed, which was accompanied by a simultaneous decrease in tryptophan fluorescence at $\sim 350 \mathrm{~nm}$. Importantly, both fluorescence signals obtained either by direct or by indirect excitation were lost upon the addition of excess unlabeled GDP or GTP but not with ADP or ATP. Thus, mant-nucleotides seem to bind specifically to the nucleotide binding pocket of elF2 where they can be replaced by the unmodified ligand. 
A
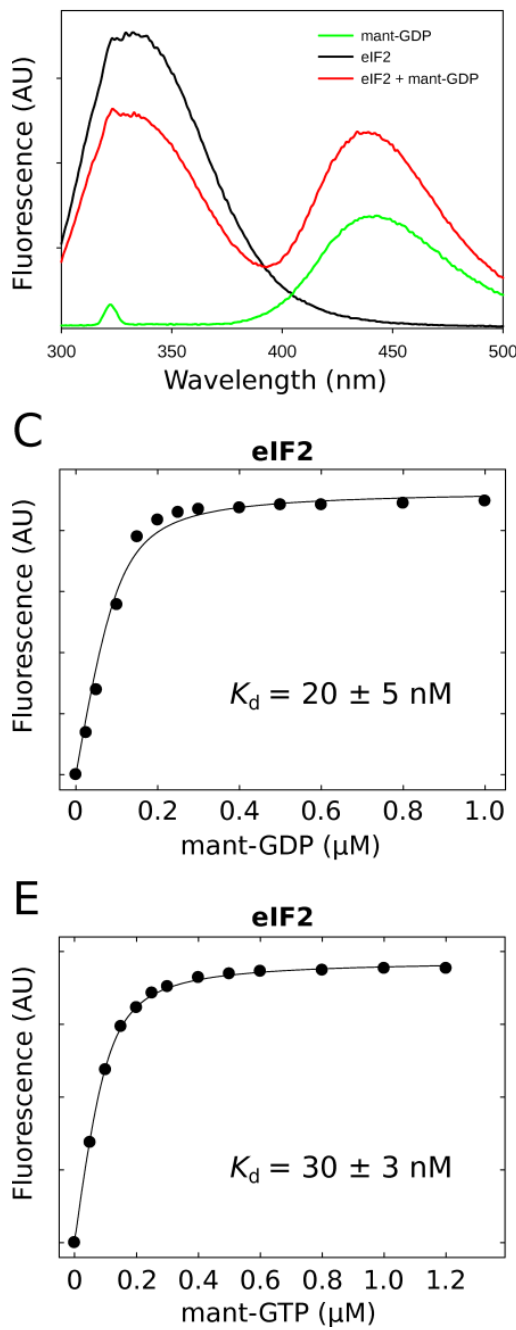

B
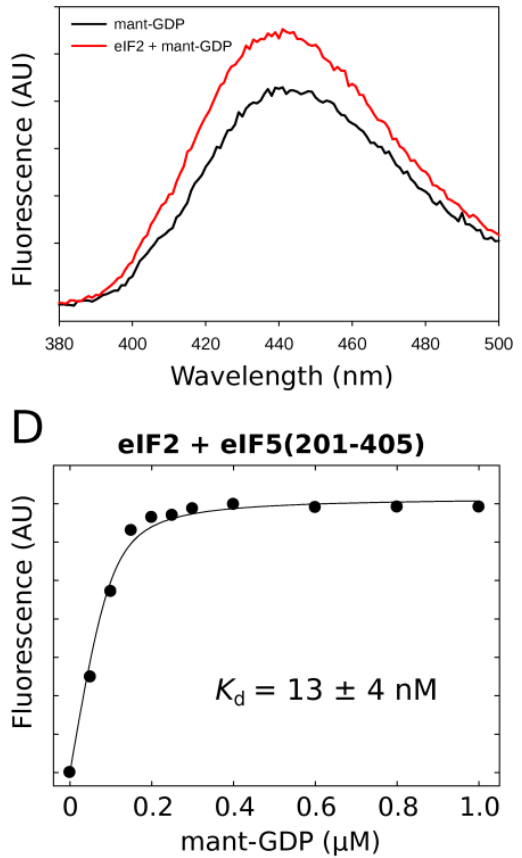

$\mathrm{F}$

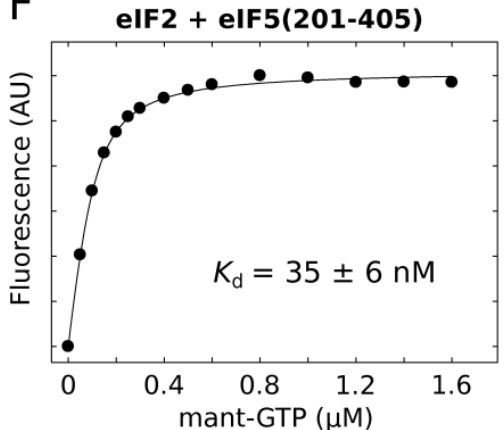

Figure 6. Fluorescence experiments for the interaction between elF2 and mant-labeled guanine-nucleotides. A) Emission spectra for $50 \mathrm{nM}$ elF2 (black), mant-GDP (green) and elF2 in the presence of mant-GDP (red) upon excitation at $280 \mathrm{~nm}$. B) Emission spectra for mant-GDP (black) and 50 $\mathrm{nM}$ of elF2 in the presence of mantGDP (red) upon direct excitation of the mant-group at a wavelength of $355 \mathrm{~nm}$. C-D) Equilibrium titrations of $100 \mathrm{nM}$ elF2 with mant-GDP either alone (C) or in the presence of $5 \mathrm{mM}$ elf5(201-405) (D). The binding reaction was monitored by FRET between elF2 (excited at $280 \mathrm{~nm}$ ) and the mant-group of the nucleotide (emission monitored at $440 \mathrm{~nm}$ ). The black line shows the fit to the data, using a quadratic one-side binding model. E-F) Equilibrium titrations of $100 \mathrm{nM}$ elF2 with mant-GTP alone (E) or in the presence of $5 \mathrm{mM}$ elF5(201405) (F). $K_{d}$ values are summarized in Table 4.

Equilibrium titrations of elF2 with the mant-nucleotides give equilibrium dissociation constants $\left(K_{\mathrm{d}}\right)$ of $\sim 20$ and $\sim 30 \mathrm{nM}$ for mant-GDP and mant-GTP, respectively (Fig. 6C/E and Table 4). For mant-GDP this value is very close to that previously measured for GDP by nitrocellulose filtration (20 $55 \mathrm{nM}$ )[97]. However, the value obtained for mant-GTP is significantly (57-fold) lower than that reported for GTP $(1700 \pm 1000 \mathrm{nM})$ [97]. Previous studies have shown that nucleotide binding by GTP-binding proteins, including the elF2 $\gamma$ paralog EF-Tu, is not perturbed by the mantgroup [98-100]. It is therefore possible that the high value previously reported for GTP might be due to the used method of nitrocellulose filtration in combination with the relatively fast dissociation rates ( $k_{\text {off }}$ ) of GTP compared to those for GDP (see below). 
A

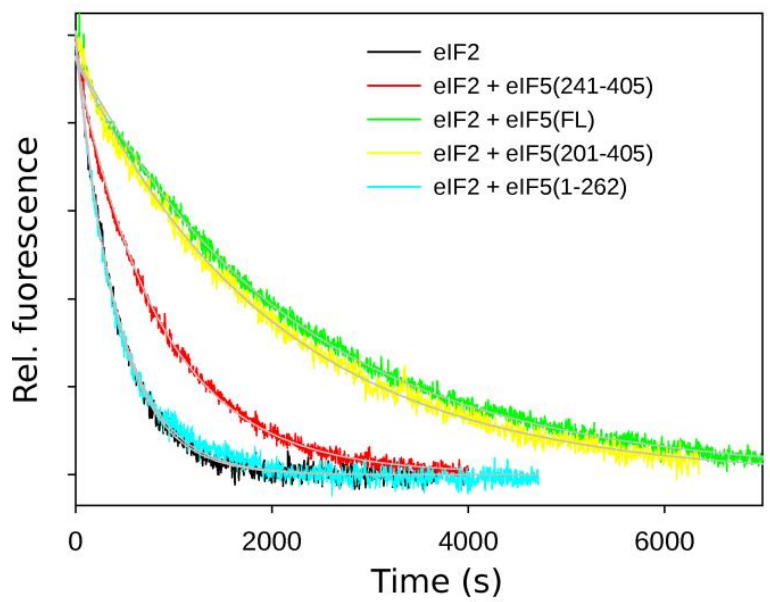

C

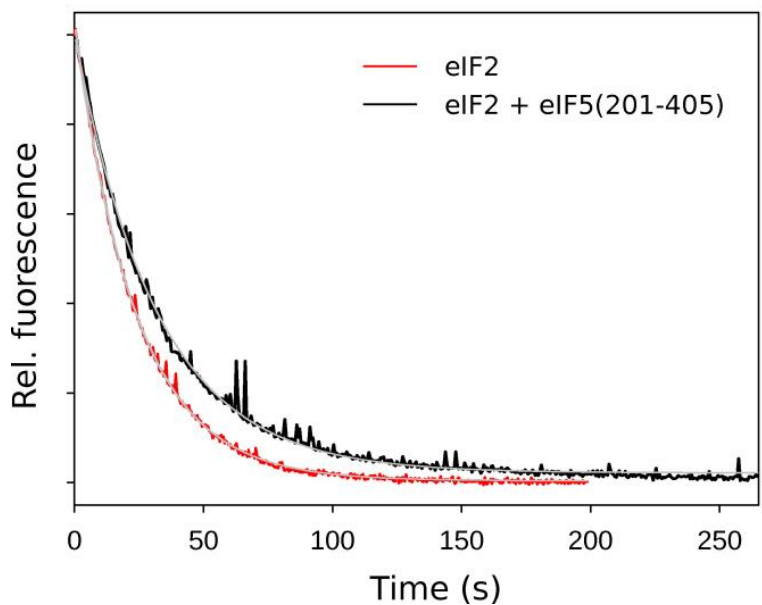

B

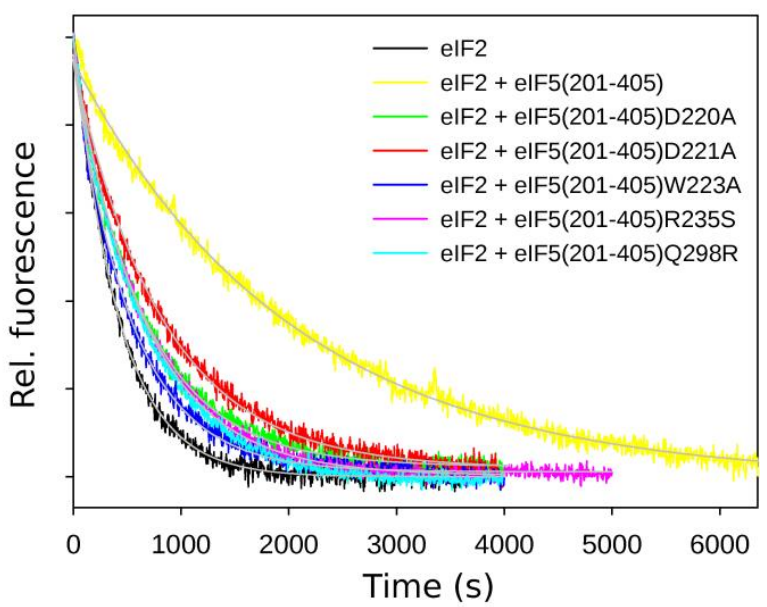

D

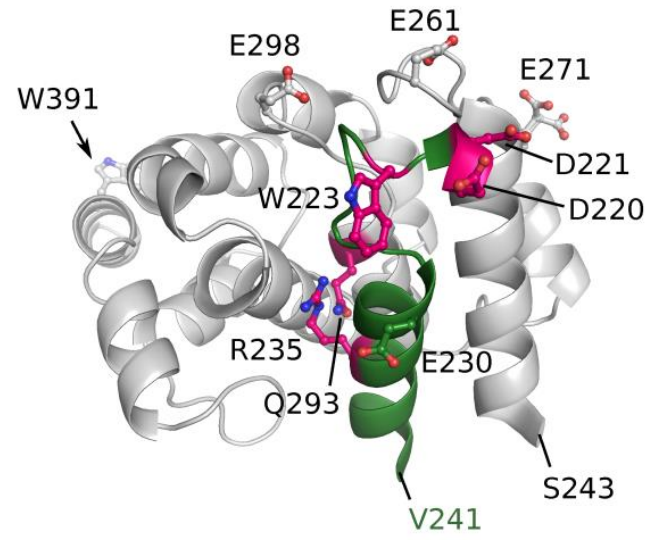

Figure 7. Influence of elF5 on the dissociation of mant-nucleotides from elF2. A) Time courses for the dissociation of mant-GDP from elF2 (100 nM elF2 with $125 \mathrm{nM}$ mant-GDP, followed by the addition of $5 \mu \mathrm{M}$ unlabeled GDP) in the absence (black) or in the presence of different elF5 constructs $(5 \mu \mathrm{M})$ : Full-length elF5 (green), elF5(201-405) (yellow), elF5(241-405) (red) and the point mutant elF5(1-262) (cyan). The grey lines represent single-exponential fits which yielded the respective apparent rate constants $k_{\text {app }}$. B) Time courses for the dissociation of mant-GDP from elF2 (100 $\mathrm{nM}$ elF2 with $125 \mathrm{nM}$ mant-GDP, followed by the addition of $5 \mu \mathrm{M}$ unlabeled GDP) in the absence (black) or in the presence of $5 \mu \mathrm{M}$ elF5(201-405) (yellow), elF5(241-405)D220A (green), elF5(241-405)D221A (red), elF5(241405)W223A (blue), elF5(241-405)R235S (pink) and elF5(241-405)Q293R (cyan). C) Time courses for the dissociation of mant-GTP from elF2 (100 nM elF2 with $125 \mathrm{nM}$ mant-GTP, followed by the addition of $5 \mu \mathrm{M}$ unlabeled GDP) in the absence (red) or in the presence (black) of elF5(201-405) (5 $\mu \mathrm{M})$. D) Structure of the elF5-CTD (with the DWEAR-motif in green modeled as part of the CTD as shown in Fig. 5A.Positions of point mutations used in the mant-GDP dissociation experiments are shown as sticks.

Dissociation rate constants for mant-GDP and mant-GTP were determined by chase experiments with excess of unlabeled GDP (Fig. 7). Under these conditions, every mant-nucleotide that dissociates from elF2 is immediately replaced by GDP, while the rate of mant-nucleotide 
Table 4. Influence of elF5 constructs on the binding of mant-GDP and mant-GTP by elF2

\begin{tabular}{|c|c|c|c|c|c|c|}
\hline & Nucl. & $\begin{array}{l}k_{\text {off }} \\
\left(\min ^{-1}\right)\end{array}$ & $k_{\text {app }}\left(\min ^{-1}\right)^{a}$ & $\begin{array}{l}k_{\text {on }}\left(\mathrm{M}^{-1} \mathrm{~min}^{-}\right. \\
\left.{ }^{1}\right)^{\mathrm{b}}\end{array}$ & $\begin{array}{l}K_{\mathrm{d}} \text { (Nucl.) } \\
(\mathrm{nM})^{\mathrm{c}}\end{array}$ & $\begin{array}{l}K_{1 / 2}(\text { elF5) } \\
(n M)^{d}\end{array}$ \\
\hline \multicolumn{7}{|l|}{ elF2 } \\
\hline & mGDP & $0.14 \pm 0.05$ & & $7 \times 10^{6}$ & $20 \pm 5$ & \\
\hline & mGTP & $2.32 \pm 0.11$ & & $7.7 \times 10^{7}$ & $30 \pm 3$ & \\
\hline \multicolumn{7}{|c|}{ + elF5(FL) } \\
\hline wt & mGDP & $0.068 \pm 0.004$ & & & & \\
\hline \multicolumn{7}{|c|}{ + elF5(201-405) } \\
\hline wt & mGDP & $0.06 \pm 0.005$ & $0.061 \pm 0.002$ & $4.6 \times 10^{6}$ & $13 \pm 4$ & $123 \pm 28$ \\
\hline wt & mGTP & & $2.0 \pm 0.1$ & $5.7 \times 10^{7}$ & $35 \pm 6$ & \\
\hline A309E & mGDP & $0.064 \pm 0.004$ & & & & $209 \pm 66$ \\
\hline K313E & mGDP & $0.066 \pm 0.006$ & & & & $640 \pm 125$ \\
\hline D220A & mGDP & & $0.088 \pm 0.002$ & & & \\
\hline D221A & mGDP & & $0.08 \pm 0.002$ & & & \\
\hline W223A & mGDP & & $0.112 \pm 0.004$ & & & \\
\hline E230A & mGDP & & $0.066 \pm 0.002$ & & & \\
\hline R235S & mGDP & & $0.088 \pm 0.001$ & & & \\
\hline E261A & mGDP & & $0.068 \pm 0.002$ & & & \\
\hline E271A & mGDP & & $0.073 \pm 0.004$ & & & \\
\hline Q293R & mGDP & & $0.105 \pm 0.003$ & & & \\
\hline E298A & mGDP & & $0.07 \pm 0.002$ & & & \\
\hline \multicolumn{7}{|c|}{ + elF5(216-405) } \\
\hline wt & $\mathrm{mGDP}$ & & $0.06 \pm 0.005$ & & & \\
\hline \multicolumn{7}{|c|}{ + elF5(241-405) } \\
\hline wt & mGDP & & $0.108 \pm 0.006$ & & & \\
\hline \multicolumn{7}{|c|}{ + elF5(1-262) } \\
\hline wt & mGDP & & $0.142 \pm 0.01$ & & & \\
\hline
\end{tabular}

${ }^{a}$ Obtained from three independent experiments with $100 \mathrm{nM}$ elF2 in the presence of $5 \mu \mathrm{M}$ of the respective elF5 construct.

${ }^{\mathrm{b}}$ The bimolecular association constant was calculated according to the relation $k_{\text {on }}=k_{\text {off }} / K_{\mathrm{d}}$

${ }^{c} K_{\mathrm{d}}$ is the equilibrium dissociation constant between elF2 and mant-GDP or mant-GTP.

${ }^{d} K_{1 / 2}$ is the apparent equilibrium dissociation constant between elF2 and the elF5 construct, obtained from the dependency of the $k_{\text {app }}$ of mant-GDP dissociation from elF2 on the elF5 concentration.

association is negligible. Thus, the rate by which the fluorescence signal decreases corresponds to the dissociation rate constant of the mant-labeled nucleotide. Single-exponential fitting of the resulting time courses gives values of $0.14 \mathrm{~min}^{-1}$ and $2.3 \mathrm{~min}^{-1}$ for mant-GDP and mant-GTP, respectively (Table 4); both values are similar to those reported previously $[44,69]$. In combination with the $K_{d}$ values obtained in equilibrium titration experiments, the obtained $k_{\text {off }}$ values give estimates for the bimolecular association constants $\left(k_{\text {on }}\right)$ of $7 \times 10^{6} \mathrm{M}^{-1} \mathrm{~min}^{-1}$ and $7.7 \times 10^{7} \mathrm{M}^{-1} \mathrm{~min}^{-1}$ for mant-GDP and mant-GTP, respectively. Taken together, these results show that mant-GDP and mant-GTP are suitable fluorescent guanine nucleotide derivatives to study the nucleotide binding properties of elF2. 


\section{The elF5-CTD interacts directly with the $y$-subunit of elF2 to stabilize the bound nucleotide.}

To test the hypothesis that elF5-CTD is directly involved in the stabilization of elF2-bound nucleotides, we used fluorescence-based chase experiments with mant-GDP. For this purpose, elF2-mant-GDP was mixed with excess of unlabeled GDP either in absence or presence of elF5, and the resulting decrease of fluorescence due to mant-GDP dissociation and replacement by GDP was monitored over time (Fig. 7). In the absence of elF5, mant-GDP dissociates from elF2 with an off rate $\left(k_{\text {off }}\right)$ of $0.14 \mathrm{~min}^{-1}$, which is decreased more than 2 -fold to 0.068 and $0.06 \mathrm{~min}^{-1}$ in the presence of full-length elF5 and elF5(201-405), respectively. At the same time, the $K_{d}$ for the elF2-mant-GDP interaction decreased nearly 2-fold to $13 \mathrm{nM}$, giving a bimolecular association constant $\left(k_{\text {on }}\right)$ of $\sim 4.6 \times 10^{6} \mathrm{M}^{-1} \mathrm{~s}^{-1}$. For the construct elF5(216-405), which lacks most of helix $\alpha 1$ but contains the DWEAR-motif and should thus retain the structural integrity of helix $\alpha 2$ in the context of the CTD, $k_{\text {off }}$ was identical to that of the longer fragment $\left(0.06 \mathrm{~min}^{-1}\right)$. By contrast, the construct lacking the DWEAR-motif (elF5(241-405)) showed a significantly reduced but not completely abolished ability to stabilize mant-GDP on elF2 $\left(k_{\text {off }}=0.11 \mathrm{~min}^{-1}\right)$. Finally, the construct elF5(1-262), which contains the entire N-terminal domain, linker region, DWEAR-motif, and helix $\alpha 3$ of the CTD, showed no stabilizing effect at all $\left(k_{\text {off }}=0.142 \mathrm{~min}^{-1}\right)$. These results indicate that, although the DWEAR-motif provides a major contribution to the GDP stabilizing function of elF5, its effect is dependent on the structural context of the CTD and, moreover, complemented by regions of the CTD that lie C-terminal to Val241 in the primary structure.

Similarly, but to a lesser degree, dissociation of mant-GTP from elF2 is as well affected by the presence of elF5 (Fig. 7C). The dissociation rate decreases from $2.32 \mathrm{~min}^{-1}$ in the absence of elF5 to $2.0 \mathrm{~min}^{-1}$ in its presence. However, this is not accompanied by an increase in the overall binding affinity $\left(K_{\mathrm{d}}=35 \mathrm{nM}\right)$, indicating that together with the decreased dissociation rate also the association rate is slightly reduced to $\sim 5.7 \times 10^{7} \mathrm{M}^{-1} \mathrm{~min}^{-1}$.

Next, we tested a number of single point mutations of residues lying within the DWEAR-motif (D220A, D221A, W223A, E230A, and R235S) as well as area III and adjacent regions (E261A, E271A, E298A and Q293R), introduced into elF5(201-405). Among the DWEAR-motif mutants only D220A, D221A, W223A and R235S had a detectable negative effect on nucleotide stabilization $\left(k_{\text {off }}=0.088\right.$, 
$0.8,0.112$ and $0.088 \mathrm{~min}^{-1}$, respectively). E261A and E298A in area III showed very slight effects $\left(k_{\text {off }}=0.068\right.$ and $0.071 \mathrm{~min}^{-1}$, respectively). Q293R exhibited a strong negative effect on the ability of the elF5-CTD construct to stabilize mant-GDP on elF2 $\left(k_{\text {off }}=0.105 \mathrm{~min}^{-1}\right)$, comparable to W223A. The importance of this observation lies in the fact that $\mathrm{Gln} 293$ lies within the hydrophobic pocket that is proposed to accommodate helix $\alpha 2$ (Fig. 5A and 7D). An arginine in this position would necessarily prevent the association of the DWEAR-motif and its placement next to area III due to sterical repulsion, resulting in the loss of the contribution by the DWEAR-motif. Consistently, Q293R shows only a residual activity corresponding to that of the elF5(241-405) construct without the DWEAR-motif. Trp-quenching and pull-down experiments with the Q293R mutant and the elF2 $\beta$-NTT show that it retains its ability to bind elF2 $\beta$-NTT and therefore seems to be correctly folded in the region C-terminal to Val241 (Fig. S5). It should be noted however that the dissociation rates for all eIF5-CTD point mutants represent only apparent rate constants, obtained at only one elF5 concentration (5 mM) at which elF2 is saturated with elF5(201-405). Thus, the observed effects for some mutants possibly reflect only a reduction in the affinity of elF5 to elF2 and not necessarily a direct involvement of the respective residues in the stabilization of the nucleotide.

The next question was whether the elF5-CTD, including the associated DWEAR-motif, mediates the stabilization of elF2-bound nucleotides indirectly or through a direct contact to the $\mathrm{Y}^{-}$ subunit.For RhoGDIs it is known that the stabilization of the nucleotide is achieved through the formation of direct contacts to the area surrounding the nucleotide binding pocket of the $G$ domain [101]. For elF5-CTD or the DWEAR-motif, however, no such direct interaction to the elF2 $\gamma$ subunit has so far been demonstrated, most likely due to the transient character of the contacts mediating GDP stabilization. To assess the spatial arrangement of elF5-CTD relative to elF2 in the elF2-elF5 complex, we performed UV-crosslinking experiments usingvarious elF5(201-405)Bpa constructs (see above). Among the DWEAR-motif constructs particularly D221Bpa, W223Bpa and A234Bpa were found to form distinct crosslink bands migrating above the $100 \mathrm{kDa}$ marker, which, according to MS analysis, represent specific crosslinks to the $\mathrm{y}$-subunit of elF2 (Fig. 8A). Finally, D220Bpa and E238Bpa as well form crosslinks to elF2 $\gamma$, but with significantly reduced efficiency. Importantly, E261Bpa in area III as well produces a strong and distinct crosslink band with the $\gamma$ subunit that is comparable to those obtained for positions 221, 223 and 234, supporting the idea 
that the DWEAR-motif and area III form a combined surface area (in the following called area III ${ }^{\text {ext }}$ for extended area III; see Fig. S4B) for their interaction with elF2 $\gamma$. A number of additional weak bands migrating at an apparent molecular weight of $130 \mathrm{kDa}$ suggest conformational heterogeneity for this position in the elF2-elF5-CTD complex (it should be noted here that all the crosslink-products elute from an analytical size exclusion column at the same volume as the uncrosslinked elF2-eIF5-CTD complex, thus indicating that the change in migration speed on the SDS-PAGE is most likely due to differences in the topology of the denatured crosslinked peptides (e.g. the crosslink occurred either at the end or the center of the target-chain) rather than an actual higher molecular weight). The only position that was found to form specific crosslinks to elF2 $\beta$ and not to the $\gamma$-subunit was V270Bpa, which produced bands migrating only slightly above that of uncrosslinked elF2 $\gamma$ (Fig. 8D, lanes 7-9). This is in good agreement with the crystal structure of the elF5-CTD-elF2 $\beta$-NTT complex (Fig. 8E) and is further validated by UV-crosslink experiments with elF2 $\beta(1-106)$ alone, which show a strong crosslink only from position V270Bpa (Fig. 10B/D).

Using ESI-MS/MS2analysis in an Orbitrapmass spectrometer, followed by an analysis of the obtained data with the StavroX software [102], we were able to identify potential target peptides for the crosslinks from positions W223Bpa, A234Bpa, E238Bpa and E261Bpa (Table 5). Several of the linkage sites were identified more than once (e.g. ${ }^{508} \mathrm{HWR}^{510}$ from position $\operatorname{Trp} 223$ or ${ }^{409} \mathrm{ADRLV}^{413}$ from positions Ala234 and Glu238), which increases the probability that the assignment of the crosslinked peptides is correct. According to a homology model of elF $2 \gamma$, based on the structure of alF $2 \gamma$ from the archaeon $S$. solfataricus, all the identified linkage sites lie in the frontal face of elF2 $\gamma$ in domains II and III and in the vicinity of switch 1 and switch 2 of the G domain (Fig. 13A). In line with their spatial proximity in helix $\alpha 1$ of elF5(201-405), positions A234Bpa and E238Bpa were found to crosslink predominantly to the same target peptide $\left({ }^{409} \mathrm{ADRLV}^{413}\right)$, which lies at the bottom of the domain II $\beta$-barrel in the elF2 $\gamma$ homology model, directly below switch 1 . The immediately preceding peptide ${ }^{404}{ }^{P T L C R}{ }^{408}$, with Arg408 as likely linkage site, was identified as additional target only for E238Bpa. For W223Bpa and E261Bpa on the other hand, the target sites were found to lie in domain III and in direct vicinity to the switch 2 region, with ${ }^{508} \mathrm{HWR}^{510}$, the main target for W223Bpa, in the last $\beta$-hairpin loop, and ${ }^{444} \mathrm{KTD}^{446}$, the identified target for E261Bpa in the extended first $\beta$-hairpin loop of domain III. 


\section{Chapter 2}
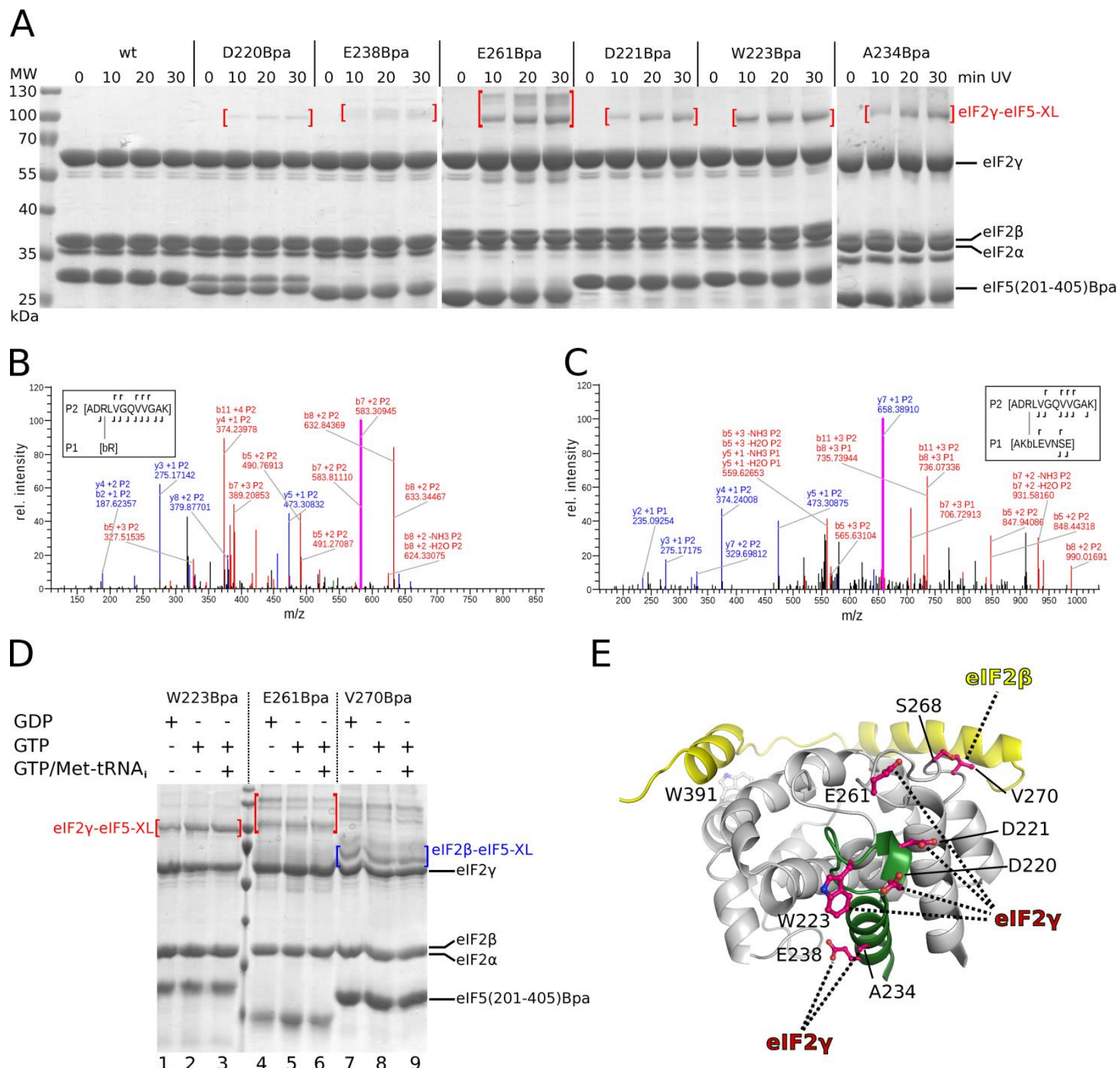

Figure 8. Mapping of the direct interactions between the elF5-CTD and elF2 $\gamma$. A) Coomassie stained SDS-gels showing the UV- and time-dependent formation of crosslinks between elF2 $\gamma$ and the indicated elF5(201-405)Bpa constructs (crosslink bands are indicated by red brackets). For each experiment, $2 \mu \mathrm{M}$ elF2 was mixed with $3 \mu \mathrm{M}$ GDP and $5 \mu \mathrm{M}$ elF5(201-405)Bpa. B-C) High-resolution MS2 fragmentation spectra for the crosslinks of A234Bpa (B) and E238Bpa (C) to their target peptide in elF2 $\gamma$. A series of $b$ and $y$ product ions were detectable for the peptides involved in the crosslinks; the relative intensity of the observed peaks is plotted against their mass-to-charge ratio $(\mathrm{m} / \mathrm{z})$. The inset shows the observed product ions mapped to the sequences of the crosslinked peptides; $b$ stands for Bpa. D) SDS-gels showing formation of crosslinks between elF2 $(2 \mu \mathrm{M})$ and the indicated elF5(201-405)Bpa constructs $(5 \mu \mathrm{M})$ in the

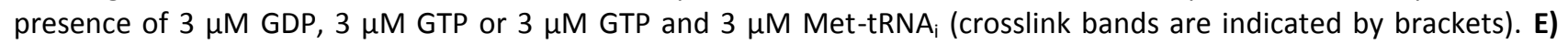
Structure of the elF5-CTD (with the DWEAR-motif in green modeled as part of the CTD as shown in Fig. 5A) in complex with the eIF2 $\beta$-NTT (yellow), indicating the positions where Bpa was introduced (residues shown as sticks). The dashed lines indicate the crosslinks to elF2 formed from the respective positions. 
Taken together, these results provide evidence for a direct interaction between the elF5-CTD and the $y$-subunit close to the nucleotide binding pocket in the free elF2-elF5 complex. Within elF5-CTD, this interaction seems to be mediated by a continuous binding interface composed of area III and the associated DWEAR-motif (forming area III ${ }^{\text {ext }}$ ) that is required for the full nucleotide stabilizing effect on elF2.

Table 5. Crosslinks between elF5(201-405)Bpa and elF2 $\gamma$.

\begin{tabular}{|c|c|c|c|c|c|c|}
\hline & \multicolumn{6}{|c|}{ Crosslinked peptides } \\
\hline Construct & Bpa peptide & Target peptide & Site & Mass (Da) & $\begin{array}{l}\text { Error } \\
(\mathrm{ppm})\end{array}$ & $\mathrm{m} / \mathrm{z}$ \\
\hline W223Bpa & $\begin{array}{l}\text { DEb-bAVMSE } \\
\underline{\mathrm{b}} \text { AVD } \\
\text { VKDDEb } \underline{\mathrm{b} A V D} \\
\underline{\mathrm{b} A V D}\end{array}$ & $\begin{array}{l}\text { VRKLEPNE } \\
\text { KHWRLIGWATIKK } \\
\text { IEKHWR } \\
\text { KHWRLIGWATIK }\end{array}$ & $\begin{array}{l}453-454 \\
509-510 \\
507-510 \\
509-512 \\
\end{array}$ & $\begin{array}{l}2111.9518 \\
2063.1183 \\
2008.9739 \\
1935.0238 \\
\end{array}$ & $\begin{array}{l}-2.9 \\
-2.0 \\
-1.1 \\
-2.0\end{array}$ & $\begin{array}{l}704.65527 \\
688.37726 \\
670.33099 \\
645.67914 \\
\end{array}$ \\
\hline A234Bpa & $\begin{array}{l}\underline{b} R \\
\underline{b} R \\
\underline{b} R\end{array}$ & 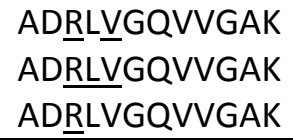 & $\begin{array}{l}411,413 \\
411-413 \\
411\end{array}$ & $\begin{array}{l}1637.9127 \\
1637.9132 \\
1619.9042 \\
\end{array}$ & $\begin{array}{l}0.3 \\
0.6 \\
1.6 \\
\end{array}$ & $\begin{array}{l}410.23355 \\
546.276 \\
540.63959 \\
\end{array}$ \\
\hline E238Bpa & 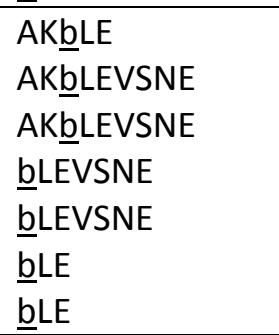 & $\begin{array}{l}\text { ADRLVGQVVGAK } \\
\text { ADRLVGQVVGAK } \\
\text { ADRLVGQVVGAK } \\
\text { ADRLVGQVVGAK } \\
\text { ADRLVGQVVGAK } \\
\text { PTLC } \\
\text { VDPTLCR }\end{array}$ & $\begin{array}{l}411-413 \\
409-412 \\
411-412 \\
411-413 \\
409-411 \\
408 \\
408\end{array}$ & $\begin{array}{l}1923.0703 \\
2352.2558 \\
2352.2569 \\
2153.1235 \\
2153.1275 \\
1139.5551 \\
1296.6265 \\
\end{array}$ & $\begin{array}{l}0.2 \\
0.0 \\
0.5 \\
-0.1 \\
1.8 \\
-0.3 \\
-2.2 \\
\end{array}$ & $\begin{array}{l}641.69495 \\
588.82007 \\
784.75824 \\
539.03632 \\
718.38037 \\
570.28119 \\
432.88034\end{array}$ \\
\hline E261Bpa & $\begin{array}{l}\text { QAG } \underline{b} D K E N L P S D \\
\text { WILEQAGㅁDKE }\end{array}$ & $\begin{array}{l}\text { RLLGVKTDGQK } \\
\text { LLGVKTD }\end{array}$ & $\begin{array}{l}447 \\
444-446\end{array}$ & $\begin{array}{l}2638.3460 \\
2184.1241\end{array}$ & $\begin{array}{l}-0.4 \\
0.8\end{array}$ & $\begin{array}{l}880.12018 \\
728.71289\end{array}$ \\
\hline
\end{tabular}

The likely target positions in the target peptides are underlined.

Potential involvement of helix $\alpha C^{\beta}$ in the elF2 $\beta-N T T$ in the pre-organization of the elF5-CTD relative to elF2 $\gamma$

According to the above results, elF5-CTD provides two distinct contact surfaces for the heterotrimeric elF2 complex in solution: On the one hand, the acidic area III ${ }^{\text {ext }}$ provides the interface for direct interactions with the $\gamma$-subunit. On the other hand, the acidic area $I$ is responsible for high affinity interactions with the eIF2 $\beta$-NTT and thus for the efficient recruitment of elF5 to elF2. Due to the apparently weak character of the direct contacts between elF5-CTD and 
A

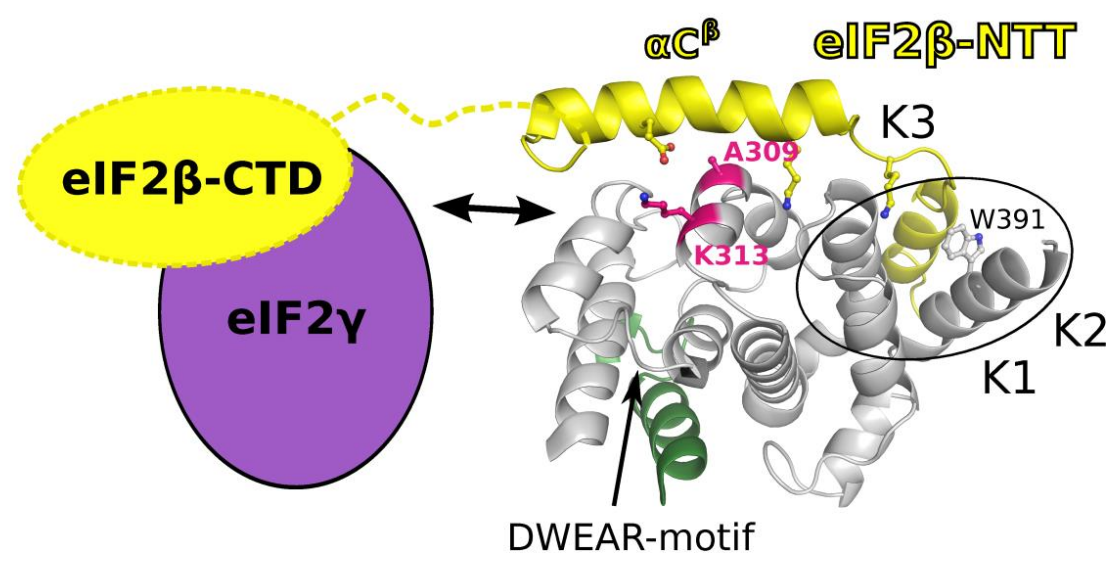

B

C
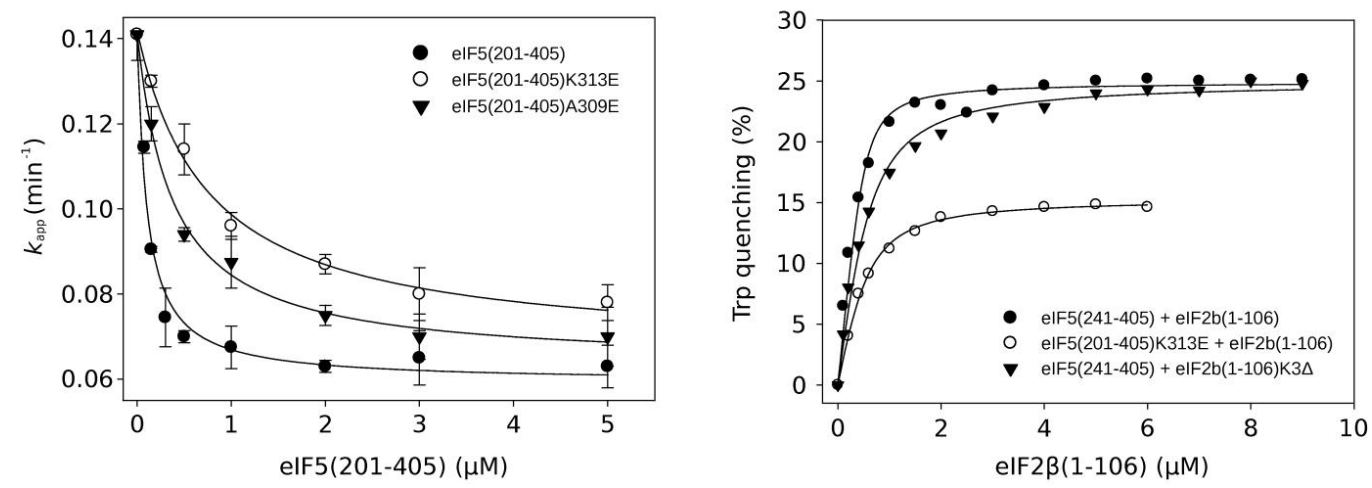

Figure 9.Role of helix $\alpha C^{\beta}$ in elF2 $\beta$-NTT in the pre-organization of the elF5-CTD relative to elF2 $\gamma$. A) Structure of the elF5-CTD (the DWEAR-motif (green) is modeled as in Fig. 5A in complex with the elF2 $\beta$-NTT (yellow). The black circle indicates area I of the elF5-CTD, which forms the contact site for K-boxes 1-3 of elF2 $\beta$. Helix $\alpha C^{\beta}$ points away from area $\mathrm{I}$, ending atop of repeat $\mathrm{R}_{\mathrm{I}}$ and next to area $\mathrm{III}^{\mathrm{ext}}$, which contains residues involved in the interactions with elF2 $\gamma$ (see Fig. 8). elF $2 \gamma$ and the associated elF2 $\beta$-CTD are shown schematically; the linker between elF2 $\beta$-CTD and elF $2 \beta$-NTT is shown as yellow dashed line. The double arrow indicates the direct interaction between elF5 and elF2 $\gamma$, which might be promoted by the directionality provided by helix $\alpha C^{\beta}$. Ala309 and Lys313 in elF5 are indicated in pink. B) Dependency of $k_{\text {app }}$ for dissociation of mant-GDP from elF2 on the concentration of elF5(201-405) (closed circles), elF5(201-405)A309E (closed triangles) and eIF5(201-405)K313E (open circles). Standard deviations, obtained from two independent experiments, are given as error bars. C) Equilibrium titrations of $0.5 \mu \mathrm{M}$ elF5(241-405) with increasing amounts of wild-type elF2 $\beta(1-106)$ (closed circles) or the K-box mutant K3 $\Delta$ (closed triangles) and of $0.5 \mu \mathrm{M}$ elF5(201405)K313E with wild-type elF2 $\beta(1-106)$ (open circles). The Trp quenching signal was plotted against the concentration of the elF2 $\beta$-NTT constructs. The $K_{\mathrm{d}}$ of the elF5(201-405)K313E-elF2 $\beta(1-106)$ complex is $212 \pm 30 \mathrm{nM}$ and thus similar to that of the elF5(241-405) -elF2 $\beta(1-106) \mathrm{K} 3 \Delta$ complex (200 nM) and $\sim 3$-fold reduced compared to the elF5(201405)K313E-elF2 $\beta(1-106)$ complex $(70 \mathrm{nM})$. It should be noted that the quenching signal for the elF5(201405)K313E-elF2 $\beta(1-106)$ complex is reduced compared to the complexes with elF5(241-405) due to the presence of one additional Trp residue (Trp223) in elF5(201-405).

elF2 $\gamma$ we asked whether the elF2 $\beta$-NTT might indirectly promote these interactions by prearranging the eIF5-CTD relative to the rest of elF2 in addition to its role as a high affinity anchor 
point for elF2. As indicated by the crystal structure of the elF5-CTD-elF2 $\beta$-NTT complex, this could involve helix $\alpha C^{\beta}$, which in full-length elF2 $\beta$ is followed by the elF $2 \gamma$-bound elF2 $\beta$-CTD and could thus induce an orientation of eIF5-CTD relative to the $\gamma$-subunit that is favorable for their direct contact (Fig. 9A). For this purpose we purified two elF5(201-405) constructs in which either A309 or K313 were exchanged against glutamate, thereby disrupting the interface for $\alpha C^{\beta}$ either by sterical or electrostatic repulsion (K313 forms a salt bridge to D114 in $\alpha C^{\beta}$; Fig. 9A and 4). These mutants were then tested for their ability to stabilize mant-GDP on elF2. Neither of the mutants had a severe negative impact on the GDP-stabilizing effect of the elF5-CTD, with off rates of 0.065 $\mathrm{min}^{-1}$ and $0.068 \mathrm{~min}^{-1}$ for $\mathrm{A} 309 \mathrm{E}$ and $\mathrm{K} 313 \mathrm{E}$, respectively, compared to $0.06 \mathrm{~min}^{-1}$ for the wild-type (Fig. 9B and Table 4). However, the concentration of the respective construct that was necessary to achieve the half maximal stabilizing effect increased relative to the wild-type construct $\left(K_{1 / 2}=123\right.$ $\mathrm{nM})$ by $\sim 2$ - and 5-fold for A309E $\left(K_{1 / 2}=209 \mathrm{nM}\right)$ and K313E $\left(K_{1 / 2}=640 \mathrm{nM}\right)$, respectively, indicative of a reduced affinity to elF2 $\gamma$. Trp-quenching experiments showed nearly wild-type affinities of the K313E mutant for elF2 $\beta(1-106)$ (Fig. 9C and Table 1), in line with its relative distance from the main interface for K-box binding in surface area I in the crystal structure. Thus, under the assumption that elF5 interacts with the isolated elF2 $\beta-N T T$ in the same way as in the heterotrimeric elF2 complex, which was supported by the Bpa crosslinking experiments from position Val270, these results indicate that these mutants increase the $k_{\text {off }} / k_{\text {on }}$ ratio in the direct interface between elF5CTD and elF2 $\gamma$, which could be explained by a reduced ability of helix $\alpha C^{\beta}$ in elF2 $\beta$ to pre-arrange elF5-CTD relative to the $\gamma$-subunit.

\section{Interactions between elF5-CTD, elF2 $\beta$-NTT, elF1 and elF3c-NTD}

Previous biochemical and genetic studies have identified the C-terminal domain of elF5 as one of the key components in eukayal translation initiation as versatile interaction partner for other initiation factors. In recent years particularly the dynamic interplay of the elF5-CTD with elF1 and elF2 came into focus as it was proposed to be pivotal for the fidelity of start codon selection and $\mathrm{P}_{\mathrm{i}}$ release from elF2 $[21,57,63,103]$. However, the exact nature of the structural rearrangements that are thought to occur between these factors upon the encounter of the AUG start codon have remained controversial, in part due to incomplete or even contradictory data concerning their 
relative arrangement on the surface of elF5-CTD. In light of our data on the interactions of elF5CTD with elF2 $\beta$ and elF $2 \gamma$, we therefore tried to gain further insight into its interactions with elF1 and the $\mathrm{N}$-terminal domain of elF3c. For this purpose we used Bpa incorporated into specific positions of elF1 or elF5 by genetic code expansion, to detect spatial proximity by UV-induced crosslinking and subsequent MS analysis.

First, we tested different elF5(201-405)Bpa constructs for their ability to form crosslinks to elF1, elF2ß(1-106) or elF3c(1-136), respectively (Fig. 10). Bpa incorporated into positions Asp220, Asp221 and Trp223 of the DWEAR-motif showed nearly no crosslinking at all (Fig. 10A-C, lanes 1-4). By contrast, the other Bpa-constructs (Glu238, Glu261, Ser268 and Val270) showed clear UV- and time-dependent crosslink bands for elF1, elF2 $\beta$-NTT as well as elF3c-NTD, however, with significant differences in the efficiency (Fig. 10A-C, lanes 5-8). For elF1 the strongest bands were obtained from positions Glu238 and Val270 (Fig. 10A, lanes 5 and 8, respectively), while slightly weaker bands were observed for Glu261 and Ser268 (Fig. 10A, lanes 6 and 7, respectively). In line with the crystal structure, the elF2 $\beta$-NTT crosslinked efficiently to S268- and V270Bpa (Fig. 10B, lanes 7 and 8, respectively), whereas E238- and E261Bpa showed significantly weaker bands in comparison (Fig. 10B, lanes 5 and 6, respectively). The opposite tendency was observed for elF3c-NTD, with the strongest crosslink occurring from position Glu238, followed by Glu261 and Val270 and virtually no crosslink from S268 (Fig. 10C, lanes 5, 6, 8, and 7, respectively). These data result in the following main conclusions:

i) E238Bpa lies close to a common binding site for elF1 and elF3c-NTD. The internal crosslinks (see above) suggest a position for E238Bpa close to the C-terminus of helix $\alpha 8$ and the loop preceding helix $\alpha 11$. This would suggest the conserved basic surface area II as most likely candidate for the interactions with elF1 and elF3c-NTD, in line with previous reports that identified this region as primary interface for both factors $[57,104]$ (e.g. the H336Q-K337Q double mutant in elF5 was found to reduce binding to elF3c and elF1 [57] and the same dipeptide is found in one of the identified target peptides ( ${ }^{331} \mathrm{FLGLE} \underline{H^{337}}$ ) for E238Bpa (Table 3)).

ii) elF1 not only binds to area II close to Glu238 but is also able to interact with a second interface in direct vicinity to Val270 on the opposite side of elF5-CTD. This would suggest overlapping interaction interfaces for elF1 and elF2 $\beta$ in this region as proposed by Luna et al.[63]. 


\section{A}

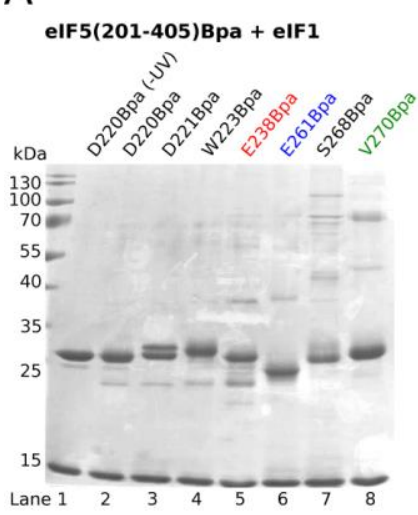

B

elF5(201-405)Bpa + elF2ß(1-106)

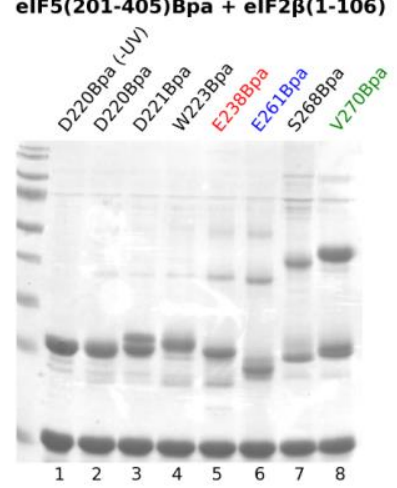

C

elF5(201-405)Bpa + elF3c(1-136)

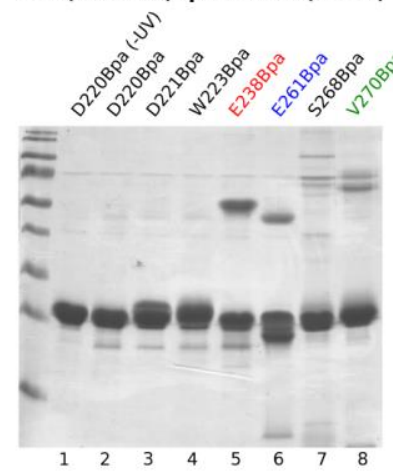

D

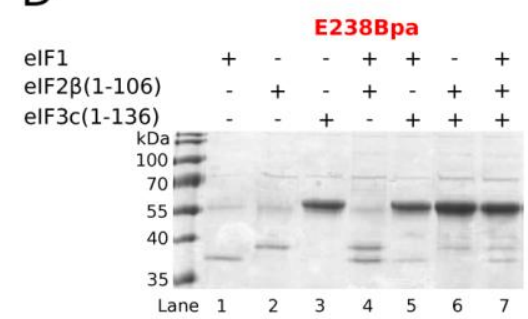

E

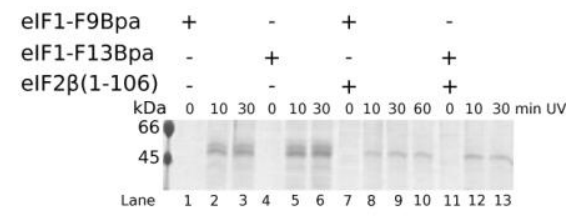

$\mathrm{F}$

elF1-F13Bpa + elF5-CTD + elF2 $3(1-106)$

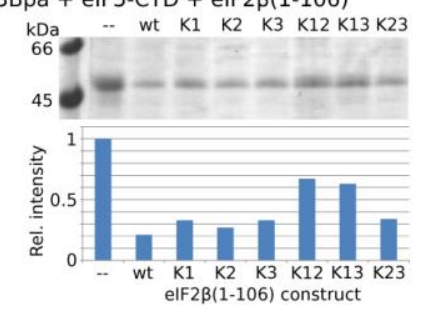

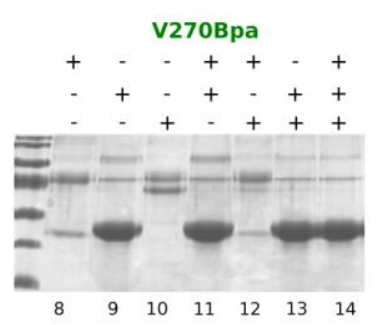

G

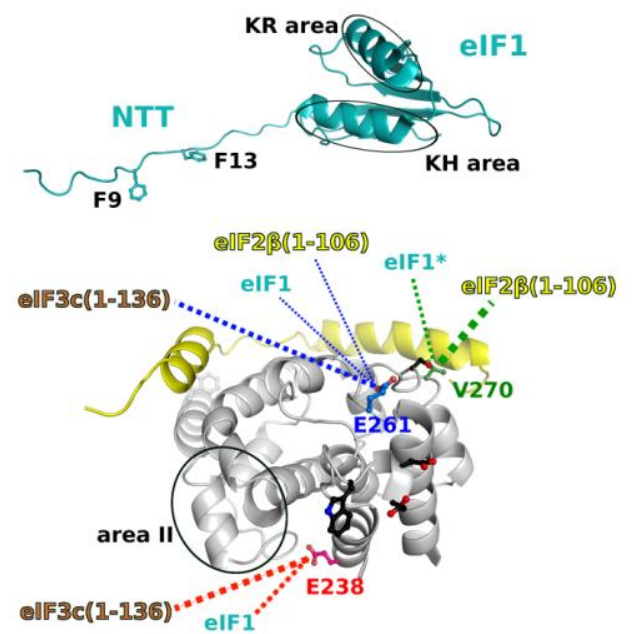

Figure 10.Mapping of the interactions between elF5CTD, elF2 $\beta-N T T$, elF1 and elF3c-NTD by crosslinking. A-C) SDS-gels showing the UV-dependent formation of crosslinks for the complexes between different elF5(201405)Bpa constructs (Bpa positions indicated above each lane) and elF1 (A), elF2 $\beta(1-106)$ (B) and elF3c(1-136) (C). In all cases (except the -UV samples), samples were exposed to UV light for $30 \mathrm{~min}$ on ice.

D) Dependency of the crosslink patterns from positions Glu238 and Val270 in elf5(201-405) on the composition of the formed complexes.

E) UV- and time dependent formation of crosslinks between elF1Bpa (with Bpa in positions Phe9 and Phe13) and elF5(201-405) in the absence (lanes 1-6) or presence of the elF $2 \beta-N T T$ (lanes 7-13).

F) Influence of K-box mutations on the ability of the elF2 $\beta$-NTT to reduce crosslinking between elF1F13Bpa and elF5(201-405). The relative intensities of the crosslink bands were determined using ImageJ. G) Structures of elF1 (cyan;

PDB: $20 G H$ ) and the elF5-CTD (the DWEAR-motif (green)is modeled as in Fig. 5A) in complex with the elF2 $\beta$-NTT (yellow), indicating the positions where Bpa was introduced (as sticks). In elF5, Asp220, Asp221, Trp223 and Ser268 are shown in black; Glu238, Glu261 and Val270 are shown in red, blue and green, respectively. Dashed lines indicate the crosslinks between the components of the minimal MFC formed from the respective positions; the thickness of the dashed lines roughly corresponds to the relative intensity of the crosslink bands shown in A-D. Black circles indicate known interaction surfaces on elF5 and elF1: area II on elF5 was proposed to interact with elF1 and elF3c; the KR area on elF1 interacts with elF3c and the ribosome; the $\mathrm{KH}$ area of elF1 was shown to interact with the elF5-CTD and/or with the elF $2 \beta-\mathrm{NTT}[55,57,104]$. The asterisk marks a crosslink that is not formed in the presence of the elF2 $\beta$-NTT.

iii) Although all three factors seem to have preferred regions for their interactions with elF5CTD, the crosslink data indicate significant overlaps between the contact surfaces in the respective 
binary complexes. This necessarily raises the question whether the interactions in these regions can occur simultaneously or are mutually exclusive.

In order to address this question, we repeated the UV-crosslinking experiments, this time forming the respective ternary and quaternary complexes of elF5(201-405)E238Bpa or -V270Bpa with elF1, elF2 $\beta$-NTT and/or elF3C-NTD. Interestingly, diametrically opposite effects were observed for the two tested positions: In case of E238Bpa the simultaneous addition of elF1 and elF2 $\beta$-NTT, each in 2-fold molar excess over elF5(201-405)Bpa, increased the crosslinking efficiency to both proteins $\sim 2$-fold relative to their respective binary complexes (Fig. 10D, compare lanes 1, 2 and 4). The addition of elF3c-NTD (equimolar to elF1) resulted in a significant loss in band intensity for elF1 as well as elF2 $\beta$; this negative effect could be slightly but reproducibly compensated in the complexes containing all four components (Fig. 10D, compare lanes 5-7). In stark contrast to these observations, the addition of elF2 $\beta$-NTT to the V270Bpa construct completely abolished any crosslinks to elF1 or elF3c-NTD (Fig. 10D, compare lanes 8, 10 and 14), while elF1 in turn was able to partially suppress the crosslinks to elF3c-NTD in the elF5(201-405)Bpa-elF1·elF3c-NTD complex (Fig. 10D, compare lanes 8, 10 and 12).

To further test these results, we then introduced Bpa into elF1 in positions Phe9 and Phe12 of the flexible N-terminal tail (NTT) (Fig. 10G). Both constructs allowed specific crosslinks to elF5(201-405), with two bands emerging in a UV- and time-dependent manner, migrating close together at $\sim 50 \mathrm{kDa}$ (Fig. 10E, lanes 1-6). The addition of elF2 $\beta$-NTT in 2-fold molar excess over elF1 caused a significant reduction of both elF5-CTD·elF1Bpa crosslinks (Fig. 10E, compare lanes 3 and 6 with lanes 9 and 13). Interestingly, this negative effect of the elF2 $\beta$-NTT on the crosslink efficiency was particularly severe on the upper band which completely disappeared in the ternary complexes. The same experiments in the presence of the single and double K-box mutants of the elF2 $\beta$-NTT showed a clear correlation between their respective affinity to elF5-CTD and their ability to reduce the crosslink efficiency between elF1Bpa and elF5 (Fig. 10F and 2). These observations indicate that the two bands originate from two alternative positions or orientations of elF1 on the surface of elF5-CTD with the upper band formed in an interaction that is incompatible with the presence of elF $2 \beta$, while the lower band is formed in an interaction which is not completely abrogated by elF $2 \beta$. It can therefore be speculated that these two interfaces between elF1 and 
elF5-CTD are the same as those that result in the crosslinks from V270Bpa and E238Bpa to elF1, respectively.

In combination with the tryptophan quenching and structural data, these observations support a model in which the respective interfaces for the interactions of elF5-CTD with other initiation factors change significantly depending on the composition of the complex. Particularly interesting is the case of elF1, for which the crosslink data indicate two distant binding sites on elF5-CTD. The first binding site (interface I) lies close to Val270 and is completely abrogated by the binding of elF2 $\beta-N T T$, presumably due to an overlap between both binding sites (Fig. 10G). According to Luna et al.[63] this interaction is independent of the elF1-NTT. Thus, elF1 seems to interact with interface I through its globular core domain, most likely involving residues within the so-called KH area and surrounding Gly97 $[63,104]$. The second binding site (interface II) lies close to Glu238 and thus most likely involves the positively charged area II. This binding site is shared with elF3c-NTD and in stark contrast to interface I this contact is not only compatible with but even slightly strengthened by the presence of the elF2 $\beta$-NTT (Fig. 10D, lane 4). The elF1-NTT, which is dispensable for the interaction with interface I [63], was shown to be required for the cooperative binding of elF1 and elF2 $\beta$ to elF5-CTD and assembly of the MFC $[55,104]$. This would suggest that the elF1-NTT, rich in acidic and aromatic side chains, is responsible for the interaction with elF5CTD in the positively charged area II (interface II), where it binds simultaneous and in close proximity (or even in direct contact) to elF3c-NTD. This placement of elF1-NTT and elF3c-NTD is, moreover, in good agreement with the crystal structure of elF2 $\beta(66-106)$ bound to elF5-CTD, in which the N-terminus of the former lies directly above area II and thereby in a suitable position to contact both factors through the linker-region between K2 and K3 (Fig. 4A and 10G). Thus, within the elF5-CTD·elF2 $\beta$-NTT-elF1·elF3c-NTD complex, the minimal MFC core, elF1 would remain directly associated with elF5-CTD area II only through its NTT. At the same time, the C-terminal domain of elF1 could remain associated with elF3c-NTD through the 'KR area' in the N-terminal $\alpha$ helix, and the $\mathrm{KH}$ area of elF1, after its displacement from elF5-CTD by the elF2 $\beta-N T T$, would become available for direct interactions with the elF2 $\beta$-NTT itself [104]. Considering the significantly higher affinity of the elF2 $\beta$-NTT for area I compared to that of elF1, it is conceivable 
that the above described interaction network represents the state in which the four initiation factors are organized in the MFC when it joins the 40S subunit.
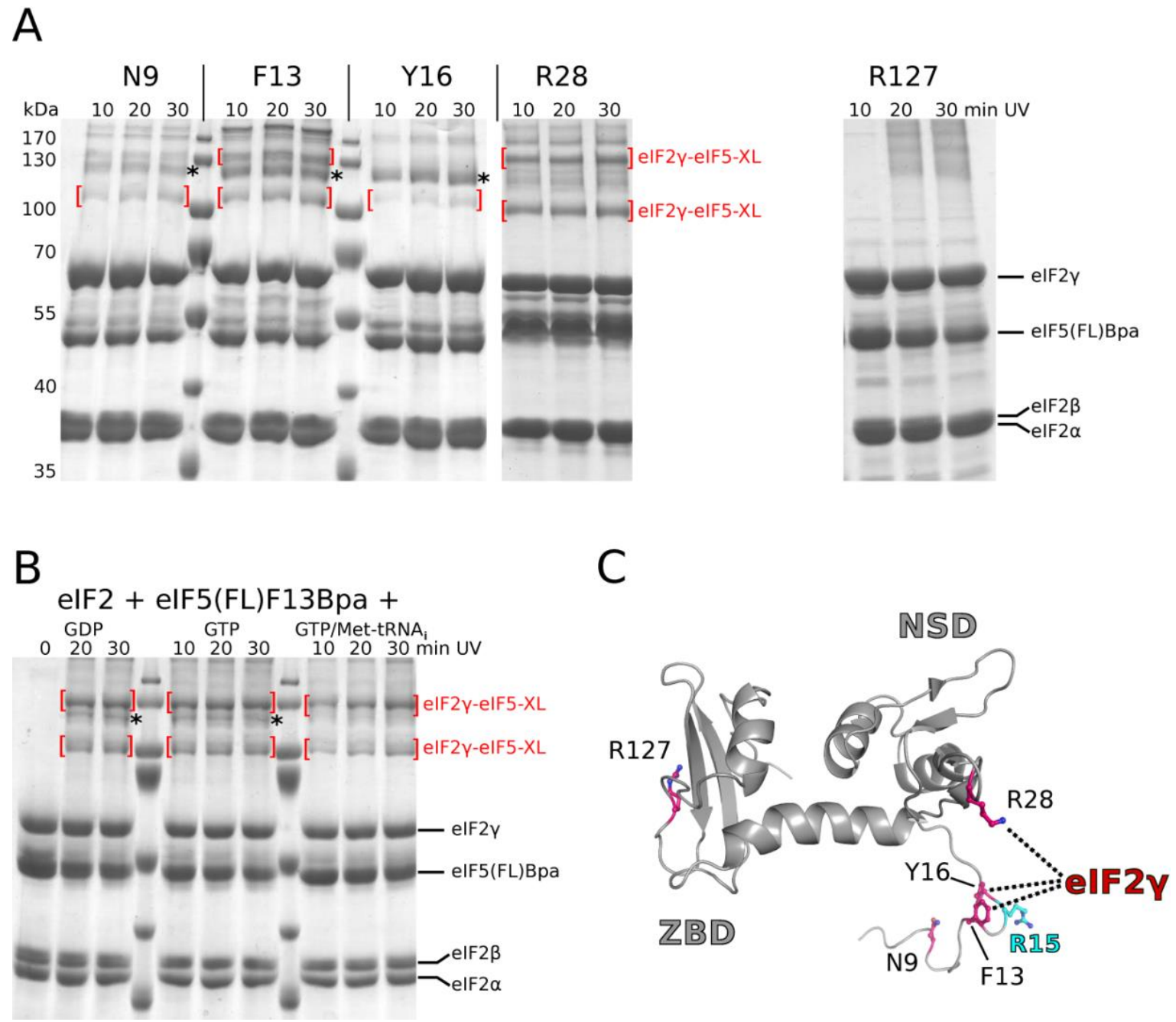

Figure 11. Mapping of the interactions between elF5-NTD and elF2 by Bpa crosslinking. A) SDS-gels showing the UVdependent formation of crosslink bands between $5 \mu \mathrm{M}$ of the different elF5(FL)Bpa constructs and $2 \mu \mathrm{M}$ elF2. Crosslinks to elF2 $\gamma$ are indicated by red brackets; the strong crosslink bands for positions Asn9, Phe13 and Tyr16 directly below the $130 \mathrm{kDa}$ marker (marked with *) also occur in the absence of elF2 and are thus likely to originate from a crosslink between two elF5 molecules. B)SDS-gel showing the UV- and time-dependent formation of crosslink bands between elF2 and elF5(FL)F13Bpa in the presence of $3 \mu \mathrm{M} \mathrm{GDP,} 3 \mu \mathrm{M}$ GTP or $3 \mu \mathrm{M} \mathrm{GTP/Met-tRNA_{i }}$ (crosslink bands are indicated by red brackets). C) Solution structure of the elF5-NTD from $\mathrm{H}$. sapiens (PDB: 2G2K) indicating the positions where Bpa was introduced in the yeast homolog (residues shown as pink sticks). Arg15 (cyan) in the flexible $\mathrm{N}$-terminal tail corresponds to the putative catalytic arginine-finger [52,53]. Dashed lines indicate crosslinks to elF2 $\gamma$. The elF5-NTD is structurally subdivided into $\mathrm{N}$-terminal subdomain (NSD) and the C-terminal zinc binding domain (ZBD). 


\section{elF5-NTD and eIF5-CTD interact with elF2 $\gamma$ in the eIF2-GTP.Met-tRNA $A_{i}$ ternary complex}

Up to this point all interaction studies were carried out in the absence of Met-tRNA $A_{i}$. It is therefore unclear, whether the direct contact between eIF5-CTD and eIF2 $\gamma$ would persist in the presence of the charged initiator tRNA and in the context of the elF2.GTP.Met-tRNA $A_{i}$ ternary complex. The UVcrosslinking experiments with Bpa constructs of the elF5-CTD were therefore repeated, this time in the presence of a 2-fold molar excess of Met-tRNA $A_{i}$ over elF2, equimolar to the Bpa construct. As shown in Figure 8D, the crosslink efficiency for the tested constructs with Bpa in positions Trp223, Glu261 or Val270 remained virtually unchanged upon the addition of the initiator tRNA. This suggests that the underlying direct interactionsof elF5-CTD to the elF2 $\gamma$-subunit andto elF2 $\beta$ exist in the complex with free elF2, as well as with the ternary complex of elF2.GTP with the Met-tRNA

Next, we studied whether it would also be possible to detect direct interactions between the elF5-NTD and elF2 in solution using UV-inducible crosslinking. For this purpose, Bpa was introduced into various positions of the NTD in full-length elF5. In the flexible N-terminal extension, the Bpa moiety was introduced in positions Asn9, Phe13 and Tyr16 and thus in direct vicinity to Arg15, the potential arginine-finger of elF5 (Fig. 11C). Moreover, Bpa was introduced in lieu of Arg28 in the first hairpin loop of the N-terminal $\beta$-sheet, and Arg127 next to the zink-finger motif. Among the three N-terminal positions N9Bpa and Y15Bpa resulted only in faint crosslink bands (Fig. 11A), the strongest of which (at $\sim 120 \mathrm{kDa}$ ) occurred even in the absence of elF2 and thus most likely represents a crosslink between two elF5 molecules. Although the same band also occurred with F13Bpa, here two additional strong crosslink bands emerged at 100 and 140 kDa in a UV- and time-dependent manner only in the presence of elF2 (Fig. 11A). A similar crosslink pattern was observed for R28Bpa, while no clear elF2-dependent crosslinks could be obtained for position R127Bpa. MS-analysis of the crosslink bands from F13Bpa and R28Bpa revealed that both positions crosslink specifically to elF $2 \gamma$ within the elF2 complex. However, it was so far not possible to identify any target peptides of the crosslink. As the weaker crosslink bands were not analyzed by mass spectrometry, it cannot be ruled out at present that some of them are due to crosslinks to either the $\alpha$ - or $\beta$-subunit. Moreover, it should be noted that none of the elF5-NTD-Bpa constructs were so far tested for their ability to stimulate GTP hydrolysis on elF2 on the ribosome; it is therefore currently not clear in how far Bpa in the various positions affects the interactions 
between elF5 and elF2. Nonetheless, the presented results indicate that the elF5-NTD within the elF2-elF5 complex is positioned relative to elF2 in a way that favors specific crosslinks to the $\gamma$ subunit particularly from the N-terminal subdomain (Fig. 11C).

Interestingly, the addition of Met-tRNA $\mathrm{A}_{i}$ apparently causes slightly reduced crosslinking efficiency from F13Bpa, which, however, seems to be accompanied by an increase in specificity for the two main crosslinks to elF2 $\gamma$ (Fig. 11B). Thus, it seems that the spatial proximity between elF5NTD and elF2 $\gamma$ persists in the ternary complex. This proximity however wasnot found to depend strongly on the nucleotide state of elF2 (except in the case of the increased specificity with the TC), with only slight increases in crosslinking efficiency for F13Bpa and R28Bpa when GTP was present. Moreover, the crosslinks were not affected by the presence of GDP and fluoroaluminates (Fig. S6). This observation is in line with the absence of any additional fluorescence signal due to the addition of $\mathrm{AlF}_{\mathrm{x}}$ to the elF2-mant-GDP.elF5 complex, and in line with the assumption that the Arg15 of eIF5 requires the structural context of the scanning $43 \mathrm{~S}$ pre-IC to perform its role.

\section{The elF5-CTD competes with elF2B $\varepsilon$-CTD for the interaction interface on elF $2 \boldsymbol{\gamma}$.}

Previous studies revealed a high degree of structural homology between elF5-CTD and the Cterminal domain of elF2BE(residues 524-712), which forms the minimal catalytically active domain of the guanine nucleotide exchange factor elF2B[1, 54, 74]. Mutational and biochemical studies demonstrated that this GEF activity is mediated by residues within the first two $\alpha$-helices (repeat $\mathrm{R}_{\mathrm{l}}$ ) of the elF2BE-CTD, whose mutagenesis directly affects its interactions to elF2 $\gamma$. In light of our own data, this indicates that elF2B $\varepsilon$-CTD and elF5-CTD contact elF2 $\gamma$ through structurally corresponding regions in $\mathrm{R}_{\mathrm{l}}$ and $\mathrm{R}_{\mathrm{l}}$ /area III, respectively. It is therefore conceivable that both domains interact with a common interface on the elF $2 \gamma$-subunit (between G domain, domain II and domain III, close to the nucleotide binding pocket) to mediate either the stabilization (elF5) or the destabilization (elF2Br) of the bound nucleotide. It has recently been proposed that elF2B $\gamma$, the second catalytically relevant subunit within the eIF2B complex, acts as a GDI displacement factor (GDF) that promotes the release of elF5. This role of elF2By was proposed to be necessary to allow productive binding of elF2Br, which on its own was unable to compete efficiently with elF5 for interactions with eIF2 [105]. This raises the question whether the DWEAR-motif in eIF5-CTD and its 
direct contact to elF2 $\gamma$ is required for the efficient competition with elF2B $\varepsilon$ for their common substrate, which in turn would indicate that the role of elF2By as GDF is to force the elF5-CTD out of its direct interaction with elF $2 \gamma$.

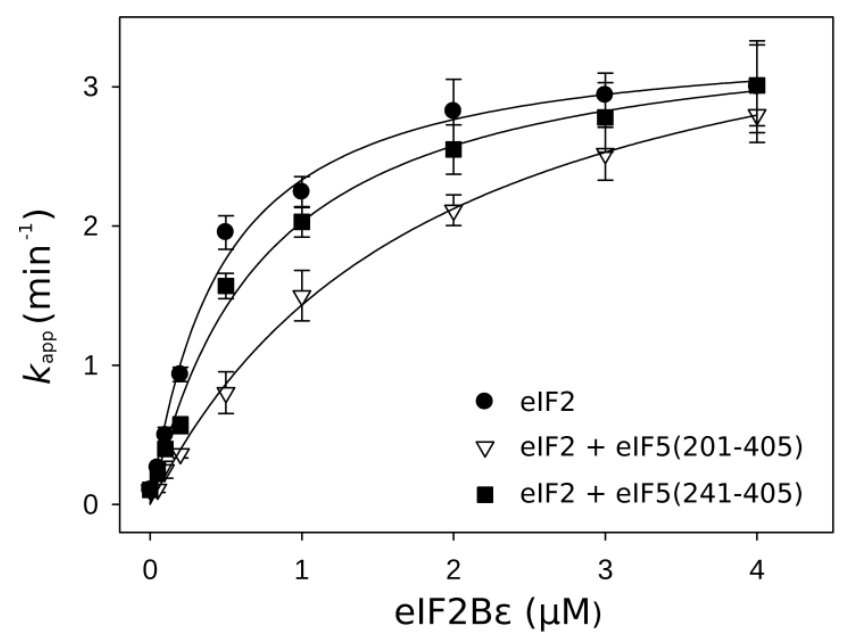

Figure 12. The DWEAR motif in the elF5-CTD is required for efficient competition with elF2B $\varepsilon$-CTD for their common substrate elF2. The plot shows the dependency of $k_{\text {app }}$ values for the dissociation of the elF2-mant-GDP complex (100 nM elF2 with 125 nM mant-GDP) on the concentration of the catalytic elF2B $\varepsilon$-CTD either in the absence of elF5 (closed circles), in the presence of $100 \mathrm{nM}$ elF5(241-405) (closed square) or in the presence of $100 \mathrm{nM}$ elF5(201-405) (open triangle). elF2B $\varepsilon$-CTD and unlabeled GDP $(5 \mu \mathrm{M})$ were added simultaneously to the mixtures of elF2-mant-GDP and elF5-CTD. Standard deviations from two independent experiments are given as error bars.

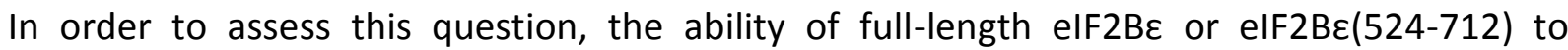
compete either with elF5(201-405) or elF5(241-405) for the interaction with elF2 was studied under steady-state conditions by monitoring the dissociation of mant-GDP from preformed elF2-elF5complex (elF5 was added equimolar to elF2) and increasing amounts of GEF. Both elF5 constructs interact identically with the elF2 $\beta-N T T$ and should therefore be able to compete equally well with elF2BE for this interaction. However, only elF5(201-405) contains the DWEAR-motif and therefore the entire interface required to interact with elF2 $\gamma$. Thus, if the above made assumption is correct, elF5(201-405) should be more efficient than elF5(241-405) in preventing elF2B $\varepsilon$ to promote mant-GDP release. As shown in Figure 12, this is indeed the case. While elF5(201-405) efficiently prevents the binding of elF2B $\varepsilon$ and reduces the apparent $K_{d}\left(K_{1 / 2}\right)$ between elF2B $\varepsilon$ and elF2·mant-GDP nearly 5-fold to $1920 \pm 350 \mathrm{nM}$, elF5(241-405) is significantly less efficient with an only 2 -fold reduction $\left(K_{1 / 2}\right.$ of $\left.700 \pm 180 \mathrm{nM}\right)$. However, in contrast to previous reports [105], we observed that the presence of either elF5 construct had no negative effect on the $k_{\text {off }}$ of mant-GDP from the elF2 complex at saturating elF2Br concentrations (Fig. 12). This indicates that the GEF at high concentrations ( $\sim 40$-fold excess over elF5) is able to out-compete elF5 from its interactions 
with elF2 efficiently, an ability that at low (physiological) GEF concentrations is limited by the direct contact between elF5 and elF2 $\gamma$.

\subsection{Discussion}

Using a combination of mutational, biochemical and structural approaches, this work provides new insights into intricate interaction network between elF2, its multifunctional effector protein elF5, elF1 and elF3c, with a particular focus on the elF2 elF5 complex. Due to the central role of these factors during the process of eukaryotic translation initiation, the presented observations have implications for nearly all steps of the initiation pathway from MFC and pre-IC assembly to scanning, start codon recognition and the general regulation of protein biosynthesis.

The elF5-CTD interacts directly with elF2y in the elF2 elF5 complex. Previous studies established that elF5 folds into two structurally independent domains, the C-terminal and $\mathrm{N}$-terminal domain, connected via a 'linker region' (LR) (Fig. 1A). The elF5-NTD (in yeast) was defined as the first 152 amino acids and contains the putative arginine-finger (Arg15) that is thought to stimulate GTP hydrolysis by elF2 in the scanning pre-IC [51-53]. The elF5-CTD was defined as comprising residues 241-405, which fold into the atypical HEAT motif shown in Figure 1B, and provides interfaces for interactions with other elFs to promote MFC and pre-IC assembly [1] (see below). The intervening residues 153-240 were proposed to form the flexible and unstructured LR, in which the conserved residues of the DWEAR-motif (residues 220-238) mediate the stabilization of the elF2-bound GDP molecule [69]. Our own data support this role of the DWEAR-motif. However, they also strongly suggest that the DWEAR-motif does not belong to the LR but in fact forms an at least transient part of the elF5-CTD. In the resulting structural model, helix $\alpha 2$ is accommodated in the hydrophobic cleft formed by the $\mathrm{N}$-terminal helices of repeats $\mathrm{R}_{\mathrm{I}}$ to $\mathrm{R}_{\mathrm{III}}$, while residues D220-E230 augment area III (Fig. 5 and S3C). Together, the DWEAR-motif and area III form an extended, mainly negatively charged surface (area III ${ }^{\text {ext }}$ ) of the elF5-CTD (Fig. S4B) that directly contacts elF2 $\gamma$ and stabilizes the bound guanine-nucleotide (Fig. 7, 8 and 13). This assumption is in line with the observation that the DWEAR-motif stimulates elF5 binding to excess elF2 $\gamma$ (and thus independently of the 
recruitment of elF5 by the elF2 $\beta-N T T$ ) only in the structural context of the CTD $[65,69]$, while this ability is lost in a construct comprising residues 1-262 (Table 4).
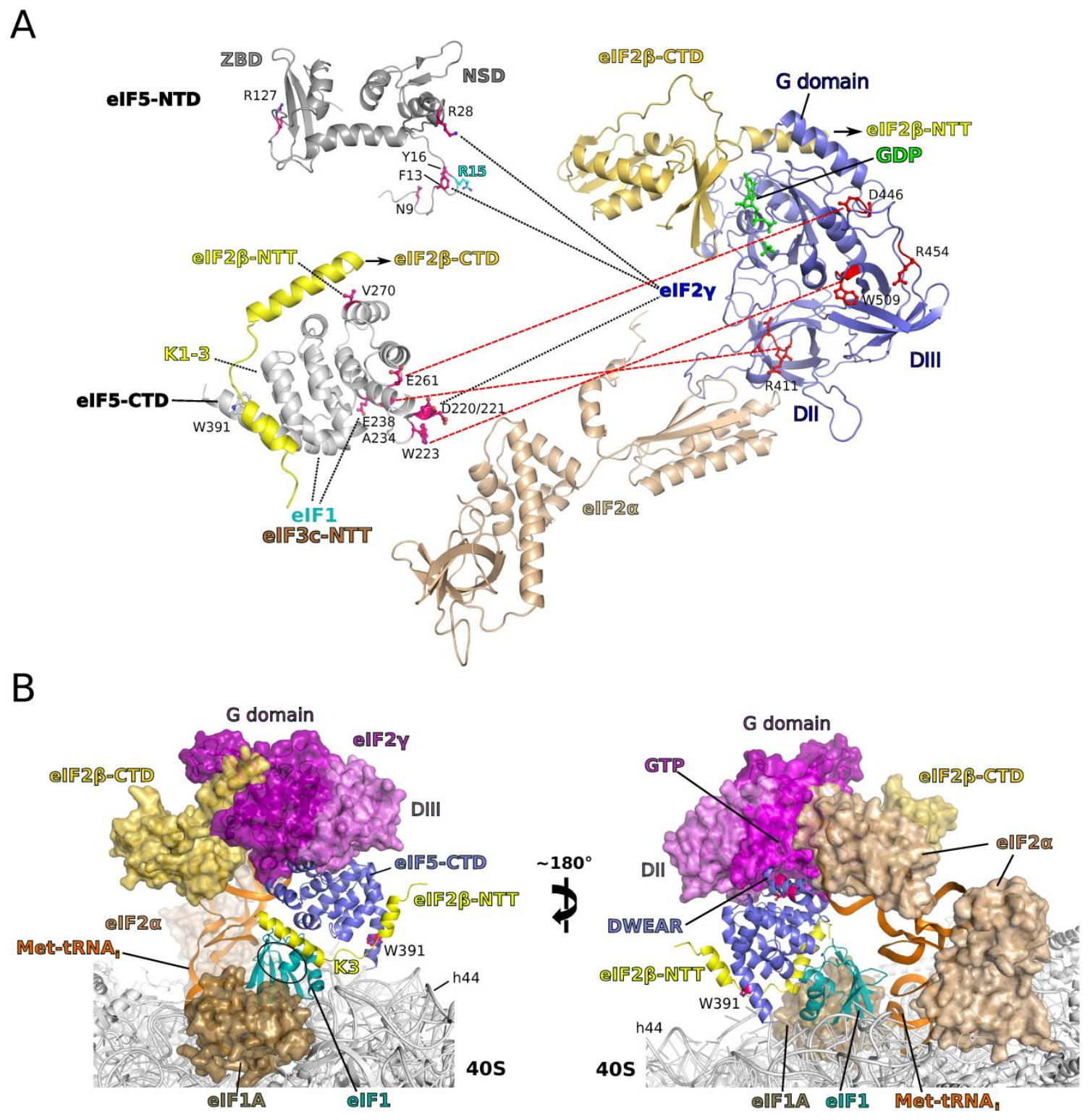

Figure 13. Model of the interactions between elF5 and elF2. A) Interaction map between elF2 and elF5 obtained by fluorescence and Bpa crosslinking experiments. elF5-NTD and -CTD are shown in dark and light grey, respectively, with Bpa positions indicated (pink sticks). The elF2 $\beta$-NTT bound to elF5-CTD and the elF2 $\beta$-CTD bound to elF $2 \gamma$ (blue, with GDP in green) are shown in light and dark yellow, respectively. elF $2 \alpha$ is colored in beige. Red dashed lines indicate identified crosslinks between elF5-CTD and elF2 $\gamma$, while black dashed lines indicate crosslinks were the target protein but no specific peptide could be identified. The heterotrimeric elF2 complex was modeled using the structures of the archaeal ortholog (aIF2) and human elF2 $\alpha$ (PDBs: 3CW2, 1Q8K). B) Model of the elF5-CTD in the context of the 43S preIC, based on the cryo-EM map of the mammalian 435 pre-IC (EMDB accession code: 5658), the crystal structures of elF1 and elF1A bound to the $40 \mathrm{~S}$ subunit $[19,50]$ and the interactions depicted in (A). The elF5-CTD (blue cartoon) thereby comes to lie in the gap between elF $2 \gamma$ (purple) and the platform of the 40 S subunit (grey), placing the elF $2 \beta$ NTT and area I in direct vicinity to the binding site for elF1 (cyan cartoon; the circle indicates the KH area) and close to elF1A (brown surface). 
Central mechanistic questions that arise from the presented data are how elF5-CTD contacts elF2 $\gamma$ and how this interaction stabilizes the bound guanine nucleotide. The mass-spectrometry analysis of the crosslink data shows that particularly the $\mathrm{N}$-terminal residues of the DWEAR-motif, as well as Glu261 from area III lie in direct vicinity to the frontal face of elF2 $\gamma$ between $G$ domain, domain II and domain III and thus close to the nucleotide binding pocket (Fig. 8 and 13). It is thus possible that elF5-CTD stabilizes the nucleotide through direct contacts with the switch regions and/or the P-loop in a manner similar to the N-terminal $\alpha$-helix bundle of RhoGDIs in complex with Rho proteins $[106,107]$ or the C-terminal $\alpha$-helical 'GoLoco' motif in regulators of $G$ protein signaling (RGS) of G $\alpha$ proteins [108-110]. However, although the mutational analysis indicates that the conserved Asp220, Asp221, Trp223 and/or Arg235 in the DWEAR-motif could play a role in nucleotide-stabilization similar to the conserved Asp45 in the RhoGDI Cdc42 [110] or the arginine residues of the GoLoco motif [109], the absence of any effect of elF5 on the fluorescence signal of elF2-bound mant-GDP/GTP (excited directly or indirectly by FRET) rather speaks against the introduction of residues directly into the nucleotide binding pocket itself. Moreover, according to our structural model of the DWEAR motif as part of the elF5-CTD (Fig. 5), the effect of Arg235 mutations on nucleotide stabilization could also be explained by the putative structural role for the formation of the interaction interface within elF5-CTD. A similar structural role for Asp220, Asp221 and/or Trp223 can also not be excluded. Instead, we assume that the elF5-CTD acts allosterically by stabilizing the overall elF2 $\gamma$ in its nucleotide-bound conformation necessarily forming direct interactions with the P-loop or the switch regions. This would be in agreement with the observation that Bpa-constructs of elF5-CTD crosslink to elF2 in its GDP-, GTP- and Met-tRNA ${ }_{i^{-}}$ bound form with virtually identical efficiency, and that the effect of elF5-CTD on nucleotide binding is not limited to GDP-bound elF2 (Fig. 7 and 8D). Both observations indicate that the contact between elF5-CTD and elF2 $\gamma$ does not depend on the conformation of the two switch regions. Moreover, they are in line with the structural studies on the archaeal elF2 ortholg (alF2), which indicate that the overall domain arrangement of alF $2 \gamma$ remains essentially unchanged between apo, GDP- and GTP-bound states with domains I-III adopting a 'closed' conformation. This conclusion results in the interesting implication that the direct contact of elF5-CTD to elF2 $\gamma$ is not 
idiosyncratic to the elF2.GDP.elF5 complex but may in fact exist during various stages of the initiation process, including assembly of pre-ICs, as well as the scanning process (see next section).

Role of the direct interaction between elF5-CTD and elF2 $\gamma$ : Although the DWEAR-motif is highly conserved among eukaryotes from yeast to human (Fig. S1), suggesting an important role in elF5 function, this role is still elusive. Hence, a critical question that arises from our own and previous data concerns the physiological relevance of the direct interaction between the elF5-CTD and elF2 $\gamma$.

Originally, the DWEAR-motif was identified as a region within elF5 that stabilizes elF2-bound GDP [69]. Based on this observation it was proposed that elF5 on the one hand physically antagonizes the GEF function of elF2B by competing for their common substrate elF2. On the other hand, it has been suggested that elF5 might acts as guanine-nucleotide dissociation inhibitor (GDI) for elF2 that antagonizes spontaneous GDP dissociation and may be necessary for tight translational control [69]. With the central difference that we assume the DWEAR-motif to belong to the elF5-CTD itself, our own results support both effects in vitro, showing that elF5 reduces the dissociation rate of elF2-bound GDP by more than 2-fold (Fig. 7 and Table 4) and antagonizes elF2Be-catalyzed GDP-dissociation at low GEF concentrations in a manner dependent on the presence of the DWEAR-motif (Fig. 12). This could indeed support a role for the elF5-CTD.elF2 $\gamma$ contact as physical antagonist of elF2B association and nucleotide exchange.

However, the claim of physiological relevance for these effects is problematic: The role of elF5 as physical antagonist for elF2B was recently questioned by the finding thatelF2B $p$ provides a second activity within the elF2B catalytic subcomplex as 'GDI displacement factor' (GDF) that effectively displaces elF5 from elF2 under physiological conditions [105]. The data presented in Figure 12 suggest that elF2B $\gamma$ may destabilize the direct contact of the elF5-CTD to elF2 $\gamma$ and thereby supports the entry of the elF2Be catalytic domain even at low (physiological) GEF concentrations. For the role of the DWEAR-motif, this poses the question for the cause-and-effect relation between the eIF5-CTD and elF2B $y$. The fact that elF2B can effectively displace elF5 under normal as well as starvation conditions (with phosphorylated elF $2 \alpha$ ) argues against a functionally relevant role for elF5 as physical antagonist [73]. In turn, this indicates that the GDF activity of 
elF2B evolved to antagonize the direct elF5-CTD elF2 $\gamma$ contact, which would thus have to serve a different function at an earlier stage in the nucleotide cycle of elF2. One of these possible functions is the inhibition of spontaneous GDP release from free elF2, thereby allowing a tighter control of translation initiation under stress conditions [73]where an efficient stress response is dependent on the reduction of elF2-TC levels [80]. Originally, this assumption was based on the observation that the W391F mutation in elF5, which reduces the GDI activity, confers a Gcn phenotype due to its inability to induce GCN4 translation under starvation conditions [69] (see introduction of chapter 4 for an explanation of the Gcn phenotype). However, our own as well as previous data show that Trp391 is directly involved in the interactions with the elF2ß-NTT (Fig. 2)[35], indicating that its mutation reduces the presumed GDI function indirectly by destabilizing the entire elF2-elF5 complex. It is thus problematic to assign the observed Gcn phenotype to the GDI function alone, as the W391F mutation is likely to affect MFC- and pre-IC formation, as well as start codon recognition. As a consequence, W391F might confer a $\mathrm{Gcn}^{-}$phenotype due to leaky-scanning on UORF1 on the GCN4 mRNA leader, similar to other elF5 mutations that destabilize its contacts to the elF2 $\beta$-NTT [58]. Moreover, the same study found that the elF5 ${ }^{\text {LR7A }}$ mutation, in which seven of the conserved DWEAR-motif residues were mutated to Ala, had an even stronger negative effect on the in vitro GDI activity of elF5, however, without conferring a Gen phenotype; this supports the idea that the loss of GDI function is not the primary origin underlying the reported W391F phenotype. Another indication for the relevance of the GDI activity is, according to Jennings and Pavitt [69], provided by the observation that the W391F and LR7A mutations in elF5 suppress the lethality of the N249K mutation in elF2B $[105]$. This was explained by the bypass of the GEF deficiency in elF2B by an increase in spontaneous GDP dissociation from elF2 due to a reduced GDI activity in elF5. The central problem with this interpretation is that it is not known how the N249K mutation affects the GEF function by eIF2B in vivo. The isolated elF2Be-N249K mutant catalyzes nucleotide exchange as efficiently as the wild-type protein [71], arguing against a direct effect on the GEF activity. Instead, the deletion of the corresponding $\mathrm{N}$-terminal region within elF2B $\varepsilon$ (residues 93-358) has a severe negative impact on the interactions with other elF2B subunits, in line with its implication in interactions with elF2B $y$ and the formation of the $\gamma_{2} \varepsilon_{2}$ tetrameric core of the functional elF2B decamer [105]. Consequently, N249K most likely reduces GEF activity in vivoby 
negatively affecting elF2Bye complex formation, which is essential for productive interactions with elF2 and the GDF function of elF2By [105]. The W391F and LR7A mutations in elF5 would thus suppress the phenotype of elF2BE-N249K by allowing the partially inactive elF2B complex to compete more efficiently for their common substrate elF2 and not due to increased spontaneous GDP dissociation. In line with this interpretation, W391F and LR7A are unable to rescue growth of the elF2BE-E569D mutant, in which complete GEF-inactivity is caused by the loss of a key catalytic residue. Thus, the increased spontaneous GDP release is not sufficient to allow efficient translation in the absence of GEF activity. Finally, it should be noted that elF5 reduces the rate of GDP dissociation only $\sim 2$-fold at saturating elF5 concentration (Fig. 9B and Table 4). As elF5 and elF2 are thought to exist in a 1:1 ratio in the cell, this effect on free elF2 would be additionally reduced to $\sim 1.5$-fold. Thus, although the elF2-elF5 complex may play a role as stable cytosolic pool for elF2 similar to the Rho-RhoGDI complex $[68,101]$ or may be relevant fora channeling process, where the potentially unstable elF2 is passed on from elF5 to elF2B, it is in our opinion unclear to what extent the stabilization of the elF2-bound guanine nucleotide is actually relevant for translation regulation.

An alternative option is that the moderate nucleotide-stabilizing effect is not a functionally relevant feature of elF5 but merely the consequence and therefore rather a side effect of the direct interaction between the elF5-CTD and elF2 $\gamma$, causing an increased internal rigidity of the latter. In this case, the actual mechanistic significance of the interaction may not lie in the delayed GDP release but in the proper arrangement of the elF5-CTD relative to the G domain of elF2. This assumption is supported by the observation that GDP-, GTP-, and GTP/Met-tRNA ${ }_{i}$-bound elF2 seem to interact equally well with the elF5-CTD (Fig. 8D).This suggests that the direct elF5-CTD.elF2 $\gamma$ contact exists not only in solution with the GDP-bound form, but my as well be formed at other stages of the initiation process in which elF2 exists in its active GTP-bound form. Hereby, a central aspect is the observation that the DWEAR-motif does not belong to the flexible linker region as previously assumed, but instead forms an element of the CTD itself - possibly stabilized in the elF5-elF2 complex. Thus, the elF5-CTD·elF2 $\gamma$ interaction might play a functional role in the context of the interactions between elF5-CTD, the elF2 $\beta-N T T$, elF1, elF1A and elF3c and their involvement in pre-IC formation, scanning and start codon recognition (see below). Interestingly, such a contact 
would be compatible with the recently reported cryo-EM model of the $43 \mathrm{~S}$ pre-IC, in which the elF5-CTD can be accommodated between elF2 $\gamma$ of the TC and the $40 \mathrm{~S}$ subunit (Fig. 13B). In agreement with previous suggestions $[21,63]$ this would position the elF5-CTD within contact distance of the 40S subunit and with areas I and II in direct vicinity to the binding sites of elF1 and elF1A. It is thus possible that the conserved elF5-CTD.elF2 $\gamma$ interaction may play a subordinate role in the communication between the ribosome, ribosome-associated initiation factors elF1 and elF1A, and the $y$-subunit of elF2. This suggestion is clearly tentative and will have to be tested experimentally in the context of the initiation machinery; however, it would be in line with the newly identified key role for the elF5-CTD in scanning and start-codon recognition and the requirement for a factor that mediates between the rearrangements occurring on the ribosome and the detached $G$ domain of elF2 $\gamma$, where $P_{i}$ release occurs in response to the formation of the codon-anticodon interaction between mRNA and initiator tRNA, reorganization of elF1A and the dissociation of elF1 from the P-site[21, 63, 64].

The eIF5-NTD interacts directly with elF2y in the elF2 elF5 complex. Another central aspect of the processes of scanning and start-site selection is the role of the elF5-NTD, the GAP domain of elF5. A previous study proposed direct interactions between the elF5-NTD and elF2 $\gamma$ [65]. However, the designated elF5-NTD of this study included residues 1-279, containing the NTD, the linker region as well as the DWEAR-motif and $R_{1}$ of the CTD. Although the elF5(1-262) construct is unable to stabilize the guanine nucleotide (Table 4 ) and is therefore most likely unable to stably contact the $Y$-subunit, it is unclear what effect the presence of the complete $R_{1}$ repeat would have. Hence, it cannot be ruled out that the observed direct contact between elF5(1-279) and elF2 $\gamma$ was at least partially mediated by its C-terminal portion and not through the actual NTD. We reassessed this question and found that the elF5-NTD in the complex with elF2 is indeed positioned in direct proximity to the $\gamma$-subunit in a way that allows specific crosslinks from positions Phe13 next to the putative arginine-finger (Arg15) in the $\mathrm{N}$-terminal tail, and from Arg28 in the first $\beta$-hairpin loop of the N-terminal subdomain, but not from Arg127 in the zinc binding domain (ZBD) (Fig. 11 and 13A). As for elF5-CTD, the crosslinks occur with free elF2 as well as the elF2-TC, indicating that the underlying interactions are independent of or even slightly more specific in the presence of bound 
Met-tRNA $_{i}$ (Fig. 11B). However, a dependency of the interactions on the presence of GTP or the transition state mimic GDP+AlF could not be observed (Fig. S6), which stands in contrast to previous reports for the mammalian factors [53] but is consistent with the dependency of GTPase activation by elF5 on the structural context of the 43S pre-IC [21]. It is thus likely that the direct interactions between elF5 and elF2 $\gamma$ that result in the observed crosslinks are of a transient nature and require additional initiation factors or the 405 subunit itself to be stabilized for productive interactions with the elF2 2 G domain to carry out its GAP activity.

The interaction network between eIF5-CTD, elF26-NTT, elF1 and elF3c-NTD. The crystal structure of the elF5(201-405)·elF2 $\beta(66-106)$ complex in combination with tryptophan quenching studies suggest a highly dynamic mode of interactions between elF5 and elF2 $\beta$ with distinct roles for each of the three conserved K-boxes. $\mathrm{K} 1$ and $\mathrm{K} 2$ seem to be mainly responsible for the high affinity interactions with a $K_{\mathrm{d}}$ of $\sim 100 \mathrm{nM}$ that center on the negatively charged area I close to Trp391 of elF5-CTD and involve residues of both AA-boxes (Fig. 2, 4 and SC). By contrast, K3 is positioned in the periphery of area I and contributes only marginally to the overall affinity (Fig. 2 and Table 1), but might, together with the adjacent helix $\alpha C^{\beta}$, serve a role by pre-arranging the elF5-CTD relative to elF2 in order to promote the weak direct interaction between area III ${ }^{\text {ext }}$ and elF2 $\gamma$ (Fig. 9).

As indicated by the mutational studies, the contributions of individual K-boxes to the interactions with elF5-CTD are mainly additive; this indicates that the elF2 $\beta-N T T$, which is presumably unfolded in the unbound state [111], does not adopt a distinct tertiary domain fold upon binding to elF5. Instead, it seems to form a peptide-domain interaction with elF5-CTD, in which only some parts adopt a secondary structure such as $\alpha N^{\beta}$ and $\alpha C^{\beta}$ flanking K-box 3 (Fig. 3 and 4). This mode of segmented peptide-domain contact provides a number of context-dependent advantages over the rigid domain-domain interaction. On the one hand, it allows rapid evolution and adaptation to interactions with varying partners, as the peptide sequence is constrained only by the need to conserve residues directly involved in these interactions and can be easily extended in length to provide new interfaces. On the other hand, it introduces the means for a high degree of internal variability in the interactions, as each interacting segment (i.e. the K-boxes) can be released and/or reorganized individually, while the remaining segments are unaffected in their 
contacts and retain reasonable affinity. This is conceivable particularly in the case of the lysine-rich $\mathrm{K}$-boxes in the eIF2 $\beta$-NTT, for which the disorder-to-order transition upon binding to elF5-CTD is most likely accompanied by a particularly large loss of internal conformational entropy, which is paid individually by the K-boxes and not collectively by the entire interface. This unfavorable entropic contribution results in an uncoupling of binding strength from specificity, giving rise to specific interactions that are at the same time relatively weak and thus reversible.

Functional relevance for this mode of interaction most likely lies in the complex series of rearrangements between elF5-CTD, elF2 $\beta$-NTT and other initiation factors such as elF1, elF1A, and eIF3c, which underlie the formation of the scanning-competent pre-IC and start codon recognition. In yeast cells, formation of the MFC is thought to provide a major intermediate step for the efficient recruitment of the eIF2-TC to the $40 \mathrm{~S}$ subunit and pre-IC assembly. The stability of the MFC and consequently its ability to promote TC recruitment was shown to depend on an intricate interaction network between elF5-CTD, eIF2 $\beta$-NTT, elF1 and elF3c-NTD, in which the loss of one of these factors negatively affects the incorporation of the others into the $43 \mathrm{~S}$ pre-IC. In this context, the eIF2 $\beta$-NTT appears to play a central role for the nucleation of MFC assembly through its tight interactions with the elF5-CTD and the promotion of elF1 and elF3c binding to elF5-CTD. In line with this scenario, our structural and biochemical studies show how the elF2 $\beta$-NTT can interact with area I of elF5-CTD (Fig. 2 and 4), while at the same time supporting the binding of the elF1NTT and elF3c-NTD in area II (Fig. 10). By contrast, the second binding site on the elF5-CTD for elF1, which lies in the vicinity of Val270, is incompatible with the simultaneous binding of the elF2 $\beta$-NTT (Fig. 10). A possible mechanistic significance of this latter observation is unclear, as the loss of this elF1-binding interface due to the mutagenesis of elF5 causes the retention of elF1 within the pre-IC and a suppressor of sui' (Ssu') phenotype (due to an increased stringency of AUG selection) rather than increased dissociation rates, most likely due to the simultaneous disruption of the binding interface between elF5 and elF2 $\beta$-NTT (see below)[21].

Apart from its involvement in cooperative MFC formation, the elF2 $\beta$-NTT is as well involved in the stabilization of elF1 on the $40 \mathrm{~S}$ subunit in its open, scanning competent conformation. However, this interaction is thought to be destabilized upon the encounter of the AUG start codon in a manner that depends on a displacement of elF1 from the elF2 $\beta$-NTT by the elF5-CTD [21, 63]. 
Thus, in light of the proposed mode of peptide-domain interaction, this could indicate that one segment of the elF2 $\beta$-NTT interacts with elF5-CTD throughout the process of pre-IC formation, scanning and start-site selection, whereas a second region switches between eIF5-CTD and elF1 in a context-dependent manner, with elF1 as the preferred interaction partner in the open, scanning competent pre-IC, while this preference switches to elF5-CTD upon the encounter of the AUG start codon and initiator tRNA-induced displacement of elF1 from the P-site [21]. In this context, the critical aspect of the nature of the peptide-domain interaction between elF2 $\beta$-NTT and elF5-CTD would be the independency of TC stabilization in the pre-IC from these rearrangements, while they at the same time provide a high degree of flexibility and the means for a tightly balanced dynamic interplay with other factors in a changing structural context. Interestingly, the placement of the eIF5-CTD in the cryo-EM structure of the mammalian $43 \mathrm{~S}$ pre-IC based on the crosslinking data, would be compatible with such rearrangements, as it would position the elF2 $\beta$-NTT directly between the eIF5-CTD and elF1 bound adjacent to the ribosomal P-site (Fig. 13B).

\subsection{Materials and Methods}

\section{Cloning}

DNA fragments corresponding to the open reading frames of elF1 (encoded by the TIF1 gene in S. cerevisiae), elF2 $\beta$ (SUI2), elF5 (TIF5) and elF2BE (GCD6) or truncations thereof were amplified by PCR using yeast genomic DNA as template and a corresponding pair of primers. These fragments were digested using restriction enzymes for which the restriction sites had been incorporated into the amplification primers. The digested DNA fragments were then ligated into the desired vectors cut with the same restriction enzymes. The ligation mixture was subsequently transformed into XL1-Blue E. coli cells (Stratagene) and plated out on LB agar plates containing the appropriate antibiotics. Several colonies of each plate were picked and subjected to a test PCR to identify positive clones, which were then individually grown over night in LB medium supplemented with antibiotics to purify the plasmid bythe Qiaprep Spin Miniprep Kit (Qiagen) according to the manufacturer's instructions. The sequences of the selected constructs were verified by DNA sequencing. 
Single or multiple point mutants of the different constructs were generated by the QickChange technique (Stratagene) using appropriate mutagenesis primers. For the incorporation of the genetically encoded unnatural amino-acid p-benzoyl-L-phenylalanine (Bpa) into a protein chain, the amber stop codon TGA was introduced into the desired position along the coding sequence. In the case of the elF5 constructs, this was preceded by the replacement of the endogenous TGA stop codon by TAA. Plasmid preparation and verification of the correct sequence were performed as described above.

\section{Protein expression and purification}

All constructs except those for the incorporation of Bpa (see below) were expressed in $E$. coliBL21(DE3) Rosetta II cells (Novagene). Cells containing the respective plasmid were grown in $2 \mathrm{XYT}$ (supplemented with antibiotics) at $37^{\circ} \mathrm{C}$ with shaking at $220 \mathrm{rpm}$ until they reached an $\mathrm{OD}_{600}$ of $\sim 0.8$ and were subsequently transferred to $16^{\circ} \mathrm{C}$. After $20 \mathrm{~min}$ the expression was induced by the addition of isopropyl- $\beta$-D-thiogalactopyranosid (IPTG) to a final concentration of 0.5-1 mM. The cells were harvested after 16 hours at $16^{\circ} \mathrm{C}$.

For the expression of proteins containing the genetically encoded unnatural amino-acid Bpa, the mutant constructs were transformed into the expression strain BL21(DE3)+pSupBpa (provided by Heinz Neumann)[94]. The cells were grown in $300 \mathrm{ml}$ cultures of LB medium, supplemented with antibiotics and $1 \mathrm{mM} \mathrm{Bpa(provided} \mathrm{by} \mathrm{Heinz} \mathrm{Neumann),} \mathrm{and} \mathrm{grown} \mathrm{at} 37^{\circ} \mathrm{C}$ until an $\mathrm{OD}_{600}$ of $\sim 0.6$ was reached. The cultures were then transferred to $25^{\circ} \mathrm{C}$ and the expression induced by the addition of 1 mM IPTG. The cells were harvested 15 hours after induction.

Purification of elF1: elF1 (wild-type and Bpa constructs) were expressed without affinity tag. The cells were resuspended in L-150 buffer (150 mM NaCl, 20 mM HEPES (pH 7.5), 5\% glycerol, 1 mM EDTA, $4 \mathrm{mM} \beta$-mercaptoethanol) supplied with a mixture of protease inhibitors including aprotinin, leupeptin, pepstatin (ALP), and PMSF. The cells were lysed in a microfluidizer (Microfluidics, USA) and cell debris was removed by centrifugation for $30 \mathrm{~min}$ at 30,000 xg. The supernatant was applied to an SP Sepharose column (GE Healthcare) equilibrated in L-150 buffer. After loading the sample and washing the column with four column volumes of L-150 buffer, the bound protein was eluted with a linear gradient of high salt buffer $(1 \mathrm{M} \mathrm{NaCl}, 20 \mathrm{mM} \mathrm{HEPES}(\mathrm{pH}$ 
7.5), $5 \%$ glycerol, $4 \mathrm{mM} \beta$-mercaptoethanol). Fractions containing elF1 (a single peak at $\sim 300 \mathrm{mM}$ $\mathrm{NaCl}$ ) were pooled and the salt concentration reduced to $\sim 150 \mathrm{mM} \mathrm{NaCl}$ by dilution with L-150 buffer containing no $\mathrm{NaCl}$. The diluted protein was applied to a HiLoad Heparin Sepharose column (GE Healthcare) equilibrated in L-150 buffer. Elution was started with a linear gradient into high salt buffer. Fractions containing elF1 were pooled and applied to a Superdex S-75 size exclusion column (GE Healthcare) equilibrated in GF-150 buffer (150 mM KCl, 10 mM HEPES (pH 7.5), 5\% glycerol, 2 mM DTT). Fractions containing the pure protein were pooled, concentrated and flashfrozen. The identity of elF1 was verified my mass spectrometry analysis.

Purification of elF2B and elF3c-NTD: Full-length elF2 $\beta$ was expressed as GST-fusion protein. Cell lysis was carried out as described above with the difference that L-500 buffer $(500 \mathrm{mM} \mathrm{NaCl}$, 20 mM HEPES (pH 7.5), 5\% glycerol, 0.1 mM EDTA, 4 mM $\beta$-mercaptoethanol) was used instead of L-150 buffer. After centrifugation, the supernatant was loaded onto a GSTrap column (GE Healthcare) equilibrated in L-500 buffer. After loading of the sample the column was washed with 2 column volumes of L-500 buffer and the bound fusion protein was eluted by washing the column with elution buffer ( $L-500$ buffer with $30 \mathrm{mM}$ reduced glutathione). Fractions containing the target protein were pooled and incubated over night at $4{ }^{\circ} \mathrm{C}$ with PreScission protease (GE Healthcare) in a 1:100 weight ratio of protease to fusion protein to remove the GST tag. After a desalting step in $200 \mathrm{mM} \mathrm{NaCl}, 20 \mathrm{mM}$ HEPES (pH 7.5), 5\% glycerol, $4 \mathrm{mM} \beta$-mercaptoethanol, the cleaved GST, PreScission protease and uncleaved protein were removed by a second GSH Sepharose step. The flow-through containing the cleaved elF2 $\beta$ was pooled, concentrated and applied to a Superdex S200 column (GE Healthcare) equilibrated in GF-150 buffer. Fractions containing the pure protein were pooled, concentrated and flash-frozen. All elF2 $\beta$ truncations and elF3c(1-136) (elF3c-NTD) were purified according to the same protocol described for full-length elF2 $\beta$ (the plasmid used for the expression of elF3c(1-136) was obtained from S. Khoshnevis).

Purification of elF5-CTD and elF2BE-CTD constructs: All C-terminal elF5 constructs and elF2Bع(524-712) were expressed as GST-fusion protein and purified similar to elF2 $\beta$ with some differences: After loading the supernatant onto the first GSTrap column and washing with L-500 buffer, the column was equilibrated in low salt buffer (100 mM NaCl, $10 \mathrm{mM}$ HEPES (pH 8), 5\% glycerol, $4 \mathrm{mM} \beta$-mercaptoethanol) before the bound fusion protein was eluted with low salt 
buffer plus $30 \mathrm{mM}$ reduced glutathione. After PreScission protease treatment, the cleaved protein was loaded onto a Source 300 column (GE Healthcare) equilibrated in low salt buffer. While GST and the protease eluted in the flow-through, the target proteins bound to the column and were eluted with a linear gradient into high salt buffer ( $1 \mathrm{M} \mathrm{NaCl}, 10 \mathrm{mM}$ HEPES ( $\mathrm{pH} 7.5), 5 \%$ glycerol, 4 $\mathrm{mM} \beta$-mercaptoethanol). Fractions containing the target protein were pooled and loaded onto a Superdex S-75 column equilibrated in GF-150 buffer. Fractions containing the pure protein were pooled, concentrated and flash-frozenfor storage at $-80^{\circ} \mathrm{C}$.

Purification of full-length elF5 and elF5(1-262): Full-length elF5 and elF5(1-262) were expressed as $\mathrm{N}$-terminally polyhistidine-tagged proteins. Cell resuspension and lysis were carried out essentially as described above for elF $2 \beta$ with the difference that L-500-His buffer additionally contained $20 \mathrm{mM}$ imidazole and no EDTA. After centrifugation the supernatant was loaded onto a HisTrap column (GE Healthcare) equilibrated in L-500-His buffer. The column was then washed with 2 column volumes of L-500-His buffer and bound protein was eluted with a linear gradient into elution buffer (L-500-His buffer with $300 \mathrm{mM}$ imidazole). Fractions containing the target protein were pooled and incubated over night at $4{ }^{\circ} \mathrm{C}$ with TEV protease in a 1:100 weight ratio of protease to target protein to remove the His-tag. After a desalting step in $250 \mathrm{mM} \mathrm{NaCl}, 20 \mathrm{mM}$ HEPES (pH 7.5), 5\% glycerol, $2 \mathrm{mM} \beta$-mercaptoethanol and $15 \mathrm{mM}$ imidazole, the cleaved His-tag, TEV protease and uncleaved protein were removed by a second Ni-NTA step. The flow-through containing the cleaved elF5 was pooled, concentrated and applied to a Superdex S-200 column equilibrated in GF-150 buffer. Fractions containing the pure protein were pooled, concentrated and flash-frozen for storage at $-80^{\circ} \mathrm{C}$.

Purification of elF2: Polyhistidine-tagged elF2 was overexpressed in S. cerevisiae strain GP3511 (a gift from G. Pavitt) containing a high-copy plasmid for the expression of all three subunits of elF2 (SUI2 (elF2 $\alpha$ ), SUI3 ( $\beta$ ), and His-tagged GCD11 ( $($ )] [112]. For expression, 15 1-L cultures of YPD media were each inoculated with $1 \mathrm{ml}$ of an overnight culture and grown for 16 hours at $30{ }^{\circ} \mathrm{C}$ with shaking at $220 \mathrm{rpm}$. The cells were harvested, resuspended in lysis buffer (500 $\mathrm{mM} \mathrm{KCl}, 50 \mathrm{mM}$ HEPES ( $\mathrm{pH}$ 7.5), $5 \%$ glycerol, $20 \mathrm{mM}$ imidazole, $1 \mathrm{mM} \mathrm{MgCl}_{2}, 4 \mathrm{mM} \beta$ mercaptoethanol) containing protease inhibitors ALP and PMSF, flash-frozen in liquid nitrogen and disrupted in an ultra centrifugal rotor mill (Retsch ZM200, \# 20.823.0001). After thawing of the cell 
powder, the lysate was clarified by centrifugation at $45,000 \mathrm{xg}$ for 45 minutes. The supernatant was filtered and applied to a HisTrap column equilibrated in lysis buffer. After washing the column with 4 column volumes of lysis buffer, bound protein was eluted with lysis buffer containing 300 $\mathrm{mM}$ imidazole. Fractions containing elF2 were pooled and the salt concentration reduced to $\sim 250$ $\mathrm{mM} \mathrm{KCl}$ by dilution with buffer containing $20 \mathrm{mM}$ HEPES (pH 7.5), 5\% glycerol and $4 \mathrm{mM} \beta$ mercaptoethanol. The diluted sample was then loaded onto a HiLoad Heparin Sepharose column equilibrated in 300 mM KCl, 20 mM HEPES (pH 7.5), 10\% glycerol and 4 mM $\beta$-mercaptoethanol. The column was washed with low salt buffer and bound protein was eluted with a linear gradient of high salt buffer (1 M KCl, 20 mM HEPES (pH 7.5), 10\% glycerol, 4 mM $\beta$-mercaptoethanol). Fractions containing elF2 were pooled, concentrated and loaded onto a Superdex S-200 column equilibrated in GF-150 buffer. The absence of residual guanine nucleotides in the elF2 preparations was verified by HPLC on a NUCLEOSIL 4000-7 PEI column (Macherey-Nagel). Nucleotide-free elF2 complex was concentrated, flash-frozen and finally stored at $-80^{\circ} \mathrm{C}$.

Purification of the yeast Methionyl-tRNA Synthetase: Yeast Methionyl-tRNA Synthetase was expressed in S. cerevisiae as GST-fusion protein from a plasmid containing a TRP auxotrophic marker (a gift from J. Lorsch). 15 1-L cultures of synthetic complete medium lacking tryptophan (SC-Trp medium) were inoculated from an overnight culture of yeast cells containing the plasmid for expression of GST-MetRS and were grown at $30^{\circ} \mathrm{C}$ for 12 hours. Harvesting and lysis of the cells was carried out as described for elF2 (see above), except for a different lysis buffer (500 mM NaCl, 50 mM HEPES (pH 7.5), 5\% glycerol, $2 \mathrm{mM} \beta$-mercaptoethanol). The cleared lysate was loaded onto a GSTrap column equilibrated in lysis buffer. After washing the column with 4 column volumes of lysis buffer, the bound protein was eluted with $20 \mathrm{mM}$ reduced glutathione. The fractions containing the GST-MetRS were pooled, concentrated and loaded onto a Superdex S-200 column equilibrated in $100 \mathrm{mM} \mathrm{KCl}, 40 \mathrm{mM}$ Tris/ $\mathrm{HCl}$ (pH 7.4), $10 \mathrm{mM} \mathrm{MgCl}$, 5\% glycerol, $2 \mathrm{mM}$ DTT. The pure GST-MetRS was flash-frozen in liquid nitrogen and stored at $-80^{\circ} \mathrm{C}$. 
Purification, crystallization and structure determination of the elf5(201-405)-elF2 $\beta(39-106)$ complex

The individual proteins, elF2 $\beta(39-106)$ and elF5(201-405), were purified as described above. The complex was formed by mixing elF5(201-405) with a 1.5-fold molar excess of elF2 $\beta(39-106)$. After incubation for $30 \mathrm{~min}$ at $20^{\circ} \mathrm{C}$ the complex was separated from excess elF2 $\beta(39-106)$ by size exclusion chromatography on a Superdex S-75 equilibrated in GF-150 buffer and finally concentrated to $\sim 20 \mathrm{mg} / \mathrm{ml}$. Other complexes that did not yield any crystals suitable for structure determination were purified by the same protocol.

Initial crystals of the elF5(201-405)-elF2 $3(39-106)$ complex grew at $20{ }^{\circ} \mathrm{C}$ within one day under a condition containing $0.4 \mathrm{M}\left(\mathrm{NH}_{4}\right)_{2} \mathrm{SO}_{4}$ and $0.05 \mathrm{M} \mathrm{Li}_{2} \mathrm{SO}_{4}$. After optimization, the best diffracting crystals were finally obtained with $12 \mathrm{mg} / \mathrm{ml}$ protein in $0.4 \mathrm{M}\left(\mathrm{NH}_{4}\right)_{2} \mathrm{SO}_{4}$ and $0.08 \mathrm{M}$ $\mathrm{Li}_{2} \mathrm{SO}_{4}$ at $10^{\circ} \mathrm{C}$.

X-ray diffraction data were collected at BL 14.1 (HZB, BESSY, Berlin) [113]. The obtained data set was processed in space group $\mathrm{P}_{2} 12$ using XDS [114] and scaled to a final resolution of $2.0 \AA$. The phase problem was solved by molecular replacement using the program PHASER [115] with the atomic coordinates of yeast elF5(241-396) (PDB: 2FUL) as search model. The initial structural model comprised two elF5(241-396) molecules per asymmetric unit. Missing regions of the elF5 construct (residues 201-240) and elF2 $\beta$ were built manually in Coot [116]. The final model was obtained gradually by iterative rounds of refinement in PHENIX [117], followed by manual model building.

\section{Synthesis and purification of Met-tRNA}

Yeast initiator tRNA was synthesized by in vitro transcription. As template, a 142 nucleotides long DNA was used that contained the T7 promoter (underlined) and a hammer-head (HH) ribozyme (italic), followed by the sequence for the tRNA itself (bold) (purchased from Sigma Aldrich), as previously described $[97,118]$ :

5'TAATACGACTCACTATAGGGAGACGCTCTGATGAGTCCGTGAGGACGAAACGGTACCCGGTACCGTCAGC GCCGTGGCGCAGTGGAAGCGCGCAGGGCTCATAACCCTGATGTCCTCGGATCGAAACCGAGCGGCGCTA CCA3' 
The use of the HH-ribozyme in front of the target RNA allows the transcription to begin with GGG instead of AGC, which results in a higher efficiency of the T7-RNA polymerase (T7-RNAP) [118]. After synthesis of the HH-tRNA fusion, the $\mathrm{HH}$-ribozyme cleaves itself off in a $\mathrm{Mg}^{2+}$ dependent reaction, leaving the native tRNA sequence.

The DNA template was amplified by PCR using 5'GGAGCCTAATACGACTCACTATAG3' as forward primer and $5^{\prime} \mathrm{T}^{\mathrm{m}} \mathrm{G}^{\mathrm{m}}$ GTAGCGCCGCTCGGTTTC3' as reverse primer. $\mathrm{T}^{\mathrm{m}}$ and $\mathrm{G}^{\mathrm{m}}$ are $2^{\prime}-\mathrm{O}$ methylated nucleotides to reduce the occurrence of $3^{\prime}$ heterogeneities due to addition of nontemplated nucleotides by the T7 polymerase [119]. The amplified template was purified by ethanol precipitation and dissolved in water.

The optimal conditions for the transcription reaction were determined in analytical scale by varying the $\mathrm{Mg}^{2+}$ concentration or the ratio of T7-RNAP to the DNA template. Preparative transcription reactions ( $7 \mathrm{ml}$ final volume) contained $1 \times$ Tris buffer, $5 \mathrm{mM} \mathrm{DTT}, 30 \mathrm{mM} \mathrm{MgCl} 2,75$ $\mu \mathrm{g} / \mathrm{ml}$ T7-RNAP, $0.54 \mu \mathrm{M}$ DNA template, and $2.5 \mathrm{mM}$ of each NTP. The reaction was allowed to continue for 4 hours at $37^{\circ} \mathrm{C}$. The mixture was then filtered and applied to a 1-ml Mono Q column (GE Healthcare). After washing the column with buffer A (400 mM Na-acetate, 0.2 mM EDTA, 100 $\mathrm{mM}$ Tris/ $\mathrm{HCl}(\mathrm{pH} 6.9))$, the elution was started by applying a $50-\mathrm{ml}$ gradient from $0-100 \%$ buffer $\mathrm{B}$ (1.5 M Na-acetate, 0.2 mM EDTA, $100 \mathrm{mM}$ Tris/HCl (pH 7.3)). The run was analyzed on a urea gel, showing that the cleaved $\mathrm{tRNA}_{i}$ eluted in a single peak containing small contaminations of $\mathrm{HH}$ ribozyme. Uncleaved $\mathrm{HH}$-tRNA and the DNA template eluted in a peak at higher salt concentrations, while the T7-RNAP eluted in the flow-through (according to SDS-PAGE analysis). Fractions containing the tRNA $\mathrm{A}_{\mathrm{i}}$ were pooled, concentrated to $\sim 2 \mathrm{ml}$ volume and applied to a Superdex S200 (16/60) column equilibrated in $25 \mathrm{mM}$ Tris/ $\mathrm{HCl}$ (pH 7.5), $30 \mathrm{mM} \mathrm{KCl}$ and $1 \mathrm{mM}$ DTT. The $t R N A_{i}$ eluted in a single peak, free of $\mathrm{HH}$-ribozyme. The pure $t R N A_{i}$ was stored at $-80{ }^{\circ} \mathrm{C}$.

In an alternative procedure, $\operatorname{tRNA}_{i}$ was in vitro transcribed and purified without $\mathrm{HH}$-ribozyme as a potential origin for a partial missfolding of the tRNA. A critical drawback of this strategy is that the T7-RNAP has to start at AGC instead of the optimal GGG in the HH-ribozyme, which results in lower initial yields. However, this is partially compensated by the fact that the $\mathrm{Mg}^{2+}$ concentration has to be optimized for the transcription efficiency alone and does not have to take the activity of the ribozyme into account as well. Moreover, no tRNA product is lost due to incomplete cleavage 
of the $H H$-tRNA $A_{i}$ fusion. For this purpose, the above reverse primer and 5'AGCCTAATACGACTCACTATAAGCGCCGTGGCGCAGTGG3' as forward primer were used to generate the new DNA template:

\section{5'AGCCTAATACGACTCACTATAAGCGCCGTGGCGCAGTGGAAGCGCGCAGGGCTCATAACCCTGATGTCC TCGGATCGAAACCGAGCGGCGCTACCA3'}

The optimal condition for the transcription reaction was found to be $16 \mathrm{mM} \mathrm{MgCl}, 50 \mu \mathrm{g} / \mathrm{ml}$ T7-RNAP and $0.68 \mu \mathrm{M}$ DNA template. The preparative reaction and subsequent purification was carried out as described above for the purification with $\mathrm{HH}$-ribozyme. From the same volume of preparative reaction mixture, the final yield of pure tRNA $i$ was usually about $25 \%$ lower than for the reaction with $\mathrm{HH}$-ribozyme.

Preparative charging of the $\operatorname{tRNA}_{i}$ with methionine was carried out essentially as described previously [97]. The stoichiometric charging reaction mixture contained $25 \mathrm{mM} \mathrm{Tris/HCl}(\mathrm{pH} 7.6)$, $10 \mathrm{mM} \mathrm{Mg}(\mathrm{OAc})_{2}, 1 \mathrm{mM}$ DTT, $2 \mathrm{mM}$ ATP, $200 \mu \mathrm{M}$ methionine, $5 \mu \mathrm{M}$ tRNA $\mathrm{A}_{i}$ and $3 \mu \mathrm{M}$ MetRS in a total volume of $4 \mathrm{ml}$. For 'hot' control reactions that were carried out parallel to the stoichiometric charging reaction, radiolabeled L-[ $\left.{ }^{35} \mathrm{~S}\right]$ methionine (Hartmann Analytic) was used to assess the amount of produced $\left[{ }^{35} \mathrm{~S}\right]$ methionyl-tRNA $i$ and thus the charging efficiency [97]. The reaction mixture was incubated at $30{ }^{\circ} \mathrm{C}$ for $20 \mathrm{~min}$, the time point at which the maximal charging efficiency, usually 34-36\%, had been reached according to the control reaction. The charged MettRNA $_{i}$ was then once extracted with buffered phenol and once with chloroform, followed by the addition of $0.3 \mathrm{M} \mathrm{NaOAc}(\mathrm{pH} 5)$ and an ethanol precipitation for 2 hours at $-20{ }^{\circ} \mathrm{C}$. The dried pellet was finally resuspended in $\mathrm{H}_{2} \mathrm{O}$ to a final concentration of $30 \mu \mathrm{M}$ Met-tRNA $A_{i}$ As the remaining uncharged tRNA $A_{i}$ was reported not to interfere significantly with the initiation process in an in vitro reconstituted system [97], a further purification of the charged initiator tRNA was not performed.

\section{UV Crosslinking Experiments with Bpa}

Crosslinking was performed by exposing Bpa-containing proteins either alone or in the presence of potential interaction partners to $365 \mathrm{~nm}$ UV light at a distance of $\sim 5 \mathrm{~cm}$ for 10-30 min on ice (Vilber Lourmat lamp, 2 X 8W, $365 \mathrm{~nm}$ tubes, 32 W, $230 \mathrm{~V}$ \#VL-208.BL). All reactions were performed in standard reaction buffer (100 mM KCl, $20 \mathrm{mM}$ Tris/ $\mathrm{HCl}(\mathrm{pH} 7.5), 3 \mathrm{mM} \mathrm{MgCl} 2$ and $2 \mathrm{mM} \mathrm{DTT})$. 
Following UV light irradiation, the crosslink products were separated by SDS-PAGE and stained with Coomassie blue. Crosslinking occurred in a UV- and time-dependent manner only in the presence of proteins containing Bpa. Protein bands of interest were excised and in-gel digested with trypsin or a mixture of trypsin and GluC as described [120] for subsequent analysis by ESIMS/MS2 (see below).

\section{Mass spectrometry analysis with nano LC-nano ESI-MS/MS2}

Nano LC - RSLCnano Ultimate 3000 system (Thermo Scientific): Peptides of $1 \mu$ l sample solution were loaded and washed on an Acclaim PepMap 100 column $(75 \mu \mathrm{m} \times 2$ cm, C18, $3 \mu \mathrm{m}, 100 \AA$, Thermo Scientific) at a flow rate of $25 \mu \mathrm{l} / \mathrm{min}$ for $6 \mathrm{~min}$ in $100 \%$ solvent $\mathrm{A}$ (98\% water, $2 \%$ acetonitrile, $0.07 \%$ TFA). Analytical peptide separation by reverse phase chromatography was performed on an Acclaim PepMap RSLC column $(75 \mu \mathrm{m} \times 25 \mathrm{~cm}, \mathrm{C} 18,3 \mu \mathrm{m}, 100 \AA$, Thermo Scientific) typically running a gradient from $98 \%$ solvent $A$ (water, $0.1 \%$ formic acid) and $2 \%$ solvent B $(80 \%$ acetonitrile, $20 \%$ water, $0.1 \%$ formic acid) to $42 \%$ solvent B within 95 min and to $65 \%$ solvent B within the next $26 \mathrm{~min}$ at a flow rate of $300 \mathrm{nl} / \mathrm{min}$ (solvents and acids from Fisher Chemicals).

Nano ESI mass spectrometry -OrbitrapVelos Pro (Thermo Scientific): Chromatographically eluting peptides were on-line ionized by nano-electrospray (nESI) using the Nanospray Flex lon Source (Thermo Scientific) at $2.4 \mathrm{kV}$ and continuously transferred into the mass spectrometer. Full scans within the mass range of 300-1850 m/z were taken from the Orbitrap-FT analyzer at a resolution of 60.000 with parallel data-dependent top 10 MS2-fragmentation with the LTQ Veleo Pro linear ion trap (CID). LCMS method programming and data acquisition was performed with the software XCalibur 2.2 (Thermo Fisher).

MS-data analysis - Protein identification with Proteome Discoverer 1.4: MS/MS2 data processing for peptides was done with the Proteome Discoverer 1.4 software (Thermo Scientific) and the Discoverer Daemon 1.4 software (Thermo Scientific) using the SequestHT and Mascot search engines (Thermo Scientific and Matrix Science, respectively) and a S. cerevisiae-specific protein databases (Saccharomyces Genome Database, SGD, Stanford University) extended by the most common contaminants. Oxidation (+ $16 \mathrm{Da})$ was considered as variable modification of 
methionine, carbamidomethylation (+ $57.02146 \mathrm{Da})$ as static modification of cysteine. False discovery rates of peptide identifications were obtained by decoy database analyses of the MS datasets. FDR was set 0.01 at maximum.

Identification of crosslinked peptides with StavroX: The search for target peptides for UV-induced crosslinks was performed using the program StavroX [102] with the following settings: Precision for precursor comparison, 10 ppm; precision of fragment comparison 0.8 Da; mass limits, 200-800 Da; used enzymes, trypsin and GluC, allowing 2 missed cleavages at Arg, Lys and Glu, and 3 missed cleavages at Asp; static modification on cystein (carbamidomethylation) and variable modification on methionine (oxidation); peaks were considered only with signal-to-noise ratio (S/N) above 2. The quality of the searches was assessed by a parallel decoy search using the reversed protein sequence to determine the occurrence of false positives, as well as by the calculation of a score, which is calculated from the number of signals above the specified $\mathrm{S} / \mathrm{N}$ value according to the algorithm given in [102].

\section{Steady-State Fluorescence Measurements}

All experiments were carried out on a Fluoromax-3 spectrophotometer (Jobin Yvon Inc., USA) using a quartz cuvette (Hellma Analytics, Germany). All reactions were done at $20 \pm 1{ }^{\circ} \mathrm{C}$ in the standard reaction buffer (100 $\mathrm{mM} \mathrm{KCl,} 20 \mathrm{mM}$ Tris/ $\mathrm{HCl}(\mathrm{pH} 7.5), 3 \mathrm{mM} \mathrm{MgCl} 2$ and $2 \mathrm{mM} \mathrm{DTT})$.

The binding of elF2 $\beta$ to elF5-CTD was monitored by tryptophan quenching experiments. Tryptophan fluorescence in elF5-CTD was excited at $280 \mathrm{~nm}$, resulting in an emission at $350 \mathrm{~nm}$, which was quenched upon the addition of elF2 $\beta$ (full-length and N-terminal domain fragments). Equilibrium dissociation constants $\left(K_{\mathrm{d}}\right)$ for the complexes were determined by titrating elF5-CTD with increasing amounts of elF2 $\beta$, until saturation was reached. The residual fluorescence signal produced by elF2 $\beta$ (which contains no tryptophan) alone was determined by blank-titrations into buffer and subsequently subtracted from the binding data.

The binding of mant-labeled guanine nucleotides to elF2 was monitored either by direct excitation at a wavelength of $355 \mathrm{~nm}$ or by Foerster Resonance Energy Transfer (FRET) from tryptophan residues excited at $280 \mathrm{~nm}$ and an emission wavelength of $440 \mathrm{~nm}$. Fluorescence emission spectra were recorded after incubating the elF2-mant-nucleotide complexes for 5 min at 
$20^{\circ} \mathrm{C}$. To estimate the $K_{\mathrm{d}}$ for elF2-mant-nucleotide complexes in presence or absence of elF5, the fluorescence of increasing amounts of mant-nucleotides was measured either in buffer alone or in the presence of $200 \mathrm{nM}$ of elF2 complex. The dilution was less than 1\%. To form the elF2 elF5 complex, 20 -fold molar excess of elF5 was added to elF2 and incubated for $10 \mathrm{~min}$ at $20^{\circ} \mathrm{C}$ prior to the experiment.

Assuming a one-site binding model for the studied binding reactions, the data obtained from the equilibrium titrations were fitted to a quadratic equation:

$$
F=F_{0}+\Delta F_{\max } \frac{\left(X+Y+K_{\mathrm{d}}\right)-\sqrt{\left(X+Y+K_{\mathrm{d}}\right)^{2}-4 X Y}}{2 X}
$$

where $F$ is the fluorescence signal of the mant-nucleotide in the absence or presence of elF2, $F_{0}$ is the initial fluorescence signal, $\Delta F_{\max }$ is the maximum fluorescence signal, $X$ is the total concentration of elF2, $Y$ is the total concentration of the added mant-nucleotide and $K_{d}$ is the equilibrium dissociation constant.

For the determination of the apparent equilibrium dissociation constants of elF2-mant-GDP to elF5 from the pre-steady-state experiments (see below), the above equation was modified as follows:

$$
k_{\mathrm{app}}=k_{\mathrm{a}}+\left(k_{\mathrm{b}}-k_{a}\right) \frac{\left(X+Z+K_{1 / 2}\right)-\sqrt{\left(X+Z+K_{1 / 2}\right)^{2}-4 X Z}}{2 X}
$$

Here, $k_{\mathrm{app}}$ is the characteristic apparent rate constant for the dissociation of the mant-nucleotide from elF2 in the presence of a specific concentration of elF5, $Z$ is the concentration of elF5, $k_{a}$ is the dissociation rate constant of mant-GDP from elF2 alone, $k_{\mathrm{b}}$ is the dissociation rate constant of mant-GDP from elF2 in the presence of saturating concentrations of elF5 and $K_{1 / 2}$ is the concentration of elF5 at which the half maximal effect on the dissociation rate of mant-GDP from elF2 is reached (the apparent equilibrium dissociation constant). Data evaluation was done with the SigmaPlot software.

\section{Pre-Steady-State fluorescence measurements}

Pre-steady-state experiments were carried out on a Fluoromax-3 spectrophotometer using a quartz cuvette with stirring magnet to allow fast mixing of the reacting components. All reactions 


\section{Chapter 2}

were done at $20 \pm 1{ }^{\circ} \mathrm{C}$ in standard reaction buffer. The fluorescence of mant-nucleotides was excited either directly at $355 \mathrm{~nm}$ or via FRET from tryptophan residues excited at $280 \mathrm{~nm}$ and monitored at an emission wavelength of $440 \mathrm{~nm}$.

To study the dissociation of nucleotides, $100 \mathrm{nM}$ elF2-mant-GDP or elF2-mant-GTP complex was formed by adding $125 \mathrm{nM}$ of the respective mant-nucleotide to $100 \mathrm{nM}$ nucleotide-free elF2, followed by an incubation for $3 \mathrm{~min}$ at $20^{\circ} \mathrm{C}$. To start the reaction, unlabeled GDP was added in excess and the decrease in mant-fluorescence was monitored over time. The data were evaluated by fitting to a single exponential function with the equation:

$$
F(t)=F_{\mathrm{o}}+A e^{-k_{\mathrm{app}} t}
$$

where $F(t)$ is the fluorescence at time $t, F_{o}$ is the final fluorescence signal, $k_{\mathrm{app}}$ is a characteristic apparent time constant and $A$ is the amplitude. Data evaluation was done with the SigmaPlot software. 


\subsection{Supplementary information}
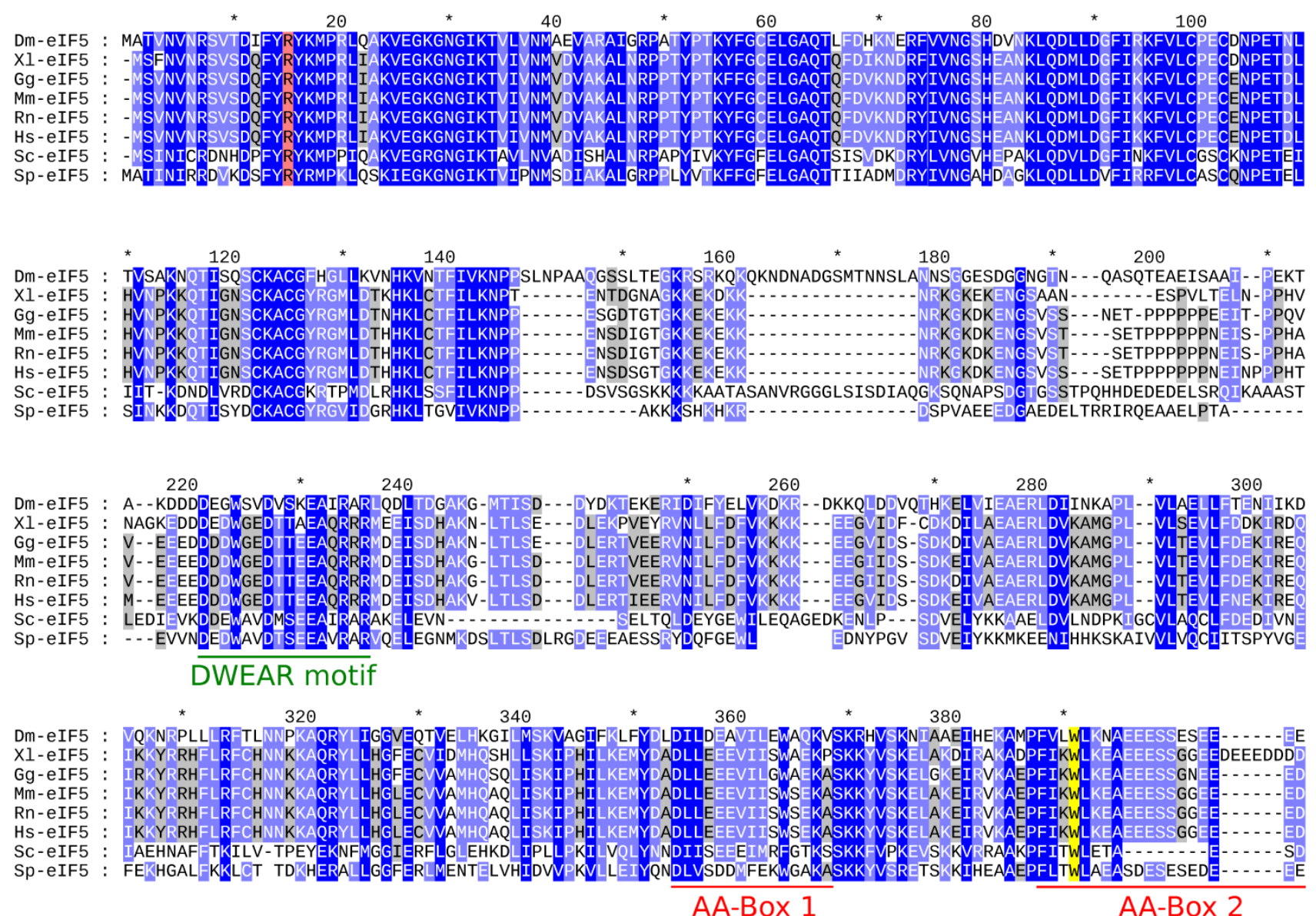

400

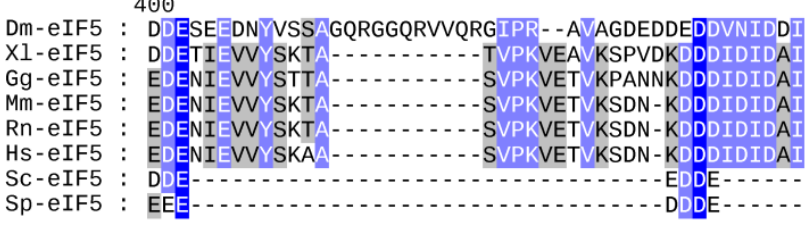

Figure S1. Multiple sequence alignment of elF5 orthologs. Highly conserved residues are highlighted in dark blue, conserved residues in light blue and variable residues in grey; the putative arginine-finger (Arg15) and Trp391 in AAbox 2 are highlighted in red and yellow, respectively. The numbering above the alignment corresponds to the yeast ortholog (Sc-elF5); the DWEAR motif and AA-boxes 1 \& 2 are indicated below the alignment. Dm, Drosophila melanogaster; XI, Xenopous laevis; Gg, Gallus gallus; Mm, Mus musculus; Rn, Rattus norvegicus; Hs, Homo sapiens; Sc, Saccharomyces cerevisiae; Sp, Schizzosaccharomyces pombe. The alignment was generated using the clustal omega algorithm. 
A

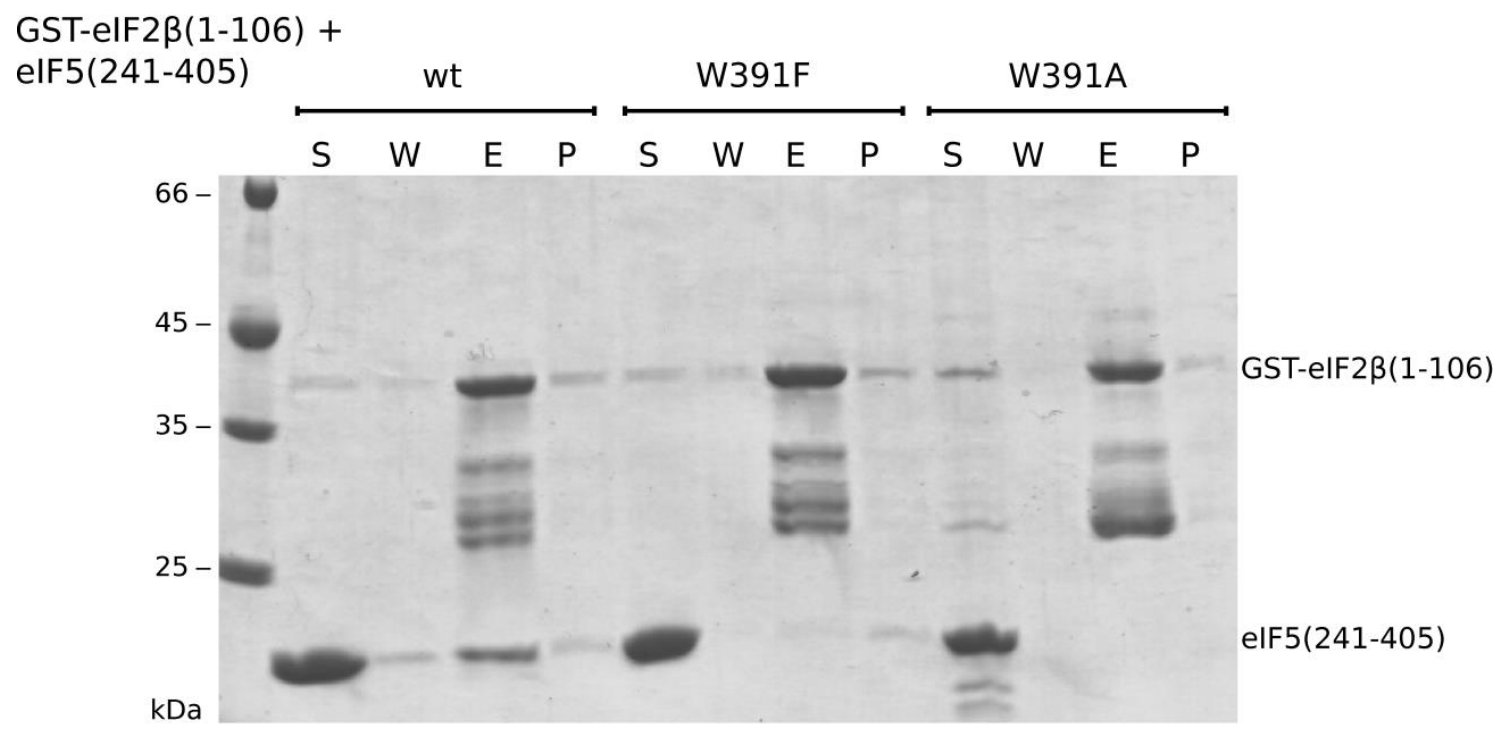

B

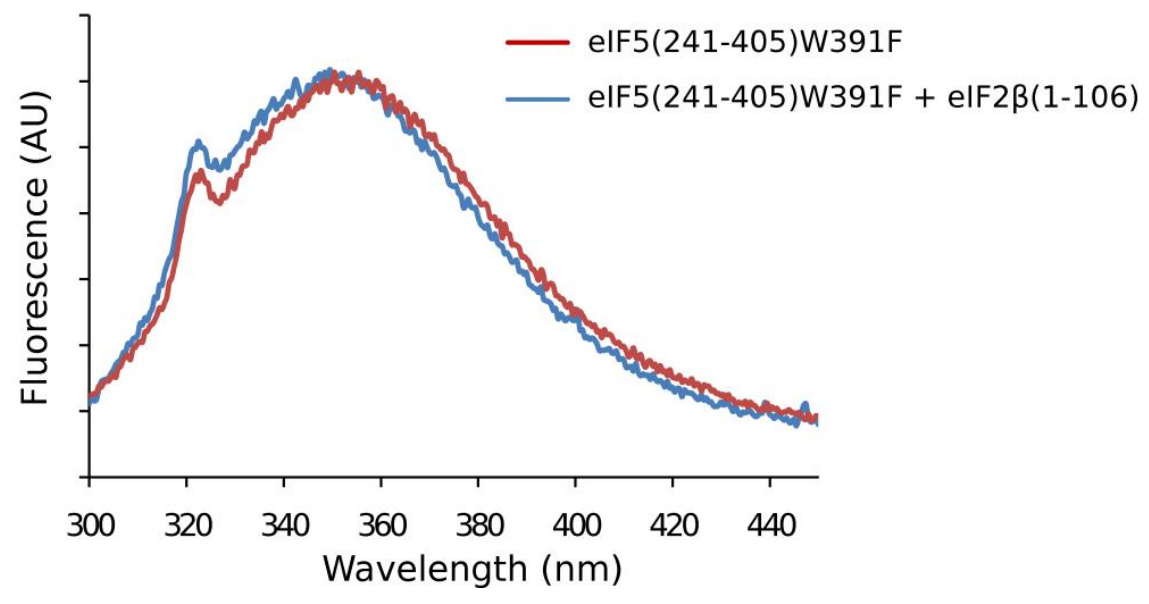

Figure S2. Effect of Trp391 mutations in elF5 on the interaction with the elF2 $\beta$-NTT. A) GST-pull down experiments between GST-elF2 $\beta(1-106)$ and elF5(241-405) wild-type (wt), elF5(241-405)W391F and elF5(241-405)W391A. For each experiment, $50 \mu \mathrm{g}$ of GST-elF2 $\beta(1-106)$ were first immobilized on GSH-Sepharose beads and subsequently incubated for $10 \mathrm{~min}$ at $20^{\circ} \mathrm{C}$ with $100 \mu \mathrm{g}$ elF5(241-405). The beads were then washed three times with washing buffer (100 mM $\mathrm{NaCl}, 20 \mathrm{mM}$ Hepes (pH 7.5), 5\% glycerol, $2 \mathrm{mM} \mathrm{DTT}$ ) and the bound proteins were finally eluted from the beads with washing buffer containing $20 \mathrm{mM}$ reduced glutathione. S, supernatant after incubation and before washing; W, supernatant after the third washing step; E, supernatant after elution; P, pellet after elution. B) Tryptophan quenching experiment between elF5(241-405)W391F and elF2 $\beta(1-106)$. The chromatogram shows the emission spectrum of elF5(241-405)W391F upon the excitation of the Trp fluorescence at $280 \mathrm{~nm}$ in the absence (red) and presence (blue) of the elF2 $\beta$-NTT. 
A

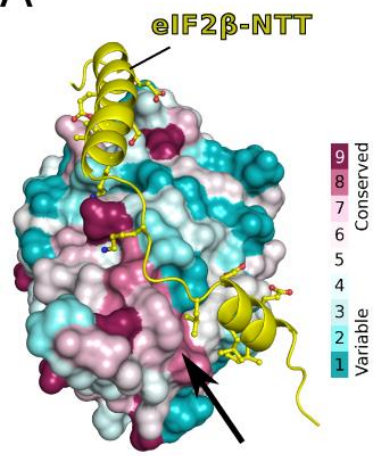

scelF5-CTD
B

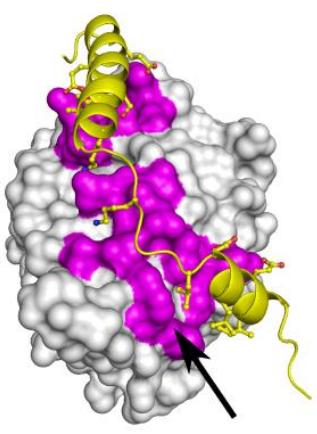

scelF5-CTD
C
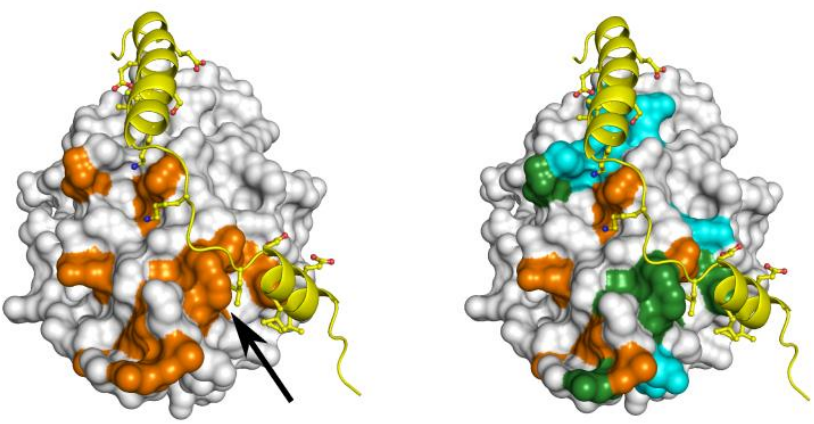

Figure S3. Binding ofthe elF2 $\beta$-NTT by elF5-CTD. A) The fragment of the elF2 $\beta$-NTT (yellow) binds elF5-CTD in a comparatively conserved area, with the highest degree of conservation occurring in the vicinity of Trp391 (arrow). B)elF5-CTD is shown as grey surface, with residues directly involved in contacts with the elF2 $\beta$-NTT (yellow) colored pink. C) Model of elF2 $\beta$-NTT (yellow) bound to the human elF5-CTD (PDB: 2IU1) according to the structure of the yeast complex (B). The hselF5-CTD is shown as grey surface; in the left panel, residues affected by elF2 $\beta(\mathrm{K} 2 \mathrm{~K} 3$ ) binding in NMR chemical shift assays are colored orange; in the right panel, residues affected only by elF2 $\beta(K 2 K 3)$ or elF1 are colored cyan and orange, respectively, and residues affected by both are colored green [63]. 


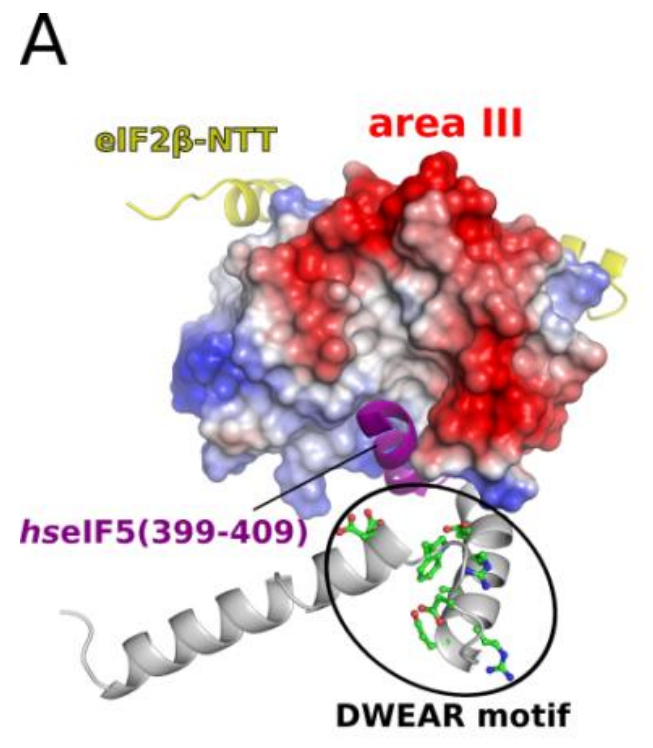

\section{B}
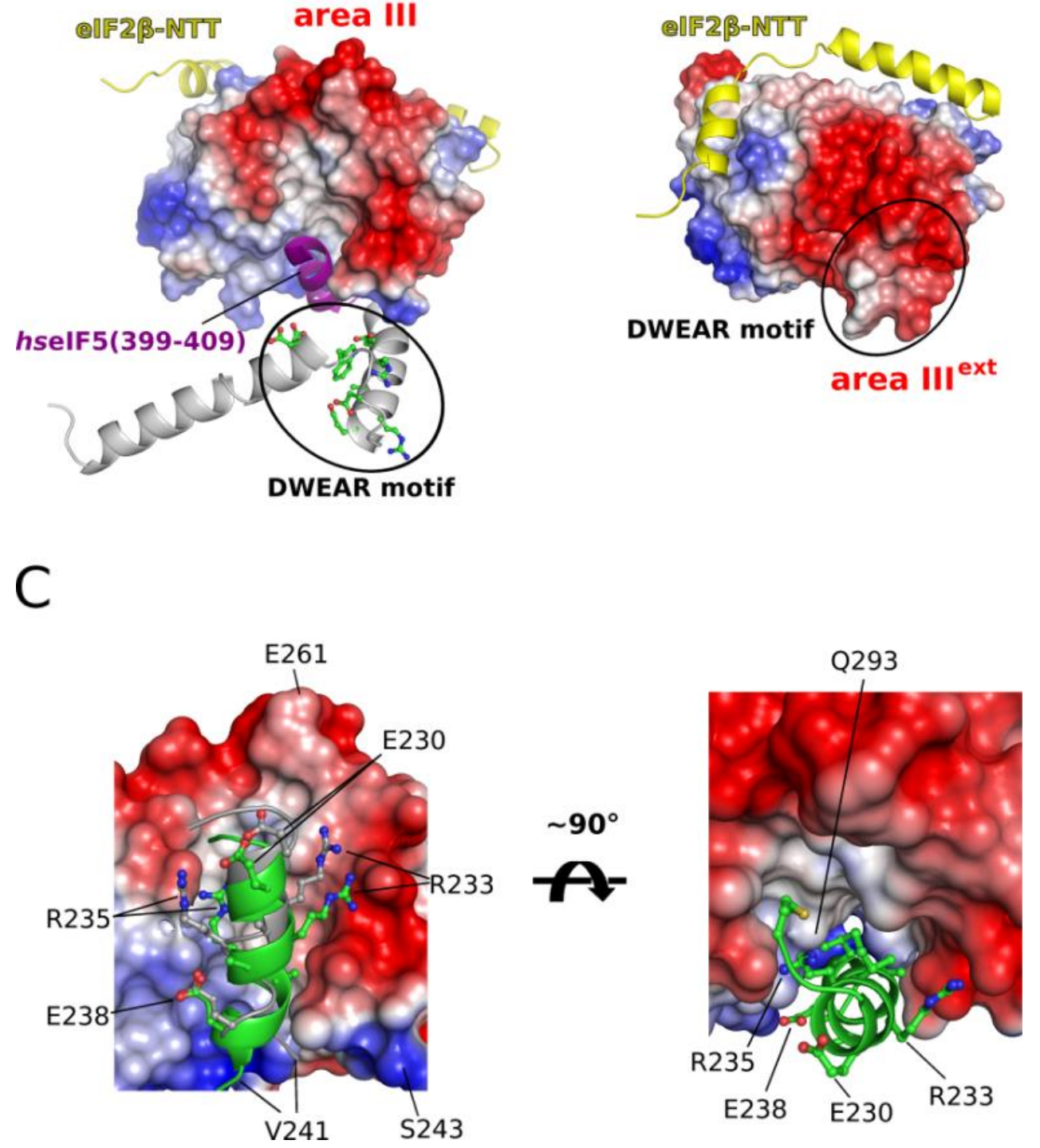

Figure S4. Model of the DWEAR-motif as element of the elF5-CTD. A) Overview of the elF5-CTD-elF2 $\beta$-NTT complex with residues 241-399 of elF5 shown in surface charge presentation; helices $\alpha 1$ and $\alpha 2$ are shown as grey cartoon with DWEAR-motif residues as green sticks (circle) and the elF2 $\beta$-NTT as yellow cartoon. In the crystal structure of human elF5-CTD (PDB: 2IU1), the hydrophobic cleft below area III is partially occupied by an $\alpha$-helix formed by the C-terminal residues 399-409 (purple), which have no counterpart in yeast (see also Fig. S1). B) Surface charge presentation of the elF5-CTD model presented in Figure 5A, with helix $\alpha 1$ and the DWEAR-motif accommodated in the hydrophobic cleft. As a consequence, the negatively charged $\mathrm{N}$-terminal portion of the DWEAR-motif comes to lie next to area III of the CTD, thereby forming the extended area $\mathrm{III}^{\mathrm{ext}}$. C) Two models of helix $\alpha 1$ (grey and green, respectively) accommodated in the hydrophobic cleft, generated by the ClusPro server (compare Fig. 5A). 

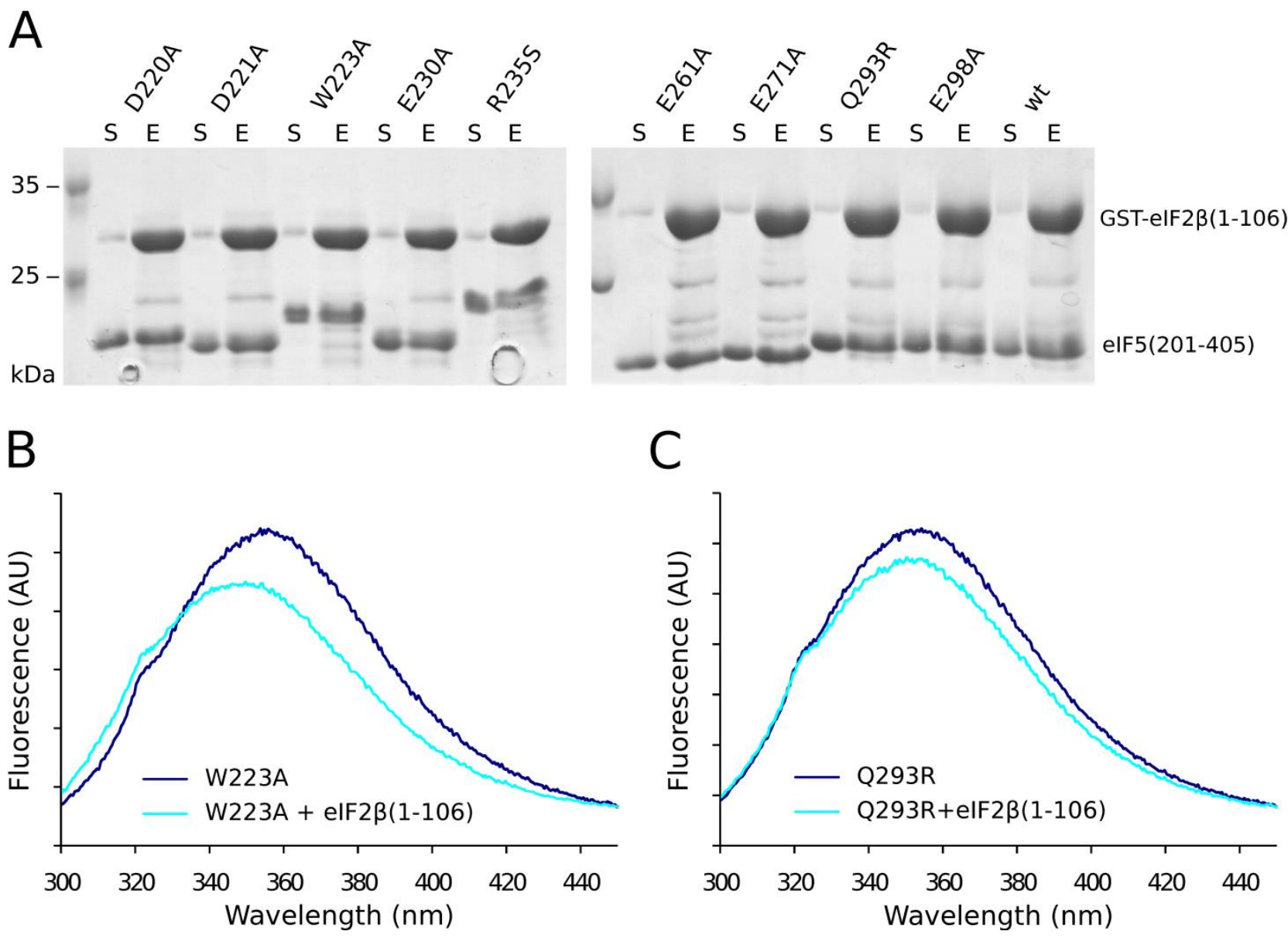

Figure S5. Effect of elF5-CTD single mutations on the interaction with the elF2 $\beta$-NTT. A)GST-pull down experiments between GST-elF2 $\beta$ (1-106) and elF5(201-405) D220A, D221A, W223A, E230A, R235S, E261A, E271A, Q293R, E298A, and the wild-type protein (wt). For each experiment, $50 \mu \mathrm{g}$ of GST-elF2 $\beta(1-106)$ were first immobilized on GSHSepharose beads and subsequently incubated for $10 \mathrm{~min}$ at $20^{\circ} \mathrm{C}$ with $100 \mu \mathrm{g}$ elF5(201-405). The beads were then washed three times with washing buffer (100 mM NaCl, $20 \mathrm{mM}$ Hepes (pH 7.5), 5\% glycerol, $2 \mathrm{mM}$ DTT) and the bound proteins were finally eluted from the beads with washing buffer containing $20 \mathrm{mM}$ reduced glutathione. $S$, supernatant after incubation and before washing; E, supernatant after elution.B-C)Tryptophan quenching by elF2 $\beta(1-106)$ upon binding to elF5(201-405)W223A (B) and elF5(201-405)Q293R (C). The chromatograms show the emission spectra of the respective elF5(241-405) construct $(1 \mu \mathrm{M})$ upon the excitation of the Trp fluorescence at $280 \mathrm{~nm}$ in the absence (blue) and presence (cyan) of the elF2 $\beta-N T T(2 \mu \mathrm{M})$. It should be noted that the quenching signal for the elF5(201405)Q293R is weaker than the one observed for elF5(241-405) (Fig. 1C) or elF5(201-405)W223A as it contains one additional Trp residues that is not directly involved in the binding reaction with the elF2 $\beta-N T T$. 

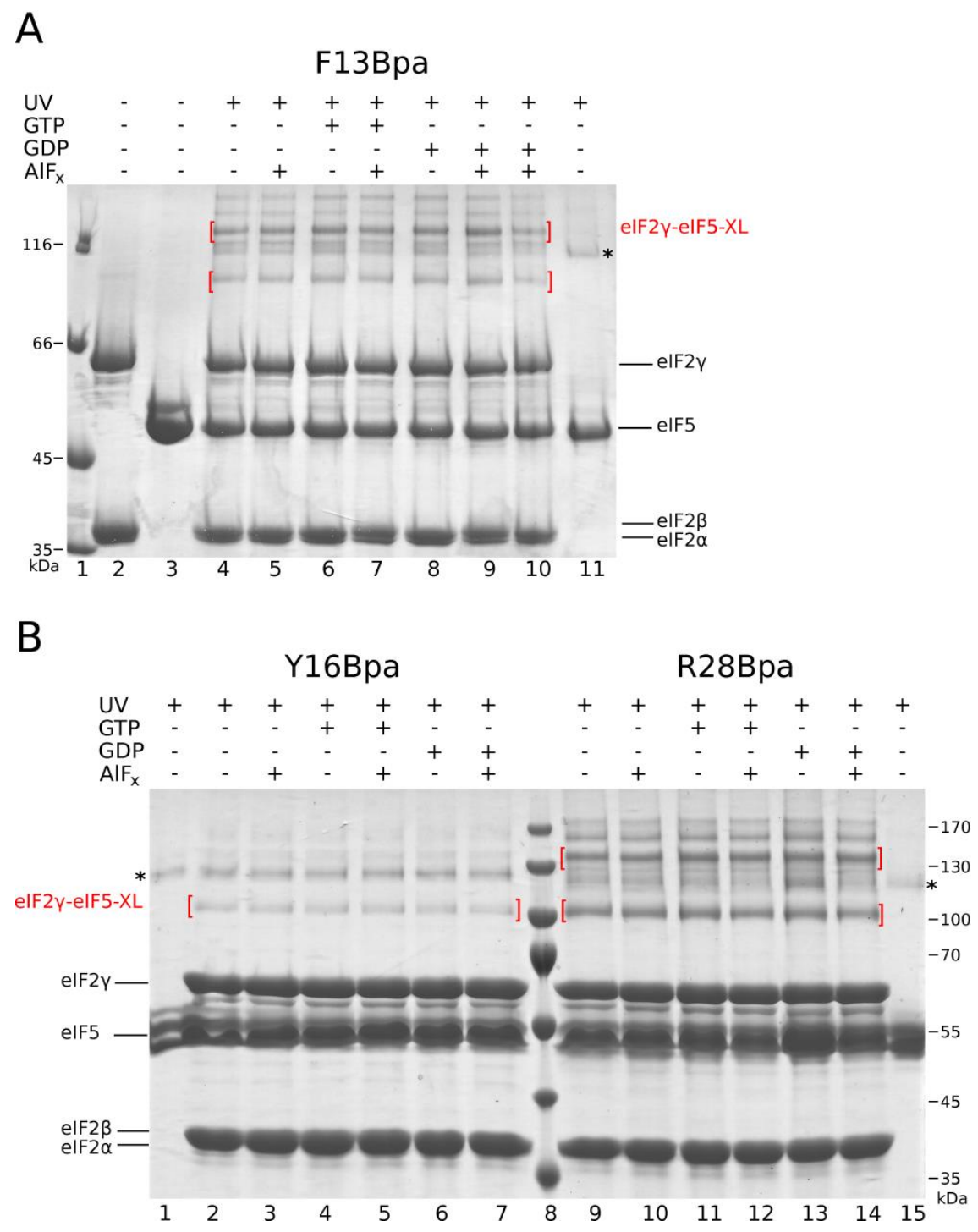

Figure S6. Dependency of the Bpa-crosslinks between the elF5-NTD and elF2 on the presence of nucleotides and fluoroaluminates. A) SDS-gel showing the UV-dependent formation of crosslink bands between $5 \mu \mathrm{M}$ elF5(FL)F13Bpa and $2 \mu \mathrm{M}$ elF2 in the absence or presence of GDP $(3 \mu \mathrm{M})$, GTP $(3 \mu \mathrm{M})$ and/or $\mathrm{AlF}_{\mathrm{x}}(2 \mathrm{mM} \mathrm{AICl} / 20 \mathrm{mM} \mathrm{NaF})$. Lane 1 contains the molecular weight marker; lane 2 contains only elF2; lanes 3 and 11 contain only elF5 without (3) and with (11) UV-irradiation; lanes 4-10 contain elF2 and elF5; lane 10 contains the same mixture as lane 9 with the difference that it contains $200 \mathrm{mM} \mathrm{KCl}$ instead of $100 \mathrm{mM}$. B) SDS-gel showing the UV-dependent formation of crosslinks between $5 \mu \mathrm{MeIF5}(\mathrm{FL}) \mathrm{Y} 16 \mathrm{Bp}$ (left) or elF5(FL)R28Bpa (right) and $2 \mu \mathrm{M}$ elF2in the absence or presence of GDP (3 $\mu \mathrm{M}$ ), GTP $(3 \mu \mathrm{M})$ and/or $\mathrm{AlF}_{\mathrm{x}}(2 \mathrm{mM} \mathrm{AlCl} / 20 \mathrm{mM} \mathrm{NaF})$. Lane 8 contains the molecular weight marker; lanes 2-7 and 9-14 contain elF2 and elF5; lanes 1 and 15 contain only elF5(FL)Y16Bpa (1) or elF5(FL)R28Bpa after UV-irradiation. For all experiments, crosslinking was performed on ice for $30 \mathrm{~min}$. Crosslinks to elF $2 \gamma$ are indicated by red brackets; the strong crosslink bands for positions directly below the 130 kDa marker (marked with *) also occur in the absence of eIF2 and are thus likely to originate from a crosslink between two elF5 molecules. 


\title{
Chapter $3 \cdot$ Interaction network between eIF2 and eIF2BE
}

This manuscript is in preparation for submission

\section{Analysis of the interaction network between eIF2 and eIF2B $\varepsilon$ and its implications for the mechanism of nucleotide exchange}

\author{
Bernhard Kuhle $^{1, \S}$, Oliver Valerius ${ }^{2}$ and Ralf Ficner ${ }^{1}$ \\ ${ }^{1}$ Abteilung für Molekulare Strukturbiologie, Institut für Mikrobiologie und Genetik, Göttinger \\ Zentrum für Molekulare Biowissenschaften, Georg-August-Universität Göttingen, D-37077 \\ Göttingen, Germany \\ ${ }^{2}$ Abteilung für Molekulare Mikrobiologie und Genetik, Institut für Mikrobiologie und Genetik, \\ Georg-August-Universität Göttingen, D-37077 Göttingen, Germany \\ \$To whom correspondence should be addressed: bkuhle@gwdg.de
}

Author contributions:

BK: designed the study and conceived the experiments; performed cloning, protein purification, crystallization, data collection and structure determination, crosslinking experiments, and biochemical studies on nucleotide binding by elF2; analyzed and interpreted the data; wrote the manuscript. OV: performed mass-spectrometry analysis of crosslinks and helped to analyze the MS-data. RF: design of the study; helped to draft the manuscript. 


\section{Abstract}

The eukaryal initiation factor 2 (elF2) is a heterotrimeric translational GTPase, responsible for the GTP-dependent delivery of the methionylated initiator tRNA to the ribosome. The guanine nucleotide cycle performed by elF2 during translation initiation is tightly regulated by the opposing effects of the GTPase activating protein (GAP) elF5 and the guanine-nucleotide exchange factor (GEF) elF2B. Here, we present a structural and biochemical analysis of the interaction network between elF2 and elF2B $\varepsilon$, the catalytic subunit of the heteropentameric elF2B complex, with the aim to gain insight into the mechanism of nucleotide exchange. On the one hand, we show that the main interaction, which is responsible for the recruitment of elF2B $\varepsilon$ to elF2, is mediated by a dynamic peptide-domain interaction between the catalytic elF2B $\varepsilon$-CTD and the elF2 $\beta \mathrm{N}$-terminal tail. On the other hand, a second interaction was mapped directly between elF2B $\varepsilon$-CTD and the immediate vicinity of the nucleotide binding pocket on elF $2 \gamma$, an interaction that is most likely responsible for the destabilization of the elF2-bound nucleotide. Together, the presented findings provide a refined model for the interactions between elF2B $\varepsilon$ and its substrate elF2, which has implications for the mechanism of nucleotide exchange and possibly provides insight into the evolutionary history of the structurally and functionally similar C-terminal domains of elF2B $\varepsilon$ and elF5.

\subsection{Introduction}

The eukaryal translation initiation factor 2 (elF2) is a G protein that plays an essential role during the initiation phase of translation in eukarya. In its GTP-bound form, elF2 specifically recognizes and binds methionylated initiator tRNA (Met-tRNA ${ }_{i}$ ) and delivers it to the $43 \mathrm{~S}$ pre-initiation complex (43S pre-IC) $[12,16]$. Upon the recognition of the correct start site for translation, the GTPase activating protein (GAP) elF5 triggers the GTPase activity in elF2, resulting in the irreversible hydrolysis of elF2-bound GTP. After the release of Met-tRNA ${ }_{i}$, the inactive elF2.GDP dissociates from the ribosome and has to be recycled back to elF2.GTP for a next round of initiation. Like other Ras-related G proteins, elF2 binds GDP tightly with an affinity in the lower nanomolar range, and the rate of spontaneous GDP dissociation is too slow to support efficient translation [44]. The exchange reaction is therefore catalyzed by the guanine-nucleotide exchange 
factor (GEF) elF2B, which promotes the dissociation of GDP, followed by binding of GTP due to its $\sim 10$-fold higher cellular concentration.

eIF2 is an unusually complex translational GTPase, formed by the three highly conserved non-identical subunits elF $2 \alpha,-\beta$, and $-\gamma[121,122]$. According to biochemical studies and crystal structure analyses of the archaeal ortholog (alF2), elF $2 \alpha$ and $-\beta$ form no direct contact to each other and bind the central elF2 $\gamma$ subunit independently [31]. The $\gamma$-subunit adopts a three-domain architecture homologous to that of elongation factors EF-Tu and eEF1A, with the nucleotide binding pocket located in the $G$ domain (domain I) [37]. Similar to other $G$ proteins, it is thought that a/elF2 $\gamma$ undergoes structural rearrangements when switching from the inactive GDP-bound to the active GTP-bound state, initiated by conformational changes in two conserved dynamic elements of the $\mathrm{G}$ domain, termed switch 1 and switch 2, that are responsible for the recognition and binding of the GTP $\gamma$-phosphate [31,33]. The $\alpha$-subunit was identified as a major factor in protein synthesis control as target for phosphorylation at a universally conserved serine (Ser51) by elF2 $\alpha$ kinases in response to stress conditions $[78,112]$. Phosphorylation of elF2 $\alpha$ is thought to convert elF2.GDP from a substrate to a competitive inhibitor of its GEF elF2B, thereby reducing the rate of translation initiation and thus protein synthesis in general [112, 123, 124].

elF2B is a large protein complex of five non-identical subunits that was reported to exist as a functional decamer of $\sim 600 \mathrm{kDa}$ in solution $[72,73]$. The homologous elF2B $\alpha,-\beta$, and $-\delta$ subunits form a regulatory subcomplex that contributes to elF2 binding through interactions with elF $2 \alpha$ [77]. These interactions are enhanced by elF $2 \alpha$-phosphorylation, thereby abrogating GEF activity by rendering phosphorylated elF2 a competitive inhibitor of elF2B [112]. The elF2B $y$ and $-\varepsilon$ subunits form the catalytic subcomplex that mediates the GEF function of elF2B [112]. Within this catalytic subcomplex, elF2B $\varepsilon$ is the actual catalytic subunit, whose activity is stimulated by elF2By.

The minimal catalytically active region within elF2B was mapped to the C-terminal domain of elF2BE (residues 524-712 in yeast), which was shown to adopt a HEAT repeat-like domain fold homologous to that of the elF5-CTD $[1,54,74]$. Like elF5-CTD, the elF2B $\varepsilon$-CTD contains two conserved aromatic/acidic motifs (AA-boxes) that interact with the lysine-rich K-boxes in the elF2 $\beta$ NTT [35] (Fig. 1 and S1). Moreover, it was found that mutagenesis of Trp699 in AA-box 2 (corresponding to Trp391 in elF5) confers lethality in yeast by weakening the interaction between 
elF2B $\varepsilon$ and its substrate elF2 [75]. A second interaction was mapped between the two $\mathrm{N}$-terminal $\alpha$-helices of elF2B $\varepsilon$-CTD and elF2 $\gamma$ itself, which was proposed to be directly involved in the catalysis of nucleotide exchange [70, 75, 76]. This assumption was based on the observation that mutagenesis of conserved surface residues in the N-terminal $\alpha$-helices, namely Thr552, Leu568, Glu569 and Ser576, caused growth defects or lethality in vivo, accompanied by a reduced or abolished catalytic activity of the elF2B $\varepsilon$ mutant in vitro. Hereby, particularly Glu569 was identified as key catalytical residue, whose mutagenesis to alanine abolishes GEF activity without affecting the affinity of the construct to elf2 $\gamma$. However, up to now it is unknown how and in which orientation the elF2B $\varepsilon$-CTD enters the elF2 complex and where the interface on the elF2 $\gamma$ subunit lies. Consequently, the actual mechanism of elF2B $\varepsilon$-catalyzed nucleotide exchange and the order of molecular events that accompany GEF-binding, GDP release and GTP rebinding are still enigmatic.

Here we used a combination of structural and biochemical methods to gain insight into the interactions between elF2B $\varepsilon$ and its substrate elF2 to provide a better understanding of the molecular events that result in nucleotide exchange. On the one hand, we studied the interactions between elF $2 B \varepsilon$ and the $\mathrm{N}$-terminal tail of elF2 $\beta$ using tryptophan quenching experiments. These indicate that the eIF $2 \beta$-NTT forms a high affinity peptide-domain interaction with the AA-boxes of elF2B $\varepsilon$-CTD very similar to that observed between elF2 $\beta$ and the elF5-CTD (see chapter 2 ). On the other hand, mass-spectrometry-coupled UV-crosslinking reveals that the $\mathrm{N}$-terminal end of elF2BECTD, containing the catalytic center, contacts the elF2 $\gamma$ subunit directly and in the immediate vicinity to the nucleotide binding pocket through an interface that overlaps with that observed for the DWEAR-motif and area III of elF5-CTD. Finally, the effect of elF2BE on nucleotide binding and dissociation was studied using fluorescent derivatives of GDP/GTP. Together, the presented findings suggest that the elF $2 B \varepsilon$-catalyzed exchange reaction follows a successive series of reversible steps involving the formation of an intermediate unstable elF2.GDP.elF2B $\varepsilon$ ternary complex, in which the GEF promotes nucleotide release by inducing conformational rearrangements in the nucleotide binding pocket in a manner similar to other known GEFs. 
A

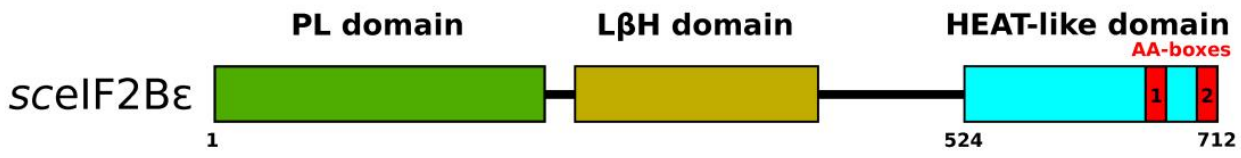

B

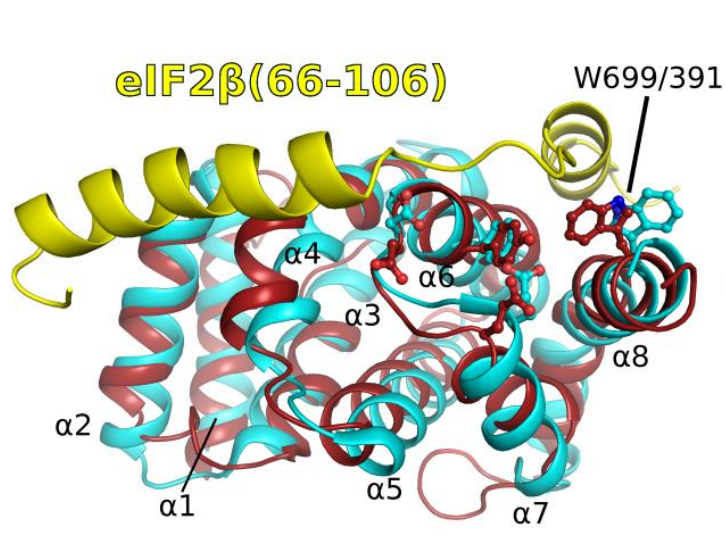

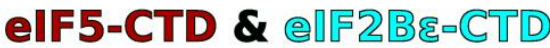

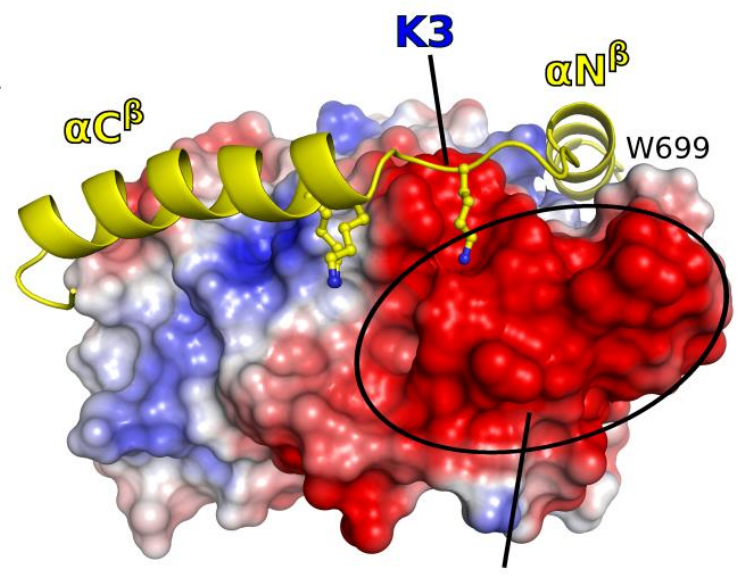

AA-box 1 \& 2

(area I)

Figure 1. Structure of the elF2B $\varepsilon$-CTD A) Schematic presentation of the proposed domain architecture in elF2B $\varepsilon$ with an $\mathrm{N}$-terminal pyrophosphorylase-like (PL) domain, a central left-handed $\beta$-helix $(\mathrm{L} \beta \mathrm{H})$ domain and the $\mathrm{C}$-terminal HEAT-like domain, which constitutes the minimal catalytic domain within the elF2B complex. B) The left panel shows the superposition of the crystal structures of the elF2BE-CTD (residues 524-712, colored in cyan; PDB: 3JUI) and elF5CTD (red) bound to a fragment of the elF2 $\beta$-NTT (yellow) (see chapter 2). Selected conserved residues of AA-boxes 1 and 2 are shown as balls and sticks, including the homologous Trp699 and Trp391 in elF2B $\varepsilon$ and elF5, respectively. As shown in the right panel, this positions K-box 3 (K3) of the elF2 $\beta$-NTT in direct vicinity to the negatively charged area I of elF2B $\varepsilon$ and allows an overall interaction between elF2B $\varepsilon$-CTD and the elF2 $\beta$-NTT very similar to that observed for the elF5-CTD (compare Figure 4 in chapter 2).

\subsection{Results}

\section{Model of the elF2 $\beta-N T T$ bound to the catalytic C-terminal domain of elF2B $\varepsilon$}

The minimal catalytically active domain of elF2BE (residues 544-712 in the S. cerevisiae ortholog) and the C-terminal domain of elF5 (residues 241-405) share structural homology over the entire HEAT repeat-like fold (Fig. 1). Despite differences in the structural details [1], this homology is complemented by the presence of the two conserved AA-boxes ( 1 \& 2 ) close to the C-terminal end, which form the negatively charged area I in the tertiary structure of both proteins. In both cases, this area was found to provide the primary contact surface for the K-box-containing $\mathrm{N}$-terminal tail 
of elF2 $\beta$ [35]. However, structural information for this interaction is so far not available for elF2B $\varepsilon$ and it is not known whether both interactions follow similar or distinct patterns.

Attempts to solve the structure of elF2BE-CTD in complex with fragments of elF2 $\beta$-NTT analogous to those for the elF5-CTD·elF2 $\beta$-NTT complex (see chapter 2 ) were unsuccessful. Instead, part of the elF2 $\beta$-NTT (residues 66-106; including K-box 3) was modeled onto elF2B $\varepsilon$-CTD according to the elF5-CTD.elF2 $\beta$-NTT complex structure (Fig. 1B). In this model, K-box 3 comes to lie in the periphery of area I, which allows the direct contact of its lysines 83 and 89 with Tyr663, Asp666 and Glu670 in elF2Be (corresponding to Tyr351, Asp354 and Glu358 in elF5, respectively). Moreover, the adjacent regions on elF2B $\varepsilon$-CTD would provide mainly hydrophobic surface areas for the binding of helices $\alpha \mathrm{N}^{\beta}$ and $\alpha \mathrm{C}^{\beta}$ flanking K-box 3, complemented by salt bridges of Lys608 and $\operatorname{Arg} 624$ in elF2B $\varepsilon$ to acidic side chains in $\alpha N^{\beta}$ and $\alpha C^{\beta}$, similar to those observed in the complex with elF5. Thus, it is conceivable from this model that the structural homology between elF2BEand elF5-CTD is as well reflected in their respective interaction with the fragment elF2 $\beta(66-106)$.

\section{Contributions of the lysine-rich K-boxes 1-3 of the elF2 $\beta$-NTT to the interactions with elF2BE.}

As in the case of elF5-CTD, elF2BE-CTD contains a universally conserved solvent exposed tryptophan residue within AA-box 2 (Trp699; Trp391 in elF5) that was previously shown to be important for interactions with elF2 $\beta[74,75]$. We therefore assumed that it is possible to determine the relative contributions of K-boxes 1,2 , and 3 in elF $2 \beta$-NTT to its interactions with elF2B $\varepsilon$-CTD by monitoring Trp-quenching upon complex formation. Unlike elF5(241-405) with its two tryptophan residues, the corresponding elF $2 B \varepsilon(524-712)$ fragment contains five tryptophan residues. Among these, Trp618, Trp676 and Trp677 are buried within the hydrophobic core, whereas Trp696 and Trp699 lie in direct vicinity to area I on the protein surface. Surprisingly, this larger number of Trp residues did not result in a smaller quenching signal due to an increased background fluorescence by unaffected residues; instead, the addition of full-length elF $2 \beta$ or elF2 $\beta(1-106)$ to elF2B $\varepsilon$-CTD resulted in a substantial reduction of the emission at $360 \mathrm{~nm}$ by $\sim 43 \%$ (compared to $\sim 24 \%$ for elF5-CTD), accompanied by a slight blueshift of the emission maximum

(Fig. 2). A possible explanation for this rather unexpectedly strong signal is that not only Trp699, but also the less conserved Trp696 and/or Trp677 are directly or indirectly affected by the binding 
of elF $2 \beta$. As the mutagenesis of Trp699 to alanine completely abolishes any quenching signal, most likely due to its inability to bind elF2 $\beta$ at all, and due to the potential structural role of the other residues, we were not able to determine the relative contributions of the individual Trp residues to the quenching signal. However, the fact that four of the five tryptophan residues are located in helices $\alpha 7$ and $\alpha 8$ in the vicinity of the negatively charged area I and Trp699, whereas the fifth tryptophan in helix $\alpha 4$ is buried in the hydrophobic core suggests that area I provides the main interface for the binding of the three K-boxes.

As observed for elF5-CTD, elF2B $\varepsilon$-CTD binds elF2 $\beta(1-106)$ and full-length elF2 $\beta$ with similar affinities ( $K_{\mathrm{d}}$ of 100 and $120 \mathrm{nM}$, respectively) and results in the same quenching signal (Table 1 ). The following experiments were therefore carried out with the isolated elF2 $\beta$-NTT (residues 1106), containing either single or double K-box mutations. The single K-box mutations exhibit only a slightly different pattern of contributions in comparison to their interactions to elF5 (see chapter 2), with 5-, 8- and 1.8-fold reductions in binding affinity for $\mathrm{K} 1 \Delta, \mathrm{K} 2 \Delta$ and $\mathrm{K} 3 \Delta$, respectively (Fig. 2B/D and Table 1). Thus, again K-boxes 1 and 2 seem to provide the primary interface for elF2B $\varepsilon$ binding with only minor contributions by K-box 3. It is, however, interesting to note thatthe contribution by K-box 1 seems to be less prominent than in the elF2 $\beta \cdot$ elF5 interaction, which is also reflected by the maintenance of the full quenching signal in $\mathrm{K} 1 \Delta$. By contrast, $\mathrm{K} 2 \Delta$ results in the loss of nearly $30 \%$ of the quenching signal, while $\mathrm{K} 3 \Delta$ shows no effect - in analogy to the observations with elF5-CTD (see chapter 2). Interestingly, the double mutants show some variations in their pattern of interactions with elF2BE-CTD compared to those observed for elF5CTD (Fig. 2C/D and Table 1). K12 $\Delta$ completely abolishes the quenching signal, in line with their strong respective contributions suggested by the single mutants and identical to the observation for elF5-CTD. K23 shows an entirely additive effect of the $\mathrm{K} 2 \Delta$ and $\mathrm{K} 3 \Delta$ single mutations on the binding affinity ( $K_{\mathrm{d}}=1.4 \mu \mathrm{M}$; 14-fold reduction) and quenching signal (loss of $\sim 30 \%$ ), suggesting that $\mathrm{K}$-box 3 in the interaction with elF2B $\varepsilon$ is not able to partially compensate the loss of K-box 2 as proposed for the elF2 $\beta \cdot$ elF5 interaction. The largest difference is exhibited by the K13 $\Delta$ mutation, which still conferred the full quenching signal at a $\sim 12$-fold reduced binding affinity $\left(K_{d}=1.2 \mu \mathrm{M}\right)$, conform with additive contributions by $\mathrm{K} 1 \Delta$ and $\mathrm{K} 3 \Delta$. 

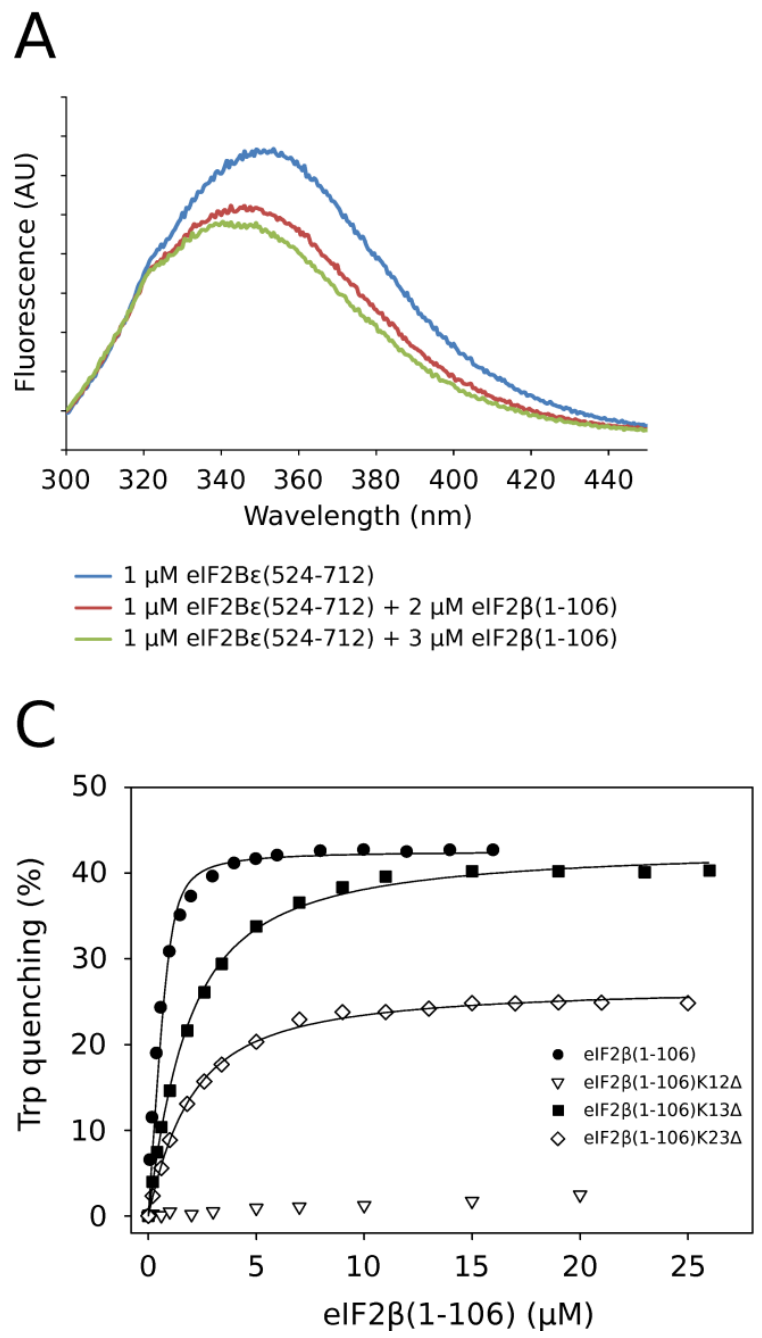

B
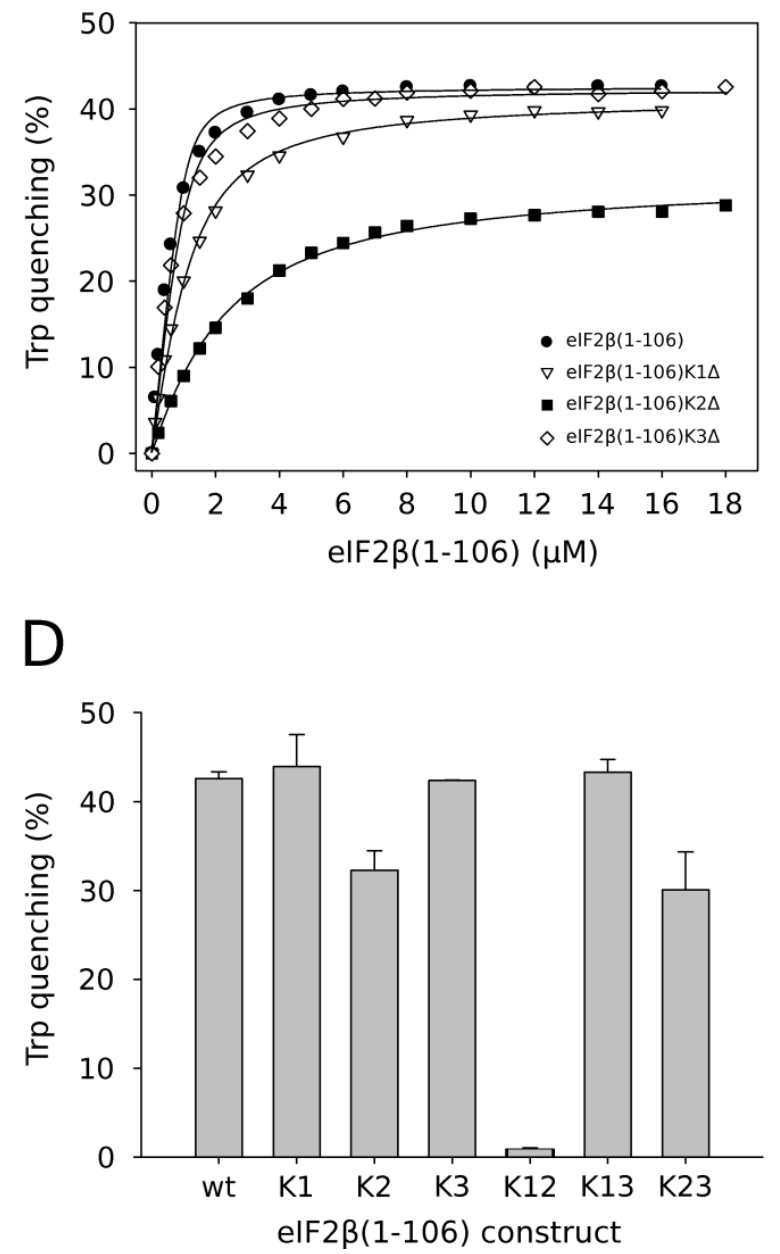

Figure 2. Trp-quenching experiments between elF2B $\varepsilon$-CTD and elF2 $\beta$-NTT. A) Trp quenching on the elF2B $\varepsilon$-CTD by the elF2 $\beta$-NTT. The chromatogram shows the emission spectrum of $1 \mu \mathrm{M}$ elF2Be(524-712) upon the excitation of the Trp fluorescence at $290 \mathrm{~nm}$ in the absence of elF2 $\beta$-NTT (blue line) or in the presence of 2 (red) or $3 \mu \mathrm{M}$ (green) elF2 $\beta$ (1106). B) Equilibrium titrations of $0.5 \mu \mathrm{M}$ elF2B $2(524-712)$ with increasing amounts of wild-type elF2 $\beta(1-106)$ or the three $\mathrm{K}$-box mutants $\mathrm{K} 1 \Delta, \mathrm{K} 2 \Delta$, and $\mathrm{K} 3 \Delta$. The Trp quenching signal (in $\%$ of the original fluorescence signal of elF2B $\varepsilon(524-712)$ ) was plotted against the concentration of the respective elF2 $\beta$-NTT constructs. The black lines represent the fit to the data using a quadratic one-side binding model. C) Equilibrium titration of $0.5 \mu \mathrm{M}$ elF2B $\varepsilon(524-$ 712 ) with wild-type elF2 $\beta(1-106)$ or the double K-box mutants $K 12 \Delta, K 13 \Delta$, and $K 23 \Delta$. C) Comparison of the Trp quenching signals for the seven elF2 $\beta(1-106)$ constructs. Standard deviations, obtained from three independent experiments, are given as error bars.

Taken together, these data suggest an interaction between the elF2 $\beta$-NTT and elF2B $\varepsilon$-CTD similar to that with the elF5-CTD, which is centered on the area surrounding Trp699 and area I and responsible for high affinity binding between the GEF domain and its substrate. The main 
contributions for the interactions are provided by K-boxes 1 and 2, while K-box 3 plays only a subordinate role.

Table 1. Trp quenching experiments between elF2BE(524-712) and eIF2 $\beta$.

\begin{tabular}{|c|c|c|c|}
\hline elF2 $\beta$ construct & $K_{\mathrm{d}}(\mathrm{nM})$ & $\begin{array}{l}\text { Reduction of affinity } \\
\text { relative to wt }\end{array}$ & $\begin{array}{l}\text { Quenching signal } \\
\text { (\%) }\end{array}$ \\
\hline elF2 $\beta(F L) w t$ & $90 \pm 41$ & -- & 41 \\
\hline elF2 $\beta(1-106) w t$ & $100 \pm 30$ & -- & 43 \\
\hline elF2 $\beta(1-106) K 1 \Delta$ & $510 \pm 65$ & 5 -fold & 44 \\
\hline elF2 $\beta(1-106) K 2 \Delta$ & $830 \pm 35$ & 8-fold & 32 \\
\hline elF2 $\beta(1-106) K 3 \Delta$ & $180 \pm 22$ & 1.8-fold & 42 \\
\hline elF2 $\beta(1-106) K 12 \Delta$ & N.D. & & 1 \\
\hline elF2 $\beta(1-106) K 13 \Delta$ & $1230 \pm 430$ & 12-fold & 41 \\
\hline elF2 $\beta(1-106) K 23 \Delta$ & $1400 \pm 380$ & 14-fold & 29 \\
\hline
\end{tabular}

$K_{\mathrm{d}}$ is the equilibrium dissociation constant.

N.D., not determined due to missing quenching signal.

elF2B $\varepsilon$-CTD contacts the $y$-subunit of elF2 directly next to the nucleotide binding pocket.

Within the elF2BE-CTD, the catalytic center was mapped to the $\mathrm{N}$-terminal helices $\alpha 1$ and $\alpha 2$ opposite to Trp699, with Thr552, Leu568, Ser576 and particularly Glu569 as critical catalytic center residues that interact directly with the elF $2 \gamma$-subunit $[74,75]$. In order to identify the binding site for the catalytic center of eIF2BE-CTD on eIF2, the photo-inducible crosslinker Bpa was introduced in various positions of elF2BE(524-712) (Asp544, GIn559, Thr572 and Met575) in the periphery of the proposed catalytic center (Fig. 3C). All four elF2BE-CTD-Bpa constructs still stimulated GDP dissociation from elF2, however, with reduced activity when compared to the wild-type protein (Fig. S2). For the first crosslinking experiments, the heterotrimeric elF2 complex in the presence of GDP was mixed with a 2-fold molar excess of purified elF2Be(524-712)Bpa and exposed to UV light. UV- and time-dependent crosslink bands emerged for all constructs, however, with significantly different efficiencies depending on the position of the crosslinker (Fig. 3A). According to MSanalysis, all crosslink bands migrating above the $70 \mathrm{kDa}$ marker were directly to the $\mathrm{\gamma}$-subunit of elF2. 

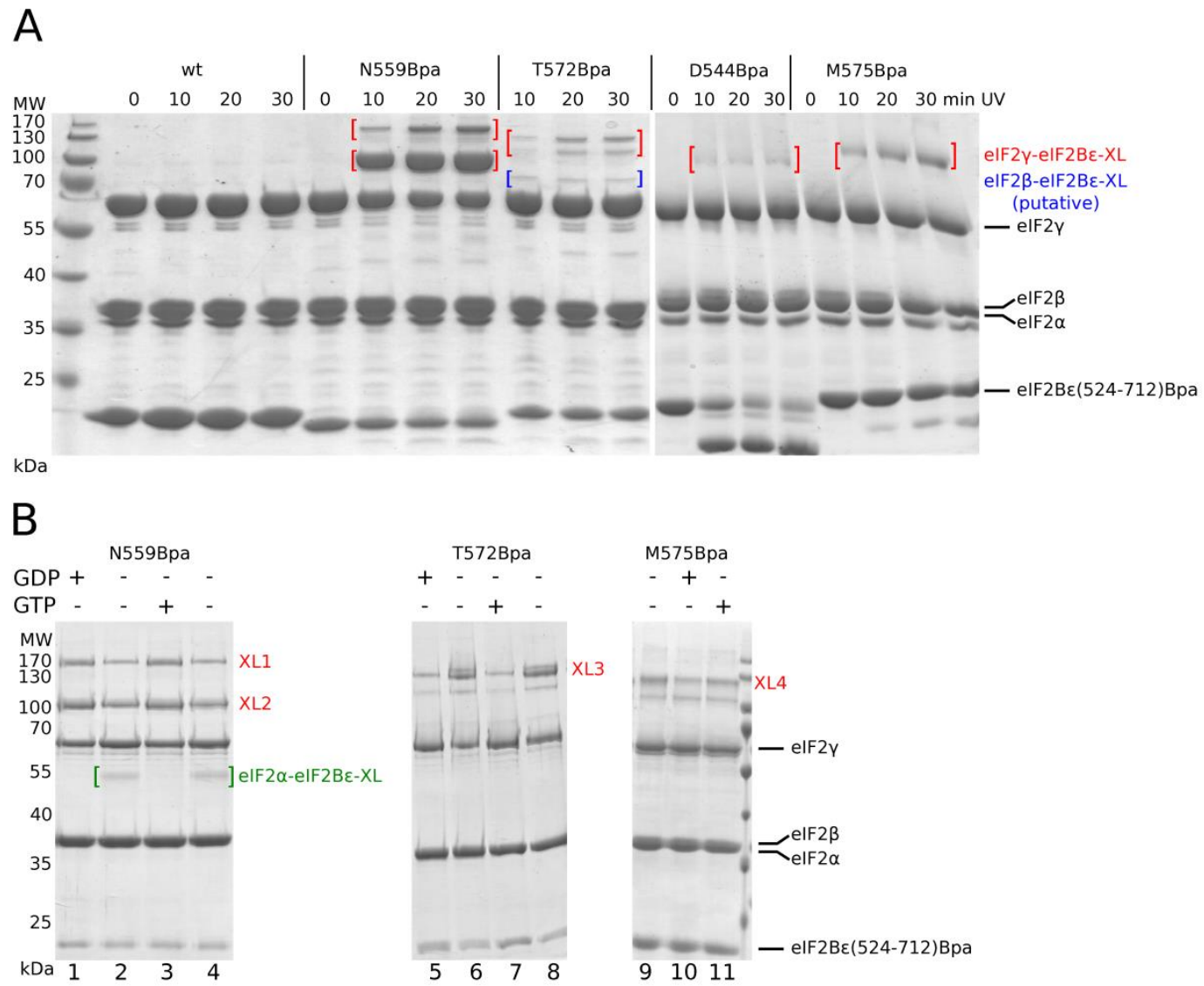

C
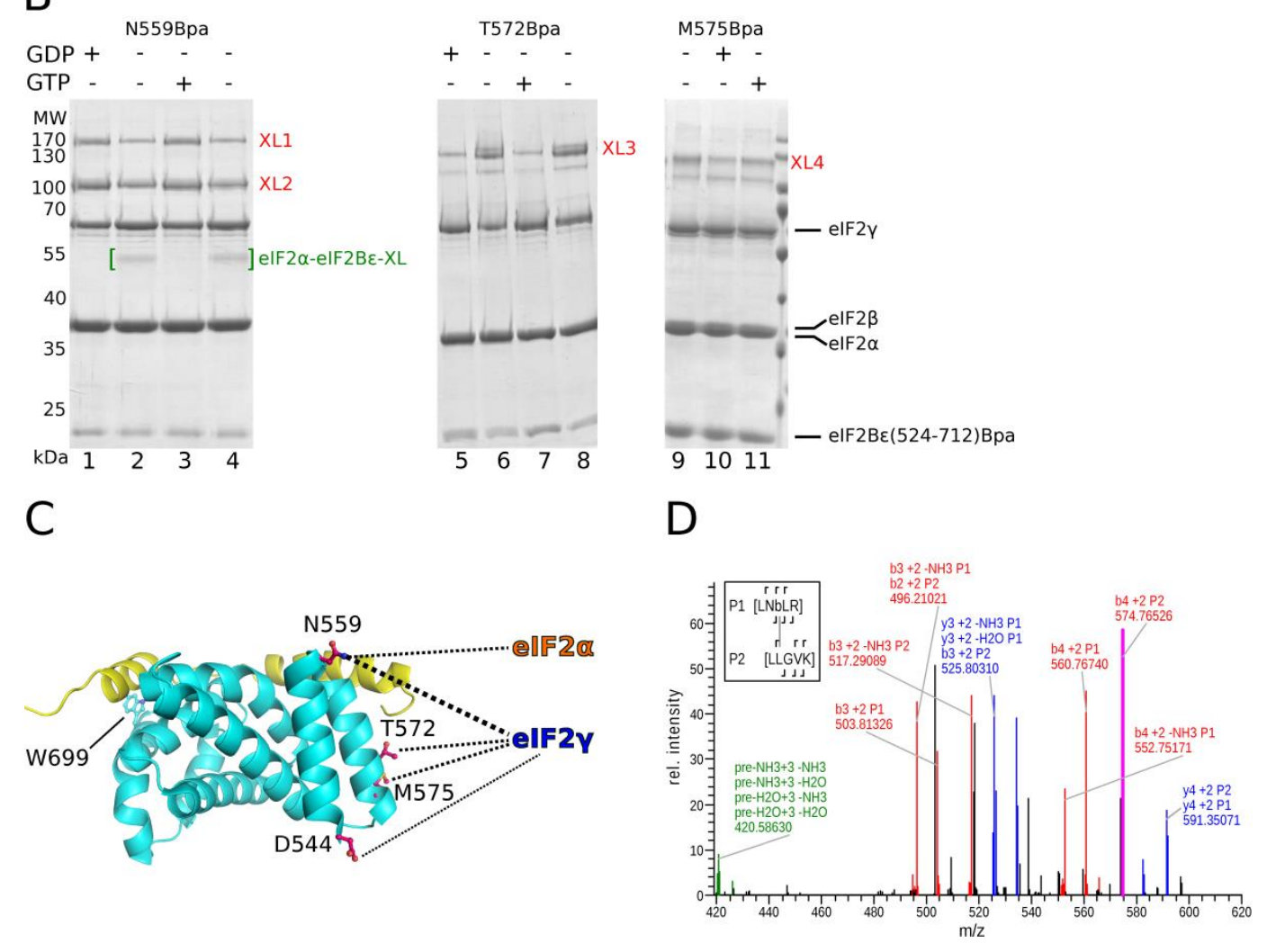

Figure 3. Mapping of the interactions between the elF2B $\varepsilon$-CTD and elF2 by UV-crosslinking. A) SDS-gels showing the UV- and time-dependent formation of crosslinks between elF $2 \gamma$ and the indicated elF2B $\varepsilon(524-712) B p a$ constructs (crosslink bands are indicated by brackets). For T572Bpa, the band migrating at $\sim 70 \mathrm{kDa}$ above elF $2 \gamma$ as well occurs in a UV-dependent manner and is most likely a crosslink between elF2Be(524-712)T572Bpa and elF2 $\beta$; an unambiguous identification of the target molecule was so far not possible. For each experiment, $2 \mu \mathrm{M}$ elF2 was mixed with $4 \mu \mathrm{M}$ elF2B $\varepsilon(524-712) B p a$. B) Nucleotide-dependency of the crosslinks between elF2B $\varepsilon(524-712) B p a$ and elF2. Crosslinking experiments were carried out with $2 \mu \mathrm{M}$ elF2 and $4 \mu \mathrm{M}$ elF2B $(524-712) B p a$ either in the absence of guaninenucleotides or in the presence of $4 \mu \mathrm{M}$ GDP or GTP. C) Structure of the elF2Be(524-712) (with the elF2 $\beta$-NTT in yellow modeled as shown in Fig. 1B), indicating the positions where Bpa was introduced (pink sticks). The dashed lines indicate the crosslinks to elF2 formed from the respective positions. D) High-resolution MS2 fragmentation spectra for the crosslink of N559Bpa to a target peptide in elF2 $\gamma$. A series of $b$ and y product ions were detectable for the peptides involved in the crosslinks; the relative intensity of the observed peaks is plotted against their mass-to-charge ratio $(\mathrm{m} / \mathrm{z})$. The inset shows product ions mapped to the sequences of the crosslinked peptides; $b$ is Bpa. 
By far the most efficient crosslink was obtained for the (nearly fully active) N559Bpa construct (Fig. S2) with Bpa at the very C-terminus of helix $\alpha 1$, which migrated at $\sim 130$ and $\sim 100 \mathrm{kDa}$, respectively (both elute from the analytical size exclusion column at the same volume as the uncrosslinked eIF2·elF2B $(524-712)$ complex). Less efficient but still significant crosslinks to elF2 $\gamma$ were observed for T572Bpa and M575Bpa, both of which produced a stronger upper band ( $\sim 120$ kDa) and a weaker lower band ( 105 kDa). The least efficient position was D544Bpa, which instead formed a strong internal crosslink.

Table 2. Crosslinks between elF2B 2 (524-712) and elF2 $\gamma$.

\begin{tabular}{|c|c|c|c|c|c|c|c|}
\hline \multirow[b]{2}{*}{ Construct } & & \multicolumn{6}{|c|}{ Crosslinked peptides } \\
\hline & & Bpa peptide & Target peptide & Site & $\begin{array}{l}\text { Mass } \\
\text { (Da) }\end{array}$ & $\begin{array}{l}\text { Error } \\
\text { (ppm) }\end{array}$ & $\mathrm{m} / \mathrm{z}$ \\
\hline \multirow[t]{7}{*}{ N559Bра } & XL1a & AMEbNHDLDTALLE & ADMAR & 484 & 2285.0215 & -0.1 & 762.3448 \\
\hline & $X L 1 b$ & AMEb̄ NHDLDTALLE & $\mathrm{ADmA} \underline{\bar{R}}$ & 484 & 2301.0157 & -0.2 & 767.6765 \\
\hline & XL1c & RAMEb $\underline{b} N H D L D T A L L E$ & PNE & 458 & 2237.0176 & -0.1 & 746.3440 \\
\hline & $X L 2 a$ & GIATVERAMEb $\underline{\text { NHHD }}$ & $\overline{\mathrm{IE}} \underline{\mathrm{R}}$ & 361 & 2223.0868 & 0.2 & 556.5271 \\
\hline & $X L 2 a$ & AmEb $\underline{b} N \mathrm{D}$ & IEIRPGIVTKD & $362-367$ & 2223.0881 & 5.9 & 556.5274 \\
\hline & $X L 2 b$ & $\underline{b} N H D$ & PTLCRAD & $408-411$ & 1410.6128 & 1.5 & 705.8107 \\
\hline & $\mathrm{XL2c}$ & AmEbNHD & PTLCRAD & $408-410$ & 1757.7259 & 0.0 & 586.5801 \\
\hline \multirow[t]{6}{*}{ T572Bpa } & XL3a & LNbLR & $\underline{\mathrm{LLGVK}}$ & $441-443$ & 1276.7775 & -0.1 & 432.2674 \\
\hline & $X L 3 b$ & LNobLR & LLGVKTDGQK & $442-443$ & 1806.0260 & -0.7 & 452.2619 \\
\hline & $X L 3 c$ & LNobLR & LLGVKTDGQK & 440 & 1806.0279 & 0.3 & 456.7647 \\
\hline & $X L 3 d$ & LNbㄴL & LLGV트 & 444 & 1276.7774 & -0.2 & 324.4524 \\
\hline & XL3e & 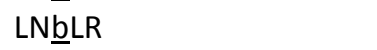 & RLLGV̄KTDGQK & 443 & 1980.1385 & -0.2 & 495.7900 \\
\hline & $X L 3 f$ & LNw $\underline{\bar{b}} L R$ & RLLGV트 & 444 & 1450.8894 & 0.1 & 363.4778 \\
\hline \multirow[t]{2}{*}{ M575Bpa } & $X L 4 a$ & LNTLRㅁSMNVTYHE & LLGVKK & 442 & 1741.8528 & -5.2 & 785.7448 \\
\hline & $X L 4 b$ & $\underline{b}$ SMNVTTYHEVRIATITALLR & $\mathrm{H} \overline{\mathrm{WR}}$ & $509-510$ & 2918.5253 & 0.2 & 730.3867 \\
\hline
\end{tabular}

Target positions in the target peptides are underlined.

Using ESI-MS/MS2analysis in an Orbitrapmass spectrometer, followed by an analysis of the obtained data with the StavroX software [102], we were able to identify potential target peptides for the crosslinks from positions N559Bpa, T572Bpa and M575Bpa (Table 2). For the upper band produced by N559Bpa (crosslink XL1), ${ }^{480} \mathrm{ADMAR}^{484}$ and ${ }^{458}{ }^{\mathrm{PNE}}{ }^{460}$ were identified as potential target peptides for the crosslink (target positions are underlined). In a homology model of yeast elF2 $\gamma$ (based on crystal structures of the archaeal alF2 $\gamma$ ) both peptides lie at the lower side of domain III adjacent to the cleft to domain II (Fig. 7). The crosslink-peptides for the stronger lower crosslink band (XL2) were found to lie in domain II, with ${ }^{361}{ }^{R P G I V T K}{ }^{367}$ and ${ }^{408}{ }^{R A D}{ }^{410}$ at the bottom of the $\beta$ - 
barrel as target sites. For the upper band of T572Bpa (XL3) ${ }^{441} \mathrm{LGVK}^{444}$ was identified as main target, which lies in the long flexible loop following the first $\beta$-strand of domain III, positioned directly next to the switch 2 region of the $G$ domain (Fig. 7). The same peptide, as well as the adjacent ${ }^{508} \mathrm{HWR}^{510}$ in domain III could also be identified as targets for M575Bpa (Table 2). For D544Bpa, no target peptides could be clearly identified so far.

\section{elF2B $\varepsilon$-CTD interacts with elF2 $\gamma$ in a nucleotide-dependent manner.}

An important further question was whether the crosslink pattern would change depending on the nucleotide status of elF2. For this purpose, the experiments were repeated either in the absence or presence of excess GDP or GTP. Interestingly, N559-, T572- and M575Bpa but not D544Bpa exhibited a strong but non-uniform dependency of their crosslinks on the nucleotide status of elF2 (Fig. 3B). For T572Bpa and M575Bpa in helix $\alpha 2$, the most efficient crosslinking was observed with apo elF2, which significantly decreased in the complex with GDP- or GTP-bound elF2 (including XL3). The opposite tendency was observed for N559Bpa, where both crosslinks to elF2 $\gamma$ (XL1 and XL2) significantly improved in the presence of either nucleotide;only with apo elF2 an additional crosslink from N559Bpa occurred at $\sim 50 \mathrm{kDa}$ at the expense of the upper elF2 $\gamma$-containing bands, which according to MS analysis was a specific crosslink to the $\alpha$-subunit of elF2.

These results indicate that the elF2-elF2BE-CTD complex undergoes a conformational rearrangement upon the transition of elF2 from the nucleotide-bound to the apo state, in which the C-terminus of helix $\alpha 2$ moves into a position that allows more efficient crosslinking to elF $2 \gamma$, while the C-terminus of helix $\alpha 1$ (with Gln559) moves into a position that allows less efficient crosslinking to elf $2 \gamma$ but instead provides access to the $\alpha$-subunit. These rearrangements, however, are insensitive to the species of the bound nucleotide and occur with GDP and GTP alike.

\section{Influence of elF2BE on the kinetics of guanine nucleotide binding by elF2}

To study the interactions of elF2 with elF2B $\varepsilon$ and GDP or GTP, we used the fluorescent derivatives of GDP/GTP, mant-GDP and mant-GTP, to monitor binding and dissociation of guanine nucleotides (see chapter 2). The affinities of mant-GDP and -GTP to elF2 in the presence of elF2B $\varepsilon$ were determined by equilibrium titrations (Fig. 4$)$. The dissociation rate constants $\left(k_{\text {off }}\right)$ were determined 
in chase experiments with excess unlabeled GDP (Fig. 5). Finally, the bimolecular association constants $\left(k_{\mathrm{on}}\right)$ were estimated from $K_{\mathrm{d}}$ and $k_{\text {off }}$ according to the relation $K_{\mathrm{d}}=k_{\text {off }} / k_{\text {on }}$. Previous reports suggested that full-length elF2B $\varepsilon$ and the C-terminal fragment elF2B $\varepsilon(524-712)$ show the same level of GEF activity [70]; we therefore used the fragment elF2B $(524-712)$ in most experiments unless stated otherwise.

A

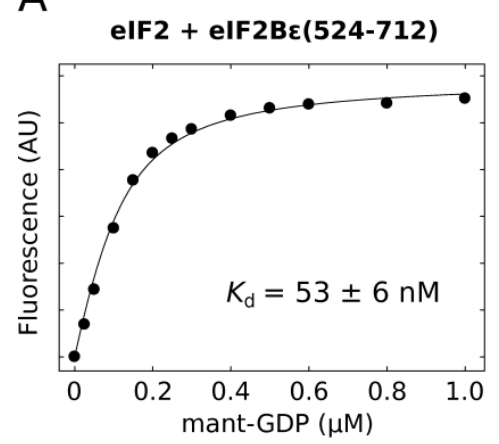

C

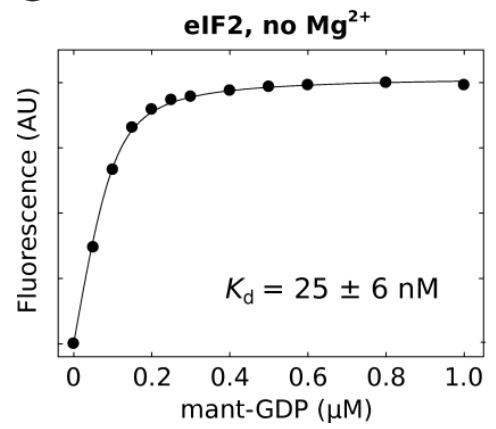

B

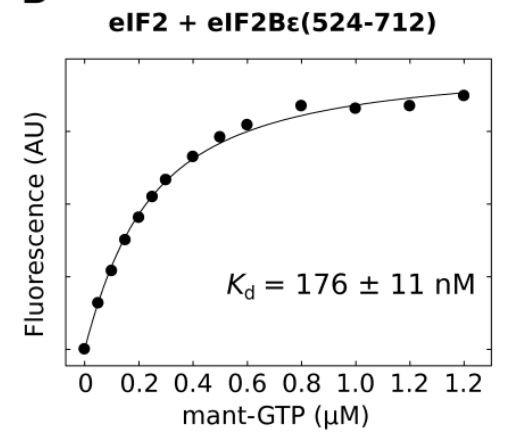

D

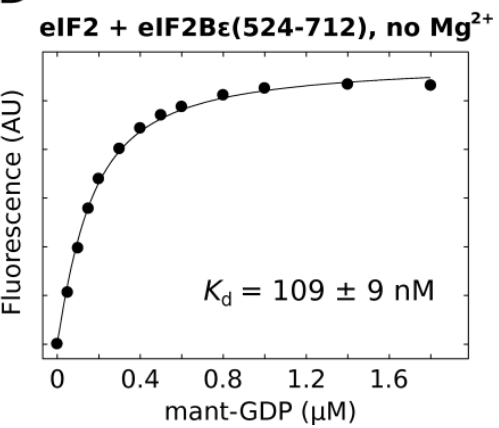

Figure 4. Influence of the elF2B $\varepsilon-C T D$ and $\mathrm{Mg}^{2+}$ ions on the binding of mant-GDP and mant-GTP by elF2. A-B) Equilibrium titrations of $100 \mathrm{nM}$ elF2 with mant-GDP (A) and mant-GTP (B) in the presence of 5 $\mu \mathrm{M}$ elF2BE(524-712). C-D) Equilibrium titrations of $100 \mathrm{nM}$ elF2 with mant-GDP without (C) or with (D) $5 \mu \mathrm{M}$ elF2Be(524712) and in the absence of $\mathrm{Mg}^{2+}$. The binding reaction was monitored by FRET between elF2 (excited at $280 \mathrm{~nm}$ ) and the mant-group of the nucleotide (emission monitored at $440 \mathrm{~nm}$ ). The black lines show the fit to the data, using a quadratic one-side binding model; the resulting values are summarized in Table 3.

Mant-GDP bound to the elF2-eIF2Be-CTD complex with a $K_{d}$ of $53 \mathrm{nM}$ and thus nearly 3-fold weaker than to the elF2 complex alone $\left(K_{d}=20 \mathrm{nM}\right)$ (Fig. 4 and Table 3). The dissociation rate constant $\left(k_{\text {off }}\right)$ was determined to be $3.42 \mathrm{~min}^{-1}$, giving an estimate for the $k_{\text {on }}$ of $6.5 \times 10^{7} \mathrm{M}^{-1} \mathrm{~min}^{-1}$. Thus, the elF2B $\varepsilon$-CTD promotes the dissociation of GDP by $\sim 25$-fold relative to the non-catalyzed reaction $\left(0.14 \mathrm{~min}^{-1}\right)$ under the used conditions, while simultaneously allowing a $\sim 10$-fold faster association (Table 3). Likewise, the affinity of elF2 for mant-GTP was reduced $\sim 6$-fold from a $K_{d}$ of $30 \mathrm{nM}$ in the absence to a $K_{\mathrm{d}}$ of $176 \mathrm{nM}$ in the presence of elF2BE-CTD. As for GDP, this reduction in affinity is accompanied by a significant increase of the dissociation rate $\left(k_{\text {off }}=2.32 \mathrm{~min}^{-1}\right.$ for elF2 alone), which, however, could not be determined reliably due to the limitations of the used technique. 

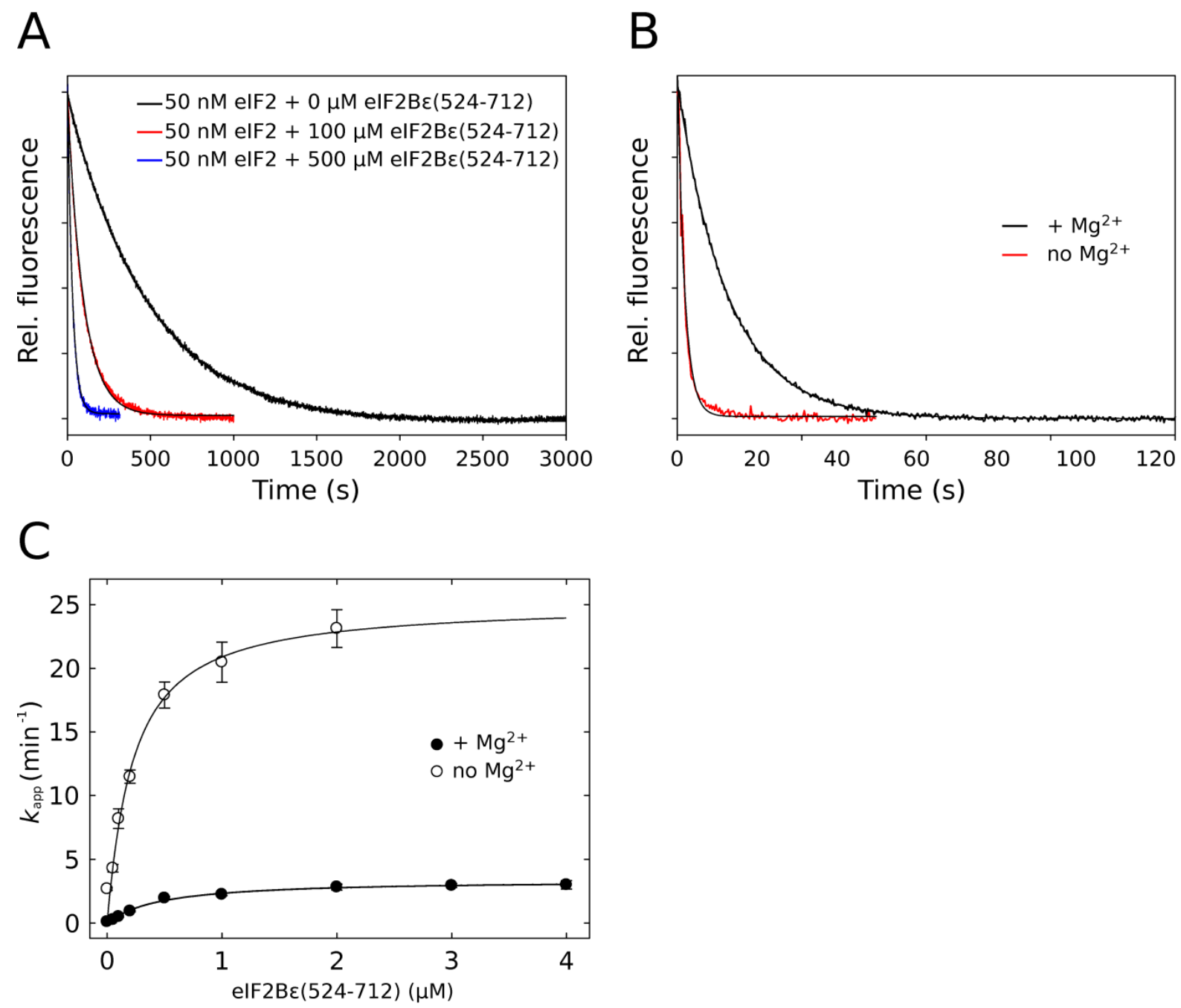

Figure 5. Effect of elF2Br(524-712) and $\mathrm{Mg}^{2+}$ on the dissociation of mant-GDP from elF2. A) Representative time courses for the dissociation of mant-GDP from elF2 ( $50 \mathrm{nM}$ elF2 with $100 \mathrm{nM}$ mant-GDP and $3 \mu \mathrm{M}$ unlabeled GDP) in the presence of 0 (black), 100 (red) and $500 \mathrm{nM}$ (blue) elF2Be(524-712). The smooth black lines represent singleexponential fits which yielded the respective apparent rate constants $k_{\text {app. }}$ B) Time courses for the dissociation of mantGDP from elF2 (50nM elF2 with $100 \mathrm{nM}$ mant-GDP and $3 \mu \mathrm{M}$ unlabeled GDP) in the presence of $200 \mathrm{nM}$ elF2B $\varepsilon(524-$ 712) either in the presence (black) or in the absence of $2.5 \mathrm{mM} \mathrm{Mg}^{2+}$ (red). C) Dependency of the dissociation of elF2-mant-GDP ( $50 \mathrm{nM}$ ) on the concentration of elF2Be(524-712) either in the presence (closed circles) or absence of $2.5 \mathrm{mM} \mathrm{Mg}^{2+}$ ions (open circles).

To determine the approximate affinity between elF2BE-CTD and GDP-bound elF2, the apparent off rates $\left(k_{\mathrm{app}}\right)$ for the dissociation of mant-GDP from elF2 at increasing concentrations of the GEF were fitted to a quadratic equation, giving an estimate for the $K_{1 / 2}$ of $423 \pm 53 \mathrm{nM}$ (Fig. 4C), which is 2.6-fold larger than the value reported previously for similar conditions ( 162 nM) [105]. 
For other GEF-catalyzed nucleotide exchange reactions it is known that the GEF contributes to the destabilization of the guanine nucleotide by destabilizing the coordination of the $\mathrm{Mg}^{2+}$ ion. To study the contribution of $\mathrm{Mg}^{2+}$ coordination to GDP binding, the affinity and dissociation rate of mant-GDP was determined in the absence of $\mathrm{Mg}^{2+}$ and the presence of $1 \mathrm{mM}$ EDTA. Although having only a minor effect on the affinity of mant-GDP to elF2 determined under steady-state condition $\left(K_{\mathrm{d}}=25 \mathrm{nM}\right)$, the absence of $\mathrm{Mg}^{2+}$ resulted in a $\sim 20$-fold increased dissociation rate $\left(k_{\text {off }}\right.$ $\left.=2.7 \mathrm{~min}^{-1}\right)$. Consequently, this implies that the association rate constant $\left(k_{\mathrm{on}}\right)$ as well increases to $1.1 \times 10^{8} \mathrm{M}^{-1} \mathrm{~min}^{-1}$. Addition of elF2B $\varepsilon$-CTD further increased the dissociation rate by a factor of 9.4 to $25.2 \mathrm{~min}^{-1}$ (7.4-fold faster than the catalyzed reaction in the presence of $\mathrm{Mg}^{2+}$ ), accompanied by a decreased affinity $\left(K_{d}=109 \mathrm{nM}\right)$ and an increased association rate constant of $3.9 \times 10^{6} \mathrm{M}^{-1} \mathrm{~s}^{-1}$.

Table 3. Influence of elF2Be(524-712) on the binding of mant-GDP and mant-GTP by elF2

\begin{tabular}{|c|c|c|c|c|c|c|}
\hline & Nucl. & $\begin{array}{l}\mathrm{MgCl} \\
(\mathrm{mM})\end{array}$ & $k_{\text {off }}\left(\min ^{-1}\right)$ & $\begin{array}{l}k_{\text {on }} \\
\left(\mathrm{M}^{-1} \min ^{-1}\right)^{a}\end{array}$ & $\begin{array}{l}K_{\mathrm{d}} \text { (Nucl.) } \\
(\mathrm{nM})^{\mathrm{b}}\end{array}$ & $\begin{array}{l}K_{1 / 2}(2 B \varepsilon) \\
(n M)^{c}\end{array}$ \\
\hline elF2 & mGDP & 2.5 & $0.14 \pm 0.05$ & $7 \times 10^{6}$ & $20 \pm 5$ & N.D. \\
\hline elF2 & mGDP & 0 & $2.7 \pm 0.15$ & $1.1 \times 10^{8}$ & $25 \pm 6$ & N.D. \\
\hline elF2 & mGTP & 2.5 & $2.32 \pm 0.11$ & $7.7 \times 10^{7}$ & $30 \pm 3$ & N.D. \\
\hline \multicolumn{7}{|l|}{ elF2 + } \\
\hline elF2BE-CTD & mGDP & 2.5 & $3.42 \pm 0.21$ & $6.5 \times 10^{7}$ & $53 \pm 6$ & $432 \pm 53$ \\
\hline elF2BE-CTD & mGDP & 0 & $25.2 \pm 0$ & $2.3 \times 10^{8}$ & $109 \pm 9$ & $195 \pm 40$ \\
\hline elF2B $\varepsilon-C T D$ & mGTP & 2.5 & N.D. & & $176 \pm 11$ & N.D. \\
\hline
\end{tabular}

\footnotetext{
${ }^{\mathrm{a}}$ The bimolecular association constant was estimated according to the relation $k_{\text {on }}=k_{\text {off }} / K_{\mathrm{d}}$

${ }^{\mathrm{b}} K_{\mathrm{d}}$ is the equilibrium dissociation constant between elF2 and mant-GDP or mant-GTP. Values for elF2 binding to mGDP or mGTP in the presence of $\mathrm{MgCl}_{2}$ are adapted from Table 4 in chapter 2.

${ }^{c} K_{1 / 2}$ is the apparent equilibrium dissociation constant between elF2 and elF2B $\varepsilon(524-712)$, estimated from the dependency of $k_{\text {app }}$ of mant-GDP dissociation from elF2 on the concentration of elF2B $\varepsilon-C T D$.

N.D., not determined
}

\section{An intermediate ternary complex of GEF, elF2 and the nucleotide is formed during the exchange reaction catalyzed by the elF2BE-CTD.}

As indicated by the crosslink data, the elF2-bound nucleotide has the ability to influence the way in which $\mathrm{elF} 2 \mathrm{~B} \varepsilon$ is arranged relative to elF2, without abolishing its binding. This suggests that nucleotide and exchange factor can bind simultaneously to elF 2 to form an intermediate ternary complex. To test this assumption, we titrated a given concentration of fluorescently labeled 
elF2-mant-GDP with increasing amounts of the exchange factor (either full-length or the CTD alone). If the binding of GDP and GEF were mutually exclusive, the latter should be able to compete with the bound mant-nucleotide, resulting in the loss of the fluorescence signal at saturating amounts of eIF2BE.
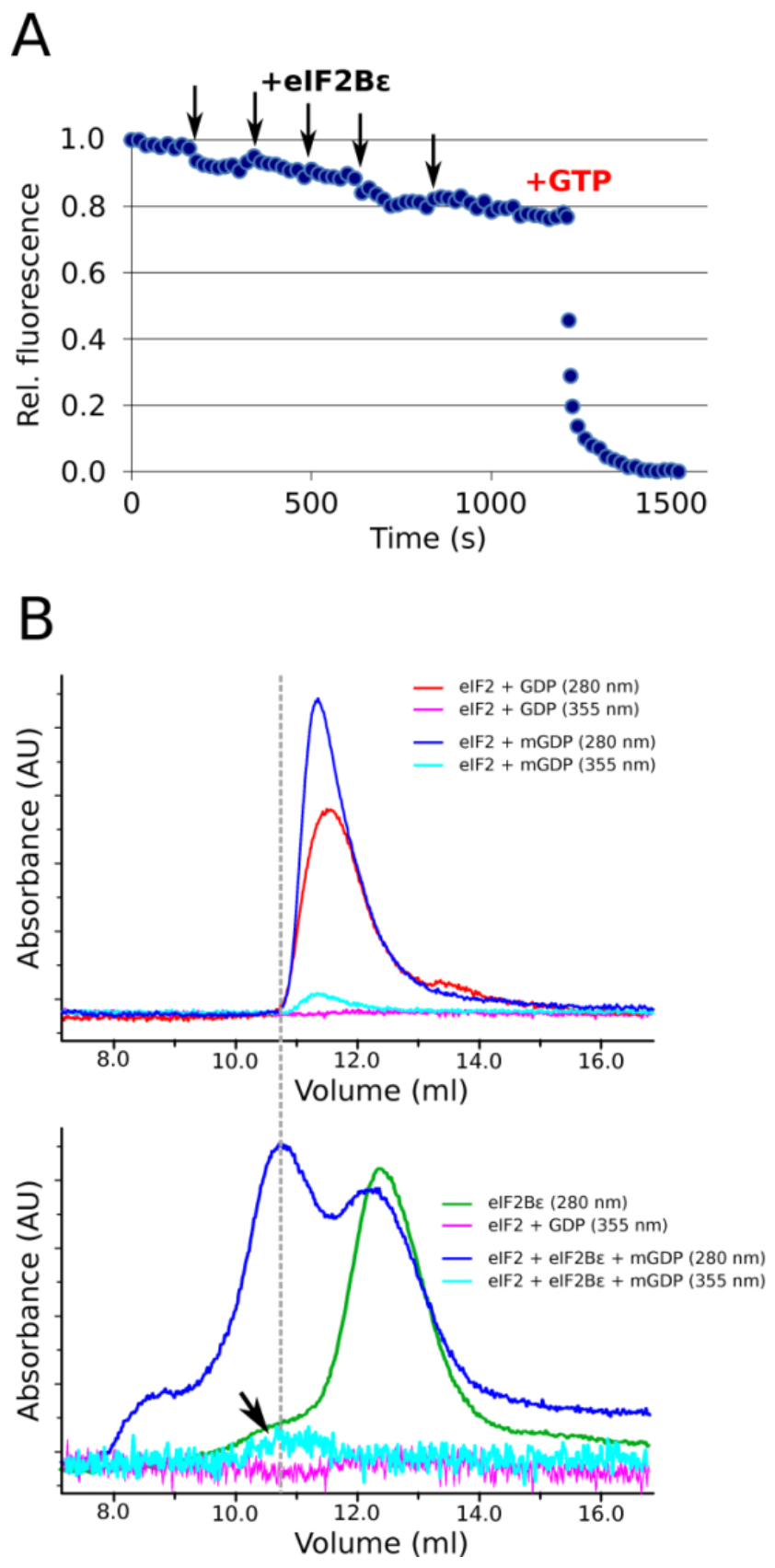

Figure 6. elF2, guanine-nucleotide and elF2B $\varepsilon$ form a ternary complex. A) Titration of elF2. mant-GDP (100 nM elF2 with $125 \mathrm{nM}$ mant-GDP) with increasing amounts of elF2B $\varepsilon$. Each of the black arrows indicates the addition of $5 \mu \mathrm{M}$ elF2Br, until $25 \mu \mathrm{M}$ (250-fold excess) was reached. '+GTP' indicates the time point at which $10 \mu \mathrm{M}$ unlabeled GTP was added to the mixture. The mant-fluorescence was excited directly at $355 \mathrm{~nm}$ and monitored at $440 \mathrm{~nm}$. B) Analytical size exclusion chromatography for elF2 in complex with guanine-nucleotides and elF2Br. The upper panel shows the runs of elF2 either with GDP (red and pink) or with mant-GDP (blue and cyan) with the absorption monitored at 280 and $355 \mathrm{~nm}$. Only for the elF2-mant-GDP complex, absorption at $280 \mathrm{~nm}$ (blue) and at $355 \mathrm{~nm}$ (cyan) is observed. The lower panel shows the complex run (blue, $280 \mathrm{~nm}$; cyan, $355 \mathrm{~nm}$ ) between elF2-mant-GDP and elF2B $\varepsilon$ (the green curve corresponds to elF2B $\varepsilon$ alone), in which the main peak is shifted $\sim 0.6$ $\mathrm{ml}$ relative to the elF2-mant-GDP peak (grey dashed line). In order to highlight the observed absorption by mantGDP (arrow) in the elF2.elF2B $\varepsilon$ containing peak, the scale for the absorption at $355 \mathrm{~nm}$ in the lower panel was reduced three-fold relative to that at $280 \mathrm{~nm}$. 
As shown in Figure $6 \mathrm{~A}$, elF2Br causes a slight decrease of the fluorescent signal, indicating either a direct effect on the fluorescence by the mant group or a reduction of the amount of mantGDP bound to elF2. After adding a 250-fold excess of elF2B $\varepsilon$ over elF2, which should be sufficient to saturate the latter with the GEF, unlabeled GTP was added in excess to determine the groundfluorescence of the unbound mant-GDP. According to this final signal, the addition of excess elF2B $\varepsilon$ resulted in the loss of only $\sim 20 \%$ of the initial fluorescence. Consistent with the crosslinking studies, this indicates that the remaining $\sim 80 \%$ originate from mant-GDP still bound to the binary complex of elF2·elF2Bc.

To further test the existence of an elF2-mant-GDP.elF2B $\varepsilon$ ternary complex we tried to monitor it by analytical size exclusion chromatography. The binary elF2-mant-GDP complex eluted at $\sim 11.4 \mathrm{ml}$ and exhibited a clear peak for the absorption at $355 \mathrm{~nm}$, the excitation wavelength for the mant moiety (Fig. 6B). A corresponding absorption at $355 \mathrm{~nm}$ was not observed for the apo elF2 complex or for elF2·GDP. We then added excess elF2B $\varepsilon$ to the preformed elF2-mant-GDP binary complex, which should yield the eIF2·mGDP.elF2B $\varepsilon$ ternary complex, and loaded it onto the column. As expected, elF2 and elF2B formed a complex which eluted at $\sim 10.7 \mathrm{ml}$ (monitored by absorption at $280 \mathrm{~nm}$ ), corresponding to a $0.7 \mathrm{ml}$ shift relative to elF2-mant-GDP alone. Importantly, a correspondingly shifted peak was observed for the absorption at $355 \mathrm{~nm}$, indicating that the elF2-elF2Br complex still contained mant-GDP and demonstrating the existence of a ternary complex of all three components as an intermediate of the nucleotide exchange reaction (Fig. 6B).

\subsection{Discussion}

\section{Implications for the mechanism of elF2BE-catalyzed nucleotide exchange on elF2.}

Like elF2, a large number of Ras-related G proteins bind GDP with high affinity and have very low rates of spontaneous GDP release. In order to allow nucleotide exchange and thus efficient activation of the $G$ protein for its function in biological time scales, GEFs are used to accelerate GDP release by several orders of magnitude [23, 25]. Although GEFs of different G protein families are structurally unrelated and bind to their substrates in different ways, it was found that they use common principles to destabilize the tightly bound guanine nucleotide, notwithstanding 
considerable differences in the molecular details how the underlying problem is solved. Initially, GEFs bind to the GTPase.GDP complex forming an unstable ternary complex in which both, the nucleotide as well as the GEF are bound with low affinity by the G protein. In the majority of cases, GDP-destabilization is primarily achieved by deformation of the nucleotide-binding site in the two switch regions and the P-loop that are responsible for $\mathrm{Mg}^{2+}$ coordination and phosphate binding. Usually, the flexible switch 1 becomes displaced by the GEF through steric hindrance, while extensive contacts are formed with switch 2 . As a result, switch 2 is usually remodeled in a way that allows the formation of a salt bridge between the aspartate of its conserved DXXG motif and the invariant P-loop lysine, causing the loss of critical contacts for the $\mathrm{Mg}^{2+}$ ion and the GDP- $\beta$ phosphate. Alternative or additional features are the introduction of side chains in cis by the $\mathrm{G}$

protein or in trans by the GEF that obstruct the binding sites for the $\mathrm{Mg}^{2+}$ ion and/or the phosphate moiety. Moreover, some GEFs distort the P-loop backbone, thereby breaking hydrogen bonds that stabilize the $\beta$-phosphate. As a consequence of these rearrangements, the GDP molecule is released more rapidly from the unstable ternary complex, followed by the formation of a stable GTPase.GEF complex. Subsequently, a GTP molecule binds, again to form an unstable ternary complex from which the GEF is finally released to yield the GTP-bound $G$ protein $[23,25,101]$.For the exchange reaction on elF2 it was so far unclear to what extent these basic principles of GEF activity also apply to the guanine-nucleotide exchange reaction catalyzed by elF2BE.

\section{A structural model for the interactions between the catalytic subdomain of elF2B $\varepsilon$ and elF2}

Interactions between elF2Be and elF26. Using tryptophan quenching experiments, we sought to provide a better understanding of the interaction between eIF $2 \beta$ and the elF2B $\varepsilon$-CTD, which was proposed to involve interactions between the three K-boxes in elF2 $\beta$ and the two conserved AAboxes in the C-terminal region of elF2BE $[35,74]$.

Our experiments show that the elF2 $\beta$-NTT provides a high-affinity binding site for the elF2BECTD within the elF2 complex with a $K_{\mathrm{d}}$ of $\sim 100 \mathrm{nM}$ (Fig. 2, Table 1), in line with the proposed role as docking site for the GEF on its substrate eIF2 $[35,74]$. This affinity is dependent on the presence of the three lysine-rich K-boxes, in particular K-boxes 1 and 2, consistent with the idea that ionic interactions with the negatively charged surfaces on eIF $2 B \varepsilon$ play a critical role in the contact. As 
indicated by the location of the solvent-exposed tryptophan residues that are likely to contribute most to the quenching signal induced by K-box 2 (Trp677, 696, and 699), area I and the acidic Cterminal tail are the most likely candidates for these interactions, in line with the high degree of conservation of their acidic residues (Fig. 1B and S1). The contributions of the three K-boxes to the overall binding affinity are entirely additive suggesting that they interact with elF2B $\varepsilon$ independently from each other via a peptide-domain interaction similar to that proposed for the elF5-CTD (see chapter 2), in which the elF $2 \beta-N T T$ does not adopt a tertiary structure upon binding, but instead adopts an extended conformation to wrap around the elF2B $\varepsilon$-CTD. Consistently, the topology and surface charge properties of the elF2B $\varepsilon$-CTD would be compatible with a mode of binding for K-box 3 and the adjacent $\alpha$-helices $\alpha N^{\beta}$ and $\alpha C^{\beta}$ similar to that seen in the elF5CTD-elF2 $\beta$-NTT complex structure (Fig. 1B). If so, it can be envisaged that $\alpha$-helix $\alpha N^{\beta}$ serves a similar role in the elF2.elF2B complex as proposed for the complex with elF5 by prearranging the elF2B $\varepsilon$-CTD relative to elF2 $\gamma$ in a way that promotes the direct interactions between the two proteins to mediate nucleotide exchange. This would provide an explanation why K-box 3 is highly conserved among elF $2 \beta$ orthologs, despite its relative marginal contribution to the overall affinity and despite the apparent absence of an evolutionary constraint imposed by the necessity to stabilize a tertiary structure fold.

Interactions between elF $2 B \varepsilon$ and elF2 $\gamma$. In previous studies the interface for direct elF2B $\varepsilon \cdot$ elF $2 \gamma$ interactions was mapped to the first two $\alpha$-helices of the elF2B $\varepsilon$-CTD; however, the binding site on elF2 $\gamma$ was unknown. We could show that the short-range UV-inducible crosslinker Bpa, introduced in various positions in the periphery of the proposed interface, crosslinks specifically to the $y$-subunit of elF2 (Fig. 3). Moreover, we were able to identify specific target peptides within elF2 $\gamma$ for the crosslinks from positions Asn559, Thr572 and Met575 in elF2BE (Fig. 7 , Table 2). These suggest that the elF2B $\varepsilon$-CTD, like elF5, contacts the frontal face of elF2 $\gamma$, with Thr572 and Met575 in helix $\alpha 2$ positioned close to residues 440-444 in domain III of elF2 $\gamma$ (green surface in Fig. 7), while Asn559 at the C-terminus of $\alpha 1$ points toward residues 362-367 in domain II and the probable binding site for elF2 $\alpha$ on elF2 $\gamma$ (golden surfaces in Fig. 7). Hence, this orientation would explain why Bpa in lieu of Asn559, but not Thr572 or Met575, is able to crosslink directly not only to elF2 $\gamma$ but also to elF2 $\alpha$ in complexes with apo elF2 (Fig. 3B). As a consequence, 
the catalytic center residues would come to lie atop of the $\mathrm{G} 3\left({ }^{194} \mathrm{DCPG}^{197}\right)$ motif and the switch 2helix (helix B in EF-Tu [38]), and in the direct vicinity to the binding sites for the $\beta$ - and $\gamma$ phosphates of the bound guanine nucleotide (Fig. 8).

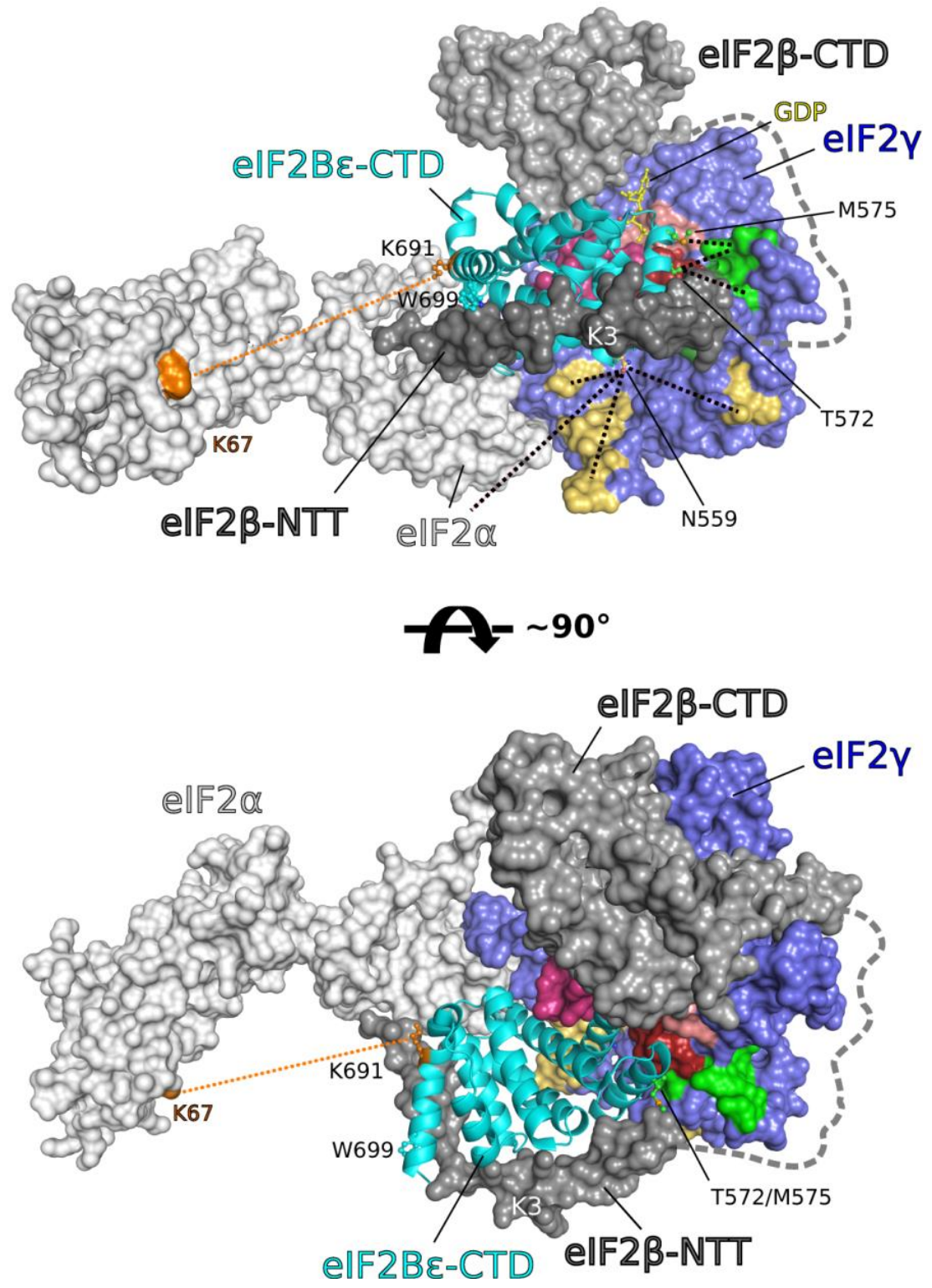

Figure 7. Model for the elF2-elF2B $\varepsilon$ CTD complex. The elF2B $\varepsilon-C T D$ is shown as cyan cartoon, between the elF2 $\beta$-NTT (dark grey surface) and elF2 $\gamma$ (blue surface, with the GDP molecule shown as yellow sticks and P-loop, switch 1 and switch 2 in light red, wine red and dark red, respectively). Target peptides for crosslinks (black dashed lines) from positions T572/M575 and N559 are highlighted as green and dark yellow surfaces on elF2 $\gamma$, respectively. N559 is oriented toward elF2 $\alpha$ (light grey), as suggested by the direct crosslink observed in the absence of a bound guanine-nucleotide. The linker peptide between the elF2 $\beta$-NTT and CTD is indicated by a thick grey dashed line next to elF2 $\gamma$. The orange dashed line between Lys67 in elF $2 \alpha$ (orange surface) and Lys691 on elF2Be (orange sticks) indicates a crosslink identified in[73]. According to this model, the elF2BE-CTD is clamped between the elF2 $\beta-N T T$, which provides the K-boxes for high affinity interactions in the area surrounding Trp699 (pointing away from elF2 $\gamma$ ), and the frontal face of elF $2 \gamma$. The putative catalytic center residues of elF2Be (located between T572/M575 and N559) in repeat $R_{l}$ are thereby positioned next to the nucleotide binding pocket in elF $2 \gamma$.

Under the assumption that the elF2BE-CTD retains its structural integrity upon binding to elF2, and that helices $\alpha 1$ and $\alpha 2$ remain associated with the rest of the domain, the resulting 
structural model predicts that the C-terminal end of elF2B $\varepsilon$ with Trp699 and area I points away from elF2 $\gamma$, which would allow the eIF2 $\beta$-NTT to clamp the eIF2B $\varepsilon$-CTD between both elF2-subunits

(Fig. 7). As a consequence, the C-terminal end of helix $\alpha N^{\beta}$ (residues 91-103) points toward domain III of elF2 $\gamma$, supporting the idea that helix $\alpha \mathrm{N}^{\beta}$ might promote the direct elF2B $\varepsilon \cdot \mathrm{elF} 2 \gamma$ interaction by prearranging the elF2B $\varepsilon$-CTD relative to the $\mathrm{G}$ protein (see above). The remaining distance of 40-50 $\AA$ to helix $\alpha 1$ (residues $127-140$ ) of the elF2 $\beta$-CTD, which anchors elF2 $\beta$ to the elF $2 \gamma$ G domain, could be readily spanned by a flexible linker formed by the missing 23 residues. Interestingly, it was reported that within the elF2-elF2B complex Lys691 of elF2B $\varepsilon$ can be crosslinked to Lys67 in the Nterminal domain of elF2 $\alpha$ using the chemical crosslinker BS3 (spacer length $11.4 \AA$ A [73]. Considering the flexibility of the elF2 $\alpha$-NTD relative to the elF $2 \gamma$-bound C-terminal domain, this finding is in excellent agreement with the proposed structural model for the elF2·elF2B $\varepsilon$-CTD complex, in which Lys691 points away from elF2 $\gamma$ and is thus freely accessible for the elF $2 \alpha-N T D$ (Fig. 7). It should, however, be mentioned that this model is incompatible with the proposal that Trp699 is directly involved in interactions with elF2 $\gamma$ and in the catalysis of nucleotide exchange $[75,76]$. Instead, our model would favor a scenario in which Trp699 is limited to a critical role in the interactions with the elF2 $\beta$-NTT, where its loss due to mutagenesis weakens the high affinity binding to elF2 through elF2 $\beta$ similar to the W391A/F mutation in elF5, and thus affects the exchange activity of eIF2B $\varepsilon$ merely indirectly by abolishing the efficient recruitment of the GEF.

\section{Implications for nucleotide-exchange by elF2B $\varepsilon$}

Does the elF2-elF2Br interaction follow the common basic principles that underlie most GEFcatalyzed exchange reactions? Positioning of the elF2BE-CTD relative to elF2 on the basis of the crosslink data suggests that helices $\alpha 1$ and $\alpha 2$ bind elF $2 \gamma$ in the direct vicinity to helix B (switch 2) (Fig. 8). This would indeed indicate that elF2BE approaches the nucleotide binding pocket from the phosphate-binding side to form contacts to the switch 2 region, similar to those found in complexes of other $\mathrm{G}$ proteins with their GEFs [25]. Moreover, elF2 forms intermediary ternary complexes with guanine-nucleotide and elF2BE (Fig. 6), however, with a reduced affinity and increased rates of dissociation for the nucleotide (Fig. 4, 5). Although the affinity between elF2B $\varepsilon$ and eIF2 $\gamma$ in these complexes was not quantified directly, the crosslink pattern between the two 
proteins was found to depend strongly on the nucleotide state of elF2, without making a difference between GDP and GTP (Fig. 3B). These observations indicate that the elF2.elF2Br complex undergoes a series of reversible conformational rearrangements from the GDP-bound state to the nucleotide-free state and finally to the GTP-bound state, in which the first and the last state adopt similar arrangements. It is therefore conceivable that the elF2B $\varepsilon$-catalyzed exchange reaction follows a multi-step process analogous to that on other $G$ proteins, involving two low-affinity ternary complexes, in which the GEF destabilizes the bound nucleotide and vice versa, as well as three high-affinity binary complexes with elF2 bound to GDP, elF2BE or GTP, respectively.
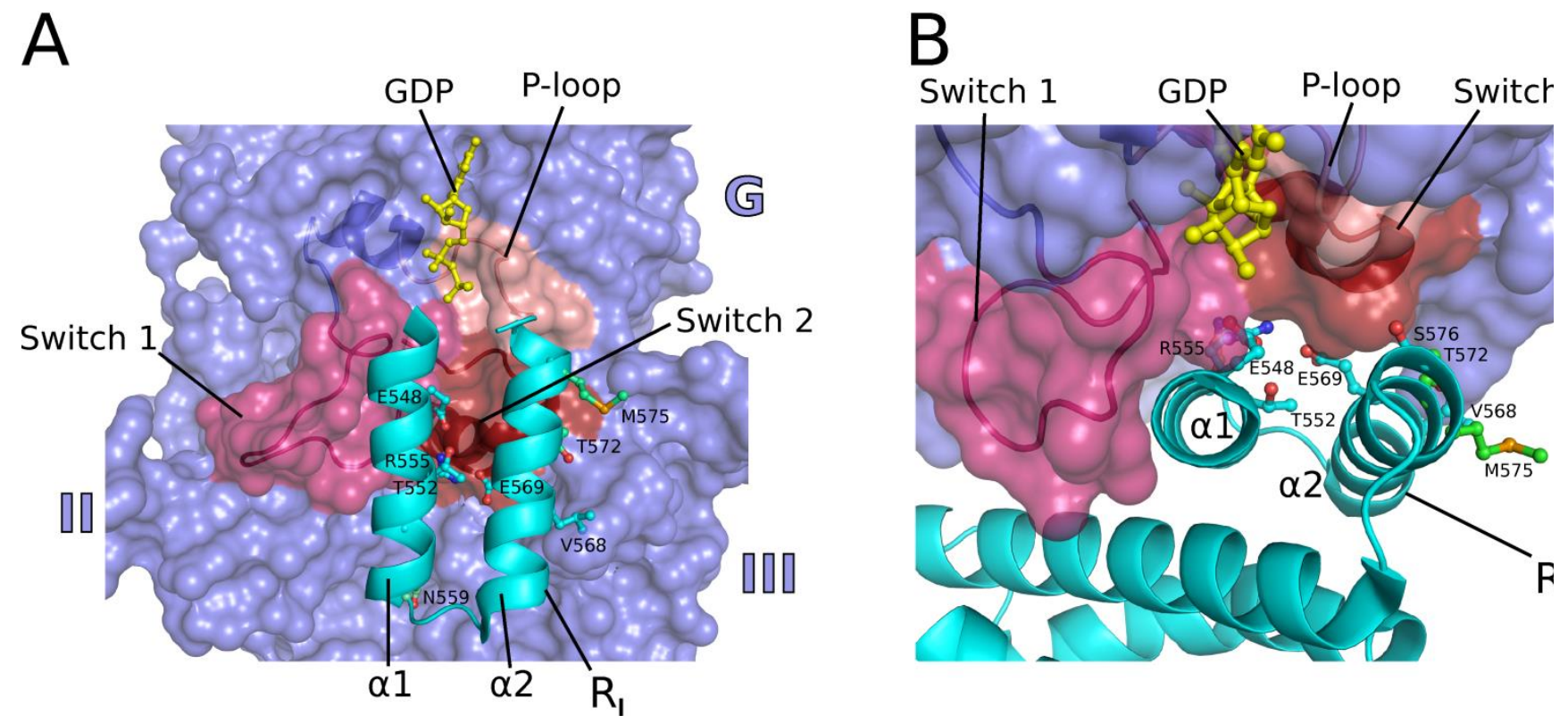

Figure 8. Detail of the modeled interactions between the elF2B $\varepsilon$-CTD and eIF2 $\gamma$. A) Front view of repeat $R_{1}$ of elF2B $\varepsilon$ (cyan cartoon) on the surface of elF2 $\gamma$ (blue surface with the GDP molecule shown as yellow sticks; P-loop, switch 1 and switch 2 are colored light red, wine red and dark red, respectively). N559, T572 and M575 are shown as green balls and sticks. Residues that are either universally conserved and/or were reported to contribute to the exchange activity of elF2B $\varepsilon$ are shown as balls and sticks in cyan. B) Top view of elF2B $\varepsilon$ on the surface of elF2 $\gamma$ (colored as in A).

One of the salient features of nucleotide destabilization by GEFs is the distortion of the $\mathrm{Mg}^{2+}$ binding site $[25,101]$. In line with previous reports [125], we found that $\mathrm{Mg}^{2+}$ ions stabilize the binding of GDP to elF2, resulting in a $\sim 20$-fold acceleration of GDP release in its absence (Fig. 4, 5, Table 3). The addition of elF2Be further accelerates GDP dissociation by $\sim 10$-fold, which 
corresponds to a 7.4-fold faster GDP release relative to the GEF-catalyzed reaction in the presence of $\mathrm{Mg}^{2+}$ ions. This indicates that the removal of $\mathrm{Mg}^{2+}$ plays a role in elF2B $\varepsilon$-catalyzed nucleotide release, consistent with the position of helices $\alpha 1$ and $\alpha 2$ in the direct vicinity of switch 2 (Fig. 8). A possible scenario for this effect is that elF $2 B \varepsilon$ stabilizes the formation of a salt bridge between aspartate 193 of the ${ }^{193} \mathrm{DCPG}^{196}(\mathrm{G} 3)$ motif in switch 2 and the invariant P-loop lysine (Lys114), an interaction that is observed in the apo states and some GDP-bound forms of trGTPases which do not require specialized GEFs, as well as in structures of the elF2 $\gamma$-orthologs eEF1A and EF-Tu in complexes with their respective GEFs eEF1B $\alpha$ and EF-Ts [126, 127].

As indicated by the acceleration of GDP release by elF2B $\varepsilon$ in the absence of $\mathrm{Mg}^{2+}$ ions, further contributions seem to arise from the deformation of the nucleotide-binding site itself. An interesting observation in this context is that GTP as well as GDP stabilize switch 1 in a way that protects it from proteolytic cleavage (Fig. S3). Thus, efficient GDP destabilization by elF2B $\varepsilon$ might requirethe displacement of switch 1 from the nucleotide binding site. According to the structural model presented in Figure 8, helix $\alpha 1$ could function as a wedge that intercalates between switch 1 and switch 2 and thereby displaces the former, similar to helix $\alpha 14$ of SOS1 in its complex with Ras (PDB: 1BKD). Notably, this positions the highly conserved Glu548 and the catalytically relevant Glu569 close to switch 2 and the $\mathrm{Mg}^{2+}$ and phosphate-binding sites, similar to Glu942 in SOS1. At the same time, the invariant Arg555 comes to lie close to the N-terminus of the switch 2-helix, which would allow a stabilizing contact to the invariant Asp198 in switch 2 following the ${ }^{193}{ }^{D C P G}{ }^{196}$ (G3) motif. This might be relevant, as one reoccurring feature of complexes between $\mathrm{G}$ proteins and their GEFs is the stabilization of switch 2 by a conserved acidic residue following the G3 (DXXG) motif through ionic interactions with the $\operatorname{GEF}[128,129]$.

Taken together, the proposed placement of elF2B $\varepsilon$-CTD relative to the nucleotide binding site on elF2 $\gamma$ places the highly conserved catalytic center residues of the GEF within contact distance to the switch regions and the P-loop and is thus compatible with their direct involvement in GDP release. It should be noted that single mutations of most of the conserved residues within helices $\alpha 1$ and $\alpha 2$, including Glu458 and Arg555, were not found to have a detectable negative impact on yeast cell growth $[74,75]$. However, is it in our opinion likely that similar to the mechanism of EF-Ts-catalzed GDP release on EF-Tu [130], most of the individual interactions 
between elF2 and eIF2B $\varepsilon$ contribute only marginally to the overall nucleotide release efficiency, but instead contribute synergistically to bring about the GDP destabilization required for its efficient release.

Due to the low resolution of our structural model for the elF2.elF2B $\varepsilon$ complex based on the crosslink data, the assumptions about the contributions by elF2B $\varepsilon$ to nucleotide exchange and the involvement of specific residues are necessarily tentative and remain speculative. However, our data clearly favor a mechanism of elF2BE-induced nucleotide exchange that proceeds via a successive series of reversible steps and the distortion of the $\mathrm{Mg}^{2+}$ and phosphate binding sites by the remodeling of the switch regions and/or the P-loop. Hence, our data support the idea that the elF2BE-CTD-catalyzed exchange reaction follows the common basic principles of nucleotide exchange known from other GEFs.

\section{Implications for the evolution of the C-terminal domains of elF2Br and elF5 and their interactions with elF2}

Within the family of trGTPases, only four factors, namely elF2 and the homologous EF-Tu, eEF1A, and aEF1A, are known to have dedicated protein-GEFs that catalyze nucleotide exchange. For all other trGTPases no GEFs are known, which is consistent with their usually low affinities for GDP and its fast dissociation rates (with the possible exception of RF3, for which the ribosome itself was proposed to act as GEF [131]). In this context, it is interesting to note that all four GEF-dependent trGTPases belong to the same monophyletic clade, with elF2 $\gamma$ most likely derived from the EF-Tu lineage [22]. An obvious conclusion would be to expect a common evolutionary history for the respective GEF functions as well. However, this is not the case. All three GEFs, namely EF-Ts, $\mathrm{a} / \mathrm{eEF} 1 \mathrm{~B}$ and $\mathrm{elF} 2 \mathrm{~B} \varepsilon$, share no mutual sequence or structural homology, and at least for EF-Ts and eEF1B it is known that their structural differences are reflected in very different ways by which they approach and bind their respective substrate trGTPases [126, 127]. Moreover, no GEF is known for the archaeal elF2 ortholog alF2. Thus, it seems evident that the catalytic activity of elF2BE-CTD evolved after the divergence of the archaeal and eukaryal lineages and independently from EF-Ts or a/eEF1B. Similarly, no structural or functional counterpart for the elF5-CTD is known from archaea, likewise implying a relatively recent evolutionary history. 
From the data presented here, it becomes evident that elF2B $\varepsilon$-CTD and elF5-CTD share two critical interactions with elF2: On the one hand the high affinity interaction between the conserved AA-boxes and the K-boxes in the elF2 $\beta$-NTT. On the other hand the direct interaction to overlapping interfaces on the frontal face of elF $2 \gamma$, which in both cases involve the regions surrounding repeat $R_{l}$ of the HEAT repeat-like fold and which have direct, though opposing, effects on the nucleotide binding properties of their common substrate elF2 $\gamma$. In light of the similarity of these interactions, combined with the structural and partial sequence homology between both proteins (Fig. S1), it is conceivable that elF2BE-CTD and elF5-CTD share a common evolutionary origin in an AA-box-containing protein, which already bound elF2 in a manner similar to the extant proteins and modulated its nucleotide binding properties - although this does not necessarily imply physiological relevance of this effect, similar to the suggestion for elf5 (see chapter 2). Unfortunately, due to the low degree of sequence conservation between elF2B $\varepsilon$-CTD and elF5CTD, their apparently ubiquitous distribution among eukarya, and the absence of homologs in archaea or bacteria which could be used as outgroups for a phylogenetic analysis, it is currently not possible to determine whether the ancestral protein was more elF5- or elF2BE-CTD-like, which would provide valuable insight into the process of evolution of the complex eukaryal initiation apparatus.

It has previously been noted that with the exception of the two AA-boxes, elF5-CTD and elF2B $\varepsilon$ CTD share very little sequence similarity (Fig. S1), which further extends to the details of the overall charge distribution and structural characteristics of the surfaces of both proteins, even in the area surrounding the two AA-boxes [1]. This divergence of sequence and structure in regions that are directly involved in an apparently highly conserved interaction with the elF2 $\beta$-NTT might be surprising at first sight. However, an explanation is provided by the proposed mode of peptidedomain interaction between elF2 $\beta-N T T$ and elF2BE-CTD or elF5-CTD, respectively. For the elF2 $\beta$ NTT it was proposed that the absence of any obvious sequence conservation outside the K-boxes is due to the absence of any evolutionary constraint that would be imposed by the necessity to adopt a stable tertiary structure fold for interactions with elF5 or elF2B $\varepsilon$ (chapter 2). Similarly, the peptide-domain interaction would also release the peptide-binding sites on both proteins from the evolutionary constraint, which would be imposed by a structurally rigid domain as common 
interaction partner for both C-terminal domains. The intrinsically flexible elF2 $\beta$-NTT would not require an in sequence and structure highly conserved interface on both proteins, but instead allows relatively rapid divergence of its two interaction partners. Consistent with this proposal of rapid evolution for elF2BE-CTD and elF5-CTD, both proteins show a low degree of sequence similarity not only between each other, but also within each of the two lineages themselves.

\subsection{Materials and Methods}

\section{Cloning}

The DNA fragment corresponding to the open reading frame of elF2BE (encoded by the GCD6 gene in S. cerevisiae) or truncations thereof were amplified by PCR using yeast genomic DNA as template and a corresponding pair of primers. Cloning, mutagenesis and verification of the different plasmids used in this study were performed as described in chapter 2(page 69).

\section{Protein expression and purification}

Expression of the various constructs and the purification of elF2 $\beta$ and elF2 was performed as described in chapter 2 (pages 70-73).

Purification of elF2BE-CTD constructs: All C-terminal elF2BE constructs (residues 524-712), including those containing Bpa, were expressed as GST-fusion protein and purified according to the protocol for the elF5-CTD constructs described in chapter 2 (page 71).

Purification of full-length elF2BE: Full-length elF2B $\varepsilon$ was expressed without affinity tag. Cell lysis was carried out as described (chapter 2, page 70) in L-150 buffer (150 mM NaCl, 20 mM HEPES (pH 7.5), 5\% glycerol, $1 \mathrm{mM}$ EDTA, $4 \mathrm{mM} \beta$-mercaptoethanol) supplied with a mixture of protease inhibitors including aprotinin, leupeptin, pepstatin (ALP), and PMSF. The cleared lysate was applied to a $Q$ Sepharose (GE Healthcare) and bound nucleic acids and proteins, including elF2B $\varepsilon$, were eluted with a linear gradient into high salt buffer (1 M NaCl, 20 mM HEPES (pH 7.5), 5\% glycerol, 4 $\mathrm{mM} \beta$-mercaptoethanol). Fractions containing the target protein were pooled and ammonium sulfate added to a final concentration of $1 \mathrm{M}$. The sample was then loaded onto a phenylsepharose column (GE Healthcare) equilibrated in $200 \mathrm{mM} \mathrm{NaCl}, 1 \mathrm{M}\left(\mathrm{NH}_{4}\right)_{2} \mathrm{SO}_{4}, 20 \mathrm{mM} \mathrm{HEPES}(\mathrm{pH}$ 7), $5 \%$ glycerol, $4 \mathrm{mM} \beta$-mercaptoethanol. While the flow through contained mainly DNA or RNA, 
elF2B $\varepsilon$ remained bound to the column and could be eluted with a linear gradient in low salt buffer containing $50 \mathrm{mM}$ HEPES (pH 7.5), 5\% glycerol and $4 \mathrm{mM} \beta$-mercaptoethanol. Peak fractions containing elF2B $\varepsilon$ were pooled and applied to a Superdex S-200 equilibrated in GF-150 buffer. The concentrated protein was flash-frozen in liquid nitrogen and stored at $-80^{\circ} \mathrm{C}$.

\section{UV Crosslinking Experiments with Bpa and analysis by mass spectrometry}

Crosslinking experiments and the subsequent analysis of the formed crosslinks by massspectrometry were performed as described inchapter 2 (pages 76-78).

\section{Steady-State Fluorescence Measurements}

All experiments were carried out on a Fluoromax-3 spectrophotometer (Jobin Yvon Inc., USA) using a quartz cuvette (Hellma Analytics, Germany). All reactions were done at $20 \pm 1{ }^{\circ} \mathrm{C}$ in the standard reaction buffer (100 mM KCl, $20 \mathrm{mM}$ Tris/ $\mathrm{HCl}(\mathrm{pH} 7.5), 3 \mathrm{mM} \mathrm{MgCl} 2$ and $2 \mathrm{mM} \mathrm{DTT})$.

The binding of elF2 $\beta$ to elF2BE-CTD was monitored by tryptophan quenching experiments. Tryptophan fluorescence in elF2BE-CTD was excited at $280 \mathrm{~nm}$, resulting in an emission at $350 \mathrm{~nm}$, which was quenched upon the addition of elF2 $\beta$ (full-length and N-terminal domain fragments). Equilibrium dissociation constants $\left(K_{\mathrm{d}}\right)$ for the complexes were determined by titrating elF2B $\varepsilon$-CTD with increasing amounts of elF2 $\beta$, until saturation was reached. The residual fluorescence signal produced by elF2 $\beta$ (which contains no tryptophan) alone was determined by blank-titrations into buffer and subsequently subtracted from the binding data.

The binding of mant-labeled guanine nucleotides to elF2 was monitored either by direct excitation at a wavelength of $355 \mathrm{~nm}$ or by Foerster Resonance Energy Transfer (FRET) from tryptophan residues excited at $280 \mathrm{~nm}$ and an emission wavelength of $440 \mathrm{~nm}$. Fluorescence emission spectra were recorded after incubating the elF2 mant-nucleotide complexes for 5 min at $20{ }^{\circ} \mathrm{C}$. To estimate the equilibrium dissociation constants $\left(K_{\mathrm{d}}\right)$ for elF2-mant-nucleotide complexes in presence or absence of elF2B $\varepsilon$, the fluorescence of increasing amounts of mant-nucleotides was measured either in buffer alone or in the presence of $200 \mathrm{nM}$ of elF2 complex. The dilution was less than $1 \%$. To form elF2-elF2B $\varepsilon$ complexes, elF2B $\varepsilon$ was added to elF2 in a 20 -fold molar excess and incubated for $10 \mathrm{~min}$ at $20^{\circ} \mathrm{C}$ prior to the experiment. 
Titrations of elF2 -mant-GDP with elF2B $\varepsilon$ were carried out by adding increasing amounts of the GEF to $1 \mathrm{ml}$ of $100 \mathrm{nM}$ eIF2-mant-GDP. The solution was carefully mixed and the fluorescence signal at $440 \mathrm{~nm}$ (after excitation at $355 \mathrm{~nm}$ ) measured until a stable value was obtained. At the end of the titration a 200-fold excess of unlabeled GDP was added to determine the contribution of the unbound mant-GDP to the observed fluorescence signal.

Assuming a one-site binding model for all studies reactions, the data obtained from equilibrium titrations were analyzed according to a quadratic binding model:

$$
F=F_{0}+\Delta F_{\max } \frac{\left(X+Y+K_{\mathrm{d}}\right)-\sqrt{\left(X+Y+K_{\mathrm{d}}\right)^{2}-4 X Y}}{2 X}
$$

where $F$ is the fluorescence signal of the mant-nucleotide in the absence or presence of elF2, $F_{0}$ is the initial fluorescence signal, $\Delta F_{\max }$ is the maximum fluorescence signal, $X$ is the total concentration of elF2, $Y$ is the total concentration of the added mant-nucleotide and $K_{d}$ is the equilibrium dissociation constant. For the determination of the apparent equilibrium dissociation constants of elF2-mant-GDP to elF2Br, the above equation was modified as follows:

$$
k_{\mathrm{app}}=k_{\mathrm{a}}+\left(k_{\mathrm{b}}-k_{a}\right) \frac{\left(X+Z+K_{1 / 2}\right)-\sqrt{\left(X+Z+K_{1 / 2}\right)^{2}-4 X Z}}{2 X}
$$

Here, $k_{\mathrm{app}}$ is the characteristic apparent rate constant of the mant-nucleotide from elF2 in the presence of a specific concentration of elF2B $\varepsilon$ (see below), $Z$ is the concentration of elF2B $\varepsilon, k_{\mathrm{a}}$ is the dissociation rate constant of mant-GDP from elF2 alone, $k_{\mathrm{b}}$ is the dissociation rate constant of mant-GDP from elF2 in the presence of saturating concentrations of elF2B $\varepsilon$ and $K_{1 / 2}$ is the concentration of elF2B $\varepsilon$ at which their respective half maximal effect on the dissociation rate of mant-GDP from elF2 is reached (the apparent equilibrium dissociation constant). Data evaluation was done with the SigmaPlot software.

\section{Pre-Steady-State Fluorescence Measurements}

Pre-Steady-State experiments were carried out on a Fluoromax-3 spectrophotometer using a quartz cuvette with stirring magnet to allow fast mixing of the reacting components. All reactions were done at $20 \pm 1{ }^{\circ} \mathrm{C}$ in standard reaction buffer. The fluorescence of mant-nucleotides was 
excited either directly at $355 \mathrm{~nm}$ or via FRET from tryptophan residues excited at $280 \mathrm{~nm}$ and monitored at an emission wavelength of $440 \mathrm{~nm}$.

To study the dissociation of nucleotides, $50 \mathrm{nM}$ elF2-mant-GDP or elF2-mant-GTP complex was formed by adding $100 \mathrm{nM}$ of the respective mant-nucleotide to $50 \mathrm{nM}$ nucleotide-free elF2, followed by an incubation for $10 \mathrm{~min}$ at $20^{\circ} \mathrm{C}$. To start the reaction, unlabeled GDP was added in excess and the decrease in mant-fluorescence was monitored over time. The data were evaluated by fitting to a single exponential function with the equation:

$$
F(t)=F_{\mathrm{o}}+A e^{-k_{\mathrm{app}} t}
$$

where $F(t)$ is the fluorescence at time $t, F_{o}$ is the final fluorescence signal, $k_{\text {app }}$ is a characteristic apparent time constant and $A$ is the amplitude. The data evaluation was done with the SigmaPlot software.

\section{Interaction studies using analytical size exclusion chromatography}

Complex formation between nucleotide-bound elF2 and elF2B $\varepsilon$ was studied by size exclusion chromatography on an analytical Superdex S-200 (10/300) column (GE Healthcare). For runs without elF2BE, $50 \mu \mathrm{g}$ elF2 complex was incubated for $5 \mathrm{~min}$ at $20{ }^{\circ} \mathrm{C}$ with $1 \mathrm{mM}$ guanine nucleotide (GDP or mant-GDP) in a total volume of $300 \mu$ l reaction buffer $(100 \mathrm{mM} \mathrm{KCl,} 20 \mathrm{mM}$ Tris/ $\mathrm{HCl}(\mathrm{pH}$ 7.5), $3 \mathrm{mM} \mathrm{MgCl}$ and $2 \mathrm{mM} \mathrm{DTT}$ ). To study complex formation between nucleotidebound elF2 and elF2B $\varepsilon, 50 \mu \mathrm{g}$ of preincubated elF2-nucleotide complexes were incubated for an additional $5 \mathrm{~min}$ at $20^{\circ} \mathrm{C}$ in the presence of $60 \mu \mathrm{g}$ of full-length elF2B $\varepsilon$ resulting in a $\sim 2$-fold molar excess of the GEF over elF2. The samples were then loaded onto the column, equilibrated in reaction buffer at $20{ }^{\circ} \mathrm{C}$. The run was monitored at absorption wavelengths of 280,254 and 355 $\mathrm{nm}$. 


\subsection{Supplementary information}
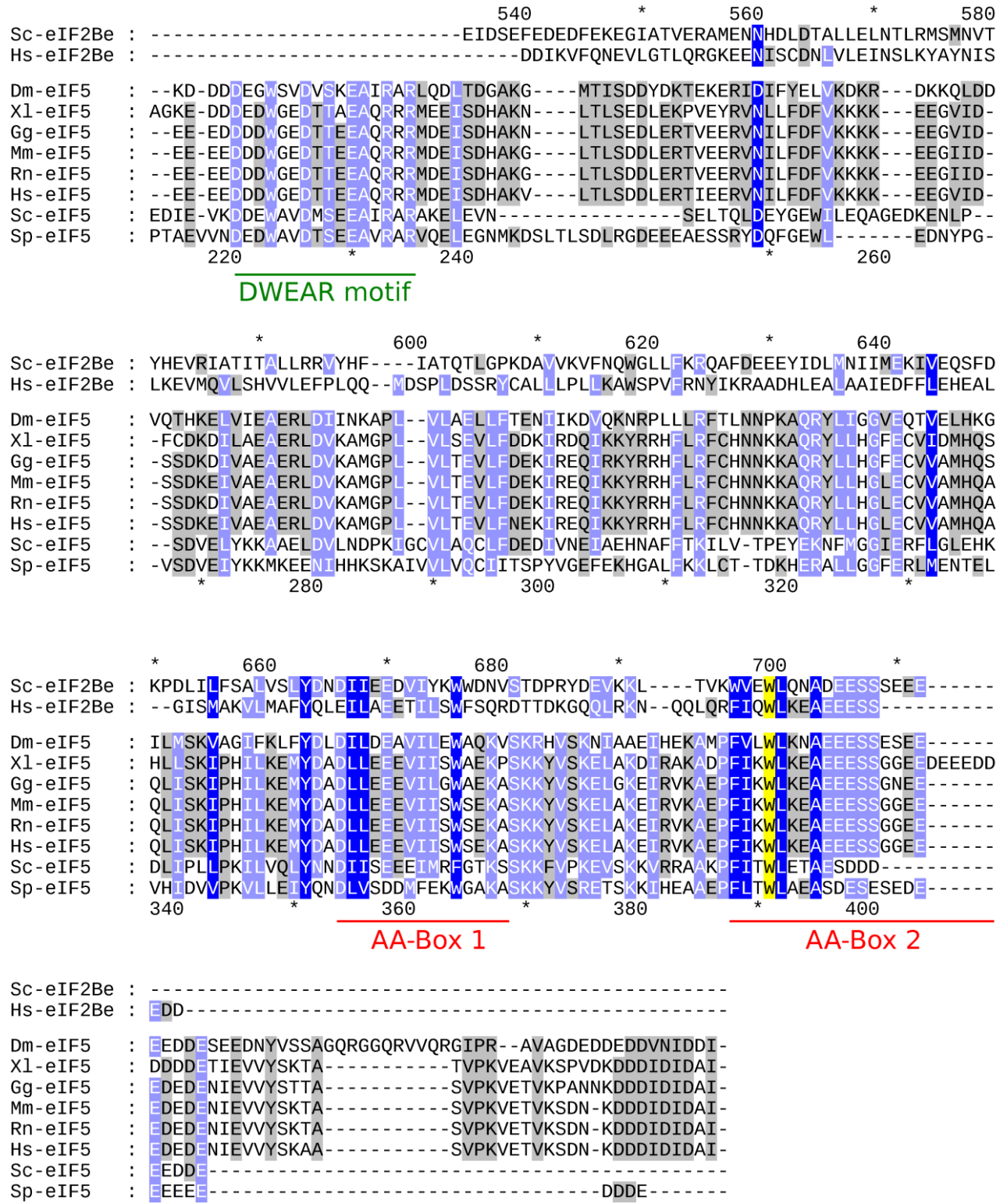

Figure S1. Multiple sequence alignment of the eIF5-CTD with elF2B $\varepsilon$-CTD.Residues conserved between both proteins are highlighted in dark blue, conserved residues in light blue and variable residues in grey; the universally conserved Trp391 (elF5) and Trp699 (elF2BE) in AA-box 2 is highlighted in yellow. The numbering above the alignment

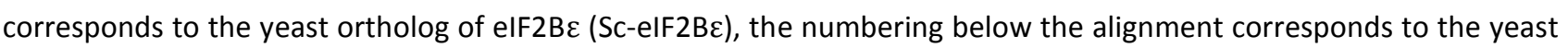
ortholog of elF5 (Sc-eIF5). The DWEAR motif and AA-boxes 1 \& 2 are indicated below the alignment. Sc, Saccharomyces cerevisiae; Hs, Homo sapiens; Dm, Drosophila melanogaster; XI, Xenopous laevis; Gg, Gallus gallus; Mm, Mus musculus; $\mathrm{Rn}$, Rattus norvegicus; Sp, Schizzosaccharomyces pombe. The alignment was initially generated using the clustal omega algorithm and subsequently corrected manually according to the structural alignment between the yeast orthologs of elF5-CTD and elF2BE-CTD (PDB: 1PAQ). 


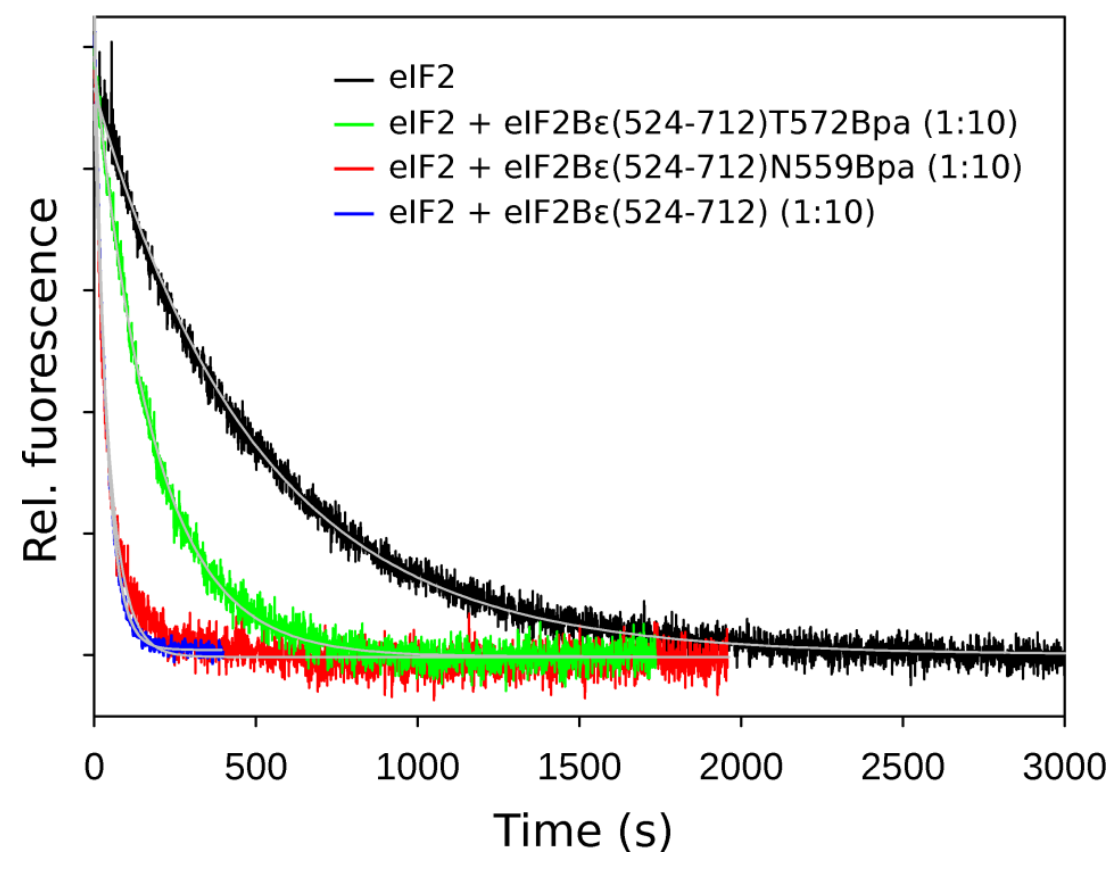

Figure S2. Influence of Bpa mutations on the guanine-nucleotide exchange activity of elF2B $\boldsymbol{\varepsilon}$-CTD. Time courses for the dissociation of mant-GDP from elF2 (50 nM elF2 with $100 \mathrm{nM}$ mant-GDP and $3 \mu \mathrm{M}$ unlabeled GDP) in the absence of elF2B $\varepsilon$ (black) and in the presence of 500 nMelF2Be(524-712)T572Bpa (green), elF2Be(524-712)N559Bpa or elF2B $\varepsilon(524-712)$. The smooth black lines represent single-exponential fits which yielded the apparent rate constants $\left(k_{\text {app }}\right.$ ) of $0.135 \mathrm{~min}^{-1}$ for elF2 alone and $0.77 \mathrm{~min}^{-1}, 1.8 \mathrm{~min}^{-1}$, and $2.3 \mathrm{~min}^{-1}$ for elF2 with elF2Bع(524-712) T572Bpa, N559Bpa or wt, respectively. 


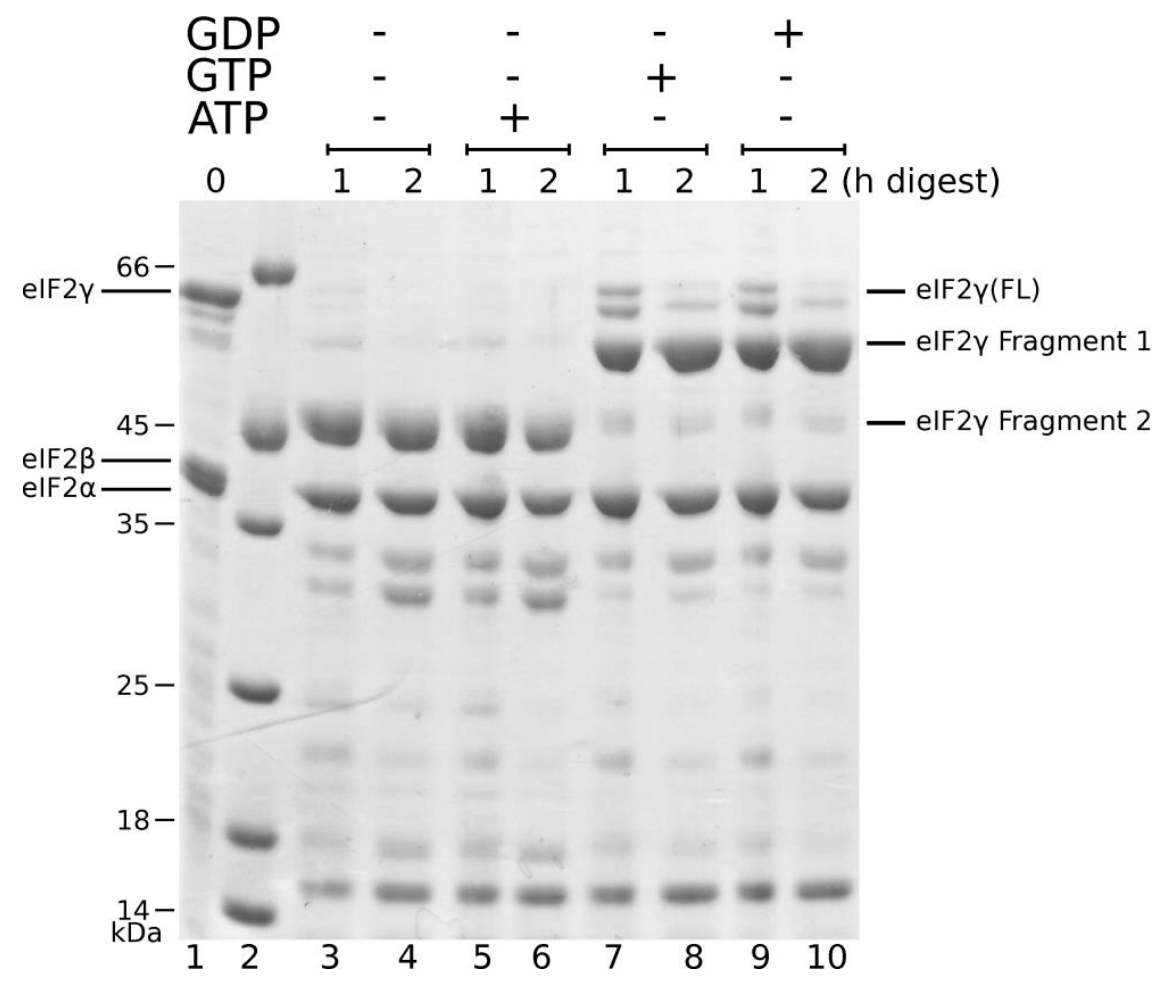

Figure S3. Influence of GDP and GTP on the limited proteolysis of elF2 by thermolysine. Depending on the presence or absence of GDP and GTP (20-fold excess over elF2), full-length elF2 $\gamma$ (lane 1; running at $\sim 65 \mathrm{kDa}$ ) is cleaved either into a fragment running at $\sim 55 \mathrm{kDa}$ (fragment 1) or a fragment running at $\sim 46 \mathrm{kDa}$ (fragment 2). According to MSanalysis, these fragments originate from a cleavage either in front of the $G$ domain (fragment 1 ) or within switch 1 (fragment 2). While ATP, like in apo elF2, is unable to protect switch 1 from proteolytic cleavage, GDP and GTP both stabilize switch 1 in a conformation that is not accessible to thermolysine. The cleavage reaction was performed for the indicated time (in hours) in $100 \mathrm{mM} \mathrm{KCl}, 20 \mathrm{mM}$ Tris (pH 7.5), $5 \%$ glycerol, $2.5 \mathrm{mM} \mathrm{MgCl}, 1 \mathrm{mM} \mathrm{DTT}$ at $20^{\circ} \mathrm{C}$ and in the presence of thermolysine at a ratio of 1:10,000 $(\mathrm{w} / \mathrm{w})$ relative to elF2. 


\section{Chapter $4 \cdot$ Structural analysis of eIF $2 B \beta$ and eIF2B $\delta$}

This manuscript is in preparation for submission

Crystal structures of eIF2B $\beta$ and eIF2B $\delta$ provide new insights into the regulatory subcomplex of eIF2B, the guanine nucleotide exchange factor for eIF2

Bernhard Kuhle ${ }^{1, \S}$ and Ralf Ficner ${ }^{1}$

${ }^{1}$ Abteilung für Molekulare Strukturbiologie, Institut für Mikrobiologie und Genetik, Göttinger Zentrum für Molekulare Biowissenschaften, Georg-August-Universität Göttingen, D-37077

Göttingen, Germany

\$To whom correspondence should be addressed: bkuhle@gwdg.de

Author contributions:

BK: designed the study and conceived the experiments; performed cloning, protein purification, crystallization, data collection and structure determination; analyzed and interpreted the data; wrote the manuscript. RF: design of the study; helped to draft the manuscript. 


\section{Abstract}

The eukaryal translation initiation factor 2B (eIF2B) acts as guanine nucleotide exchange factor (GEF) for elF2 and forms a central target for pathways regulating translation initiation. elF2B consists of five nonidentical subunits $(\alpha-\varepsilon)$, which assemble into a catalytic subcomplex ( $\gamma$ and $\varepsilon$ ) that catalyzes nucleotide exchange on elF2 and a regulatory subcomplex $(\alpha, \beta$ and $\delta)$ that regulates elF2B function under stress conditions by tight binding of elF2. Here, we provide new structural insight into the regulatory subcomplex of elF2B from the fungus Chaetomium thermophilum, obtained by its in vitro reconstitution from the individually purified subunits and by solving the first high-resolution crystal structures of elF2B $\beta$ and elF2B $\delta$. These structures confirm the proposed structural homology between the three regulatory subunits, but at the same time reveal critical differences that may be relevant for the assembly of the elF2B $\alpha \beta \delta$ complex. Based on the presented findings, we propose a structural model for the elF2B regulatory subcomplex, which constitutes a vantage point for further structural and functional studies on elF2B.

\subsection{Introduction}

The initiation of ribosomal protein synthesis in eukarya is a highly regulated process, which requires the concerted action of at least twelve different auxiliary protein factors, called eukaryal translation initiation factors (elFs) [12]. One of the central hubs for translational regulation during the initiation phase is the eukaryal translation initiation factor 2B (elF2B), which functions as guanine nucleotide exchange factor (GEF) for elF2 to regenerate the latter in its GTP-bound form $[80,112]$.

The translation initiation cycle in eukarya starts when GTP-bound elF2 specifically forms a ternary complex (TC) with methionylated initiator tRNA (Met-tRNA ${ }_{i}$ ) and, supported by other elFs, delivers it to the P-site of the $40 \mathrm{~S}$ ribosomal subunit to form the $43 \mathrm{~S}$ pre-initiation complex (preIC). The $43 \mathrm{~S}$ pre-IC is subsequently recruited to the $5^{\prime}$-end of an mRNA to be translated and enters the scanning phase of translation initiation, during which it searches the $5^{\prime}$-untranslated region (5'UTR) of the mRNA for the start site of translation. Upon recognition of the correct AUG start codon, elF2 hydrolyzes the bound GTP and dissociates from the pre-IC in its GDP-bound form. For a new round of translation initiation elF2 has to be recycled back into its 'active' GTP-bound form. 
Due to the high affinity of the eIF2.GDP complex and the low rate of spontaneous GDP release, the exchange of GDP for GTP on elF2 depends on the catalytic activity of the GEF elF2B [12, 112].

Consistent with this central position in the guanine-nucleotide cycle of elF2, elF2B is one of the main targets for global regulation of translation in eukarya [132]. The best characterized mechanism to control elF2B activity is the phosphorylation of serine 51 (S51-P) in the $\alpha$-subunit of elF2 (elF2 $\alpha)$ by kinases in response to stress or starvation conditions [78-80]. elF2 $\alpha$ phosphorylation results in a significantly increased affinity of elF2( $\alpha-P)$ for elF2B, thereby converting elF2 from a substrate into a competitive inhibitor of its GEF, and giving rise to the formation of non-productive elF2( $\alpha$-P)·elF2B complexes $[78,112,123,124]$. As a consequence, inhibition of elF2B function reduces the cellular levels of TCs that are available for translation initiation, thus causing the down-regulation of overall protein synthesis. However, at the same time, elF $2 \alpha$ phosphorylation causes the up-regulation of certain mRNAs via a mechanism called translation re-initiation through upstream open reading frames (uORFs) in the 5'-leader region. One well studied example for such an mRNA is that for the transcription factor GCN4 in the yeast Saccharomyces cerevisiae, whose expression is up-regulated due to elF $2 \alpha$ phosphorylation by the kinase GCN2 in response to amino-acid starvation, and which in turn stimulates the expression of enzymes involved in amino-acid synthesis $[79,80]$.

Compared to the GEFs of most other Ras-related G proteins, elF2B is an unusually large and complex exchange factor, comprising five non-identical subunits $(\alpha, \beta, \gamma, \delta$, and $\varepsilon)$ which form two subcomplexes that bind elF2 independently from each other [112].elF2B $\gamma$ and elF2B $\varepsilon$ together form the catalytic subcomplex, in which the C-terminal domain (CTD) of the $\varepsilon$-subunit constitutes the minimal catalytically active fragment $[65,74,112]$. The $\mathrm{N}$-terminal regions of elF2B $\varepsilon$ exhibit sequence similarity to elF2B $\mathrm{B}$ and are most likely involved in their mutual interaction [133, 134]. elF2B $\alpha,-\beta$, and $-\delta$ form the regulatory subcomplex of elF2B $[112,135]$, in which all three subunits share mutual sequence similarity over the entire length of elF2B $\alpha$ [136]. While the disruption of the genes for elF2B $\beta-\varepsilon$ is lethal in mammals and yeast, elF2B $\alpha$ is not essential [137]. However, elF2B $\alpha$ was found to be critical for the sensitivity of the elF2B regulatory subcomplex to elF $2 \alpha$ phosphorylation and thus de-repression of GCN4 expression in yeast. The essential roles for elF2B $\beta$ and $-\delta$ within the elF2B complex are still not entirely clear. Apart from their putative structural 
roles in complex assembly, elF2B $\beta$ was found to promote elF 2 binding and to contribute to the efficiency in nucleotide exchange, while elF2B $\delta$ was proposed to play a role in Met-tRNA $A_{i}$ recruitment to elF2.GTP [77].

For all five elF2B subunits, a number of mutations have been identified that affect the activity of the elF2B complex. Here, two main phenotypes can be distinguished in S. cerevisiae. On the one hand, mutations that decrease the GEF activity of eIF2B and thereby lower the cellular TC concentration, resulting in GCN4 derepression independently of elF $2 \alpha$ phosphorylation and aminoacid starvation. This phenotype is called general control de-repression (Gcd) and has been found for mutations in all five elF2B subunits. On the other hand, mutations that prevent the expression of GCN4 even under stress conditions by rendering elF2B insensitive to elF $2 \alpha$ phosphorylation are called general control non-derepressible $\left(\mathrm{GCn}^{-}\right)$. Such a phenotype was found for mutations in the three subunits of the regulatory subcomplex, which is thought to be indicative of a large interface for elF $2 \alpha$ binding on the elF2B $\alpha \beta \delta$ complex $[80,135]$.

Despite considerable efforts over the last decades to understand elF2B function, the architecture of the eIF2B complex remains elusive, thereby hampering the interpretation of available biochemical and genetic observations at the molecular level. This is exemplified by the lack of high-resolution structural insight into the elF2B complex, which is limited to the isolated structures of the C-terminal catalytic domain of elF2B from yeast and human and the human elF2B $\alpha$ subunit. Moreover, until recently it was thought that elF2B forms a hetero-pentamer containing one copy of each of the five subunits. However, recent studies indicate that elF2B in yeast and mammals in fact forms a functional decamer in solution that is composed of two elF2B $\alpha \beta \delta \gamma \varepsilon$ hetero-pentamers. The way in which the contact between the two pentamers is mediated is still not clear, as the available data contradict each other in this respect, suggesting that decamerization is either mediated by an elF $2 B \alpha_{2}$ dimer $[72,138]$ or an $\operatorname{elF} 2 \mathrm{~B}(\gamma \varepsilon)_{2}$ tetramer [73]. Similarly, the proposed arrangement of the individual subunits differs considerably between the studies, illustrating the problem to provide a clear picture of the elF2B complex and its putative interactions with the substrate elF 2 on the basis of the available data.

The aim of this study was to provide structural insight into the molecular architecture of the elF2B regulatory subcomplex. We were able to purify the three orthologs of elF2B $\alpha$, elF2B $\beta$ and elF2B $\delta$ 
from the thermophilic fungus Chaetomium thermophilum, which allowed us to reconstitute the elF2B regulatory subcomplex in vitro. Analytical size exclusion chromatography experiments suggest that this subcomplex exists as an elF $2 \mathrm{~B} \alpha_{2}(\beta \delta)_{2}$ hexamer in solution, in which an elF2B $\alpha_{2}$ dimer most likely mediates the dimerization of two elF2B $\alpha \beta \delta$ heterotrimers. Moreover, we solved the first high-resolution crystal structures of elF2B $\beta$ and $\operatorname{elF} 2 \mathrm{~B} \delta$, which demonstrate their structural homology to each other and to elF2B $\alpha$. Using the presented results in combination with previous biochemical and genetic data, we generate a structural model for the elF2B regulatory subcomplex. The possibility to in vitro reconstitute the elF2B regulatory subcomplex in a pure form and in large amounts, as well as the availability of structural models for elF $2 B \alpha,-\beta$, and $-\delta$ provides a promising basis for future structural and functional studies on the elF2B complex.

\subsection{Results}

\section{Overall structure of elF2B $\beta(\Delta 123-148)$}

Initial trials to crystallize wild-type elF2B $\beta$ from $C$. thermophilum were unsuccessful. We therefore designed a construct lacking residues 123-148 (elF2B $\beta(\Delta 123-148)$ ), which, according to secondary structure predictions and sequence comparison with orthologs from other species, form a nonconserved unstructured loop region between two conserved $\alpha$-helical regions. Unlike the wild-type protein, elF2B $\beta(\Delta 123-148)$ readily crystallized in a condition containing $100 \mathrm{mM}$ HEPES $(\mathrm{pH}$ 6.8) and 1.33 $\mathrm{M}$ tri-sodium citrate. The structure of $C$. thermophilumelF2B $\beta(\Delta 123-148)$ was solved by molecular replacement and refined at a resolution of $2.54 \AA$ (Table 1). The final model contains two elF2B $\beta(\Delta 123-148)$ molecules (molecules $\mathrm{A}$ and $\mathrm{B}$ ) per asymmetric unit, consisting of an $\mathrm{N}$-terminal $\alpha$-helical domain (residues 10-202) and a C-terminal Rossmann-fold-like domain (residues 203-419) (throughout the manuscript we will follow the residue-count for wild-type elF2B $\beta$, ignoring the internal deletion) (Fig. 1). The $\mathrm{N}$-terminal domain folds into six $\alpha$-helices, with helices $\alpha 1,2,4,5$, and 6 assembled around helix $\alpha 3$ through hydrophobic interactions. Residues 1-9 are not resolved in the electron density and are therefore considered to be disordered. Likewise, residues 106-180 are not resolved in molecule B of the asymmetric unit. In molecule A residues 106-112 and 160-174 are not resolved, whereas the region comprising residues 113-122 and 149-159 (in which residues 122 and 149 are directly fused by a peptide-bond) adopt two additional $\alpha$-helices ( $\alpha 5^{\prime}$ and $\alpha 5^{\prime \prime}$ ) 
Table 1. Crystallization, X-ray data collection and refinement statistics for structures of Chaetomium thermophilum elF2B subunits $\beta$ and $\delta$

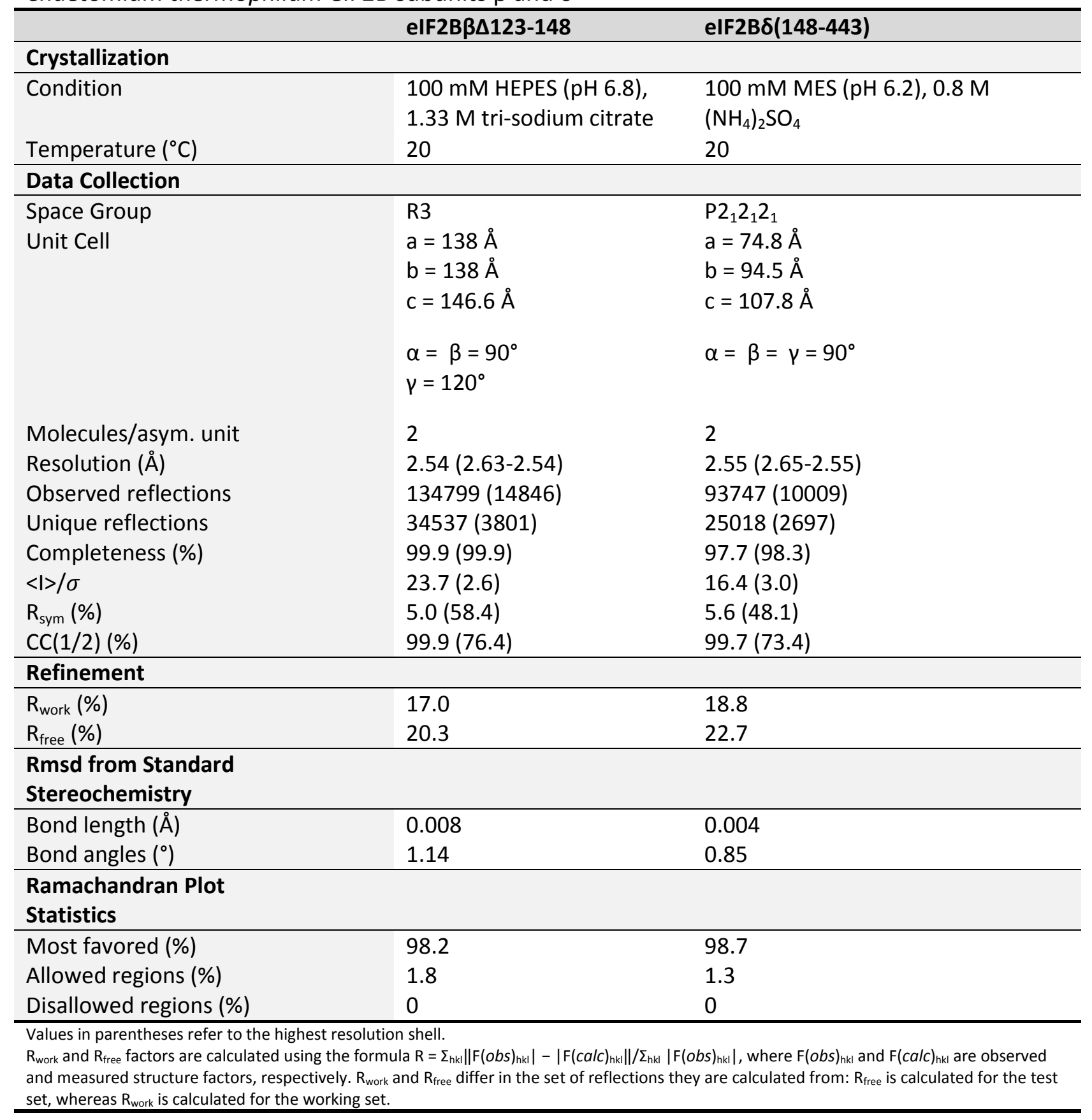

that form contacts to symmetry related molecules in the crystal packing. The C-terminal Rossmann-fold-like domain is composed of a seven-stranded $\beta$-sheet with the first six $\beta$-strands parallel and the seventh oriented antiparallel to the rest. The $\beta$-sheet is sandwiched between six $\alpha$ helices, three on each side. A second three-bladed antiparallel $\beta$-sheet (strands $\beta 5, \beta 6$, and $\beta 8$ ) is 
packed against strands $\beta 4, \beta 7$, and $\beta 9$ of the first $\beta$-sheet, with the strands of the two sheets lying orthogonal to each other. An additional $\alpha$-helix ( $\alpha 13)$ forms the C-terminal end of elF2B $\beta$. Two loop regions comprising residues 253-258 and 347-373 (denoted the 'arm-region'), respectively, are flexible and mainly disordered in both C-terminal domains of the asymmetric unit.
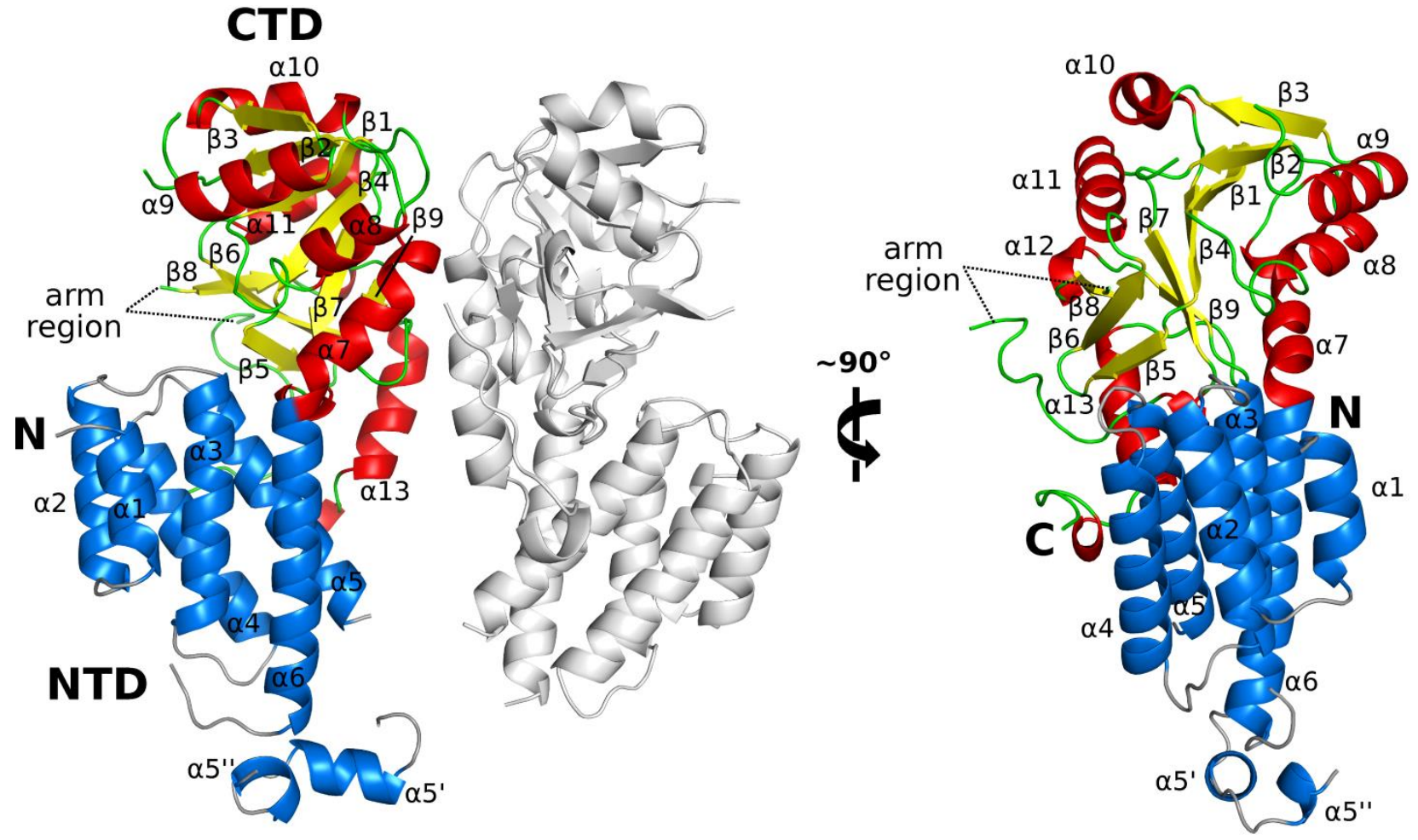

Figure 1. Overall crystal structure of $C$. thermophilumelF2B $\beta(\Delta \mathbf{1 2 3}-148)$.elF2B $\beta$ is shown in cartoon presentation with the N-terminal $\alpha$-helical domain (residues 10-202) colored blue, and the helices and $\beta$-sheets of the C-terminal Rossmann-fold-like domain (residues 203-419) are colored red and yellow, respectively. The arm region is only partially resolved in the electron density. In the left panel, the grey colored molecule represents the second copy of elF2B $\beta$ in the asymmetric unit, which is omitted in the right panel. Helix $\alpha 5^{\prime}$, which is stabilized through contacts to symmetry related molecules in the crystal packing, contains the fusion between residues 122 and 149.

The two monomers of the asymmetric unit are arranged back-to-back to each other (Fig. 1), with the mainly hydrophobic dimer-interface formed solely by the backside of the C-terminal domains, involving helices $\alpha 7$ and $\alpha 12$ and strand $\beta 9$ of the large $\beta$-sheet of each monomer. The individual $\mathrm{N}$ - and C-terminal domains of the two molecules can be superimposed well with rmsd values of $0.23 \AA$ for $98 \mathrm{C}_{\alpha}$ atoms of the $\mathrm{N}$-terminal domain and $0.28 \AA$ for $145 \mathrm{C}_{\alpha}$ atoms of the C- 
terminal domain. The overall proteins differ more significantly ( $r$ rmsd of $0.59 \AA$ over $270 C_{\alpha}$ atoms) due to different angles between $\mathrm{N}$ - and $\mathrm{C}$-terminal domains, as indicated by a slight increase in the angle between the connecting helices $\alpha 6$ and $\alpha 7$ from $\sim 40^{\circ}$ in molecule $A$ to $\sim 45^{\circ}$ in molecule $B$ (Fig. 3B). In both molecules, this conformation is stabilized through hydrophobic, as well as ionic interactions between helices $\alpha 5$ and $\alpha 6$ in the NTD and helix $\alpha 13$ in the CTD. As a consequence, the $\mathrm{N}$ - and C-terminal domains together form a deep cleft at the front of the molecule, with a mainly negatively charged platform formed by the connecting $\alpha 2-\alpha 3$ and $\alpha 4-\alpha 5$ loops in the NTD and a mainly positively charged lid formed by the CTD (Fig 3E).

\section{Overall structure of elF2B $\delta(148-443)$}

During purification, elF2B $\delta$ usually degraded into two fragments of $\sim 35 \mathrm{kDa}$ and $\sim 20 \mathrm{kDa}$ according to SDS-PAGE analysis (see Materials and Methods for details). Crystallization trials were performed for the full-length protein, as well as for the $35 \mathrm{kDa}$ fragment. Only the latter yielded high quality crystals that could be used for data collection and allowed the determination of the crystal structure of $C$. thermophilumelF2B $\delta(148-443$ ) by molecular replacement (Table 1). The final model, refined at $2.55 \AA$ resolution, contains two elF2B $8(148-443)$ molecules (molecules A and B) per asymmetric unit, which superimpose well with each other with an rmsd of $0.2 \AA$ over $239 \mathrm{C}_{\alpha}$ atoms (Fig. 2). Each monomer is formed by an N-terminal $\alpha$-helical domain (residues 148-266) and a C-terminal Rossmann-fold-like domain (residues 267-443). The $\mathrm{N}$-terminal domain folds into five $\alpha$-helices that lie nearly parallel to each other, assembled around a hydrophobic core. The $\mathrm{N}$ terminal 147 residues are not resolved in the electron density. As the theoretical masses of this fragment and that of the crystallized protein correspond well to the two degradation fragments observed during purification, we assume that the $\mathrm{N}$-terminal residues were lost during purification due to a proteolytic event, which would also be consistent with the low R-factors of 18.8 and 22.7\% (Table 1). The C-terminal Rossmann-fold-like domain is composed of a six-stranded parallel $\beta$-sheet ( $\beta 1-\beta 4, \beta 7$ and $\beta 9)$, sandwiched between three $\alpha$-helices on each side. A second threebladed antiparallel $\beta$-sheet (strands $\beta 5, \beta 6$, and $\beta 8$ ) is packed against strands $\beta 4, \beta 7$, and $\beta 9$ of the first $\beta$-sheet, with the strands of the two sheets lying orthogonal to each other. The long loop region ('arm-region') comprising residues $402-420$ is disordered in both molecules of the 
asymmetric unit. Likewise, the C-terminal 23 residues are not resolved in the electron density, indicating that they are either flexible or were lost during purification.

Together with helix $\alpha 2$, helices $\alpha 5$ and $\alpha 6$ form the $\sim 3800 \AA^{2}$ large interface for homodimer formation between the two molecules of the asymmetric unit, with the helices of one monomer oriented nearly orthogonal to those of the other (Fig. 2, 5A). The most intimate contact is thereby formed at the kink-regions between helices $\alpha 5$ and $\alpha 6$ of the two protomers.

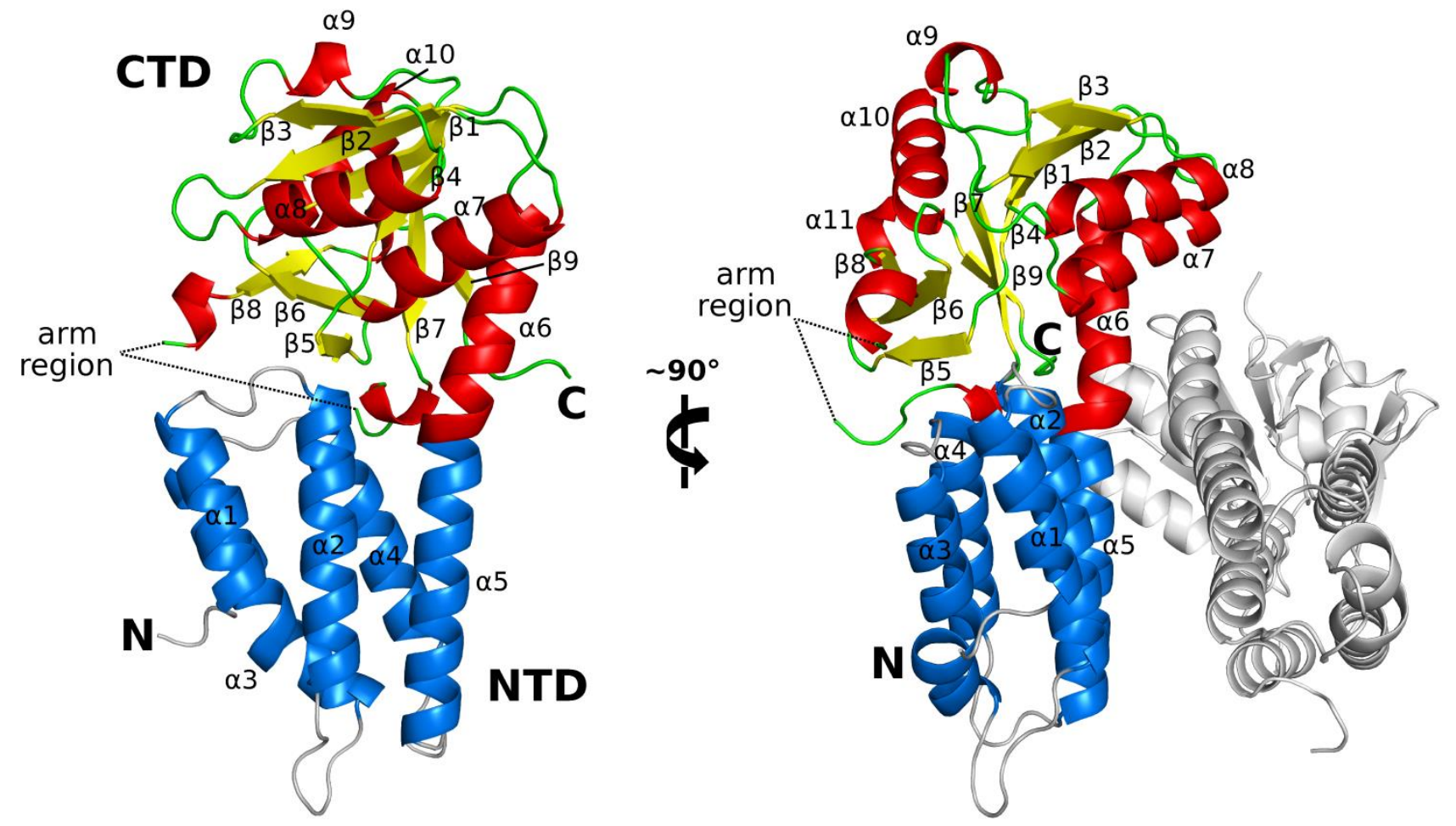

Figure 2. Overall crystal structure of $C$. thermophilumelF2B $\delta(\mathbf{1 4 8 - 4 4 3 ) . e l F 2 B \delta}$ is shown in cartoon presentation with the N-terminal $\alpha$-helical domain (residues 148-266) colored blue, and the helices and $\beta$-sheets of the $\mathrm{C}$-terminal Rossmann-fold-like domain (residues 267-443) colored red and yellow, respectively. The arm region is mainly disordered and therefore not resolved in the electron density. In the right panel, the grey colored molecule represents the second copy of elF2B $\delta$ in the asymmetric unit, which is omitted in the left panel. 


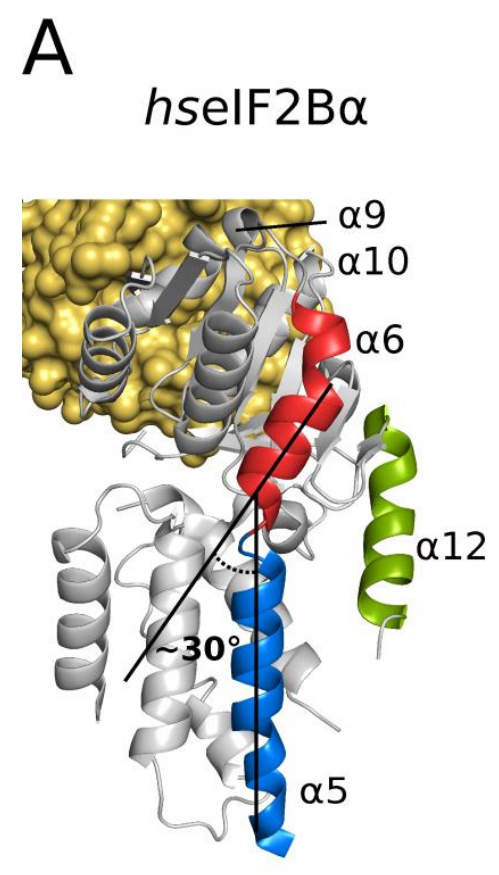

D

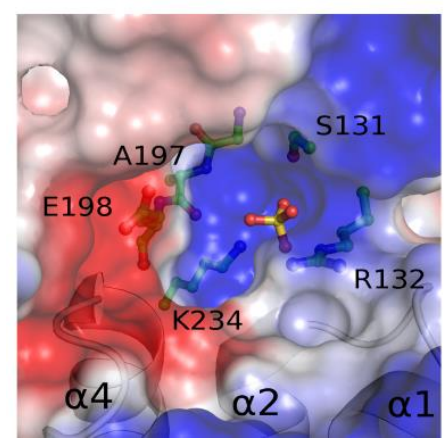

B

$$
\text { ctelF2B } \beta
$$
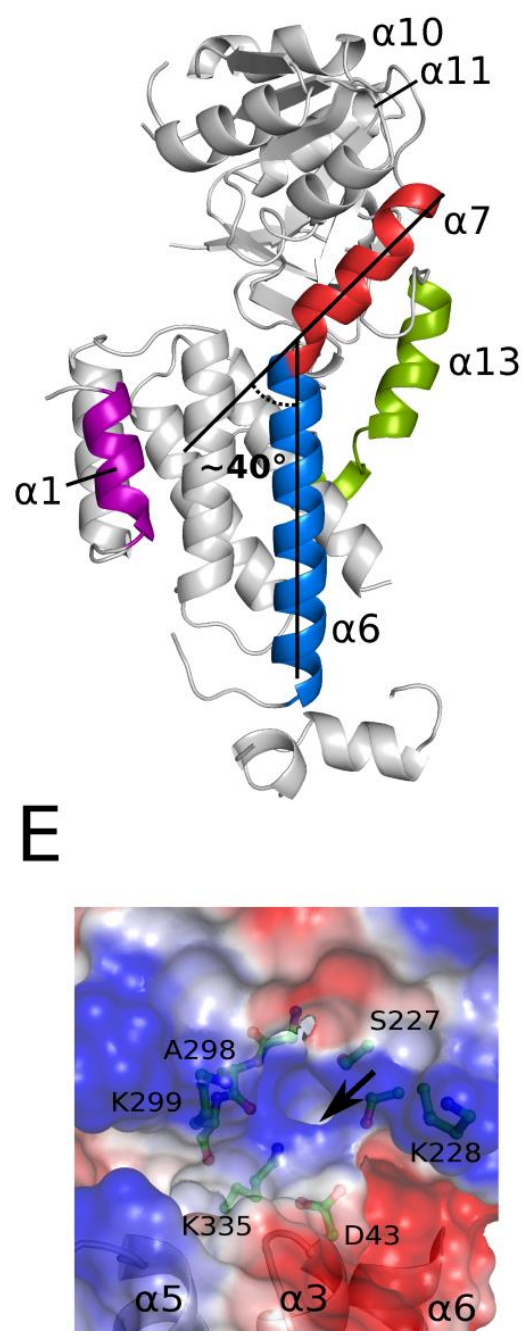

C

\section{ctelF2Bס}

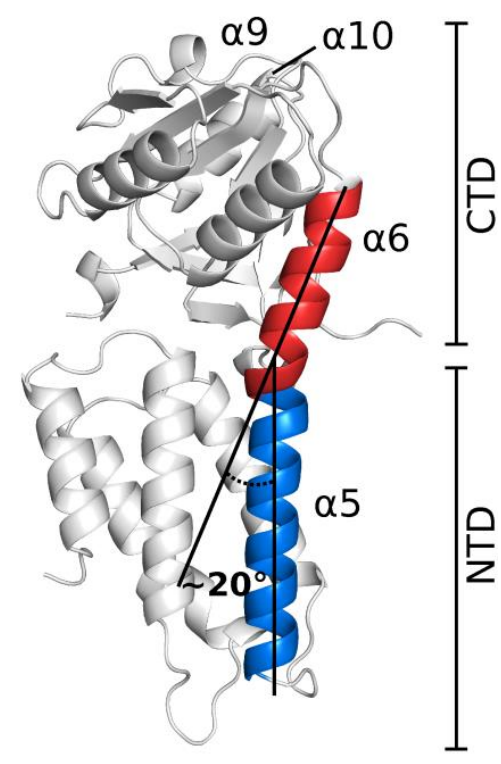

F

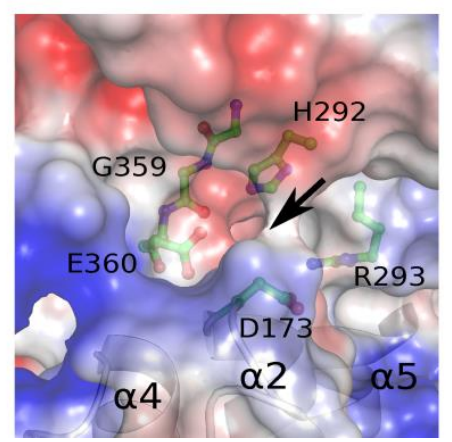

Figure 3. Comparison of the structures of human elF2B $\alpha$ with $C$. thermophilum elF2B $\beta$ and elF2B $\delta$. A-C) Compared to ctelF2B $\delta(C)$, hselF2B $\alpha$ (PDB: 3ECS) (A) and ctelF2B $\beta$ (B) exhibit significantly increased angles between the two long connecting helices between $\mathrm{N}$ - and C-terminal domains (colored blue and red, respectively), which might be dependent on the presence of the additional C-terminal $\alpha$-helix (green) that forms direct contacts to the NTD and which is absent in the ctelF2B $\delta$ structure. ctelF2B $\beta$ (B) exhibits an additional N-terminal $\alpha$-helix ( $\alpha 1$; purple), which is not found in $h s$ IF $2 \mathrm{~B} \alpha$ or ctelF2B $\delta$. Homodimer formation by $h s$ elF2B $\alpha$ through helices $\alpha 9$ and $\alpha 10$ of the C-terminal domain is indicated by the second $h s e l F 2 \mathrm{~B} \alpha$ molecule of the asymmetric unit as golden surface. D-E) Surface charge presentation (negative, red; positive, blue) of the cleft between $\mathrm{N}$ - and C-terminal domains in $h s$ elF2B $\alpha$ (D), ctelF2B $\beta$ (E) and ctelF2B $\delta(F)$. The crystal structure of hselF2B $\alpha$ accommodates a sulfate ion at the bottom of the cleft, surrounded by positively charged residues. Although some of these residues are conserved also in ctelF2B $\beta$ and ctelF2B $\delta$, both structures do not contain a sulfate ion in the corresponding position (arrows). 


\section{Structural comparison between the subunits of the elF2B regulatory subcomplex}

Earlier analyses revealed mutual sequence similarities between the three elF2B $\alpha, \beta$, and $\delta$ subunits [136]. Consistently, our structural analysis for elF2B $\beta$ and elF2B $\delta$ from $C$. thermophilum demonstrate that both subunits share a common overall fold, comprising an $\mathrm{N}$-terminal helix bundle and a C-terminal $\alpha / \beta$ Rossmann-like fold, which is, moreover, homologous to the previously reported structure of elF2B $\alpha$ from Homo sapiens[139].

Notwithstanding their overall structural similarity, each of the three structures reveals features that are either idiosyncratic among the three proteins, or shared with only one of the other subunits (Fig. 3). One prominent example is the first of the six $\alpha$-helices of the $\mathrm{N}$-terminal domain in subunit $\beta(\alpha 1)$, which replaces the four-stranded antiparallel $\beta$-sheet that usually occupies this position in 5-methylthioribulose 1-phosphate isomerases (5M1PIs), ribose-1,5bisphosphate isomerases (RBPIs) or archaeal elF2B-like proteins (e.g. PDBs 1T50, 1W2W), proteins which were found to be in sequence and structure homologous to elF $2 B \alpha,-\beta$ and $-\delta[28,139,140]$. Neither the additional $\alpha$-helix nor the $\beta$-sheet is observed in the elF2B $\alpha$ - or $\delta$-subunit. It should be noted, however, that the sequence conservation between elF2B $\beta$ orthologs is low in this region [136], suggesting that helix $\alpha 1$ might not be universally conserved.

Another distinctive feature is the relative orientation of the five conserved helices of the $\mathrm{N}$ terminal helix bundle (Fig. 3A-C). In elF2B $\beta$ and $-\delta$ all five $\alpha$-helices lie nearly parallel to each other. A similar arrangement is found in 5M1PIs, RBPIs and elF2B-like proteins. By contrast, helices $\alpha 1-\alpha 5$ in elF2B $\alpha$ are significantly less parallel to each other, particularly with helix $\alpha 1$ reoriented relative to the following helices by nearly $50^{\circ}$, thereby allowing a direct contact between the $\alpha 1-\alpha 2$ loop to helix $\alpha 7$ in the C-terminal domain that is not observed in the structurally related homologues [139].

In agreement with the relative high degree of sequence similarity, the C-terminal Rossmannlike domain of the elF2B subunits exhibits a higher degree of structural homology and only minor differences in the arrangement of helices $\alpha 8$ and $\alpha 9$ ( $\alpha 9$ and $\alpha 10$ in ctelF2B $\beta$ ). One of the most prominent distinctive features within the C-terminal domain is the C-terminal $\alpha$-helix found in the structures of elF2B $\alpha$ (helix $\alpha 12$ ) and elF2B $\beta$ (helix $\alpha 13$ ) (Fig. 3A/B). This $\alpha$-helix is not present in elF2B (Fig. $3 C$ ), despite the fact that it contains conserved residues at its C-terminus that would correspond to this $\alpha$-helix in the other two subunits [136], and despite the fact that the crystal 
packing would allow its formation in the same position. In elF2B $\alpha$ and $-\beta$, this helix forms mainly hydrophobic contacts to the $\mathrm{N}$-terminal domain, thereby stabilizing one of the most salient features of both proteins: the strong kink in the transition of helix $\alpha 5$ of the NTD to helix $\alpha 6$ of the CTD (helices $\alpha 6$ and $\alpha 7$ in elF2B $\beta$ ). The angle between the axes through both helices is $\sim 30^{\circ}$ in elF2B $\alpha$ and $40-45^{\circ}$ in elF2B $\beta$. By contrast, these helices are nearly coaxially fused in elF2B $\delta$ with an angle of close to $20^{\circ}$, suggesting that the contact to the C-terminal $\alpha$-helix observed in the other two subunits is a requirement for the conformational rearrangement to the kinked state (Fig. 3AC). This is supported by the structures of $5 \mathrm{M} 1 \mathrm{PI} / \mathrm{elF} 2 \mathrm{~B}$-like structures, which usually lack a structural element corresponding to the $C$-terminal helix in elF2B $\alpha$ and $-\beta$, resulting in nearly straight connecting helices. An exception from this rule is the RBPI from Thermococcus kodakarensis, which contains a homologous C-terminal $\alpha$-helix that forms contacts to the $\mathrm{N}$ terminal domain as observed in elF2B $\alpha$ and $-\beta$ and thereby stabilizes an angle of $\sim 50^{\circ}$ between helices $\alpha 5$ and $\alpha 6$ (PDB: 3A11)[141].

As a consequence of the increased angle, the cleft formed by the $\mathrm{N}$ - and $\mathrm{C}$-terminal domains is considerably larger in elF $2 B \alpha$ and elF2B $\beta$ than in the $\delta$-subunit, which in the case of elF2B $\alpha$ allows the coordination of a sulfate ion in a pocket of the cleft $\sim 16 \AA$ from its entrance [139] (Fig. 3D). Sulfate ions have also been observed in the narrower clefts of several 5M1PI/elF2B-like proteins. However, in these cases the sulfate ion is positioned significantly closer to the entrance and coordinated by a different set of residues [139]. Although some of the residues responsible for sulfate ion binding in elF2B $\alpha$ are conserved in subunits $\beta$ and $\delta$ [139], and despite the presence of $0.8 \mathrm{M}$ sulfate ions in the crystallization condition for elF2B (Table 1), neither of the two structures exhibited an electron density in the corresponding position that could be explained by a sulfate ion (Fig. 3E/F). This apparent inability of subunits $\beta$ and $\delta$ to coordinate the sulfate ion can be explained by the fact that elF2B $\beta$ and elF2B $\delta$ contain residues (Asp43 and His292, respectively) that would overlap with the putative sulfate ion binding site, causing electrostatic and/or steric repulsion (Fig. 3D-F).

Another important aspect of the newly determined structures is their implication for the oligomeric state of elF2B $\beta$ and elF2B $\delta$. So far studied eIF2B-like proteins, 5M1PIs, as well as RBPIs were found to form homodimers in solution $[138,139]$. All crystal structures of these proteins that 
have so far been determined revealed a structurally nearly identical dimer interface, in which two adjacent $\alpha$-helices of the $\mathrm{C}$-terminal domain in one monomer pack against the same helices of the other [138]. Additionally, a structurally conserved loop (the 'arm-region') protrudes from one CTD and packs against the other, thereby extending the large central $\beta$-sheet of the Rossmann-like fold. The same dimer interface was found in the crystal structure of $H$. sapiens elF2B $\alpha$ (Fig. 4A), and recent biochemical studies suggest that elF2B $\alpha$ uses this interface to form a physiological homodimer in solution $[72,138]$. It is therefore noteworthy that the structures of elF2B $\beta$ and elF2B presented here constitute an exception among their homologues (elF2B $\alpha /$ elF2Blike/5M1PIs/RBPIs) as they apparently do not form stable homodimers along this interface. For elF2B $\beta$, the crystal structure does not suggest an alternative interaction that might allow stable dimerization, in line with our observation that ctelF2B $\beta$ exists as a monomer in solution (see below). By contrast, ctelF2B seems to exist as a mixture of monomers and homodimers in solution (see below), which could indicate that the monomeric form crystallized under the used condition, and that an unstable homodimer could still be formed through the same interface as in elF2B $\alpha$. However, unlike eIF2B $\beta$, the two monomers in the asymmetric unit of the elF2B $\delta$ structure do interactvia a large interface formed by helices $\alpha 2, \alpha 5$, and $\alpha 6$, which could thus provide an alternative means to form a homodimer in solution (Fig. 5A).

\section{Protein interaction studies by analytical size exclusion chromatography}

To study the solution state and interactions between the individual elF2B subunits $\alpha$, $\beta$, and $\delta$ from Chaetomium thermophilum, we used analytical size exclusion chromatography (SEC) (Fig. 6).

CtelF2B $\alpha$ exists as a homo-dimer in solution. We found that ctelF2B $\alpha(\sim 41 \mathrm{kDa})$ alone elutes from the analytical SEC column in two peaks. The smaller peak elutes at $10.8 \mathrm{ml}$, while the main peak elutes at $12.5 \mathrm{ml}$, corresponding to apparent molecular weights of $\sim 250$ and $95 \mathrm{kDa}$, respectively. No peak was observed at an elution volume that would correspond to a monomer ( $\sim 14 \mathrm{ml})$. This suggests that ctelF2Ba most likely forms mainly dimers ( $\sim 82 \mathrm{kDa})$ in solution, while a small fraction forms higher oligomeric states, possibly homo-tetramers.

CtelF2B 3 exists as a monomer in solution. In contrast to ctelF2B $\alpha$, ctelF2B $\beta$ ( $\sim 45 \mathrm{kDa}$ ) alone elutes from the analytical SEC column in one peak at $14.2 \mathrm{ml}$, corresponding to an apparent 
molecular weight of $\sim 40 \mathrm{kDa}$, as expected for a monomer. An additional small shoulder elutes at 12.5-13 ml, which could correspond to a small fraction of homo-dimeric ctelF2B in solution.

A

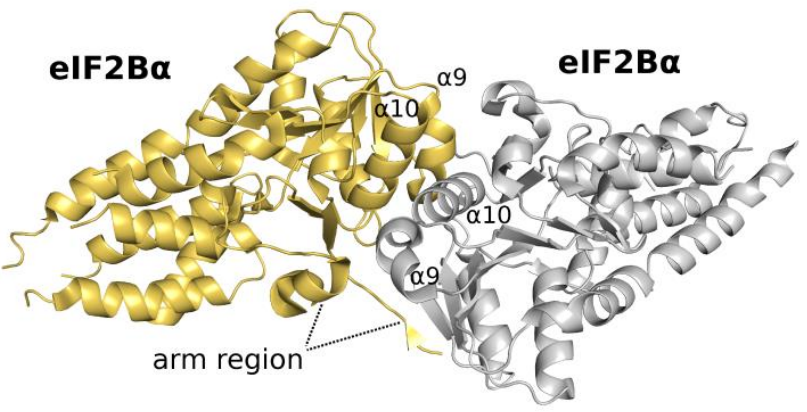

B

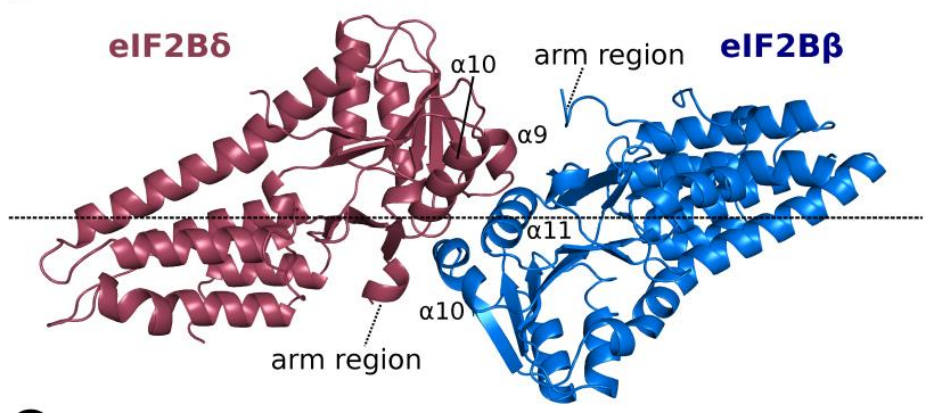

C

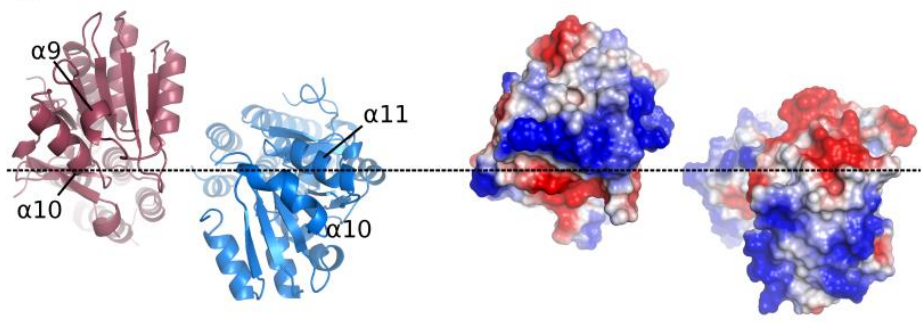

Figure 4.Model for the heterodimer between elF2B $\boldsymbol{\beta}$ and elF2B $\delta$. A) The $h s$ elF2B $\alpha$ homodimer (PDB: 3ECS). Like in the homologous 5M1PIs and RBPIs, the dimer interface is mainly formed by helices $\alpha 9$ and $\alpha 10$ of the CTD in one monomer, which pack against the same helices of the second molecule. The interface is complemented by the 'arm-region', which protrudes from one CTD to extend the large central $\beta$-sheet of the Rossmann-like fold in the CTD of the second monomer. B) Model for the elF2B $\beta \cdot e l F 2 B \delta$ heterodimer along the same interface as seen for elF2B $\alpha$. In the structures of the individual subunits, the helices corresponding to $\alpha 9$ and $\alpha 10$ in elF2B $\alpha$ are not occupied by homodimer formation and are thus available for heterodimer formation. C) The left panel shows the view directly onto the hypothetical elF2B $\beta \cdot$ elF2B $\delta$ heterodimer interfaces of elF2B $\beta$ (blue) and elF2B $\delta$ (dark red), respectively. The right panel shows the same view on both proteins in surface charge presentation (red, negative; blue, positive). The horizon indicated by the dashed line corresponds to that shown in (B), demonstrating that the surface charge distribution on the two proteins would be compatible with heterodimer formation.

CtelF2B $\delta$ exists as a mixture of monomers and homo-dimers in solution. Finally, full-length ctelF2B $(\sim 49 \mathrm{kDa})$ alone elutes in two main peaks, the first at $12.1 \mathrm{ml}$ and the second at $14.1 \mathrm{ml}$, corresponding to apparent molecular weights of $\sim 120$ and $\sim 40 \mathrm{kDa}$, respectively. This suggests that ctelF2B $\delta$ exists as a mixture in solution of nearly equal amounts of monomers and dimers ( $\sim 98$ $k D a)$, with the equilibrium slightly shifted in favor of dimer-formation. 

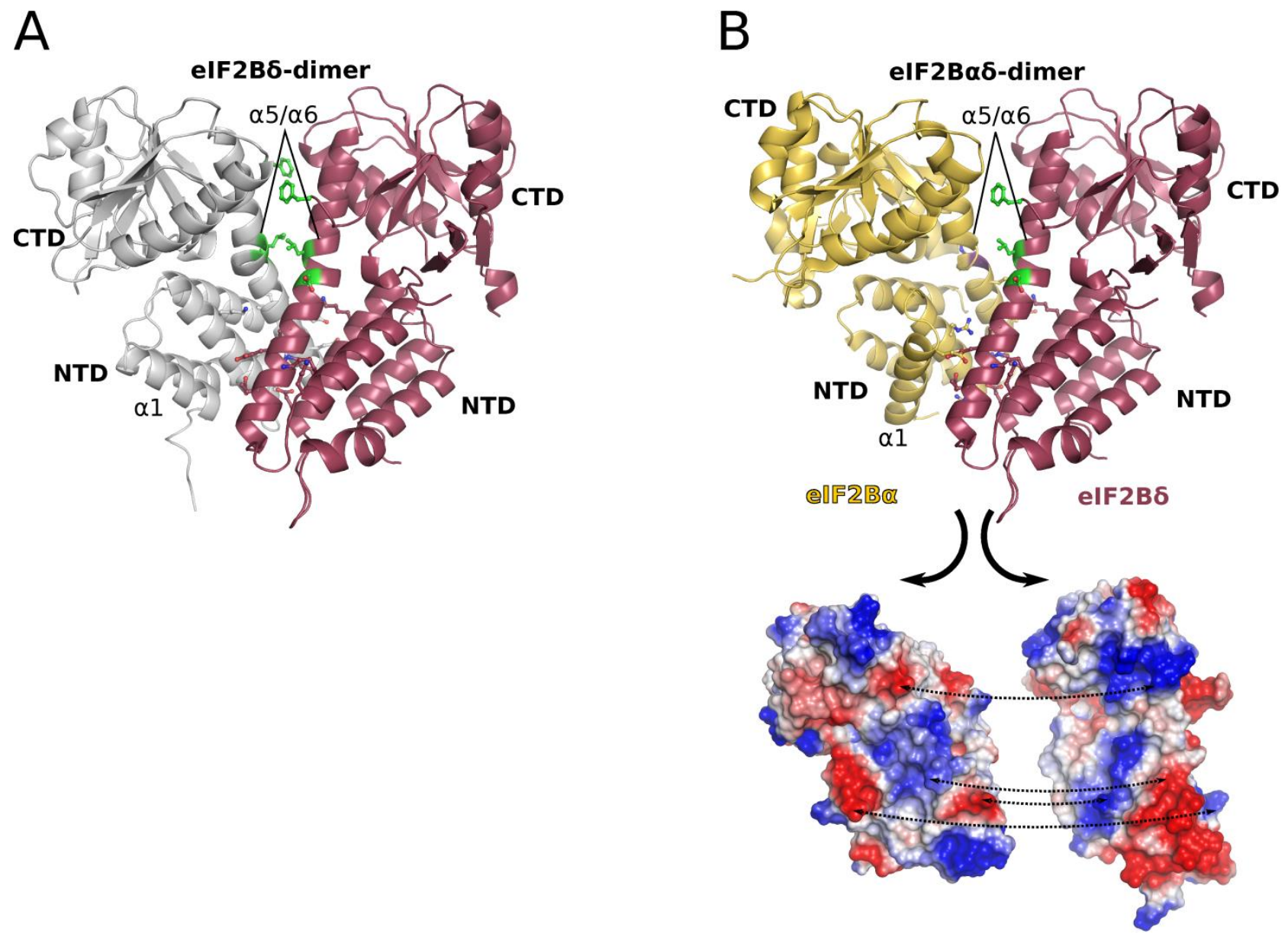

Figure 5. Model for the heterodimer between elF2B $\alpha$ and elF2B $\delta$. A) The ctelF2B $\delta$ homodimer observed in the asymmetric unit of the crystal structure (see also Fig. 2). In both molecules, the interface is mainly formed by helices $\alpha 2, \alpha 5$ and $\alpha 6$, which are oriented nearly orthogonal between the two protomers. The most intimate contacts are formed in the area of the kink-region between helices $\alpha 5$ and $\alpha 6$, where the green residues indicate positions of $\mathrm{G} c n$ mutations. The contact between the two ctelF2B $\delta$ molecules is mediated mainly by polar interactions between complementary charged surfaces (charged residues are shown as sticks). B) Model for the elF2B $\alpha \cdot e F I 2 B \delta$ heterodimer, in which the grey copy of elF2B from $A$ ) is exchanged for a homology model of ctelF2B $\alpha$ (golden; based on the hselF2B $\alpha$ structure, generated by the Phyre2 modeling server). The lower panel shows the direct view onto the potential binding interfaces of elF2B $\alpha$ (left) and elF2B $\delta$ (right) in surface charge presentation, with dashed arrows indicating the areas that contact each other in the model shown in the upper panel (red, negative; blue, positive).

Formation of the ctelF2B $\alpha B \delta$ complex depends on the presence of all three subunits. Mixing ctelF2B $\alpha$ with either ctelF2B $\beta$ (full-length or $\Delta 123-148$ ) or with ctelF2B $\delta$ alone did not result in complex formation between the respective proteins, as indicated by the elution profiles of the SEC (Fig. 6A/B). In the case of the ctelF2B $\alpha$-ctelF2B $\delta$ mixture it cannot be excluded that a hetero-dimer is formed that migrates at approximately the same volume as the respective homo-dimers; however, the elution profile is in good agreement with what would be expected for an interference 
of the individual runs (blue line in Fig. 6B), and the amount of monomeric ctelF2B $\delta$ seems to be unchanged between the presence or absence of ctelF2B $\alpha$. Thus, neither ctelF2B $\beta$ nor ctelF2B $\delta$ seem to interact with cteIF $2 B \alpha$ individually. By contrast, mixing ctelF2B $\beta$ with ctelF2B $\delta$ in the absence of ctelF2B $\alpha$ results in the complete precipitation of both proteins, leaving in solution only the protein that had been added in excess over the other. Indirectly, this indicates that elF2B $\beta$ and $\delta$ interact with each other independently of elF2B $\alpha$ and form a 1:1 complex with low solubility.

Finally, we tried to in vitro reconstitute the entire $c t e I F 2 B \alpha \beta \delta$ regulatory subcomplex by first mixing elF2B $\beta$ with a $\sim 2$-fold molar excess of elF2B $\alpha$, followed by the addition of elF2B $\delta$ in $~ 1.5-$ fold excess over the $\beta$-subunit. This time, no precipitation occurred and the analytical SEC run clearly shows the formation of a new peak, eluting at a volume of $10.31 \mathrm{ml}$ (Fig. 6C), which corresponds to an apparent molecular weight of $\sim 320 \mathrm{kDa}$. SDS-PAGE analysis of the peak fractions demonstrates that they contain all three subunits in approximately a 1:1:1 ratio (Fig. 6C, right panel). The calculated molecular mass of a hetero-trimer of the three subunits is $\sim 135 \mathrm{kDa}$, which is significantly smaller than the size of the observed complex in solution as indicated by SEC. Instead, the obtained results would be in better agreement with a hexamer of elF2B $\alpha, \beta$, and $\delta$ in a 2:2:2 ratio (elF $2 \mathrm{~B} \alpha_{2} \beta_{2} \delta_{2}$ ), which would add up to a complex of $\sim 270 \mathrm{kDa}$. Importantly, this putative elF2B $\alpha_{2} \beta_{2} \delta_{2}$ complex also contained small amounts of the upper degradation product of elF2B $\delta$ (35 kDa), which contains the crystallized fragment elF2Bס(148-443) (Fig. 6C), while the second smaller degradation product elutes separately from the complex peak at a higher volume. Moreover, the construct elF2B $\beta(\Delta 123-148)$, used for structure determination (see above), formed the elF2B $\alpha \beta \delta$ complex like the wild-type protein. 

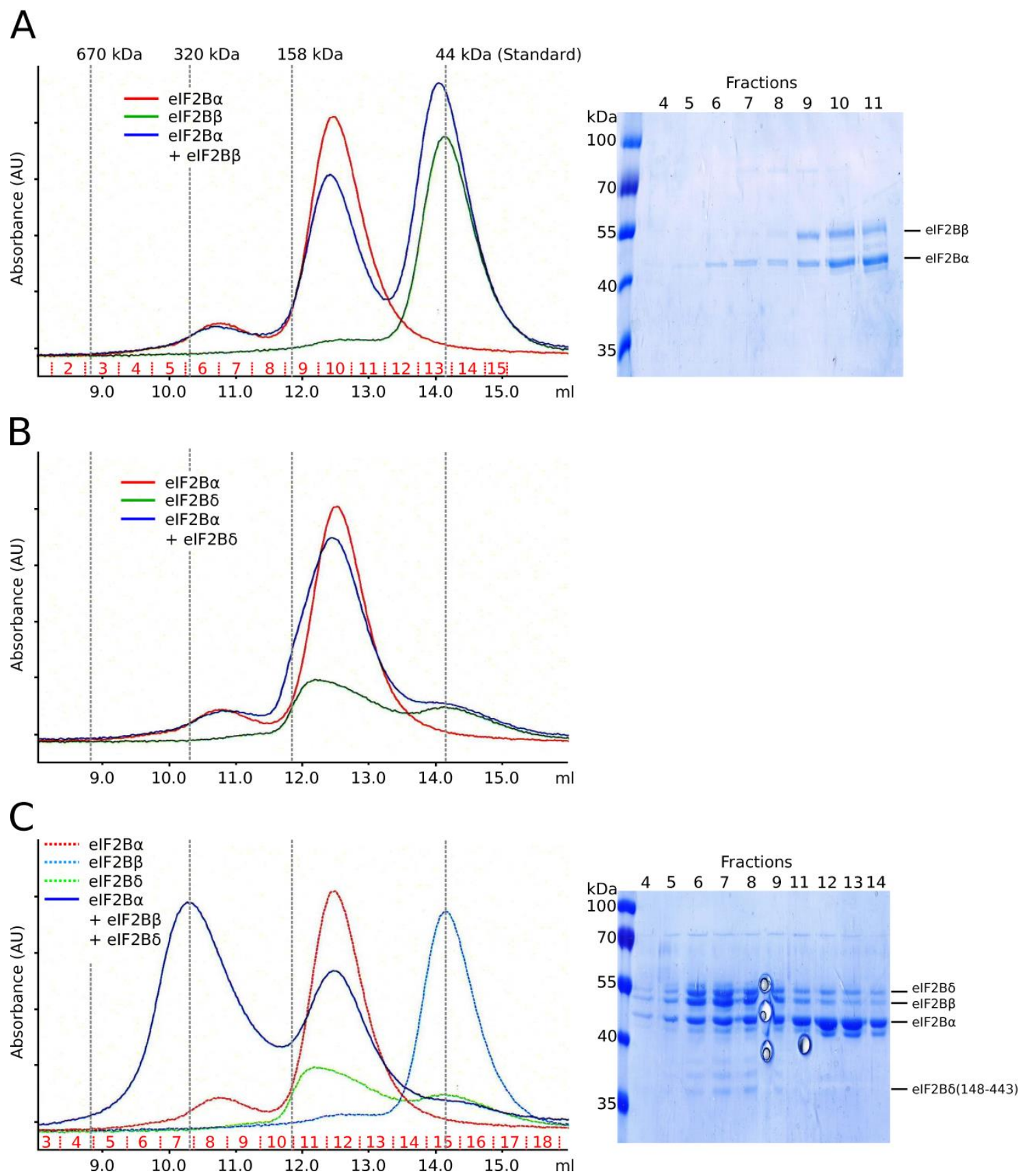

Figure 6. Reconstitution of the elF2B regulatory subcomplex from $C$. thermophilum by analytical size exclusion chromatography. A)Chromatograms for ctelF2B $\alpha$ (red curve), ctelF2B $\beta$ (green curve) and the mixture of ctelF2B $\alpha$ and ctelF2B $\beta$ (blue curve). While ctelF2B $\alpha$ elutes as an apparent dimer at $\sim 12.5 \mathrm{ml}$, corresponding to a size of $\sim 95 \mathrm{kDa}$, ctelF2B $\beta$ elutes at $14.2 \mathrm{ml}$, corresponding to an apparent molecular weight of $\sim 40 \mathrm{kDa}$, as expected for a monomer. No shift in the elution volume is observed for either peak when both subunits are mixed, indicating that no complex formation takes place (as confirmed by the SDS-PAGE). B)Chromatograms for ctelF2B $\alpha$ (red curve), ctelF2B $\delta$ (green curve) and the mixture of ctelF2B $\alpha$ and ctelF2B (blue curve). ctelF2B $\delta$ elutes in two peaks at $12.1 \mathrm{ml}$ and $14.1 \mathrm{ml}$, respectively, corresponding to apparent molecular weights of $\sim 120$ and $\sim 40 \mathrm{kDa}$, respectively. No shift in the elution volume is observed for either peak when both subunits are mixed, indicating that no complex formation takes place. C) When all three regulatory subunits are mixed, a new peak emerges at an elution volume of $10.31 \mathrm{ml}$ (blue line), which corresponds to an apparent molecular weight of $\sim 320 \mathrm{kDa}$. SDS-PAGE analysis of the peak fractions (right panel) demonstrates that they contain all three subunits in approximately a 1:1:1 ratio. 


\subsection{Discussion}

In comparison to most known GEFs of Ras-related G proteins, elF2B, the GEF for elF2, is an unusually large and complicated protein complex composed of five non-identical subunits. Despite considerable efforts over the last decades, little is known about the actual architecture of this large protein complex, the arrangement of the individual subunits relative to each other, and the way in which they bind their substrate elF2. Previously available high-resolution structural information for the elF2B complex was limited to the isolated $\sim 20 \mathrm{kDa}$ C-terminal catalytic domain of elF2B $\varepsilon$ and the elF2B $\alpha$ regulatory subunit from $H$. sapiens $[74,139]$. Thus, the lack of detailed structural information for the individual elF2B subunits, as well as for their mutual interactions is one of the key problems that currently hamper the interpretation of available mutational and genetic data to obtain a detailed picture of the molecular mechanisms that underlie elF2 binding by elF2B, nucleotide exchange and its regulation under stress conditions.

The motivation for this present work was toprovide insight into the architecture of the elF2B regulatory subcomplex in its molecular details. For this purpose, the orthologs of the three subunits elF2B $\alpha,-\beta$, and $-\delta$ from the thermophilic fungus Chaetomium thermophilum were recombinantly expressed and purified in their respective full-length forms that were active in in vitro reconstitution experiments for the regulatory subcomplex (Fig. 6). Initial trials to crystallize the entire regulatory subcomplex were not successful. However, it was possible to improve the crystallizability of the isolated ctelF2B $\beta$ by deleting a non-conserved predicted loop region within the $\mathrm{N}$-terminal domain, resulting in the first high-resolution crystal structure of an elF2B $\beta$ ortholog (elF2Bß( $\Delta 123-148)$ ) (Fig. 1). Moreover, by using a spontaneously occurring degradation product containing only the conserved C-terminal part of elF2Bס, we solved the first crystal structure of an elF2B ortholog (elF2Bס(148-443)) (Fig. 2). Importantly, both constructs were found to be incorporated into the elF2B $\alpha \beta \delta$ regulatory subcomplex with wild-type elF2B $\alpha$, indicating that the missing regions are not critical for its formation.

Together with the structure of $H$. sapiens elF $2 B \alpha$, the newly determined structures provide a vantage point for further structural studies on the architecture of the regulatory subcomplex. In line with predictions on the basis of sequence homology [136], both crystal structures confirmed the structural homology between the three eIF2B regulatory subunits, with an all-helical $\mathrm{N}$ - 
terminal domain and a C-terminal Rossmann-like $\alpha / \beta$ domain. One of the most striking observations from the crystal structures of elF2B $\beta$ and elF2B $\delta$ is the fact that they constitute the first examples from this protein family that do not stably homodimerize via the usual interface in the C-terminal domain. Hence, the same interface used for homodimerization in elF2B $\alpha$, archaeal elF2B-like proteins, 5M1PIs and RBPIs [138] would be available in elF2B $\beta$ and elF2B $\delta$ for heterodimerization either with each other or with elF2B $\alpha$. The latter option is unlikely, since elF2B $\alpha$ from $C$. thermophilum (Fig. 6) as well as from yeast forms stable homodimers in solution using this interface [138] and does not form stable complexes with the isolated elF2B $\beta$ or elF2B $\delta$ (Fig. 6A/B). By contrast, the analytical SEC experiments revealed that eIF2B $\beta$ forms a monomer and elF2B $\delta$ is present as a mixture of monomers and homodimers in solution, which would allow the formation of heterodimers between both proteins. Consistently, elF2B $\beta$ or elF2B $\delta$ interact with each other, most likely forming a tight 1:1 complex in solution that, however, was not soluble under the used experimental conditions. Heterodimerization between elF2B $\beta$ and elF2B $\delta$ via the usual homodimerization interface has been suggested previously [138] and would be consistent with the complementary surface charge distribution on both $C$. thermophilum proteins (Fig. 4B/C). Moreover, phylogenetic analyses suggest a common clade for these two proteins within the family of elF2B regulatory subunits [140], which indicates that they could constitute a derived homodimer that originates in a duplication event of their ancestral gene and a subsequent derivatization of the two lines without losing their ability to interact. The specific interaction between elF2B $\beta$ and elF2B $\delta$ is further supported by previous studies that demonstrated co-depletion of elF2B $\delta$ together with the genetic depletion of elF2B $\beta$ in $S$. cerevisiae, indicating that the latter is required to stabilize elF2B $\delta$ in the cellular context [77]. 
A
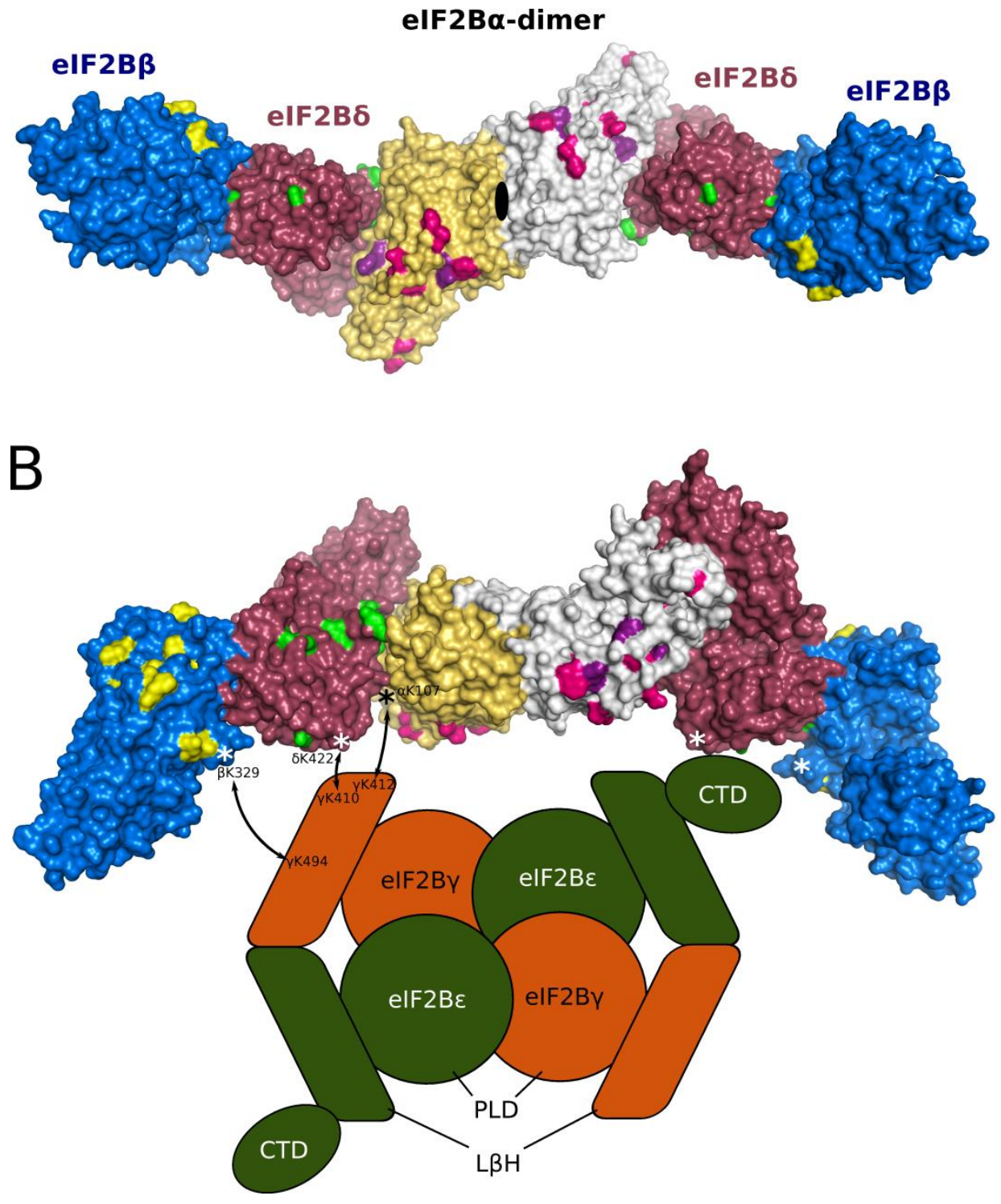

Figure 7. Structural model for the elF2B regulatory subcomplex. A)According to the model, the regulatory elF2B subomplex consists of one central elF2 $\mathrm{Ba}_{2}$ homodimer (gold and grey surfaces) which mediates the interactions between two elF2B $\beta \delta$ heterodimers (blue and red surfaces) through direct contact to the $\delta$-subunit (as in Fig. 5B). Pink, green and yellow surfaces indicate positions of $\mathrm{Gcn}$ - mutations in elF2B $\alpha$, elF2B $\beta$ and elF2B $\delta$, respectively[123, 135 , 142 , 143]. The complex has a two-fold symmetry axis through the dimerization interface of the elf2B $\alpha_{2}$ dimer (indicated by the black ellipsis). B) The resulting half-moon structure of the regulatory subcomplex suggest that the elF $2 \mathrm{~B} \gamma_{2} \varepsilon_{2}$ catalytic subcomplex (presented as by Gordiyenko et al.[73]) becomes bound between the two elF2B $\beta \delta$ heterodimers and thus in the vicinity of the previously identified Gcn mutations. The asterisks mark positions of lysine residues that were found to be crosslinked to specific positions on the elF2B $\gamma_{2} \varepsilon_{2}$ complex (indicated by black arrows) [73]. elF2B $\gamma$ and elF2B $\varepsilon$ each consist of a pyrophosphorylase-like domain (PLD) and a left-handed $\beta$-helix (L $\beta H$ ) domain, to which the additional catalytic C-terminal domain (CTD) is attached only in the $\varepsilon$-subunit. 
An interesting question that arises from our own and previous data is that of the topology/connectivity within the eIF $2 \mathrm{~B} \alpha \beta \delta$ regulatory subcomplex. In line with recent biochemical and mass-spectrometry data $[72,138]$, the analytical SEC experiments shown in Fig. 6 suggest that the regulatory subcomplex is not a heterotrimer as previously thought but in fact a dimer of heterotrimers, forming an elF $2 \mathrm{~B} \alpha_{2}(\beta \delta)_{2}$ complex. The necessity to allow further binding of two additional elF2B $\mathrm{\gamma} \varepsilon$ subcomplexes assembled as a symmetrical $\gamma_{2} \varepsilon_{2}$ complex [73], as well as the problem to avoid aggregation between elF2B complexes favors an arrangement of the two elF2B $\alpha \beta \delta$ trimers in a way that allows their superposition by a simple rotational symmetry operation. Within this complex, neither elF2B $\beta$, nor elF2B $\delta$ are individually sufficient to interact with elF2B $\alpha$ but require the structural context of the elF2B $\beta \delta$ complex to allow the formation of the hexamer. This suggests that the elF2B $\beta \delta$ complex either forms a combined interaction surface for the $\alpha$-subunit or that one subunit induces conformational changes within the other that render it active for interactions with elF2B $\alpha$. In both cases, the assumption that elF2B $\alpha_{2}$ and elF2B $\beta \delta$ each dimerize via the usual interface of the C-terminal domain (Fig. 4) makes it necessary to search for an alternative interface to mediate the association between the two dimers.

One interesting candidate for such an interaction is the interface between the two elF2B $\delta$ protomers within the asymmetric unit of the crystal structure (Fig. 5). This interface along helices $\alpha 5$ and $\alpha 6$ is sufficiently large $\left(\sim 7600 \AA^{2}\right.$ of combined buried surface area) to be physiologically relevant. Interestingly, yeast elF2B $\alpha$ and elF2B 6 , but not elF2B $\beta$, both contain sites of $\mathrm{Gcn}^{-}$and $\mathrm{Gcd}^{-}$mutants in the kink-region between helices $\alpha 5$ and $\alpha 6$ that forms the area for the most intimate contact within the elF2B dimer interface (Fig. 5B). Thus, an appealing option would be that each of the two elF2B $\beta \delta$ dimers associates with one copy of elF2B $\alpha$ in the elF2B $\alpha_{2}$ homodimer through the $\delta$-subunit and via an interface formed by helices $\alpha 5$ and $\alpha 6$ in both proteins (Fig. 7A). As an increased kink between helices $\alpha 5$ and $\alpha 6$ in elF2B $\delta$ would allow a more intimate contact to its interaction partner elF2B $\alpha$, it is conceivable that elF2B $\beta$-binding might stabilize the C-terminal helix in elF2B $\delta$ (which is disordered in the crystal structure of elF2B $\delta$ and stabilizes the large angles between $\alpha 5$ and $\alpha 6$ in elF2B $\alpha$ and elF2B $\beta$ (Fig. 3A-C)), thereby providing a possible explanation for the apparent cooperativity in their binding to elF2B $\alpha$. In this scenario, the inability of the $\mathrm{Gcn}^{-}$ mutants in the kink-regions to respond to elF2 $\alpha$ phosporylation would thus be due to the 
destabilization or even loss of elF2B $\alpha$, the primary sensor of elF2 $\alpha$ (S51-P), from the elF2B complex by interrupting the contact to elF2B $\delta$.

In the resulting model, elF2Ba would play a critical role by mediating hexamer formation in the regulatory subcomplex (Fig. 7), consistent with previous studies that demonstrated that elF2B $\alpha$ promotes decamer formation between two elF2B $\beta \delta \gamma \varepsilon$ complexes [72]. It should be noted however that data presented by Gordiyenko et al.[73] suggest that decamer formation is mediated between two elF2B $\gamma \varepsilon$ dimers, whereas the two regulatory subcomplexes, each comprising elF2B $\alpha \beta \delta$ in a 1:1:1 stoichiometry would not contact each other directly [73]. This is ostensibly at odds with the data by Wortham et al.[72] and our own observations and would require the dissociation of the conserved stable elF2B $\alpha_{2}$ dimer upon complex formation. Although the studies by Wortham et al.[72] and by Gordiyenko et al.[73] were done with proteins from different species (mammals and yeast, respectively), it is in our opinion unlikely that the observed differences are due to speciesspecific modes of decamerization, particularly as our own data for the fungus $C$. thermophilum support the data from the mammalian system. To reconcile both data sets it is in our opinion conceivable that decamerization is in fact mediated by the elF2B $\alpha_{2}$ homodimer, as well as the elF $2 \mathrm{~B}(\gamma \varepsilon)_{2}$ tetramer, with each $\gamma \varepsilon$ dimer binding independently to a regulatory subcomplex formed by one copy of elF2B $\alpha$ and the elF2B $\beta \delta$ heterodimer (Fig. 7B). This possibility would explain why Wortham and coworkers [72] found that the disruption of the elF2Ba dimer by mutagenesis does not abolish elF2B $\alpha$-dependent decamerization [72], while the data by Gordiyenko et al. [73] indicate that the presence of elF2B $\alpha$ stabilizes elF2B $(\beta \delta)_{2}(\nu \varepsilon)_{2}$ formation [73]. The resulting halfmoon shape of the elF $2 \mathrm{~B} \alpha_{2}(\beta \delta)_{2}$ model would be consistent with an elF2B $(\nu \varepsilon)_{2}$ tetramer assembled on the inner side (Fig. 7B). Moreover, most identified Gen mutations would as well map to the inner side of the model. Finally, Gordiyenko and coworkers [73] found that K107 in yeast elF2B $\alpha$ and $K 422$ in yeast elF2B $\delta$ crosslink to $K 412$ and $K 410$ in the left-handed $\beta$-helix $(L B H)$ domain of elF2B $\gamma$, respectively. As K107 in elF2B $\alpha$ and K422 in elF2B $\delta$ occupy adjacent positions within our structural model ( $\sim 17 \AA$ apart) and are both orientated toward the inner side of the half-moon, these data add further support to the structural model presented in Fig. 7. 


\subsection{Materials and Methods}

\section{Cloning}

The coding sequences of elF2B subunits $\alpha, \beta$ and $\delta$ were amplified by PCR as individual exons from Chaetomium thermophilum genomic DNA and introduced into the expression vector according to the InFusion protocol (Clontech). The coding sequence for full-length elF2B $\alpha$ was introduced into PET22b vector cleaved with the restriction enzymes Ncol and Xhol. The coding sequences for fulllength elF2B $\beta$ and $\delta$ were introduced into the pGEX-6P1 vector cleaved with BamHI and Xhol. The InFusion mixture was transformed into XL1-Blue E. coli cells (Stratagene) and plated out on LB agar plates containing appropriate antibiotics. Several colonies of each plate were picked and subjected to a test PCR to identify positive clones. Positive colonies were grown over night in LB medium supplemented with antibiotics and the plasmid was purified withthe Qiaprep Spin Miniprep Kit (Qiagen) according to the manufacturer's instructions. The sequences of the selected constructs were verified by DNA sequencing.

The construct encoding elF2B $\beta \Delta 123-148$ was generated by deletion of the corresponding codons from the original plasmid (wild-type eIF2B $\beta$ in pGEX-6P1) according to the QickChange technique (Stratagene) using appropriate mutagenesis primers. The plasmid was prepared and verified by sequencing as described above.

\section{Protein expression and purification}

Expression: All three constructs were expressed in E. coliBL21(DE3) Rosetta II cells (Novagene). Cells containing the respective plasmid were grown in 2xYT medium (supplemented with antibiotics) at $37^{\circ} \mathrm{C}$ with shaking at $220 \mathrm{rpm}$ until they reached an $\mathrm{OD}_{600}$ of $\sim 0.8$. Subsequently the cell cultures were transferred to $16{ }^{\circ} \mathrm{C}$, and the expression was induced after 20 min by the addition of isopropyl- $\beta$-D-thiogalactopyranosid (IPTG) to $0.5 \mathrm{mM}$ final concentration. The cells were harvested after 16 hours at $16^{\circ} \mathrm{C}$.

Purification of elF2B $\alpha$ : Cells containing C-terminally His-tagged elF2B $\alpha$ were resuspended in L-100-His buffer (20 mM Hepes (pH 7.5), 100 mM KCl, 10\% glycerol, 15 mM imidazole, 2 mM $\beta$ mercaptoethanol) containing a mixture of protease inhibitors including aprotinin, leupeptin, pepstatin (ALP), and PMSF. Cell lysis was performed in a microfluidizer (Microfluidics, USA), and 
cell debris was removed by centrifugation for $30 \mathrm{~min}$ at 30,000 $\mathrm{xg}$. The supernatant was loaded onto a HisTrap column (GE Healthcare) equilibrated in L-100-His buffer. The column was then washed with 2 column volumes of L-100-His buffer and bound protein was eluted with a linear gradient into elution buffer (L-100-His buffer with $350 \mathrm{mM}$ imidazole). Fractions containing the target protein were pooled, concentrated to a volume of $5 \mathrm{ml}$ and applied to a Superdex S-200 gelfiltration column (GE Healthcare) equilibrated in G-100 buffer (10 mM Hepes (pH 7.5), 100 mM $\mathrm{KCl}, 10 \%$ glycerol and $2 \mathrm{mM} \mathrm{DTT})$. The pure protein was concentrated to $20 \mathrm{mg} / \mathrm{ml}$, flash-frozen in liquid nitrogen and stored at $-80^{\circ} \mathrm{C}$.

Purification of elF2BB, elF2BB( $\triangle 123-148)$ and elF2BS: All three constructs were expressed as $\mathrm{N}$-terminally GST-tagged fusion proteins. Cell lysis was performed as described for elF2B $\alpha$ with the difference that L-500 buffer (20 mM Hepes (pH 7.5), $500 \mathrm{mM} \mathrm{KCl}, 10 \%$ glycerol, $4 \mathrm{mM} \beta$ mercaptoethanol) was used to resuspend the cells. After removal of cell debris by centrifugation, the supernatant was applied to a GSTrap column (GE Healthcare) equilibrated in L-500 buffer. After washing the column with 4 column volumes of L-500 buffer, the bound fusion protein was eluted with $30 \mathrm{mM}$ reduced glutathione in L-500 buffer. Fractions containing the target protein were pooled and desalted on a HiPrep Desalting column (GE Healthcare) equilibrated in desalting buffer (10 mM Hepes (pH 7.5), $200 \mathrm{mM} \mathrm{KCl,} \mathrm{10 \%} \mathrm{glycerol,} 4 \mathrm{mM} \beta$-mercaptoethanol). The pooled protein was incubated over night at $4{ }^{\circ} \mathrm{C}$ with Prescission protease in a 1:100 weight ratio of protease to target protein to remove the GST-tag. In order to remove the GST-tagged protease, cleaved GST and uncleaved fusion protein the sample was applied to a second GSTrap column equilibrated in desalting buffer. The flow-through was pooled and concentrated to $5 \mathrm{ml}$ before loading onto a Superdex S-200 gelfiltration column equilibrated in G-100 buffer. elF2B $\beta$ and elF2B $\beta(\Delta 123-148)$, which eluted as pure protein in a single peak, were concentrated to $15-20 \mathrm{mg} / \mathrm{ml}$ and stored at -80 ${ }^{\circ} \mathrm{C}$. elF2B $\delta$ usually suffered strong degradation resulting in the cleavage of the full-length protein into two fragments migrating at $\sim 36 \mathrm{kDa}$ and $\sim 20 \mathrm{kDa}$, respectively, on the SDS-PAGE. As a consequence, elF2B $\delta$ eluted in two major peaks from the $\mathrm{S}-200$ column containing either the fulllength protein or the $36-\mathrm{kDa}$ fragment. Both fragments were pooled independently, concentrated to $10-15 \mathrm{mg} / \mathrm{ml}$ and stored at $-80^{\circ} \mathrm{C}$. 


\section{Protein crystallization and structure determination}

eIF2BB. Initial crystallization trials for full-length elF2B $\beta$ were performed by sitting-drop vapor diffusion with commercially available standard screens. No crystals were obtained at any of the tested protein concentrations (between 6 and $15 \mathrm{mg} / \mathrm{ml}$ ) or temperatures $\left(4\right.$ and $20^{\circ} \mathrm{C}$ ). In order to improve the crystallizability of the protein, we decided to remove residues 123 to 148 from the peptide-chain by deletion of the corresponding coding sequence from the expression vector. According to multiple sequence alignments of $C$. thermophilum elF2B $\beta$ with its homologs from other species, these residues are not conserved and seem to correspond to a loop region between two conserved $\alpha$-helices that is idiosyncratic to ctelF2B $\beta$. Thus, we reasoned that its removal would have no negative impact on the overall structure of the protein, which is supported by the fact that it still forms a complex with elF2B subunits $\alpha$ and $\delta$ (see below). In initial crystallization trials with the construct elF2B $\triangle 123-148$, crystals were obtained after 5 days at $20{ }^{\circ} \mathrm{C}$ in a condition containing $100 \mathrm{mM}$ HEPES ( $\mathrm{pH} 7.5$ ) and 1.0 M tri-sodium citrate. After optimization, diffraction quality crystals were obtained with $9.5 \mathrm{mg} / \mathrm{ml}$ protein at $20^{\circ} \mathrm{C}$ in $100 \mathrm{mM} \mathrm{HEPES}(\mathrm{pH} 6.8)$ and 1.33 $M$ tri-sodium citrate. X-ray diffraction data were collected at BL 14.1 (HZB, BESSY, Berlin) [113]. The phase problem was solved by molecular replacement using the program PHASER [115] and the atomic coordinates of the N-terminal domain of the Bacillus subtilis 5-methylthioribose 1phosphate isomerase (PDB: 2YVK) and the C-terminal domain of Homo sapiens elF2Ba (PDB: 3ECS) as independent search models. The structure was refined in trigonal space group R3 at a resolution of $2.54 \AA$ using the program PHENIX [117]. Missing regions of the peptide chain were built manually in Coot [116]. The final model contains two molecules per asymmetric unit (see Table 1 for details of data collection and refinement).

elF2BS. Initial crystallization trials for elF2B $\delta$ were performed by sitting-drop vapor diffusion with commercially available standard screens. With the truncated version of elF2B $(35 \mathrm{kDa}$ fragment) initial crystals grew with 10 and $12 \mathrm{mg} / \mathrm{ml}$ protein at $20^{\circ} \mathrm{C}$ in a condition containing 100 $\mathrm{mM}$ MES $(\mathrm{pH} 6.0)$ and $1.0 \mathrm{M}\left(\mathrm{NH}_{4}\right)_{2} \mathrm{SO}_{4}$. After optimization, diffraction quality crystals were obtained in $100 \mathrm{mM}$ MES $\left(\mathrm{pH}\right.$ 6.2) and $0.8 \mathrm{M}\left(\mathrm{NH}_{4}\right)_{2} \mathrm{SO}_{4}$. X-ray diffraction data were collected at $\mathrm{BL}$ 14.1 (HZB, BESSY, Berlin) [113]. The phase problem was solved by molecular replacement using PHASER [115]. As independent search models we used the atomic coordinates of $\alpha$-helices 2-4 
(residues 27-93) of the N-terminal domain of Homo sapiens elF2B $\alpha$ (PDB: 3ECS), and residues 116295 of its C-terminal domain. The structure was refined in primitive orthorhombic space group $\mathrm{P} 2{ }_{1} 2_{1} 2_{1}$ at a resolution of $2.54 \AA$ using the program PHENIX [117]. The final model contains two molecules per asymmetric unit (see Table 1 for details of data collection and refinement).

\section{Interaction studies by analytical size exclusion chromatography}

Complex formation between elF $2 B \alpha,-\beta$ and $-\delta$ was studied by size exclusion chromatography on an analytical Superdex S-200 (10/300) column (GE Healthcare). For the individual standard runs for the subunits, $50 \mu \mathrm{g}$ protein in a total volume of $300 \mu \mathrm{l}$ was loaded onto the column equilibrated in running buffer (100 mM KCl, $20 \mathrm{mM}$ Tris/HCl (pH 7.5) and $2 \mathrm{mM} \mathrm{DTT).} \mathrm{For} \mathrm{the} \mathrm{analysis} \mathrm{of} \mathrm{binary}$ complex formation between two subunits, $50 \mu \mathrm{g}$ of each protein were mixed in $300 \mu \mathrm{l}$ running buffer and incubated for $5 \mathrm{~min}$ at $20^{\circ} \mathrm{C}$ before loading onto the column. In case of the elF2B $\beta$ elF2B $\delta$ mixture, the simultaneous presence of both proteins resulted in their complete precipitation until only the subunit added in excess over the other was still in solution. To study complex formation between all three subunits, elF2B $\beta(50 \mu \mathrm{g})$ was first mixed with a 2 -fold excess of elF2B $\alpha(100 \mu \mathrm{g})$, followed by the addition of elF2B $\delta$ in $\sim 1.5$-fold excess over the $\beta$-subunit (80 $\mu \mathrm{g})$. The mixture was incubated for $5 \mathrm{~min}$ at $20^{\circ} \mathrm{C}$ in $300 \mu$ l running buffer before loading onto the column. The runs were monitored at absorption wavelengths of 280 and $254 \mathrm{~nm}$. 


\section{Chapter $5^{\bullet}$ The domain release mechanism of eIF5B}

This manuscript has originally been published in The EMBO Journal

\section{eIF5B employs a novel domain release mechanism to catalyze ribosomal subunit joining}

Bernhard Kuhle ${ }^{1, \S}$ and Ralf Ficner ${ }^{1}$

${ }^{1}$ Abteilung für Molekulare Strukturbiologie, Institut für Mikrobiologie und Genetik, Göttinger Zentrum für Molekulare Biowissenschaften, Georg-August-Universität Göttingen, D-37077 Göttingen, Germany

\$To whom correspondence should be addressed: bkuhle@gwdg.de

Keywords: crystal structure; molecular machines; GTPase; ribosome; subunit joining; translation initiation

Received 6 November 2013 | Revised 6February 2014 | Accepted 20 February 2014 | Published online 31 March 2014

The EMBO Journal (2014) 33: 1177-1191 (DOI 10.1002/embj.201387344)

Author contributions:

BK: Conceived the study and designed the experiments; prepared and crystallized proteins, collected X-ray data andsolved the structures;performed ITC experiments; created figures; analyzed data and wrote the manuscript. RF: analyzed data and helped to draft the manuscript. 


\section{Abstract}

elF5B is a eukaryal translational GTPase that catalyzes ribosomal subunit joining to form elongation competent ribosomes. Despite its central role in protein synthesis, the mechanistic details that govern the function of elF5B or its archeal and bacterial (IF2) orthologs remained unclear. Here, we present six high resolution crystal structures of elF5B in its apo, GDP- and GTP-bound form that, together with an analysis of the thermodynamics of nucleotide binding, provide a detailed picture of the entire nucleotide cycle performed by eIF5B. Our data show that GTP binding induces significant conformational changes in the two conserved switch regions of the $\mathrm{G}$ domain, resulting in the reorganization of the GTPase center. These rearrangements are accompanied by the rotation of domain II relative to the $\mathrm{G}$ domain and release of domain III from its stable contacts to switch 2, causing an increased intrinsic flexibility in the free GTP-bound elF5B. Based on these data, we propose a novel domain release mechanism for elF5B/IF2 activation that explains how elF5B and IF2 fulfill their catalytic role during ribosomal subunit joining.

\subsection{Introduction}

Translation is the fundamental cellular process in which the ribosome synthesizes proteins according to genetically encoded information. Among the individual steps, translation initiation is the most complex and most divergent in the three domains of life, which is highlighted by the different number of initiation factors (IFs) employed by eukaryal ( 12 elFs) or bacterial cells (three IFs) to accomplish the same goals during ribosome assembly [9]. The major differences between eukaryal and bacterial translation initiation concern the formation of the 48S/30S pre-initiation complex (pre-IC) where the small 40S/30S ribosomal subunit is assembled at the AUG start codon of an mRNA with the charged initiator-tRNA (Met-tRNA ${ }_{i}^{\text {Met }} /$ fMet-tRNA $^{\text {fMet }}$ ) in its $P$ site [9]. This is followed by the formation of the elongation competent 80S/70S ribosome, which is achieved by the joining of the large 60S/50S ribosomal subunit, catalyzed by the orthologous GTPases elF5B, alF5B and IF2 in eukarya, archea and bacteria, respectively [15, 144, 145].

Together with the elongation factors EF-Tu and EF-G the initiation factors elF5B, alF5B and IF2 form the set of canonical translational GTPases (trGTPases) that are ubiquitous in extant 
cellular life. This suggests that the function of elF5B and its orthologs was fixed at an early stage of cellular evolution before the onset of speciation, reflecting the importance of subunit joining as the final control step in the initiation pathway. Up to now, however, the structural dynamics between the active and inactive factor that govern the process of subunit joining, and the degree of their conservation across the three domains of life remain unclear.

Earlier crystal structures of alF5B and IF2 revealed similar architectures for both proteins with an N-terminal GTP binding (G) domain and a $\beta$-barrel domain II as structural core, followed by domains III and IV [82-84]. IF2 and elF5B contain an additional N-domain, which displays little conservation in sequence and length and was shown to be dispensable for the function of yeast elF5B [85].

Like all trGTPases elF5B and its orthologs belong to the family of guanine nucleotide-binding (G) proteins [24]. Consequently, their mechanism has to be viewed as a specific variation of the classical concept of $G$ proteins as molecular switches that alternate between an inactive GDP- and a structurally distinct active GTP-bound state. The transition between the two states is defined by conformational changes in two dynamic elements of the $\mathrm{G}$ domain, termed switch 1 and switch 2, which specifically interact with the $\gamma$-phosphate of the GTP molecule [23]. Only in the GTP-bound state the $G$ protein interacts tightly and productively with effector molecules. Accordingly, the functional cycle of the G protein ends when it is 'switched off' by GTP hydrolysis and the following structural transition to the inactive GDP-bound state [23, 24].

For elF5B it was demonstrated that it interacts with the ribosomal subunits and catalyzes $80 \mathrm{~S}$ ribosome formation in a GTP-dependent manner $[145,146]$. It was further shown that subunit joining occurs catalytically in the presence of GTP but only stoichiometrically with the nonhydrolyzable GTP analog GDPNP. This indicates that GTP hydrolysis is required for the release of the factor from the 80S ribosome, in line with the observation that GDPNP but not GTP slows down the dissociation of elF5B from $80 \mathrm{~S}$ ribosomes and inhibits the peptidyl-transfer reaction [85, 145]. Similarly, it was found that IF2 promotes subunit joining much more efficiently in the presence of GTP and GDPNP than with GDP, and that GTP hydrolysis is required for the dissociation of the factor and subsequent peptide-bond formation [147-149]. Based on this evidence, it was 
suggested that elF5B and IF2 employ a similar mechanism to promote subunit joining [147] which is compatible with the classical concept of $G$ protein function.

However, this assumption stands in sharp contrast to other structural and biochemical data. It was found that GDP- and GTP-bound IF2 catalyze subunit joining nearly equally well, and that GTP hydrolysis is not required for the release of IF2 from the ribosome [150, 151]. Based on these observations, it was proposed that IF2 functions differently from elF5B and as a non-classical GTPase with apparently no role for GTP hydrolysis $[27,82,150]$, raising the question why the catalytic machinery required for GTP binding and hydrolysis is universally conserved in IF2.

So far available structure-based models for elF5B/IF2 function do not provide explanations for these contradictory results. The 'articulated lever model' for elF5B/IF2 function, which is based on crystal structures of alF5B, assumes that a GTP-induced $\sim 2 \AA$ shift in switch 2 is amplified by an en bloc rearrangement of domains II to IV into a $\sim 5$ Å movement of domain IV [84]. According to this model, neither switch 1 nor switch 2 undergoes the conformational changes or form the direct contacts to the $\gamma$-phosphate that are typical for the classical molecular switch. However, low resolution cryo-EM reconstructions of bacterial and eukaryal 70S/80S ICs revealed conformations of IF2 and elF5B that are incompatible with the articulated lever model [91, 152]. In order to reconcile the contradictory experimental data with the classical concept of molecular switching it was suggested that elF5B and IF2 follow a mechanism of 'conditional switching', in which GTP binding alone is insufficient to activate eIF5B/IF2 but requires the ribosome as a cofactor that shifts the equilibrium between an inactive and an active GTP-bound form toward the latter [153, 154]. More recently it was proposed that IF2 does not have an 'effecter domain' like other trGTPases and therefore behaves different from elF5B and not as a classical GTPase [82], which, however, leaves open the question how the nucleotide status of the $G$ domain is communicated into domains III and IV.

For alF5B as well as IF2, crystal structures have previously been solved in the GDPNP- and GTP-bound state, respectively $[83,84]$. However, in both cases the $G$ domain remained in the apo/GDP conformation despite the presence of the $\psi$-phosphate. Thus, the knowledge of elF5B and IF2 function is limited by the fact that up to now no high resolution structural information is available for their GTP-bound forms that is in agreement with the classical concept of molecular 
switching. Though of paramount importance to the understanding of elF5B/IF2 function, it is therefore not known what distinguishes the active from the inactive state of the $G$ domain and how these differences modulate the affinity of the overall elF5B/IF2 to ribosomal effector complexes or influence the mechanism of ribosome induced GTP hydrolysis.

Here we present thermodynamic data and high resolution structures of elF5B that provide a detailed picture of its entire nucleotide cycle. We determined six crystal structures of elF5B from Saccharomyces cerevisiae and Chaetomium thermophilum containing the $\mathrm{G}$ domain and domains IIIV or substructures thereof in the apo, GDP- and GTP-bound states. These structures unambiguously demonstrate that the $G$ domain of free elF5B follows the classical switch mechanism involving large structural rearrangements of the two switch regions. The GTP-induced changes result in the formation of a catalytic GTPase center similar to that in EF-Tu suggesting a possible scenario for ribosome dependent GTPase activation in elF5B. Most importantly, the various structures in combination with an analysis of the thermodynamics of nucleotide binding suggest a mechanism for elF5B activation in which the local switch within the $G$ domain is

propagated into the rest of the factor through the release of domain III, resulting in an increase of intrinsic flexibility that is necessary for efficient subunit joining. Based on these observations, we propose a domain release mechanism for elF5B activation, which represents a novel variation from the classical paradigm of $\mathrm{G}$ proteins and suggests a unified picture of subunit joining by a/elF5B and IF2.

\subsection{Results}

\section{Overall structure and domain arrangement in apo elF5B}

Six different crystal structures of elF5B from Chaetomium thermophilum (Ct) and Saccharomyces cerevisiae (Sc) were solved either in the apo form or cocrystallized with GDP or GTP. All structures were solved by means of molecular replacement. A summary of structures, crystallographic details and data statistics is presented in Table I and Fig. S1.

The topology of the individual domains as well as the overall domain arrangement of apo elF5B is similar to that of the archeal ortholog alF5B [84] (Fig. 1). Domains I-III form the core structure that is assembled as triangle around switch 2 of domain I (G domain). Domains I and II 
Table 1. Crystallization, $\mathrm{X}$-ray data collection and refinement statistics

\begin{tabular}{|c|c|c|c|c|c|c|}
\hline \multirow{2}{*}{$\begin{array}{l}\text { Organism } \\
\text { Construct (purified) }\end{array}$} & \multicolumn{4}{|c|}{ C. thermophilum } & \multicolumn{2}{|c|}{ S. cerevisiae } \\
\hline & elF5B(517C) & elF5B(517C) & elF5B(517-970) & elF5B(517-970) & elF5B(399-852) & elF5B(399-852) \\
\hline $\begin{array}{l}\text { Construct in } \\
\text { structure }\end{array}$ & $\begin{array}{l}\text { elF5B(517C) } \\
\text { apo }\end{array}$ & elF5B(870C) & $\begin{array}{l}\text { elF5B(520- } \\
970) \cdot G D P\end{array}$ & elF5B(517-860)·GTP & $\begin{array}{l}\text { elF5B(401-852) } \\
\text { apo }\end{array}$ & $\begin{array}{l}\text { elF5B(401- } \\
852) \cdot G D P\end{array}$ \\
\hline \multicolumn{7}{|l|}{ Crystallization } \\
\hline Temperature $\left({ }^{\circ} \mathrm{C}\right)$ & $\begin{array}{l}100 \mathrm{mM} \text { MES }(\mathrm{pH} \\
6.8), 12 \% \mathrm{PEG} \\
20000,10 \mathrm{mM} \\
\text { Na-lactate } \\
4\end{array}$ & $\begin{array}{l}100 \mathrm{mM} \text { MES (pH } \\
6.8), 12 \% \text { PEG } \\
20000,10 \mathrm{mM} \\
\text { Na-lactate } \\
4\end{array}$ & $\begin{array}{l}15 \% \text { PEG } 8000 \\
\text { and } 0.5 \mathrm{M} \mathrm{Li}_{2} \mathrm{SO}_{4}\end{array}$ & $\begin{array}{l}100 \mathrm{mM} \text { Hepes }(\mathrm{pH} \\
7), 13 \% \text { PEG } 4000 \\
\text { and } 100 \mathrm{mM} \mathrm{NaOAc} \\
20\end{array}$ & $\begin{array}{l}20 \% \text { ethylene } \\
\text { glycol, } 5 \% \text { PEG } 3350 \\
\text { and } 20 \mathrm{mM} \mathrm{MgCl}_{2} \\
4\end{array}$ & $\begin{array}{l}8 \% \text { PEG } 8000 \text { and } \\
0.37 \mathrm{M} \mathrm{Li}_{2} \mathrm{SO}_{4}\end{array}$ \\
\hline \multicolumn{7}{|l|}{ Data Collection } \\
\hline \multirow[t]{2}{*}{$\begin{array}{l}\text { Space Group } \\
\text { Unit Cell }\end{array}$} & $\begin{array}{l}\mathrm{P}_{2} 21 \\
\mathrm{a}=\mathrm{b}=111.5 \AA \\
\mathrm{c}=115.2 \AA\end{array}$ & $\begin{array}{l}\mathrm{P}_{1} 21 \\
\mathrm{a}=\mathrm{b}=98.2 \AA \\
\mathrm{c}=97.4 \AA\end{array}$ & $\begin{array}{l}\mathrm{P} 2{ }_{2} 2_{1} 2_{1} \\
\mathrm{a}=66.9 \AA \\
\mathrm{b}=72.9 \AA \\
\mathrm{c}=199.2 \AA\end{array}$ & $\begin{array}{l}\mathrm{P} 2_{1} \\
\mathrm{a}=55.4 \AA \\
\mathrm{b}=114.8 \AA \\
\mathrm{c}=65.9 \AA\end{array}$ & $\begin{array}{l}\mathrm{P} 41 \\
\mathrm{a}=\mathrm{b}=118.0 \AA \\
\mathrm{c}=77.5 \AA\end{array}$ & $\begin{array}{l}\mathrm{P} 2{ }_{1} 2_{1} 2_{1} \\
\mathrm{a}=73.6 \AA \\
\mathrm{b}=119.5 \AA \\
\mathrm{c}=120.7 \AA ;\end{array}$ \\
\hline & $\begin{array}{l}\alpha=\beta=90^{\circ} \\
\gamma=120^{\circ}\end{array}$ & $\begin{array}{l}\alpha=\beta=90^{\circ} \\
\gamma=120^{\circ}\end{array}$ & $\alpha=\beta=\gamma=90^{\circ}$ & $\begin{array}{l}\alpha=90^{\circ} \\
\beta=102.3^{\circ} \\
\gamma=90^{\circ}\end{array}$ & $\alpha=\beta=\gamma=90^{\circ}$ & $\alpha=\beta=\nu=90^{\circ}$ \\
\hline $\begin{array}{l}\text { Molecules/asym. } \\
\text { unit }\end{array}$ & 1 & 1 & 2 & 2 & 2 & 2 \\
\hline Resolution (Å) & $2.75(2.85-2.75)$ & $3.2(3.3-3.2)$ & $2.12(2.21-2.12)$ & $1.87(1.97-1.87)$ & $1.83(1.93-1.83)$ & $3.02(3.12-3.02)$ \\
\hline Observed reflections & 168769 (15898) & $59836(5506)$ & 288203 (38832) & 251805 (36887) & 426259 (62736) & 108724 (10591) \\
\hline Unique reflections & 22063 (2217) & $9288(800)$ & 56555 (7213) & 66297 (9605) & 93360 (13677) & 21521 (1972) \\
\hline $\begin{array}{l}\text { Completeness (\%) } \\
<1>/ \sigma\end{array}$ & $\begin{array}{l}99.9(100) \\
32.66(3.29)\end{array}$ & $\begin{array}{l}99.8(100) \\
32.12(4.31)\end{array}$ & $\begin{array}{l}99.7(99.9) \\
17.79(2.97)\end{array}$ & $\begin{array}{l}99.8(99.8) \\
20.53(2.38)\end{array}$ & $\begin{array}{l}99.8(99.9) \\
21.05(3.11)\end{array}$ & $\begin{array}{l}99.2(99.5) \\
23.3(3.52)\end{array}$ \\
\hline $\mathrm{R}_{\text {sym }}(\%)$ & $3.7(58.9)$ & $3.4(57.4)$ & $5.6(63.1)$ & $4.4(62.0)$ & $3.9(52.5)$ & $5.3(60.6)$ \\
\hline \multicolumn{7}{|l|}{ Refinement } \\
\hline $\begin{array}{l}\mathrm{R}_{\text {work }}(\%) \\
\mathrm{R}_{\text {free }}(\%)\end{array}$ & $\begin{array}{l}19.3 \\
23.8\end{array}$ & $\begin{array}{l}19.0 \\
22.0\end{array}$ & $\begin{array}{l}21.9 \\
25.2\end{array}$ & $\begin{array}{l}16.7 \\
20.6\end{array}$ & $\begin{array}{l}16.8 \\
19.4\end{array}$ & $\begin{array}{l}24.9 \\
28.7\end{array}$ \\
\hline $\begin{array}{l}\text { Rmsd from } \\
\text { Standard } \\
\text { Stereochemistry }\end{array}$ & & & & & & \\
\hline $\begin{array}{l}\text { Bond length }(\AA ̊) \\
\text { Bond angles }\left({ }^{\circ}\right)\end{array}$ & $\begin{array}{l}0.005 \\
0.82\end{array}$ & $\begin{array}{l}0.003 \\
0.66\end{array}$ & $\begin{array}{l}0.005 \\
0.95\end{array}$ & $\begin{array}{l}0.019 \\
1.75\end{array}$ & $\begin{array}{l}0.008 \\
1.17\end{array}$ & $\begin{array}{l}0.004 \\
0.99\end{array}$ \\
\hline \multicolumn{7}{|l|}{ Ramachandran Plot } \\
\hline Most favored (\%) & 98.0 & 98.0 & 98.8 & 98.3 & 98.5 & 97.0 \\
\hline Allowed regions (\%) & 2.0 & 2.0 & 1.2 & 1.7 & 1.5 & 3.0 \\
\hline $\begin{array}{l}\text { Disallowed regions } \\
\text { (\%) }\end{array}$ & 0 & 0 & 0 & 0 & 0 & 0 \\
\hline
\end{tabular}

are tightly associated through hydrophobic contact areas composed of $\beta 2$ (part of switch 1), $\beta 3$ and $\beta 4$ in the $G$ domain as well as $\beta 9, \beta 10$ and $\beta 16$ in domain II (Fig. 1). The orientation of domain II relative to the $\mathrm{G}$ domain is nearly identical in Ct-elF5B and Sc-elF5B and similar to that found in IF2, EF-G or GDPNP-bound EF-Tu $[48,83,155]$. 


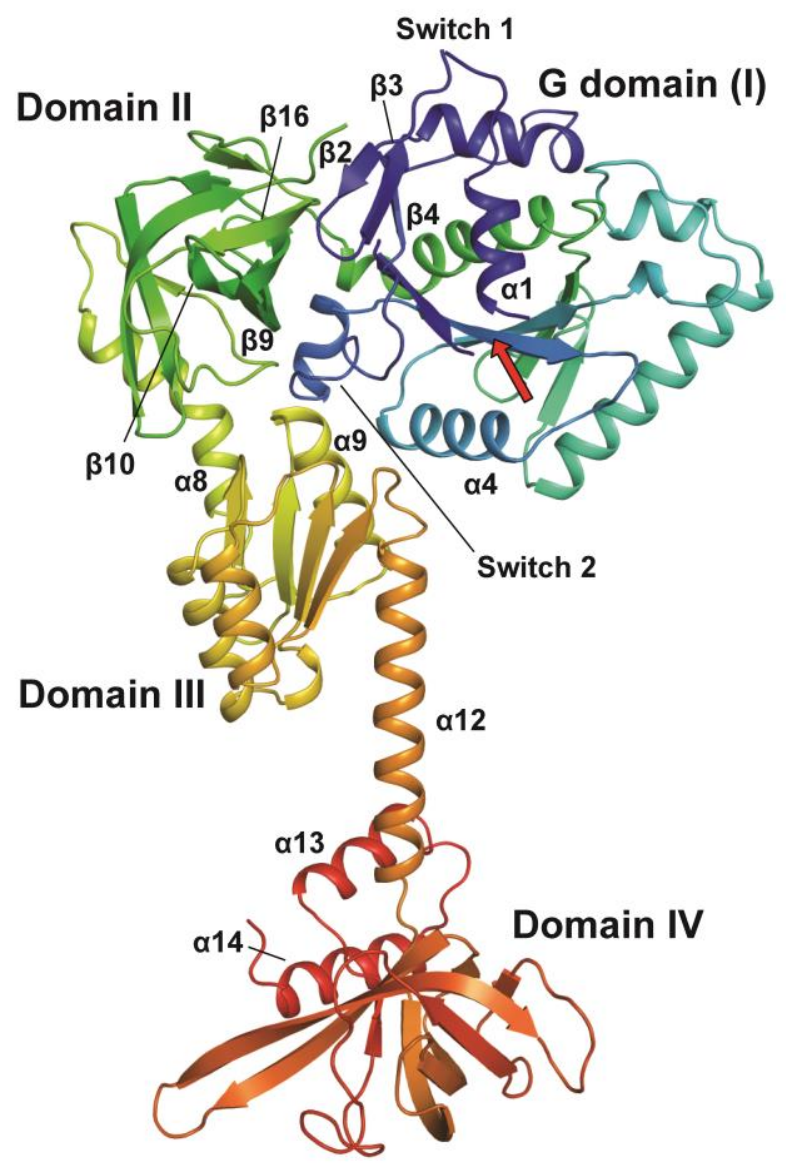

Figure 1.Front view of the overall structure of $C$. thermophilumelF5B (517C) in the apo form. The $C_{\alpha}$ trace is shown in rainbow coloring from the $\mathrm{N}$ (blue) to the C-terminus (red). The functional core of elF5B is composed of the $\mathrm{G}$ domain (I) with the nucleotide binding site (arrow), domain II, domain III and domain IV.
Stable interactions of domain III with other domains are restricted to the area surrounding the $\mathrm{N}$-terminal half of helix $\alpha 9$ (Fig. 1 and 3A/B). Van der Waals contacts are formed with domain II and the linker-helix $\alpha 8$ with a buried surface area of $\sim 700 \AA^{2}$. Helix $\alpha 9$ also interacts with switch 2 as the only stable contact partner for domain III within the $\mathrm{G}$ domain. Further contacts are formed between the $\mathrm{N}$-terminus of helix $\alpha 12$ and $\alpha 4$; however, these do not seem to be functionally relevant, as they differ between the various apo structures. Consequently, the orientation of domain III relative to the $G$ domain differs considerably in Sc-elF5B, Ct-elF5B and alF5B, which amounts to a displacement of the C-terminus of $\alpha 12$ by $12-22 \AA$ between the three apo structures (Fig. S2A). In a direct superposition of domain III from alF5B, CtelF5B(517C) and Ct-elF5B(870C) the C-terminal ends of $\alpha 12$ lie only 3-3.5 $\AA$ apart. However, domain IV adopts significantly different orientations relative to $\alpha 12$ and domain III, indicating a high degree of flexibility (Fig. S2B).

\section{Conformational changes in the $\mathbf{G}$ domain of elF5B upon GTP binding}

GTP is bound by elF5B in the way common for G proteins involving five conserved sequence motifs termed G1-G5 [24] (Fig. 2 and S3). The base is in contact with the ${ }^{530} \mathrm{NKID}^{533}$ (G4) and ${ }^{598} \mathrm{SAx}^{600}$ (G5) motifs (unless stated otherwise, S. cerevisiae sequence numbering will be used throughout) and 
the phosphates of the nucleotide are stabilized by main- and side-chain interactions with the $P$ loop (G1).

A

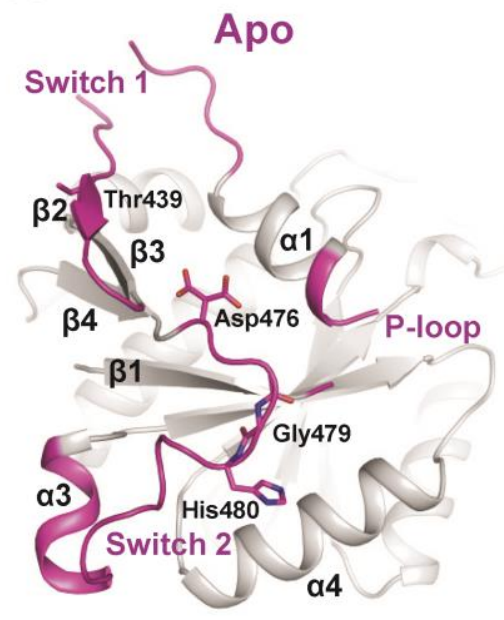

D

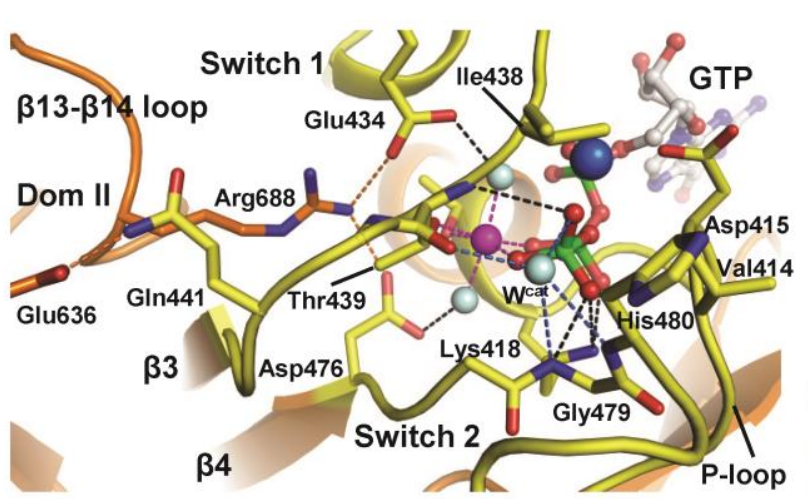

B

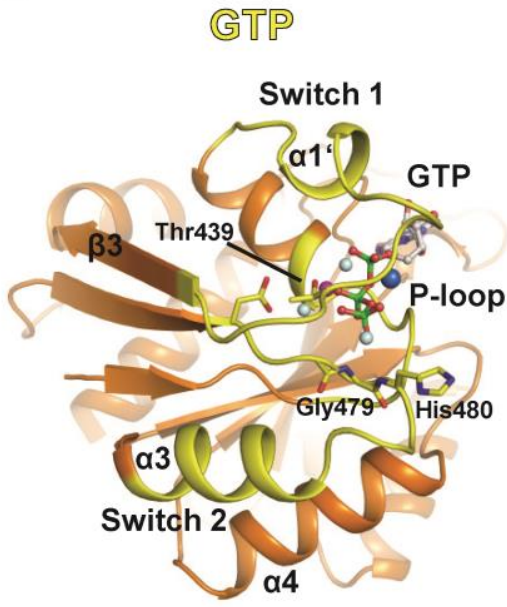

E
C

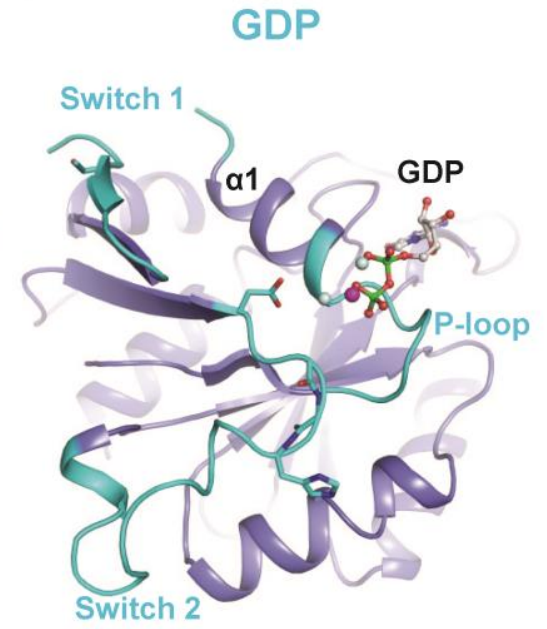

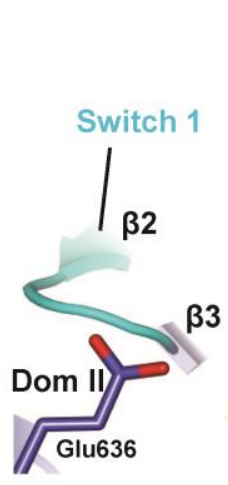

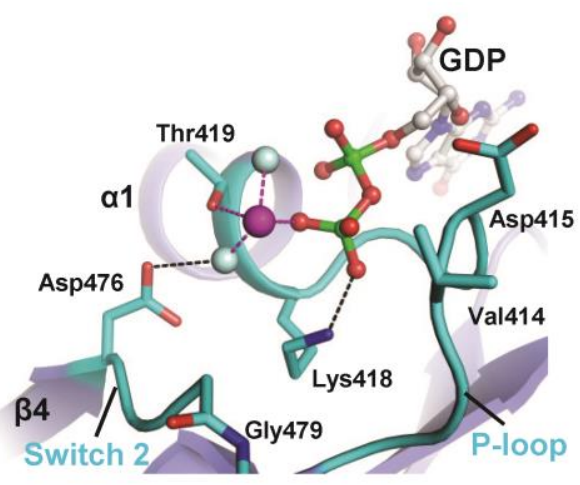

Figure 2.The nucleotide-dependent conformational switch in the $G$ domain of elF5B. A-C) Structural transition of the G domain from its apo form (A) to the GTP- (B) and the GDP-bound (C) states. P-loop, switch 1 and switch 2 are colored pink in the apo state, yellow in the GTP-bound state and cyan in the GDPbound state. Thr439, Asp476, Gly479 and His480 (S. cerevisiae numbering) are shown as sticks; the $\mathrm{Mg}^{2+}$ ion, $\mathrm{Na}^{+}$ion and water molecules are shown as spheres in magenta, blue and grey, respectively; nucleotides are shown as balls and sticks. D, E) Network of interactions in the nucleotide binding pocket of the GTP- (D) and GDP-bound (E) factor. Direct interactions are indicated by dashed lines.

The most severe conformational changes are observed for the two switch regions which contain the ${ }^{437} \mathrm{GIT}^{439}$ (G2) and ${ }^{476} \mathrm{DTPG}^{479}$ (G3) motifs that function as sensors for the presence of the $\gamma$-phosphate (Fig. 2). In the apo state most of switch 1 (residues 427-443) forms an unstructured loop, which points away from the nucleotide binding pocket. Only within its last third 
the inactive switch 1 forms a short $\beta$ strand ( $\beta 2$ ) oriented antiparallel to $\beta 3$ as part of the interface with domain II. Upon GTP binding, switch 1 flips over by $180^{\circ}$ using Gln427 and Gly443 as hinges (Fig. 2A/B and S4A). As a result its $\mathrm{N}$-terminal part is oriented antiparallel to helix $\alpha 1$ toward the nucleotide binding pocket where it forms a one-turn $\alpha$-helix $\left(\alpha 1^{\prime}\right)$ above the $\alpha$-phosphate, followed by a turn toward the $\beta$ - and $\gamma$-phosphates that continues into $\beta 3$. The critical Thr439 is displaced from its position in $\beta 2$ of the apo state by nearly $20 \AA$ to form direct contacts to the $\mathrm{Mg}^{2+}$ ion and the $\gamma$-phosphate. In this new position switch 1 has almost no contacts outside the nucleotide binding pocket with the exceptions of a salt bridge between Glu434 and Arg688 of the $\beta 13-\beta 14$ loop and a hydrogen bond of Gln441 to the conserved Glu636 in domain II (Fig. 2D).

Switch 2 (476-492) undergoes a substantial rearrangement as well. In the apo state the G3 motif in switch 2 runs parallel to $\beta 4$ as far as Gly479, where the peptide backbone makes a sharp turn of $>90^{\circ}$ and continues through the inter-domain cleft formed by domains I-III toward the back of the protein (Fig. 2A and $3 \mathrm{~B}$ ). Here switch 2 turns a second time toward the dorsal side of the $G$ domain forming the two-turn helix $\alpha 3$. In this conformation switch 2 makes van der Waals contacts to domain II but mainly interacts with domain III: The backbone CO of Ser484 accepts a hydrogen bond from Gly763, Arg487 forms a hydrogen bond and salt bridges to Glu766 and Asp770, and Arg489 forms a strong salt bridge to Asp740 (Fig. 3A/B).

Upon GTP binding Asp476 moves $3.1 \AA$ toward the $\gamma$-phosphate and forms a hydrogen bond to one of the water molecules coordinating the $\mathrm{Mg}^{2+}$ ion (Fig. 2B/D and S4B). The universally conserved Gly479 of switch 2 moves $\sim 8 \AA$ toward the $\gamma$-phosphate; concomitantly, the peptide bond between Pro478 and Gly479 flips by $\sim 160^{\circ}$. In its new position Gly479 interacts directly with the $\mathrm{\gamma}$-phosphate and the putative catalytic water molecule $\left(\mathrm{W}^{\mathrm{cat}}\right)$. The movement of the $\mathrm{G} 3$ motif has a profound impact on the rest of switch 2: His480 moves by $12 \AA$ and forms part of a loop at the front of the $\mathrm{G}$ domain that is stabilized by Arg487 (Fig. S4B). In its new position His480 lies next to Val414 (P loop) with the imidazole moiety pointing outward, away from $\mathrm{W}^{\text {cat }}$ (Fig. 2D). The rest of switch 2 (484-493) forms the extended helix $\alpha 3$ next to $\alpha 4$, with some residues up to $15 \AA$ relocated from their original position in apo elF5B; the axis through $\alpha 3$ is thereby rotated by more than $90^{\circ}$ (Fig. S4A). In order to achieve this conformational change in switch 2 all its interactions to domain III in the apo state are necessarily broken (Fig. 3A/B). 

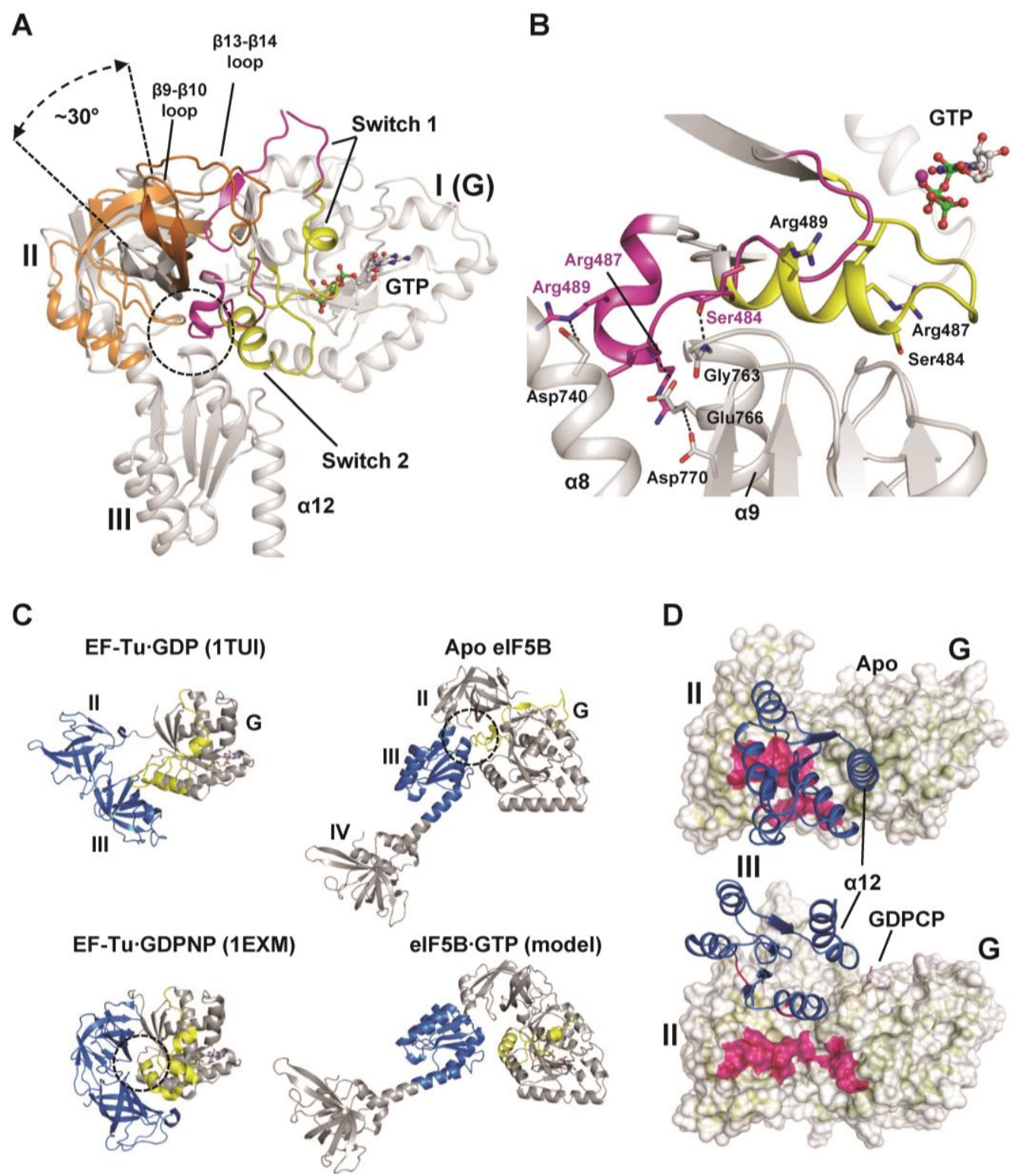

Figure 3.Nucleotide-dependent conformational changes in elF5B. A) G domain-based superposition of domains I (G), II and III from Ct-elF5B in the apo and GTP-bound state. Both G domains are shown in grey; otherwise the same color code as in Fig. 2. The GTP-induced rearrangements result in the loss of interactions between switch 2 and helices $\alpha 8$ and $\alpha 9$ (circle) and ultimately in the release of domain III from the $\mathrm{G}$ domain. Domain II rotates by $\sim 30^{\circ}$ relative to the $\mathrm{G}$ domain and is stabilized in its new orientation by the newly formed contact between $\beta 13-\beta 14$ loop and domain I. B) In apo elF5B the inactive switch 2 (pink) forms stable contacts to helices $\alpha 8$ and $\alpha 9$ of domain III which are broken upon the GTP-induced transition of switch 2 to its active state (yellow). C) Comparison of the molecular switch mechanisms in EF-Tu (left) and elF5B (right). Both trGTPases are shown in their inactive apo or GDP-bound (top) and GTP-bound (bottom) states, respectively. Functionally relevant interactions between the switch regions (yellow) of the $\mathrm{G}$ domain and downstream functional domains (blue) are indicated by dashed circles. D) The contact surface (pink) found between domain III (blue) and domains I and II in apo elF5B (top) is entirely lost in ribosome-bound elF5B.GDPCP (bottom; PDB: 4BVX [91]), where domains III and IV become stabilized between SRL and Met$\operatorname{tRNA}_{i}^{\text {Met }}$ (not shown). 
Switch 1 and switch 2 are stabilized in their active GTP-bound conformations through a

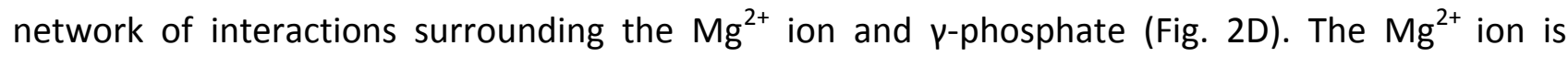
coordinated by six oxygen ligands with octahedral coordination geometry; two of the ligands are water molecules, two come from the $\beta$ - and $\gamma$-phosphates and two are provided by the side chains of Thr419 and Thr439. The $\mathrm{p}$-phosphate is further stabilized by Lys418 (P loop), Thr439 and Gly479. $W^{\text {cat }}$ lies $2.8 \AA$ from the outward pointing $\gamma$-phosphate oxygen in position for an in-line attack on the -phosphate, stabilized by the backbone amides of Gly479 and His480 and the backbone CO of Thr439. Next to the $\mathrm{y}$-phosphate an additional strong electron density was observed, which was assigned to a $\mathrm{Na}^{+}$ion. Its pentagonal coordination shell with the typical bond lengths of 2.3-2.5 [156] is constituted by two oxygens from the $\alpha$ - and $\gamma$-phosphates, the $\beta$ - $\gamma$-bridging oxygen, the caboxylate of Asp415 (P loop) and the CO from Gly437 in switch 1. Together, P loop, switch 1 and switch 2 form a closed $\sim 10 \AA$ deep pocket that accommodates the $\mathrm{Mg}^{2+}$ and $\mathrm{Na}^{+}$ions as well as all three phosphates with the $\gamma$-phosphate and $W^{\text {cat }}$ at its bottom. His480 and bulk solvent are excluded from this pocket by a gate formed by Val414 and Ile438.

A comparison with the structure of EF-Tu·GDPNP [157] shows a strikingly high degree of similarity between the catalytic centers in GTP/GDPNP-bound EF-Tu and elF5B with nearly identical positions for all conserved residues with a pair-wise $C_{\alpha}$ rmsd of $1.1 \AA$ over 99 residues as well as for $\mathrm{W}^{\text {cat }}$ and the $\mathrm{Mg}^{2+}$ ion (Fig. $4 \mathrm{~A}$ and S5A). However, the position occupied by the $\mathrm{Na}^{+}$ion in elF5B is vacant in the EF-Tu.GDPNP structure and a perfect agreement between elF5B and EF-Tu is restricted to residues that are directly involved in nucleotide binding or implicated in GTPase activity. Switch 1 in elF5B lacks the second helix ( $\left.A^{\prime \prime}\right)$ that serves factor specific functions in EF-Tu [48].

\section{Domain rearrangements in elF5B upon GTP binding}

Upon GTP binding domain II performs two main movements that result from the activation of the $\mathrm{G}$ domain (Fig. $3 \mathrm{~A}$ ): on the one hand the dorsal portion of domain II tilts inward, following the movement of $\beta 3$ and $\beta 4$ that was induced by the $3.1 \AA$ shift of Asp476 toward the $\mathrm{Mg}^{2+}$ ion (Fig. S4); on the other hand domain II rotates by $\sim 30^{\circ}$ causing the front portion to move upward and the ventral side to move parallel to switch 2 toward the front. The rotated orientation of domain II 
is stabilized by a newly formed interaction of the $\beta 13-\beta 14$ loop (residues 684-689) with the $G$ domain, which takes over the position next to $\beta 3$ that was originally occupied by $\beta 2$ and left vacant after the GTP-induced rearrangement of switch 1 (Fig. 2 and 3A).

As described above, all residues of switch 2 that are involved in contacts with domains II and III in the apo structure are rearranged and move by an average of $14 \AA$ upon GTP binding (Fig. 3B and S4). Since switch 2 is the main contact area for domain III in the G domain either a completely new set of interactions has to be formed or the interaction of domain III to the $\mathrm{G}$ domain is entirely lost. As domain III is not present in the structure of Ct-elF5B.GTP, direct information about its position in free elF5B.GTP is not available. However, our ITC experiments described below point toward a scenario in which domain III is released from the G domain without forming stable new contacts with the reorganized G domain in the free form of elF5B·GTP (see below).

\section{Conformational changes in elF5B during the transition from the GTP- to the GDP-bound state}

Subsequent to GTP hydrolysis and release of $\mathrm{P}_{\mathrm{i}}$ most conformational rearrangements that followed GTP binding are reversed in elF5B.GDP (Fig. 2). Switch 1 flips back to its original position, thereby displacing the $\beta 13-\beta 14$ loop. Switch 2 loses part of its $\alpha$-helical structure and retracts toward the back of the protein to adopt a conformation nearly identical to that found in the apo form (Fig. S4). However, Asp476 in the G3 motif of Ct-elF5B.GDP remains in its activated position in contact with the $\mathrm{Mg}^{2+}$ ion. Likewise, Thr477 and Pro478 retain their activated positions, whereas Gly479 is rotated back. As a consequence, the entire switch 2 is still shifted $\sim 3 \AA$ relative to its apo position but already forms the interactions to domains II and III found in apo elF5B (Fig. S4). Accordingly, $\alpha 9$ and therewith domain III are shifted 3-4 A relative to their apo state position. Finally, also the ventral side of domain II is still shifted forward, however, no upward movement of its frontal face occurs, indicating that the loss of interactions between domains I and III is a prerequisite for this domain rearrangement (Fig. S4C).

In summary, the transition from the GTP to the GDP state allows domain III to reassociate with the core domains of elF5B and reverses the rotation of domain II. Interestingly, binding of GDP and $\mathrm{Mg}^{2+}$ seems to be able to partially activate switch 2 (including the peptide flip of Gly479), which induces conformational strain on the switch 2-domain III interactions. 
A

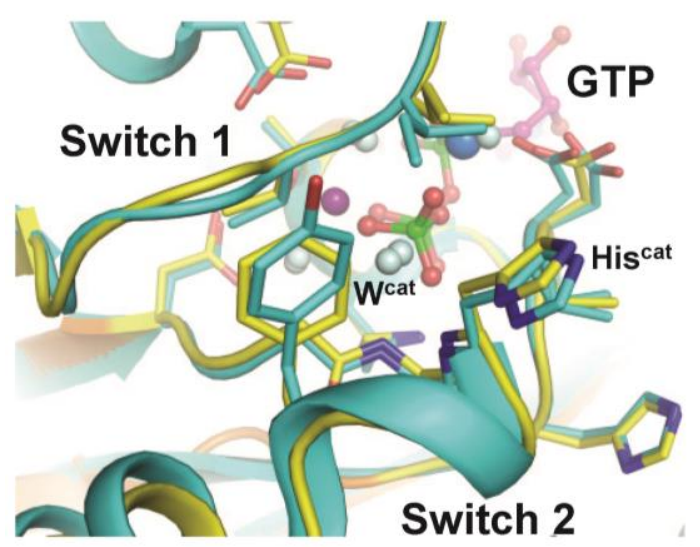

C

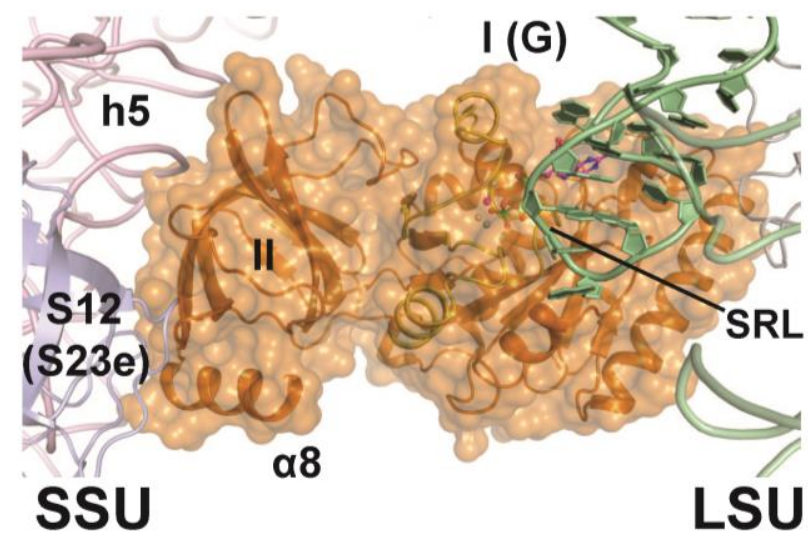

B

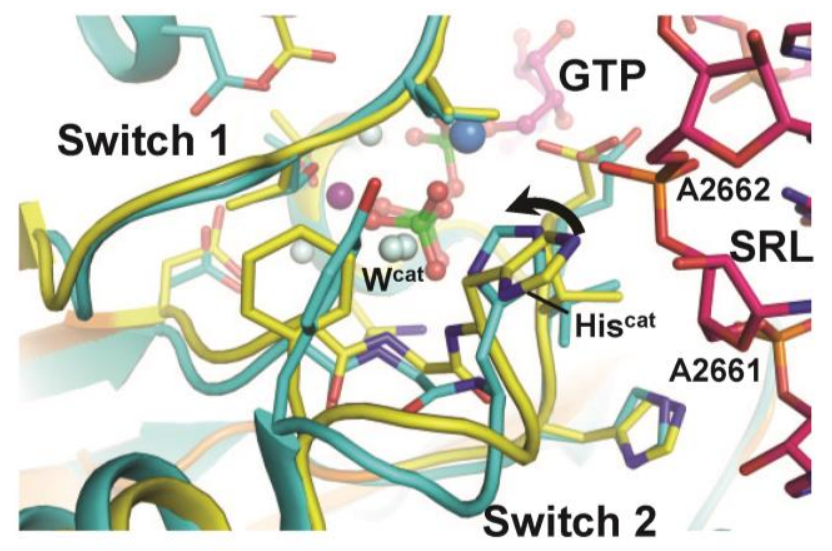

D

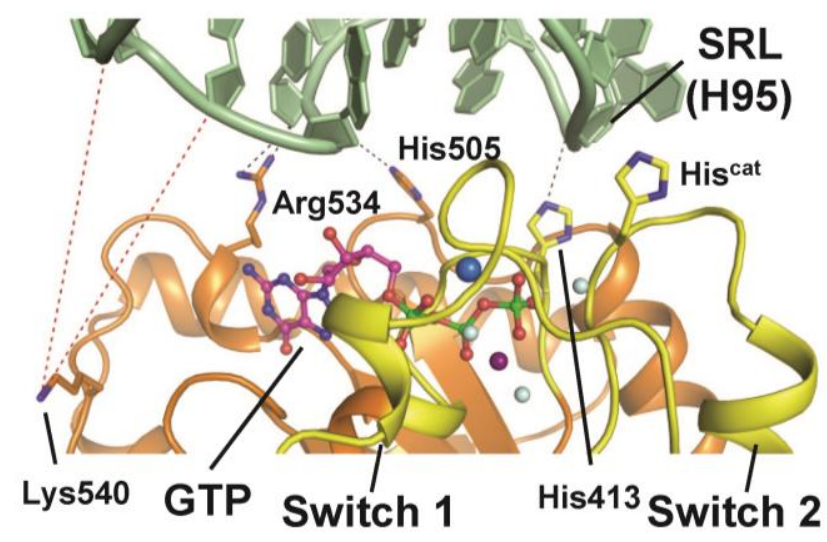

Figure 4. Structural model of elF5B.GTP on the ribosome.A) Superposition of the catalytic centers of elF5B.GTP (yellow) and free EF-Tu.GDPNP (cyan; PDB: 1EXM). Conserved residues are shown as sticks; GDPNP is omitted for clarity. B) Superposition of the catalytic centers of elF5B.GTP (yellow) and ribosomebound EF-Tu.GDPCP (PDB: 2XQD, 2XQE) with the sarcin-ricin loop (SRL) in pink. Structural alterations relative to free elF5B.GTP and EF-Tu.GDPNP are limited to His ${ }^{\text {cat }}$ of EF-Tu, which is reoriented (arrow) into its active position between A2662 and $W^{\text {cat }}$. C) Model of domains I and II of elF5B (orange) on the ribosome, based on the superposition with EF-Tu.GDPCP. Similar to elF5B.GDPCP in the cryo-EM model of the 80S IC (see Fig. S5B) the G domain is associated with the SRL of the large subunit (LSU; green), while domain II interacts with the body of the small subunit (SSU; light pink). D) Putative interactions between the $\mathrm{G}$ domain and the SRL/H95 (green). Direct interactions are indicated by black dashed lines; red dashed lines indicate the positions in $\mathrm{H} 95$ that are cleaved by Fe(II)-BABE introduced in the position of Lys540 [90]. His505 lies only $3.5 \AA$ from $\mathrm{H} 95$, explaining why the $\mathrm{H} 505 \mathrm{Y}$ mutation results in a reduced affinity for the ribosome and GTPase deficiency in elF5B [85] (see also Table S1). The conserved Arg534 likely contributes to the interactions with H95. 


\section{Thermodynamics of the interactions between elF5B and guanine nucleotides}

In order to gain further insight into the domain rearrangements during the nucleotide cycle of elF5B we performed ITC experiments to determine the thermodynamic parameters of elF5B binding to GTP and GDP in the temperature interval of $5-30{ }^{\circ} \mathrm{C}$ (a summary of the data is given in Tables II and III; Fig. 5 and S6).

To probe the conformational changes in eIF5B upon GTP binding and to test the particular influence of domain III we performed ITC experiments with two different constructs, one comprising domains I-IV (Ct-elF5B(517C)), the other comprising only domains I and II (CtelF5B(517-858)). In both cases, GTP binding was driven by favorable negative changes in binding enthalpy $\left(\Delta \mathrm{H}=-9.34 \mathrm{kcal} / \mathrm{mol}\right.$ for domains I-IV and $-18.8 \mathrm{kcal} \cdot \mathrm{mol}^{-1}$ for domains I-II at $\left.30^{\circ} \mathrm{C}\right)$ and opposed by unfavorable entropic contributions (Table II).

For both constructs, $\Delta \mathrm{H}$ plotted against the temperature results in a straight line with negative slope (Fig. $5 A)$ representing the change in heat capacity $\left(\Delta C_{p}\right)$ which can be used as estimate for the change in solvent accessible surface area $(\triangle A S A)$ upon complex formation (see Materials and Methods). For Ct-elF5B(517C) a $\Delta \mathrm{C}_{\mathrm{p}}$ of $-155 \mathrm{cal} \cdot \mathrm{mol}^{-1} \cdot \mathrm{K}^{-1}$ is calculated, corresponding to 344 to $646 \AA^{2}$ of surface area that become buried upon GTP binding (Table III). In contrast, GTP binding to Ct-elF5B(517-858), the construct lacking domains III and IV, gives a $\Delta C_{p}$ of $-553 \mathrm{cal} \cdot \mathrm{mol}^{-}$ ${ }^{1} \cdot \mathrm{K}^{-1}$, corresponding to a GTP-dependent surface burial of 1229 to $2304 \AA^{2}$, in agreement with the $\sim 1800 \AA^{2}$ that become buried by switch 1 and the $\beta 13-\beta 14$ loop in domains I and II according to the crystal structures (Table III). Thus, the presence of domains III and IV contributes to the overall $\Delta \mathrm{C}_{\mathrm{p}}$ of $-155 \mathrm{cal} \cdot \mathrm{mol}^{-1} \cdot \mathrm{K}^{-1}$ in $\mathrm{Ct}-\mathrm{elF5B}(517 \mathrm{C}) \cdot \mathrm{GTP}$ with $+398 \mathrm{cal} \cdot \mathrm{mol}^{-1} \cdot \mathrm{K}^{-1}$ to compensate the contribution of $-553 \mathrm{cal} \cdot \mathrm{mol}^{-1} \cdot \mathrm{K}^{-1}$ by domains I and II alone. This corresponds to a $\triangle \mathrm{ASA}$ of 884 to $1658 \AA^{2}$ that are exposed upon GTP binding simultaneously to the burial of $\sim 1800 \AA^{2}$ (or 1229 to $2304 \AA^{2}$ ) in domains I and II. The only reasonable candidates that can account for this compensatory effect are the surface areas buried between domain III and the $\mathrm{G}$ domain $(\sim 1150$ $\AA^{2}$ ) and domain II ( 700 $\left.\AA^{2}\right)$, respectively, in apo elF5B (Fig. 5B). Thus, these data indicate that domain III is released from most or all its contacts with the $\mathrm{G}$ domain and domain II in response to the GTP-induced rearrangement of switch 2. 
Table 2.Thermodynamic parameters of elF5B binding to GDP and GTP at different temperatures and in presence or absence of $\mathrm{Mg}^{2+}$ ions.

\begin{tabular}{cccccccc}
\hline Construct & Ligand & $\begin{array}{c}\mathrm{MgCl}_{2} \\
{[\mathrm{mM}]}\end{array}$ & $\begin{array}{c}\mathrm{T} \\
{\left[{ }^{\circ} \mathrm{C}\right]}\end{array}$ & $\begin{array}{c}\mathrm{K}_{\mathrm{d}} \\
{[\mu \mathrm{M}]}\end{array}$ & $\begin{array}{c}\Delta \mathrm{H} \\
{[\mathrm{cal} / \mathrm{mol}]}\end{array}$ & $\begin{array}{c}\Delta \mathrm{G} \\
{[\mathrm{kcal} / \mathrm{mol}]}\end{array}$ & $\begin{array}{c}\mathrm{T} \Delta \mathrm{S} \\
{[\mathrm{kcal} / \mathrm{mol}]}\end{array}$ \\
\hline Ct-elF5B(517C) & & & & & & & \\
& GDP & 2.5 & 5 & 2.09 & -6286 & -7.23 & 1.0 \\
& GDP & 2.5 & 10 & 1.92 & -5057 & -7.4 & 2.3 \\
& GDP & 2.5 & 15 & 2.61 & -5240 & -7.36 & 2.0 \\
& GDP & 2.5 & 20 & 2.9 & -5520 & -7.43 & 1.9 \\
& GDP & 2.5 & 25 & 3.3 & -6058 & -7.48 & 1.4 \\
& GDP & 2.5 & 30 & 3.45 & -6650 & -7.58 & 0.9 \\
& GDP & 0 & 5 & 0.8 & -1855 & -7.76 & 5.9 \\
& GDP & 0 & 10 & 1.0 & -2385 & -7.77 & 5.4 \\
& GDP & 0 & 15 & 1.14 & -2805 & -7.84 & 5.0 \\
& GDP & 0 & 25 & 1.96 & -3912 & -7.79 & 3.9 \\
& GTP & 2.5 & 5 & 4.21 & -5346 & -6.84 & 1.5 \\
& GTP & 2.5 & 10 & 4.83 & -6192 & -6.89 & 0.7 \\
& GTP & 2.5 & 15 & 5.68 & -7033 & -6.92 & 0.12 \\
& GTP & 2.5 & 20 & 6.02 & -7423 & -7.0 & -0.6 \\
& GTP & 2.5 & 25 & 6.20 & -8426 & -7.1 & -1.3 \\
& GTP & 2.5 & 30 & 7.04 & -9344 & -7.03 & -2.2 \\
\hline Ct-elF5B(517-858) & & & & & & & -11.3 \\
\hline & GTP & 2.5 & 5 & 1.34 & -4860 & -7.47 & 2.6 \\
& GTP & 2.5 & 10 & 1.58 & -7822 & -7.52 & -0.3 \\
& GTP & 2.5 & 20 & 2.12 & -12985 & -7.61 & -5.4 \\
& GTP & 2.5 & 30 & 4.07 & -18810 & -7.48 & -11.3 \\
\hline
\end{tabular}

All measurements were performed two to four times; for GTP binding to both constructs and for GDP binding in the presence of $\mathrm{Mg}^{2+}$ the experiments were done with two independent purifications of the respective construct; for GDP binding in the absence of $\mathrm{Mg}^{2+}$ the experiments were done with protein from one purification.

$\mathrm{K}_{\mathrm{d}}$, dissociation equilibrium constant; calculated as $1 / \mathrm{K}_{\mathrm{a}}$.

$\mathrm{K}_{\mathrm{a}}$, association equilibrium constant; standard deviation did not exceed $\pm 15 \%$.

$\Delta \mathrm{H}$, standard enthalpy change; standard deviation did not exceed $\pm 10 \%$.

$\Delta G$, Gibbs energy; calculated from equation $\Delta G=-R T \ln K_{a}$.

$\mathrm{T} \Delta \mathrm{S}$, standard entropy change; calculated from equation $\Delta \mathrm{G}=\Delta \mathrm{H}-\mathrm{T} \Delta \mathrm{S}$.

GDP binding to Ct-elF5B(517C) was driven by favorable contributions of both, binding enthalpy and entropy $\left(\Delta \mathrm{H}=-6.65 \mathrm{kcal} / \mathrm{mol}\right.$ and $\mathrm{T} \Delta \mathrm{S}=0.9 \mathrm{kcal} / \mathrm{mol}$ at $\left.30^{\circ} \mathrm{C}\right)($ Table II). In contrast to GTP binding, the temperature dependency of $\Delta \mathrm{H}$ was not linear for GDP binding; instead the data between 10 and $30{ }^{\circ} \mathrm{C}$ fit better to a second order polynomial function, indicating a strong temperature dependency of $\Delta C_{p}$ (Fig. 5A). This suggests that the amount of contact surface within the formed complex changes over the used temperature range. At higher temperatures $\left(30{ }^{\circ} \mathrm{C}\right)$ 
Table 3. Changes in heat capacity and solvent accessible surface area for elF5B binding to GDP and GTP

\begin{tabular}{|c|c|c|c|c|c|}
\hline Construct & Ligand & $\begin{array}{c}\Delta \mathrm{C}_{\mathrm{p}} \\
{[\mathrm{cal} / \mathrm{mol} \cdot \mathrm{K}]}\end{array}$ & $\begin{array}{c}\Delta \mathrm{ASA}_{\min } \\
{\left[\AA^{2}\right]}\end{array}$ & $\begin{array}{c}\triangle \mathrm{ASA}_{\max } \\
{\left[\AA^{2}\right]}\end{array}$ & $\begin{array}{c}\Delta \mathrm{ASA}_{\text {calc }} \\
{\left[\AA^{2}\right]}\end{array}$ \\
\hline \multicolumn{6}{|l|}{ Ct-elF5B(517C) } \\
\hline & $\mathrm{GDP}+\mathrm{Mg}$ & $-140 \pm 23^{a}$ & -311 & -583 & \multirow[t]{3}{*}{$-400^{c}$} \\
\hline & GDP-Mg $_{-M}$ & $-102 \pm 5^{b}$ & -227 & -425 & \\
\hline & $\mathrm{GTP}_{+\mathrm{Mg}}$ & $-155 \pm 8^{b}$ & -344 & -646 & \\
\hline \multicolumn{6}{|c|}{ Ct-elF5B(517-858) } \\
\hline & $\mathrm{GTP}_{+\mathrm{Mg}}$ & $-553 \pm 11^{b}$ & -1229 & -2304 & $-1800^{c}$ \\
\hline \multicolumn{6}{|c|}{$\Delta C_{p}$, heat capacity change; obtained from $\Delta H / d T$} \\
\hline \multicolumn{6}{|c|}{$\begin{array}{l}\triangle \mathrm{ASA}_{\min } \text { and } \triangle \mathrm{ASA}_{\max } \text {, changes in solvent accessible surface areas assuming that all changes were conferred by either apolar or } 70 \% \text { apolar and } 30 \% \\
\text { polar surfaces, respectively } \\
\text { a Calculated for } 30^{\circ} \mathrm{C} \text { from the first derivative of the second order polynomial fit to } \Delta \mathrm{H} \text { measured at five different temperatures between } 10 \text { and } 30 \\
{ }^{\circ} \mathrm{C} \text {. }\end{array}$} \\
\hline
\end{tabular}

GDP binding results in a $\Delta \mathrm{C}_{\mathrm{p}}$ of $-140 \mathrm{cal} \cdot \mathrm{mol}^{-1} \cdot \mathrm{K}^{-1}$, corresponding to a surface burial of 311 to $583 \AA^{2}$ (Table III), which agrees well with a $\triangle \mathrm{ASA}$ of $\sim 400 \AA^{2}$ for GDP binding according to the crystal structures. However, the negative value for $\Delta C_{p}$ decreases with lower temperatures. Below $10{ }^{\circ} \mathrm{C}$ the second order polynomial behavior of $\Delta \mathrm{H}$ breaks down and $\Delta \mathrm{C}_{\mathrm{p}}$ changes sign, indicating a net exposure of ASA upon GDP binding. Here, three observations based on the elF5B.GDP structures are of particular interest: i) $\mathrm{GDP} / \mathrm{Mg}^{2+}$ is able to partially activate switch 2 and to induce conformational strain on its interactions to domains II and III (Fig. S4), ii) domain III contacts switch 2 primarily through ionic interactions (Fig. 3B) which are destabilized at low temperatures [158, 159], and iii) domain III is released from the $\mathrm{G}$ domain in molecule B of Sc-elF5B.GDP for which crystals were obtained at $10{ }^{\circ} \mathrm{C}$ (Table I; Fig. S1E and S7A). Since switch 1 and the $\beta 13-\beta 14$ loop remain flexible in this structure, the release of domain III upon GDP binding results in a positive $\triangle \mathrm{ASA}$ corresponding to a positive contribution to $\Delta \mathrm{C}_{\mathrm{p}}$ as observed in the ITC experiments. The nonlinear behavior of $\Delta \mathrm{C}_{\mathrm{p}}$ above $10{ }^{\circ} \mathrm{C}$ would thus indicate that domain III always has the propensity to be released in elF5B.GDP due to the partial activation of switch 2 in the presence of $\mathrm{Mg}^{2+}$, however, with a reduced tendency to do so with increasing temperatures at which the ionic interactions to the $\mathrm{G}$ domain become increasingly stable $[158,159]$. In line with this interpretation, 
we found that the temperature dependency of $\Delta \mathrm{H}$ does not break down at low temperatures when the ITC experiments are repeated with GDP in the absence of $\mathrm{Mg}^{2+}$ (Fig. 5A). Instead, $\Delta \mathrm{H}$ plotted against the temperature results in a straight line with a slope $\left(\Delta \mathrm{C}_{\mathrm{p}}\right)$ of $-102 \mathrm{cal} \cdot \mathrm{mol}^{-1} \cdot \mathrm{K}^{-1}$, which is comparable to that for the elF5B.GDP complex in the presence of $\mathrm{Mg}^{2+}$ at higher temperatures (Table III).

A

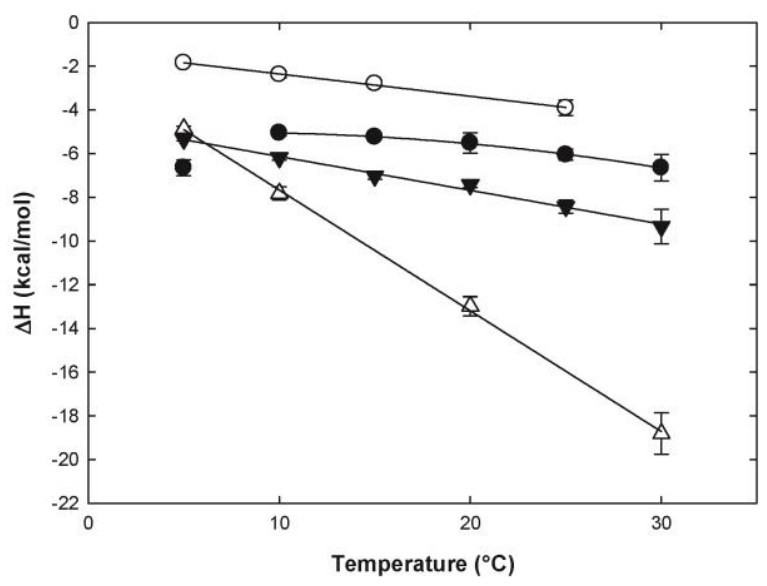

B

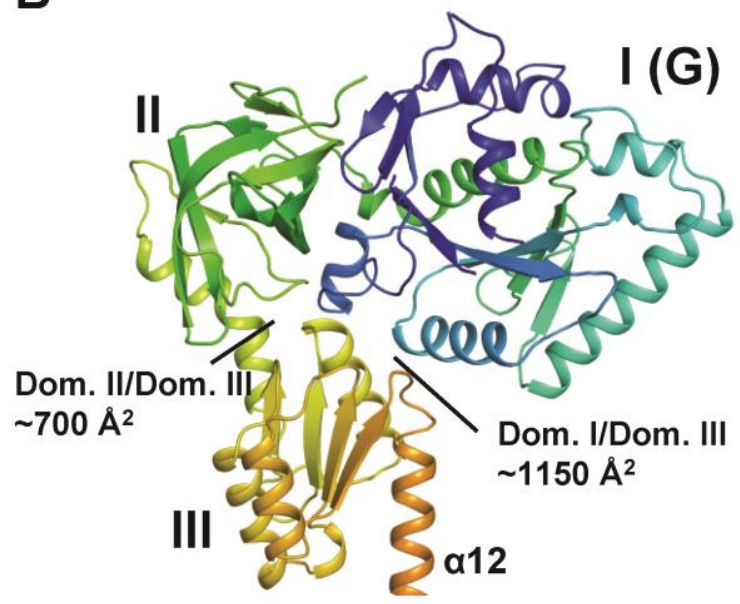

Figure 5.elF5B interactions with guanine nucleotides measured by ITC.A) Heat capacity changes upon elF5B interaction with GDP or GTP. Temperature dependency of binding enthalpy changes $(\Delta \mathrm{H})$ upon $\mathrm{Ct}$ elF5B(517C) interactions with GDP in the presence $(\bullet)$ or absence $(0)$ of $\mathrm{MgCl}_{2}$ and of Ct-elF5B(517C) ( $\left.\mathbf{\nabla}\right)$ and Ct-elF5B(517-852) $(\Delta)$ with GTP in the presence of $\mathrm{MgCl}_{2}$. Standard deviations are given by error bars (in some cases not visible because they are smaller than the symbol size). B) Domains I-III of apo Ct-elF5B. Indicated are the contact areas of domain III to domains I and II, respectively.

Taken together, these data support the idea that the conserved Asp476 in the G3 motif plays a critical role in the reorganization of switch 2 in response to nucleotide binding and indicate a direct connection between the $\mathrm{Mg}^{2+}$ ion and the temperature dependency of $\Delta \mathrm{C}_{\mathrm{p}}$ in the elF5B.GDP complex. The fact that GTP binding in contrast to GDP binding shows no temperature dependency of $\Delta C_{p}$ indicates that the interactions of domain III to domains I and II in apo elF5B are broken upon GTP binding regardless of the temperature at which the reaction takes place. However, here the 
positive contribution to $\Delta \mathrm{C}_{\mathrm{p}}$ is compensated by the large negative contribution due to the burial of ASA in the GTP-bound G domain.

\subsection{Discussion}

The molecular switch between the GDP- and GTP-bound states of elF5B: the domain release mechanism

The structural and thermodynamic data presented here provide the first detailed picture of the entire nucleotide cycle of elF5B. The structures of elF5B in its apo, GTP- and GDP-bound states reveal that its $\mathrm{G}$ domain follows the classical molecular switch mechanism, oscillating between an inactive (apo and GDP-bound) and a structurally distinct active GTP-bound form (Fig. 2). The GTPinduced transition from the inactive to the active conformation of the $G$ domain is characterized by marked rearrangements in the two switch regions, which allow the conserved Thr439 of switch 1 and Gly479 of switch 2 to directly contact the $\mathrm{y}$-phosphate and $\mathrm{W}^{\text {cat }}$, resulting in the formation of the catalytic GTPase center (Fig. 2B/D). This observation is in line with previous findings that the mutagenesis of Thr439 or Gly479 to Ala results in severe functional defects in elF5B, including GTPase deficiency $[85,160]$ (see also Table S1). In switch 2 the initial signal of GTP binding experiences a considerable amplification along its way through the inter-domain cleft toward the back of the protein with a movement of $3 \AA$ at Asp476, $8 \AA$ in Gly479, $12 \AA$ in His480 and finally $\sim 14 \AA$ in Arg487 and Arg489, which form the primary contact surface for domain III in apo elF5B (Fig. 3A/B and S4A/B). As a result, domain III is released from the activated $G$ domain, accompanied by the counter-clockwise rotation of domain II with respect to the $\mathrm{G}$ domain (Fig. 3A). As indicated by the ITC data, domain III remains released in free elF5B.GTP and does not form stable new contacts to the reorganized switch regions. Thus, the signal of GTP binding is amplified from a relatively small conformational change in the nucleotide binding pocket into the release of domain III and thereby ultimately translated into a gain of conformational freedom and higher structural flexibility of domains III and IV relative to domains I and II.

This mechanism for the activation of elF5B in solution contradicts earlier assumptions that GTP alone is insufficient to induce the conformational switch in free elF5B in the absence of the ribosome as auxiliary cofactor, which forms the basis for the hypothesis that elF5B follows a 
mechanism of conditional switching [153]. Moreover, the domain release mechanism is in stark contrast to the previously proposed non-classical articulated lever model for elF5B/IF2 function, in which the GTP-induced conformational changes in the G domain are limited to a $\sim 2 \AA$ shift in switch 2 . This causes a rigid body movement of domains III and IV and a displacement of the latter by $\sim 5 \AA$ as ultimate result of elF5B activation $[84,144]$. In contrast to the release mechanism, this involves neither a conformational change in switch 1 or switch 2 to form the canonical catalytic GTPase center nor does it require the loss or the formation of contacts between $\mathrm{G}$ domain and domains II and III at any stage of the activation process (Fig. S8). The articulated lever model therefore does not explain why switch 1 and switch 2 are universally conserved among elF5B orthologs and why the mutagenesis of conserved residues in both motifs results in severe functional defects in elF5B $[85,161,162]$.

\section{elF5B combines the classical GTP operated switch mechanism in the Gomain with a novel mechanism of activation for a trGTPase}

TrGTPases such as elF5B and EF-Tu are multidomain proteins which consist of a universally conserved structural core composed of the $\mathrm{G}$ domain and domain II that is supplemented with additional functional domains related to the respective role of the GTPase during translation. The activation of trGTPases by GTP is therefore not merely restricted to the $\mathrm{G}$ domain but involves a reorganization of the overall domain arrangement, induced by a modulation of the interactions between $\mathrm{G}$ domain and the downstream functional domains.

This principle was first established for the elongation factor EF-Tu. Here, the GTP-induced transition from the inactive apo form to the GTP-bound state depends on the rearrangement of the switch regions in the $G$ domain that follows the canonical switch mechanism of Ras-like GTPases [163]. As a consequence, domain II, which is separated from the G domain in inactive EF$\mathrm{Tu}$, stably associates with the reorganized $\mathrm{G}$ domain involving the newly formed surface of the activated switch 2 [163] (Fig. 3C). Only in this more compact GTP-conformation EF-Tu is able to form a stable ternary complex with aminoacyl-tRNA - involving also the reorganized switch 1 - for delivery of the latter to the ribosome [48, 164-167]. Thus, the presence of the defined 
activeconformation of switch 1 and 2 functions as the critical signal and thereby is a necessity for the overall activation of EF-Tu (Fig. 3C).

The same basic principle of activation also applies to elF5B. The $G$ domain follows the classical molecular switch mechanism (Fig. 2) and the signal of $G$ domain activation is propagated into domains II-IV through the reorganization of the switch regions. However, the mechanism by which this signal transduction is achieved appears to be different from that in EF-Tu and so far unprecedented in trGTPases: in eIF5B the GTP-induced absence of the inactive conformation of switch 2 and the resulting release of domain III seems to be the decisive signal that renders elF5B.GTP activated for productive interactions with the ribosome (Fig. 3). This does not imply that the GTP-bound conformation of the $G$ domain is irrelevant for elF5B function but is most likely required for tight interactions of the $G$ domain with the large ribosomal subunit and GTPase activity (Fig. 4) as well as to prevent the reassociation of domain III before GTP hydrolysis. Instead, this means that the defined GTP-conformation is not as critical for productive interactions between elF5B and its effector molecules as it is for EF-Tu.

This scenario is in line with earlier biochemical studies that identified mutations in the $G$ domain and domain III that are able to partially activate elF5B by destabilizing the interactions between domain III and switch 2 in inactive elF5B. The mutation of Gly479 in switch 2 to Ala was found to reduce GTP binding and to impair subunit joining and ribosome dependent GTP hydrolysis [160]. Our structural analysis shows that this Gly residue undergoes a peptide flip of $\sim 160^{\circ}$ during the transition of switch 2 from its inactive to the active state (Fig. 4B), a conformational change that is energetically not allowed for any other residue. G479A would thus stabilize the inactive switch 2 preventing the formation of the GTPase center and the release of domain III, ultimately causing the inability of the mutant to promote subunit joining and to hydrolyze GTP. A444V and D740R were identified as two independent intragenic suppressor mutants for G479A that restore nucleotide binding, GTP hydrolysis and subunit joining activities in elF5B [160]. Interestingly, Asp740 is located in domain III $\sim 30 \AA$ apart from the nucleotide binding pocket and forms a direct salt bridge to the conserved Arg489 of the inactive switch 2 which moves $\sim 15 \AA$ upon GTP binding (Fig. 3B and S7B/C). Consequently, D740R would result in a steric and electrostatic repulsion of the inactive switch 2 and thereby a destabilization of the domain III-switch 2 contact in apo elF5B. 
A444V is located at the $\mathrm{N}$-terminus of strand $\beta 3$ close to Asp476 in the G3 motif and most likely causes the constitutive reduction of the energy barrier that has to be overcome by GDP and GTP to move Asp476 into a GTP-like position thereby facilitating the distortion of the interactions between switch 2 and domain III [160] (Fig. S7C). Particularly interesting is that A444V does not only restore GTP dependency in eIF5B but even allows GDP to activate elF5B for stable interactions with the ribosome [160]. This demonstrates that the full GTP-conformation in the G domain is not an absolute requirement for stable interactions between elF5B and the ribosome. Instead, it seems to be critical that the suppressor mutations overcome the increased energy barrier introduced by G479A by destabilizing the inactive conformation and thereby the contact between switch 2 and domain III either directly in the case of D740R or indirectly in the case of A444V (see also Table S1).

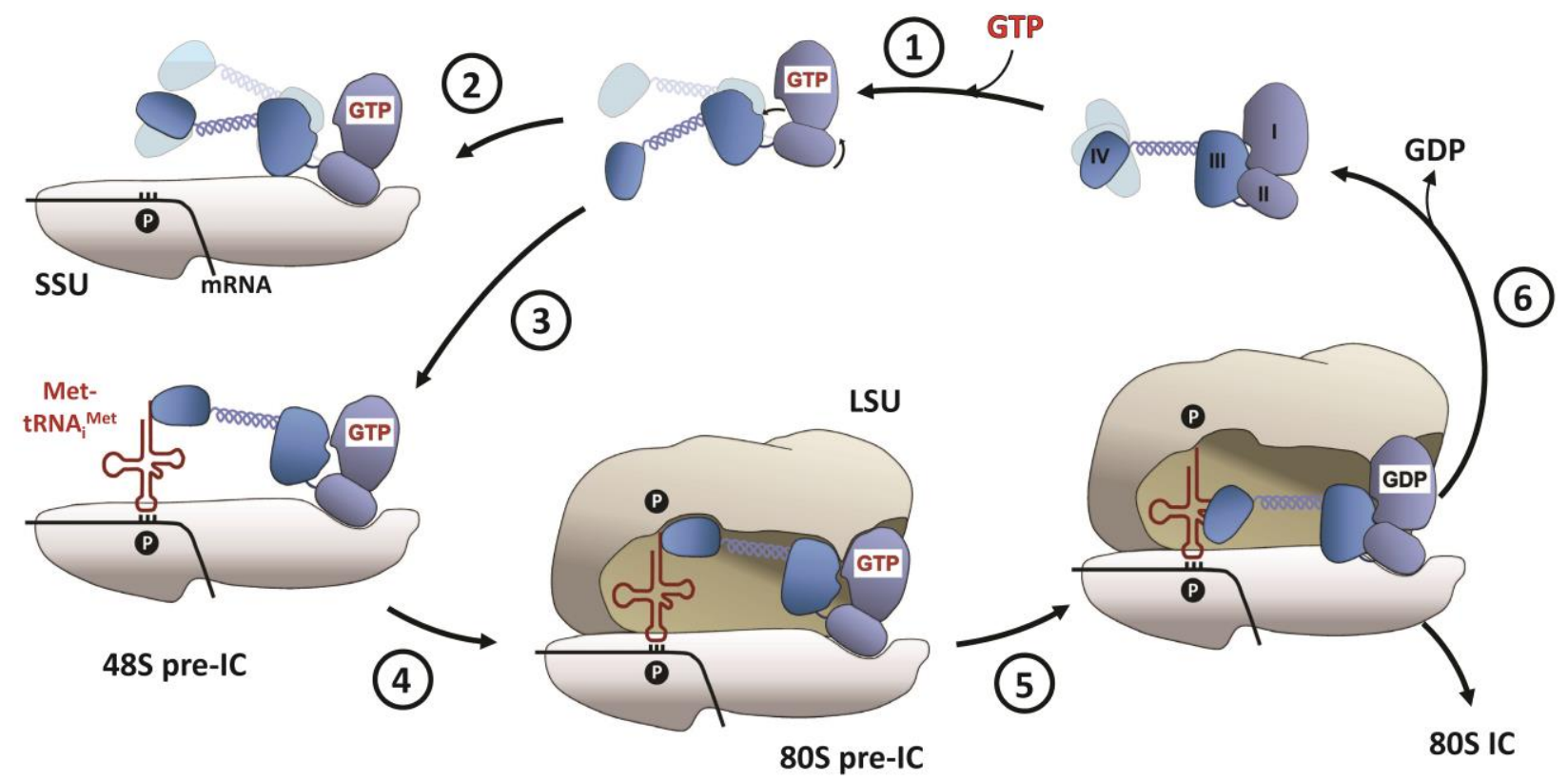

Figure 6.Schematic model of the of nucleotide cycle of elF5B during subunit joining.elF5B with domains I (G) to IV is shown in blue. (1) GTP binding activates elF5B by release of domain III and rotation of domain II relative to the $G$ domain. (2) Binding of elF5B.GTP to the small subunit (SSU) in the absence of Met-tRNA ${ }_{i}^{\text {Met }}$ results in a nonproductive complex in which elF5B is not able to stimulate subunit joining. (3) In the correctly preassembled $48 \mathrm{~S}$ pre-IC the subunit joining competent conformation of elF5B.GTP is stabilized by the $P$ site-bound initiator-tRNA, (4) resulting in the recruitment of the large ribosomal subunit (LSU). (5) Formation of the $80 \mathrm{~S}$ pre-IC triggers GTP hydrolysis in elF5B, which reverts back into its inactive conformation, (6) followed by the dissociation of elF5B.GDP from the elongation competent $80 \mathrm{~S}$ ribosome. 


\section{Implications of the domain release mechanism for ribosomal subunit joining}

elF5B interacts with the ribosomal subunits and catalyzes subunit joining in a GTP-dependent manner $[145,146]$. This indicates that elF5B undergoes a structural transition from the inactive apo-conformation to a GTP-bound state that allows productive interactions with its ribosomal effector complexes. However, it was shown in kinetic experiments that even in its GTP-bound state, elF5B is unable to catalyze subunit joining unless the Met-tRNA ${ }_{i}{ }^{\text {Met }}$ has been positioned in the $P$ site of the 40S subunit [146]. This seemingly paradoxical situation is compatible with the domain release mechanism of elF5B activation. On the one hand the GTP-induced release of domain III allows domain II to stably interact with the 18S rRNA (without steric hindrance by domain III as discussed below; see also Fig. S5C). On the other hand the conformational freedom of domains III and IV relative to each other (Fig. S2B), relative to domains I and II, as well as to the $40 \mathrm{~S}$ subunit, prevent elF5B from self-supporting a conformation that allows efficient subunit docking on the 40S-elF1A complex without Met-tRNA ${ }_{i}^{\text {Met }}$ (Fig. 6). Consequently, for the domain release model a distinction has to be drawn between the activated state of elF5B.GTP in solution and its subunit joining competent conformation on the $48 \mathrm{~S}$ pre-IC, which is only one of the many possible conformations accessible to the free elF5B.GTP, and which requires the reduction of conformational freedom of domains III and IV and their stabilization in the correct orientation. This proposed dependency of elF5B.GTP on the ribosomal effector complex resembles the hypothesis of conditional switching for elF5B [153]. However, the critical conceptual difference is that elF5B does not require the ribosome as cofactor to induce the GTP-dependent conformational switch, but instead depends on the ribosomal effector complex to stabilize the sole conformation of GTPbound elF5B (among the many possible) capable to promote the association of the 60S subunit. The recent cryo-EM structures of elF5B on the $80 \mathrm{~S}$ ribosome demonstrate that this stability is primarily provided by the methionylated $3^{\prime}$-CCA end of Met-tRNA ${ }_{i}{ }^{\text {Met }}{ }^{[91]}$. In agreement with the domain release mechanism, the cryo-EM structures show that the activation of the $G$ domain by GDPCP is sufficient to induce the release of domain III from switch 2 and domain II, but insufficient to stabilize domains III and IV in a defined conformation, as they remain disordered in the absence of amino-acylated tRNA. Only through the interactions between domain IV and the amino-acylated tRNA, domain III and IV become stabilized in their subunit joining competent conformation, in 
which domain III is released from all its contacts to the G domain and domain II that are also found in the apo state of elF5B and is reoriented relative to domain I and II by $\sim 65^{\circ}$ (Fig. 3D and S5C) [91].

Taken together, according to the presented domain release mechanism the preparation of elF5B for the catalysis of subunit joining involves a two-step process: i) activation of the $G$ domain by GTP binding, resulting in the release of domain III and an increased intrinsic flexibility that allows productive binding to the effector complexes and ii) stabilization of elF5B.GTP in the subunit joining competent conformation by the $48 \mathrm{~S}$ pre-IC through a reduction of conformational entropy within the factor (Fig. 6). According to this scenario, elF5B requires GTP as well as the correctly assembled 40S.Met-tRNA ${ }_{i}^{\text {Met }}$ effector complex for its function. Thus, the domain release mechanism seems evolved to ensure the formation of productive 80S ICs by discriminating against pre-ICs that do not contain Met-tRNA ${ }_{i}^{\text {Met }}$.

\section{Ribosome induced GTP hydrolysis by elF5B}

Crystal structures of EF-G.GDPCP and EF-Tu.GDPCP on the ribosome suggest a common mechanism of GTPase activation for both elongation factors [168, 169]. According to the current model, the $G$ domain of the translation factor binds to the sarcin-ricin loop (SRL) of the $50 S$ ribosomal subunit and the GTPase activity is triggered as His ${ }^{\text {cat }}$ (His84 in EF-Tu) rotates inward, where it is stabilized by hydrogen bond interactions with the phosphate of A2662 (SRL) and $W^{\text {cat }}$ which becomes subsequently activated for the in-line attack on the $\gamma$-phosphate [168].

Our own structural investigations show that the catalytic centers in elF5B.GTP and free EFTu.GDPNP exhibit nearly identical positions for residues implicated in ribosome binding and GTP hydrolysis (Fig. 4). In line with the recent cryo-EM model of ribosome bound elF5B [91], this suggests that domains I and II of elF5B bind the $80 \mathrm{~S}$ ribosome in the same way as the bacterial elongation factor (Fig. 4 and S5A/B). As for His84 in free EF-Tu.GDPNP, the imidazole moiety of His480 ( His $^{\text {cat }}$ ) in elF5B points outward and therefore requires rearrangement in order to contact $W^{\text {cat }}$ (Fig. 4B). In combination with earlier studies that highlight the importance of His480 for GTP hydrolysis [161, 162], these observations strongly suggest that the ribosome dependent GTPase 
activation of initiation factor elF5B follows the same basic mechanism as employed for the elongation factors EF-Tu and EF-G.

\section{Dissociation of elF5B.GDP from the $80 \mathrm{~S}$ ribosome}

In accordance with the function of $G$ proteins as molecular switches, the formation of the elongation competent $80 \mathrm{~S}$ ribosome by eIF5B.GTP results in GTP hydrolysis and $\mathrm{P}_{\mathrm{i}}$ release, followed by the dissociation of elF5B.GDP $[145,161]$. Thus, the reduced affinity for the ribosome and dissociation of elF5B depends on its structural transition from the GTP-bound state to the inactive GDP-bound conformation.

Our data show that upon transition from the GTP- to the GDP-bound state the G domain of elF5B switches back into its inactive conformation (Fig. 2 and S4A). As a result, domain III is retrieved to interact with switch 2 and domain II, causing the latter to rotate clockwise relative to the $\mathrm{G}$ domain (Fig. 3A and $\mathrm{S} 4 \mathrm{C}$ ). This reorientation relative to each other necessarily disrupts the interactions of the $\mathrm{G}$ domain and domain II with the $60 \mathrm{~S}$ and $40 \mathrm{~S}$ subunits, respectively, which were formed by elF5B.GTP in the rotated state (the rotation is also observable in the cryo-EM structure of eIF5B in the 80S IC [91]). Under the assumption that the interactions between domain II and the 18S rRNA are the last to be broken (as they do not directly depend on the presence of GTP as do those of the G domain with the SRL), domain III moves away from the SRL and domain IV moves away from the peptidyl-transferase center (PTC) toward the 40S subunit and helix H38 (60S) (Fig. S5C). This position of elF5B.GDP is not stable as domains III and IV would clash with the ribosomal protein S23e and elF1A, respectively, and the G domain is rotated into the SRL. As a result, elF5B would have to retreat from the factor binding site toward h6 of the 40S subunit as seen in the cryo-EM structure of IF2.GDP on the 70S ribosome [170], followed by its dissociation (Fig. 6).

\section{IF2 might function according to the domain release mechanism}

Despite a large body of experimental data for IF2, its precise mode of function during subunit joining in bacteria is still unclear $[27,82,147,150,151]$. For the reasons discussed below it is in our opinion reasonable to assume that the domain release mechanism also applies to IF2. Nearly all 
residues involved in the contacts between switch 2 and domain III in elF5B, particularly also those in the N-terminus of helix $\alpha 9$, are highly conserved in a/elF5B/IF2 orthologs (Fig. S3). In structures of the IF2 G domain switch 2 is usually flexible with only partial helical character $[82,83,171]$ and would thus be easily accessible for interactions with domain III. Moreover, from the high degree of structural and sequence homology it can be inferred that the G domain of IF2, like in elF5B, follows the classical switch mechanism, resulting in the formation of the canonical active site. The transition of IF2 between the GDP- and GTP-bound states would therefore result in the release of domain III and an increase in the overall flexibility of the factor, and the reduction of flexibility and retraction of domain III upon GTP hydrolysis. Indeed this becomes apparent in cryo-EM structures of bacterial initiation complexes, where domain III is associated with domains I and II in IF2.GDP whereas the GTP/GDPNP-bound forms adopt an elongated shape to contact the $P$ site-bound fMet-tRNA $^{\text {fMet }}[152,170,172,173]$.

The assumption that the domain release mechanism applies to IF2 seems to be contradicted by two recent structural studies $[82,83]$. The only so far available high resolution structure of GTPbound IF2 appears to indicate that its $\mathrm{G}$ domain does not follow the classical switch mechanism as switch 1 remains virtually unchanged upon GTP binding and switch 2 undergoes only a small local rearrangement without forming a contact to the $\gamma$-phosphate [83]. However, it is important to note that this IF2.GTP structure was obtained by soaking GTP into crystals of apo IF2, in which both switch regions are fixed by extensive contacts to symmetry related molecules. A reorganization of the $G$ domain that would allow the $G 2$ and $G 3$ motifs to contact the GTP molecule in the classical way is thus most likely prevented by crystal contacts and not due to a non-classical behavior of IF2. The recent crystal structure of $T$. thermophilus IF2(3-467) in the apo and GDP-bound state as well seems to argue against the domain release mechanism for IF2 as domain III has no direct contact to either of the switch regions [82]. The authors propose that the increased length of helix $\alpha 8$ (the linker between domains II and III) compared to $\alpha 8$ in alF5B accounts for the inability of domain III to contact switch 2 [82]. However, the lengths of helix $\alpha 8$ and the following flexible linker to domain III are actually compatible with a direct contact between the N-terminal half of helix $\alpha 9$ and switch 2 as observed in apo a/elF5B (Fig. 3A/B). Instead, the crystal packing shows that the position occupied by domain III in a/elF5B is occupied by symmetry related molecules in the IF2 
crystals. Crystallization would therefore be selective for the state in which domain III is released, irrespective of its fraction among the IF2 molecules in solution. As we show by means of ITC and observe in the Sc-elF5B.GDP structure (Fig. S1E and S7A), domain III in elF5B has the ability to dissociate from the $G$ domain even in the absence of GTP. This, we suggest, also applies to IF2.

As for elF5B, the domain release model can explain why IF2 requires GTP for efficient interactions with ribosomal complexes and the fMet-tRNA ${ }^{\text {fMet }}$ in the context of the $30 \mathrm{~S}$ pre-IC [147, 174], why IF2.GTP is unable to promote subunit docking in the absence of the initiator-tRNA [175] and finally, why even GDP is able to partially activate IF2 for ribosome binding and subunit joining in in vitro studies despite its inability to stabilize the GTP-conformation of the G domain. With the critical conceptual difference that in the domain release mechanism the GTP-dependent conformational switch in IF2 precedes and is therefore not a consequence of its interaction with the ribosome, this hypothesis of a common mechanism for eIF5B and IF2 is in agreement with previous proposals of a stepwise activation mechanism for IF2 by GTP and the 30S.fMet-tRNA ${ }^{\text {fMet }}$ complex made on the basis of biochemical experiments $[147,154]$.

\subsection{Materials and Methods}

\section{Protein preparation, crystallization and structure determination}

The N-terminally His-tagged versions of elF5B from C. thermophilum (Ct-elF5B(517-1116), -(517970) and -(517-858)) and S. cerevisiae (Sc-elF5B(399-852)) were expressed in E. coli and purified using standard procedures. Crystals used for structure determination were obtained by sittingdrop vapor diffusion using standard screens either in the presence or absence of GDP or GTP (for details see Table I and supplementary text).

X-ray diffraction data were collected using synchrotron radiation. For all structures, the phase problem was solved by molecular replacement using the program PHASER [115]. Structures were refined to reasonable R-values and stereochemistry using the program PHENIX [117]. Data collection and refinement statistics are summarized in Table I. See supplementary text for details. 


\section{Isothermal Titration Calorimetry}

The thermodynamic parameters of Ct-elF5B binding to GDP or GTP were measured using a MicroCal VP-ITC instrument (GE Healthcare). Experiments were carried out essentially as described previously [176]. The obtained values for enthalpy changes $(\Delta H)$ at different temperatures were used to estimate the change in heat capacity $\left(\Delta C_{p}\right)$ for the various protein-nucleotide complexes. These $\Delta C_{p}$ values were then used to estimate the conformational changes occurring in Ct-elF5B upon GDP or GTP binding, using the empirically determined relation $\Delta \mathrm{C}_{\mathrm{p}}=\Delta \mathrm{c}_{\mathrm{ap}} \cdot \Delta \mathrm{ASA}_{\mathrm{ap}}+\Delta \mathrm{c}_{\mathrm{p}}$. $\Delta \mathrm{ASA}_{\mathrm{p}}$ (where $\Delta \mathrm{c}_{\mathrm{ap}}$ and $\Delta \mathrm{c}_{\mathrm{p}}$ are the area coefficients in $\mathrm{cal} \cdot \mathrm{K}^{-1} \cdot\left(\text { mole } \cdot \AA^{2}\right)^{-1}$ for contributions of apolar or polar side chains to the change in solvent accessible surface area ( $\triangle A S A)$, respectively) [177]. For more details see supplementary text.

\section{Coordinates}

Coordinates have been deposited in the PDB: Apo Ct-elF5B(517C) (4N3N); Ct-elF5B(870C) (4N3G); Ct-elF5B(517-970)·GDP (4NCL); Ct-elF5B(517-858).GTP (4NCN); Apo Sc-elF5B(399-852) (4N3S); ScelF5B(399-852)·GDP (4NCF).

\section{Supplementary Information}

The Supplementary Information includes extended Materials and Methods, eight figures and one table and is available at The EMBO Journal Online.

\section{Acknowledgments}

We thank the beam line scientists at BESSY (Berlin) and EMBL/DESY (Hamburg) as well as P. Neumann for support during X-ray diffraction data collection, M. Franke for technical assistance and L. K. Dörfel and A. Dickmanns for critical reading of the manuscript.

\section{Author Contributions}

Author contributions: BK designed the experiments, prepared and crystallized proteins, collected X-ray data, solved structures, performed ITC experiments, created figures. BK and RF analyzed the data and wrote the manuscript. 


\section{Conflict of interest}

The authors declare that they have no conflict of interest.

\subsection{Supplementary information}

\section{Supplementary Materials and Methods}

\section{Protein preparation and crystallization}

The plasmid for the expression of N-terminally His-tagged C. thermophilum (Ct-)elF5B(517C) (comprising residues 517-1116 that form the G domain, domain II, III and IV) was transformed into E. coli BL21 (DE3) cells (Stratagene) by heat shock. Transformed cells were grown in 1 I cultures of $2 Y T$ medium at $37{ }^{\circ} \mathrm{C}$ to an $\mathrm{OD}_{600}$ of $0.6-0.8$, followed by the induction of protein expression with $0.5 \mathrm{mM}$ isopropyl- $\beta$-D-thiogalactopyranoside (IPTG). Cells were grown for an additional $18 \mathrm{~h}$ at 16 ${ }^{\circ} \mathrm{C}$ before harvesting. The harvested cells were resuspended in buffer $\mathrm{A}$ (40 mM Hepes (pH 7.5), $500 \mathrm{mM} \mathrm{KCl}, 10 \%$ glycerol, $30 \mathrm{mM}$ imidazole, $2 \mathrm{mM} \beta$-mercaptoethanol), lysed using a Microfluidizer (Microfluidics) and clarified by ultracentrifugation. The supernatant was loaded onto two HisTrap columns (GE Healthcare), equilibrated in buffer A. After the elution of bound proteins with a linear gradient of imidazole $(30-300 \mathrm{mM})$, elF5B-containing fractions were pooled and desalted in buffer $\mathrm{B}(20 \mathrm{mM}$ Hepes ( $\mathrm{pH} 7.5), 200 \mathrm{mM} \mathrm{KCl}, 10 \%$ glycerol, $30 \mathrm{mM}$ imidazole and 2 $\mathrm{mM} \beta$-mercaptoethanol) for subsequent TEV-protease cleavage at $4{ }^{\circ} \mathrm{C}$ over night to remove the His-Tag. Uncleaved protein was removed by a second HisTrap in buffer B and the flow-through was pooled and concentrated for the final size exclusion chromatography step on a Superdex 200 column (GE Healthcare), equilibrated in buffer C (10 mM Hepes (pH 7.5), $50 \mathrm{mM} \mathrm{KCl,} 3 \mathrm{mM} \mathrm{MgCl} 2$ and $1 \mathrm{mM}$ DTT). The purified elF5B was finally pooled and concentrated to $15-20 \mathrm{mg} / \mathrm{ml}$. Other versions of Ct-elF5B containing residues $517-970$ or 517-858 and of S. cerevisiae (Sc-) elF5B containing residues 399-852 were purified according to the same protocol.

For ITC experiments truncated versions of Ct-elF5B were purified according to the above protocol with the difference that ITC buffer (see below) was used in the size exclusion chromatography step. The concentration of the protein was determined by absorbance at $280 \mathrm{~nm}$ (with extinction coefficients for the different Ct-elF5B constructs: $\varepsilon_{517 \mathrm{C}}=30370 \mathrm{M}^{-1} \mathrm{~cm}^{-1}, \varepsilon_{517-970}=20400 \mathrm{M}^{-1} \mathrm{~cm}^{-1}$ and $\left.\varepsilon_{517-852}=14440 \mathrm{M}^{-1} \mathrm{~cm}^{-1}\right)$. The protein was $>95 \%$ pure as judged by SDS-PAGE and free of nucleotides as determined by HPLC.

Crystals of Ct-elF5B(517C) without nucleotide were obtained after two days at $4{ }^{\circ} \mathrm{C}$ via sitting-drop vapor diffusion against $100 \mathrm{mM}$ MES (pH 6.8), 12\% PEG 20000 and $10 \mathrm{mM} \mathrm{Na-lactate.} \mathrm{The} \mathrm{crystals}$ grow in primitive hexagonal space group $\mathrm{P}_{2} 21$ with one molecule per asymmetric unit (unit cell: a $=b=111.47 \AA, c=115.21 \AA, \alpha=\beta=90^{\circ}, y=120^{\circ}$; diffraction limit = $2.75 \AA$ ). 
Despite extensive trials we were not able to obtain crystals of the Ct-elF5B(517C) construct in the GDP- or GTP-bound forms. However in the presence of GTP another type of crystals was obtained after 5 days at $4{ }^{\circ} \mathrm{C}$ under the above conditions. These crystals grew in the primitive hexagonal space group P3 121 (unit cell: $a=b=98.23 \AA$, $c=97.42 \AA, \alpha=\beta=90^{\circ}, y=120^{\circ}$; diffraction limit = 3.2 $\AA$ ) with one molecule per asymmetric unit comprising only domains III and IV of Ct-elF5B (residues 870-1116).

Crystals of Ct-elF5B(517-970) in the presence of $2 \mathrm{mM}$ GDP grew within 4 days at room temperature against 15\% PEG 8000 and $0.5 \mathrm{M} \mathrm{Li}_{2} \mathrm{SO}_{4}$ in primitive orthorhombic space group $\mathrm{P} 2{ }_{1} 2_{1} 2_{1}$ with two protein-GDP complexes per asymmetric unit (unit cell: $a=66.9 \AA$, $b=72.96 \AA$, $c=$ 199.23 $\AA, \alpha=\beta=\gamma=90^{\circ}$; diffraction limit $=2.1 \AA$ ).

Crystals of Ct-elF5B(517-970) in the presence of $2 \mathrm{mM} \mathrm{GTP}$ grew over night at room temperature against $100 \mathrm{mM}$ Hepes (pH 7), 13\% PEG 4000 and $100 \mathrm{mM}$ sodium acetate in primitive monoclinic space group $\mathrm{P} 2{ }_{1}$ with two protein-GTP complexes per asymmetric unit (unit cell: $a=55.4 \AA, b=$ $114.83 \AA, c=65.85 \AA, \alpha=90^{\circ}, \beta=102.3^{\circ}, \gamma=90^{\circ}$; diffraction limit $=1.87 \AA$ ).

Crystals of the nucleotide free form of Sc-elF5B(399-852) grew after 2 days at $4{ }^{\circ} \mathrm{C}$ against $20 \%$ ethylene glycol, 5\% PEG 3350 and $20 \mathrm{mM} \mathrm{MgCl}_{2}$ in primitive tetragonal space group P41 with two molecules per asymmetric unit (unit cell: $a=b=118.01 \AA, c=77.51 \AA$, $\alpha=\beta=\gamma=90^{\circ}$; diffraction limit $=1.83 \AA$ ) . The data were twinned with a twin fraction of $18 \%$ (twin law $h,-k,-1)$.

Finally, crystals of the GDP-bound form of Sc-elF5B(399-852) could be obtained over night at $10^{\circ} \mathrm{C}$ against $8 \%$ PEG 8000 and $0.37 \mathrm{M} \mathrm{Li}_{2} \mathrm{SO}_{4}$ in primitive orthorhombic space group $\mathrm{P} 2{ }_{1} 2_{1} 2_{1}$ with two protein-GDP complexes per asymmetric unit (unit cell: $a=73.56 \AA, b=119.46 \AA, c=120.73 \AA, \alpha=\beta$ $=\gamma=90^{\circ}$; diffraction limit $=3.02 \AA$ ) .

\section{$X$-ray data collection, structure determination and refinement}

For the structure of Ct-eIF5B.GTP X-ray diffraction data were collected at BL 14.1 (HZB, BESSY, Berlin) [113]. All other structures were solved using X-ray diffraction data collected at P13 beamline (EMBL, PETRA III, Hamburg). The structure of free Ct-elF5B(517C) was solved by molecular replacement using the program PHASER [115] using the atomic coordinates of the archeal ortholog of elF5B from $M$. thermoautotrophicum (PDB: 1G7R) with the individual domains as independent search models. The structure was refined using the program PHENIX [117]. The other structures where solved by means of molecular replacement using the individual domains of the newly determined structure as search model as described below (Data collection and refinement statistics are summarized in Table I).

The second structure obtained from Ct-elF5B(517C), this time in the presence of GTP, was solved using domains I to IV of Ct-elF5B(517C) as independent search models. A solution was found only for domains III and IV and the final model after refinement comprises residues 870 to 1116 (domains III and IV). A Coomassie Blue-stained SDS-PAGE gel of dissolved crystals confirmed that domains I and II are missing, probably due to a proteolytic event during crystallization. 
The structures of Ct-elF5B(517-970) in the GDP- and GTP-bound state and of free Sc-elF5B(399852) were solved using domains I to III of Ct-elF5B(517C) as independent search models. The resulting models of Ct-elF5B(517-970) in complex with either GDP or GTP contains two copies of Ct-elF5B(517-970), each bound to a $\mathrm{Mg}^{2+}$ ion and GDP or GTP, respectively. In the final model of CtelF5B(517-970).GTP residues 517-859 are resolved in the electron density, however the entire domain III (residues 860-970) is missing, probably due to a proteolytic event during crystallization as indicated by a Coomassie Blue-stained SDS-PAGE gel of dissolved crystals. The final model of the nucleotide-free form of Sc-elF5B(399-852) contains two copies of Sc-elF5B(399-852).

Finally, the structure of Sc-elF5B.GDP was solved using domains I to III of free Sc-elF5B(399-852) as independent search models. The final model contains two Sc-elF5B.GDP complexes in the asymmetric unit.

\section{Isothermal Titration Calorimetry}

The thermodynamic parameters of elF5B binding to GDP or GTP were measured by means of ITC using a MicroCal VP-ITC instrument (GE Healthcare). Experiments were carried out in ITC buffer (30 $\mathrm{mM}$ Hepes pH 7.5, $100 \mathrm{mM} \mathrm{KCl}, 10 \%$ glycerol, $4 \mathrm{mM} \beta$-mercaptoethanol, $0.01 \%$ tween 20, $2.5 \mathrm{mM}$ $\mathrm{MgCl}_{2}$ ) at six different temperatures $\left(5,10,15,20,25\right.$ and $\left.30^{\circ} \mathrm{C}\right) .14-\mu$ l aliquots of $200-400 \mu \mathrm{M}$ ligand were injected into the $1.42 \mathrm{ml}$ cell containing 10-30 $\mu \mathrm{M}$ elF5B. The heat of dilution was measured by injecting the ligand into the buffer solution without protein; the values were then subtracted from the heat of the individual binding reactions to obtain the effective heat of binding. The final titration curves were fitted using the 'Origin' based MicroCal software, assuming one binding site per protein molecule. For each isotherm the binding stoichiometry $(\mathrm{N})$, enthalpy changes $(\Delta \mathrm{H})$ and the association constants $\left(\mathrm{K}_{\mathrm{a}}\right)$, were obtained by a nonlinear regression fitting procedure. These directly measured values were then used to estimate the Gibbs energy $(\Delta G)$ from the relation $\Delta G=-R \cdot T \cdot I n K_{a}$ and the entropy changes $(\Delta S)$ through $\Delta G=\Delta H-T \cdot \Delta S$.

To investigate the influence of the $\mathrm{Mg}^{2+}$ ion on the binding of GDP to elF5B, experiments were repeated in a modified ITC buffer, containing $0.25 \mathrm{mM}$ EDTA instead of $\mathrm{MgCl}_{2}\left(\mathrm{Mg}^{2+}\right.$ was omitted also from the buffers used during purification).

In order to estimate the change in heat capacity $\left(\Delta C_{p}\right)$ upon complex formation, the measured $\Delta H$ values were plotted against the temperature $[178,179]$. Depending on whether a linear or nonlinear temperature dependency was applicable for $\Delta \mathrm{H}$, the data were fitted either to a linear or a second-order polynomial function. In the first case, the slope of the fitted line directly represents the $\Delta C_{p}$ of the binding reaction, whereas in the second case the $\Delta C_{p}$ for a given temperature can be calculated from the first derivative of the polynomial function.

$\Delta C_{p}$ can be used as an estimate for the change in solvent-accessible surface area ( $\triangle A S A$ ) upon complex formation as it was found to be proportional to the size of the area which is either exposed to or excluded from the aqueous environment during the binding event $[176,177,180$ 183]. $\Delta C_{p}$ and $\triangle A S A$ are connected by the empirically determined relation $\Delta C_{p}=\Delta C_{a p} \cdot \Delta A S A_{a p}+\Delta C_{p}$ 
$\cdot \Delta \mathrm{ASA}_{\mathrm{p}}$ where $\Delta \mathrm{c}_{\mathrm{ap}}$ and $\Delta \mathrm{c}_{\mathrm{p}}$ are the area coefficients in cal $\cdot \mathrm{K}^{-1} \cdot\left(\text { mole } \cdot \AA^{2}\right)^{-1}$ for the contributions of apolar or polar side chains to $\triangle A S A$, respectively [177]. Studies on the dissolution of model compounds and protein unfolding suggest a negative contribution due to the burial of apolar surfaces $\left(\triangle A S A_{a p}\right)$ and a positive contribution upon burial of polar surfaces $\left(\triangle A S A_{p}\right)$ with values for $\Delta c_{\text {ap }}$ and $\Delta c_{p}$ of 0.45 and -0.26 , respectively $[177,182,184]$. Since binding of guanine nucleotides can be expected to involve both, apolar and polar residues, a treatment of the obtained $\Delta C_{p}$ values solely based on nonpolar contributions according to Connelly et al. (1992) would be insufficient. At the same time, the differential treatment by calculating the individual contributions of apolar and polar groups to the total surface area often proved to be inaccurate, especially in cases where the interactions did not conform to a rigid-body binding model [185-188]. We therefore use two values for the area coefficients to estimate the total surface area upon ligand binding: $\Delta c_{\max }=0.24$ (= $0.7 \cdot \Delta c_{\text {ap }}+0.3 \cdot \Delta c_{p}$ ) as the upper limit case, assuming $\sim 70 \%$ apolar and $\sim 30 \%$ polar groups contributing to the total $\triangle A S A$ (calculated from the crystal structures of Ct-elF5B in its apo, GDP and GTP forms using the program AREAIMOL from the CCP4 Program Suite 6.3.0) and $\Delta c_{\min }=0.45$ as the lower limit case in which all involved residues are apolar. 


\section{Supplementary Images}

A

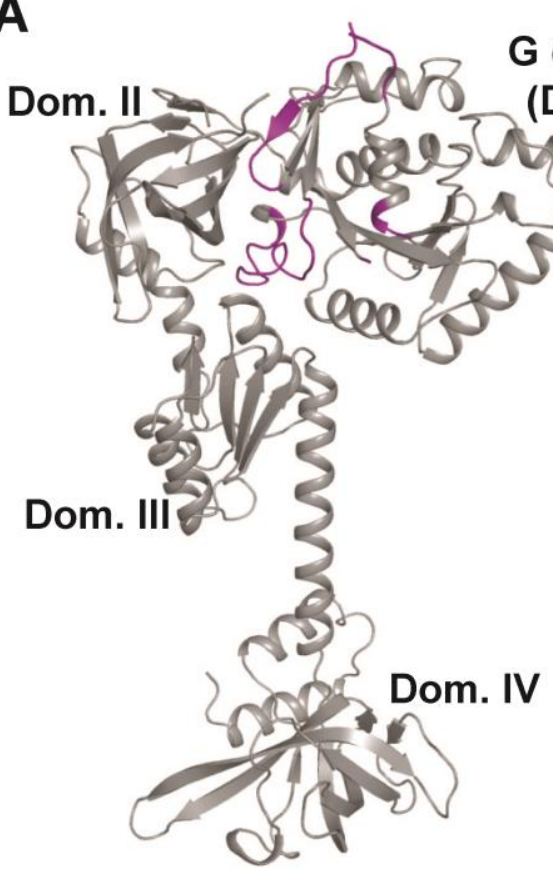

D

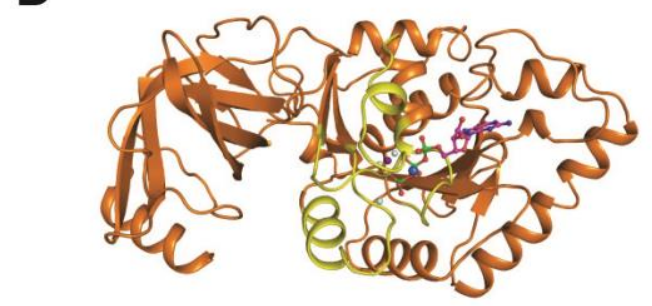

$\mathbf{F}$

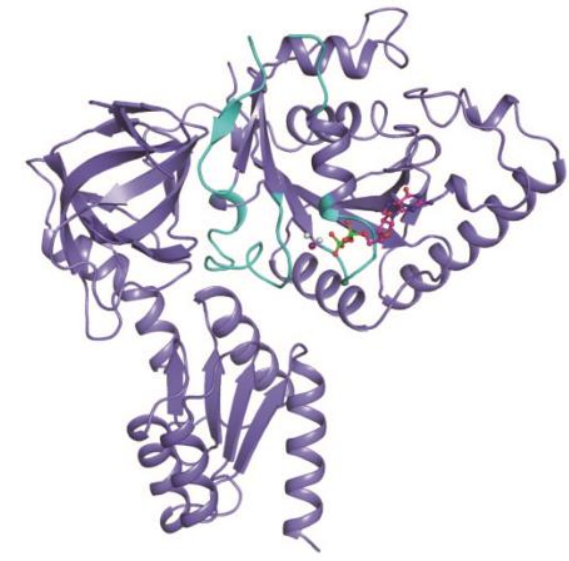

C

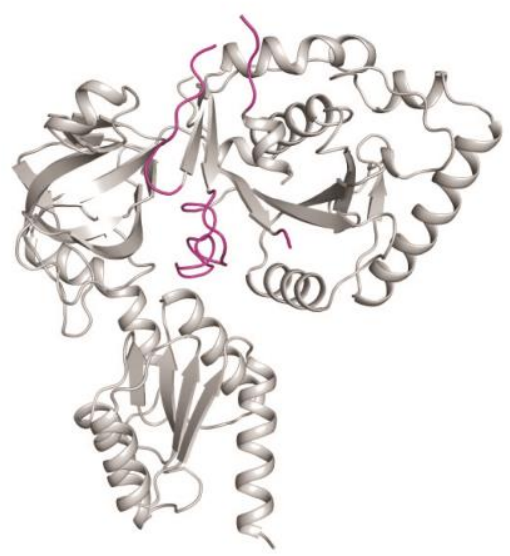

m. I)

\section{B}

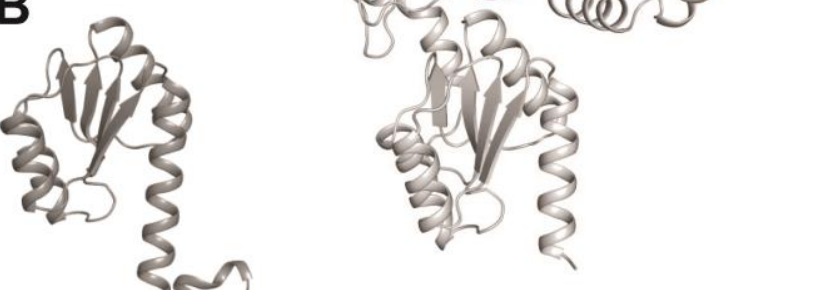

E

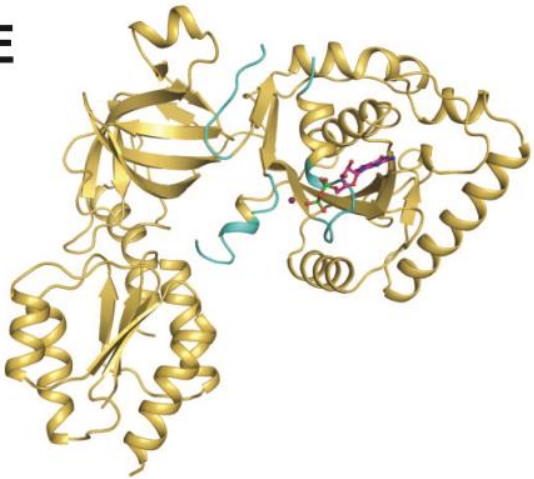

G

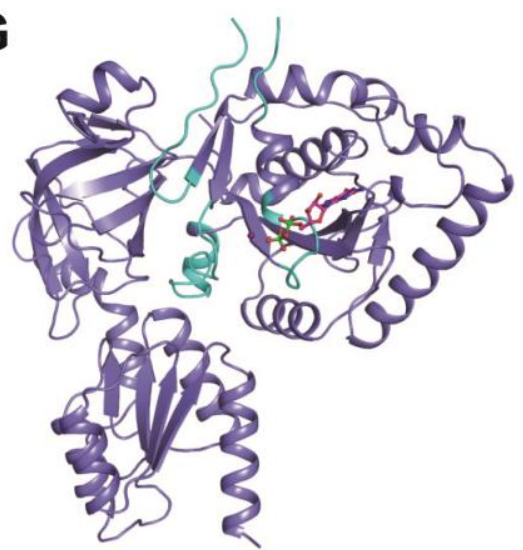

Figure S1.Structures of elF5B from C. thermophilum and S. cerevisiae.A) Structure of apo Ct-elF5B(517C) containing residues 517-1116 comprising the G domain (domain I) and domains II, III and IV. The P-loop, switch 1 and 2 are colored pink. B) Structure of Ct-elF5B(870C) with residues 870-1116, comprising domains 
III and IV. C) Structure of apo Sc-elF5B(401-852) comprising the G domain, domain II and domain III. Switch 1 and switch 2 are shown in pink. D) Structure of Ct-elF5B(517-859) in the GTP-bound state comprising G domain and domain II. The switch regions and the P-loop are shown in yellow; the $\mathrm{Mg}^{2+}$ ion, $\mathrm{Na}^{+}$ion and water molecules are shown as spheres in magenta, blue, and grey, respectively; GTP is shown as balls and sticks. E) Molecule B in the asymmetric unit of the Sc-elF5B.GDP structure containing residues 401-852, comprising G domain and domains II and III. P-loop and the switch regions are colored cyan; the GDP molecule is shown as balls and sticks. F) Structure of Ct-eIF5B(520-970).GDP comprising G domain, domain II and domain III. P-loop and switch regions are colored cyan, the GDP molecule is shown as balls and sticks. G) Molecule $A$ in the asymmetric unit of the Sc-elF5B.GDP structure containing residues 401-852, comprising the $\mathrm{G}$ domain, domain II and domain III. Same color code as in F).
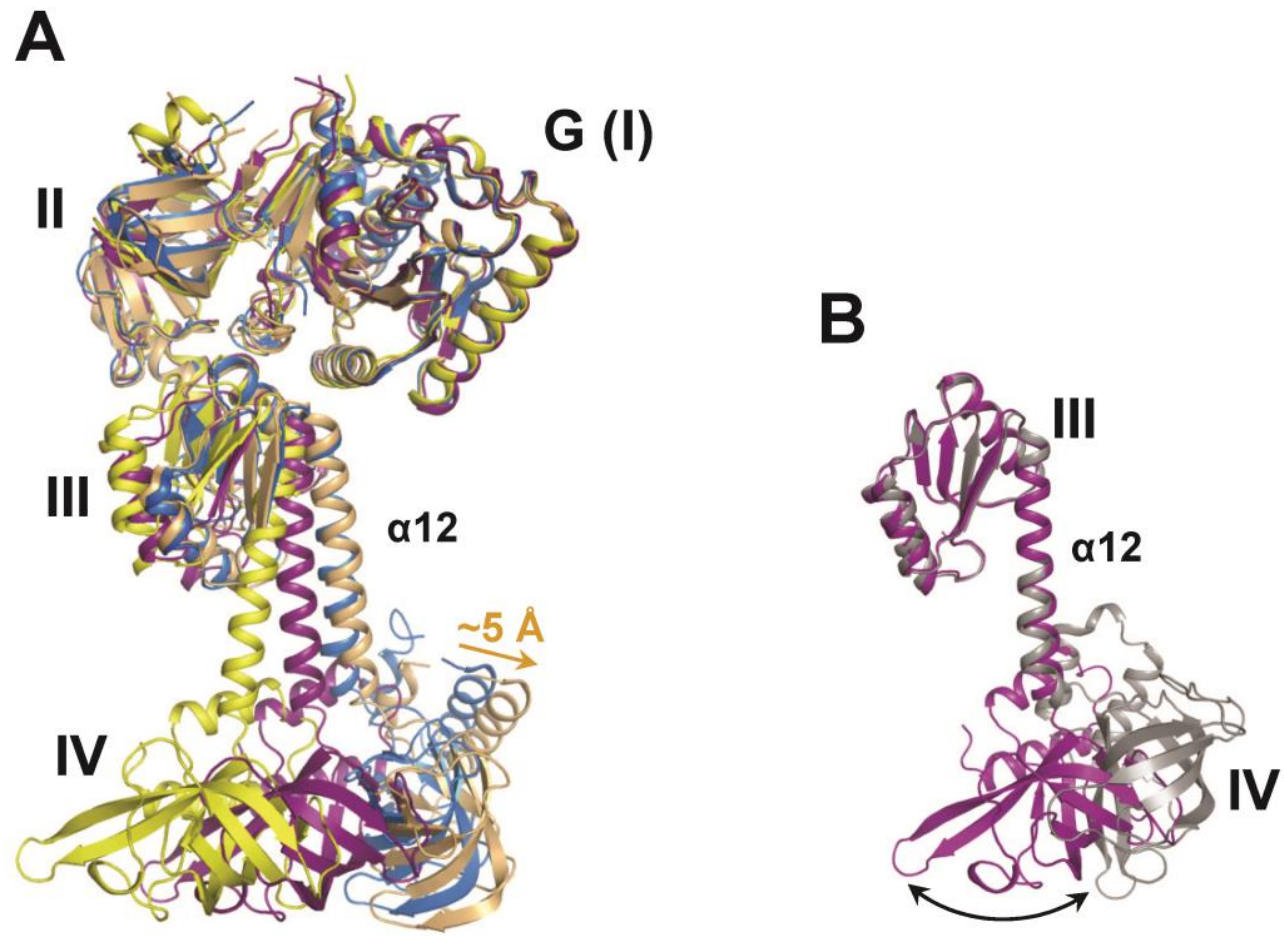

Figure S2.A)G domain based superposition of the apo structures of elF5B from C.thermophilum (purple), S.cerevisiae (yellow) and alF5B from $M$. thermoautotrophicum (blue; PDB: 1G7R), showing the conformational flexibility of domain III relative to domain I in the absence of a bound nucleotide. The Cterminus of helix $\alpha 12$ in apo alF5B lies 12 and $22 \AA$ apart from that in Ct-elF5B and Sc-elF5B, respectively. The GDPNP-bound form of alF5B is also shown (wheat; PDB: 1G7T), highlighting the relatively changes observed in the archeal ortholog upon binding of the GDPNP (see also Fig. S8). As the structure of the $S$. cerevisiae ortholog does not contain the second half of helix $\alpha 12$ and domain IV, domains III and IV of the Ct-elF5B structure were superimposed onto domain III of the Sc-elF5B structure. B) Superposition of domains III and IV of the apo Ct-elF5B(517C) structure (purple) with the structure of Ct-elF5B(870C) (grey), based on domain III. Domain III and the following helix $\alpha 12$ are nearly identical in both structures; in contrast, the orientation of domain IV relative to domain III differs significantly, indicating a high degree of conformational flexibility in the linker between helix $\alpha 12$ and domain IV. 

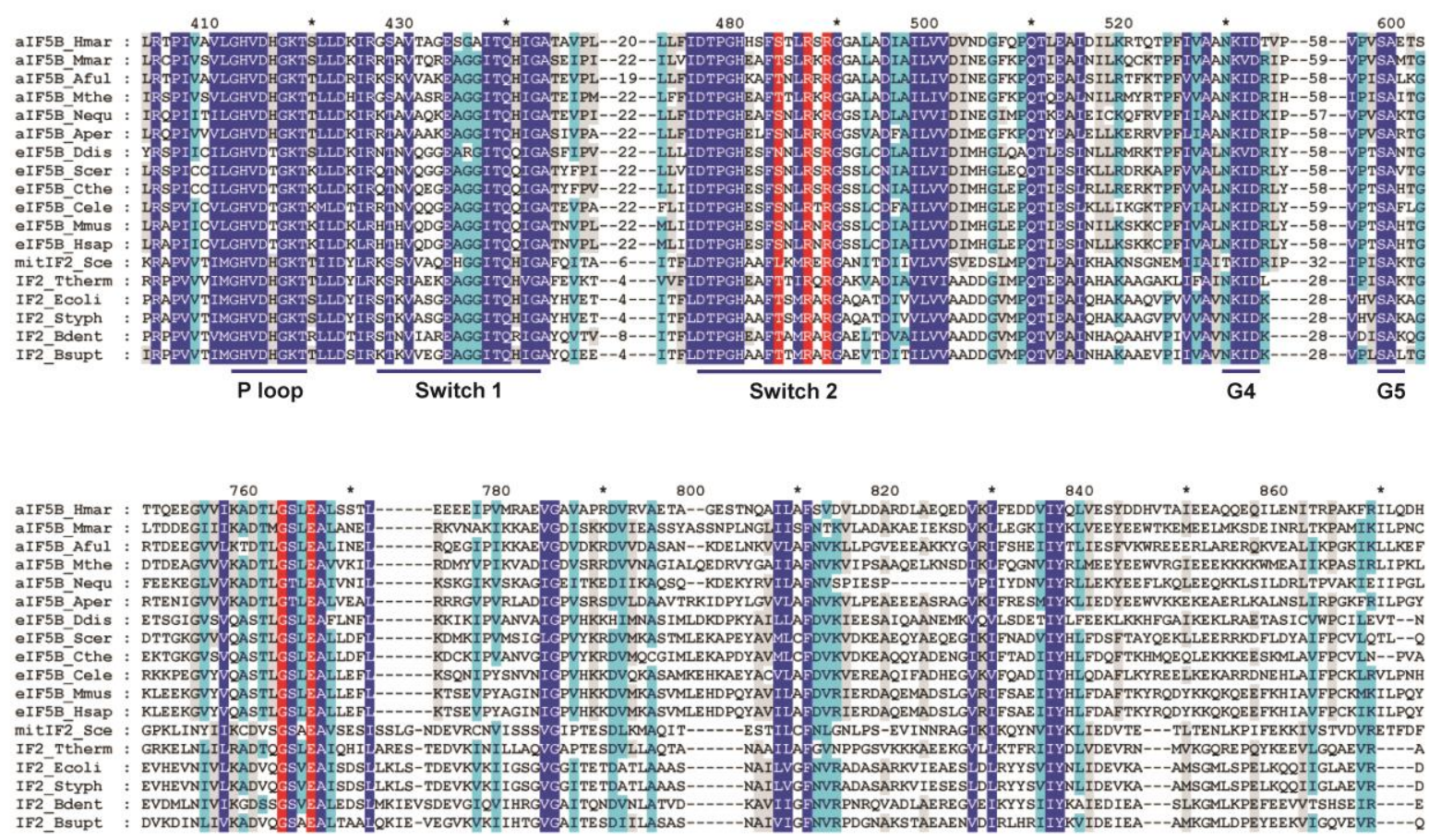

Figure S3. Sequence alignment of the $G$ domains (upper panel) and domains III (lower panel) from different aIF5B, elF5B and IF2 homologues. Highly conserved residues are highlighted in blue; conserved residues that are directly involved in the stable contacts between switch 2 and domain III in inactive (apo and GDP-bound) elF5B are highlighted in red. P-loop (G1 motif), switch 1 (with the G2 motif containing Thr439), switch 2 (with the G3 motif "DTPG") and motifs G4 and G5 in the G domain are indicated. The numbering above the sequences is according to the elF5B homologue from Saccharomyces cerevisiae. Residues within the $\mathrm{G}$ domain that are implicated in nucleotide binding and the GTP-dependent molecular switch in elF5B are universally conserved among all three orthologs. Domain III is significantly less well conserved than the $\mathrm{G}$ domain; however, the $\mathrm{N}$-terminal part of helix $\alpha 9$ (residues 763-768 in elF5B) is highly conserved, particularly in those residues involved in the interactions with switch 2. Species names are abbreviated as follows: Hmar, Haloarculamarismortui; Mmar, Methanococcusmaripaludis; Aful, Archaeoglobusfulgidus; Mthe, Methanothermobacterthermoautotrophicum; Nequi, Nanoarchaeumequitans; Aper, Aeropyrumpernix; Ddis, Dictyosteliumdiscoideum; Scer, Saccharomycescerevisiae; Cter, Chaetomiumthermophilum; Cele, Caenorhabditiselegans; Mmus, Musmusculus; Hsap, Homosapiens; Ttherm, Thermusthermophilus; Ecoli, Escherichiacoli; Styph, Salmonellatyphimurium; Bdent, Bifidobacteriumdentium; Bsupt, Bacillussubtilis; mitIF2_Sce, mitochondrial IF2 from S. cerevisiae. 
A

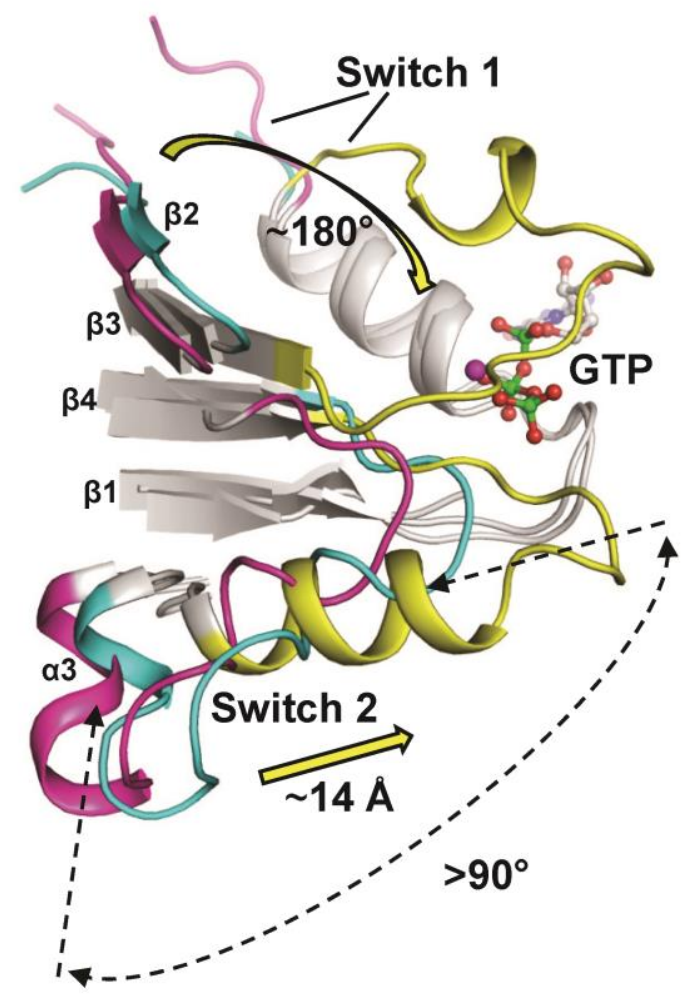

B

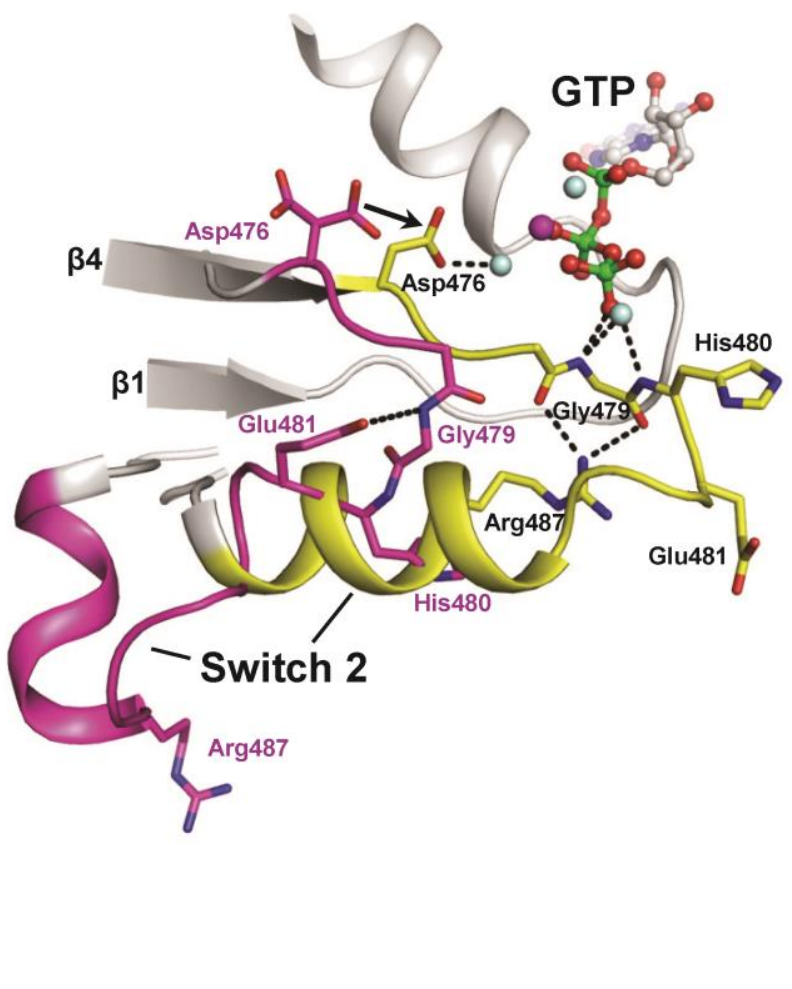

C

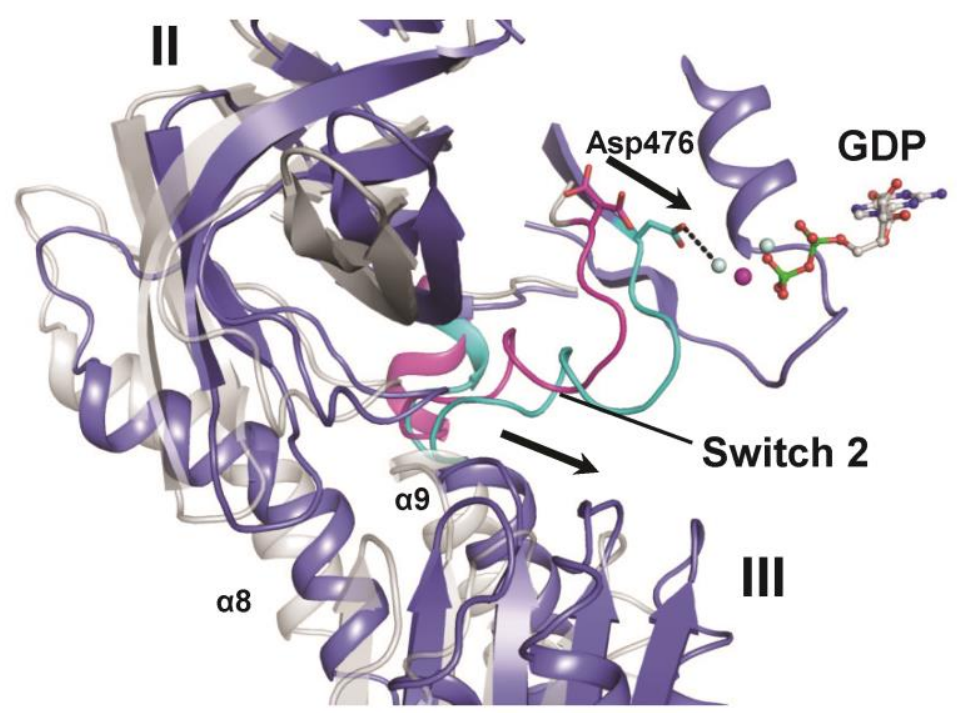

Figure S4.Nucleotide-dependent conformational changes in switch 1 and switch 2 of elF5B.A) Overview of the GTP-induced conformational changes in the G domain. P loop, switch 1 and switch 2 are colored pink for apo elF5B, cyan for elF5B.GDP and yellow for elF5B.GTP; the $\mathrm{Mg}^{2+}$ ion is shown as sphere in magenta; GTP is shown in balls and sticks; GDP is omitted for clarity. In the apo state switch 1 points away from the nucleotide binding pocket and is partially disordered. Switch 2 adopts a loop conformation with most residues positioned at the backside of the $\mathrm{G}$ domain (left). Upon GTP binding the switch regions become 


\section{Chapter 5}

reorganized (yellow arrows) to form the catalytic center around the GTP- $\nu$-phosphate. Switch 1 flips over by $\sim 180^{\circ}$ relative to its position in the apo state and the residues forming strand $\beta 2$ are repositioned to form part of the coordination sphere for the $\mathrm{Mg}^{2+}$ ion. Switch 2 moves up to $15 \AA$ in some residues, resulting in an extended helix $\alpha 3$, which is reoriented relative to $\alpha 3$ in the apo state by more than $90^{\circ}$. The transition from the GTP- to GDP-bound state reverses these changes and results in conformations of switch 1 and 2 like for the apo form, however, with switch 2 shifted $\sim 3 \AA$ towards the nucleotide binding pocket. B) Details of the transition between apo and GTP-bound forms of switch 2. The color code is the same as in A); water molecules are shown as grey spheres; hydrogen bonds indicated as dashed lines. Upon GTP binding Asp476 moves $\sim 3 \AA$ towards the GTP molecule (arrow). Gly479 moves towards the $\gamma$-phosphate (meanwhile the peptide bond to the preceding Pro478 flips by $\sim 160^{\circ}$ ) resulting in a reorganization of the rest of switch 2 including His480 ( $\mathrm{His}^{\mathrm{cat}}$ ) and Arg487, which is involved in interactions with domain III in the apo and GDPbound states of the factor (see also Fig. 3A/B). C) As shown in A), switch 2 in Ct-elF5B.GDP (blue with switch 2 in cyan) remains $\sim 3 \AA$ closer to the nucleotide binding pocket than in the apo form (grey with switch 2 in pink) but forms the same contacts to domains II and III as found in apo elF5B. As a consequence, also domain III and the ventral side of domain II are shifted in the same direction as switch 2 (arrows). 
A

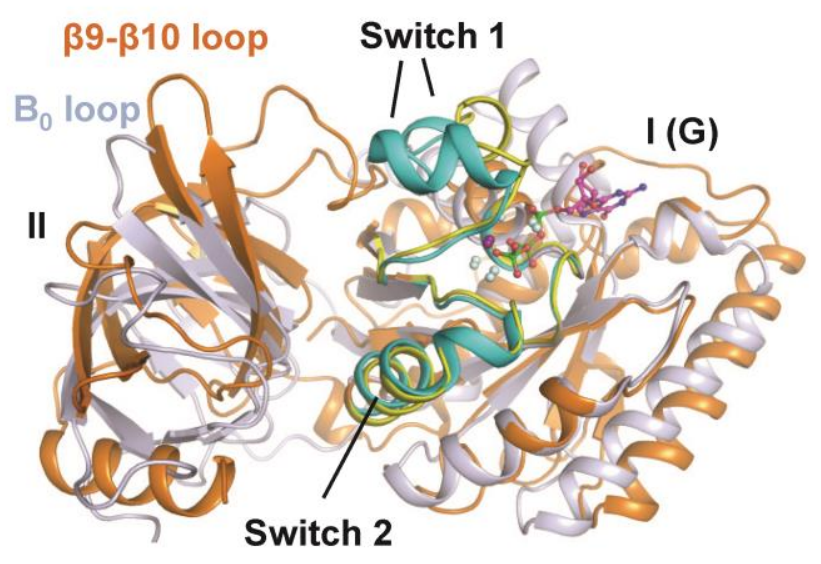

B

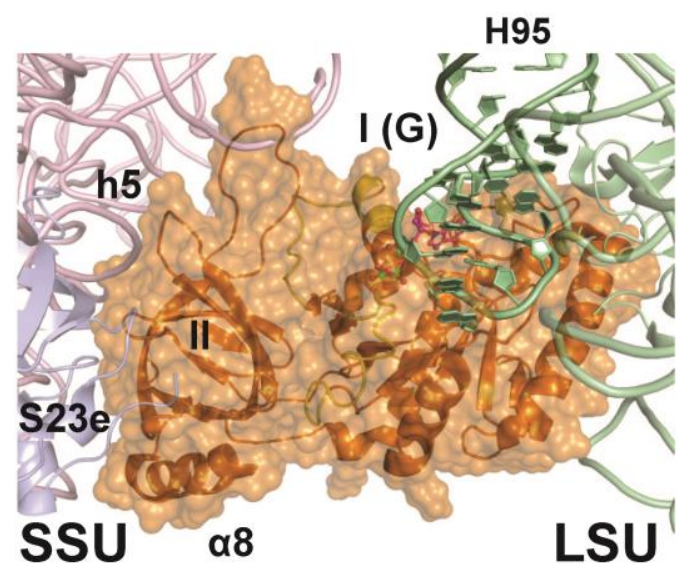

C

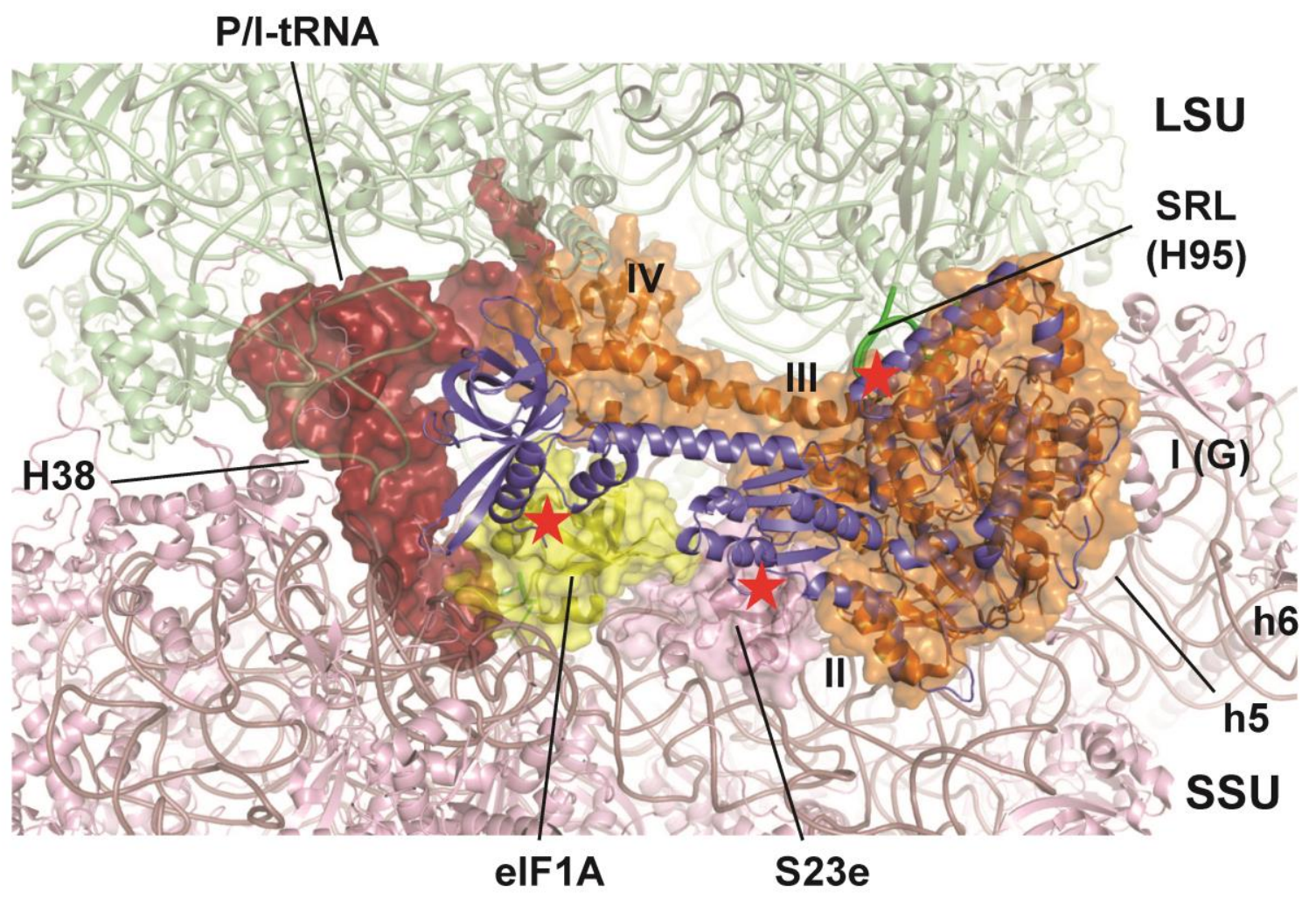

Figure S5. A) Superposition of elF5B.GTP (orange with P loop, switch 1 and switch 2 in yellow) and free EFTu.GDPNP (light blue with P loop, switch 1 and switch 2 in cyan; PDB code: 1EXM), based on their respective catalytic centers. Domains I and II from elF5B and EF-Tu show the same overall dimensions and exhibit very similar arrangements in regions involved in nucleotide binding (switch 1 and switch 2) or in interactions with the small ribosomal subunit (e.g. the $\beta 9-\beta 10$ loop in elF5B and the $B_{0}$ loop in EF-Tu). B) Cryo-EM model of domains I and II of elF5B.GDPCP (orange) on the $80 \mathrm{~S}$ ribosome [91]. The large ribosomal subunit (LSU) is shown in green; the small ribosomal subunit (SSU) is shown in light pink. C) Comparison of elF5B.GDPCP in 


\section{Chapter 5}

the $80 \mathrm{~S} \mathrm{IC} \mathrm{(orange;} \mathrm{[91])} \mathrm{with} \mathrm{inactive} \mathrm{elF5B} \mathrm{(blue).} \mathrm{The} \mathrm{structure} \mathrm{of} \mathrm{inactive} \mathrm{elF5B} \mathrm{was} \mathrm{modeled} \mathrm{by}$ superposition of its domain II onto domain II of elF5B.GDPCP. According to this model, GTP hydrolysis and subsequent $P_{i}$ release causes domain IV to move away from the acceptor end of the tRNA (dark red) as domain III associates with domains I and II. Simultaneously, the G domain rotates with respect to domain II by $\sim 30^{\circ}$ towards the SRL. These movements result in clashes (red stars) of domains I, III and IV with the SRL, ribosomal protein $\mathrm{S} 23 \mathrm{e}$ and $\mathrm{A}$ site-bound elF1A (yellow; modeled according to the structure of elF1 and eIF1A on the 40S subunit (PDB: 4BPE)), respectively, which reduce the affinity of elF5B.GDP to the ribosome and facilitate its dissociation.

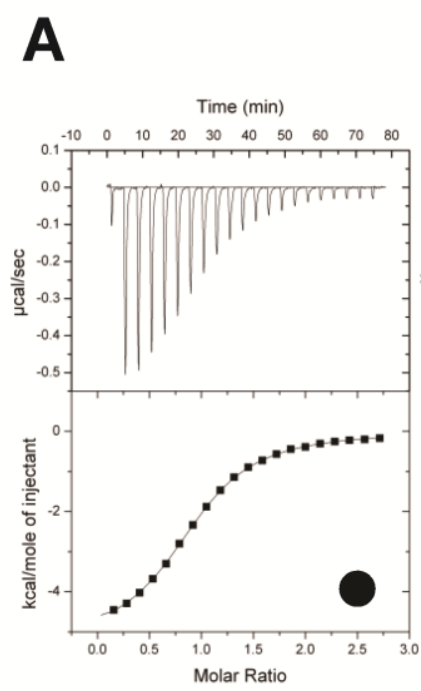

B

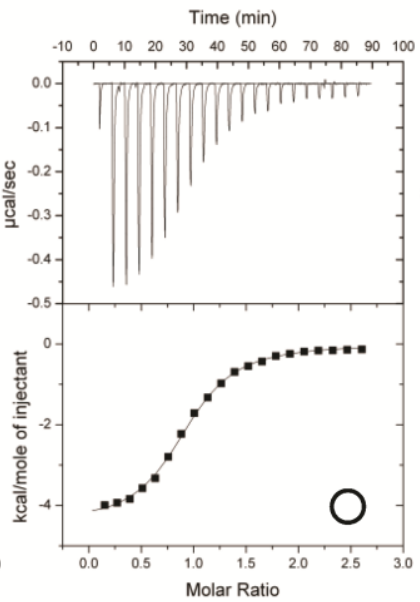

C

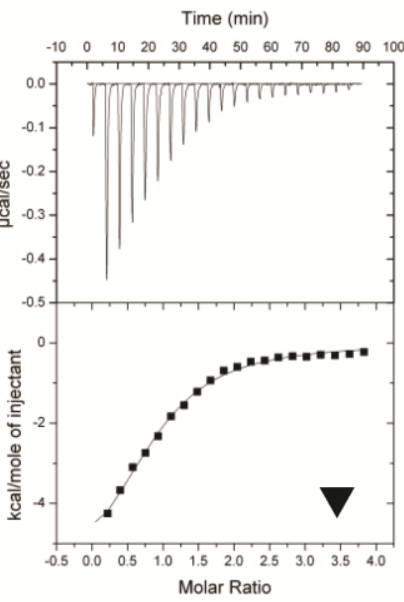

D

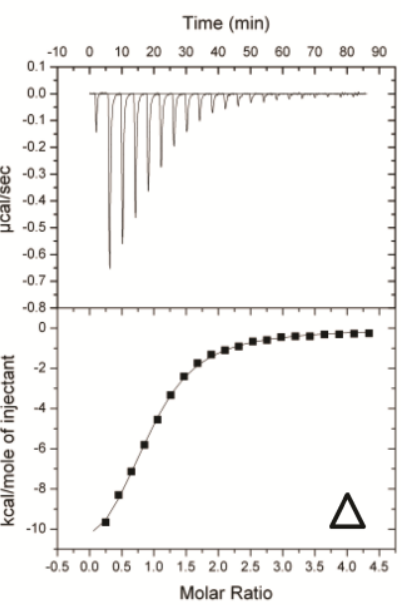

Figure S6. A-D) Titration curves (upper panels) and binding isotherms (lower panels) of elF5B interactions with GDP or GTP.A) Ct-elF5B(517C) with GDP at $20{ }^{\circ} \mathrm{C}$ in the presence of $\mathrm{MgCl}_{2} ; \mathrm{B}$ ) Ct-elF5B(517C) with GDP at $25{ }^{\circ} \mathrm{C}$ in the absence of $\mathrm{MgCl}_{2} ; \mathrm{C}$ ) Ct-elF5B(517C) with GTP at $20{ }^{\circ} \mathrm{C}$ in the presence of $\mathrm{MgCl}_{2}$; D) CtelF5B(517-852) with GTP at $20^{\circ} \mathrm{C}$ in the presence of $\mathrm{MgCl}_{2}$. 

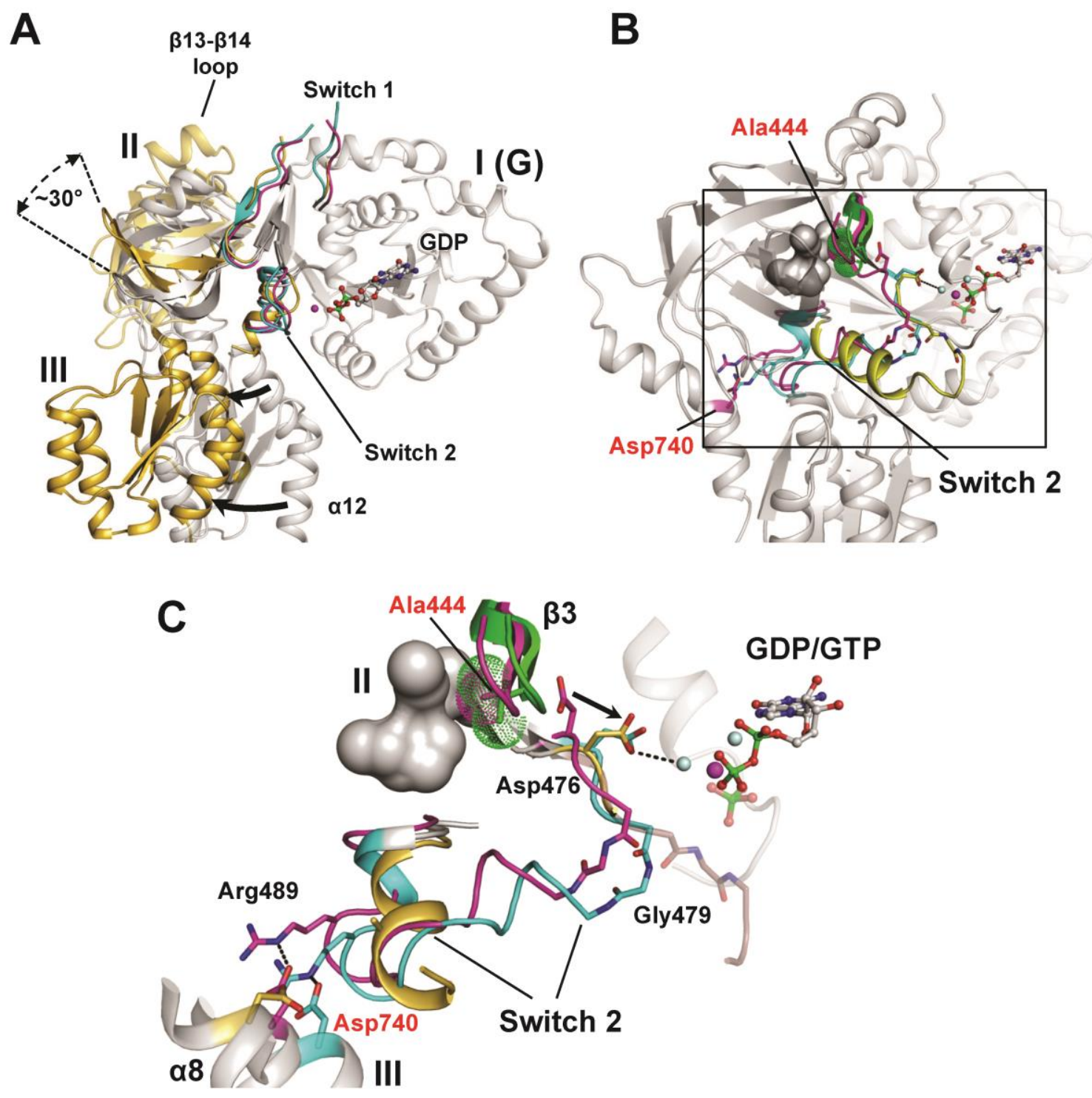

Figure S7. A) G domain-based superposition of elF5B domains I-III from S. cerevisiae in its apo and GDPbound states. The $\mathrm{G}$ domain is colored grey; the switch regions are colored pink for the apo state, cyan for molecule A of Sc-elF5B.GDP and dark yellow for molecule B of Sc-elF5B.GDP. While domains II and III of molecule A in the Sc-elF5B.GDP structure superimpose well with domains II and III of the apo form (grey), domain III in molecule $B$ (dark yellow) is moved away from the $G$ domain (arrows) and has no contact to the partially disordered switch 2 . Domain II of molecule B is in its rotated state with the $\beta 9-\beta 10$ loop moved upwards; switch 1 and the $\beta 13-\beta 14$ loop remain in their respective inactive conformations. B) Overview of apo elF5B with the locations of Ala444 and Asp740 relative to the nucleotide binding pocket and switch 2. Switch 2 is shown in its apo (pink), GDP- (cyan) and GTP-bound forms (yellow). C) Detail of the area indicated in B) (box). Switch 2 is shown in four different states: i) The inactive apo state (pink), in which Asp476 and Gly479 point away from the nucleotide binding pocket and Arg489 forms a salt bridge to Asp740 of domain III. ii) The partially activated GDP state in Ct-elF5B.GDP (cyan), in which Asp476 interacts with the $\mathrm{Mg}^{2+}$ ion. Gly479 has undergone the peptide flip relative to the apo state. iii) The partially activated GDP state in molecule B of Sc-elF5B.GDP (dark yellow). Asp476 interacts with the $\mathrm{Mg}^{2+}$ ion; switch 2 is shifted towards the GDP molecule and has lost its contacts to domain III (see also Fig. S5A). iv) The G3 motif 
of the activated switch 2 in Ct-elF5B.GTP (brown; the rest of switch 2 is not shown). Gly 479 has undergone the peptide flip relative to the apo state and contacts the $\gamma$-phosphate. Mutagenesis of Gly479 to Ala most likely prevents the peptide flip and thereby stabilizes the inactive conformation of switch 2. Mutagenesis of Asp740 to Arg would result in the steric and electrostatic repulsion of switch 2 in its inactive conformation. Mutagenesis of Ala444 to Val would result in a steric clash with domain II (grey surface), most likely causing strand $\beta 3$ to move towards Asp476 (green model). To avoid a clash with the repositioned strand $\beta 3$ Asp476 would have to retreat towards the nucleotide binding pocket, thereby facilitating the partial or full activation of switch 2 by GDP as well as GTP.

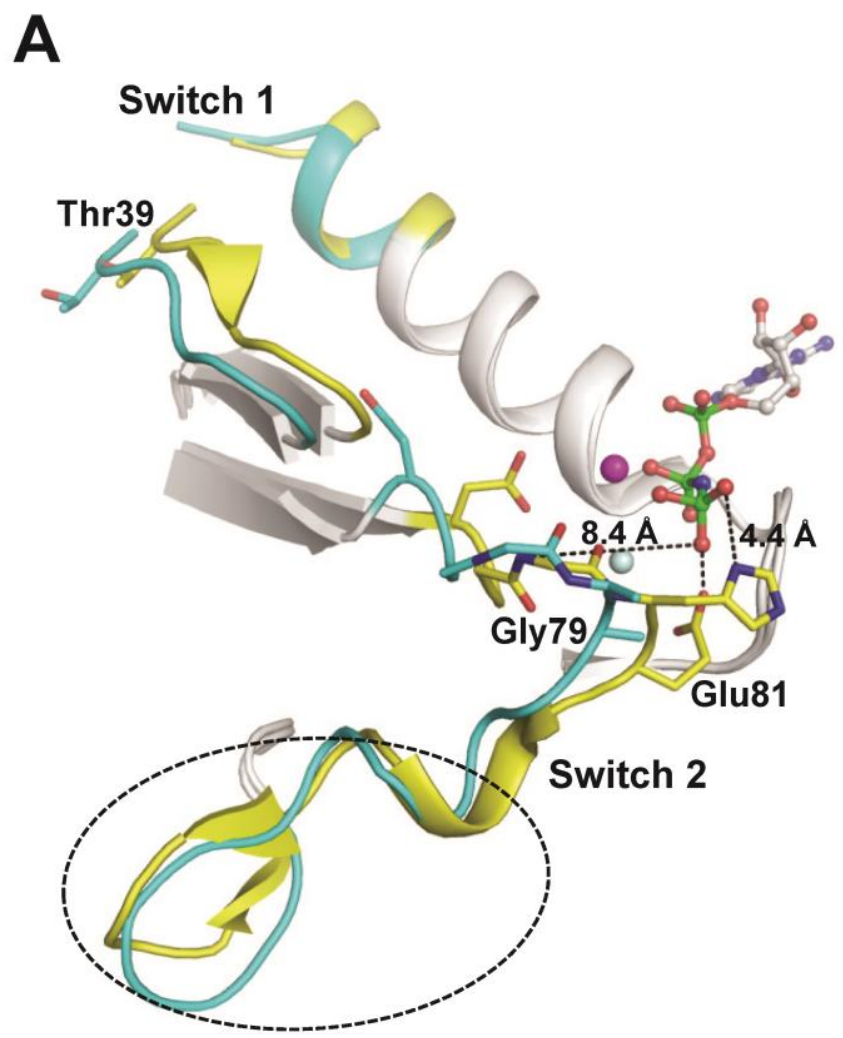

B

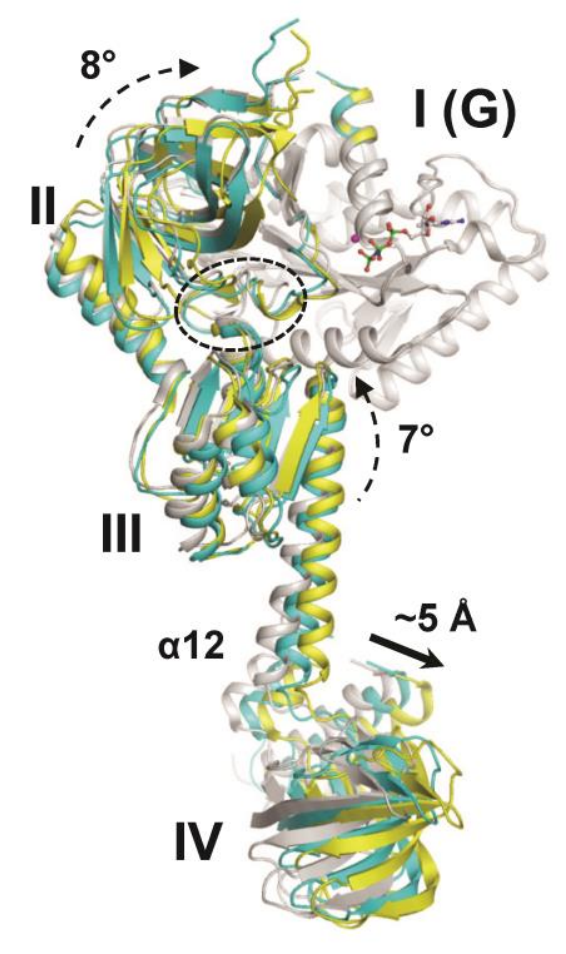

Figure S8. The articulated lever model for elF5B/IF2 activation [84, 144].A) Superposition of switch 1 and switch 2 from GDP- (cyan) and GDPNP-bound (yellow) alF5B from M. thermoautotrophicum (PDB: 1G7S, 1G7T). The structural transition from the GDP-bound state to the GDPNP-bound form is limited to the Nterminal half of switch 2, which moves $\sim 2 \AA$ towards the nucleotide binding pocket, while switch 1 remains unaltered. Neither Thr39 in the G2 motif nor Gly79 in G3 form a direct contact to the $y$-phosphate; the only potential interaction occurs between Glu81 and the $y$-phosphate. The region of switch 2 forming the contacts to domain III (dashed circle) remains virtually unchanged. Compare also to Fig. S4. B) The GDPNPinduced shift in switch 2 is thought to cause a concerted rearrangement of domains II and III (dashed arrows), resulting in a $\sim 5 \AA$ shift of domain IV (arrow). The interaction interface between switch 2 and domains II and III (dashed circle) remains nearly unchanged. The switch regions are colored as in A); domains II-IV are colored grey for apo alF5B (PDB: 1G7R), cyan for alF5B·GDP and yellow for alF5B.GDPNP. Compare also to Fig. S2A. 


\section{Chapter $6 \cdot$ Recognition of amino-acylated initiator tRNA by eIF5B in the 80S IC}

This manuscript has been accepted for publication in BMC Structural Biology

Structural insight into the recognition of amino-acylated initiator tRNA by eIF5B in the $80 S$ initiation complex

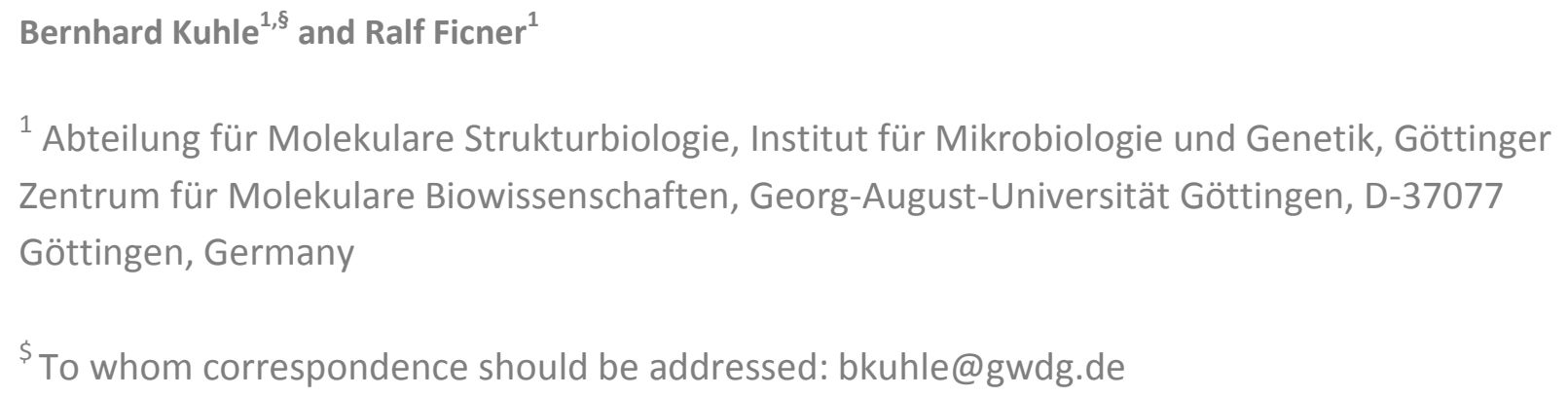




\section{Background}

From bacteria to eukarya, the specific recognition of the amino-acylated initiator tRNA by the universally conserved translational GTPase eIF5B/IF2 is one of the most central interactions in the process of translation initiation. However, the molecular details, particularly also in the context of ribosomal initiation complexes, are only partially understood.

\section{Results}

A reinterpretation of the $6.6 \AA$ resolution cryo-electron microscopy (cryo-EM) structure of the eukaryal $80 \mathrm{~S}$ initiation complex using the recently published crystal structure of elF5B reveals that domain IV of elF5B forms extensive interaction interfaces with the Met-tRNA $A_{i}$, which, in contrast to the previous model, directly involve the methionylated 3' CCA-end of the acceptor stem. These contacts are mediated by a conserved surface area, which is homologous to the surface areas mediating the interactions between IF2 and fMet-tRNA ${ }^{\text {fMet }}$ as well as between domain II of EF-Tu and amino-acylated elongator tRNAs.

\section{Conclusions}

The reported observations provide novel direct structural insight into the specific recognition of the methionylated acceptor stem by elF5B domain IV and demonstrate its universality among elF5B/IF2 orthologs in the three domains of life.

\subsection{Background}

The process of translation initiation results in the formation of an elongation-competent ribosome with the start codon of an mRNA in its P site, base paired to the amino-acylated initiator tRNA. In bacteria and eukarya this process follows significantly different mechanisms, highlighted by different numbers of auxiliary protein factors (initiation factors or IFs) that are employed by bacterial (three IFs) or eukaryal cells (at least 12 elFs) for correct ribosome assembly [9]. Only two of these factors, a/eIF1A/IF1 and the translational GTPase a/eIF5B/IF2, are universally conserved in the three domains of life [81]. In bacteria, IF2 plays a critical role throughout the initiation pathway. In the early stages, IF2 binds to the 30S subunit in a GTP-dependent manner and stimulates the recruitment of the $\mathrm{N}$-formylmethionylated initiator tRNA (fMet-tRNA ${ }^{f M e t}$ ) to the $P$ site of the $30 \mathrm{~S}$ ribosomal subunit to form the $30 \mathrm{~S}$ pre-initiation complex (pre-IC). Finally, IF2.GTP 
catalyzes the joining of the $50 \mathrm{~S}$ ribosomal subunit to form the elongation-competent ribosome [15, 189]. Speed and accuracy of both processes depend of the specific recognition of the aNH-blocked methionine esterified to the 3' CCA-end of tRNA ${ }^{\text {fMet }}$ [190-193]. Biochemical studies showed that all determinants required for this interaction are located in domain IV of IF2, which consists of a sixstranded $\beta$ barrel [194-197]. Domain IV of IF2 exhibits a marked structural homology to domain II of EF-Tu that, together with the G domain, forms the universally conserved structural core among translational GTPases [198] and in EF-Tu constitutes part of the binding pocket for the aminoacylated acceptor arm of elongator tRNAs [48, 163, 197]. Based on this observation it was suggested that IF2 domain IV and EF-Tu domain II use similar interfaces for their interactions with the tRNA [197]. This assumption is at least partially corroborated by mutational and NMR spectroscopy analyses [196]. Cryo-EM structures of bacterial 30 S pre-ICs and 70S IC containing

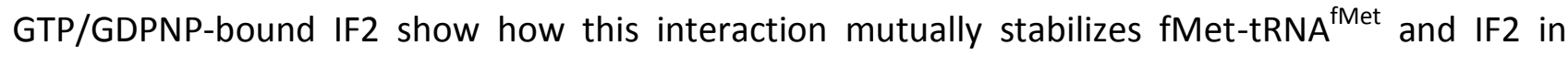
conformations that allow the efficient association of the 50 S subunit $[152,172]$. However, none of these structures were determined at sufficiently high resolution to give any detailed insight into the interaction that would allow a correlation with the biochemical data.

In contrast to bacterial IF2, the role of a/elF5B in eukarya and archaea seems to be confined to the GTP-dependent promotion of subunit joining, the last step of the initiation process [145, 161, 199]. The recruitment of the charged initiator tRNA (Met-tRNA $A_{i}$ ) to the small ribosomal subunit is carried out by the heterotrimeric a/elF2, a specialized EF-Tu paralog that has no counterpart in bacteria [200]. Accordingly, a/eIF5B.GTP binds to the small ribosomal subunit already containing the $P$ site-bound Met-tRNA ${ }_{i}$, which invokes the question whether a/elF5B still has to interact with Met-tRNA $A_{i}$ to promote joining of the large ribosomal subunit, and whether this interaction would involve a specific recognition of the methionylated acceptor end, similar to the recognition of the fMet-tRNA ${ }^{\text {fMet }}$ by IF2. Genetic, biochemical and structural studies point toward essentially the same mechanisms for elF5B and IF2 catalyzed subunit joining [84, 91, 145, 154, 199, 201]. Crystal structures of aIF5B and elF5B revealed a six-stranded $\beta$ barrel fold for domain IV, homologous to domain IV in IF2 [84, 201]. Indirect biochemical assays showed that a/elF5B binds Met-tRNA $A_{i}$ in solution, however, with very low affinity and specificity for the methionyl moiety in case of elF5B $[84,202]$. NMR studies revealed a weak but specific interaction between methionine- 
ethyl ester (mimicking the ester bond between tRNA and the methionly moiety) and elF5B domain IV in the area corresponding to the surface on IF2 that is affected by $\mathrm{N}$-formylmethionine binding [86]. Finally, the recently determined $6.6 \AA$ resolution cryo-EM structure of the yeast 80 S IC (EMDatabank: EMD-2422) demonstrates that, like IF2 in the corresponding bacterial 70 S complex [152], elF5B and the $P$ site-bound Met-tRNA $A_{i}$ stabilize each other in their subunit joining-competent conformations through the direct contact between domain IV and acceptor stem [91]. Surprisingly however, according to the structural model this contact does not involve the methionylated $3^{\prime}$ CCA-end of the tRNA [91]. Instead, the CCA-end points away from domain IV, placing the methionyl moiety $\sim 23 \AA$ from the protein. Thus, this model is clearly at odds with the observations from biochemical studies $[86,202]$ and fails to explain why deacylation of the initiator tRNA results in the loss of its ability to stabilize elF5B [91].

Here, we provide an analysis and reinterpretation for the cryo-EM density of the yeast 80S IC [91] for domain IV and its contact to the initiator tRNA. We show that the original structural model for this region, based on the fit of the archaeal alF5B ortholog, is only partially consistent with the available density. Fitting of the recently determined structure of elF5B domain IV from $C$. thermophilum, which shows a significantly higher degree of sequence similarity to the S. cerevisiae ortholog, allows a reinterpretation of the $6.6 \AA$ resolution density. The resulting model demonstrates a direct contact between the methionylated CCA-end of the TRNA and a conserved surface area of domain IV that directly corresponds to the binding sites for the tRNA acceptor arm on domain IV of IF2 or domain II of EF-Tu $[48,196,197]$. Thus, we show that the high-quality cryoEM density of the $80 \mathrm{~S}$ complex not only provides the first direct structural indications for the EFTu-like interactions between elF5B/IF2 domain IV and the initiator tRNA but also for their universality among a/elF5B/IF2 orthologs in the three domains of life. Finally, we use our observations to propose a possible scenario for the evolution of the translational $\beta$ barrel fold in eIF5B/IF2 and EF-Tu and its interactions with tRNAs. 
A

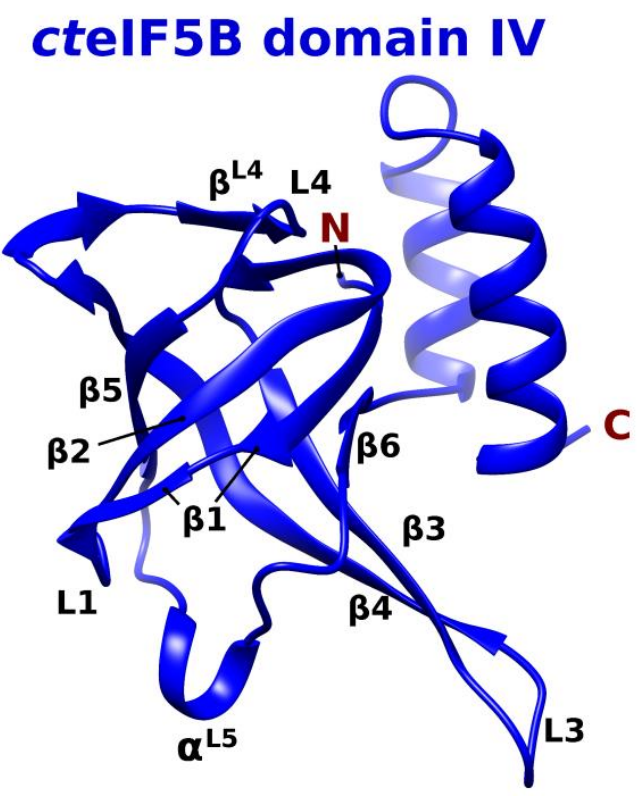

B mtalF5B domain IV
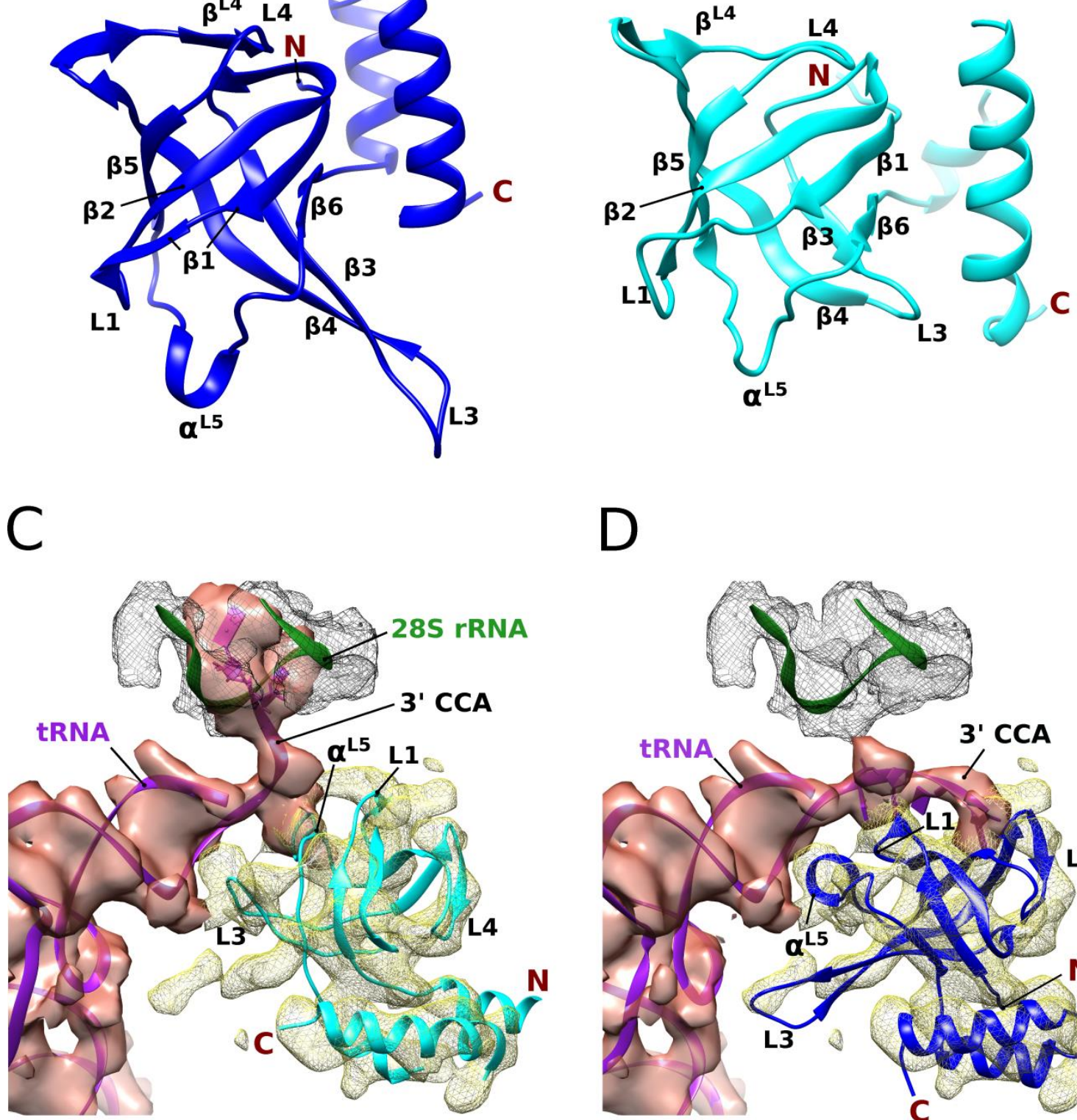

D

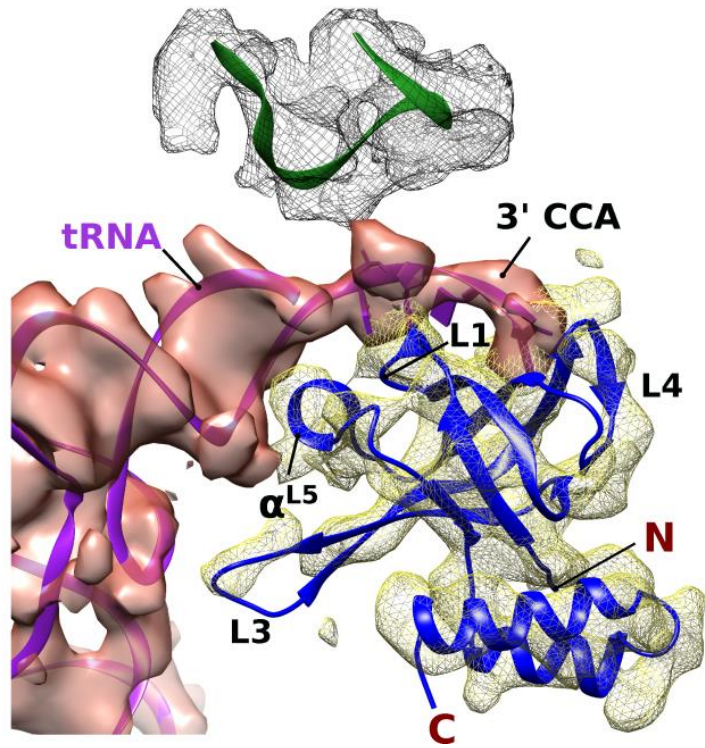

Figure 1. Model for the interactions between elF5B domain IV and Met-tRNA in the 80S IC. A) Crystal structure of ctelF5B domain IV (PDB: 4N3G). The most marked differences to domain IV of alF5B from the archaeon $M$. thermoautotrophicum (PDB: 1G7T) (B) are found in the lengths of the $\beta 3-\beta 4$ hairpin and loop L5 as well as in the arrangement of the two C-terminal $\alpha$ helices. C) Original model for the interactions between domain IV (cyan) and Met-tRNA $A_{i}$ (purple), fitted into the cryo-EM density of the 80S IC (EMD-2422) [91]. D) New model for the interactions between domain IV (blue) and Met-tRNA $A_{i}$ (purple), based on the rigid-body fitting of the crystal structure of domain IV from ctelF5B. The 3' CCA-end now forms a direct contact with the surface of domain IV, and $\alpha^{L 5}$ occupies a position in the major groove of the acceptor stem. 


\subsection{Results and Discussion}

\section{Model of elF5B domain IV and the acceptor end of Met-tRNA $A_{i}$ in the 80 S IC.}

Recently, we were able to solve two structures of elF5B domain IV from the fungus C. thermophilum [201]. It consists of six antiparallel $\beta$ strands ( $\beta 1-\beta 6)$ forming a closed $\beta$ barrel that is followed by two $\alpha$ helices (Figure 1A). At its top and bottom, the $\beta$ barrel is closed by an additional short $\beta$ strand $\left(\beta^{L 4}\right)$ and a one-turn $\alpha$ helix $\left(\alpha^{L 5}\right)$, respectively. Despite relatively low sequence similarity, it is structurally very similar to domain IV of alF5B from the archaeon $M$. thermoautotrophicum (rmsd of $2.2 \AA$ for $\mathrm{C}_{\alpha}$ atoms with $\sim 20 \%$ identity and $\sim 30 \%$ similarity). However, in the cteIF5B ortholog the $\beta$ hairpin formed by $\beta$ strands 3 and 4 and the loop following strand $\beta 5$ (L5) contain 9 and 5 additional amino acids, respectively (Figure 1A/B). Further differences can be found in the organization of the two C-terminal $\alpha$ helices that are rotated by $\sim 25^{\circ}$ with respect to the $\beta$ barrel.

Compared to the archaeal ortholog, domain IV from ctelF5B shows a relatively high sequence similarity to the yeast ortholog (19\% identity and $30 \%$ similarity for mtalF5B compared to $49 \%$ identity and 65\% similarity for cteIF5B) including the $\beta 3$ - $\beta 4$ hairpin, L5 and the two C-terminal $\alpha$ helices. Based on this observation, we assumed that the ctelF5B structure allows a better fit to the recently determined cryo-EM density of the yeast 80S IC with initiator TRNA and elF5B-GDPCP than obtained with the mtalF5B structure [91] (Figure 1C). Rigid-body fitting of ctelF5B domain IV (crosscorrelation coefficient (CCC) of 73\%) results in an improved correlation between structural model and density (Figure 1D): In contrast to the original fit of $m$ talF5B [91], no clashes occur between the ribosomal RNA and ctelF5B domain IV, as the loop between $\beta$ strands 1 and 2 (L1) is moved away from C2284-U2286 and now lies next to the acceptor stem of the tRNA. Compared to the original model [91] the $\beta$ barrel is rotated by $\sim 30^{\circ}$, causing the conserved helix $\alpha^{\mathrm{L} 5}$ at the bottom of the $\beta$ barrel to displace the $\beta 3-\beta 4$ hairpin in the major groove of the initiator tRNA acceptor stem. In turn, the long, poorly conserved $\beta 3-\beta 4$ hairpin now occupies previously unexplained density close to the C-terminus of the last $\alpha$ helix and forms apparently no direct contacts to the tRNA (Figure 1D).

An interesting consequence of this reorganization of domain IV is the emergence of a well defined but unexplained density packed alongside the $\beta$ barrel, directly opposite to the C-terminal 
$\alpha$ helices (Figure 1D). This density starts next to the very C-terminus of $\beta$ strand 4 and the following loop (L4) and runs across strands $\beta 5, \beta 2$ and finally $\beta 1$ where it directly leads into the continuous density of the phosphate backbone of the initiator tRNA at A73. Interestingly, this same position (A73) also marks the starting point for the distortion of the following 3' CCA-end in the original model that is markedly different from its canonical conformation [91]. For the following reasons, it is unlikely that this original model gives the correct conformation for the tRNA acceptor arm in the 80S pre-IC: First, C75 and A76 clash extensively with the ribosomal RNA between G2615 and C2625 (Figure 1C). Second, the CCA-end is oriented away from elF5B domain IV, resulting in a distance of $\sim 23 \AA$ between the ribose of A76 (which carries the methionyl moiety) and the nearest parts of domain IV. This is clearly inconsistent with the observation that deacylation of the tRNA results in the loss of its contact to elF5B in the $80 \mathrm{~S}$ complex [91] and is at odds with the expected direct contact between the methionyl moiety and domain IV [86, 199, 202]. Remodeling of the 3' CCAend into the vacant density next to the $\beta$ barrel of elF5B avoids the clashes with the rRNA and, moreover, allows a direct recognition of the aminoacyl group by the protein by placing the $3^{\prime}$ end of the tRNA directly on top of the conserved Ala1056 of strand $\beta 5$ (Figure 1D). It is important to note, that there is no alternative density present that could accommodate the entire CCA-end without causing a sterical conflict with the rRNA. Independent support for this new placement of the CCA-end is provided by the just recently published lower resolution (8-9 A) cryo-EM model of the mammalian $80 \mathrm{~S}$ pre-IC with elF5B bound on HCV-IRES RNA, which suggests a direct contact between the acceptor end of the Met-tRNA $A_{i}$ and domain IV of elF5B [203].

As reported previously for mtalF5B [84], the $\beta$ barrel of elF5B is structurally homologous to domain IV (C2 domain) of bacterial IF2 [197] and domain II of EF-Tu homologs, despite an overall low sequence similarity (Figure 2 and 3). Using site directed mutagenesis and NMR spectroscopy, it was shown that IF2 interacts with the $\alpha \mathrm{NH}$-formylmethionylated CCA-end of fMet-tRNA ${ }^{\text {fMet }}$ through a surface of domain IV that overlaps with that used by EF-Tu domain II to interact with the acceptor end of elongator tRNAs [48, 163, 196, 197]. The superposition of domain IV of ctelF5B with domain IV of IF2 from Bacillus stearothermophilus[197] and domain II of EF-Tu from Thermus aquaticus in complex with Phe-tRNA ${ }^{\text {Phe }}[48]$ reveals that these surface areas coincide perfectly with those occupied by the CCA-end in our cryo-EM density-based model (Figure 2). Consistently, a 
structure-based sequence alignment reveals the highest degree of sequence conservation between the elF5B orthologs and EF-Tu domain II in those residues implicated in TRNA binding in IF2 and EFTu (Figure 3).

A

B

C

$\mathrm{D}$
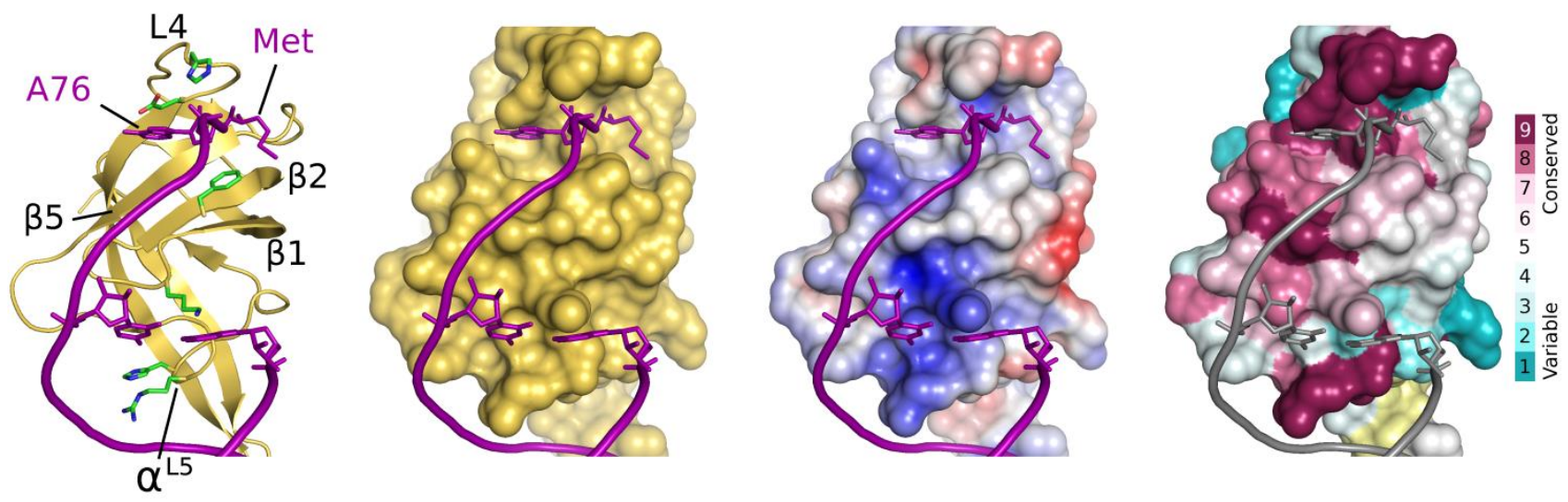

$E$

$\mathrm{F}$
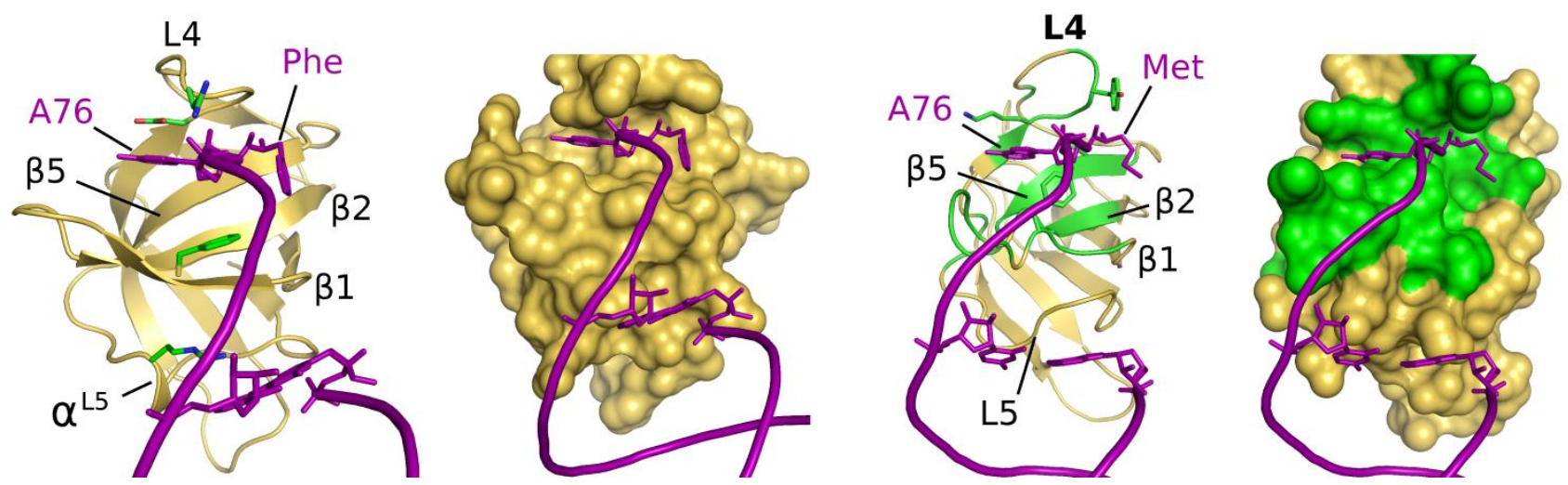

Figure 2. The interaction interface between the acceptor stem of Met-tRNA $A_{i}$ and domain IV of elF5B.

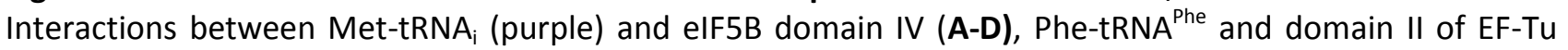
(PDB: 1TTT) (E) and fMet-tRNA ${ }^{\text {fMet }}$ and IF2 domain IV (F). A) Domain IV of elF5B in ribbon presentation (yellow) with residues potentially involved in interactions with the tRNA as green sticks. B) Surface presentation of domain IV, revealing the two well defined pockets below loop L4 that are also visible on the surfaces of EF-Tu domain II (E, right) and IF2 domain IV (F, right), and might accommodate A76 and the methionyl moiety of the 3' CCA-end. C) Electrostatic surface potential of domain IV. D) Conservation of residues lying in the proposed interaction interface to the acceptor stem. E) Position of the acceptor stem of Phe-tRNA ${ }^{\text {Phe }}$ (purple) on the surface of domain II of EF-Tu (yellow; PDB: 1TTT). F) Model of domain IV of IF2 (PDB: 1D1N) and the initiator tRNA positioned as in A). The green surfaces indicate residues of IF2 that were shown to interact with fMet-tRNA ${ }^{\text {fMet }}[196,197]$. 
The analysis of the surface area in ctelF5B reveals two pockets next to the modeled 3' end of the tRNA (Figure 2 and 4). The first formed by Val999, Gly1037, Glu1039 and the aliphatic part of Lys1058, corresponding to the EF-Tu residues Val237, Gly269, Glu271 and Leu289, respectively, which accommodate the base of $A 76$ in Phe-tRNA ${ }^{\text {Phe }}[48]$. A similar pocket is found on the surface of IF2, whose residues are directly affected by fMet-tRNA ${ }^{f M e t}$ binding $[196,197]$. The second pocket is separated from the first by the methyl group of Ala1056 (corresponding to the conserved Gly287 in EF-Tu and Gly715 in bsIF2) and is formed on the one side by the hydrophobic Val989, Ala990, Phe992, Gly1001 and Ala1054 and on the other by the peptide backbone of Glu1039 to His1042. The position of this pocket corresponds to the localization of the aminoacyl groups in ternary complexes of EF-Tu, and residues of this area were found to interact specifically with $\mathrm{N}$ formylmethionine in IF2 [196] and methionine-ethyl ester in elF5B [86]. Consistently, this second pocket is compatible with the binding of a methionyl moiety in size as well as electrostatic surface properties (Figure 2 and 4). Notably, in both available crystal structures of ctelF5B domain IV, this pocket is occupied by a large additional electron density. Due to the absence of alternatives in the crystallization condition (100 mM MES, 12\% PEG 20000, 10 mM Na-lactate; ethylene glycol was used for cryo protection), this density was originally assigned to a lactate molecule [201]. However, refinement with the lactate molecule still results in positive difference electron density. A simulated annealing omit map for this area gives a density too large for a lactate (Figure 4B). Thus, the density would be compatible with the size of a methionine or other similarly large amino-acids whose $\alpha$-carboxylate and $\alpha$-amino groups form hydrogen bonds to the amide proton of Asp1041 and the main chain CO of Ala1054, respectively, corresponding to His273 and Asn285 that form similar contacts to the aminoacyl group in ternary complexes of EF-Tu [48, 204] (Figure 4D). However, the resolution of the structures (2.75 and $3.02 \AA)$ necessarily does not allow an unambiguous assignment of the densities to a certain ligand, and a possible origin for a putative amino acid in this position remains elusive, as the weak binding between IF2 and fMet or elF5B and methionine-ethyl ester $[86,196]$ makes a co-purification unlikely. The critical point, however, is the observation that the described pocket is evidently suited to accommodate organic molecules of a size similar to that of methionine and could thus accommodate the methionyl moiety of the MettRNA $_{i}$ in a way analogous to domains II in EF-Tu and alF2 $\gamma$ (Figure 4). 


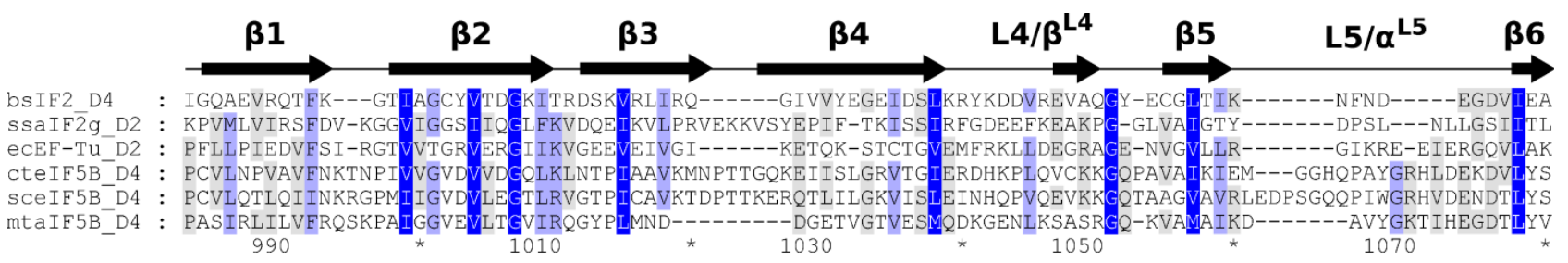

Figure 3.Partial structure-based sequence alignment of the $\beta$ barrel fold of domain IV (D4). The aligned sequences are from B. stearothermophilus IF2, M. thermoautotrophicum alF5B, C. thermophilum elF5B and S. cerevisiae elF5B with domain II (D2) from E. coli EF-Tu and S. solfataricus alF2 $\gamma$ (GenBank: CAA27987, AAB84765, EGS21143, AAC04996, CAA40370, AAK40740, respectively). Highly conserved residues are highlighted in dark blue, conserved residues in light blue and similar residues in grey. Sequence numbering and secondary structure elements correspond to the ctelF5B structure (PDB: 4N3G). As there is no structure of scelF5B available so far, its sequence was aligned directly with that of ctelF5B.

In ternary complexes of EF-Tu, the binding site for the 3' CCA-end on domain II is complemented by the conserved Phe229 in strand $\beta 1$ that stacks against C4 and C5 of the A76 ribose and by Arg274 (sometimes Gln or Lys) in the flexible loop following strand $\beta 4$ that interacts with the phosphate of A76 [48, 204] (Figure 4C). The density assigned to the CCA-end of the MettRNA $_{i}$ suggests similar interactions for the conserved Phe992 (in few cases Tyr or lle) and His1042 in ctelF5B (Figure 4A). According to the model, the rest of the acceptor stem of the tRNA adopts a slightly different orientation relative to the $\beta$ barrel than observed for aa-tRNA bound to EF-Tu. In good agreement with the predictions made for IF2 [196], C75 and C74 seem to be rotated $\sim 20^{\circ}$ toward the L1 loop (Figure 2). Interestingly, the orientation of the $\beta$ barrel would allow several positively charged residues to interact directly with the acceptor stem. Lys994 in the L1 loop could contact the initiator tRNA specific A1:U72 base pair. The conserved Arg1070 and His1071 in helix $\alpha^{\mathrm{L5}}$, positioned in a well defined density in the cryo-EM map (Figure 1D), are within contact distance to the phosphate backbone at G68 and C69 in the major groove. Notably, EF-Tu domain II contains a corresponding short helix $\alpha^{\mathrm{L5}}$ in which the conserved Arg295 as well forms a contact to the acceptor arm of the bound tRNA [48, 204] (Figure 2E). Based on the comparison with EF-Tu it was previously assumed that a similar contact might be formed between Lys725 and Glu726 of bsIF2 and fMet-tRNA ${ }^{\text {fMet }}$. However, such an interaction could not be observed by NMR spectroscopy in solution [196]. It is therefore conceivable that these interactions are formed only in the context of the ribosomal pre-IC, where the tRNA is stabilized in a specific orientation relative to domain IV of elF5B/IF2. 
A

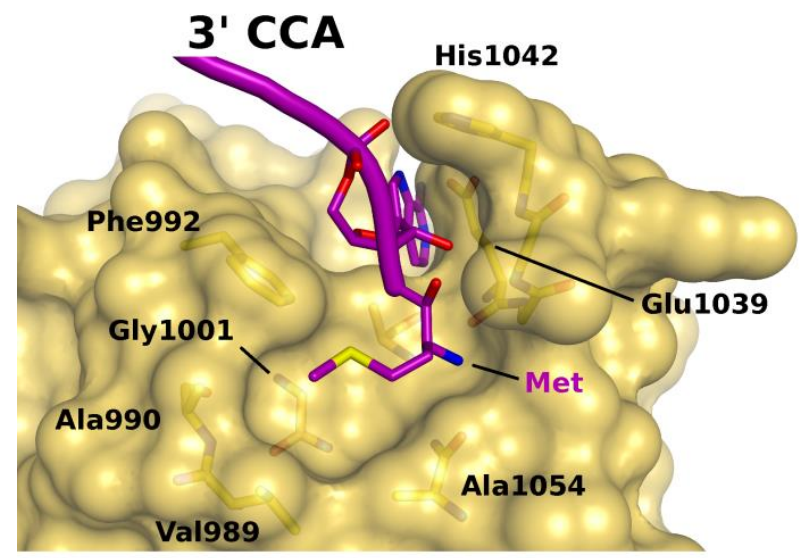

C

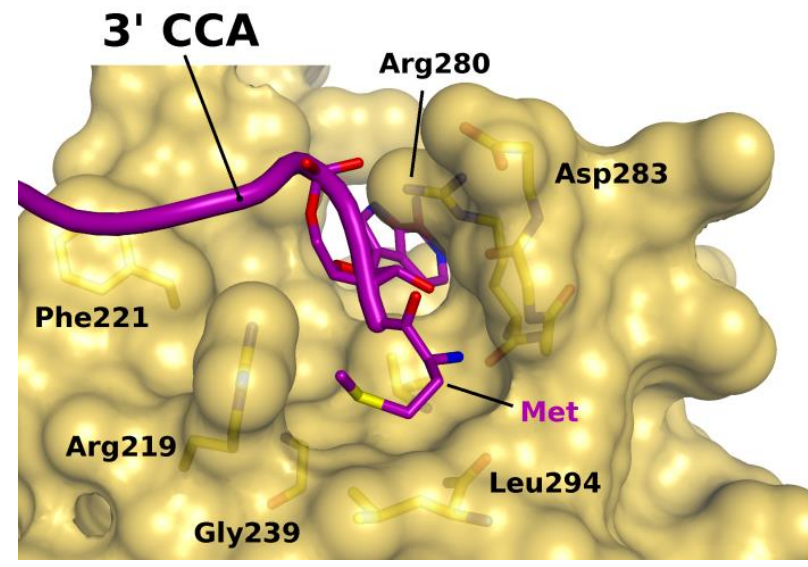

B

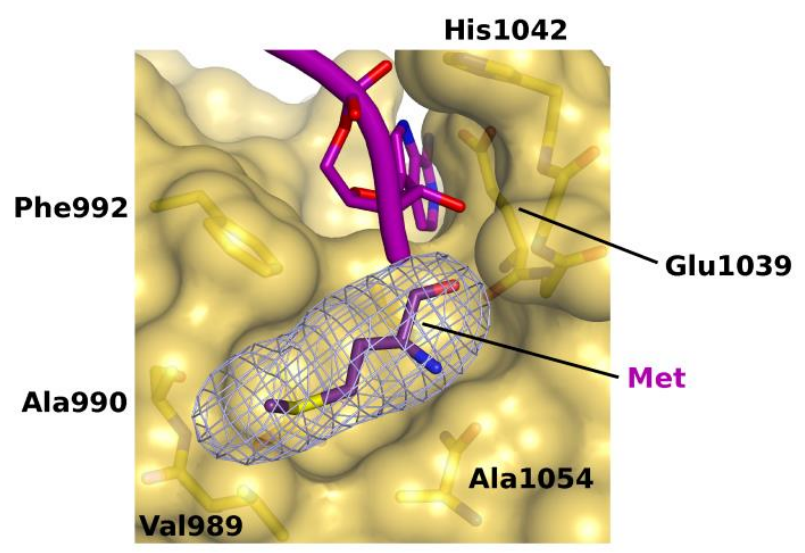

D

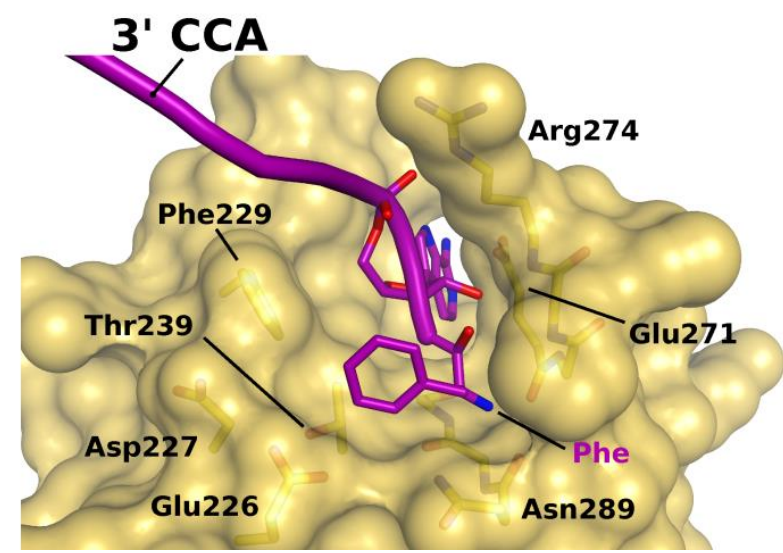

Figure 4. Recognition of the methionylated 3' CCA-end by elF5B domain IV. A) CCA arm, A76 and the methionly moiety of Met-tRNA $A_{i}$ modeled into the two surface pockets on elF5B domain IV according to the cryo-EM density. Residues implicated in the interactions with the $3^{\prime}$ end are indicated. B) Simulated annealing $f_{o}-f_{c}$ omit map for the putative methionyl-binding pocket in elF5B domain IV (blue mesh; contoured at $3 \sigma$ ). C) Met-tRNA $A_{i}$ bound to alF2 $\gamma$ (PDB: 3V11). D) Phe-tRNA ${ }^{\text {Phe }}$ bound to EF-Tu (PDB: 1TTT).

Biological relevance of this domain IV-tRNA interaction lies in the mutual stabilization of initiator tRNA and elF5B in conformations that allow efficient recruitment of the large ribosomal subunit and insertion of the acceptor arm into the peptidyl-transferase center (PTC). In the 80S complex domain IV stabilizes the tRNA in a non-canonical P/I orientation [91] that according to our model places the $3^{\prime}$ end $\sim 20 \AA$ from the PTC without inducing a major distortion of the CCA-end from its canonical conformation (Figure 5). The following GTP hydrolysis and dissociation of elF5B would thus allow the acceptor stem to rotate into the PTC while the overall tRNA relaxes into its 
canonical P site conformation. Through the specificity of domain IV for the methionylated acceptor arm, which might be more pronounced in the context of the preassembled 40S.Met-tRNA $A_{i}$ complex than in solution [202], this interaction would mark a final checkpoint in the initiation process that allows subunit joining only on correctly assembled 48S pre-ICs with a charged initiator tRNA bound in the $P$ site $[146,201]$.

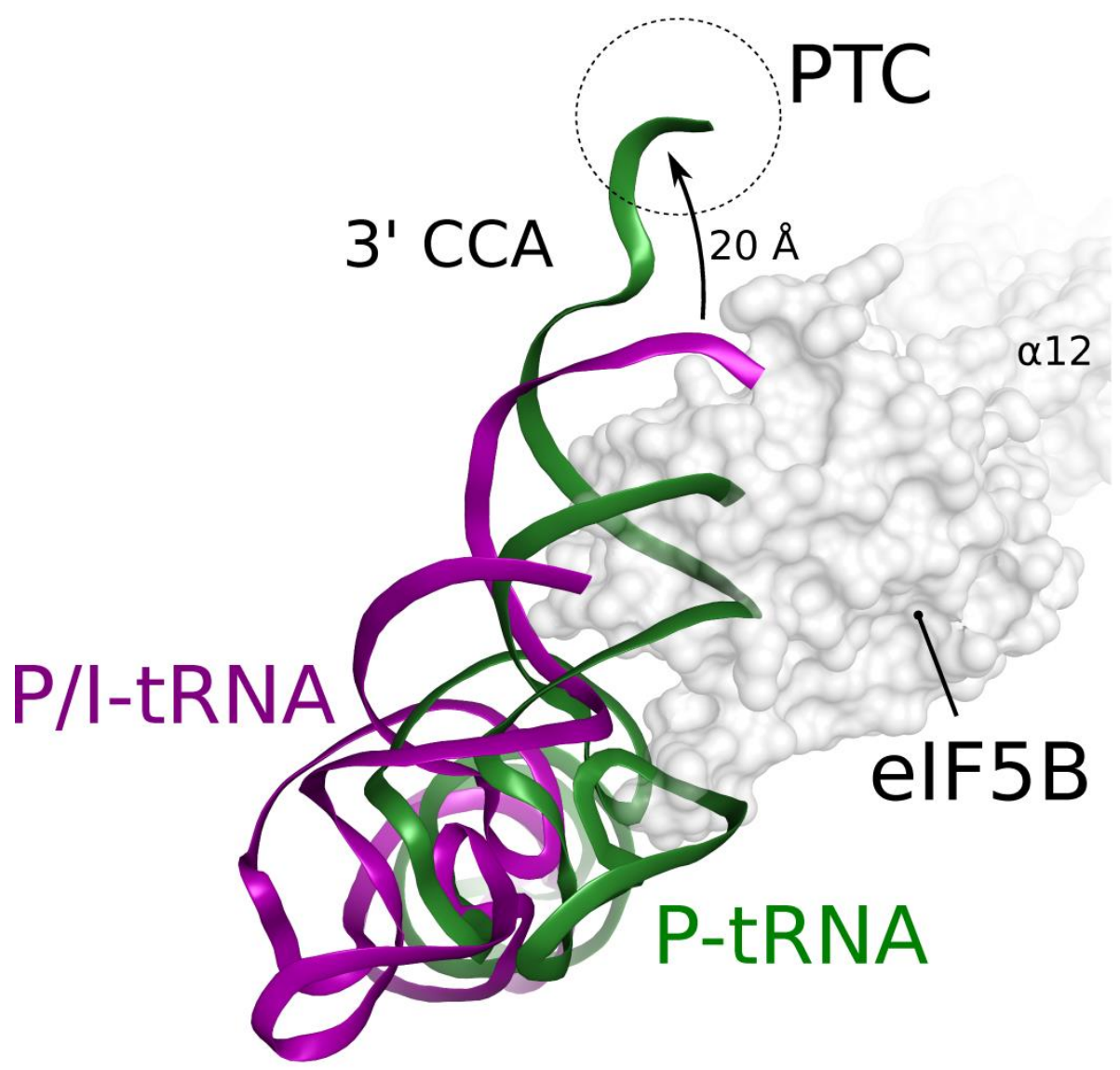

Figure 5. Conformational rearrangement of Met-tRNA $A_{i}$ on the initiation complex. elF5B stabilizes the initiator tRNA (purple) in a non-canonical P/I conformation [91] with the 3' CCA-end outside of the PTC. Upon GTP hydrolysis in elF5B and the release of the 3' CCA-end from its contacts to domain IV, the initiator tRNA rearranges into the canonical P site conformation, involving a $20 \AA$ repositioning of the $3^{\prime}$ end into the PTC.

\section{Implications for the evolution of the translational $\beta$ barrel fold}

As reported previously, domain IV of IF2 and the structurally homologous domain II of EF-Tu use similar surface areas to interact with amino-acylated tRNAs [196, 197] (Figure 2E/F). The structure of the ternary complex of aIF2 shows the same interface for the interactions between domain II of 
alF2 $\gamma$ (a paralog of EF-Tu) and the Met-tRNA $[$ [42] (Figure 4C). Our observations provide structural evidence that this also applies to domain IV of elF5B. This common binding interface for the $3^{\prime}$ CCA-end on the translational $\beta$ barrel fold is centered on $\beta$ strands 1,2 and 5 and framed by the flexible loops L1 and L4 (Figure 2 and 4). In all cases additional interactions are made by the short capping $\alpha$-helix that provides positively charged residues for contacts to the phosphate backbone of the acceptor stem, while at the same time allowing substantially different overall orientations of the tRNA relative to the $\beta$ barrel, irrespective of an identical polarity of the bound CCA-end (Figure 6). Despite the low average sequence identity over the various $\beta$ barrel folds (Figure 3), the significant structural and functional parallels in their interactions with amino-acylated tRNAs clearly point toward a common evolutionary origin. As elF5B/IF2 and EF-Tu are both universally conserved in the three domains of life, their divergence and thus the origin for their respective tRNA-binding domains most likely lies long before the onset of speciation; this raises the interesting question of potential homologies to other ancient RNA-binding protein folds.

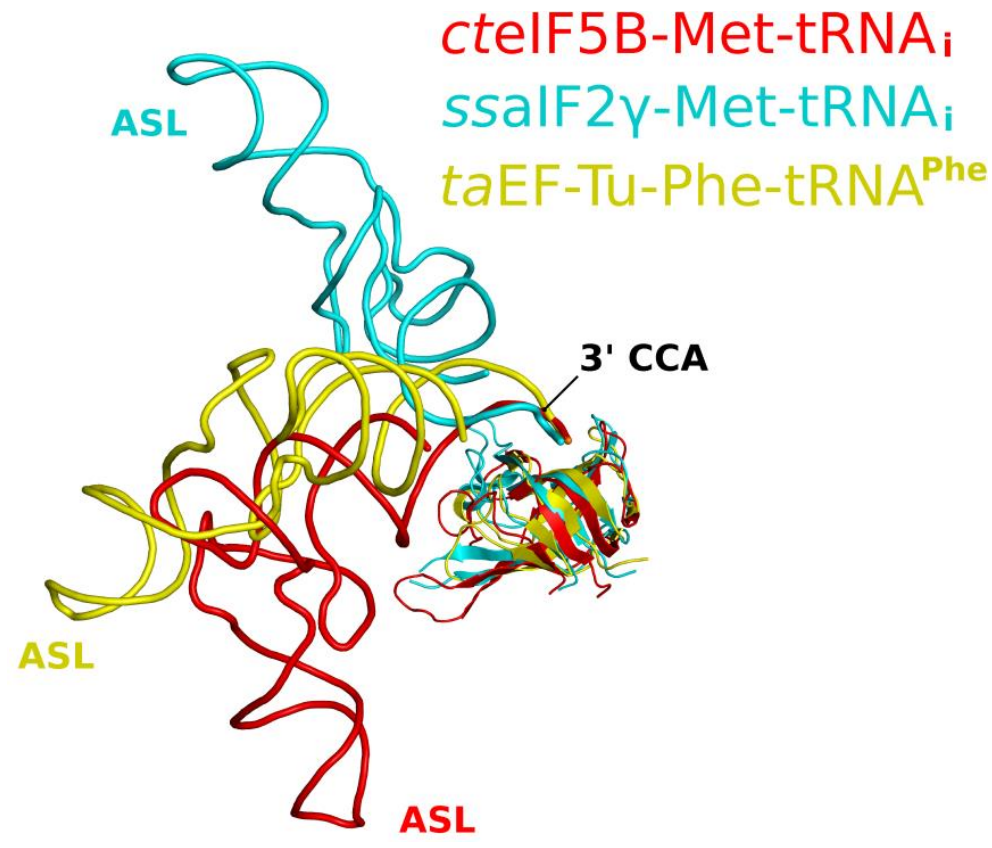

Figure 6.Comparison of the interactions between elF5B, EF-Tu and alF2 $\gamma$ with tRNA. Despite the same polarity and similar interfaces for the interactions between the translational $\beta$ barrel fold and the single-stranded 3' CCA-end, the tRNAs adopt significantly different overall orientations relative to the protein in ribosome-bound elF5B (red) or the ternary complexes of $S$. solfataricus alF2 (cyan; PDB: 3V11) and T. aquaticus EF-Tu (yellow; PDB: 1TTT). ASL is anticodon stem loop.

A central question for the problem of cellular evolution is the appearance of the basic protein-folding types and of domains as functional building blocks for proteins. Folded proteins adopt only a limited number of folding structures; however, whether these folds emerged by 
divergent evolution from a single ancestor or independently by convergent evolution from different lineages is unclear. In this context, it is interesting that the characteristic features of tRNA binding by the translational $\beta$ barrel fold show significant parallels to those between OB-fold domains and single-stranded nucleic acids (Figure 7). The OB-fold is a five-stranded mixed $\beta$ barrel, capped on one end by an $\alpha$-helix [205]. Most known OB-fold domains are involved in interactions with single-stranded RNA or DNA [206]. Despite very low sequence similarity among its members, the OB-fold superfamily is thought to be an ancient domain structure that derived by divergent evolution from a common ancestral protein - an assumption that is based on the common features of their fold-related ligand-binding interface [206, 207]. Despite a different overall topology of the OB-fold (Figure 7C/D) and a different classification in the SCOP (Structural Classification of Proteins) database, this interface, composed of $\beta 1-L 1-\beta 2-\beta 3-L 3 / \alpha^{L 3}-\beta 4-L 4$ (Figure 7B), shows an intriguing structural and functional correspondence to the identically arranged but differently connected building blocks of $\beta 1-\mathrm{L} 1-\beta 2$ and $\beta 4-\mathrm{L} 4-\beta 5-\mathrm{L} 5 / \alpha^{\mathrm{L5}}-\beta 6$ in the translational $\beta$ barrel fold that are responsible for its interactions with tRNA (Figure 7A).

These similarities might merely be a functional analogy between both protein families that arose by convergent evolution from two distinct starting points. However, by the argument of a common descent based on a fold-related ligand-binding interface, the evident similarities might as well be indicative of a common evolutionary origin for the two equally ancient protein folds. For this hypothesis, two previously proposed theories are of particular interest: i) The emergence of domain folds by polyphyletic evolution from self-assembling short peptide ancestors, whose remnants (in sequence, structure or function) still exist in extant proteins [208]; and ii) the theory of a chemoautotrophic origin of life on volcanic iron-sulfur surfaces, according to which protein domains emerged from functional peptides that used metal ions as folding determinants or formed surface-bonded $\beta$-sheets that finally detached from the stabilizing surfaces (e.g. to form $\beta$-barrel domains) in the course of progressing cellularization [209-211]. In both theories, the transition from the peptide- to the independently folding protein-domain proceeds concomitant to the refinement of the genetic machinery that allows the synthesis of increasingly long polypeptides with sufficiently high fidelity $[208,210,211]$. 
A
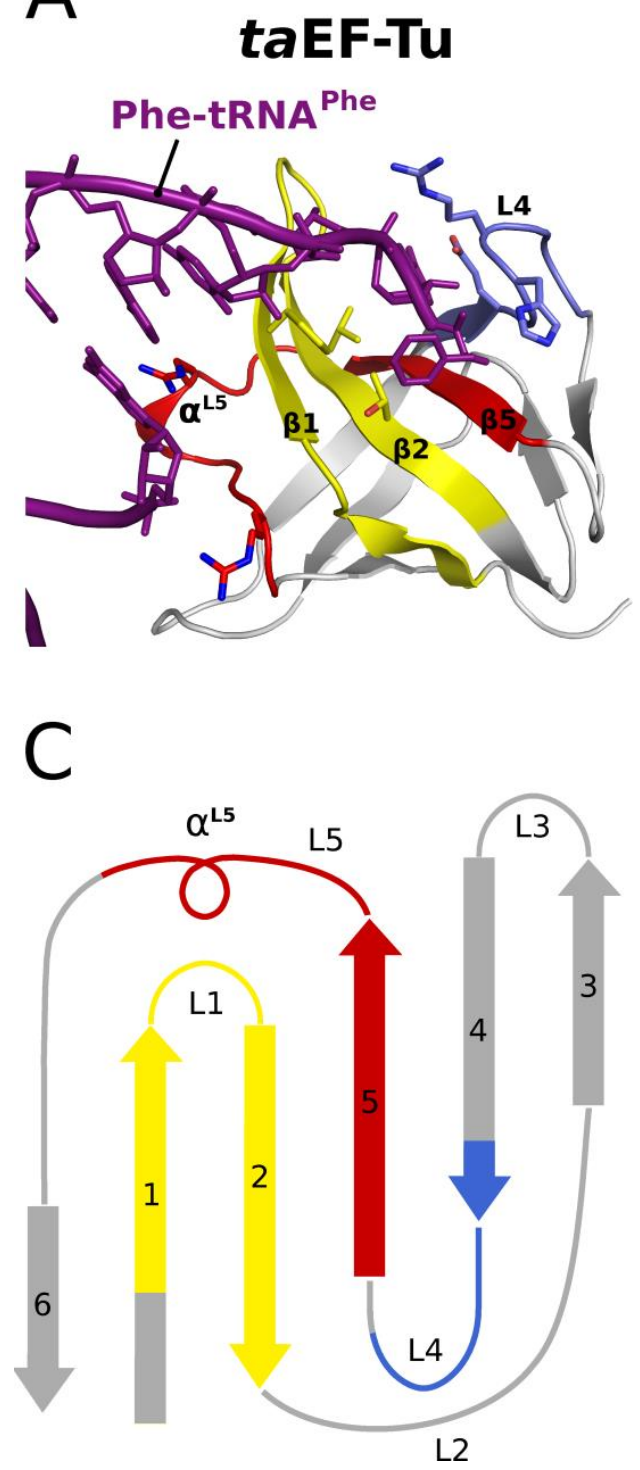

B

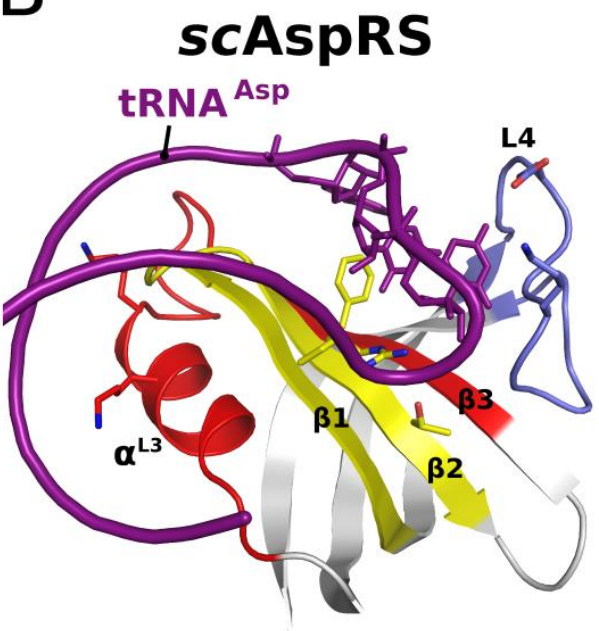

D

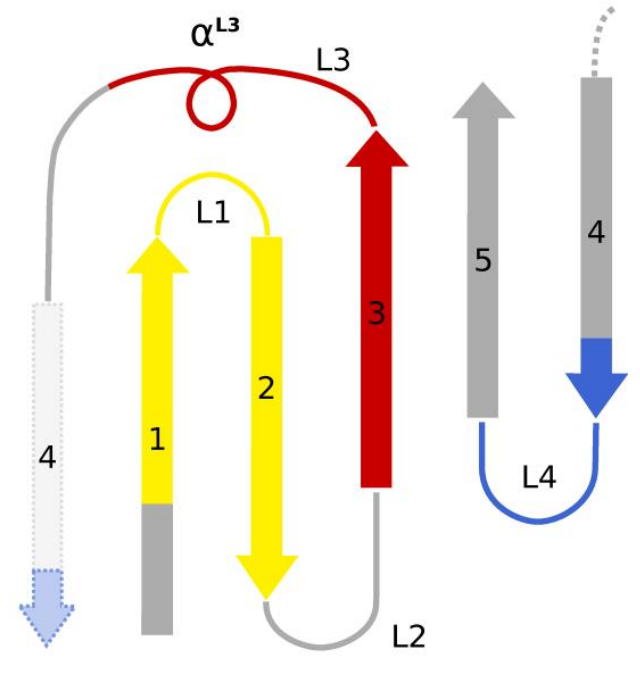

Figure 7. Translational $\beta$ barrel fold and OB-fold share the same fold related ligand-binding interface. A and C) The interaction interface between the 3' CCA-end of the tRNA and the translational $\beta$ barrel (here Phe-tRNA $^{\text {Phe }}$ (purple) bound to T. aquaticus EF-Tu domain II (PDB: 1TTT)) centers on $\beta$ strands 1,2 and 5 and is augmented by loops $L 1, L 4$ and $L 5$, containing helix $\alpha^{L 5}$. B and D) A related ligand-binding interface is found in single-stranded nucleic acid binding OB-folds (here the anticodon binding domain of the aspartyltRNA synthetase (PDB: 1ASZ) from S. cerevisiae[212]). Similar to the interactions observed for EF-Tu and despite a different topology, the bases of the anticodon stem loop (purple) point toward the surface of the $\beta$ barrel, centered on $\beta$ strands 1,2 and 3, while the flexible loops $L 1, L 4$ and $L 3$ with $\alpha^{L 3}$ form additional contacts to the phosphate backbone.

In light of these hypotheses, we suggest a possible common polyphyletic origin of both foldrelated RNA binding interfaces discussed above. At the earliest stages of cellular evolution, when the fidelity of the primordial translation apparatus allowed the synthesis only of short peptides, 
nucleic acid-peptide interactions most likely played an essential role, particularly for the genetic machinery. In this context, it would be conceivable that the common ligand-binding interface in the ancient lineages of OB-fold proteins and translational GTPases has arisen as an ancient structural entity formed by individually synthesized peptides, associating with single-stranded nucleic acids as folding determinants, similar to metallo-peptides as precursors for metalloproteins [209-211]; during the gradual replacement of peptides by their fusion into independently folding proteins, this would ensure the conservation of the nucleic acid-binding interface, while at the same time allowing a substantially different connectivity of the individual building blocks in the emerging protein families.

\section{Conclusions}

In this study, we used the recently reported medium resolution cryo-EM density of the yeast 80S IC [91] and high resolution crystal structures of elF5B from $C$. thermophilum to propose a new model for the interactions between elF5B domain IV and the Met-tRNA $A_{i}$ in the context of the ribosome (Figure 1). According to this model, domain IV forms direct interactions with the phosphate backbone in the major groove of the acceptor arm, the initiator tRNA specific A1:U72 base pair and - most importantly - with the methionylated 3' CCA-end. The relevance of these findings lies in the novel insight into the specific recognition of the amino-acylated initiator tRNA by elF5B/IF2 in the context of pre-initiation complexes, which, as a final checkpoint for ribosomal subunit joining, is one of the central interactions in the process translation initiation. Finally, the identified binding interface between elF5B and Met-tRNA $A_{i}$ directly corresponds to that reported earlier for the interaction between the homologous domains in IF2, EF-Tu and alF2 $\gamma$ with their respective tRNA ligands $[48,197]$ and exhibits a striking structural and functional similarity to the fold-related ligand-binding interface of OB-fold domains, possibly reflecting a common evolutionary origin of the two ancient domain folds. 
Chapter 6

\subsection{Methods}

\section{Model building}

Rigid-body fitting of ctelF5B domain IV (residues 382-1116; PDB codes 4N3N and 4N3G) was performed using UCSF Chimera [213]. Despite entirely different sets of crystal contacts for domain IV in the two X-ray structures they are very similar to each other ( $r m s d$ of 0.34 ), indicating a high degree of structural rigidity. Thus, although domain IV might undergo minor conformational changes upon interacting with ribosome and tRNA, particularly in the loop regions, we decided not to include any flexible fitting procedures to avoid overfitting of the model. Manual rebuilding of the acceptor stem of the Met-tRNA $A_{i}$ between bases $G 70$ and A76 into the density next to domain IV was done in COOT [116]. Figures were prepared using UCSF Chimera [213] or Pymol (http://www.pymol.org).

We would like to mention here that in our hands the isolated domain IV of the mtalF5B crystal structure (PDB: 1G7R) is fitted to the cryo-EM density in the same way as the ctelF5B structure (with a CCC of 67\%), supporting the newly proposed fit shown in Figure 1D. However, the mtalF5B domain IV of the cryo-EM based model (PDB: 4BYX) is fitted as presented in [91] with a CCC of $78.7 \%$ (Figure 1C), most likely as the result of a combination of rigid-body and flexible fitting procedures [91], which gave rise to rmsds of $5.8 \AA$ and $7.5 \AA$ (over $106 \mathrm{C}_{\alpha}$ atoms) relative to the crystal structures of mtelF5B and ctelF5B, respectively, while the two crystal structures themselves

differ only by an rmsd of $2.2 \AA$. Thus, the higher CCC for the cryo-EM-based model is most likely due to its distortion from the original rigid structure of $m$ talF5B and is therefore not comparable to the CCC values obtained for our rigid-body fit.

\section{Sequence alignments}

Multiple sequence alignments were done using the iterative alignment program MUSCLE [214]. Structural sequence alignments were done using the DALI server [215]. 


\section{Author contributions}

BK designed the study, analyzed structures and cryo-EM density, performed the structural modeling and sequence alignments, analyzed and interpreted the data and wrote the manuscript. $\mathrm{RF}$ analyzed the data and helped to draft the manuscript. All authors read and approved the final manuscript.

\section{Acknowledgments}

We thank L. K. Dörfel for critical reading of the manuscript.

\section{List of abbreviations}

IF, initiation factor; elF, eukaryal initiation factor; alF, archaeal initiation factor; cryo-EM, cryoelectron microscopy; pre-IC, pre-initiation complex; EMD, EM-Databank; EF-Tu, elongation factorTu; ct, Chaetomium thermophilum; mt, Methanococcus thermoautotrophicum; sc, Saccharomyces cerevisiae; bs, Bacillus stearothermophilus; PDB, protein data bank; CCC, cross-correlation coefficient; SCOP, Structural Classification of Proteins; OB-fold, oligo-nucleotide/oligo-saccharide binding fold; rmsd, root mean square deviation; PTC, peptidyl-transferase center; ASL, anticodon stem loop.

\section{Competing interests}

The authors declare that they have no competing interests. 


\title{
Chapter $7 \bullet A$ monovalent cation as cofactor in translational GTPases
}

This manuscript has originally been published in The EMBO Journal

\author{
A Monovalent Cation Acts as Structural and Catalytic Cofactor in Translational \\ GTPases \\ Bernhard Kuhle $^{1, \S}$ and Ralf Ficner ${ }^{1}$ \\ ${ }^{1}$ Abteilung für Molekulare Strukturbiologie, Institut für Mikrobiologie und Genetik, Göttinger \\ Zentrum für Molekulare Biowissenschaften, Georg-August-Universität Göttingen, D-37077 \\ Göttingen, Germany \\ \$To whom correspondence should be addressed: bkuhle@gwdg.de
}

Keywords: translation, catalytic mechanism, monovalent cation, crystal structure, GTPase

Received 18 March 2014 | Revised 1 July 2014 | Accepted 14 August 2014

Author contributions:

BK: Conceived the study and designed the experiments; prepared and crystallized proteins, collected X-ray data andsolved the structures;performed ITC and fluorescence experiments; created figures; analyzed data and wrote the manuscript. RF: analyzedthe data and helped to draft the manuscript. 


\section{Abstract}

Translational GTPases are universally conserved GTP hydrolyzing enzymes, critical for fidelity and speed of ribosomal protein biosynthesis. Despite their central roles, the mechanisms of GTPdependent conformational switching and GTP-hydrolysis that govern the function of trGTPases remain poorly understood. Here, we provide biochemical and high-resolution structural evidence that elF5B and aEF1A/EF-Tu bound to GTP or GTPyS coordinate a monovalent cation $\left(\mathrm{M}^{+}\right)$in their active site. Our data reveal that $\mathrm{M}^{+}$ions form constitutive components of the catalytic machinery in trGTPases acting as structural cofactor to stabilize the GTP-bound 'on' state. Additionally, the $\mathrm{M}^{+}$ ion provides a positive charge into the active site analogous to the arginine-finger in the RasRasGAP system indicating a similar role as catalytic element that stabilizes the transition state of the hydrolysis reaction. In sequence and structure, the coordination shell for the $\mathrm{M}^{+}$ion is, with exception of elF2 $\gamma$, highly conserved among trGTPases from bacteria to human. We therefore

propose a universal mechanism of $\mathrm{M}^{+}$-dependent conformational switching and GTP-hydrolysis among trGTPases with important consequences for the interpretation of available biochemical and structural data.

\subsection{Introduction}

Translation - the ribosome-catalyzed synthesis of biologically functional polypeptides according to genetically encoded information - is one of the most fundamental and complex biochemical processes in extant cellular life. In all stages of protein biosynthesis the ribosome depends on a set of auxiliary guanine nucleotide binding (G) proteins (termed translational GTPases or trGTPases) that include initiation factor 2 (IF2), which catalyzes ribosomal subunit joining and formation of the elongation-competent ribosome, elongation factor Tu (EF-Tu), which delivers aminoacyl-tRNA (aatRNA) to the ribosomal A site, as well as elongation factor $\mathrm{G}$ (EF-G) that catalyzes the translocation of the mRNA-tRNA complex on the ribosome $[8,9]$. Despite the differences in their respective functions, trGTPases from bacteria to eukarya share a common evolutionarily conserved structural core composed of the $\mathrm{G}$ domain and domain II, which is supplemented with additional factor specific domains. The $G$ domain forms the functional center in all trGTPases that couples GTP 
binding and hydrolysis to the specific biological function of the translation factor [9]. This present work focuses on so far unresolved aspects of the universal molecular mechanisms that govern the functional cycle of the $\mathrm{G}$ domain in trGTPases and that are therefore central to the understanding of the process of translation as well as its evolution.

In sequence and architecture the $\mathrm{G}$ domain in trGTPases is related to small Ras-like $\mathrm{G}$ proteins and thus belongs to the superfamily of P-loop GTPases [24]. According to the classical view, G proteins are thought to act as molecular switches that oscillate between a GDP-bound 'off' state and a structurally distinct GTP-bound 'on' state [23, 24]. The exchange of GDP for GTP is accompanied by conformational changes in two conserved dynamic elements termed switch 1 and switch 2 that form specific interactions with the GTP- $\gamma$-phosphate. Conversion back to the GDPbound state requires the hydrolysis of GTP and release of inorganic phosphate $\left(P_{i}\right)$, allowing switch 1 and 2 to relax back into their inactive conformations. These structural changes ensure that only the GTP-bound form of a G protein is able to interact productively with effector molecules to carry out its biological function [23].

Despite its importance for the understanding of the translation process, the mechanism of GDP/GTP-dependent conformational switching has remained obscure for nearly all trGTPases. Over the years, high resolution structures have become available for various trGTPases in their apo states as well as in complex with GDP and the nonhydrolyzable GTP analogs GDPNP or GDPCP ( $\beta-\gamma-$ imidoguanosine 5 -triphosphate and $\beta$ - $\gamma$-methyleneguanosine $5^{\prime}$-triphosphate, respectively). Surprisingly, however, from these structures a highly heterogeneous picture emerged for the mechanisms of conformational switching in the G domains of trGTPases that is at odds with their evolutionary and structural homology: While only EF-Tu exhibited clearly distinct GDP- and GDPNPbound conformations in agreement with the classical concept $[38,163]$, EF-G, alF5B and eRF3 adopted virtually identical structures in their respective GDP- and GDPNP/GDPCP-bound forms, invoking suggestions about non classical mechanisms in these $G$ proteins $[84,216,217]$. SelB, finally, seemed to constitute an intermediate case with switch 2 undergoing conformational changes upon GDPNP binding, while switch 1 remained mainly flexible similar to the GDP-bound state [218]. In order to reconcile the structural data with the classical model of GTPase function it was proposed that some of these apparently non-classical trGTPases follow a mechanism of 
'conditional switching' [153]. According to this concept, the GTP alone is insufficient to induce the GTP-conformation in the $G$ domain but requires the ribosome as additional cofactor for the efficient conformational switch to the 'on' state. However, biochemical experiments as well as a recent crystal structure of elF5B bound to GTP indicate that EF-G, SelB and elF5B in fact do undergo significant structural rearrangements in the presence of true GTP instead of GDPNP or GDPCP, indicating that they conform to the classical model without the requirement of the ribosome or aa-tRNA for the conformational switch $[183,219,220]$. These observations are paralleled by a large body of biochemical data indicating that GDPNP is not an authentic GTP analog for nearly all trGTPases $[153,183,221-224]$ and that the use of nonhydrolyzable analogs in structural experiments most likely accounts for the apparent deviation from the classical switch mechanism [153]. Hence, it seems evident that the stable conformational switch is dependent on critical contributions by the GTP molecule that are, however, not provided by the structurally similar GDPNP/GDPCP. It is therefore a central conceptual assumption of this present work that the identification of this so far unknown contribution by the GTP molecule provides a key to a more unified view on the GDP/GTP cycle in trGTPases, in agreement with their common evolutionary descent.

Another central and yet unresolved problem in the functional cycle of trGTPases is the molecular mechanism of ribosome-induced GTP hydrolysis. Similar to other Ras-like G proteins, trGTPases possess a low intrinsic GTPase activity which is accelerated by several orders of magnitude upon productive binding to the ribosome [27, 225]. In analogy to the systems of the GTPase activating protein (RasGAP) of Ras or the regulators of $G$ protein signaling (RGS) of G $\alpha$ proteins, it was shown that the ribosome stimulates rapid GTP hydrolysis in trGTPases by the precise positioning of an invariant histidine ( $\mathrm{His}^{\text {cat }}$ ) in switch 2 (corresponding to $\mathrm{Gln} 61$ in Ras) in its catalytically active conformation $[168,169]$. However, the actual origin of the catalytic effect in the activated system has remained controversial [168, 226-229]. That is, it remained unclear how the nucleophilic water $\left(\mathrm{W}^{\mathrm{cat}}\right)$ is activated for its attack on the $\mathrm{y}$-phosphate and how the subsequent stabilization of the transition state (TS) is achieved. In the Ras system RasGAPs further stimulate GTP hydrolysis by supplying an additional catalytic residue, the 'arginine-finger', into the active site, which is responsible for a $\sim 2000$-fold acceleration of the GTPase reaction by direct 
electrostatic stabilization of developing negative charges in the TS [106]. Analogous catalytic elements provided in cis or in trans have been identified in P-loop GTPases from Ga proteins to MnmE, dynamin and the signal recognition particle [25, 230-232]. However, up to now, trGTPases seemed to be an exception among Ras-related G proteins, as the search for an arginine-finger or an analogous catalytic element has been unsuccessful [233-236].

Here, we discuss the unresolved questions concerning the GTP-induced conformational switch and GTP hydrolysis in trGTPases in light of the novel assumption that trGTPases utilize a monovalent cation ( $\mathrm{M}^{+}$ion) as structural and catalytic cofactor. We show that elF5B (eukaryal IF2 ortholog) as well as aEF1A (archaeal EF-Tu ortholog) coordinate an $\mathrm{M}^{+}$ion in their active sites in the same position as known $\mathrm{M}^{+}$-dependent GTPases, thus placing a positive charge analogous to the guanidino group of the arginine-finger in the Ras-RasGAP complex. The coordination shell for the $\mathrm{M}^{+}$ion is, with the notable exception of elF2 $\gamma$, universally conserved among trGTPases from bacteria to humans and directly involves the oxygen atom of GTP that is replaced in GDPNP and GDPCP but not in the slowly hydrolyzable GTPYS. In combination with mutational, biochemical and isothermal titration calorimetry (ITC) data, these findings provide the conceptual framework and a significant explanatory power for the interpretation of a large body of previously unexplained observations for trGTPases, resulting in the conclusions that i) the $\mathrm{M}^{+}$ion acts as structural cofactor that stabilizes the 'on' state of the G domain and thus contributes to the conformational switch in trGTPases; ii) GDPNP and GDPCP are unable to coordinate the $\mathrm{M}^{+}$ion and thereby destabilize the GTP-conformation; iii) GTPYS is able to coordinate the $\mathrm{M}^{+}$ion and thus a suitable GTP analog for trGTPases; iv) the $\mathrm{M}^{+}$ion participates in the GTP hydrolysis reaction, most likely by stabilizing its TS; v) with few exceptions $\mathrm{M}^{+}$-dependency is universal among canonical trGTPases.

\subsection{Results}

\section{GTP-bound elF5B coordinates an $\mathrm{M}^{+}$ion in its catalytic center}

Recently we reported the crystal structure of GTP-bound elF5B from $C$. thermophilum, solved at $1.9 \AA$ resolution (protein data bank (PDB): 4NCN) [220]. This structure revealed a $\mathrm{Na}^{+}$ion next to the GTP- $\gamma$-phosphate in a catalytically relevant position of the active site, which has so far never been reported for a trGTPase (Fig. 1A and E1A). In line with previously reported values [156], this 
$\mathrm{Na}^{+}$ion is penta-coordinated with coordination distances between 2.3 and $2.5 \AA$ by two oxygens from the $\alpha$ - and $\psi$-phosphates, the $\beta$ - $\gamma$-bridging oxygen, the carboxylate group of Asp533 in the Ploop (which we denote $\mathrm{Asp}^{\mathrm{MC}}$ for aspartate involved in monovalent cation-binding) and the backbone CO from Gly555 in switch 1 (Gly ${ }^{\mathrm{MC}}$ ). The latter is part of a short peptide backbone excursion of switch 1 (formed by Gly554 and Gly555) which approaches the $\mathrm{Na}^{+}$ion opposite to the $\beta$ - $\gamma$-bridging oxygen and is denoted 'MC-loop' (Fig. 1).

\section{GTPYS but not GDPNP supports crystallization of elF5B in its GTP-bound conformation.}

The position of the $\mathrm{M}^{+}$ion in elF5B.GTP suggests that it provides a direct contribution to the stabilization of the GTP-bound switch 1 by forming a stable link to the GTP molecule (Fig. 1A). To test the relevance of this contribution, crystallization trials were performed with the construct ctelF5B(517-858) in the presence of GTP, GDPNP and GTPYS. If the $\mathrm{M}^{+}$is relevant for the conformational switch in elF5B, GTP and GTP $\gamma$ S but not GDPNP, in which the $\beta$ - $\nu$-bridging oxygen is replaced by an $\mathrm{NH}$-group (Fig. 2A), should readily stabilize the GTP-conformation and allow crystallization of ctelF5B(517-858) in an optimization screen for the original crystallization condition (0.1 M HEPES/NaOH (pH 7); 13\% PEG 4000; $0.1 \mathrm{M} \mathrm{NaOAc)} \mathrm{(see} \mathrm{Expanded} \mathrm{View} \mathrm{for}$ details).

In line with our assumption, elF5B(517-858).GTP and elF5B(517-858).GTPyS readily crystallized, while no crystals grew in the presence of GDPNP. The structure of elF5B(517-858).GTP, solved at $1.55 \AA$ resolution, is very similar to that reported previously [220] (see Table 1 for details of data collection and refinement). The electron densities for both $\mathrm{Na}^{+}$ions in the asymmetric unit are well defined with occupancies of $100 \%$.

The structure of elF5B(517-858).GTPYS was solved at $1.53 \AA$ A resolution, exhibiting an overall structure and nucleotide binding virtually identical to that of the GTP-bound form (Fig. E1B/D). The sulfur atom of the $\psi$-phosphate points outward and thus replaces the non-bridging oxygen atom that is involved in the coordination of the $\mathrm{M}^{+}$ion in elF5B.GTP. Nonetheless, both elF5B.GTPYS complexes in the asymmetric unit contain the $\mathrm{Na}^{+}$ion in the pentameric coordination shell with coordination distances of 2.2-2.6 $\AA$ to the four oxygen ligands from the GTP molecule, Asp ${ }^{\mathrm{MC}}$ and Gly ${ }^{\mathrm{MC}}$, and $2.83 \AA$ to the sulfur atom (in agreement with the theoretical distance of $2.8 \AA$, 
calculated from the van der Waals radius of sulfur $(1.8 \AA)$ and the effective ion radius of $\mathrm{Na}^{+}(1.0 \AA$ for coordination number 5) [237, 238]).

Table 1. Crystallization, X-ray data collection and refinement statistics for structures of wild-type elF5B(517-858)

\begin{tabular}{|c|c|c|c|}
\hline & elF5B(517-858) $\cdot \mathrm{GTP} \cdot \mathrm{Na}^{+}$ & $\begin{array}{l}\text { elF5B(517- } \\
858) \cdot G T P \gamma S \cdot \mathrm{Na}^{+}\end{array}$ & $\begin{array}{l}\text { elF5B(517- } \\
858) \cdot G T P \gamma S \cdot K^{+}\end{array}$ \\
\hline \multicolumn{4}{|l|}{ Crystallization } \\
\hline Condition & $\begin{array}{l}100 \mathrm{mM} \text { HEPES }(\mathrm{pH} \\
7.25), 12 \% \text { PEG 4000, } \\
100 \mathrm{mM} \mathrm{NaOAc}\end{array}$ & $\begin{array}{l}100 \mathrm{mM} \text { HEPES (pH 7), } \\
\text { 13\% PEG 4000, } 125 \mathrm{mM} \\
\text { NaOAc }\end{array}$ & $\begin{array}{l}11 \% \text { PEG } 8000,6 \% \\
\text { glycerol, } 50 \mathrm{mM} \mathrm{KCl}\end{array}$ \\
\hline Temperature $\left({ }^{\circ} \mathrm{C}\right)$ & 20 & 20 & 20 \\
\hline \multicolumn{4}{|l|}{ Data Collection } \\
\hline Space Group & $\mathrm{P} 2_{1}$ & $\mathrm{P} 2_{1}$ & $\mathrm{P} 4{ }_{1} 2_{1} 2$ \\
\hline Unit Cell & $\begin{array}{l}a=55.6 \AA \\
b=116.5 \AA \\
c=66.2 \AA \\
\alpha=\beta=90^{\circ} \\
\beta=101.1^{\circ}\end{array}$ & $\begin{array}{l}a=55.6 \AA \\
b=115.9 \AA \\
c=66.1 \AA \\
\alpha=\beta=90^{\circ} \\
\beta=101.4^{\circ}\end{array}$ & $\begin{array}{l}a=116.1 \AA \\
b=116.1 \AA \\
c=120.3 \AA \\
\alpha=\beta=\gamma=90^{\circ}\end{array}$ \\
\hline Molecules/asym. unit & 2 & 2 & 2 \\
\hline Resolution $(\AA ̊)$ & $1.55(1.64-1.55)$ & $1.53(1.63-1.53)$ & $2.28(2.42-2.28)$ \\
\hline Observed reflections & $405616(64352)$ & $510862(86738)$ & 307464 (49685) \\
\hline Unique reflections & 118431 (18849) & 121606 (20942) & $38004(6021)$ \\
\hline Completeness (\%) & $98.8(97.7)$ & $98.5(98.4)$ & $99.9(99.7)$ \\
\hline$<1>/ \sigma$ & $15.5(2.3)$ & $18.8(2.8)$ & $19.3(3.8)$ \\
\hline $\mathrm{R}_{\text {sym }}(\%)$ & $4.6(50.5)$ & $3.7(49.6)$ & $6.3(54.2)$ \\
\hline $\mathrm{CC}(1 / 2)(\%)$ & $99.9(75.1)$ & $99.9(82.2)$ & $99.9(99.1)$ \\
\hline \multicolumn{4}{|l|}{ Refinement } \\
\hline $\mathrm{R}_{\text {work }}(\%)$ & 15.7 & 16.3 & 20.0 \\
\hline$R_{\text {free }}(\%)$ & 18.4 & 18.8 & 23.9 \\
\hline \multicolumn{4}{|l|}{$\begin{array}{l}\text { Rmsd from Standard } \\
\text { Stereochemistry }\end{array}$} \\
\hline Bond length $(\AA)$ & 0.019 & 0.02 & 0.005 \\
\hline Bond angles $\left({ }^{\circ}\right)$ & 1.8 & 1.9 & 1.0 \\
\hline \multicolumn{4}{|l|}{$\begin{array}{l}\text { Ramachandran Plot } \\
\text { Statistics }\end{array}$} \\
\hline Most favored (\%) & 98.9 & 98.1 & 98.5 \\
\hline Allowed regions (\%) & 1.1 & 1.9 & 1.5 \\
\hline Disallowed regions (\%) & 0 & 0 & 0 \\
\hline
\end{tabular}

These results demonstrate that GTP $\gamma$ S is a suitable structural GTP analog that is able to coordinate the $\mathrm{M}^{+}$ion and to stabilize the GTP-conformation in elF5B. In contrast, GDPNP seems 
unable to stabilize efficiently the same conformation (and thus to provide the same surfaces for crystal contacts) as GTP, resulting in the inability of the elF5B.GDPNP complex to crystallize under the same conditions as elF5B.GTP. The likely explanation for this observation is that, while GTP and GTPYS provide all the ligands required for the coordination of the $\mathrm{Na}^{+}$ion and thus allow the stable association of switch 1, GDPNP is unable to provide the $\mathrm{M}^{+}$ion as structural cofactor due to the replacement of the $\beta$ - $\gamma$-bridging oxygen of GTP with a $\beta$ - - -bridging imido (NH) group (Fig. $2 \mathrm{~A}$ ).

\section{A}
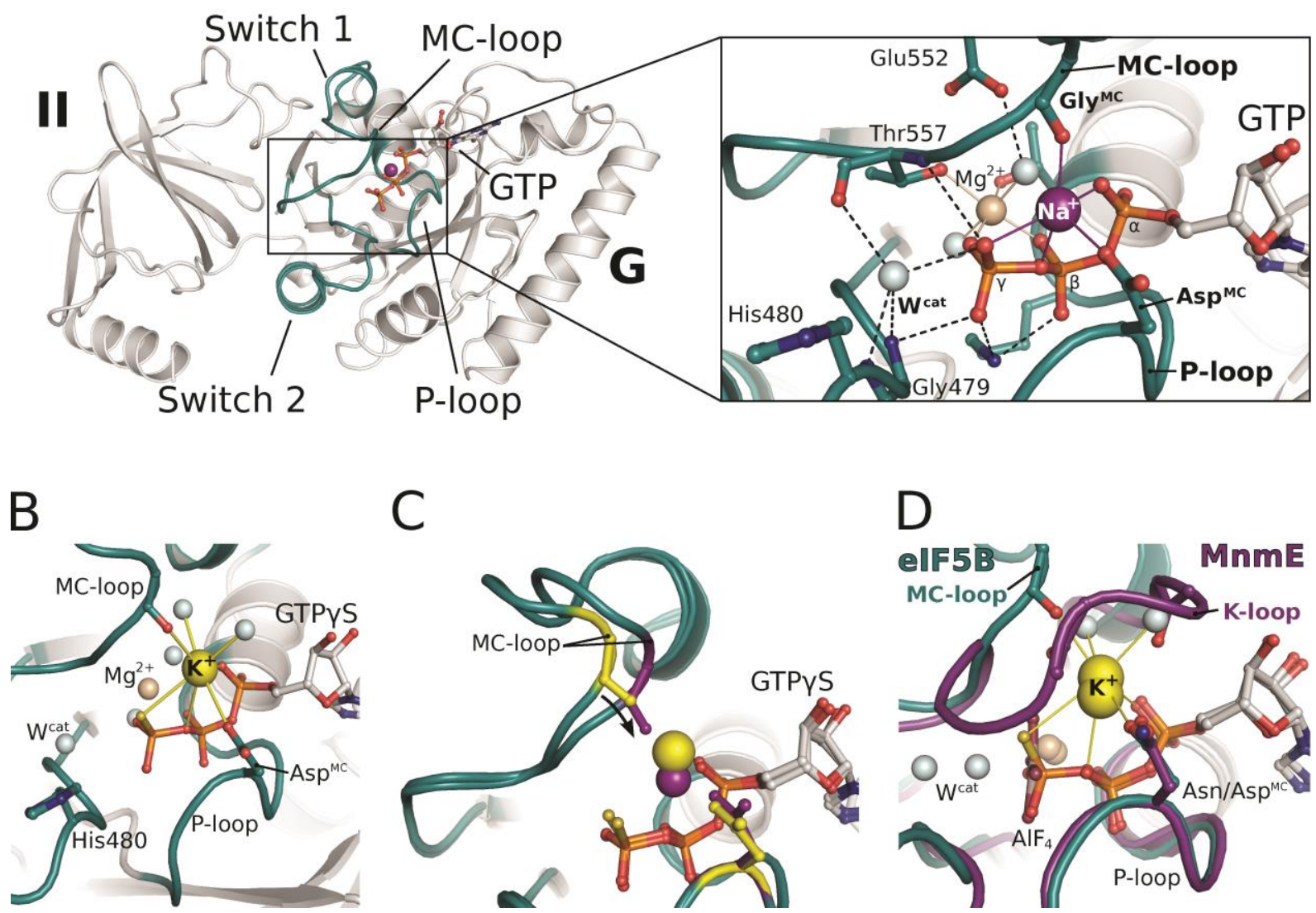

Figure 1. GTP-bound elF5B coordinates an $\mathrm{M}^{+}$ion in its GTPase center. A) Overview of $\mathrm{G}$ domain and domain II of GTP-bound eIF5B with a $\mathrm{Na}^{+}$ion (purple sphere) in the active site. P-loop and switch regions are shown in cyan; GTP is shown as balls and sticks. The $\mathrm{Na}^{+}$ion is bound by a pentameric coordination sphere (inset; indicated by purple lines) formed by GTP, $\mathrm{Asp}^{\mathrm{MC}}$ and $\mathrm{Gly}^{\mathrm{MC}} \cdot \mathrm{Mg}^{2+}$ and water molecules are shown as spheres in light brown and grey, respectively; the catalytic histidine (His480) and highly conserved residues that interact with GTP or the cations are indicated; hydrogen bonds are shown as dashed lines. B) The active site of elF5B.GTP $\gamma S$ with a $\mathrm{K}^{+}$ion (yellow sphere) bound in a heptameric coordination sphere. C) Superposition of eIF5B.GTP $y$ S structures with either $\mathrm{Na}^{+}$(purple sphere) or $\mathrm{K}^{+}$(yellow sphere) bound in the active site. Due to the shorter coordination distances to the $\mathrm{Na}^{+}$ion, $\mathrm{Asp}^{\mathrm{MC}}$ and $\mathrm{Gly}^{\mathrm{MC}}$ (purple sticks) are drawn closer to the GTP molecule than in the $\mathrm{K}^{+}$-structure (yellow sticks). D) Superposition of elF5B.GTP $\mathrm{S} \cdot \mathrm{K}^{+}$and MnmE (purple; PDB: $2 \mathrm{GJ} 8$ ) bound to GDP, $\mathrm{AlF}_{4}$ and $\mathrm{K}^{+}$. 
A

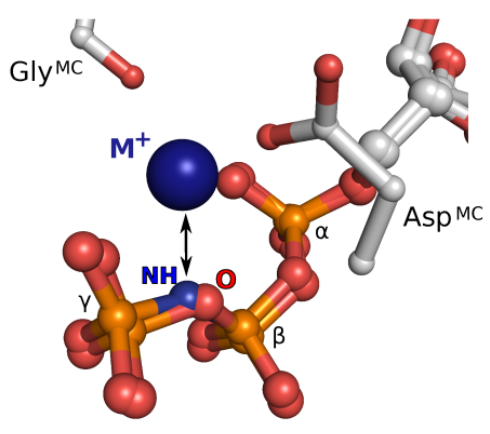

C

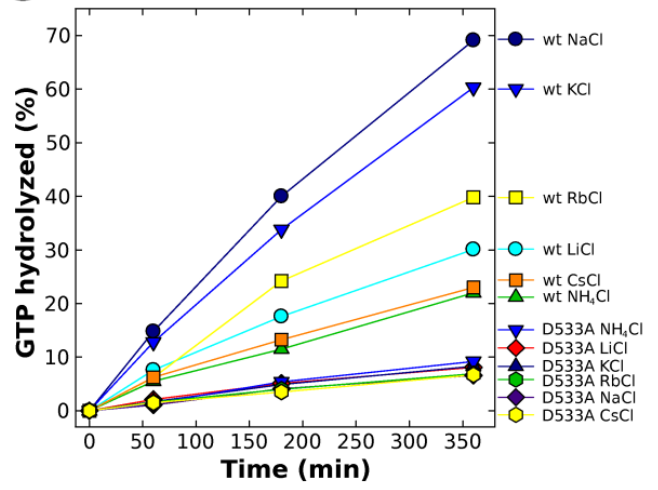

E

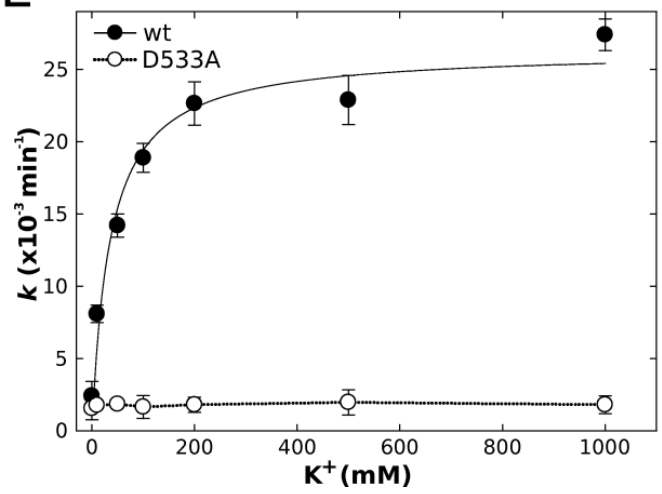

B

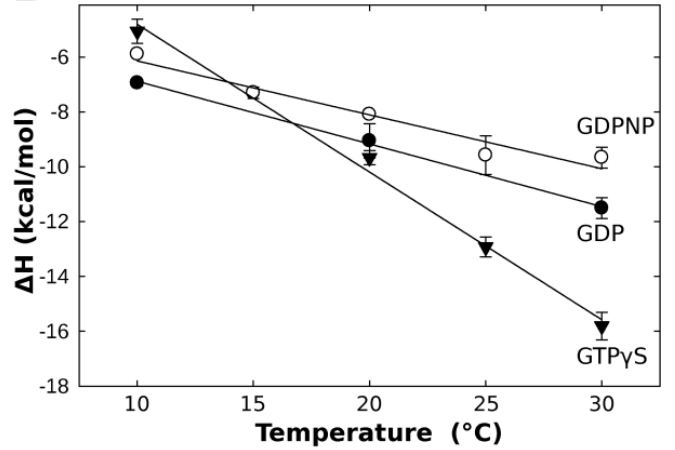

D

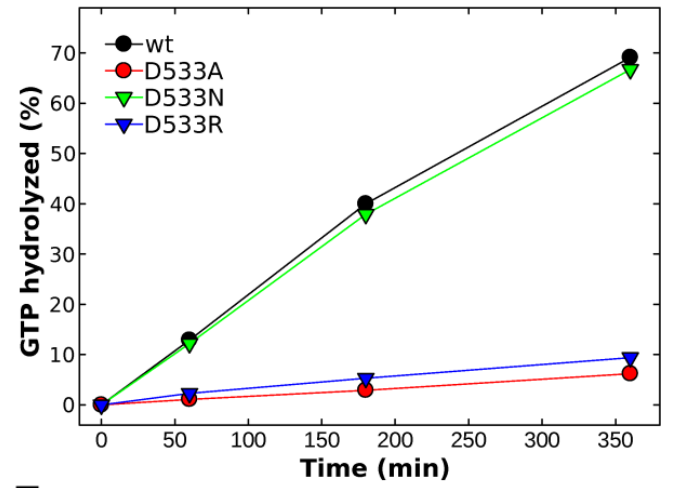

$\mathrm{F}$

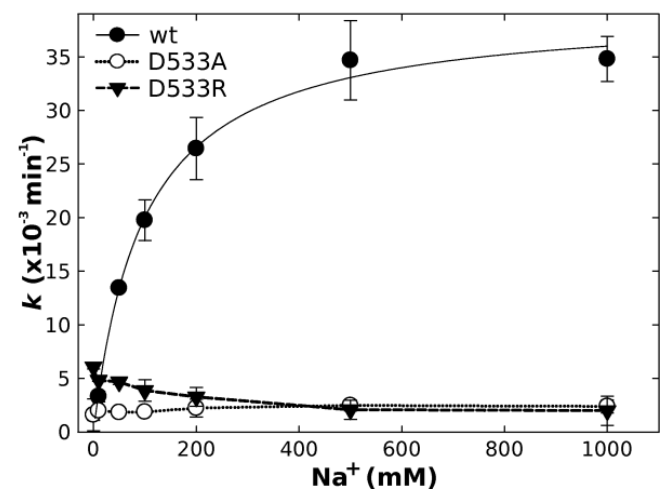

Figure 2. Biochemical characterization of nucleotide binding and GTP hydrolysis by elF5B.A) GDPNP disrupts the coordination sphere for the $\mathrm{M}^{+}$ion (blue sphere) formed by $\mathrm{Asp}^{\mathrm{MC}}$, Gly ${ }^{\mathrm{MC}}$ and three oxygens from GTP by replacing the $\beta$ - $\gamma$-bridging oxygen (O) with an NH group. B) Temperature dependency of binding enthalpy changes $(\Delta \mathrm{H})$ upon elF5B(517-858) interaction with GDP $(\bullet)$, GDPNP $(0)$ or GTPyS $(\boldsymbol{\nabla})$ measured by ITC. C) Intrinsic GTPase activity of elF5B(517-858) (wild-type (wt) or the D533A mutant) determined at $35{ }^{\circ} \mathrm{C}$ in the presence of $200 \mathrm{mM}$ of the indicated salts, $25 \mu \mathrm{M}$ protein and $300 \mu \mathrm{M}$ GTP and subsequent analysis by HPLC. The order in which the combinations are given on the right corresponds to the relative rates of GTP hydrolysis. D) Intrinsic GTPase activity of elF5B(517-858) wild-type (black), D533A (red), D533N (green), and D533R (green) in the presence of $\mathrm{NaCl}$ under conditions as in C). E and F) Dependency

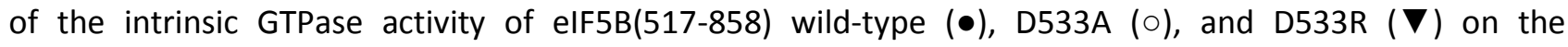
concentration of $\mathrm{KCl}(\mathrm{E})$ or $\mathrm{NaCl}(\mathrm{F})$. Experiments were repeated two to three times; standard deviations are given by error bars (in some cases not visible because they are smaller than the symbol size). 
GTPYS but not GDPNP is able to substitute for GTP to induce the conformational switch of elF5B in solution.

GTP binding to apo elF5B induces a substantial rearrangement of the switch regions, involving a $\sim 180^{\circ}$ flip of switch 1 and the burial of $\sim 1800 \AA^{2}$ of solvent accessible surface area (ASA) within G domain and domain II, accompanied by significant changes in heat capacity $\left(\Delta \mathrm{C}_{\mathrm{p}}=-553 \mathrm{cal} \cdot \mathrm{mol}^{-1} \mathrm{~K}^{-}\right.$ $\left.{ }^{1}\right)$ [220]. Our structural data argue for a scenario in which the $\mathrm{M}^{+}$ion provides a direct contribution as structural cofactor to stabilize the 'on' state of elF5B. In order to test this assumption for free elF5B we probed the conformational changes in ctelF5B(517-858) upon binding of GDP, GTP $\gamma$ S or GDPNP using ITC under conditions reported earlier for GTP binding [220] (Table E1, Fig. 2B and E2).

The affinities of GDP, GTPYS and GDPNP to ctelF5B(517-858) were measured at different temperatures between 10 and $30{ }^{\circ} \mathrm{C}$. At $30^{\circ} \mathrm{C}$ GDP binds to ctelF5B(517-858) with an equilibrium dissociation constant $\left(K_{\mathrm{d}}\right)$ of $10.4 \mu \mathrm{M}, 2.5$-fold weaker than GTP $(4.1 \mu \mathrm{M})$. GDPNP binds with a $K_{\mathrm{d}}$ of $20.8 \mu \mathrm{M}$ (at $30^{\circ} \mathrm{C}$ ), $\sim 5$-fold weaker than GTP. GTPyS has an about 4-fold higher affinity to ctelF5B(517-858) $\left(0.92 \mu \mathrm{M}\right.$ at $\left.30^{\circ} \mathrm{C}\right)$ than GTP. Comparable values were reported from fluorescence experiments with mammalian elF5B and mant-nucleotides [239].

The interactions of ctelF5B(517-858) with GTPYS, GDPNP and GDP result in significant exothermic heat effects $\left(\Delta \mathrm{H}=-15.8,-9.7\right.$ and $-11.5 \mathrm{kcal} \cdot \mathrm{mol}^{-1}$, respectively, at $\left.30^{\circ} \mathrm{C}\right)$. As for GTP, their binding is driven by favorable changes in binding enthalpy and opposed by unfavorable entropic contributions $\left(T \Delta S=-7.4,-3.2\right.$ and $-4.6 \mathrm{kcal} \cdot \mathrm{mol}^{-1}$ for GTP $\psi \mathrm{S}, \mathrm{GDPNP}$ and GDP at $30{ }^{\circ} \mathrm{C}$, respectively) (Table E1).

In the temperature range between 10 and $30{ }^{\circ} \mathrm{C} \Delta \mathrm{H}$ is temperature dependent. When $\Delta \mathrm{H}$ was plotted against the temperature, straight lines were obtained with the slopes representing the changes in heat capacity $\left(\Delta C_{p}\right)$ upon complex formation (Table 2 and Fig. 2B) [179]. GTPyS causes a $\Delta \mathrm{C}_{\mathrm{p}}$ of $-539 \mathrm{cal} \cdot \mathrm{mol}^{-1} \cdot \mathrm{K}^{-1}$, very similar to the value observed for GTP $\left(-553 \mathrm{cal} \cdot \mathrm{mol}^{-1} \cdot \mathrm{K}^{-1}\right)[220]$. Significantly smaller changes in heat capacity were obtained for GDPNP and GDP binding $\left(\Delta \mathrm{C}_{\mathrm{p}}=-\right.$ $197 \mathrm{cal} \cdot \mathrm{mol}^{-1} \cdot \mathrm{K}^{-1}$ and $-228 \mathrm{cal} \cdot \mathrm{mol}^{-1} \cdot \mathrm{K}^{-1}$, respectively). $\Delta \mathrm{C}_{\mathrm{p}}$ can be used as an estimate for the change in solvent accessible surface area ( $\triangle A S A)$ upon complex formation. The burial of surface area was shown to be associated with a negative change in heat capacity, with $\Delta C_{p}$ being proportional to the size of the surface area involved in the ligand binding process [177, 180, 240$]$. 
According to this correlation, GTPYS binding induces major structural rearrangements in ctelF5B(517-858) similar to those observed for GTP binding (Table 2), suggesting nearly identical structures for ctelF5B(517-858)-GTP and ctelF5B(517-858)·GTPyS in solution. In contrast, GDPNP induces significantly smaller changes which are similar to those for GDP (Table 2), indicating a GDPlike conformation for the ctelF5B(517-858).GDPNP complex. These results are in line with the observations from the crystal structures of GTP-, GTPYS- and GDP-bound elF5B and GDPNP-bound alF5B [84], as well as the assumption of a specific inability of GDPNP to efficiently stabilize the conformational switch of the $G$ domain by repulsion of the $\mathrm{M}^{+}$ion (Fig. 2A).

Table 2. Changes in heat capacity and solvent accessible surface area for ctelF5B(517-858) binding to GTP, GTPYS, GDPNP and GDP

\begin{tabular}{|c|c|c|c|}
\hline Ligand & $\begin{array}{c}\Delta \mathrm{C}_{\mathrm{p}} \\
{\left[\mathrm{cal} \mathrm{mol} \mathrm{mo}^{-1} \cdot \mathrm{K}^{-1}\right]}\end{array}$ & $\begin{array}{l}\Delta \mathrm{ASA}_{\min }\left[\AA^{2}\right] \\
\text { (with } \Delta \mathrm{c}_{\min }=0.45 \text { ) }\end{array}$ & $\begin{array}{c}\Delta \mathrm{ASA}_{\max }\left[\AA^{2}\right] \\
\text { (with } \Delta \mathrm{c}_{\max }=0.24 \text { ) }\end{array}$ \\
\hline GTP & $-553 \pm 11^{a}$ & 1229 & 2304 \\
\hline GTPYS & $-539 \pm 30^{b}$ & 1198 & 2254 \\
\hline GDPNP & $-197 \pm 26^{b}$ & 438 & 820 \\
\hline GDP & $-228 \pm 10^{b}$ & 507 & 950 \\
\hline \multicolumn{4}{|c|}{$\begin{array}{l}\triangle A S A_{\min } \text { and } \triangle A S A_{\max } \text {, changes in solvent accessible surface areas assuming that all changes were conferred by either apolar or } 70 \% \text { apolar and } \\
30 \% \text { polar surfaces, respectively. } \\
\text { a Obtained from [220]. }\end{array}$} \\
\hline
\end{tabular}

The structure of elF5B bound to GTPYS and potassium.

Most $\mathrm{M}^{+}$-dependent enzymes show a preference for potassium $\left(\mathrm{K}^{+}\right)$over other $\mathrm{M}^{+}$ions as cofactor. A preference for $\mathrm{K}^{+}$is known also for the translation apparatus in general and the function of trGTPases in particular [241-244]. We therefore assumed that $\mathrm{K}^{+}$is able to substitute for $\mathrm{Na}^{+}$as cofactor in the GTP-bound form of elF5B. Consistently, we were able to obtain crystals of elF5B(517-858).GTPyS in space group $\mathrm{P}_{1}{ }_{1}{ }_{1} 2$ that grew within two weeks at $20{ }^{\circ} \mathrm{C}$ under a condition containing $50 \mathrm{mM} \mathrm{KCl}$ (Table 1). The structure was solved at $2.28 \AA$ resolution and contains two elF5B.GTPYS complexes in the asymmetric unit. The two water molecules in the coordination shell of the $\mathrm{Mg}^{2+}$ ion and $\mathrm{W}^{\text {cat }}$ are weakly defined in the electron density, with the latter lying 3.1-3.2 $\AA$ from the outward pointing sulfur atom of GTPYS. On the other side of the 
sulfur atom, opposite to $\mathrm{W}^{\text {cat }}$, a strong electron density peak was observed that was assigned to a $\mathrm{K}^{+}$ion with a coordination shell nearly identical to that of the $\mathrm{Na}^{+}$ion in elF5B.GTPyS (Fig. 1B and E1C). However, additional weak densities were observed on the solvent exposed side of the cation, suggesting that two water molecules contribute to a heptameric coordination shell. As expected for a $\mathrm{K}^{+}$ion [156], the coordination distances to most oxygen ligands lie between 2.7 and $3.0 \AA$ and are thus clearly different from those observed for the $\mathrm{Na}^{+}$ions. The distance to the $\beta$ - $\gamma$-bridging oxygen is slightly increased ( $3.47 \AA$ ), most likely due to the large sulfur atom that coordinates the $\mathrm{K}^{+}$ion at a distance of $3.2 \AA$ (in good agreement with the theoretical distance of $3.26 \AA$, calculated from the van der Waals radius of sulfur $(1.8 \AA)$ and effective ion radius of $K^{+}(1.46 \AA$ for coordination number 7) $[237,238])$.

Despite the different crystallization conditions and a different set of crystal contacts, the overall structure of elF5B.GTPYS bound to $\mathrm{K}^{+}$is nearly identical to that of elF5B.GTP/GTP $\mathrm{S}$ bound to $\mathrm{Na}^{+}$with the switch regions stabilized in their typical GTP-conformation. Significant differences are limited to Gly ${ }^{\mathrm{MC}}$ and the $\mathrm{MC}$-loop of switch 1 which is moved $\sim 2 \AA$ away from the guanine nucleotide, owing to the increased coordination distances to the $\mathrm{K}^{+}$ion (Fig. 1C). This demonstrates that the position of the MC-loop in switch 1 is directly influenced by the species of the $\mathrm{M}^{+}$ions coordinated next to the nucleotide, indicating a direct contribution of the $\mathrm{M}^{+}$ion to the stabilization of the activated switch 1 conformation.

\section{elF5B coordinates the $\mathrm{M}^{+}$ion in the same position as known $\mathbf{M}^{+}$-dependent GTPases.}

Superposition of the eIF5B.GTP/GTPYS structures with MnmE and dynamin reveals that the $\mathrm{K}^{+}$and $\mathrm{Na}^{+}$ions in elF5B are coordinated in the same position as the catalytic $\mathrm{K}^{+}$and $\mathrm{Na}^{+}$ions in the presence of GDP-AlF $F_{x}$ in the two known $\mathrm{M}^{+}$-dependent GTPases [231, 232] (Fig. 1D and E1E). Asp ${ }^{\mathrm{MC}}$ replaces an identically positioned and conserved Asn or Ser, and the backbone CO of Gly ${ }^{\mathrm{MC}}$ replaces two backbone COs from the 'K-loop' in switch 1 which circles around the position of the MC-loop of elF5B. The contacts formed between the $\mathrm{K}^{+}$and $\mathrm{Na}^{+}$ions and GTP in elF5B are virtually identical to those of the $\mathrm{K}^{+}$ion in $\mathrm{MnmE}$ or the $\mathrm{Na}^{+}$ion in dynamin with the transition state mimic GDP-AlF $F_{x}$ where a $\beta$-phosphate oxygen of GDP represents the former $\beta$ - $\psi$-bridging oxygen and the outward pointing non-bridging $\gamma$-phosphate oxygen is mimicked by a fluoride ion. 


\section{The structural elements required for $\mathrm{M}^{+}$coordination in elF5B are highly conserved among trGTPases}

The G domains of trGTPases are highly conserved in sequence and structure. In order to investigate whether $\mathrm{M}^{+}$ion binding may be a common characteristic among trGTPases we analyzed sequences and available structures with regard to key features involved in $\mathrm{M}^{+}$ion binding in elF5B. The key determinants for $\mathrm{M}^{+}$ion binding are $\mathrm{Asp}^{\mathrm{MC}}$ in the P-loop and Gly ${ }^{\mathrm{MC}}$ in the MC-loop of switch 1. Sequence analysis reveals that both residues, $\mathrm{Asp}^{\mathrm{MC}}$ and $\mathrm{Gly}{ }^{\mathrm{MC}}$, are universally conserved among orthologs of elF5B, EF-Tu, SelB, alF2 $\gamma$, eRF3, EF-G, RF3 and LepA from bacteria to human (Fig. 3A). The only notable exception among trGTPases is elF2 $\gamma$, where ssp $^{\mathrm{MC}}$ and $\mathrm{Gly}^{\mathrm{MC}}$ are replaced by Ala and Asn, respectively.

Structurally, the P-loop is well conserved among trGTPases. In contrast, switch 1 shows a high degree of variability in sequence and structure, which is, however, limited to regions lying $\mathrm{N}$ terminally of the MC-loop. In a superposition of elF5B.GTP with EF-Tu.GDPNP or the ribosomebound EF-G.GDPCP (Fig. 3B/C), the nucleotide binding motifs occupy virtually identical positions including all residues directly involved in the coordination of the nucleotide and $\mathrm{Mg}^{2+}$ ion. Importantly, the structural homology extends to $\mathrm{Asp}^{\mathrm{MC}}$ as well as Gly ${ }^{\mathrm{MC}}$, the latter of which invariably forms part of the characteristic MC-loop excursion of switch 1 at the end of helix $A^{\prime \prime}$ in EF-Tu and EF-G, placing its carbonyl group in the correct position to coordinate the cation.

\section{GTP-bound aEF1A coordinates an $\mathrm{M}^{+}$ion in its catalytic center}

Most known structures of trGTPases that were reported to be in the GTP-conformation do not contain GTP but GDPNP or GDPCP that contain either an $\mathrm{NH}$ or a $\mathrm{CH}_{2}$ group in lieu of the $\beta-\gamma-$ bridging oxygen. Both prevent the coordination of the $\mathrm{M}^{+}$ion as observed in structures of known $\mathrm{M}^{+}$-dependent GTPases [245, 246] (Fig. 2A and E4). We therefore searched the PDB for structures of trGTPases that were cocrystallized with GTP and found two structures, both of the archaeal EFTu ortholog aEF1A either in complex with pelota (PDB: 3AGJ) or with release factor RF1 (PDB: 3VMF). 
A

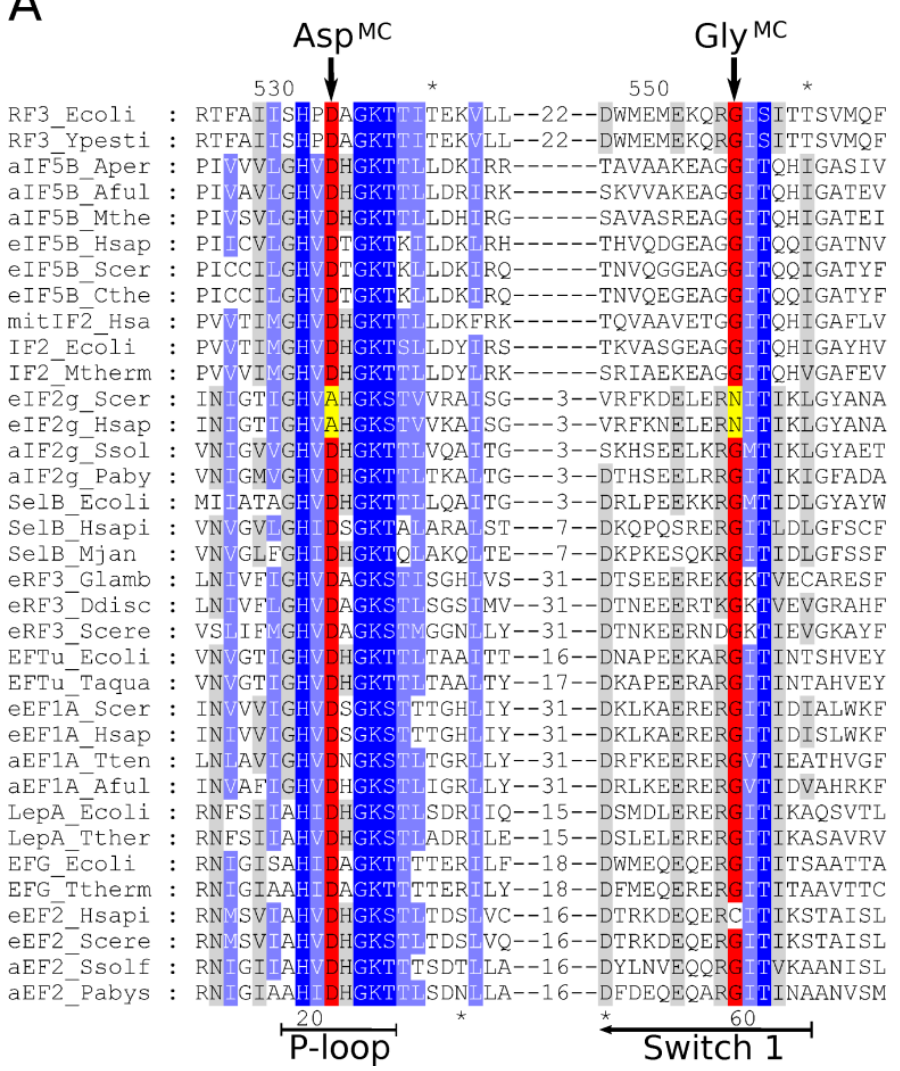

B
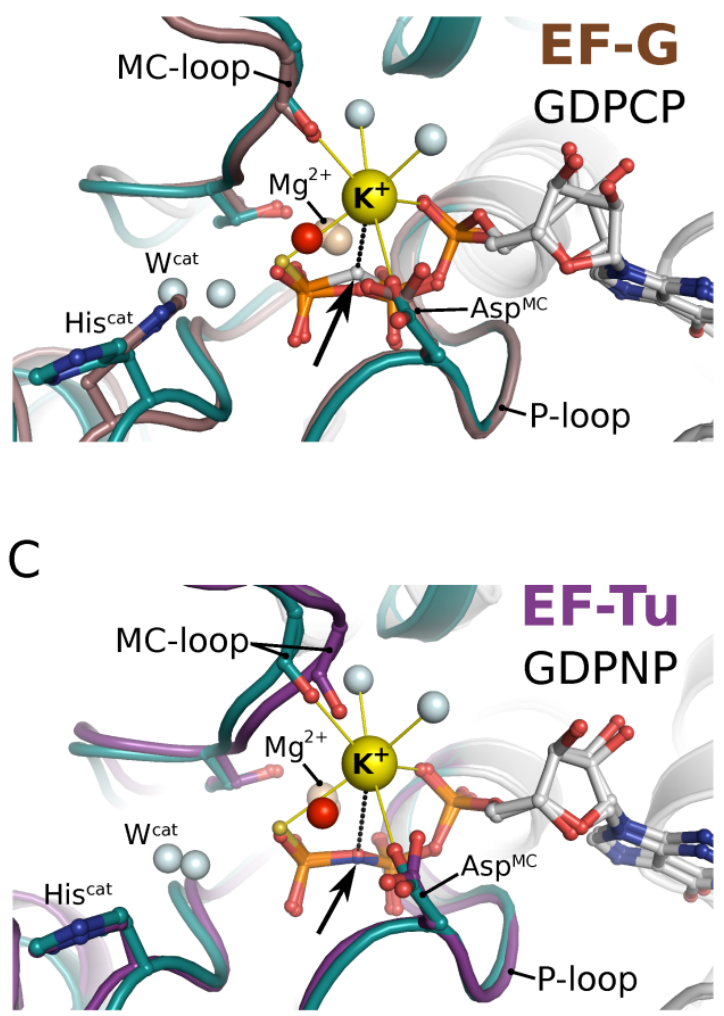

Figure 3. Structural elements required for $\mathbf{M}^{+}$-coordination in elF5B are universally conserved among trGTPases.A) Excerpt of a multiple sequence alignment of different trGTPases (orthologs of RF3, elF5B, elF2 $\gamma$, SelB, eRF3, EF-Tu, LepA, EF-G) showing P-loop and switch 1. The upper and lower numbering corresponds to $C$. thermophilum elF5B (Cthe) and E. coli EF-Tu (Ecoli), respectively. Highly conserved residues are highlighted in dark blue, conserved residues in light blue. Asp ${ }^{\mathrm{MC}}$ and $\mathrm{Gly}^{\mathrm{MC}}$ are highlighted in red, residues in elF2 $\gamma$ that replace $A s p^{\mathrm{MC}}$ and $\mathrm{Gly}^{\mathrm{MC}}$ are highlighted in yellow. B) Superposition of elF5B.GTPYS. $\mathrm{K}^{+}$(colored as in Fig. 1) with ribosome-bound EF-G.GDPCP (brown; PDB: 4JUW). Ribosomebound EF-G provides all structural elements to bind the $\mathrm{M}^{+}$ion; however, its coordination is prevented by the $\mathrm{CH}_{2}$ group of GDPCP in lieu of the $\beta$ - $\gamma$-bridging oxygen (arrow). A water molecule (red sphere) is bound next to the $\mathrm{M}^{+}$-binding site instead. C) Similarly, EF-Tu.GDPNP (purple; PDB: 2C78) provides all structural elements to bind the $\mathrm{M}^{+}$ion; however, its coordination is prevented by the NH group of GDPNP (arrow). A water molecule (red sphere) is bound next to the $\mathrm{M}^{+}$binding site instead.

Crystals of the aEF1A/pelota complex contained four aEF1A molecules (chains $A, C, E, G$ ) per asymmetric unit, each bound to GTP. The structure had been determined at $2.3 \AA$ resolution[247]. Each of the aEF1A molecules contains a water molecule modeled in the position occupied by the $\mathrm{M}^{+}$ion in elF5B.GTP. Accordingly, the supposed water molecules are coordinated by five hydrogen bond acceptors with the sphere formed by the outward pointing $\alpha$ - and $\gamma$-phosphate oxygens and the $\beta$ - $\gamma$-bridging oxygen of GTP, Asp16 in the P-loop (Asp ${ }^{\mathrm{MC}}$ ) and the CO of Gly69 (Gly ${ }^{\mathrm{MC}}$ ) in switch 
1. The MC-loop lies at the end of helix $A^{\prime \prime}$ in switch 1 and adopts the same conformation found in elF5B (Fig. 1A and 4A/B). The coordination distances of the supposed water molecules lie between 2.2 and $2.7 \AA$ with an overall average of $2.45 \AA$. Thus, the coordination pattern as well as the distances is in better agreement with those expected for a $\mathrm{Na}^{+}$ion than for water [156]. Reevaluation of the experimental X-ray diffraction data (downloaded from the PDB (3AGJ)) revealed positive difference electron density for the supposed water molecules in aEF1A molecules $C$ and $E$ in the asymmetric unit, indicating a higher density of electrons in these positions than provided by $\mathrm{H}_{2} \mathrm{O}$ (Fig. E3A). Replacement of the four water molecules by $\mathrm{Na}^{+}$and subsequent refinement results in occupancies of 92 and $94 \%$ for the $\mathrm{Na}^{+}$ions in molecules $\mathrm{A}$ and $\mathrm{G}$, respectively, and $100 \%$ in molecules $C$ and $E$. Since the crystals used for structure analysis were grown in the presence of $\mathrm{NaCl}$ [247], these observations indicate that the supposed water molecules next to Asp16 of aEF1A are most likely $\mathrm{Na}^{+}$ions (Fig. 4A and E3C).

The structure of the aEF1A/aRF1 complex was solved at $2.3 \AA$ resolution and contains one copy of GTP-bound aEF1A per asymmetric unit [248]. This structure as well has been built with a water molecule in the pentameric coordination sphere described above. Its contact distances lie between 2.6 and $3.1 \AA$ ( 2.7 to $3.0 \AA$ after reevaluation of the experimental data, downloaded from the PDB (3VMF)) which are typical for the coordination of a $\mathrm{K}^{+}$ion [156]. However, the electron density in this position does not correspond to $\mathrm{K}^{+}$. This observation is supported by the fact that the crystallization condition did not contain $\mathrm{K}^{+}$but instead $200 \mathrm{mM}$ of $\mathrm{NH}_{4}^{+}$ions [248]. $\mathrm{NH}_{4}^{+}$has a similar ion radius as $\mathrm{K}^{+}(1.46 \AA$ and $1.38 \AA$, respectively) and was shown to substitute for the latter structurally and functionally in the $\mathrm{K}^{+}$-dependent GTPase MnmE [231]. Thus, the properties of the observed coordination point toward an $\mathrm{NH}_{4}^{+}$ion lying in the same position as the $\mathrm{K}^{+}$and $\mathrm{Na}^{+}$ions in elF5B.GTP and the aEF1A.GTP/pelota complex (Fig. 4B and E3D).

\section{The intrinsic GTPase activity of elF5B depends on monovalent cations}

The position of the observed $\mathrm{M}^{+}$ion in elF5B suggests a direct involvement in the catalysis of GTP hydrolysis in analogy to MnmE and dynamin. To test this possibility, we monitored the intrinsic GTPase activity of either wild-type elF5B(517-858) or various Asp ${ }^{\mathrm{MC}}$ mutants (D533A, D533R and D533N) under different salt conditions (Fig. 2C-F and Table 3). 
A

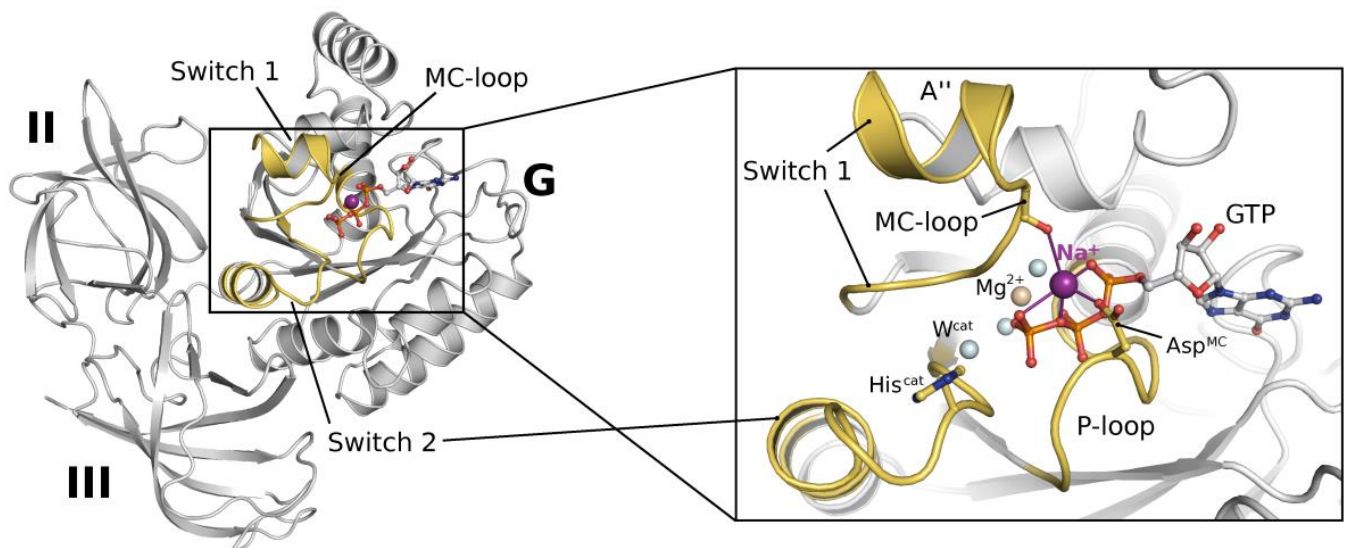

B

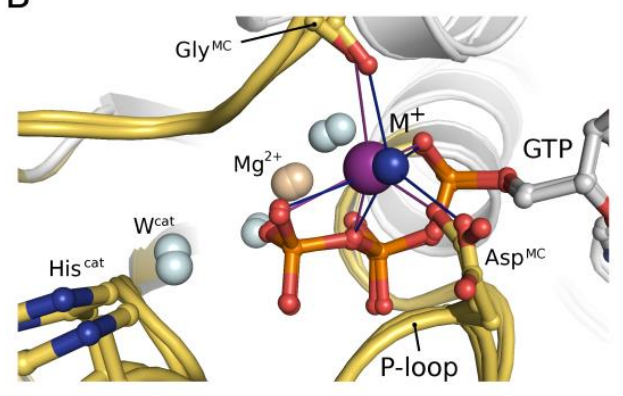

D

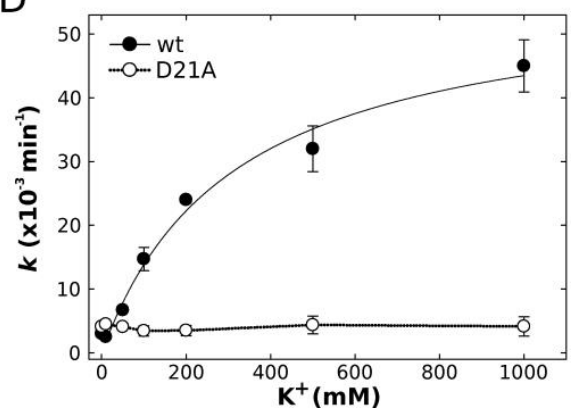

C

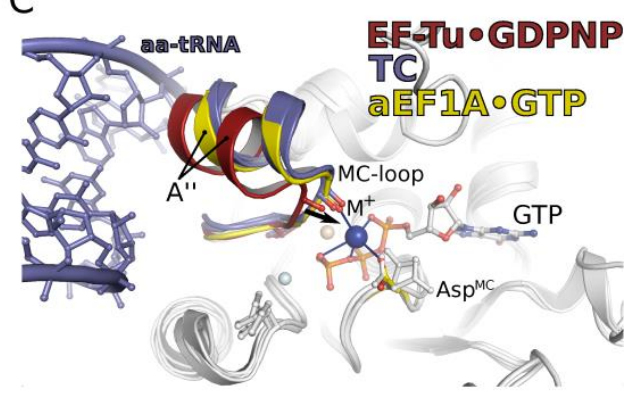

$\mathrm{E}$

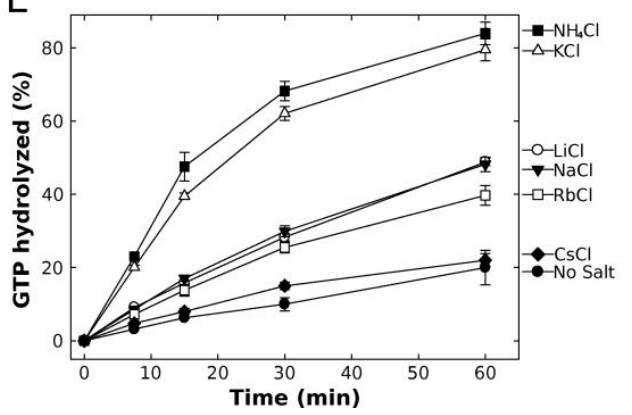

Figure 4.The GTP-bound EF-Tu ortholog aEF1A coordinates an $\mathbf{M}^{+}$ion in its GTPase center.A) Overview of domains I (G) to III of GTP-bound aEF1A with a $\mathrm{Na}^{+}$ion (purple sphere) in the active site. P-loop and switch regions are shown in yellow. The inset shows a detailed view on the active site with the coordination sphere of the $\mathrm{Na}^{+}$ion (indicated by purple lines) formed by GTP, Asp ${ }^{\mathrm{MC}}$ and Gly ${ }^{\mathrm{MC}}$ in the MC-loop. B) Superposition of GTP-bound aEF1A with either a Na${ }^{+}$(purple sphere) or $\mathrm{NH}_{4}{ }^{+}$ion (blue sphere) in the active site. Both ions are coordinated by the identical sphere, however, with different coordination distances. C) Superposition of EF-Tu.GDPNP (switch 1 in red; PDB: 1EXM) with EF-Tu.GDPNP in the ternary complex (TC) with aa-tRNA (blue; PDBs: 1TTT, 1OB2) and aEF1A.GTP.M $\mathrm{M}^{+}\left(\mathrm{M}^{+}\right.$in blue; switch 1 in yellow). The $\mathrm{M}^{+}$ion stabilizes a conformation of helix $A^{\prime \prime}$ that is required for stable TC formation. D) Dependency of the intrinsic GTPase activity of E. coli EF-Tu wild-type $(\bullet)$ or the D21A mutant (O) on the concentration of $\mathrm{K}^{+}$ions, determined in the presence of $0-1 \mathrm{M} \mathrm{KCl}$ under single turnover conditions $\left(20 \mu \mathrm{M}\right.$ GTP-bound EF-Tu) at $30{ }^{\circ} \mathrm{C}$ and subsequent analysis by HPLC. E) Intrinsic GTPase activity of E. coli EF-Tu determined in the presence of 200 $\mathrm{mM}$ of the indicated salts under single turnover conditions. The order in which the combinations are given on the right corresponds to the relative rates of GTP hydrolysis. Experiments were repeated two to three times; standard deviations are given by error bars (in some cases not visible because they are smaller than the symbol size). 
First, we tested whether GTPase stimulation in elF5B depends on the species of the available $\mathrm{M}^{+}$ion (at $200 \mathrm{mM}$ ) (Fig. 2C). The experiments revealed that the GTPase activity is stimulated most in the presence of $\mathrm{Na}^{+}$and $\mathrm{K}^{+}$, followed by $\mathrm{Rb}^{+}, \mathrm{Li}^{+}$and finally $\mathrm{Cs}^{+}$and $\mathrm{NH}_{4}^{+}$with the lowest degree of activation. Importantly, this dependency was lost for the elF5B mutant carrying Ala in lieu of $\mathrm{Asp}^{\mathrm{MC}}$. In the presence of $200 \mathrm{mM} \mathrm{K}^{+}$or $\mathrm{Na}^{+}$, wild-type elF5B catalyzed GTP hydrolysis at rates of 0.023 and $0.027 \mathrm{~min}^{-1}$, respectively. The Ala mutant of $A s p^{M C}$ exhibited significantly reduced rates of $0.0018 \mathrm{~min}^{-1}$ in $\mathrm{K}^{+}$or $\mathrm{Na}^{+}$, corresponding to a 13- to 15 -fold reduction of the intrinsic GTPase activity. Similar results were obtained for the substitution of Asp $^{\mathrm{MC}}$ by Arg (D533R) (Table 3). By contrast, an Asn mutant retained the ability to catalyze GTP hydrolysis nearly at wild-type rates (Fig. 2D), in line with the assumption that an Asn but not an Ala or Arg residue would retain the ability to coordinate the $\mathrm{M}^{+}$ion. This is corroborated by crystal structures of the respective mutants in complex with GTP (or GTP $\gamma$ S), which reveal that D533N contains the $\mathrm{M}^{+}$ion in its active site, whereas the cation is not present in D533R and D533A and exchanged for a water molecule in the latter (Fig. E5). Moreover, fluorescence measurements with mant-labeled GTP show that all three mutants bind the nucleotide with a $K_{d}$ comparable to that of the wild-type protein, speaking against the possibility that the observed effects on the GTPase activity are due to reduced affinities to the substrate (Fig. E5E-H).

Next, we studied the effect of increasing $\mathrm{Na}^{+} / \mathrm{K}^{+}$ion concentrations (Fig. 2E/F). In the absence of $\mathrm{M}^{+}$ions ( $\sim 600 \mu \mathrm{M} \mathrm{Na}{ }^{+}$were added with the GTP), elF5B hydrolyzed GTP with a rate of 0.002 $\mathrm{min}^{-1}$. This rate successively increased with increasing concentrations of $\mathrm{K}^{+}$or $\mathrm{Na}^{+}$ions, resulting in an 11- to 12 -fold rate enhancement at $200 \mathrm{mM}$ salt (Table 3). By contrast, the rate of GTP hydrolysis in the D533A mutant was found to be insensitive to the salt concentration with invariably low rates in the absence or presence of $\mathrm{M}^{+}$ions, whereas D533R exhibited a slightly increased GTPase activity in the absence of $\mathrm{M}^{+}$ions $\left(0.005 \mathrm{~min}^{-1}\right)$, which successively decreased with increasing salt concentrations (Fig. 2F).

\section{The intrinsic GTPase activity of EF-Tu depends on monovalent cations}

The GTPase center of bacterial EF-Tu is virtually identical to that of its archaeal ortholog and therefore most likely coordinates an identically positioned $\mathrm{M}^{+}$ion in its GTP-bound form (Fig. E3B). 
To test the generality of our assumption of $\mathrm{M}^{+}$-dependency in trGTPases, we studied the influence of $\mathrm{M}^{+}$ions on the intrinsic GTPase activity of $E$. coli EF-Tu by monitoring GTP hydrolysis at different $\mathrm{K}^{+}$-concentrations (Fig. 4D). As observed for elF5B, the rate of GTP hydrolysis successively increased with increasing concentrations of $\mathrm{K}^{+}$, corresponding to a nearly 10-fold rate enhancement for the intrinsic GTPase reaction at $200 \mathrm{mM}$ (Table 3). This dependency is lost when Asp21 (Asp ${ }^{M C}$ ) in EF-Tu is mutated to Ala (D21A), consistent with a role of Asp ${ }^{M C}$ as key ligand for the coordination of an $\mathrm{M}^{+}$ion in EF-Tu at physiological salt concentration as observed for GTPbound aEF1A (see above; Fig. 4A/B).

Table 3.Intrinsic GTPaseactivities of wt and mutant cteIF5B(517-858) and ecEF-Tu.

\begin{tabular}{|c|c|c|c|c|}
\hline Protein & Construct & $\mathrm{KCl}(m M)$ & $k\left(\min ^{-1}\right)$ & $\begin{array}{l}\text { Fold reduction compared } \\
\text { to wild-type at } 200 \mathrm{mM} \mathrm{KCl}\end{array}$ \\
\hline \multirow[t]{6}{*}{ ctelF5B $^{\mathrm{a}}$} & wt & 200 & $0.023 \pm 0.0015$ & \\
\hline & wt & 0 & $0.0021 \pm 0.001$ & 11 \\
\hline & D533A & 200 & $0.0018 \pm 0.0005$ & 13 \\
\hline & D533A & 0 & $0.0016 \pm 0.0008$ & 14 \\
\hline & D533N & 200 & $0.017 \pm 0.002$ & 1.4 \\
\hline & D533R & 200 & $0.0028 \pm 0.0007$ & 8 \\
\hline \multirow[t]{4}{*}{ ecEF-Tu ${ }^{b}$} & wt & 200 & $0.026 \pm 0.0005$ & \\
\hline & wt & 0 & $0.003 \pm 0.0002$ & 9 \\
\hline & D21A & 200 & $0.0035 \pm 0.0008$ & 7 \\
\hline & D21A & 0 & $0.0041 \pm 0.0001$ & 6 \\
\hline
\end{tabular}

In order to identify the preferences of EF-Tu for GTPase stimulation, we analyzed the effect of different alkali salts $\left(\mathrm{Li}^{+}, \mathrm{Na}^{+}, \mathrm{K}^{+}, \mathrm{Rb}^{+}, \mathrm{Cs}^{+}\right.$) or $\mathrm{NH}_{4}^{+}$(each at $200 \mathrm{mM}$ ) on the intrinsic hydrolysis rates (Fig. 4E). The strongest stimulating effect was observed for $\mathrm{NH}_{4}^{+}$and $\mathrm{K}^{+} . \mathrm{Na}^{+}, \mathrm{Li}^{+}$and $\mathrm{Rb}^{+}$ions showed only a slight activation; virtually no effect was observed for the large $\mathrm{Cs}^{+}$ion. Importantly, none of the tested $\mathrm{M}^{+}$ion species show a significant stimulating effect on the D21A mutant of EF$\mathrm{Tu}$, which, moreover, shows no preference for $\mathrm{NH}_{4}{ }^{+}$or $\mathrm{K}^{+}$as observed for the wild-type protein (Fig. E6). 


\subsection{Discussion}

\section{$\mathrm{M}^{+}$-dependent conformational switching of elF5B}

Elucidation of the structural dynamics - that is the conformational switch - of the G domain in response to GDP/GTP exchange and GTP hydrolysis is one of the central problems to understand the functional cycle of GTPases. Here we identified a monovalent cation $\left(\mathrm{Na}^{+} / \mathrm{K}^{+}\right)$as structural cofactor in the active site of GTP-bound elF5B, which stabilizes the GTP-dependent reorganization of its $\mathrm{G}$ domain (Fig. 1 and 5). The $\mathrm{M}^{+}$ion is bound in a highly conserved coordination shell formed by two non-bridging $\alpha$ - and $\gamma$-phosphate oxygens and the $\beta$ - $\gamma$-bridging oxygen of the GTP molecule, $\mathrm{Asp}^{\mathrm{MC}}$ in the P-loop and Gly ${ }^{\mathrm{MC}}$ in the MC-loop of switch 1. P-loop and GTP molecule thus form a stable socket for the $\mathrm{M}^{+}$ion, which in turn constitutes an anchor point for the reorganized switch 1 in the 'on' state. Structural and thermodynamic data demonstrate that GTP $\gamma S$ is a faithful replacement for GTP that coordinates the $\mathrm{M}^{+}$ion and is thus able to stabilize the GTP-bound 'on' state of elF5B (Fig. 1 and E4A). In contrast, replacement of GTP with GDPNP disrupts the coordination shell, resulting in the inability of the analog to stabilize the GTP-conformation in elF5B through the $\mathrm{M}^{+}$ion (Fig. $2 \mathrm{~A} / \mathrm{B}$ and $5 \mathrm{~A}$ ). As a consequence, the equilibrium between 'on' state and GDP-like 'off' state is shifted toward the latter in elF5B.GDPNP allowing the GDPNP-bound factor to crystallize in the GDP-conformation, as observed for alF5B.GDPNP from $M$. thermoautotrophicum [84] (Fig. 5A and E4A).

This interpretation is supported by the parallels between our observations and previous reports on $\mathrm{M}^{+}$-dependent GTPases, distantly related to trGTPases. In MnmE an identically positioned $\mathrm{K}^{+}$ion (Fig. 1D) is required for the rearrangement of switch 1 and subsequent dimerization [231]. $\mathrm{K}^{+}$-dependent dimerization in MnmE was found to be induced by GTP and GTP $y$, while GDPNP and GDPCP failed to support the stable switching of the G domain in biochemical as well as structural experiments [231, 249]. Similarly, GDPCP was found to be unable to induce stable $\mathrm{G}$ domain dimerization in dynamin or the conformational switch in EngA GD2, with structural changes limited to a shift of switch 2 [232, 246, 250]. Moreover, FeoB was crystallized in the presence of GDP-AIF with switch 1 stabilized in the 'on' state by a $\mathrm{K}^{+}$ion [245], whereas the GDPNP-bound structures either remained in the 'off' state (2WIC) or did switch to the 'on' state containing either a water molecule or nothing at all in place of the $M^{+}$ion (3B1X; $\left.3 L X 5\right)$. These 
findings support the idea that GDPNP and GDPCP are incompatible with the coordination of an $\mathrm{M}^{+}$ ion and thereby destabilize the GTP-conformation of $\mathrm{M}^{+}$-dependent GTPases as observed for a/elF5B.

A

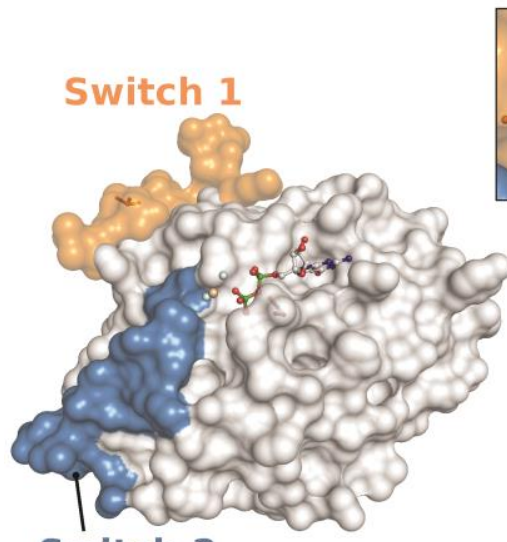

Switch 2

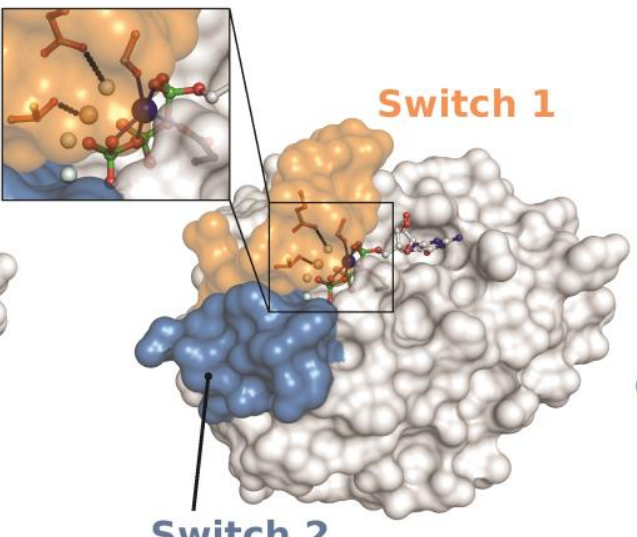

Switch 2

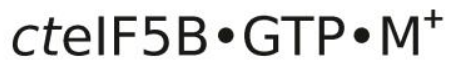

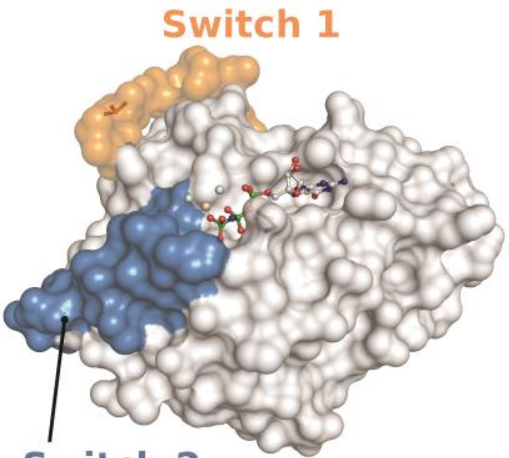

Switch 2

\section{ctelF5B・GDP}

B

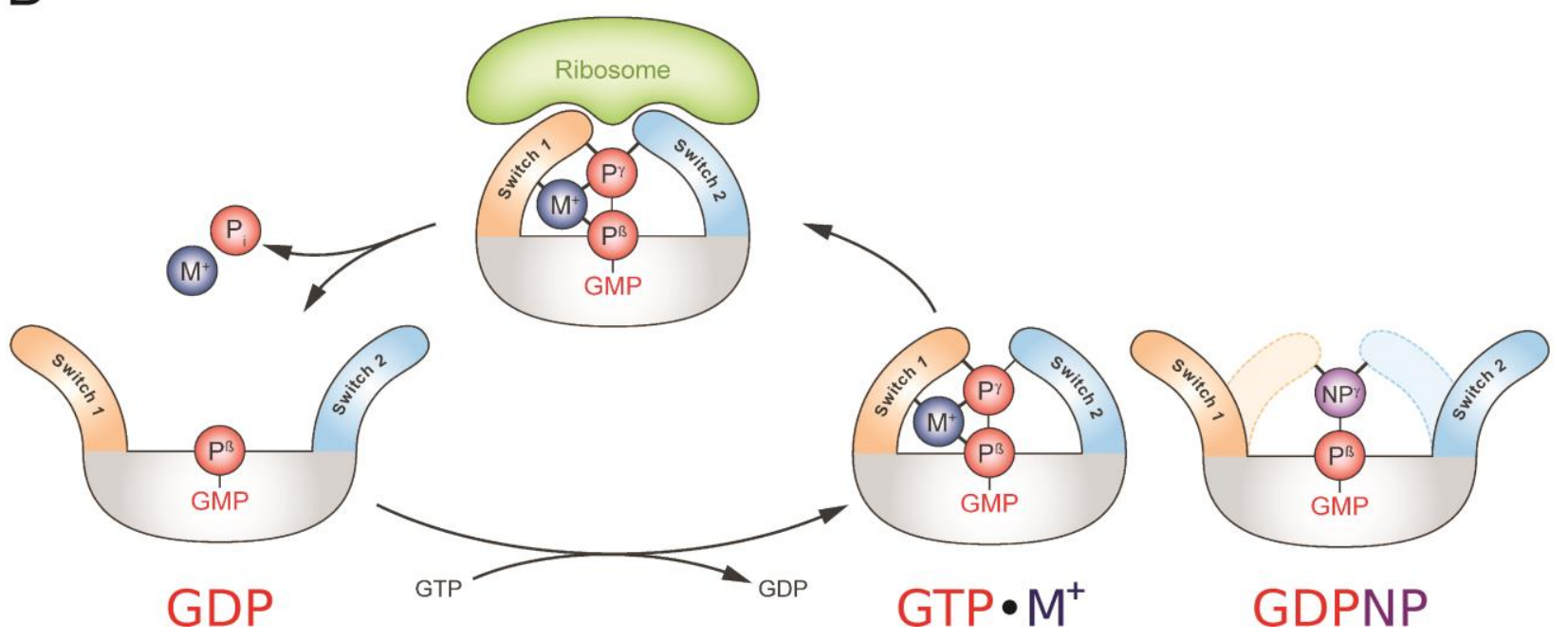

Figure 5. The mechanism of $\mathbf{M}^{+}$-dependent conformational switching in trGTPases. A) The nucleotidedependent conformational switch in the G domain of a/elF5B. In elF5B.GDP, switch 1 and 2 are oriented away from the nucleotide binding pocket. Upon exchange of GDP for GTP, the switch regions undergo a large conformational rearrangement that results in direct contacts with the $\gamma$-phosphate. Here, the $\mathrm{M}^{+}$ion (blue sphere) provides a direct contribution to the stabilization of switch 1 (inset). This contribution is missing in alF5B.GDPNP (PDB: 1G7T), allowing it to crystallize in the GDP-like 'off' state conformation. B) Schematic presentation of the $\mathrm{M}^{+}$-dependent conformational switch mechanism in trGTPases. 
Based on the presented data, we propose an $\mathrm{M}^{+}$-dependent conformational switch mechanism for elF5B in solution (Fig. 5) similar to that in $\mathrm{MnmE}$ [231], where the $\mathrm{M}^{+}$ion in tandem with $\mathrm{Mg}^{2+}$ acts as structural cofactor that supports binding of the GTP molecule and helps to reorganize and close the active site around the substrate. The $\mathrm{M}^{+}$ion would thus form a constitutive component in the preorganized active site, required to stabilize the 'on' state conformation of the trGTPase for productive interactions with the ribosome.

\section{Universality of $\mathrm{M}^{+}$-dependent conformational switching among trGTPases}

Like for elF5B, the mechanism of nucleotide-dependent conformational switching has remained obscure for most other trGTPases. Crystal structures of isolated EF-G and eRF3 reveal essentially identical conformations for their respective apo, GDP- and GDPNP-bound forms [216, 217] (Fig. E4). In SelB, the canonical GDPNP-induced structural rearrangements are limited to switch 2, while switch 1 is only partially reorganized and remains mainly flexible [218] (Fig. E4C). In the crystal structure, GDPNP is bound by SelB in the canonical way and provides the $\mathrm{Mg}^{2+}$ ion as well as its coordinating water molecules that usually contribute to the association of switch 1 . However, although Thr46 (Thr557 in ctelF5B) interacts with $\mathrm{Mg}^{2+}$ in the canonical way, the preceding residues starting with Ile45 and Gly44 (Gly ${ }^{\mathrm{MC}}$ in ctelF5B) remain flexible together with the rest of switch 1 [218]. This observation cannot be explained by the loss of the hydrogen bond between the $\beta$ - $\gamma$-bridging atom of the nucleotide and the backbone $\mathrm{NH}$ of the $\mathrm{P}$-loop, but can be readily explained by the loss of the $\mathrm{M}^{+}$ion as structural cofactor, required to stabilize Gly44 (Gly ${ }^{\mathrm{MC}}$ ) and thereby the rest of switch 1 in the 'on' state. This is corroborated by ITC experiments with SelB, showing that GDP and GDPNP binding result in similar structures for SelB.GDP and SelB·GDPNP, whereas GTP and GTPyS induce substantial structural rearrangements [183]. Similarly, ITC experiments with EF-G indicate large differences between the GTP-bound and apo conformation [219]. In both cases, these results were interpreted as the GTP-induced burial of surface areas by switch 1 and 2 that, however, do not become apparent from the crystal structures.

The parallels to the observations discussed above for a/elF5B are obvious. It is moreover evident from sequence comparison that nearly all trGTPases, including EF-Tu, EF-G, SelB, and eRF3 contain the highly conserved $\mathrm{Asp}^{\mathrm{MC}}$ and $\mathrm{Gly}^{\mathrm{MC}}$ and would thus be able to provide the coordination 
shell for the $\mathrm{M}^{+}$ion (Fig. 3). The loss of the $\mathrm{M}^{+}$ion as structural cofactor between nucleotide and switch 1 due to the use of GDPNP or GDPCP therefore provides a general explanation for the observations for EF-G, SelB and eRF3, as well as other previously reported discrepancies in the behavior of trGTPases toward GTP or its nonhydrolyzable analogs [166, 183, 221-224]. The common evolutionary origin of trGTPases and their functional homology during the translation process argue in favor of a systematic origin rather than individual reasons why GDPNP and GDPCP but not GTPYS are generally unable to substitute for GTP. In light of the above presented observations, we therefore propose that with few exceptions (elF2 $\gamma$ ) trGTPases utilize $\mathrm{M}^{+}$ions as structural cofactor and that the mechanism of $\mathrm{M}^{+}$-dependent conformational switching as suggested for elF5B (Fig. 5B) is universal among canonical trGTPases.

This proposed commonality of $\mathrm{M}^{+}$-dependency is corroborated by the example of elongation factor Tu (EF-Tu). In line with our prediction on the basis of sequence and structural homology to elF5B, we found that the active site of the archaeal EF-Tu ortholog aEF1A bound to GTP as well contains an $\mathrm{M}^{+}$ion $\left(\mathrm{Na}^{+} / \mathrm{NH}_{4}{ }^{+}\right)$, bound by an identical coordination shell as observed for elF5B.GTP (Fig. 4 and E3). Likewise, $\mathrm{M}^{+}$ion binding was demonstrated for free EF-Tu by the finding that its intrinsic GTPase activity is accelerated by $\mathrm{M}^{+}$ions in a manner dependent on Asp21 (Asp ${ }^{\mathrm{MC}}$ ), in line with its role as key ligand for $\mathrm{M}^{+}$ion coordination (Fig. $4 \mathrm{D} / \mathrm{E}$ ). The usage of $\mathrm{M}^{+}$ions as structural cofactor in EF-Tu provides a simple explanation why ternary complexes (TC) of EF-Tu with aa-tRNA formed in the presence of GDPNP exhibit decreased stability compared to those formed with GTP $[166,224]$ due to the loss of the $\mathrm{M}^{+}$-dependent allosteric stabilization of the GTP-conformation required for aa-tRNA binding. This is highlighted by the comparison of EF-Tu.GDPNP with the structures of EF-Tu-GDPNP.aa-tRNA complexes and aEF1A.GTP, which indicates that the $\mathrm{M}^{+}$ion specifically stabilizes a conformation of switch 1 in which helix $A^{\prime \prime}$ is drawn toward the GTP molecule, which seems necessary for stable TC formation (Fig. 4C). Hence, in the absence of the $\mathrm{M}^{+}$ ion, the aa-tRNA itself has to overcome the entropic penalty to arrange switch 1 in the correct conformation that would otherwise be paid by the $\mathrm{M}^{+}$ion in the correctly assembled active site with GTP. 


\section{The $\mathrm{M}^{+}$ion as catalytic element in the GTPase reaction of trGTPases}

Apart from the GTP-induced conformational switch, the mechanism of GTP hydrolysis is another unresolved problem in the universal functional cycle of trGTPases. Although it has been established that the precise ribosome-induced positioning of the invariant $\mathrm{His}^{\mathrm{cat}}$ from the inactive ground state to the catalytically active conformation is critical for GTP hydrolysis [168], it remained obscure how the ribosome-bound trGTPase stabilizes the transition state (TS) of the hydrolysis reaction in the absence of an arginine-finger.

As demonstrated above, $\operatorname{trGTPases}$ coordinate an $\mathrm{M}^{+}$ion next to the GTP- $\gamma$-phosphate in a conserved coordination shell, where it forms a structurally relevant component of the catalytic center (Fig. 1 and 4). The $\mathrm{M}^{+}$ion thus adds another positive charge to the preorganized active site of trGTPases that together with the invariant lysine of the $\mathrm{P}$-loop and the $\mathrm{Mg}^{2+}$ ion forms a triangle of positively charged moieties around the $\beta$ - $\nu$-bridging oxygen of the GTP molecule (Fig. 6A). Following the ribosome-induced activation of His ${ }^{\text {cat }}$ (which leaves the coordination shell for the $\mathrm{M}^{+}$ ion intact (Fig. 3B) [168]), the $\mathrm{M}^{+}$ion would thus be in a suitable position to neutralize negative charges of the TS in the $y$-phosphate as well as the designated leaving group (GDP) (Fig. 6A/B). This suggests that the $\mathrm{M}^{+}$ion might function as the so far elusive catalytic element in trGTPases that acts in the second step of the ribosome-dependent GTPase reaction and contributes to rapid GTP hydrolysis by providing electrostatic stabilization for the TS, in analogy to the arginine-finger in the Ras-RasGAP system (Fig. 6) or the $\mathrm{M}^{+}$ion in MnmE (Fig. 1D).

Structurally, this role for the $\mathrm{M}^{+}$ion in trGTPases is supported by the superposition of GTPbound eIF5B and aEF1A with MnmE, dynamin or the Ras-RasGAP complex where the catalytic $\mathrm{M}^{+}$ ions and the guanidino group are located in virtually identical positions (Fig. 1D, 6C and E1E) [106, $231,232]$. For $\mathrm{Asp}^{\mathrm{MC}}$ this indicates a role of vital importance in the universal functional cycle of trGTPases as key ligand for the $\mathrm{M}^{+}$ion that provides an explanation for its invariant conservation (Fig. 3A). Consistently, we found that the loss of $A s p^{M C}$ in the D533A mutant results in $\mathrm{M}^{+}$independency of the intrinsic GTPase activity in elF5B, whereas the $\mathrm{M}^{+}$-dependency of the wildtype protein is retained in the D533N mutant. The marked effect of Asp ${ }^{\mathrm{MC}}$ mutations in elF5B, which directly correlates with the ability of the substituted residue to coordinate $\mathrm{M}^{+}$ions, clearly argues in favor of a direct contribution by the $\mathrm{M}^{+}$ion to the GTPase reaction. 
A

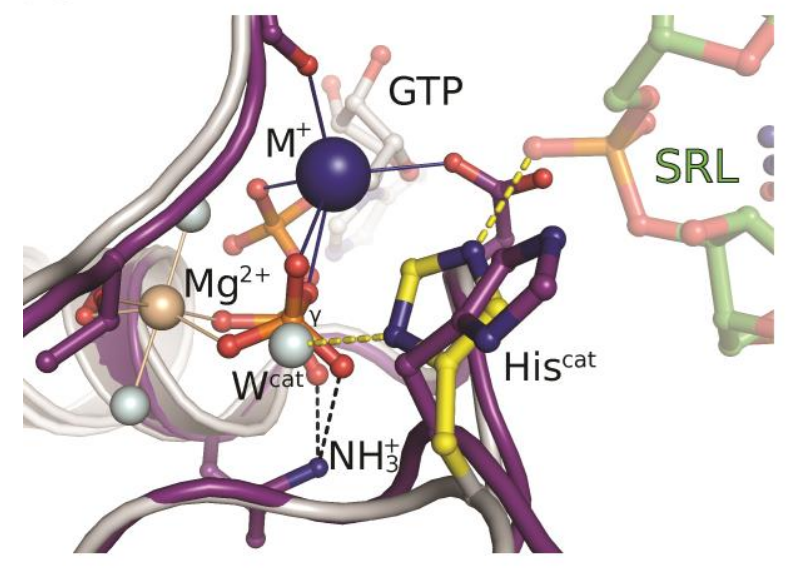

C

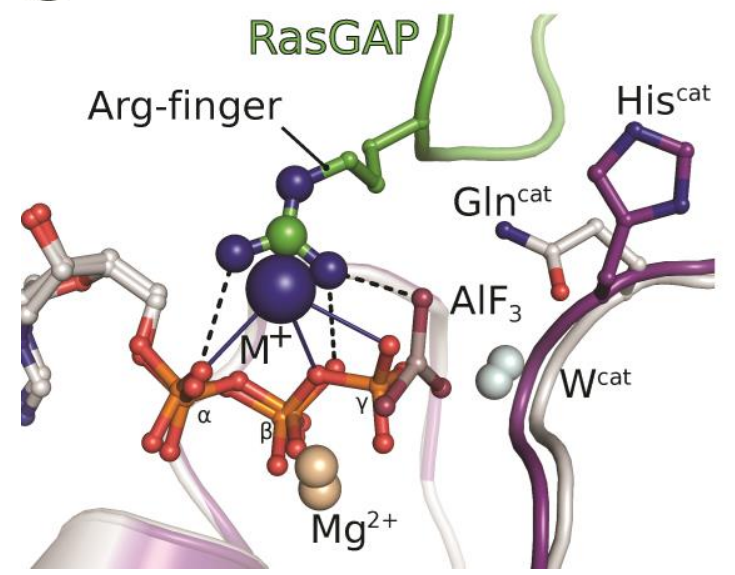

B

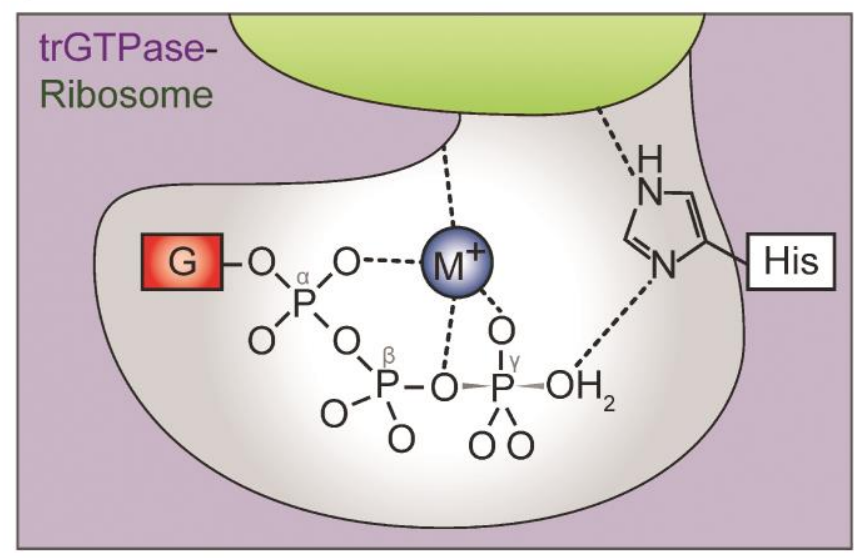

D

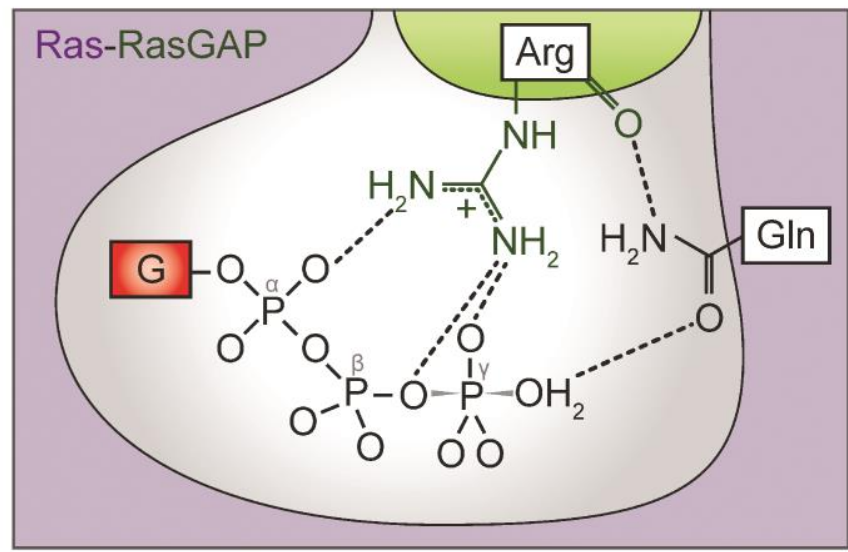

Figure 6. The $\mathrm{M}^{+}$ion as catalytic element in the GTP hydrolysis reaction.A) Model of aEF1A.GTP.M $\mathrm{M}^{+}$on the ribosome (the sarcin-ricin loop (SRL) is shown as green sticks), based on a superposition with ribosomebound EF-Tu (grey; PDB: 2XQD). Upon productive interactions with the SRL, the imidazole moiety of $\mathrm{His}^{\text {cat }}$ is reoriented from its inactive ground state (purple) to the active position (yellow) in which it forms a hydrogen bond to $\mathrm{W}^{\text {cat }}$ [168]. The invariant $\mathrm{P}$-loop lysine, the $\mathrm{Mg}^{2+}$ ion (light brown sphere) and the $\mathrm{M}^{+}$ion (blue sphere) form a triangle of positively charged moieties around the $\beta$ - $\nu$-bridging oxygen. The $\mathrm{M}^{+}$ion is thus suitably positioned to stabilize negative charges that develop in the TS of the hydrolysis reaction. B) Schematic presentation of the active site of a ribosome-bound trGTPase with GTP in the transition state of the hydrolysis reaction stabilized by the $\mathrm{M}^{+}$ion (blue). TrGTPase and ribosome are colored in purple and green, respectively; negative charges, the P-loop lysine and the $\mathrm{Mg}^{2+}$ ion are omitted for clarity. For simplicity, $\mathrm{His}^{\text {cat }}$ is shown in its neutral form, although it might be double protonated in its activated state [226-229]. $C$ and D) The $\mathrm{M}^{+}$ion in elF5B and aEF1A (purple) is coordinated in a position analogous to the arginine-finger in the complex of Ras (grey) and RasGAP (green) (Fig. B and D are modified from [25, 233]).

Further evidence is provided by experiments with bacterial EF-Tu. In agreement with earlier observations from Parmeggiani and coworkers [244] we found the intrinsic GTPase activity of EF-Tu to depend on the concentration as well as the species of $\mathrm{M}^{+}$ions, with a preference for $\mathrm{K}^{+}$and $\mathrm{NH}_{4}^{+}$ (Fig. 4D/E and E6). As for elF5B, the reduced activity in the absence of $\mathrm{M}^{+}$ions and the loss of $\mathrm{M}^{+}$- 
dependency in the D21A (Asp ${ }^{\mathrm{MC}}$ ) mutant argues for a direct effect on GTP hydrolysis. Moreover, kinetic experiments indicated that mutations of Asp21 in E. coli EF-Tu result in a significant reduction of the GTPase activity in the EF-Tu.GTP-aa-tRNA complex in the presence of the correct codon on the ribosome (C. Maracci and M.V. Rodnina, personal communication). This observation as well is consistent with a role of $\mathrm{Asp}^{\mathrm{MC}}$ as key ligand for a catalytic $\mathrm{M}^{+}$ion in EF-Tu, involved in ribosome-dependent GTP hydrolysis. Moreover, the ribosome-dependent GTPase activity of EF-Tu, EF-G as well as IF2 was reported to depend on $\mathrm{M}^{+}$ions $[149,243,244]$. However, in all these cases the GTPase activity reaches maximum values between 20 and $100 \mathrm{mM} \mathrm{M}^{+}$ions, most likely reflecting compensating effects of the salt concentration e.g. on the stability and/or conformation of the ribosomal complexes and their interactions with trGTPases [251] that necessarily obscure a direct effect of $\mathrm{M}^{+}$ions on the GTPase reaction. Importantly, similar observations are known from dynamins, where the stimulating effect of $\mathrm{M}^{+}$ions on GTP hydrolysis is compensated at increasing salt concentrations due to an inhibition of oligomerization and consequently dimerizationdependent GTPase activation [246, 252, 253].

The question, whether trGTPases are specific for $\mathrm{K}^{+}$ions under physiological conditions cannot be unambiguously answered at present. The structural and biochemical analyses indicate that elF5B and EF-Tu/aEF1A have slightly different specificities in their usage of $\mathrm{M}^{+}$ions in vitro. However, given the general preference of the translation apparatus for $\mathrm{K}^{+}$or ions with similar ionic radii $[149,241-244]$, the similarly strong stimulation of the GTPase activity in elF5B and EF-Tu by $\mathrm{K}^{+}$, and the usually high cellular $\mathrm{K}^{+} / \mathrm{Na}^{+}$ratio, we assume that $\mathrm{K}^{+}$is preferred by $\operatorname{trGTPases}$ under physiological conditions.

An interesting case that provides further indirect evidence for $\mathrm{M}^{+}$-dependency among trGTPases is the $\gamma$-subunit of eukaryal initiation factor 2 (elF2 $\gamma$ ). Notably, elF2 $\gamma$ is the only trGTPase for which a specific GAP (elF5) has been identified that was reported to provide an arginine-finger as catalytic element to promote GTP hydrolysis in elF2 $[53,254]$. The coordination sphere for the $\mathrm{M}^{+}$ion would therefore not be required in elF2 $\gamma$. Consistently, elF2 $\gamma$ is the only trGTPase that contains neither an $\mathrm{Asp}^{\mathrm{MC}}$ nor Gly ${ }^{\mathrm{MC}}$ (Fig. 3A). Instead, $\mathrm{Asp}^{\mathrm{MC}}$ is replaced by Ala, which might be necessary to allow the introduction of the bulky guanidino group into the active site instead of the 
$\mathrm{M}^{+}$ion. Importantly, the archaeal ortholog, alF2 $\gamma$, contains $\mathrm{Asp}^{\mathrm{MC}}$ and Gly ${ }^{\mathrm{MC}}$ and no GAP is known indicating that alF $2 \gamma$ in contrast to elF $2 \gamma$ may be $\mathrm{M}^{+}$-dependent.

\section{Implications for the evolution of trGTPases}

At the center of this work stands the conclusion that trGTPases belong to the group of $\mathrm{M}^{+}$dependent $G$ proteins. This establishes trGTPases as a functionally distinct subfamily among GTPases in which the ribosome, as an RGS-like GAP, stabilizes the active conformation of the catalytic machinery of the GTPase [168] which includes an $\mathrm{M}^{+}$ion as an additional trans-acting catalytic element, constitutively bound in the active site. Conceptually, this places trGTPases between the classical GAP- or RGS-activated GTPases and GTPases activated by homodimerization (GADs) (e.g. MnmE and dynamin), which, like trGTPases, directly couple the GTPase reaction to their biological function in the cell [230].

The proposed universality of $\mathrm{M}^{+}$-dependency among canonical trGTPases is particularly interesting from the evolutionary perspective, as it points toward a common origin in an ancestral $\mathrm{M}^{+}$-dependent trGTPase. At the same time, usage of $\mathrm{M}^{+}$ions as structural and catalytic cofactor constitutes a functional link to other known $\mathrm{M}^{+}$-dependent GTPases that - like trGTPases - usually belong to particularly ancient lineages of the TRAFAC class, associated with basic cellular functions such as tRNA modification or ribosome assembly $[22,255]$. It is therefore conceivable that $\mathrm{M}^{+}-$ dependency might represent the primordial form of catalysis of GTP hydrolysis in GTPases of the TRAFAC class that antedates the convergent occurrence of arginine-finger-dependent catalysis.

\subsection{Materials and Methods}

\section{Protein purification, crystallization and structure determination}

C. thermophilum elF5B constructs containing residues 517-858 (ctelF5B(517-858)) were purified as previously described [220]. E. coli EF-Tu was prepared essentially as described in [256]. E. coli EF-Ts was prepared using standard procedures (see Expanded View for details).

Crystallization trials were performed by sitting-drop vapor diffusion method using either self-made optimization screens for the original crystallization condition for ctelF5B.GTP (0.1 M HEPES/NaOH (pH 7); 13\% PEG 4000; $0.1 \mathrm{M} \mathrm{NaOAc)} \mathrm{[220]} \mathrm{or} \mathrm{standard} \mathrm{screens} \mathrm{in} \mathrm{the} \mathrm{presence} \mathrm{of}$ 
$\mathrm{Mg}^{2+}$ and varying concentrations of guanine nucleotides. Crystals of cteIF5B(517-858)·GTP used for structure determination grew over night at $20^{\circ} \mathrm{C}$ in $100 \mathrm{mM}$ HEPES (pH 7.25), 12\% PEG 4000 and $100 \mathrm{mM} \mathrm{NaOAc}$ with $10 \mathrm{mg} / \mathrm{ml}$ of protein in the presence of $2 \mathrm{mM} \mathrm{GTP}$. Crystals of ctelF5B(517858) bound to GTP $\gamma S$ grew over night at $20^{\circ} \mathrm{C}$ under similar conditions (100 mM HEPES (pH 7), $13 \%$ PEG 4000 and $125 \mathrm{mM} \mathrm{NaOAc}$ ) with $12 \mathrm{mg} / \mathrm{ml}$ protein and $2 \mathrm{mM}$ GTPyS. The crystals for both proteins grew in space group $\mathrm{P} 2_{1}$.

Crystals of ctelF5B(517-858).GTPyS with a potassium ion bound in the active site were obtained after two weeks at $20{ }^{\circ} \mathrm{C}$ in $11 \%$ PEG 8000, 6\% glycerol, and $50 \mathrm{mM} \mathrm{KCl}$. The best diffracting crystals grew when using $10 \mathrm{mg} / \mathrm{ml}$ protein in the presence of $2 \mathrm{mM} \mathrm{GTP} \gamma \mathrm{S}$. The crystals grew in space group $\mathrm{P} 4{ }_{1} 2{ }_{1} 2$.

Crystals of the Asp ${ }^{\mathrm{MC}}$ mutant ctelF5B(517-858)D533N were obtained with $8 \mathrm{mg} / \mathrm{ml}$ protein and $3 \mathrm{mM} \mathrm{GTP}$ at $20^{\circ} \mathrm{C}$ in a condition containing $100 \mathrm{mM} \mathrm{HEPES}(\mathrm{pH}$ 7), 13\% PEG 4000 and 125 $\mathrm{mM} \mathrm{NaOAc}$. As for the wild-type protein crystals that were obtained under similar conditions, the crystals of ctelF5B(517-858)D533N belonged to space group P2 $2_{1}$. Initially, no crystals were obtained for the mutants D533A and D533R. However, high-quality crystals for both mutants could finally be obtained by repeated microseeding experiments (see Expanded View for details). Crystals of ctelF5B(517-858)D533A (with $15 \mathrm{mg} / \mathrm{ml}$ protein and $6 \mathrm{mM} \mathrm{GTPyS)} \mathrm{grew} \mathrm{in} \mathrm{space} \mathrm{group} \mathrm{P2}{ }_{1}$ in a condition containing $0.1 \mathrm{M} \mathrm{HEPES} / \mathrm{NaOH}$ (pH 7.3), 15\% PEG 4000, and $150 \mathrm{mM} \mathrm{NaOAc.} \mathrm{Crystals} \mathrm{of}$ ctelF5B(517-858)D533R (with $15 \mathrm{mg} / \mathrm{ml}$ protein and $4 \mathrm{mM}$ GTP $\gamma$ S) grew in space group P4 ${ }_{1} 2{ }_{1} 2$ in a condition containing 100 mM MES (pH 6.7), 13\% PEG 8000, 225 mM NaOAc.

X-ray diffraction data were collected using synchrotron radiation. For all structures, the phase problem was solved by molecular replacement using the program PHASER [115]. Structures were refined to reasonable R-values and stereochemistry using the program PHENIX [117]. Data collection and refinement statistics for the structures of wild-type elF5B(517-858) and for the three Asp $^{\mathrm{MC}}$ mutants are summarized in Tables 1 and E2, respectively. See Expanded View for details.

\section{Isothermal Titration Calorimetry}

The thermodynamic parameters of elF5B binding to GDP or GDPNP were measured using a MicroCal VP-ITC instrument (GE Healthcare). Experiments were carried out as previously described 
[220] in ITC buffer (30 mM HEPES/KOH (pH 7.5), $100 \mathrm{mM} \mathrm{KCl,} 10 \%$ glycerol, $4 \mathrm{mM} \beta-$ mercaptoethanol, $0.01 \%$ tween $\left.20,2.5 \mathrm{mM} \mathrm{MgCl}_{2}\right)$ at different temperatures $(10,15,20,25$ or $30^{\circ} \mathrm{C}$ ). $14-\mu \mathrm{l}$ aliquots of $200-400 \mu \mathrm{M}$ ligand were injected into the $1.42 \mathrm{ml}$ cell containing $10-30 \mu \mathrm{M}$ ctelF5B(517-858). The heat of dilution was measured by injecting the ligand into the buffer solution without protein; the values were then subtracted from the heat of the individual binding reactions to obtain the effective heat of binding. The final titration curves were fitted using the 'Origin' based MicroCal software, assuming one binding site per protein molecule. For each isotherm the binding stoichiometry $(\mathrm{N})$, enthalpy changes $(\Delta \mathrm{H})$ and the association constants $\left(\mathrm{K}_{\mathrm{a}}\right)$, were obtained by a nonlinear regression fitting procedure. These directly measured values were used to estimate the Gibbs energy $(\Delta G)$ from the relation $\Delta G=-R \cdot T \cdot \ln K_{a}$ and the entropy changes $(\Delta \mathrm{S})$ through $\Delta \mathrm{G}=\Delta \mathrm{H}-\mathrm{T} \cdot \Delta \mathrm{S}$.

In order to estimate the change in heat capacity $\left(\Delta C_{p}\right)$ upon complex formation between elF5B and guanine nucleotides, the measured $\Delta H$ values were plotted against the temperature, where the slope of the fitted line directly represents the $\Delta C_{p}$ of the binding reaction $[178,179]$. See Expanded View for details about the correlation between $\Delta C_{p}$ and the change in solvent accessible surface area $(\triangle \mathrm{ASA})$.

\section{Analysis of the GTPase reaction of elF5B and EF-Tu by HPLC}

The intrinsic GTP hydrolysis of $C$. thermophilumeIF5B(517-858) and E. coli EF-Tu was analyzed by HPLC (GE Healthcare). Nucleotides were separated on a NUCLEOSIL 4000 PEI (Macherey Nagel) in $10 \mathrm{mM}$ Tris $/ \mathrm{HCl}(\mathrm{pH}$ 8.0) with a linear gradient from 0-1 M NaCl. For EF-Tu, the reactions were followed under single-turnover conditions, for which $25 \mu \mathrm{M}$ EF-Tu.GTP was incubated at $30{ }^{\circ} \mathrm{C}$ in $25 \mathrm{mM}$ Tris/ $\mathrm{HCl}(\mathrm{pH} 7.5), 7 \mathrm{mM} \mathrm{MgCl}, 2 \mathrm{mM} \mathrm{DTT}$ and alkali salts at different concentrations. At various time points, $50 \mu \mathrm{l}$ aliquots were taken and incubated at $96{ }^{\circ} \mathrm{C}$ for 2 min to stop the reaction. Denatured protein was removed by centrifugation and the supernatant applied to the HPLC.

For elF5B, the reactions were followed under multiple-turnover conditions, for which $25 \mu \mathrm{M}$ nucleotide-free elF5B was incubated with $300 \mu \mathrm{M}$ GTP at $35^{\circ} \mathrm{C}$ in $25 \mathrm{mM}$ Tris/ $\mathrm{HCl}(\mathrm{pH} 7.5), 3 \mathrm{mM}$ 
$\mathrm{MgCl}_{2}, 2 \mathrm{mM}$ DTT and alkali salts at different concentrations. $50 \mu \mathrm{l}$ aliquots were taken at various time points and treated as described above.

\section{Multiple sequence alignments}

Multiple sequence alignments were done using the iterative alignment program MUSCLE [214].

\section{Coordinates}

Coordinates and structure factors have been deposited in the PDB: 4TMW(ct5B(517-858).GTP.Na $\left.{ }^{+}\right)$; 4TMV $\quad\left(c t 5 B(517-858) \cdot G T P y S \cdot \mathrm{Na}^{+}\right) ; \quad 4 T M Z \quad\left(c t 5 B(517-858) \cdot G T P y S \cdot K^{+}\right) ; \quad 4 T M T \quad$ (ct5B(517-

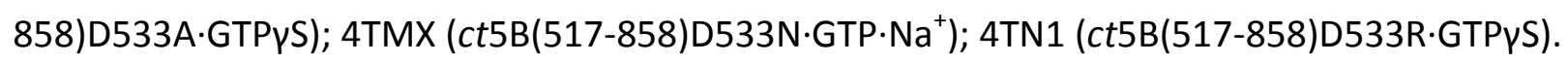

\section{Expanded View}

Expanded View information is available at The EMBO Journal Online and includes six figures, two tables and extended Materials and Methods.

\section{Acknowledgments}

We thank the beam line scientists at EMBL/DESY (Hamburg) and ESRF (Grenoble) as well as P. Neumann for support during X-ray diffraction data collection and L. Hsu for help with figures. We are also grateful to L. K. Dörfel and A. Dickmanns for critical reading of the manuscript as well as C. Maracci and M.V. Rodnina for comments on the manuscript and for sharing data before publication.

\section{Author Contributions}

Author contributions: BK designed the experiments, prepared and crystallized proteins, collected X-ray data, solved structures, performed GTPase and ITC experiments, created figures. BK and RF analyzed the data and wrote the manuscript.

\section{Conflict of interest}

The authors declare that they have no conflict of interest. 


\subsection{Expanded View}

\section{Expanded View Materials and Methods}

\section{Protein purification}

The purification of Chaetomium thermophilum elF5B(517-858) was done as previously described [220]. The plasmid for the expression of N-terminally His-taggedctelF5B(517-858) (comprising G domain and domain II) was transformed into E. coli BL21(DE3) cells (Stratagene) by heat shock. Transformed cells were grown in $1 \mathrm{I}$ cultures of $2 \mathrm{YT}$ medium at $37{ }^{\circ} \mathrm{C}$ to an $\mathrm{OD}_{600}$ of $0.6-0.8$, followed by the induction of protein expression with $0.5 \mathrm{mM}$ isopropyl- $\beta$-D-thiogalactopyranoside (IPTG). Cells were grown for an additional $18 \mathrm{~h}$ at $16{ }^{\circ} \mathrm{C}$ before harvesting. Harvested cells were

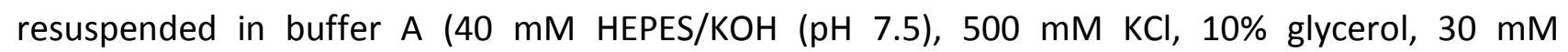
imidazole, $2 \mathrm{mM} \beta$-mercaptoethanol), lysed using a Microfluidizer (Microfluidics) and clarified by $30 \mathrm{~min}$ ultracentrifugation at 30,000 $\mathrm{xg}$. The supernatant was loaded onto two HisTrap columns (GE Healthcare), equilibrated in buffer A. After the elution of bound proteins with a linear gradient of imidazole (30-300 mM), elF5B-containing fractions were pooled and desalted in buffer B (20 mM HEPES/KOH (pH 7.5), $200 \mathrm{mM} \mathrm{KCl,} 10 \%$ glycerol, $30 \mathrm{mM}$ imidazole and $2 \mathrm{mM} \beta$-mercaptoethanol) for subsequent TEV-protease cleavage at $4{ }^{\circ} \mathrm{C}$ over night to remove the His-Tag. Uncleaved protein was removed by a second HisTrap in buffer B and the flow-through was pooled and concentrated for the final size exclusion chromatography step on a Superdex 200 column (GE Healthcare), equilibrated in buffer $\mathrm{C}(10 \mathrm{mM} \mathrm{HEPES} / \mathrm{KOH}(\mathrm{pH} 7.5), 50 \mathrm{mM} \mathrm{KCl}, 3 \mathrm{mM} \mathrm{MgCl}$ and $1 \mathrm{mM}$ DTT). The absence of copurified guanine nucleotides was confirmed by HPLC. The purified apo elF5B was pooled, concentrated to $15-20 \mathrm{mg} / \mathrm{ml}$, and finally stored at $-80^{\circ} \mathrm{C}$.

Mutant constructs of ctelF5B, in which Asp533 (Asp ${ }^{\mathrm{MC}}$ ) was substituted by alanine (D533A), asparagine (D533N) or arginine (D533R), respectively, were generated by site-directed mutagenesis according to the QuikChange protocol (Stratagene, Instruction Manual, 2006). All mutants were expressed and purified according to the above described protocol for wild-type ctelF5B(517-858) [220].

For ITC experiments ctelF5B(517-858) was purified according to the above protocol with the difference that ITC buffer (30 mM HEPES/KOH (pH 7.5), $100 \mathrm{mM} \mathrm{KCl,} 10 \%$ glycerol, $4 \mathrm{mM} \beta$ mercaptoethanol, $0.01 \%$ tween $20,2.5 \mathrm{mM} \mathrm{MgCl}_{2}$ ) was used in the final gelfiltration step.

For GTPase activity assays under different salt conditions, purified ctelF5B(517-858) (wild-type or mutant) was desalted in the desired buffer (Tris/HCl (pH 7.5), $3 \mathrm{mM} \mathrm{MgCl}, 2 \mathrm{mM}$ DTT and alkali salts at different concentrations) prior to the experiment (see below).

E. coli EF-Ts was expressed with an N-terminal His-tag in E. coliBL21(DE3)pLysS cells (Stratagene). Transformed cells were grown in 1 I cultures of $L B$ medium at $37^{\circ} \mathrm{C}$ to an $O_{600}$ of $\sim 0.8$, followed by the induction of protein expression with $0.5 \mathrm{mM}$ IPTG. Cells were grown for an additional $4 \mathrm{~h}$ at 37 ${ }^{\circ} \mathrm{C}$ before harvesting. Purification of EF-Ts (including the removal of the His-tag) was performed according to the protocol described above for ctelF5B(517-858), with the difference that a 
Superdex 75 column (GE Healthcare), equilibrated in a buffer containing $25 \mathrm{mM}$ Tris (pH 7.5), 30 $\mathrm{mM} \mathrm{KCl}, 70 \mathrm{mM} \mathrm{NH}_{4} \mathrm{Cl}$ and $7 \mathrm{mM} \mathrm{MgCl}_{2}$, was used in the final size exclusion chromatography step. Purified protein was flash-frozen in liquid nitrogen and stored at $-80^{\circ} \mathrm{C}$.

E. coli EF-Tu was prepared essentially as described previously [256]. The final gelfiltration buffer contained $20 \mathrm{mM}$ Tris/ $\mathrm{HCl}$ (pH 7.5), $50 \mathrm{mM} \mathrm{KCl}, 7 \mathrm{mM} \mathrm{MgCl}$ and $2 \mathrm{mM}$ DTT. GTP-bound EF-Tu was prepared by incubating $100 \mu \mathrm{M}$ purified EF-Tu with $3 \mathrm{mM}$ GTP, $3 \mathrm{mM}$ phosphoenolpyruvate (Roche), $0.05 \mathrm{mg} / \mathrm{ml}$ pyruvate kinase (Roche) and $0.04 \mu \mathrm{M} \mathrm{EF-Ts} \mathrm{for} 1 \mathrm{~h}$ at $37^{\circ} \mathrm{C}$. To remove excess GTP and phosphoenolpyruvate, GTP-bound EF-Tu was desalted on a HiTrap Desalting column (GE Healthcare) at $4{ }^{\circ} \mathrm{C}$ in the desired buffer $\left(25 \mathrm{mM}\right.$ Tris/ $\mathrm{HCl}\left(\mathrm{pH} 7.5\right.$ at $\left.25^{\circ} \mathrm{C}\right), 7 \mathrm{mM} \mathrm{MgCl}, 2 \mathrm{mM} \mathrm{DTT}$ and alkali salts at different concentrations).

\section{Crystallization and structure determination}

The first crystallization trials with ctelF5B(517-858) were performed in an optimization screen for the condition under which the original crystals of GTP-bound cteIF5B(517-860) had been obtained using a fragment comprising residues 517-970 (0.1 M HEPES/NaOH (pH 7); 13\% PEG 4000; $0.1 \mathrm{M}$ $\mathrm{NaOAc}$ [220]. 8, 10 or $12 \mathrm{mg} / \mathrm{ml}$ of protein were mixed with GTP, GTPYS or GDPNP at final concentrations of 1, 2 or $3 \mathrm{mM}$ (up to $10 \mathrm{mM}$ for GDPNP in the presence of $10 \mathrm{mM} \mathrm{MgCl}$ ) of the respective guanine nucleotides. Crystallization was performed at 4 and $20{ }^{\circ} \mathrm{C}$ by vapor diffusion using a 1:1 or 2:1 ratio of protein to reservoir solution. For ctelF5B(517-858).GDPNP no crystals were obtained under any of the tested conditions. Crystals of ctelF5B(517-858).GTP and ctelF5B(517-858).GTPYS grew over night in most conditions, irrespective of the temperature or used guanine nucleotide concentration. In both cases the best diffracting crystals that were finally used for structure determination grew at $20^{\circ} \mathrm{C}$ in primitive monoclinic space group $\mathrm{P} 2{ }_{1}$.

To find a crystallization condition for ctelF5B(517-858) in the presence of potassium, crystallization trials with $10 \mathrm{mg} / \mathrm{ml}$ protein were performed in the presence of $2 \mathrm{mM} \mathrm{GTPYS}$ using standard screens. Diffraction quality crystals were obtained after two weeks at $20{ }^{\circ} \mathrm{C}$ in a condition containing 11\% PEG 8000, 6\% glycerol, and $50 \mathrm{mM} \mathrm{KCl}$. The crystals used for structure determination grew in space group $\mathrm{P}_{1} 2_{1} 2$ and contained two protein-GTPYS complexes per asymmetric unit.

Initial crystallization trials for the Asp ${ }^{\mathrm{MC}}$ mutants of ctelF5B(517-858) (D533A, D533N and D533R) were performed with $8 \mathrm{mg} / \mathrm{ml}$ protein in the presence of $3 \mathrm{mM}$ GTP or GTP $\psi \mathrm{S}$ using fine-screens around the two conditions described above. Only for the D533N mutant high quality crystals in space group $\mathrm{P} 2{ }_{1}$ grew over night that could be directly used to determine the structure of ctelF5B(517-858)D533N bound to GTP and a $\mathrm{Na}^{+}$ion. No crystals were initially obtained for the other mutants D533A and D533R. We then performed microseeding experiments for both mutants, in which crystals of the wild-type protein were destroyed and used as crystallization nuclei to induce crystallization. At protein concentrations of $15 \mathrm{mg} / \mathrm{ml}$ and $6 \mathrm{mM}$ guanine nucleotide, this resulted in the formation of thin needle clusters for D533A in conditions containing 
$0.1 \mathrm{M} \mathrm{HEPES} / \mathrm{NaOH}$ ( $\mathrm{pH} 7.3$ ), 15\% PEG 4000, and $150 \mathrm{mM} \mathrm{NaOAc}$. These were then used in an additional round of microseeding, finally yielding large enough crystals for structure determination. As the wild-type protein, the D533A mutant crystallized in space group $\mathrm{P} 2{ }_{1}$ and the structure was finally solved at a resolution of $1.58 \AA$. In contrast to D533A, no crystals were obtained for D533R in the initial microseeding experiments but only small spherolite-like aggregates which could not be used as seeds. We therefore performed new crystallization trials for the D533R mutant in various commercially available grid screens, in combination with microseeding. This yielded thin, plate-shaped crystals in a condition containing $100 \mathrm{mM}$ MES (pH 6.5), 11\% PEG 8000, and $150 \mathrm{mM}$ $\mathrm{NaOAc}$. Using these crystals as microseeds in an optimized screen, we were finally able to obtain ctelF5B(517-858)D533R crystals in the presence of GTPYS suited for structure determination. Despite the fact that the D533R mutant crystals were obtained in the presence of $\mathrm{Na}^{+}$ions, they grew in space group $\mathrm{P}_{1}{ }_{1}{ }_{1} 2$ as did those obtained in the presence of $\mathrm{K}^{+}$. The structure of ctelF5B(517-858)D533R could finally be solved at a resolution of $2.75 \AA$.

Based on the observations for the ctelF5B mutants, additional crystallization trials were performed with wild-type ctelF5B(517-858) in the presence of GDPNP and microseeds. In the fine-screens, microseeding resulted in the formation of large spherolytes, which, however, did not yield any crystals when used as seeds themselves.

For the structures of wild-type ctelF5B(517-858) bound to GTP or GTPyS and $\mathrm{Na}^{+}, \mathrm{X}$-ray diffraction data were collected at P13 beamline at PETRA III (EMBL, Hamburg). For the structures of wild-type ctelF5B(517-858) bound to GTPYS and $\mathrm{K}^{+}$and all Asp ${ }^{\mathrm{MC}}$ mutants, X-ray diffraction data used for structure determination were collected at beamline ID23-1 at ESRF (Grenoble). For all structures, the phase problem was solved by molecular replacement using the program PHASER [115] with the original structure of ctelF5B.GTP as search model. Structures were refined to reasonable R-values and stereochemistry using the program PHENIX [117]. Data collection and refinement statistics are summarized in Table 1 and Table E2.

Structure factors and coordinates for the two aEF1A structures were obtained from the protein data bank (PDB: 3AGJ, 3VMF). The structures were refined using the program PHENIX [117]. Manual model rebuilding was performed against electron density maps in Coot [116]. Figures were prepared using Pymol (http://www.pymol.org).

\section{Isothermal Titration Calorimetry}

The thermodynamic parameters of elF5B binding to GDP or GDPNP were measured using a MicroCal VP-ITC instrument (GE Healthcare). Experiments were carried out as previously described [220] in ITC buffer at different temperatures $\left(10,15,20,25\right.$ or $\left.30^{\circ} \mathrm{C}\right)$. 14- $\mu$ l aliquots of 200-400 $\mu \mathrm{M}$ ligand were injected into the $1.42 \mathrm{ml}$ cell containing 10-30 $\mu \mathrm{M}$ ctelF5B(517-858). The heat of dilution was measured by injecting the ligand into the buffer solution without protein; the values were then subtracted from the heat of the individual binding reactions to obtain the effective heat of binding. The final titration curves were fitted using the 'Origin' based MicroCal software, 
assuming one binding site per protein molecule. For each isotherm the binding stoichiometry $(\mathrm{N})$, enthalpy changes $(\Delta \mathrm{H})$ and the association constants $\left(\mathrm{K}_{\mathrm{a}}\right)$, were obtained by a nonlinear regression fitting procedure. These directly measured values were used to estimate the Gibbs energy $(\Delta G)$ from the relation $\Delta G=-R \cdot T \cdot \ln K_{a}$ and the entropy changes $(\Delta S)$ through $\Delta G=\Delta H-T \cdot \Delta S$.

In order to estimate the change in heat capacity $\left(\Delta C_{p}\right)$ upon complex formation, the measured $\Delta H$ values were plotted against the temperature $[178,179]$. The slope of the fitted line directly represents the $\Delta C_{p}$ of the binding reaction. $\Delta C_{p}$ can be used as an estimate for the change in solvent accessible surface area ( $\triangle \mathrm{ASA}$ ) upon complex formation as it was found to be proportional to the size of the area which is either exposed to (associated with a positive value for $\Delta C_{p}$ ) or removed from (negative value for $\Delta C_{p}$ ) the aqueous environment during the binding event [177, 180-182]. $\Delta C_{p}$ and $\triangle A S A$ are connected by the empirically determined relation $\Delta C_{p}=\Delta c_{a p} \cdot \Delta A_{S A}+$ $\Delta c_{p} \cdot \Delta A S A_{p}$ where $\Delta c_{a p}(0.45)$ and $\Delta c_{p}(-0.26)$ are the area coefficients in $\mathrm{cal} \cdot \mathrm{K}^{-1} \cdot\left(\mathrm{mol} \cdot \AA^{2}\right)^{-1}$ for the contributions of apolar or polar side chains to $\triangle A S A$, respectively $[177,182,184]$. As previously described [220], we use two values for the area coefficients to estimate the change in surface area upon ligand binding: $\Delta c_{\max }=0.24\left(=0.7 \cdot \Delta c_{a p}+0.3 \cdot \Delta c_{p}\right)$ as the upper limit case and $\Delta c_{\min }=0.45$ as the lower limit case in which all involved residues are apolar.

\section{Analysis of the GTPase reaction of elF5B and EF-Tu by HPLC}

The intrinsic GTP hydrolysis of $C$. thermophilumelF5B(517-858) and E. coli EF-Tu was analyzed by HPLC (GE Healthcare). Nucleotides were separated on a NUCLEOSIL 4000 PEI (Macherey Nagel) in $10 \mathrm{mM}$ Tris/ $\mathrm{HCl}$ ( $\mathrm{pH}$ 8.0) with a linear gradient from 0-1 M NaCl. For EF-Tu, the reactions were followed under single-turnover conditions, for which $25 \mu \mathrm{M}$ EF-Tu.GTP was incubated at $30{ }^{\circ} \mathrm{C}$ in $25 \mathrm{mM}$ Tris/ $\mathrm{HCl}(\mathrm{pH} \mathrm{7.5)}, 7 \mathrm{mM} \mathrm{MgCl}, 2 \mathrm{mM}$ DTT and alkali salts at different concentrations. At various time points, $50 \mu$ laliquots were taken and incubated at $96{ }^{\circ} \mathrm{C}$ for 2 min to stop the reaction. Denatured protein was removed by centrifugation and the supernatant applied to the HPLC.

For elF5B, the reactions were followed under multiple-turnover conditions, for which $25 \mu \mathrm{M}$ nucleotide-free elF5B was incubated with $300 \mu \mathrm{M} \mathrm{GTP}$ at $35^{\circ} \mathrm{C}$ in $25 \mathrm{mM}$ Tris/ $\mathrm{HCl}(\mathrm{pH} 7.5), 3 \mathrm{mM}$ $\mathrm{MgCl}_{2}, 2 \mathrm{mM}$ DTT and alkali salts at different concentrations. $50 \mu \mathrm{l}$ aliquots were taken at various time points and treated as described above.

\section{Steady-state fluorescence measurements}

Fluorescence measurements were carried out on a Fluoromax-3 spectrophotometer (Jobin Yvon Inc.) using a $1 \mathrm{ml}$ quartz cuvette with magnet stirrer. Titrations of elF5B constructs with mant-GTP

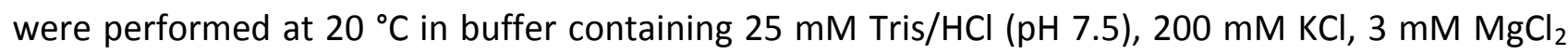
and $2 \mathrm{mM}$ DTT. Binding of mant-GTP to elF5B constructs was monitored by tryptophan Foerster Resonance Energy Transfer (FRET) using an excitation wavelength of $290 \mathrm{~nm}$ and an emission wavelength of $440 \mathrm{~nm}$. In order to estimate the equilibrium dissociation constant $\left(K_{\mathrm{d}}\right)$ between 


\section{Chapter 7}

elF5B constructs and mant-GTP, $2 \mu \mathrm{M}$ of the protein was titrated with increasing amounts of the mant-nucleotide (dilution was less than $1 \%$ ). The resulting signal was corrected for the contribution of unbound nucleotide by titrating mant-GTP into buffer and subsequently subtracting these values from the signals obtained with protein. The titration data were analyzed using a quadratic binding model:

$$
F=F_{0}+\Delta F_{\max } \frac{\left(X+Y+K_{\mathrm{d}}\right)-\sqrt{\left(X+Y+K_{\mathrm{d}}\right)^{2}-4 X Y}}{2 X}
$$

where $F$ is the fluorescence signal of the mant-nucleotide in the presence of elF5B, $F_{0}$ is the initial fluorescence signal, $\Delta F_{\max }$ is the maximum fluorescence signal, $X$ is the total concentration of elF5B, $Y$ is the total concentration of the added mant-nucleotide and $K_{d}$ is the equilibrium dissociation constant. 


\section{Expanded ViewFigures}

A

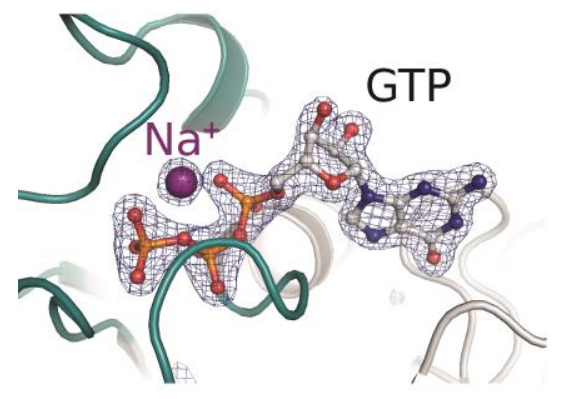

$\mathrm{D}$

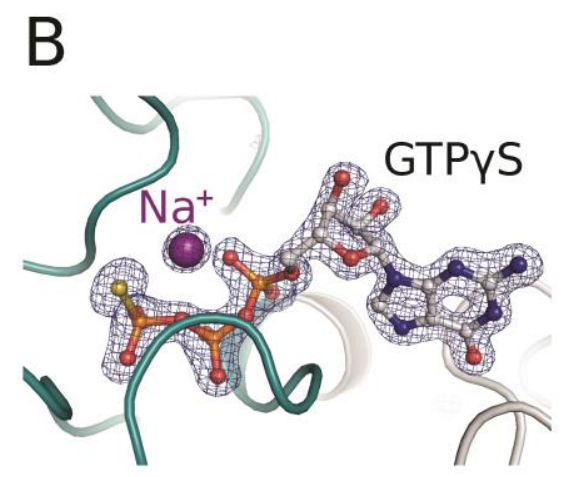

C

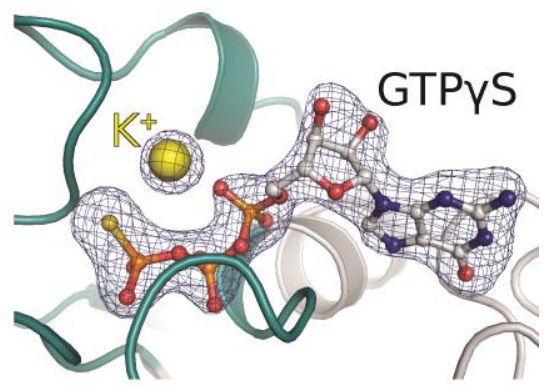

$\mathrm{E}$
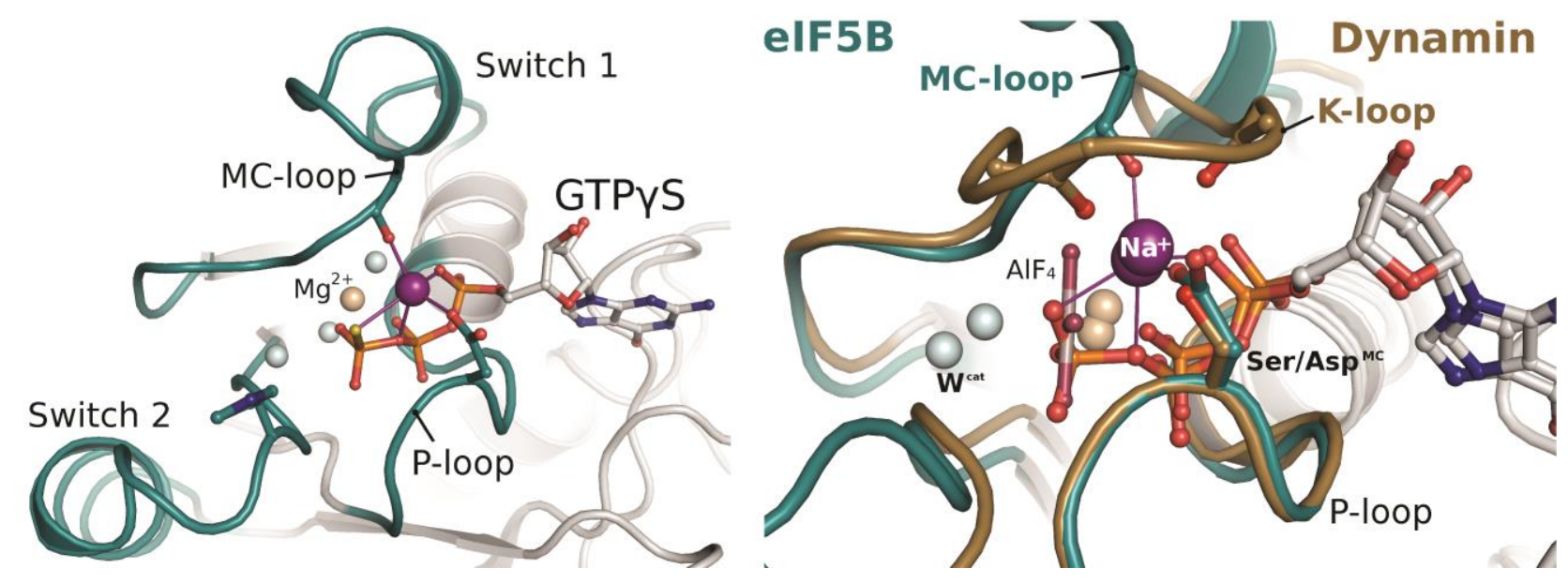

Figure E1. The GTPase center of elF5B bound to GTP or GTPyS. A-C) $F_{o}-F_{c}$ omit maps for GTP and $\mathrm{Na}^{+}(\mathrm{A})$,

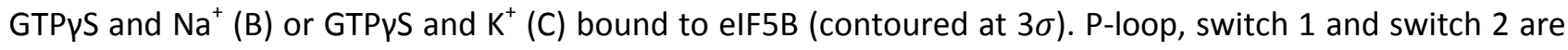
colored in cyan. The electron density maps for GTP $\gamma S$ clearly indicate that the sulfur atom of the $\gamma$ phosphate is oriented outward and interacts with the respective $\mathrm{M}^{+}$ion. D) Overview of the active site of elF5B bound to GTP $\gamma S$ and $\mathrm{Na}^{+}$(purple sphere). The pentameric coordination sphere is indicated by purple lines. GTP, Asp ${ }^{\mathrm{MC}}$, Gly ${ }^{\mathrm{MC}}$ and His ${ }^{\mathrm{cat}}$ are shown as balls and sticks; P-loop, switch 1 and switch 2 are colored in cyan; the $\mathrm{Mg}^{2+}$ ion and water molecules are shown as spheres in light brown and grey, respectively. E) Superposition of elF5B.GTP. $\mathrm{Na}^{+}$(coloring as in D) and dynamin (brown) bound to GDP, $\mathrm{AlF}_{4}$ and $\mathrm{Na}^{+}$(PDB: $2 \mathrm{X} 2 \mathrm{E}$ ). The $\mathrm{M}^{+}$ions are coordinated by both proteins in nearly identical positions, despite differences in the ligands forming the coordination shell: $A s \mathrm{p}^{\mathrm{MC}}$ is replaced by a Ser residue and two backbone oxygens from the K-loop replace Gly ${ }^{\mathrm{MC}}$ from the MC-loop. 

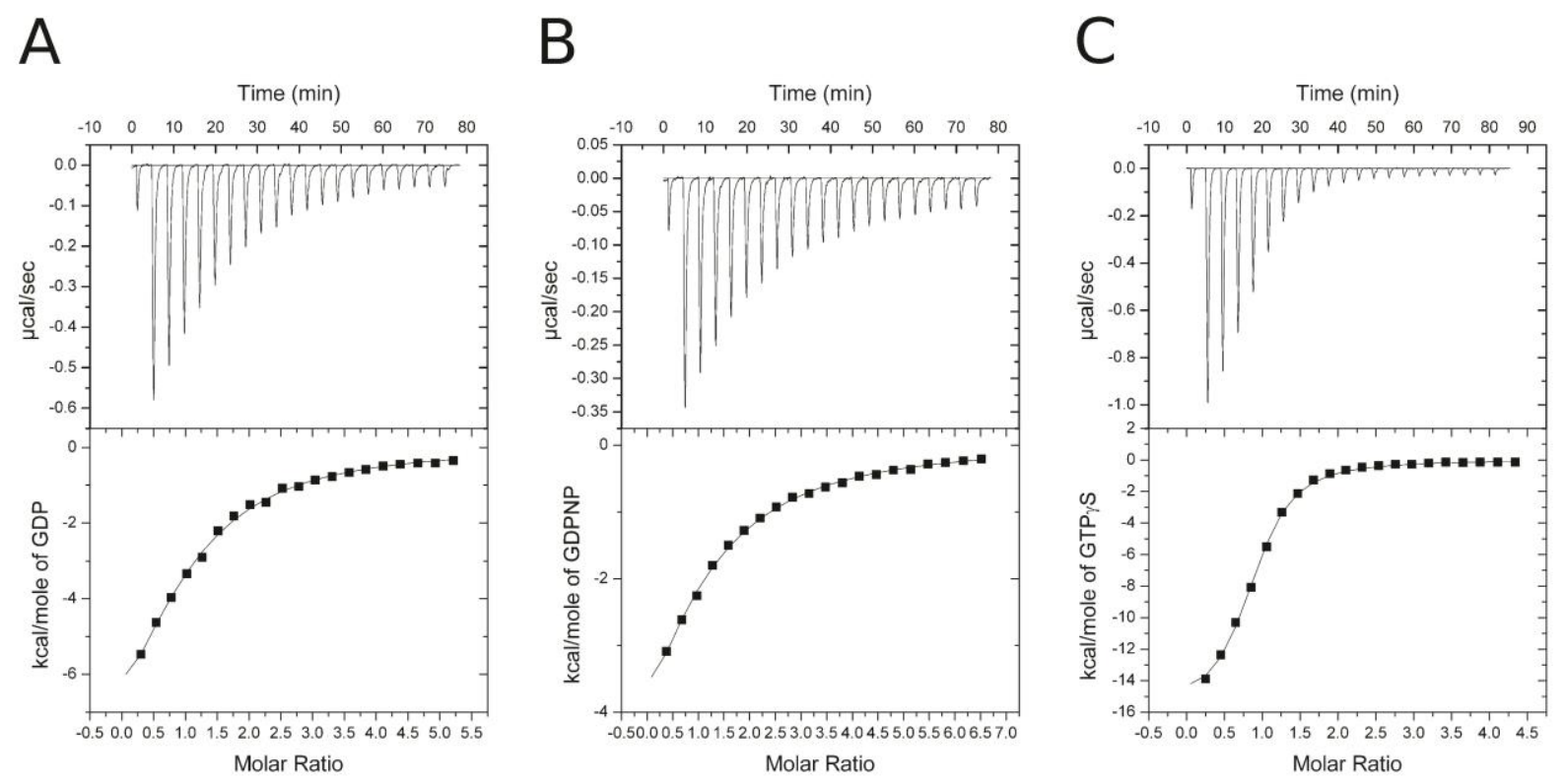

Figure E2. A-C) Titration curves (upper panels) and binding isotherms (lower panels) of elF5B(517-858) interactions with GDP (A), GDPNP (B) or GTP $\mathrm{S}(\mathrm{C})$ at $30^{\circ} \mathrm{C}$. 
A

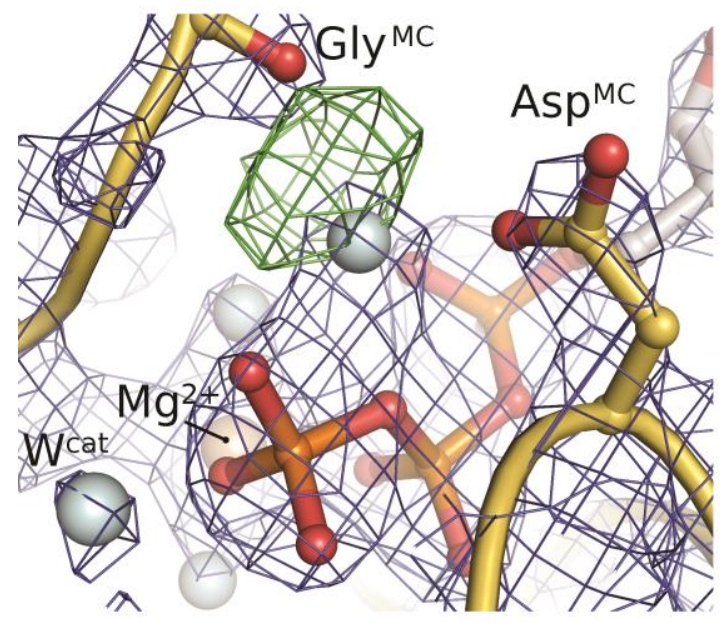

C

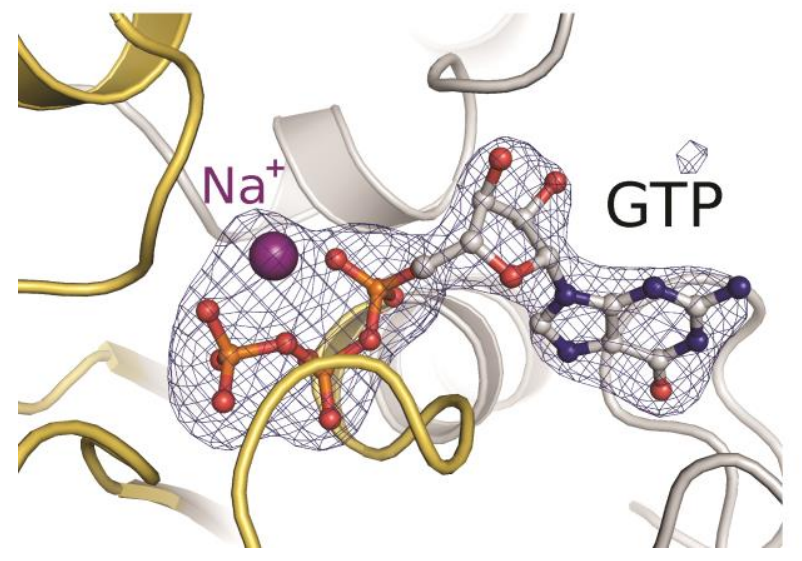

B

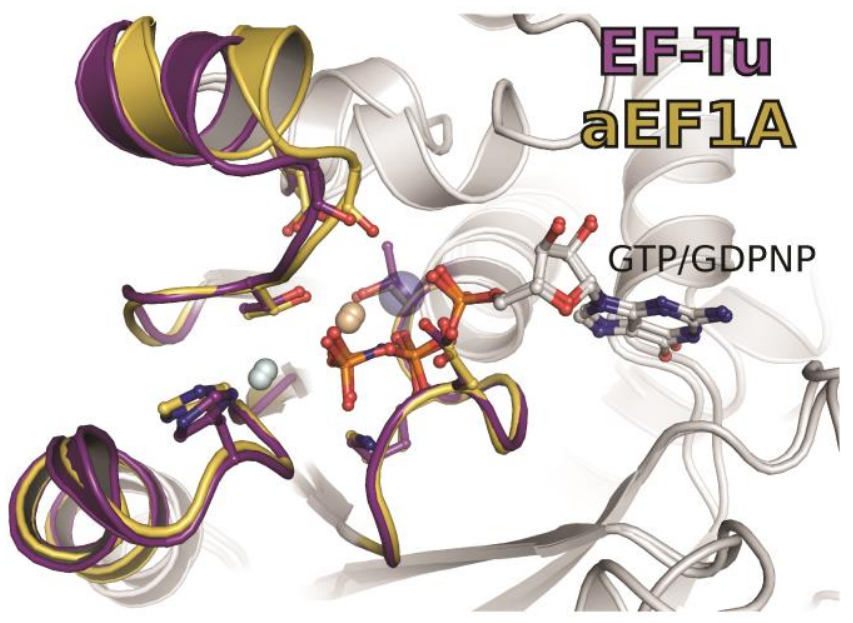

$\mathrm{D}$

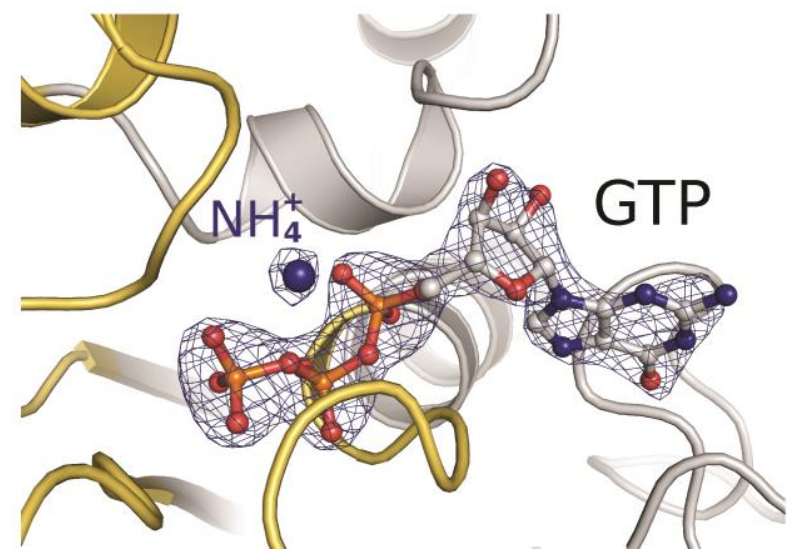

Figure E3.Analysis of $\mathbf{M}^{+}$ion coordination in structures of GTP-bound aEF1A.A) $2 \mathrm{mFo-DFc}$ map (blue mesh) and mFo-DFc difference map (green mesh) for GTP and its surrounding in aEF1A molecule $E$ of the

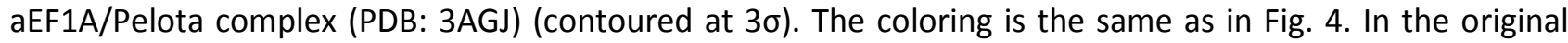
structure a water molecule is modeled into the pentameric coordination sphere between $\mathrm{Asp}^{\mathrm{MC}}$ and $\mathrm{Gly}^{\mathrm{MC}}$ and the phosphates of the GTP molecule with coordination distances of $\sim 2.45 \AA$. Despite its solvent exposed position, the electron density for this water molecule is significantly stronger than that for the catalytic water $\left(\mathrm{W}^{\text {cat }}\right)$ or the water molecules coordinating the $\mathrm{Mg}^{2+}$ ion in the center of the active site. The positive difference electron density for the supposed water molecule (green mesh) indicates a higher density of electrons in this position than provided by $\mathrm{H}_{2} \mathrm{O}$. B) The GTPase center in EF-Tu in its GDPNP conformation is virtually identical to that of aEF1A.GTP. With the exception of the $\beta$ - $\gamma$-bridging oxygen of GTP EF-Tu.GDPNP provides all structural elements that are involved in $\mathrm{M}^{+}$coordination in the archaeal ortholog. The $\mathrm{M}^{+}$ion is indicated as semi-transparent blue sphere. Some universally conserved residues involved in GTP/GDPNP binding or GTP hydrolysis are shown as sticks. $C$ and D) $F_{o}-F_{c}$ omit maps for GTP and

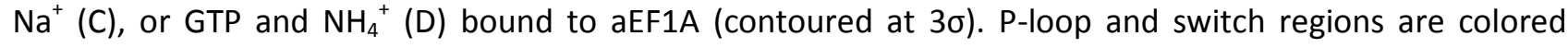
yellow. 

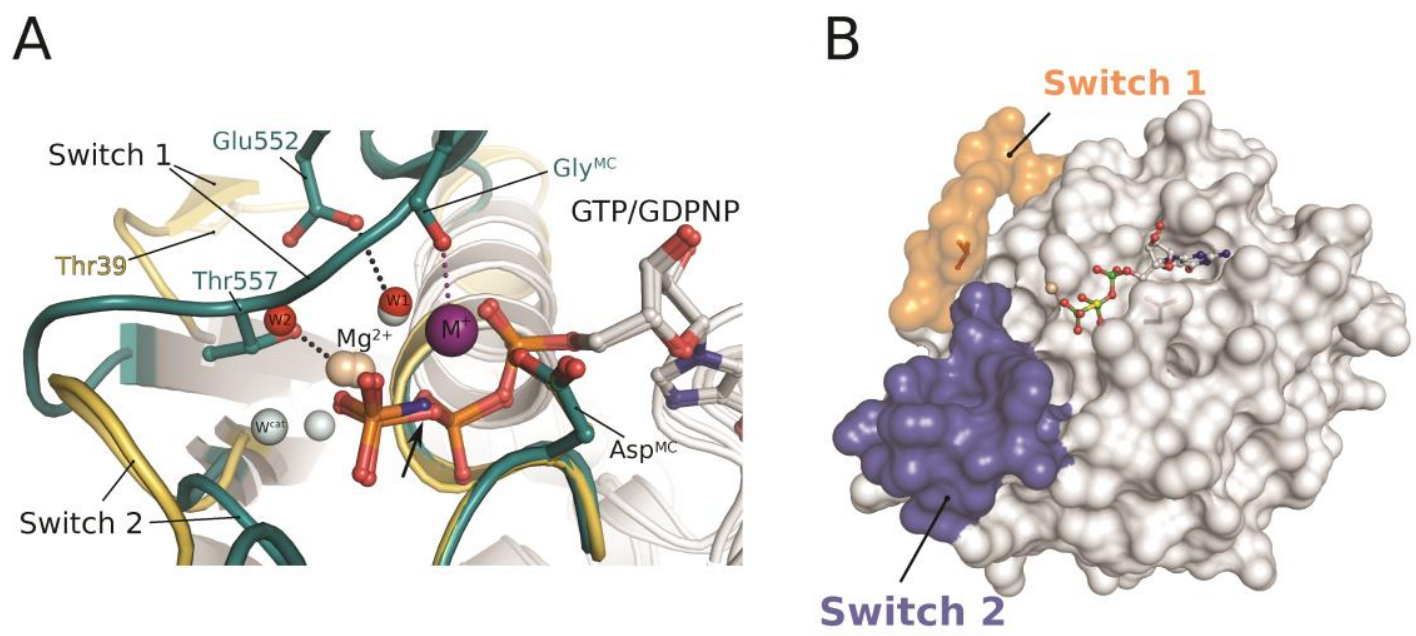

\section{$\mathrm{EF}-\mathrm{G} \cdot \mathrm{GDPCP}$}

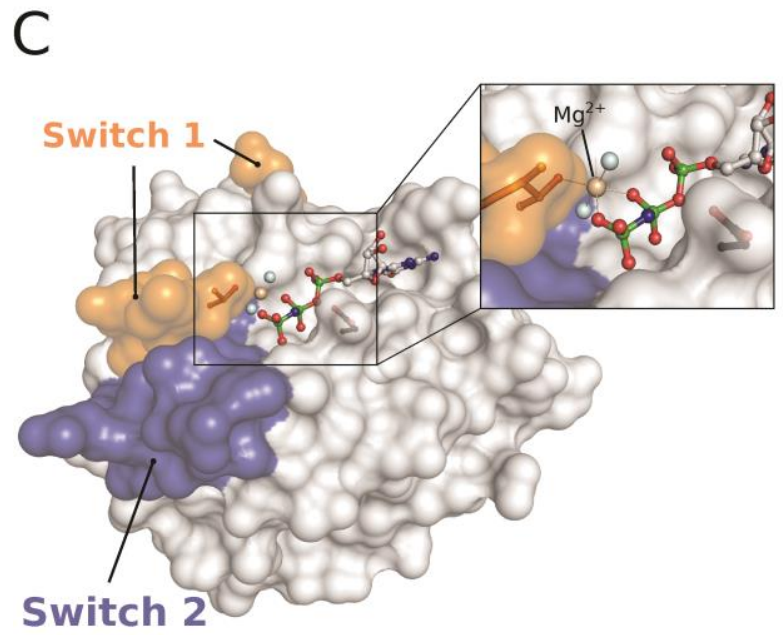

\section{$\mathrm{SelB} \cdot \mathrm{GDPNP}$}

D

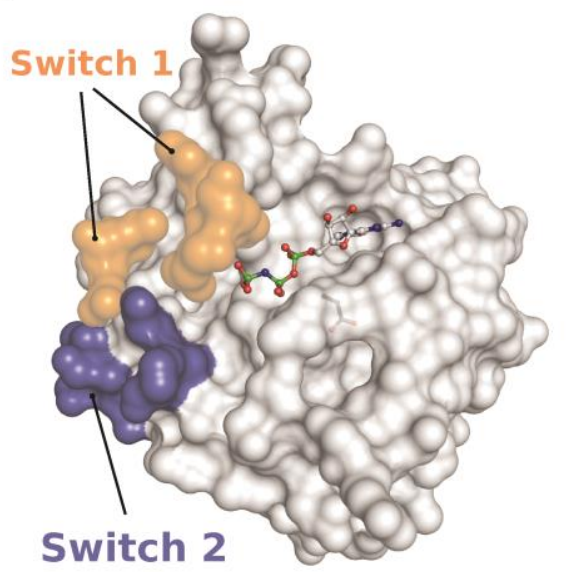

$\mathrm{eRF} 3 \cdot \mathrm{GDPNP}$

Figure E4.GDPNP and GDPCP prevent the coordination of the $\mathrm{M}^{+}$ion, which results in their inability to stably induce the conformational switch in trGTPases.A) Detailed view on the nucleotide binding pocket in a superposition of elF5B.GTP (P-loop and switch regions in cyan) and alF5B-GDPNP (P-loop and switch regions in yellow). GDPNP provides the $\gamma$-phosphate oxygens, the $\mathrm{Mg}^{2+}$ ion (light brown sphere) and its water ligands (red sphere; W1) in the identical positions as GTP for interactions with switch 2 and the stable association of switch 1 through Glu552 and Thr557 (C. thermophilum numbering). However, in contrast to the GTP-structure switch 1 and switch 2 remain in their GDP-like conformation in GDPNP-bound alF5B and the position of Thr39 (corresponding to Thr557) is instead occupied by an additional water molecule (red sphere; W2). The only significant difference between GTP and GDPNP is the inability of the latter to coordinate the $\mathrm{M}^{+}$ion (purple sphere), resulting in the loss of its contribution as structural cofactor to stabilize the GTP-conformation of switch 1 through the interaction with Gly ${ }^{\mathrm{MC}}$ (purple dashed line). (It is important to note that in the originally downloaded structure file for alF5B.GDPNP (PDB: 1G7T), W1 (red sphere) is interpreted as the $\mathrm{Mg}^{2+}$ ion, whereas a water (number 844) was modeled in the position which is here indicated as $\mathrm{Mg}^{2+}$ ion (light brown sphere). Our reinterpretation is based on the coordination geometry as well as the coordination distances for these two positions (coordination distances between 2.1 and $2.4 \AA$ 
for water 844 and between 2.6 and $3.4 \AA$ for the supposed $\mathrm{Mg}^{2+}$ ion). This reinterpretation is consistent with previously reported values for the coordination of $\mathrm{Mg}^{2+}$ ions [257]. As the structure factors are not deposited in the PDB, we were unable to examine whether our reinterpretation is consistent with the experimental data). B) Surface presentation of the $G$ domain in free EF-G.GDPCP (PDB: 2J7K). As for alF5B.GDPNP, switch 1 (orange) and switch 2 (blue) remain in their 'off' state conformation despite the presence of the GTP analog. Like in the case of GDPNP, the coordination of the $\mathrm{M}^{+}$ion is prevented by the $\beta$ $\gamma$-bridging $\mathrm{CH}_{2}$ group instead of the required oxygen ligand. C) In SelB.GDPNP switch 2 (blue) interacts with the $\gamma$-phosphate in the canonical way. Switch 1 as well undergoes a conformational change, resulting in the canonical direct contact between Thr46 (corresponding to Thr557 in ctelF5B) and the $\mathrm{Mg}^{2+}$ ion (inset). However, despite the stabilization of Thr46, the preceding regions including the residues corresponding to the MC-loop in elF5B and aEF1A remain flexible and are not defined in the electron density, most likely due to the loss of the $\mathrm{M}^{+}$ion as stable interaction partner. D) Surface presentation of GDPNP-bound eRF3. As in the cases of alF5B and EF-G, both switch regions remain in their 'off' state conformation. 
A
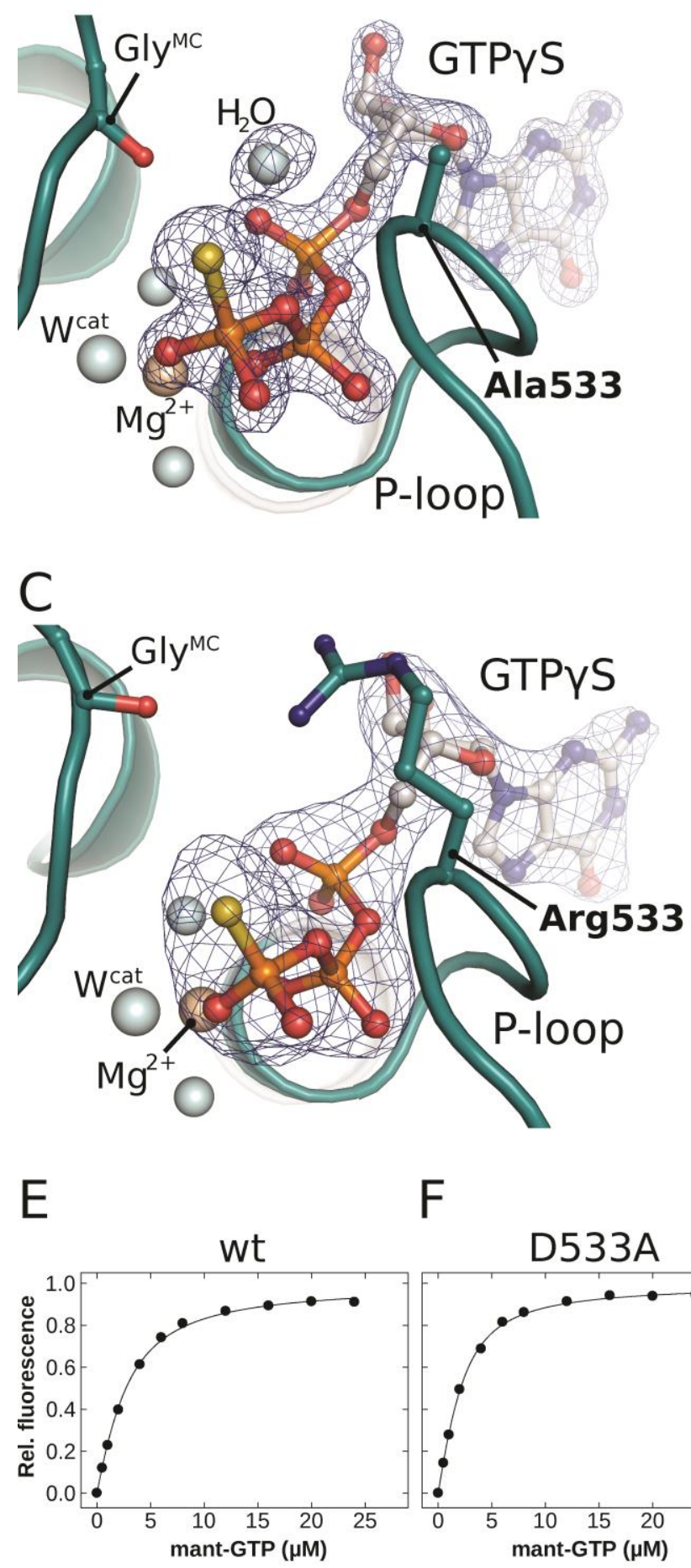

$\mathrm{F}$
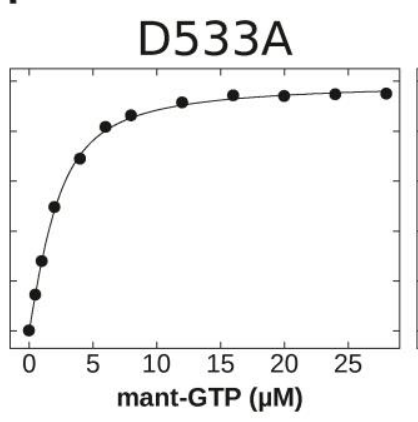

$$
K_{\mathrm{d}}=1.7 \pm 0.09 \mu \mathrm{M} \quad K_{\mathrm{d}}=1.08 \pm 0.05 \mu \mathrm{M}
$$

B
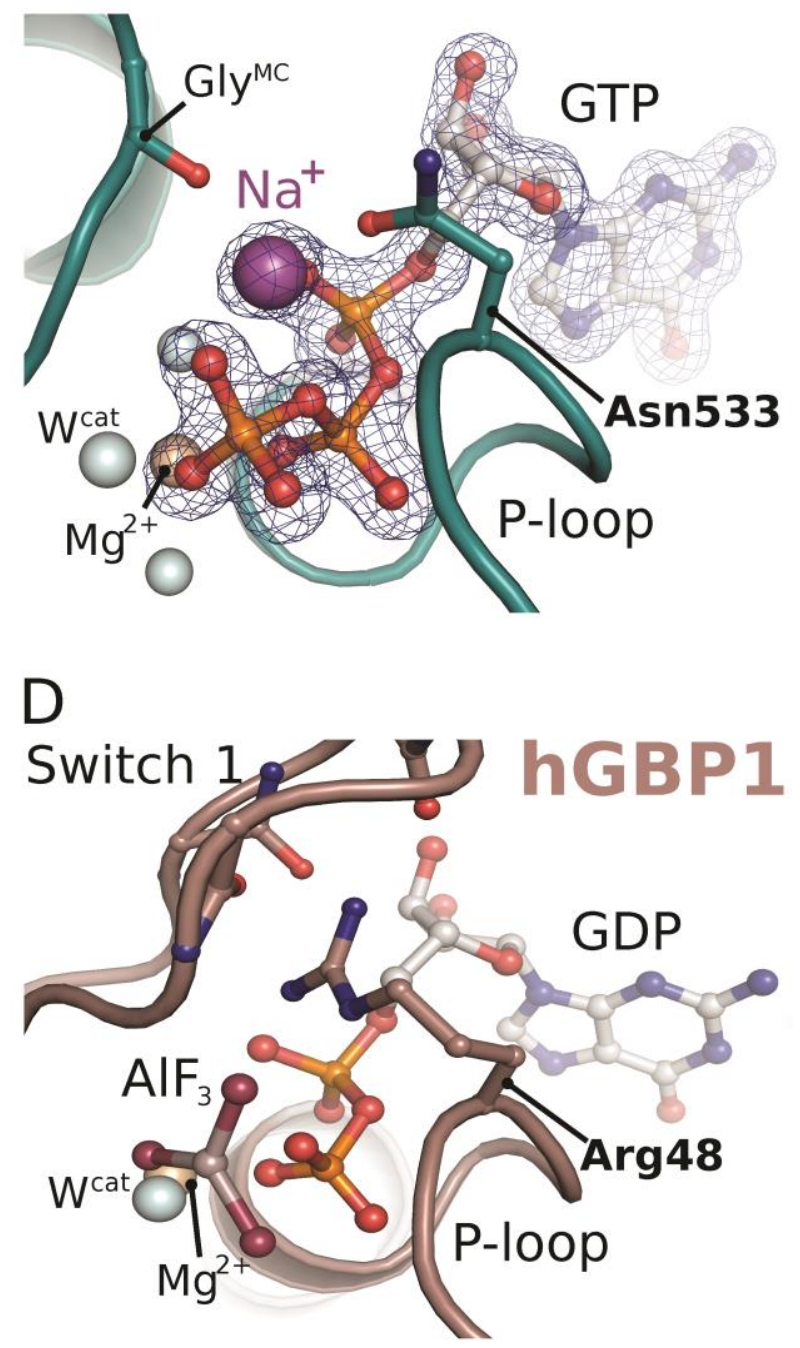

G

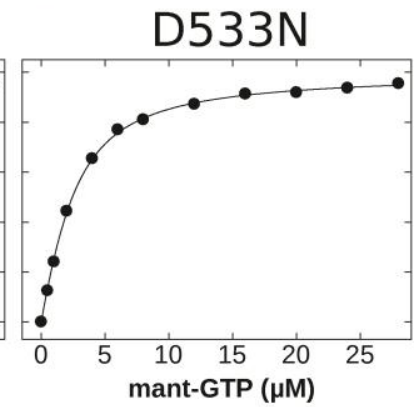

$\mathrm{H}$

Figure E5.Crystalstructures of elF5B Asp ${ }^{\mathrm{MC}}$ (D533) mutants bound to GTP or GTPyS. The coloring is the same as in Figure E1. A) Nucleotide binding pocket of elF5B-D533A bound to GTPYS. Due to the replacement of $\mathrm{Asp}^{\mathrm{MC}}$ by $\mathrm{Ala}$, one of the key ligands and thus the pentagonal coordination sphere for the $\mathrm{M}^{+}$ion is lost. Accordingly, a water molecule $\left(\mathrm{H}_{2} \mathrm{O}\right)$ is now asymmetrically coordinated by four ligands provided by Gly ${ }^{\mathrm{MC}}$ in the P-loop and the GTPYS molecule instead of the $\mathrm{M}^{+}$ion. The coordination distances lie between $2.8 \AA$ (to 
the $\alpha$-phosphate) and $3.2 \AA$ (to Gly ${ }^{\mathrm{MC}}$ ) to the oxygen ligands and $\sim 3.3 \AA$ to the sulfur atom. These distances to the oxygen ligands are significantly too large for a $\mathrm{Na}^{+}$ion ( $2.42 \AA$ [156]). The $F_{o}-F_{c}$ omit map is shown for GTPYS and the water molecule, contoured at $3 \sigma$. B) The nucleotide binding pocket of elF5B-D533N bound to GTP and $\mathrm{Na}^{+}$. Unlike the D533A mutant, Asn in lieu of $\mathrm{Asp}^{\mathrm{MC}}$ still provides the oxygen ligand required for the coordination of the $\mathrm{M}^{+}$ion, analogous to the P-loop Asn in MnmE (see Figure 1D). Consistently, the crystal structure of elF5B-D533N was found to coordinate a $\mathrm{Na}^{+}$ion virtually identically to the wild-type protein (see Figure 1A). The coordination distances in the pentameric coordination shell lie between $2.22 \AA$ (to Gly ${ }^{\mathrm{MC}}$ ) and $2.45 \AA$ (to the $\alpha$-phosphate), consistent with the expected values for a $\mathrm{Na}^{+}$ion [156]. The $F_{o}-F_{c}$ omit map is shown for GTP and the $\mathrm{Na}^{+}$ion, contoured at $3 \sigma$. C) The nucleotide binding pocket of elF5BD533R bound to GTPYS. In both elF5B molecules of the asymmetric unit, the side chain of Arg533 is oriented toward the nucleotide and forms a hydrogen bond to Gly ${ }^{\mathrm{MC}}$ (in one of the elF5B molecules, Arg533 adopts a second (alternative) conformation, in which the guanidino group is oriented away from the nucleotide (not shown)). As expected from the disruption of the coordination sphere, no $\mathrm{M}^{+}$ion is bound in the active site. Moreover, no water molecule seems to be bound in lieu of the $\mathrm{M}^{+}$ion as seen in the D533A mutant (A). Although the guanidino group in this structure does not form a direct contact to the phosphate moieties of GTPYS, it is conceivable that - particularly at low salt concentrations - Arg533 may transiently adopt a conformation similar to that observed for Arg48, the cis-acting arginine-finger, in the GTPase hGBP1 (D) [258], to allow the observed slightly stimulating effect on the intrinsic GTPase activity (see Fig. 2F). The $F_{o}-F_{c}$ omit map is shown for GTPYS, contoured at $3 \sigma$. D) The nucleotide binding pocket of hGBP1 (with P-loop and switch 1 in brown) bound to the transition state mimic GDP/AIF 3 (PDB: 2B92). Several hydrogen bonds to switch 1 stabilize Arg48 in its active conformation, in which the guanidino group forms direct contacts to GDP and $\left.\mathrm{AlF}_{3} . \mathrm{E}-\mathrm{H}\right)$ Steady-state fluorescence measurements for the affinity of wild-type elF5B(517-858) and Asp ${ }^{\mathrm{MC}}$ mutants to mant-GTP. Increasing amounts of mant-GTP were titrated to $2 \mu \mathrm{M}$ wild-type elF5B (E) or the Asp ${ }^{\mathrm{MC}}$ mutants D533A (F), D533N (G) and D553R (H) at $20^{\circ} \mathrm{C}$ in buffer containing $25 \mathrm{mM}$ Tris/ $\mathrm{HCl}(\mathrm{pH}$ 7.5), $200 \mathrm{mM} \mathrm{KCl}, 3 \mathrm{mM} \mathrm{MgCl}$ and $2 \mathrm{mM}$ DTT. Under these conditions, wild-type elF5B(517-858) bound mant-GTP with a $K_{\mathrm{d}}$ of $1.7 \pm 0.09 \mu \mathrm{M}$. A similar value was obtained for the D533N mutant $\left(K_{\mathrm{d}}\right.$ of $1.5 \pm 0.05$ $\mu \mathrm{M})$, while D533A and D533R showed slightly lower $K_{d}$ values of $1.08 \pm 0.05 \mu \mathrm{M}$ and $0.97 \pm 0.06 \mu \mathrm{M}$, respectively. The $K_{\mathrm{d}}$ value for each construct was calculated from three independent equilibrium titrations. 


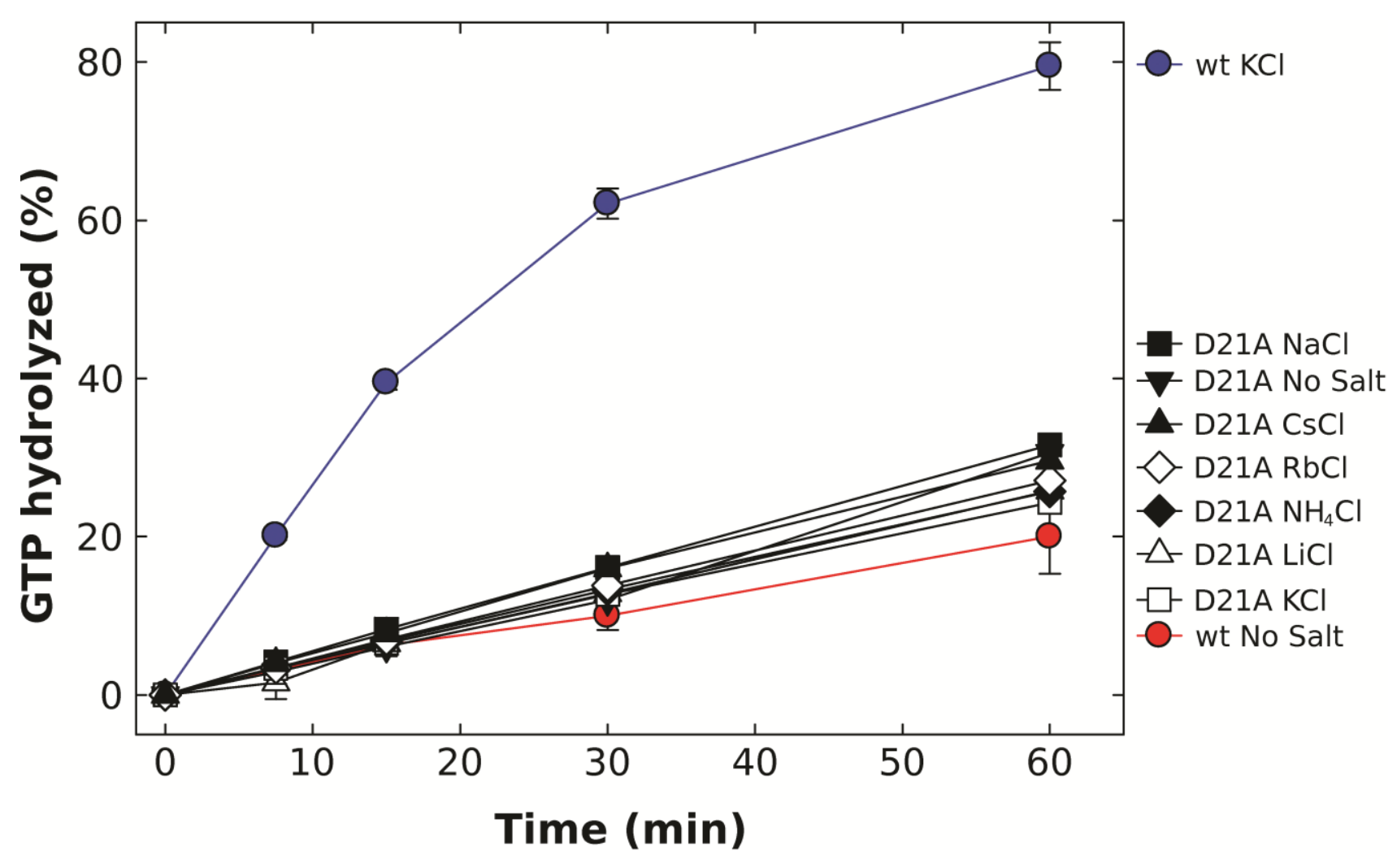

Figure E6. Comparison of the effect of different species of $\mathrm{M}^{+}$ions on the intrinsic GTPase activity of the $E$. coli EF-Tu D21A mutant (black and white) and wild-type EF-Tu (red and blue). The intrinsic GTPase activity was determined in the presence of $200 \mathrm{mM}$ of the indicated salts under single turnover conditions. The order in which the combinations are given on the right corresponds to the relative rates of GTP hydrolysis. Experiments were repeated two to three times; standard deviations are given by error bars (in some cases not visible because they are smaller than the symbol size). 


\section{Expanded ViewTables}

Table E1. Thermodynamic parameters of ctelF5B(517-858) binding to GTPYS, GDPNP and GDP at different temperatures.

\begin{tabular}{lllllll}
\hline Ligand & $\begin{array}{l}\mathrm{T} \\
{\left[{ }^{\circ} \mathrm{C}\right]}\end{array}$ & $\begin{array}{l}\mathrm{K}_{\mathrm{d}} \\
{[\mu \mathrm{M}]}\end{array}$ & $\begin{array}{l}\Delta \mathrm{H} \\
{[\mathrm{kcal} / \mathrm{mol}]}\end{array}$ & $\begin{array}{l}\Delta \mathrm{G} \\
{[\mathrm{kcal} / \mathrm{mol}]}\end{array}$ & $\begin{array}{l}\mathrm{T} \Delta \mathrm{S} \\
{[\mathrm{kcal} / \mathrm{mol}]}\end{array}$ \\
\cline { 2 - 6 } & 10 & 0.58 & -5.06 & -8.06 & 3.0 \\
\cline { 2 - 6 } GTPyS & 20 & 0.67 & -9.67 & -8.27 & -1.4 \\
\cline { 2 - 6 } GTPyS & 25 & 0.82 & -12.93 & -8.83 & -4.1 \\
\cline { 2 - 6 } GTPyS & 30 & 0.92 & -15.81 & -8.41 & -7.4 \\
\hline GTPyS & 10 & 10.67 & -5.89 & -6.44 & 0.55 \\
\hline GDPNP & 15 & 12.3 & -7.31 & -6.48 & -0.83 \\
GDPNP & 20 & 15.47 & -8.09 & -6.49 & -1.6 \\
GDPNP & 25 & 15.0 & -9.58 & -6.68 & -2.9 \\
\hline GDPNP & 30 & 20.8 & -9.67 & -6.47 & -3.2 \\
\hline GDPNP & 10 & 3.37 & -6.94 & -7.09 & 0.15 \\
\hline GDP & 20 & 5.85 & -9.06 & -7.76 & -2.3 \\
GDP & 30 & 10.4 & -11.51 & -6.91 & -4.6 \\
\hline
\end{tabular}

Measurements were performed two to three times.

$\mathrm{K}_{\mathrm{d}}$, dissociation equilibrium constant; calculated as $1 / \mathrm{K}_{\mathrm{a}}$.

$\mathrm{K}_{\mathrm{a}}$, association equilibrium constant; standard deviation did not exceed $\pm 15 \%$.

$\Delta \mathrm{H}$, standard enthalpy change; standard deviation did not exceed $\pm 15 \%$.

$\Delta \mathrm{G}$, Gibbs energy; calculated from equation $\Delta \mathrm{G}=-\mathrm{R} \cdot \mathrm{T} \cdot \ln \mathrm{K}_{\mathrm{a}}$.

$\mathrm{T} \Delta \mathrm{S}$, standard entropy change; calculated from equation $\Delta \mathrm{G}=\Delta \mathrm{H}-\mathrm{T} \Delta \mathrm{S}$. 
Table E2. Crystallization, X-ray data collection and refinement statistics for structures of elF5B(517-858) mutants

\begin{tabular}{|c|c|c|c|}
\hline 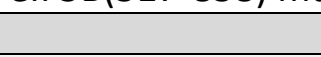 & elF5B(517-858)D533A.GTPyS & elF5B(517-858)D533R.GTPYS & elF5B(517-858)D533N.GTP.Na ${ }^{+}$ \\
\hline \multicolumn{4}{|l|}{ Crystallization } \\
\hline Condition & $\begin{array}{l}100 \mathrm{mM} \text { HEPES (pH 7.3), } \\
15 \% \text { PEG 4000, } 150 \mathrm{mM} \\
\mathrm{NaOAc}\end{array}$ & $\begin{array}{l}100 \mathrm{mM} \text { MES (pH 6.7), } \\
13 \% \text { PEG 8000, } 225 \mathrm{mM} \\
\text { NaOAc }\end{array}$ & $\begin{array}{l}100 \text { mM HEPES (pH 7), 13\% } \\
\text { PEG 4000, } 125 \text { mM NaOAc }\end{array}$ \\
\hline Temperature $\left({ }^{\circ} \mathrm{C}\right)$ & 20 & 20 & 20 \\
\hline \multicolumn{4}{|l|}{ Data Collection } \\
\hline Space Group & $\mathrm{P} 2_{1}$ & $\mathrm{P} 4_{1} 2_{1} 2$ & $\mathrm{P} 2_{1}$ \\
\hline Unit Cell & $\begin{array}{l}a=55.6 \AA \\
b=115.8 \AA \\
c=65.9 \AA \\
\alpha=90^{\circ} \\
\beta=102.3^{\circ} \\
\gamma=90^{\circ}\end{array}$ & $\begin{array}{l}a=115.7 \AA \\
b=115.7 \AA \\
c=119.8 \AA \\
\alpha=90^{\circ} \\
\beta=90^{\circ} \\
\gamma=90^{\circ}\end{array}$ & $\begin{array}{l}a=55.4 \AA \\
b=115.9 \AA \\
c=66.1 \AA \\
\alpha=90^{\circ} \\
\beta=101.4^{\circ} \\
\gamma=90^{\circ}\end{array}$ \\
\hline $\begin{array}{l}\text { Molecules/asym. } \\
\text { unit }\end{array}$ & 2 & 2 & 2 \\
\hline Resolution $(\AA ̊)$ & $1.58(1.68-1.58)$ & $2.75(2.92-2.75)$ & $1.5(1.59-1.5)$ \\
\hline $\begin{array}{l}\text { Observed } \\
\text { reflections }\end{array}$ & $374970(60270)$ & $154138(23582)$ & $542902(84681)$ \\
\hline Unique reflections & $110416(17665)$ & 21704 (3403) & 131458 (20989) \\
\hline Completeness (\%) & $98.8(98.0)$ & $99.8(99.1)$ & $99.2(98.2)$ \\
\hline$<1>/ \sigma$ & $17.7(2.2)$ & $19.35(3.11)$ & $24.4(2.9)$ \\
\hline $\mathrm{R}_{\text {sym }}(\%)$ & $3.9(51.5)$ & $7.8(56.8)$ & $3.0(46.3)$ \\
\hline $\mathrm{CC}(1 / 2)(\%)$ & $99.9(73.9)$ & $99.9(86.1)$ & $100(82.8)$ \\
\hline \multicolumn{4}{|l|}{ Refinement } \\
\hline $\mathrm{R}_{\text {work }}(\%)$ & 16.9 & 20.6 & 15.5 \\
\hline $\mathrm{R}_{\text {free }}(\%)$ & 19.4 & 25.7 & 18.2 \\
\hline \multicolumn{4}{|l|}{$\begin{array}{l}\text { Rmsd from Standard } \\
\text { Stereochemistry }\end{array}$} \\
\hline Bond length $(\AA)$ & 0.012 & 0.005 & 0.014 \\
\hline Bond angles $\left({ }^{\circ}\right)$ & 1.5 & 1.0 & 1.6 \\
\hline \multicolumn{4}{|l|}{$\begin{array}{l}\text { Ramachandran Plot } \\
\text { Statistics }\end{array}$} \\
\hline Most favored (\%) & 98.7 & 98.2 & 98.5 \\
\hline $\begin{array}{l}\text { Allowed regions } \\
\text { (\%) }\end{array}$ & 1.3 & 1.8 & 1.5 \\
\hline $\begin{array}{l}\text { Disallowed regions } \\
\text { (\%) }\end{array}$ & 0 & 0 & 0 \\
\hline
\end{tabular}




\section{Chapter $8 \cdot$ Synopsis and outlook}

Translation is an intriguing problem from two different perspectives: the mechanistic aspect of the translation apparatus as a biochemical process, and the evolutionary aspect of translation as the result of selective historical constellations. Necessarily, both aspects are inseparably connected with each other, with the accumulated mechanistic details being relicts of the former selective constellations under which the translation apparatus emerged. Hence, the problem to understand translation as an evolved mechanism, the relationship between the different stages of the translation process and the emergence of domain-specific variations cannot be derived from sequence-based phylogenetic analysis alone but must be based on an understanding of its structural and functional details, which, moreover, remain an intriguing problem in themselves. In turn, a full understanding of the mechanistic details and the underlying principles that govern translation in extant cells is not possible without simultaneously treating it as an evolutionary problem. This intersection is exemplified by translational GTPases, an ancient protein family with common evolutionary descent, whose members became involved in a variety of steps in the translation process.

The principle aim of this thesis was the structural and functional characterization of elF2 and elF5B, the two translational GTPases involved in eukaryal translation initiation, which, in comparison with their homologs from bacteria and archaea or from other stages of the translation process, provide two disparate examples for the intersection between mechanistic and evolutionary aspects of translation.

The first part of this thesis (chapters 2-4) was dedicated to the structural and functional characterization of the interactions between elF2 and its effector proteins elF5 and elF2B $\varepsilon$, which 
perform essential and opposing roles during the guanine-nucleotide cycle of elF2. On the one hand, a refined model for the intricate interaction network in the elF2.elF5 complex could be derived using a combination of complementary structural and biochemical approaches, which included the first crystal structure of a complex between the elF5-CTD and the elF2 $\beta$-NTT. This model suggests that elF5 uses three distinct binding interfaces with the elF2 complex, two of which are formed by the elF5-CTD that becomes clamped between the elF2 $\beta$-NTT and elF2 $\gamma$ and the third formed between the elF5-NTD and elF2 $\gamma$, which might become relevant at overlapping stages of the initiation process (chapter 2). On the other hand, an analogous interaction model could be derived for the complex between elF2 and the catalytic subdomain of its GEF elF2BE (chapter 3), which provided insight into the mechanism by which the isolated elF2B $\varepsilon$ (outside the structural context of the eIF2B holo-complex) binds its substrate to promote GDP release from elF2 and its exchange against GTP. The comparison between the two proposed binding models for the interactions between elF2 and its effector proteins finally gave rise to the hypothesis that the structurally similar C-terminal domains of elF5 and elF2B $\varepsilon$ share a common evolutionary descent from an ancestral form which has already been engaged in elF2 binding and the modulation of its nucleotide-binding properties (chapter 3).

Both binding models are currently based on a number of complementary biochemical and structural approaches used in this work as well as previous biochemical and genetic studies. However, several conclusions or suggestions presented in the two chapters only form the vantage point for further studies. In this context, interesting future aims would be the model-based introduction of additional Bpa-sites in the various proteins and the identification of additional crosslinking sites (e.g. the crosslinking target of elF2BE(524-712)N569Bpa on elF2 $\alpha$ ) or a structural confirmation of the proposal that the DWEAR-motif forms part of the C-terminal domain of elF5 by solving a crystal structure with helix $\alpha 1$ accommodated in the hydrophobic cleft as suggested in figure 5 of chapter 2 . The ultimate goal, however, remains the structural analysis of the entire elF2-elF5 and elF2·elF2B $\varepsilon$ complexes either in isolation or in the structural contexts of pre-ICs or the eIF2-elF2B complex by means of X-ray crystallography or cryo-EM, in order to correlate biochemical and genetic data with structural information. Particularly interesting would be the 
structure of a $43 \mathrm{~S}$ pre-IC containing the TC as well as elF5 to test the hypotheses presented in chapter 2 concerning the location and involvement of its C-terminal domain.

Chapter 4 presented the vantage point to solve the problem of elF2B function with the in vitro reconstitution of the elF $2 \mathrm{~B} \alpha \beta \delta$ regulatory subcomplex and the determination of the first crystal structures of eIF2B $\beta$ and elF2B $\delta$ from the thermophilic fungus $C$. thermophilum. Unlike the usually used S. cerevisiae system, all three subunits can be purified recombinantly in large amounts and like in other known cases, the thermophilic origin of the source organism appears to result in structural properties of the proteins that are favorable for crystallization. It would thus be an interesting scheme for future studies to obtain a high-resolution structural model for the elF2B regulatory subcomplex. Necessarily, the next step would be the reconstitution of the elF2B holocomplex including eIF2B $\varepsilon$ and elF2By, which is, however, currently hampered by the insolubility of elF2B $\gamma$ when expressed recombinantly in E. coli.

The fist two chapters of the second part of the thesis (chapters 5 and 6) were dedicated to the structural and functional characterization of elF5B. Specific aspects of the findings for elF5B subsequently served as the basis to develop the hypothesis of universal monovalent cation dependency among trGTPases, which was presented in chapter 7.

The initial question at hand was the specific function of elF5B in the process of subunit joining and its relation to the functional characteristics of the archaeal and bacterial orthologs. The starting point for this study was the observation that previously proposed structural and functional models for elF5B were incompatible with the classical concepts of $\mathrm{G}$ protein function, at odds with the universal conservation of the nucleotide-binding motifs within a/elF5B/IF2 homologs and finally incompatible with most available biochemical and structural data. The structural and biochemical studies presented in chapter 5 revealed that the activation mechanism of the elF5B G domain for ribosomal subunit joining indeed follows the classical paradigms of G protein function as molecular switches. Moreover, this mechanism was found to be coupled to a so far unprecedented mode of overall activation of elF5B, called domain release mechanism, which provides elF5B·GTP with the means to discriminate against erroneously assembled pre-ICs by the 
requirement of stabilizing interactions with the unique $P$ site bound initiator tRNA (chapters 6 ) to promote the formation of an elongation competent 80S IC (chapter 5).

Chapter 7 was finally dedicated to the hypothesis of $\mathrm{M}^{+}$ion-dependent conformational switching and GTP hydrolysis among trGTPases. This hypothesis is so far based on biochemical and structural studies on the canonical trGTPases elF5B, aEF1A and EF-Tu, the common descent of trGTPases as reflected in the high degree of sequence and structural homology in their G domains, as well as the immense explanatory power of $\mathrm{M}^{+}$dependency for previously unresolved problems concerning the function of trGTPases. It is clear however that the claim of universality for this hypothesis, although in agreement with the available data, will have to be confirmed by structural and biochemical studies on the various trGTPases it has been proposed for. In this context, a number of additional projects were initiated in the course of this thesis, which included attempts to crystallize the GTP/GTPYS-bound forms of T. thermophilus IF2 and EF-Tu, alF2 $\gamma$ and aEF2 from $S$. solfataricus, as well as eRF3 from C. thermophilum either in isolation or in a complex with eRF1 (data not shown). Up to now, none of the desired structures could be determined. However, for several of the projects crystallization conditions could be identified as promising starting points for future studies. Moreover, future work will have to comprise mutational analyses, in particular of the key $\mathrm{P}$ loop aspartate $\left(\mathrm{Asp}^{\mathrm{MC}}\right.$ ) in terms of its influence on the intrinsic and ribosome-dependent GTPase activity of the various trGTPases. However, as for the work concerning elF2 function, the ultimate goal would be to provide high-resolution structural evidence for $\mathrm{M}^{+}$coordination by a trGTPase in the context of the ribosome, which, due to the limitations of nonhydrolyzable GTP analogs as discussed in chapter 7 , would require the usage of GDP in combination with transition state mimics such as fluoroaluminates or berylliumfluorides to trap the $\mathrm{M}^{+}$-bound state. 


\section{References}

1. Wei, Z., et al., Crystal structure of the C-terminal domain of S.cerevisiae elF5. J Mol Biol, 2006. 359(1): p. 1-9.

2. Ramakrishnan, V., Ribosome structure and the mechanism of translation. Cell, 2002. 108(4): p. 55772.

3. Ban, N., et al., The complete atomic structure of the large ribosomal subunit at $2.4 \mathrm{~A}$ resolution. Science, 2000. 289(5481): p. 905-20.

4. Wimberly, B.T., et al., Structure of the 30 S ribosomal subunit. Nature, 2000. 407(6802): p. 327-39.

5. Ben-Shem, A., et al., The structure of the eukaryotic ribosome at 3.0 A resolution. Science, 2011. 334(6062): p. 1524-9.

6. Klinge, S., et al., Atomic structures of the eukaryotic ribosome. Trends Biochem Sci, 2012. 37(5): p. 189-98.

7. Schmeing, T.M. and V. Ramakrishnan, What recent ribosome structures have revealed about the mechanism of translation. Nature, 2009. 461(7268): p. 1234-42.

8. Voorhees, R.M. and V. Ramakrishnan, Structural basis of the translational elongation cycle. Annu Rev Biochem, 2013. 82: p. 203-36.

9. Marintchev, A. and G. Wagner, Translation initiation: structures, mechanisms and evolution. Q Rev Biophys, 2004. 37(3-4): p. 197-284.

10. Rodnina, M.V. and W. Wintermeyer, Recent mechanistic insights into eukaryotic ribosomes. Curr Opin Cell Biol, 2009. 21(3): p. 435-43.

11. Dever, T.E. and R. Green, The elongation, termination, and recycling phases of translation in eukaryotes. Cold Spring Harb Perspect Biol, 2012. 4(7): p. a013706.

12. Hinnebusch, A.G. and J.R. Lorsch, The mechanism of eukaryotic translation initiation: new insights and challenges. Cold Spring Harb Perspect Biol, 2012. 4(10).

13. Kozak, M., Initiation of translation in prokaryotes and eukaryotes. Gene, 1999. 234(2): p. 187-208.

14. Shine, J. and L. Dalgarno, The 3'-terminal sequence of Escherichia coli 16S ribosomal RNA: complementarity to nonsense triplets and ribosome binding sites. Proc Natl Acad Sci U S A, 1974. 71(4): p. 1342-6.

15. Gualerzi, C.O., et al., Initiation factors in the early events of mRNA translation in bacteria. Cold Spring Harb Symp Quant Biol, 2001. 66: p. 363-76.

16. Jackson, R.J., C.U. Hellen, and T.V. Pestova, The mechanism of eukaryotic translation initiation and principles of its regulation. Nat Rev Mol Cell Biol, 2010. 11(2): p. 113-27.

17. Biou, V., F. Shu, and V. Ramakrishnan, X-ray crystallography shows that translational initiation factor IF3 consists of two compact alpha/beta domains linked by an alpha-helix. EMBO J, 1995. 14(16): p. 4056-64.

18. Fletcher, C.M., et al., Structure and interactions of the translation initiation factor elF1. EMBO J, 1999. 18(9): p. 2631-7.

19. Weisser, M., et al., The crystal structure of the eukaryotic 40S ribosomal subunit in complex with elF1 and elF1A. Nat Struct Mol Biol, 2013. 20(8): p. 1015-7.

20. Lomakin, I.B. and T.A. Steitz, The initiation of mammalian protein synthesis and mRNA scanning mechanism. Nature, 2013. 500(7462): p. 307-11. 
21. Nanda, J.S., et al., Coordinated movements of eukaryotic translation initiation factors elF1, elF1A, and elF5 trigger phosphate release from eIF2 in response to start codon recognition by the ribosomal preinitiation complex. J Biol Chem, 2013. 288(8): p. 5316-29.

22. Leipe, D.D., et al., Classification and evolution of P-loop GTPases and related ATPases. J Mol Biol, 2002. 317(1): p. 41-72.

23. Vetter, I.R. and A. Wittinghofer, The guanine nucleotide-binding switch in three dimensions. Science, 2001. 294(5545): p. 1299-304.

24. Bourne, H.R., D.A. Sanders, and F. McCormick, The GTPase superfamily: conserved structure and molecular mechanism. Nature, 1991. 349(6305): p. 117-27.

25. Bos, J.L., H. Rehmann, and A. Wittinghofer, GEFs and GAPs: critical elements in the control of small $G$ proteins. Cell, 2007. 129(5): p. 865-77.

26. Margus, T., M. Remm, and T. Tenson, Phylogenetic distribution of translational GTPases in bacteria. BMC Genomics, 2007. 8: p. 15.

27. Rodnina, M.V., et al., GTPases mechanisms and functions of translation factors on the ribosome. Biol Chem, 2000. 381(5-6): p. 377-87.

28. Kyrpides, N.C. and C.R. Woese, Archaeal translation initiation revisited: the initiation factor 2 and eukaryotic initiation factor 2B alpha-beta-delta subunit families. Proc Natl Acad Sci U S A, 1998. 95(7): p. 3726-30.

29. Yatime, L., et al., Structure-function relationships of the intact alF2alpha subunit from the archaeon Pyrococcus abyssi. Biochemistry, 2005. 44(24): p. 8749-56.

30. Ito, T., A. Marintchev, and G. Wagner, Solution structure of human initiation factor elF2alpha reveals homology to the elongation factor eEF1B. Structure, 2004. 12(9): p. 1693-704.

31. Schmitt, E., M. Naveau, and Y. Mechulam, Eukaryotic and archaeal translation initiation factor 2: a heterotrimeric tRNA carrier. FEBS Lett, 2010. 584(2): p. 405-12.

32. Naveau, M., et al., tRNA binding properties of eukaryotic translation initiation factor 2 from Encephalitozoon cuniculi. Biochemistry, 2010. 49(40): p. 8680-8.

33. Yatime, L., et al., Structural switch of the gamma subunit in an archaeal alF2 alpha gamma heterodimer. Structure, 2006. 14(1): p. 119-28.

34. Sokabe, M., et al., Structure of archaeal translational initiation factor 2 betagamma-GDP reveals significant conformational change of the beta-subunit and switch 1 region. Proc Natl Acad Sci U S A, 2006. 103(35): p. 13016-21.

35. Asano, K., et al., Conserved bipartite motifs in yeast elF5 and elF2Bepsilon, GTPase-activating and GDP-GTP exchange factors in translation initiation, mediate binding to their common substrate elF2. EMBO J, 1999. 18(6): p. 1673-88.

36. Das, S., et al., Specific interaction of eukaryotic translation initiation factor 5 (elF5) with the betasubunit of elF2. J Biol Chem, 1997. 272(50): p. 31712-8.

37. Schmitt, E., S. Blanquet, and Y. Mechulam, The large subunit of initiation factor alF2 is a close structural homologue of elongation factors. EMBO J, 2002. 21(7): p. 1821-32.

38. Polekhina, G., et al., Helix unwinding in the effector region of elongation factor EF-Tu-GDP. Structure, 1996. 4(10): p. 1141-51.

39. Abel, K., et al., An alpha to beta conformational switch in EF-Tu. Structure, 1996. 4(10): p. 1153-9.

40. Erickson, F.L., et al., Functional analysis of homologs of translation initiation factor 2gamma in yeast. Mol Gen Genet, 1997. 253(6): p. 711-9.

41. Naveau, M., et al., Roles of yeast elF2alpha and elF2beta subunits in the binding of the initiator methionyl-tRNA. Nucleic Acids Res, 2013. 41(2): p. 1047-57.

42. Schmitt, E., et al., Structure of the ternary initiation complex alF2-GDPNP-methionylated initiator tRNA. Nat Struct Mol Biol, 2012. 19(4): p. 450-4. 
43. Yatime, L., et al., Structure of an archaeal heterotrimeric initiation factor 2 reveals a nucleotide state between the GTP and the GDP states. Proc Natl Acad Sci U S A, 2007. 104(47): p. 18445-50.

44. Kapp, L.D. and J.R. Lorsch, GTP-dependent recognition of the methionine moiety on initiator tRNA by translation factor elF2. J Mol Biol, 2004. 335(4): p. 923-36.

45. Erickson, F.L. and E.M. Hannig, Ligand interactions with eukaryotic translation initiation factor 2: role of the gamma-subunit. EMBO J, 1996. 15(22): p. 6311-20.

46. Kapp, L.D., S.E. Kolitz, and J.R. Lorsch, Yeast initiator tRNA identity elements cooperate to influence multiple steps of translation initiation. RNA, 2006. 12(5): p. 751-64.

47. Farruggio, D., et al., The A1 x U72 base pair conserved in eukaryotic initiator tRNAs is important specifically for binding to the eukaryotic translation initiation factor elF2. Mol Cell Biol, 1996. 16(8): p. 4248-56.

48. Nissen, P., et al., Crystal structure of the ternary complex of Phe-tRNAPhe, EF-Tu, and a GTP analog. Science, 1995. 270(5241): p. 1464-72.

49. Shin, B.S., et al., Initiation factor elF2gamma promotes elF2-GTP-Met-tRNAi(Met) ternary complex binding to the 40 S ribosome. Nat Struct Mol Biol, 2011. 18(11): p. 1227-34.

50. Hashem, Y., et al., Structure of the mammalian ribosomal 435 preinitiation complex bound to the scanning factor DHX29. Cell, 2013. 153(5): p. 1108-19.

51. Conte, M.R., et al., Structure of the eukaryotic initiation factor (elF) 5 reveals a fold common to several translation factors. Biochemistry, 2006. 45(14): p. 4550-8.

52. Das, S., R. Ghosh, and U. Maitra, Eukaryotic translation initiation factor 5 functions as a GTPaseactivating protein. J Biol Chem, 2001. 276(9): p. 6720-6.

53. Paulin, F.E., et al., Eukaryotic translation initiation factor 5 (elF5) acts as a classical GTPase-activator protein. Curr Biol, 2001. 11(1): p. 55-9.

54. Bieniossek, C., et al., The crystal structure of the carboxy-terminal domain of human translation initiation factor elF5. J Mol Biol, 2006. 360(2): p. 457-65.

55. Singh, C.R., Y. Yamamoto, and K. Asano, Physical association of eukaryotic initiation factor (elF) 5 carboxyl-terminal domain with the lysine-rich elF2beta segment strongly enhances its binding to elF3. J Biol Chem, 2004. 279(48): p. 49644-55.

56. Asano, K., et al., Multiple roles for the C-terminal domain of elF5 in translation initiation complex assembly and GTPase activation. EMBO J, 2001. 20(9): p. 2326-37.

57. Yamamoto, Y., et al., The eukaryotic initiation factor (eIF) 5 HEAT domain mediates multifactor assembly and scanning with distinct interfaces to elF1, elF2, elF3, and elF4G. Proc Natl Acad Sci U S A, 2005. 102(45): p. 16164-9.

58. Singh, C.R., et al., Eukaryotic translation initiation factor 5 is critical for integrity of the scanning preinitiation complex and accurate control of GCN4 translation. Mol Cell Biol, 2005. 25(13): p. 548091.

59. Asano, K., et al., A multifactor complex of eukaryotic initiation factors, elF1, elF2, elF3, elF5, and initiator tRNA(Met) is an important translation initiation intermediate in vivo. Genes Dev, 2000. 14(19): p. 2534-46.

60. Dennis, M.D., M.D. Person, and K.S. Browning, Phosphorylation of plant translation initiation factors by CK2 enhances the in vitro interaction of multifactor complex components. J Biol Chem, 2009. 284(31): p. 20615-28.

61. Sokabe, M., C.S. Fraser, and J.W. Hershey, The human translation initiation multi-factor complex promotes methionyl-tRNAi binding to the 40 S ribosomal subunit. Nucleic Acids Res, 2011. 40(2): p. 905-13.

62. Valasek, L., et al., Interactions of eukaryotic translation initiation factor 3 (elF3) subunit NIP1/c with elF1 and elF5 promote preinitiation complex assembly and regulate start codon selection. Mol Cell Biol, 2004. 24(21): p. 9437-55. 
63. Luna, R.E., et al., The C-terminal domain of eukaryotic initiation factor 5 promotes start codon recognition by its dynamic interplay with elF1 and elF2beta. Cell Rep, 2012. 1(6): p. 689-702.

64. Algire, M.A., D. Maag, and J.R. Lorsch, Pi release from elF2, not GTP hydrolysis, is the step controlled by start-site selection during eukaryotic translation initiation. Mol Cell, 2005. 20(2): p. 251-62.

65. Alone, P.V. and T.E. Dever, Direct binding of translation initiation factor elF2gamma-G domain to its GTPase-activating and GDP-GTP exchange factors elF5 and elF2B epsilon. J Biol Chem, 2006. 281(18): p. 12636-44.

66. Hashimoto, N.N., L.S. Carnevalli, and B.A. Castilho, Translation initiation at non-AUG codons mediated by weakened association of eukaryotic initiation factor (elF) 2 subunits. Biochem J, 2002. 367(Pt 2): p. 359-68.

67. Huang, H.K., et al., GTP hydrolysis controls stringent selection of the AUG start codon during translation initiation in Saccharomyces cerevisiae. Genes Dev, 1997. 11(18): p. 2396-413.

68. Singh, C.R., et al., An elF5/elF2 complex antagonizes guanine nucleotide exchange by elF2B during translation initiation. EMBO J, 2006. 25(19): p. 4537-46.

69. Jennings, M.D. and G.D. Pavitt, elF5 has GDI activity necessary for translational control by elF2 phosphorylation. Nature, 2010. 465(7296): p. 378-81.

70. Gomez, E., S.S. Mohammad, and G.D. Pavitt, Characterization of the minimal catalytic domain within elF2B: the guanine-nucleotide exchange factor for translation initiation. EMBO J, 2002. 21(19): p. 5292-301.

71. Gomez, E. and G.D. Pavitt, Identification of domains and residues within the epsilon subunit of eukaryotic translation initiation factor $2 B$ (elF2Bepsilon) required for guanine nucleotide exchange reveals a novel activation function promoted by elF2B complex formation. Mol Cell Biol, 2000. 20(11): p. 3965-76.

72. Wortham, N.C., et al., Analysis of the subunit organization of the elF2B complex reveals new insights into its structure and regulation. FASEB J, 2014. 28(5): p. 2225-37.

73. Gordiyenko, Y., et al., elF2B is a decameric guanine nucleotide exchange factor with $a$ gamma2epsilon2 tetrameric core. Nat Commun, 2014. 5: p. 3902.

74. Boesen, T., et al., Structure of the catalytic fragment of translation initiation factor $2 B$ and identification of a critically important catalytic residue. J Biol Chem, 2004. 279(11): p. 10584-92.

75. Mohammad-Qureshi, S.S., et al., Critical contacts between the eukaryotic initiation factor 2B (elF2B) catalytic domain and both elF2beta and -2gamma mediate guanine nucleotide exchange. Mol Cell Biol, 2007. 27(14): p. 5225-34.

76. Mohammad-Qureshi, S.S., M.D. Jennings, and G.D. Pavitt, Clues to the mechanism of action of elF2B, the guanine-nucleotide-exchange factor for translation initiation. Biochem Soc Trans, 2008. 36(Pt 4): p. 658-64.

77. Dev, K., et al., The beta/Gcd7 subunit of eukaryotic translation initiation factor $2 B$ (elF2B), a guanine nucleotide exchange factor, is crucial for binding elF2 in vivo. Mol Cell Biol, 2010. 30(21): p. 5218-33.

78. Hinnebusch, A.G., Gene-specific translational control of the yeast GCN4 gene by phosphorylation of eukaryotic initiation factor 2. Mol Microbiol, 1993. 10(2): p. 215-23.

79. Sonenberg, N. and A.G. Hinnebusch, Regulation of translation initiation in eukaryotes: mechanisms and biological targets. Cell, 2009. 136(4): p. 731-45.

80. Hinnebusch, A.G., Translational regulation of GCN4 and the general amino acid control of yeast. Annu Rev Microbiol, 2005. 59: p. 407-50.

81. Kyrpides, N.C. and C.R. Woese, Universally conserved translation initiation factors. Proc Natl Acad Sci U S A, 1998. 95(1): p. 224-8.

82. Eiler, D., et al., Initiation factor 2 crystal structure reveals a different domain organization from eukaryotic initiation factor $5 B$ and mechanism among translational GTPases. Proc Natl Acad Sci U S A, 2013. 
83. Simonetti, A., et al., Structure of the protein core of translation initiation factor 2 in apo, GTP-bound and GDP-bound forms. Acta Crystallogr D Biol Crystallogr, 2013. 69(Pt 6): p. 925-33.

84. Roll-Mecak, A., et al., X-Ray structures of the universal translation initiation factor IF2/elF5B: conformational changes on GDP and GTP binding. Cell, 2000. 103(5): p. 781-92.

85. Shin, B.S., et al., Uncoupling of initiation factor elF5B/IF2 GTPase and translational activities by mutations that lower ribosome affinity. Cell, 2002. 111(7): p. 1015-25.

86. Marintchev, A., et al., Mapping the binding interface between human eukaryotic initiation factors $1 \mathrm{~A}$ and 5B: a new interaction between old partners. Proc Natl Acad Sci U S A, 2003. 100(4): p. 1535-40.

87. Acker, M.G., et al., Interaction between eukaryotic initiation factors $1 A$ and $5 B$ is required for efficient ribosomal subunit joining. J Biol Chem, 2006. 281(13): p. 8469-75.

88. Fringer, J.M., et al., Coupled release of eukaryotic translation initiation factors $5 B$ and $1 A$ from $80 S$ ribosomes following subunit joining. Mol Cell Biol, 2007. 27(6): p. 2384-97.

89. Olsen, D.S., et al., Domains of elF1A that mediate binding to elF2, elF3 and elF5B and promote ternary complex recruitment in vivo. EMBO J, 2003. 22(2): p. 193-204.

90. Unbehaun, A., et al., Position of eukaryotic initiation factor elF5B on the 80 S ribosome mapped by directed hydroxyl radical probing. EMBO J, 2007. 26(13): p. 3109-23.

91. Fernandez, I.S., et al., Molecular architecture of a eukaryotic translational initiation complex. Science, 2013. 342(6160): p. 1240585.

92. Karaskova, M., et al., Functional characterization of the role of the $\mathrm{N}$-terminal domain of the $\mathrm{c} / \mathrm{Nip} 1$ subunit of eukaryotic initiation factor 3 (elF3) in AUG recognition. J Biol Chem, 2012. 287(34): p. 28420-34.

93. Das, S. and U. Maitra, Mutational analysis of mammalian translation initiation factor 5 (elF5): role of interaction between the beta subunit of elF2 and elF5 in elF5 function in vitro and in vivo. Mol Cell Biol, 2000. 20(11): p. 3942-50.

94. Ryu, Y. and P.G. Schultz, Efficient incorporation of unnatural amino acids into proteins in Escherichia coli. Nat Methods, 2006. 3(4): p. 263-5.

95. Kozakov, D., et al., PIPER: an FFT-based protein docking program with pairwise potentials. Proteins, 2006. 65(2): p. 392-406.

96. Kozakov, D., et al., Achieving reliability and high accuracy in automated protein docking: ClusPro, PIPER, SDU, and stability analysis in CAPRI rounds 13-19. Proteins, 2010. 78(15): p. 3124-30.

97. Acker, M.G., et al., Reconstitution of yeast translation initiation. Methods Enzymol, 2007. 430: $p$. 111-45.

98. Gromadski, K.B., H.J. Wieden, and M.V. Rodnina, Kinetic mechanism of elongation factor Tscatalyzed nucleotide exchange in elongation factor Tu. Biochemistry, 2002. 41(1): p. 162-9.

99. Wagner, A., et al., Interaction of guanosine nucleotides and their analogs with elongation factor Tu from Thermus thermophilus. Biochemistry, 1995. 34(39): p. 12535-42.

100. Lenzen, C., R.H. Cool, and A. Wittinghofer, Analysis of intrinsic and CDC25-stimulated guanine nucleotide exchange of p21ras-nucleotide complexes by fluorescence measurements. Methods Enzymol, 1995. 255: p. 95-109.

101. Cherfils, J. and M. Zeghouf, Regulation of small GTPases by GEFs, GAPs, and GDIs. Physiol Rev, 2013. 93(1): p. 269-309.

102. Gotze, M., et al., StavroX--a software for analyzing crosslinked products in protein interaction studies. J Am Soc Mass Spectrom, 2012. 23(1): p. 76-87.

103. Luna, R.E., et al., The interaction between eukaryotic initiation factor $1 A$ and elF5 retains elF1 within scanning preinitiation complexes. Biochemistry, 2013. 52(52): p. 9510-8.

104. Reibarkh, M., et al., Eukaryotic initiation factor (elF) 1 carries two distinct elF5-binding faces important for multifactor assembly and AUG selection. J Biol Chem, 2008. 283(2): p. 1094-103. 
105. Jennings, M.D., et al., elF2B promotes elF5 dissociation from elF2*GDP to facilitate guanine nucleotide exchange for translation initiation. Genes Dev, 2014. 27(24): p. 2696-707.

106. Scheffzek, K., et al., The Ras-RasGAP complex: structural basis for GTPase activation and its loss in oncogenic Ras mutants. Science, 1997. 277(5324): p. 333-8.

107. Grizot, S., et al., Crystal structure of the Rac1-RhoGDI complex involved in nadph oxidase activation. Biochemistry, 2001. 40(34): p. 10007-13.

108. Kimple, R.J., et al., Structural determinants for GoLoco-induced inhibition of nucleotide release by Galpha subunits. Nature, 2002. 416(6883): p. 878-81.

109. Willard, F.S., R.J. Kimple, and D.P. Siderovski, Return of the GDI: the GoLoco motif in cell division. Annu Rev Biochem, 2004. 73: p. 925-51.

110. Hoffman, G.R., N. Nassar, and R.A. Cerione, Structure of the Rho family GTP-binding protein Cdc42 in complex with the multifunctional regulator RhoGDI. Cell, 2000. 100(3): p. 345-56.

111. Gai, Z., et al., The binding mechanism of elF2beta with its partner proteins, elF5 and elF2Bepsilon. Biochem Biophys Res Commun, 2012. 423(3): p. 515-9.

112. Pavitt, G.D., et al., elF2 independently binds two distinct elF2B subcomplexes that catalyze and regulate guanine-nucleotide exchange. Genes Dev, 1998. 12(4): p. 514-26.

113. Mueller, U., et al., Facilities for macromolecular crystallography at the Helmholtz-Zentrum Berlin. J Synchrotron Radiat, 2012. 19(Pt 3): p. 442-9.

114. Kabsch, W., Xds. Acta Crystallogr D Biol Crystallogr, 2010. 66(Pt 2): p. 125-32.

115. McCoy, A.J., et al., Phaser crystallographic software. J Appl Crystallogr, 2007. 40(Pt 4): p. 658-674.

116. Emsley, P., et al., Features and development of Coot. Acta Crystallogr D Biol Crystallogr, 2010. 66(Pt 4): p. 486-501.

117. Adams, P.D., et al., PHENIX: a comprehensive Python-based system for macromolecular structure solution. Acta Crystallogr D Biol Crystallogr, 2010. 66(Pt 2): p. 213-21.

118. Fechter, P., et al., Ribozyme processed tRNA transcripts with unfriendly internal promoter for T7 RNA polymerase: production and activity. FEBS Lett, 1998. 436(1): p. 99-103.

119. Kao, C.C., P. Singh, and D.J. Ecker, De novo initiation of viral RNA-dependent RNA synthesis. Virology, 2001. 287(2): p. 251-60.

120. Shevchenko, A., et al., Mass spectrometric sequencing of proteins silver-stained polyacrylamide gels. Anal Chem, 1996. 68(5): p. 850-8.

121. Levin, D.H., D. Kyner, and G. Acs, Protein initiation in eukaryotes: formation and function of a ternary complex composed of a partially purified ribosomal factor, methionyl transfer RNA, and guanosine triphosphate. Proc Natl Acad Sci U S A, 1973. 70(1): p. 41-5.

122. Barrieux, A. and M.G. Rosenfeld, Characterization of GTP-dependent Met-tRNAf binding protein. J Biol Chem, 1977. 252(11): p. 3843-7.

123. Krishnamoorthy, T., et al., Tight binding of the phosphorylated alpha subunit of initiation factor 2 (elF2alpha) to the regulatory subunits of guanine nucleotide exchange factor elF2B is required for inhibition of translation initiation. Mol Cell Biol, 2001. 21(15): p. 5018-30.

124. Sudhakar, A., et al., Phosphorylation of serine 51 in initiation factor 2 alpha (elF2 alpha) promotes complex formation between eIF2 alpha(P) and eIF2B and causes inhibition in the guanine nucleotide exchange activity of elF2B. Biochemistry, 2000. 39(42): p. 12929-38.

125. Panniers, R., A.G. Rowlands, and E.C. Henshaw, The effect of $\mathrm{Mg} 2+$ and guanine nucleotide exchange factor on the binding of guanine nucleotides to eukaryotic initiation factor 2. J Biol Chem, 1988. 263(12): p. 5519-25.

126. Andersen, G.R., et al., Crystal structures of nucleotide exchange intermediates in the eEF1AeEF1Balpha complex. Nat Struct Biol, 2001. 8(6): p. 531-4.

127. Kawashima, T., et al., The structure of the Escherichia coli EF-Tu.EF-Ts complex at 2.5 A resolution. Nature, 1996. 379(6565): p. 511-8. 
128. Rossman, K.L., et al., A crystallographic view of interactions between Dbs and Cdc42: PH domainassisted guanine nucleotide exchange. EMBO J, 2002. 21(6): p. 1315-26.

129. Boriack-Sjodin, P.A., et al., The structural basis of the activation of Ras by Sos. Nature, 1998. 394(6691): p. 337-43.

130. Schummer, T., K.B. Gromadski, and M.V. Rodnina, Mechanism of EF-Ts-catalyzed guanine nucleotide exchange in EF-Tu: contribution of interactions mediated by helix B of EF-Tu. Biochemistry, 2007. 46(17): p. 4977-84.

131. Zavialov, A.V., R.H. Buckingham, and M. Ehrenberg, A posttermination ribosomal complex is the guanine nucleotide exchange factor for peptide release factor RF3. Cell, 2001. 107(1): p. 115-24.

132. Pavitt, G.D., elF2B, a mediator of general and gene-specific translational control. Biochem Soc Trans, 2005. 33(Pt 6): p. 1487-92.

133. Wang, X., et al., Identification of residues that underpin interactions within the eukaryotic initiation factor (elF2) 2B complex. J Biol Chem, 2012. 287(11): p. 8263-74.

134. Reid, P.J., S.S. Mohammad-Qureshi, and G.D. Pavitt, Identification of intersubunit domain interactions within eukaryotic initiation factor (elF) $2 B$, the nucleotide exchange factor for translation initiation. J Biol Chem, 2012. 287(11): p. 8275-85.

135. Pavitt, G.D., W. Yang, and A.G. Hinnebusch, Homologous segments in three subunits of the guanine nucleotide exchange factor elF2B mediate translational regulation by phosphorylation of elF2. Mol Cell Biol, 1997. 17(3): p. 1298-313.

136. Price, N.T., et al., elF2B, the guanine nucleotide-exchange factor for eukaryotic initiation factor 2. Sequence conservation between the alpha, beta and delta subunits of elF2B from mammals and yeast. Biochem J, 1996. 318 ( Pt 2): p. 637-43.

137. Craddock, B.L. and C.G. Proud, The alpha-subunit of the mammalian guanine nucleotide-exchange factor elF-2B is essential for catalytic activity in vitro. Biochem Biophys Res Commun, 1996. 220(3): p. 843-7.

138. Bogorad, A.M., et al., Insights into the architecture of the elF2Balpha/beta/delta regulatory subcomplex. Biochemistry, 2014. 53(21): p. 3432-45.

139. Hiyama, T.B., et al., Crystal structure of the alpha subunit of human translation initiation factor 2B. J Mol Biol, 2009. 392(4): p. 937-51.

140. Dev, K., et al., Archaeal alF2B interacts with eukaryotic translation initiation factors elF2alpha and elF2Balpha: Implications for aIF2B function and elF2B regulation. J Mol Biol, 2009. 392(3): p. 701-22.

141. Nakamura, A., et al., Dynamic, ligand-dependent conformational change triggers reaction of ribose1,5-bisphosphate isomerase from Thermococcus kodakarensis KOD1. J Biol Chem, 2012. 287(25): p. 20784-96.

142. Vazquez de Aldana, C.R. and A.G. Hinnebusch, Mutations in the GCD7 subunit of yeast guanine nucleotide exchange factor elF-2B overcome the inhibitory effects of phosphorylated elF-2 on translation initiation. Mol Cell Biol, 1994. 14(5): p. 3208-22.

143. Hannig, E.M., et al., The translational activator GCN3 functions downstream from GCN1 and GCN2 in the regulatory pathway that couples GCN4 expression to amino acid availability in Saccharomyces cerevisiae. Genetics, 1990. 126(3): p. 549-62.

144. Roll-Mecak, A., et al., Engaging the ribosome: universal IFs of translation. Trends Biochem Sci, 2001. 26(12): p. 705-9.

145. Pestova, T.V., et al., The joining of ribosomal subunits in eukaryotes requires elF5B. Nature, 2000. 403(6767): p. 332-5.

146. Acker, M.G., et al., Kinetic analysis of late steps of eukaryotic translation initiation. J Mol Biol, 2009. 385(2): p. 491-506.

147. Antoun, A., et al., The roles of initiation factor 2 and guanosine triphosphate in initiation of protein synthesis. EMBO J, 2003. 22(20): p. 5593-601. 
148. Benne, R., et al., Recycling of initiation factors IF-1, IF-2 and IF-3. Eur J Biochem, 1973. 32(2): p. 37280.

149. Dubnoff, J.S., A.H. Lockwood, and U. Maitra, Studies on the role of guanosine triphosphate in polypeptide chain initiation in Escherichia coli. J Biol Chem, 1972. 247(9): p. 2884-94.

150. Tomsic, J., et al., Late events of translation initiation in bacteria: a kinetic analysis. EMBO J, 2000. 19(9): p. 2127-36.

151. Fabbretti, A., et al., Translation initiation without IF2-dependent GTP hydrolysis. Nucleic Acids Res, 2012. 40(16): p. 7946-55.

152. Allen, G.S., et al., The cryo-EM structure of a translation initiation complex from Escherichia coli. Cell, 2005. 121(5): p. 703-12.

153. Hauryliuk, V., S. Hansson, and M. Ehrenberg, Cofactor dependent conformational switching of GTPases. Biophys J, 2008. 95(4): p. 1704-15.

154. Pavlov, M.Y., et al., Activation of initiation factor 2 by ligands and mutations for rapid docking of ribosomal subunits. EMBO J, 2011. 30(2): p. 289-301.

155. Laurberg, M., et al., Structure of a mutant EF-G reveals domain III and possibly the fusidic acid binding site. J Mol Biol, 2000. 303(4): p. 593-603.

156. Harding, M.M., Metal-ligand geometry relevant to proteins and in proteins: sodium and potassium. Acta Crystallogr D Biol Crystallogr, 2002. 58(Pt 5): p. 872-4.

157. Hilgenfeld, R., J.R. Mesters, and T. Hogg, The Ribosome: Structure, Function, Antibiotics, and Cellular Interactions. ASM Press, 2000.

158. Hendsch, Z.S. and B. Tidor, Do salt bridges stabilize proteins? A continuum electrostatic analysis. Protein Sci, 1994. 3(2): p. 211-26.

159. Elcock, A.H., The stability of salt bridges at high temperatures: implications for hyperthermophilic proteins. J Mol Biol, 1998. 284(2): p. 489-502.

160. Shin, B.S., et al., Intragenic suppressor mutations restore GTPase and translation functions of a eukaryotic initiation factor 5B switch II mutant. Mol Cell Biol, 2007. 27(5): p. 1677-85.

161. Lee, J.H., et al., Initiation factor elF5B catalyzes second GTP-dependent step in eukaryotic translation initiation. Proc Natl Acad Sci U S A, 2002. 99(26): p. 16689-94.

162. Shin, B.S. and T.E. Dever, Molecular genetic structure-function analysis of translation initiation factor elF5B. Methods Enzymol, 2007. 429: p. 185-201.

163. Berchtold, H., et al., Crystal structure of active elongation factor Tu reveals major domain rearrangements. Nature, 1993. 365(6442): p. 126-32.

164. Abrahamson, J.K., et al., Direct determination of the association constant between elongation factor Tu X GTP and aminoacyl-tRNA using fluorescence. Biochemistry, 1985. 24(3): p. 692-700.

165. Louie, A. and F. Jurnak, Kinetic studies of Escherichia coli elongation factor Tu-guanosine 5'triphosphate-aminoacyl-tRNA complexes. Biochemistry, 1985. 24(23): p. 6433-9.

166. Delaria, K., et al., Stabilization of the Escherichia coli elongation factor Tu-GTP-aminoacyl-tRNA complex. Arch Biochem Biophys, 1991. 286(1): p. 207-11.

167. Romero, G., V. Chau, and R.L. Biltonen, Kinetics and thermodynamics of the interaction of elongation factor Tu with elongation factor Ts, guanine nucleotides, and aminoacyl-tRNA. J Biol Chem, 1985. 260(10): p. 6167-74.

168. Voorhees, R.M., et al., The mechanism for activation of GTP hydrolysis on the ribosome. Science, 2010. 330(6005): p. 835-8.

169. Tourigny, D.S., et al., Elongation factor $G$ bound to the ribosome in an intermediate state of translocation. Science, 2013. 340(6140): p. 1235490.

170. Myasnikov, A.G., et al., Conformational transition of initiation factor 2 from the GTP- to GDP-bound state visualized on the ribosome. Nat Struct Mol Biol, 2005. 12(12): p. 1145-9. 
171. Wienk, H., et al., Structural dynamics of bacterial translation initiation factor IF2. J Biol Chem, 2012. 287(14): p. 10922-32.

172. Simonetti, A., et al., Structure of the 30 S translation initiation complex. Nature, 2008. 455(7211): $p$. 416-20.

173. Julian, P., et al., The Cryo-EM structure of a complete 305 translation initiation complex from Escherichia coli. PLoS Biol, 2011. 9(7): p. e1001095.

174. Antoun, A., et al., Ribosome formation from subunits studied by stopped-flow and Rayleigh light scattering. Biol Proced Online, 2004. 6: p. 35-54.

175. Antoun, A., et al., How initiation factors tune the rate of initiation of protein synthesis in bacteria. EMBO J, 2006. 25(11): p. 2539-50.

176. Mitkevich, V.A., et al., Termination of translation in eukaryotes is mediated by the quaternary eRF1*eRF3*GTP*Mg2+ complex. The biological roles of eRF3 and prokaryotic RF3 are profoundly distinct. Nucleic Acids Res, 2006. 34(14): p. 3947-54.

177. Perozzo, R., G. Folkers, and L. Scapozza, Thermodynamics of protein-ligand interactions: history, presence, and future aspects. J Recept Signal Transduct Res, 2004. 24(1-2): p. 1-52.

178. Prabhu, N.V. and K.A. Sharp, Heat capacity in proteins. Annu Rev Phys Chem, 2005. 56: p. 521-48.

179. Jelesarov, I. and H.R. Bosshard, Isothermal titration calorimetry and differential scanning calorimetry as complementary tools to investigate the energetics of biomolecular recognition. $\mathrm{J}$ Mol Recognit, 1999. 12(1): p. 3-18.

180. Spolar, R.S. and M.T. Record, Jr., Coupling of local folding to site-specific binding of proteins to DNA. Science, 1994. 263(5148): p. 777-84.

181. Connelly, P.R. and J.A. Thomson, Heat capacity changes and hydrophobic interactions in the binding of FK506 and rapamycin to the FK506 binding protein. Proc Natl Acad Sci U S A, 1992. 89(11): p. 4781-5.

182. Murphy, K.P. and E. Freire, Thermodynamics of structural stability and cooperative folding behavior in proteins. Adv Protein Chem, 1992. 43: p. 313-61.

183. Paleskava, A., A.L. Konevega, and M.V. Rodnina, Thermodynamics of the GTP-GDP-operated conformational switch of selenocysteine-specific translation factor SelB. J Biol Chem, 2012. 287(33): p. 27906-12.

184. Murphy, K.P., et al., Molecular basis of co-operativity in protein folding. III. Structural identification of cooperative folding units and folding intermediates. J Mol Biol, 1992. 227(1): p. 293-306.

185. Frisch, C., et al., Thermodynamics of the interaction of barnase and barstar: changes in free energy versus changes in enthalpy on mutation. J Mol Biol, 1997. 267(3): p. 696-706.

186. Faergeman, N.J., et al., Thermodynamics of ligand binding to acyl-coenzyme $A$ binding protein studied by titration calorimetry. Biochemistry, 1996. 35(45): p. 14118-26.

187. Holdgate, G.A., et al., The entropic penalty of ordered water accounts for weaker binding of the antibiotic novobiocin to a resistant mutant of DNA gyrase: a thermodynamic and crystallographic study. Biochemistry, 1997. 36(32): p. 9663-73.

188. Schrift, G.L., et al., Molecular basis for nucleotide-binding specificity: role of the exocyclic amino group "N2" in recognition by a guanylyl-ribonuclease. J Mol Biol, 2006. 355(1): p. 72-84.

189. Milon, P. and M.V. Rodnina, Kinetic control of translation initiation in bacteria. Crit Rev Biochem Mol Biol, 2012. 47(4): p. 334-48.

190. Sundari, R.M., et al., Interaction of bacterial initiation factor 2 with initiator tRNA. J Biol Chem, 1976. 251(11): p. 3338-45.

191. Petersen, H.U., et al., Specific interaction of initiation factor IF2 of E. coli with formylmethionyl-tRNA fMet. Biochem Biophys Res Commun, 1979. 91(3): p. 1068-74.

192. Boelens, R. and C.O. Gualerzi, Structure and function of bacterial initiation factors. Curr Protein Pept Sci, 2002. 3(1): p. 107-19. 
193. Antoun, A., et al., How initiation factors maximize the accuracy of tRNA selection in initiation of bacterial protein synthesis. Mol Cell, 2006. 23(2): p. 183-93.

194. Krafft, C., et al., Interaction of fMet-tRNA(fMet) with the C-terminal domain of translational initiation factor IF2 from Bacillus stearothermophilus. FEBS Lett, 2000. 471(2-3): p. 128-32.

195. Spurio, R., et al., The C-terminal subdomain (IF2 C-2) contains the entire fMet-tRNA binding site of initiation factor IF2. J Biol Chem, 2000. 275(4): p. 2447-54.

196. Guenneugues, M., et al., Mapping the fMet-tRNA(f)(Met) binding site of initiation factor IF2. EMBO J, 2000. 19(19): p. 5233-40.

197. Meunier, S., et al., Structure of the fMet-tRNA(fMet)-binding domain of B. stearothermophilus initiation factor IF2. EMBO J, 2000. 19(8): p. 1918-26.

198. Avarsson, A., Structure-based sequence alignment of elongation factors $T u$ and $G$ with related GTPases involved in translation. J Mol Evol, 1995. 41(6): p. 1096-104.

199. Choi, S.K., et al., Promotion of met-tRNAiMet binding to ribosomes by yIF2, a bacterial IF2 homolog in yeast. Science, 1998. 280(5370): p. 1757-60.

200. Pain, V.M., Initiation of protein synthesis in eukaryotic cells. Eur J Biochem, 1996. 236(3): p. 747-71.

201. Kuhle, B. and R. Ficner, elF5B Employs a Novel Domain Release Mechanism to Catalyze Ribosomal Subunit Joining. EMBO J, 2014.

202. Guillon, L., et al., Initiator tRNA binding by e/aIF5B, the eukaryotic/archaeal homologue of bacterial initiation factor IF2. Biochemistry, 2005. 44(47): p. 15594-601.

203. Yamamoto, H., et al., Structure of the mammalian 80 S initiation complex with initiation factor $5 B$ on HCV-IRES RNA. Nat Struct Mol Biol, 2014. 21(8): p. 721-7.

204. Nissen, P., et al., The crystal structure of Cys-tRNACys-EF-Tu-GDPNP reveals general and specific features in the ternary complex and in tRNA. Structure, 1999. 7(2): p. 143-56.

205. Murzin, A.G., OB(oligonucleotide/oligosaccharide binding)-fold: common structural and functional solution for non-homologous sequences. EMBO J, 1993. 12(3): p. 861-7.

206. Theobald, D.L., R.M. Mitton-Fry, and D.S. Wuttke, Nucleic acid recognition by OB-fold proteins. Annu Rev Biophys Biomol Struct, 2003. 32: p. 115-33.

207. Arcus, V., OB-fold domains: a snapshot of the evolution of sequence, structure and function. Curr Opin Struct Biol, 2002. 12(6): p. 794-801.

208. Lupas, A.N., C.P. Ponting, and R.B. Russell, On the evolution of protein folds: are similar motifs in different protein folds the result of convergence, insertion, or relics of an ancient peptide world? J Struct Biol, 2001. 134(2-3): p. 191-203.

209. Huber, C. and G. Wachtershauser, Peptides by activation of amino acids with $\mathrm{CO}$ on (Ni,Fe)S surfaces: implications for the origin of life. Science, 1998. 281(5377): p. 670-2.

210. Wachtershauser, G., Before enzymes and templates: theory of surface metabolism. Microbiol Rev, 1988. 52(4): p. 452-84.

211. Wachtershauser, G., On the chemistry and evolution of the pioneer organism. Chem Biodivers, 2007. 4(4): p. 584-602.

212. Cavarelli, J., et al., The active site of yeast aspartyl-tRNA synthetase: structural and functional aspects of the aminoacylation reaction. EMBO J, 1994. 13(2): p. 327-37.

213. Pettersen, E.F., et al., UCSF Chimera--a visualization system for exploratory research and analysis. J Comput Chem, 2004. 25(13): p. 1605-12.

214. Edgar, R.C., MUSCLE: a multiple sequence alignment method with reduced time and space complexity. BMC Bioinformatics, 2004. 5: p. 113.

215. Holm, L. and C. Sander, Protein structure comparison by alignment of distance matrices. J Mol Biol, 1993. 233(1): p. 123-38.

216. Hansson, S., et al., Crystal structure of a mutant elongation factor $G$ trapped with a GTP analogue. FEBS Lett, 2005. 579(20): p. 4492-7. 
217. Kong, C., et al., Crystal structure and functional analysis of the eukaryotic class II release factor eRF3 from S. pombe. Mol Cell, 2004. 14(2): p. 233-45.

218. Leibundgut, M., et al., Selenocysteine tRNA-specific elongation factor SelB is a structural chimaera of elongation and initiation factors. EMBO J, 2005. 24(1): p. 11-22.

219. Hauryliuk, V., et al., The pretranslocation ribosome is targeted by GTP-bound EF-G in partially activated form. Proc Natl Acad Sci U S A, 2008. 105(41): p. 15678-83.

220. Kuhle, B. and R. Ficner, elF5B employs a novel domain release mechanism to catalyze ribosomal subunit joining. EMBO J, 2014. 33(10): p. 1177-91.

221. Paleskava, A., A.L. Konevega, and M.V. Rodnina, Thermodynamic and kinetic framework of selenocysteyl-tRNASec recognition by elongation factor SelB. J Biol Chem, 2010. 285(5): p. 3014-20.

222. Wilden, B., et al., Role and timing of GTP binding and hydrolysis during EF-G-dependent tRNA translocation on the ribosome. Proc Natl Acad Sci U S A, 2006. 103(37): p. 13670-5.

223. Hauryliuk, V., et al., Class-1 release factor eRF1 promotes GTP binding by class-2 release factor eRF3. Biochimie, 2006. 88(7): p. 747-57.

224. Burnett, B.J., et al., Elongation factor Ts directly facilitates the formation and disassembly of the Escherichia coli elongation factor Tu.GTP.aminoacyl-tRNA ternary complex. J Biol Chem, 2013. 288(19): p. 13917-28.

225. Mesters, J.R., et al., Synergism between the GTPase activities of EF-Tu.GTP and EF-G.GTP on empty ribosomes. Elongation factors as stimulators of the ribosomal oscillation between two conformations. J Mol Biol, 1994. 242(5): p. 644-54.

226. Liljas, A., M. Ehrenberg, and J. Aqvist, Comment on "The mechanism for activation of GTP hydrolysis on the ribosome". Science, 2011. 333(6038): p. 37; author reply 37.

227. Wallin, G., S.C. Kamerlin, and J. Aqvist, Energetics of activation of GTP hydrolysis on the ribosome. Nat Commun, 2013. 4: p. 1733.

228. Aleksandrov, A. and M. Field, Mechanism of activation of elongation factor Tu by ribosome: catalytic histidine activates GTP by protonation. RNA, 2013. 19(9): p. 1218-25.

229. Adamczyk, A.J. and A. Warshel, Converting structural information into an allosteric-energy-based picture for elongation factor Tu activation by the ribosome. Proc Natl Acad Sci U S A, 2011. 108(24): p. 9827-32.

230. Gasper, R., et al., It takes two to tango: regulation of $G$ proteins by dimerization. Nat Rev Mol Cell Biol, 2009. 10(6): p. 423-9.

231. Scrima, A. and A. Wittinghofer, Dimerisation-dependent GTPase reaction of MnmE: how potassium acts as GTPase-activating element. EMBO J, 2006. 25(12): p. 2940-51.

232. Chappie, J.S., et al., $G$ domain dimerization controls dynamin's assembly-stimulated GTPase activity. Nature, 2010. 465(7297): p. 435-40.

233. Rodnina, M.V., Visualizing the protein synthesis machinery: new focus on the translational GTPase elongation factor Tu. Proc Natl Acad Sci U S A, 2009. 106(4): p. 969-70.

234. Kubarenko, A.V., P.V. Sergiev, and M.V. Rodnina, GTPases of translational apparatus. Mol Biol (Mosk), 2005. 39(5): p. 746-61.

235. Mohr, D., W. Wintermeyer, and M.V. Rodnina, GTPase activation of elongation factors Tu and $G$ on the ribosome. Biochemistry, 2002. 41(41): p. 12520-8.

236. Wieden, H.J., W. Wintermeyer, and M.V. Rodnina, A common structural motif in elongation factor Ts and ribosomal protein $\mathrm{L} 7 / 12$ may be involved in the interaction with elongation factor Tu. J Mol Evol, 2001. 52(2): p. 129-36.

237. Bondi, A., van der Waals Volumes and Radii. J. Phys. Chem., 1964. 68(3): p. 441-451.

238. Shannon, R.D., Revised Effective Ionic Radii and Systematic Studies of Interatomie Distances in Halides and Chaleogenides. Acta Crystallogr A, 1976. 32(Pt 5): p. 751-767. 
239. Pisareva, V.P., C.U. Hellen, and T.V. Pestova, Kinetic analysis of the interaction of guanine nucleotides with eukaryotic translation initiation factor elF5B. Biochemistry, 2007. 46(10): p. 2622-9.

240. Gomez, J., et al., The heat capacity of proteins. Proteins, 1995. 22(4): p. 404-12.

241. Conway, T.W., On the Role of Ammonium or Potassium Ion in Amino Acid Polymerization. Proc Natl Acad Sci U S A, 1964. 51: p. 1216-20.

242. Conway, T.W. and F. Lipmann, Characterization of a Ribosome-Linked Guanosine Triphosphatase in Escherichia Coli Extracts. Proc Natl Acad Sci U S A, 1964. 52: p. 1462-9.

243. Parmeggiani, A. and G. Sander, Properties and regulation of the GTPase activities of elongation factors Tu and $G$, and of initiation factor 2. Mol Cell Biochem, 1981. 35(3): p. 129-58.

244. Fasano, O., E. De Vendittis, and A. Parmeggiani, Hydrolysis of GTP by elongation factor Tu can be induced by monovalent cations in the absence of other effectors. J Biol Chem, 1982. 257(6): p. 314550.

245. Ash, M.R., et al., The initiation of GTP hydrolysis by the G-domain of FeoB: insights from a transitionstate complex structure. PLoS One, 2011. 6(8): p. e23355.

246. Chappie, J.S., et al., A pseudoatomic model of the dynamin polymer identifies a hydrolysisdependent powerstroke. Cell, 2011. 147(1): p. 209-22.

247. Kobayashi, K., et al., Structural basis for mRNA surveillance by archaeal Pelota and GTP-bound EF1alpha complex. Proc Natl Acad Sci U S A, 2010. 107(41): p. 17575-9.

248. Kobayashi, K., et al., Structural basis for translation termination by archaeal RF1 and GTP-bound EF1alpha complex. Nucleic Acids Res, 2012. 40(18): p. 9319-28.

249. Meyer, S., et al., Kissing $G$ domains of MnmE monitored by X-ray crystallography and pulse electron paramagnetic resonance spectroscopy. PLoS Biol, 2009. 7(10): p. e1000212.

250. Foucher, A.E., et al., Potassium acts as a GTPase-activating element on each nucleotide-binding domain of the essential Bacillus subtilis EngA. PLoS One, 2012. 7(10): p. e46795.

251. Nagel, K. and J. Voigt, Regulation of the uncoupled GTPase activity of elongation factor $G$ (EF-G) by the conformations of the ribosomal subunits. Biochim Biophys Acta, 1993. 1174(2): p. 153-61.

252. Praefcke, G.J. and H.T. McMahon, The dynamin superfamily: universal membrane tubulation and fission molecules? Nat Rev Mol Cell Biol, 2004. 5(2): p. 133-47.

253. Warnock, D.E., J.E. Hinshaw, and S.L. Schmid, Dynamin self-assembly stimulates its GTPase activity. J Biol Chem, 1996. 271(37): p. 22310-4.

254. Das, S. and U. Maitra, Functional significance and mechanism of elF5-promoted GTP hydrolysis in eukaryotic translation initiation. Prog Nucleic Acid Res Mol Biol, 2001. 70: p. 207-31.

255. Ash, M.R., et al., The cation-dependent G-proteins: in a class of their own. FEBS Lett, 2012. 586(16): p. 2218-24.

256. Perla-Kajan, J., et al., Properties of Escherichia coli EF-Tu mutants designed for fluorescence resonance energy transfer from tRNA molecules. Protein Eng Des Sel, 2009. 23(3): p. 129-36.

257. Harding, M.M., Geometry of metal-ligand interactions in proteins. Acta Crystallogr D Biol Crystallogr, 2001. 57(Pt 3): p. 401-11.

258. Ghosh, A., et al., How guanylate-binding proteins achieve assembly-stimulated processive cleavage of GTP to GMP. Nature, 2006. 440(7080): p. 101-4. 


\section{Acknowledgements}

This doctoral thesis was written during my time at the Department for Molecular Structural Biology of the Georg-August University Göttingen. First and foremost, I am grateful to Prof. Dr. Ralf Ficner for providing me with excellent working conditions and for his continous and unreserved support throughout my time in his department. I would like to thank Prof. Dr. Marina Rodnina for the ecceptance to be second reviewer of my thesis and for her comments on my work during the thesis committee meetings. Achim Dickmanns I thank for his support and discussions on countless occasions and his comments on various chapters presented in this thesis. Particularly indepted I am to Piotr Neumann for invaluable help during X-ray data collection and structure determination, as well as to Oliver Valerius for his contribution to the analysis of the crosslinking data. Finally, I thank Michael Franke for his excellent technical assistance. All former and current members of the research group for Applied Synthetic Biology and the MSB department, in particular my lab collegue Yi Liu, made the considerable amount of time I spent in lab or office worthwile.

My parents provided me with indispensible advice and support. Moreover, I express my deep gratitude to Lili Dörfel, who contributed significantly to this thesis with her patience, her open ear regarding every detail of my work and for her critical comments on the many previous drafts of this manuscript. 


\section{Curriculum vitae}

\section{Persönliche Daten}

Name: Bernhard Kuhle

Geburtsdatum: 06.12.1984

Geburtsort: Göttingen

Nationalität: deutsch

E-Mail: bkuhle@gwdg.de

\section{Ausbildung}

\section{Schulbidung}

1992-1996

1996-1998

2000-2001

1998-2004

2004

Studium

2004-2005

2005-2010

2010

\section{Promotion}

seit $07 / 2010$
Grundschule Waake

Orientierungsstufe Weende-Nord, Göttingen

Duluth Central High School, Minnesota, USA

Theodor-Heuss Gymnasium, Göttingen

Allgemeine Hochschulreife (Abitur)
Physikstudium (Freie Universität Berlin)

Biologiestudium (Georg-August-Universität Göttingen)

Diplom (Georg-August-Universität Göttingen)

Promotion (Georg-August-Universität Göttingen) 